\title{
Philosophers of the World Unite! Theorising Digital Labour and Virtual
}

Work - Definitions, Dimensions and Forms

Edited by Marisol Sandoval, Christian Fuchs, Jernej A. Prodnik, Sebastian Sevignani, Thomas Allmer

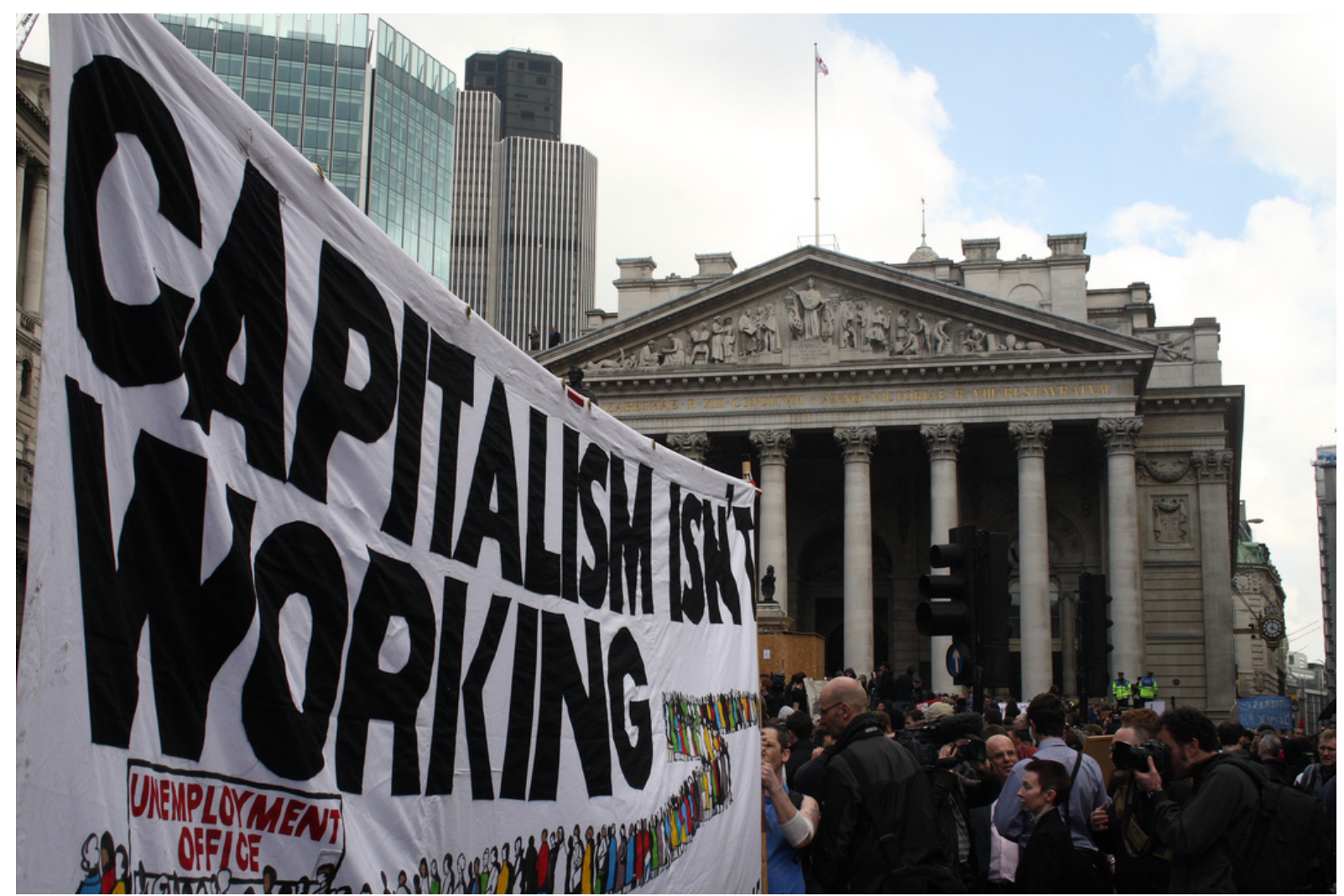

\section{triple communication, triple capitalism \& critique}

Volume 12 (2014), Number 2: page 464-801

Cover image:

By Jonny White (G20 April 1st) [CC-BY-2.0

(http://creativecommons.org/licenses/by/2.0)], via Wikimedia Commons 



\section{Table of Contents}

Introduction: Philosophers of the World Unite! Theorising Digital Labour and Virtual Work-Definitions, Dimensions, and Forms

Marisol Sandoval, Christian Fuchs, Jernej A. Prodnik, Sebastian Sevignani, Thomas Allmer, 464-467

Work and Labour as Metonymy and Metaphor

Olivier Frayssé, 468-485

Digital Workers of the World Unite! A Framework for Critically Theorising and Analysing Digital Labour

Christian Fuchs, Marisol Sandoval, 486-563

Circuits of Labour: A Labour Theory of the iPhone Era

Jack Linchuan Qiu, Melissa Gregg, Kate Crawford, 564-581

Concepts of Digital Labour: Schelling's Naturphilosophie

Kevin Michael Mitchell, 582-598

Digital Labour and the Use-value of Human Work. On the Importance of Labouring Capacity for understanding Digital Capitalism

Sabine Pfeiffer, 599-619

The Ideological Reproduction: (Free) Labouring and (Social) Working within Digital Landscapes

Marco Briziarelli, 620-631

Alienation and Digital Labour-A Depth-Hermeneutic Inquiry into Online Commodification and the Unconscious

Steffen Krüger, Jacob Johanssen, 632-647

Production Cultures and Differentiations of Digital Labour

Yujie Chen, 648-667

Digital Labour in Chinese Internet Industries

Bingqing Xia, 668-693

Will Work For Free: The Biopolitics of Unwaged Digital Labour Brian Brown, 694-712 
Toward a Political Economy of 'Audience Labour' in the Digital Era

Brice Nixon, 713-734

Playing, Gaming, Working and Labouring: Framing the Concepts and Relations

Arwid Lund, 735-801

\section{Acknowledgement:}

This special issue has been published in the context of the EU COST Action IS1202 Dynamics of Virtual Work, http://dynamicsofvirtualwork.com/ 


\title{
Introduction: Philosophers of the World Unite! Theorising Digital Labour and Virtual Work-Definitions, Dimensions, and Forms
}

\author{
Marisol Sandoval", Christian Fuchs ${ }^{\dagger}$, Jernej A. Prodnik ${ }^{\ddagger}$, Sebastian \\ Sevignani ${ }^{\S}$, Thomas Allmer ${ }^{\pi}$
}

"City University London, UK, marisol.sandoval.1@city.ac.uk

${ }^{\dagger}$ University of Westminster, UK, c.fuchs@westminster.ac.uk

${ }^{\ddagger}$ Charles University in Prague, Czech Republic; Faculty of Social Sciences, Ljubljana, Slovenia, jernej.amon-prodnik@fdv.uni-lj.si

$\S$ University of Jena, Germany, sebastian.sevignani@uni-jena.de

"UTI Research Group, Austria, thomas.allmer@uti.at

In 1845, Karl Marx $(1845,571)$ formulated the 11th Feuerbach Thesis: "The philosophers have only interpreted the world in various ways; the point is to change it." Today, interpreting the world has become an important form of labour that is expressed on and with the help of digital media. In this context it has become common to talk about digital labour and virtual work. Yet the changes that digital, social, and mobile media bring about in the world of labour and work have thus far only been little theoretically interpreted. In order to change the information society for the better, we first have to interpret digital labour with the help of critical theories. Social theorists of the world from different fields, backgrounds, interdisciplines, transdisciplines, and disciplines have to unite for this collective philosophical task.

This special issue of tripleC: Communication, Capitalism \& Critique aims to contribute to building a theoretical framework for the critical analysis of digital labour, virtual work, and related concepts that can initiate further debates, inform empirical studies, and inspire social struggles connected to work and labour in and beyond digital capitalism. The papers collected in this special issue (a) provide systematic definitions of digital labour, (b) analyse its specific dimension, and (c) discuss different forms of digital labour.

\section{(a) Definitions of Digital Labour}

The first group of papers focuses on conceptualising and defining the concept of digital labour. The contributions included in this section examine the relation between work and labour, discuss how digital labour should be defined and highlight implications of different definitions of digital labour. Olivier Frayssé's paper Work and Labour as Metonymy and Metaphor, which opens the special issue, offers an etymological contextualization of the digital labour debate. Based on literary analysis and linguistics Frayssé traces the roots and the meanings of the concepts of work and labour in different languages. The following three papers move on to defining digital labour in particular. Based on Raymond Williams' approach to cultural materialism Christian Fuchs and Marisol Sandoval in Digital Workers of the World Unite! A Framework for Critically Theorising and Analysing Digital Labour argue for a broad definition of digital labour that takes into account the various forms of mental and manual labour that are needed for the production, circulation and use of digital media. Jack Linchuan Qiu, Melissa Gregg and Kate Crawford in their paper Circuits of Labour: A Labour Theory of 
the iPhone Era also advocate an inclusive understanding of digital labour. They suggest a "circuit of labour" model as a holistic framework for studying labour and ICTs and apply it to the case of Foxconn. Kevin Michael Mitchell's contribution, Concepts of Digital Labour: Schelling's Naturphilosophie, takes a philosophical perspective on defining digital labour based on Schelling's Naturphilosophie and argues for a materialist perspective on the digital.

\section{(b) Dimensions of Digital Labour}

By looking at some of the specific dimensions of digital labour such as exploitation, use value and exchange value, commodification, ideology, and subjectivity the papers included in the second section of this special issue further deepen the engagement with digital labour. Sabine Pfeiffer in Digital Labour and the Use-value of Human Work. On the Importance of Labouring Capacity for Understanding Digital Capitalism focuses on Marx's concept of labouring capacity (Arbeitsvermögen) as opposed to labour power and highlights its implications for analysing digital labour. Marco Briziarelli's article The Ideological Reproduction: (Free) Labouring and (Social) Working within Digital Landscapes examines (neo-)liberal ideology as an important dimension in reproducing digital labour, using Facebook as an example. Steffen Krüger's and Jacob Johanssen's contribution Alienation and Digital Labour-a DepthHermeneutic Inquiry into Online Commodification and the Unconscious shifts the focus towards the subjective dimension of the digital labour debate. They take a psychoanalytic perspective to interpret user posts on Facebook's Site Governance Page, and add to ongoing discussions of alienation on social media. Finally, Yujie Chen in her contribution Production Cultures and Differentiations of Digital Labour reviews various dimensions of digital labour including exploitation, surveillance, productive versus unproductive labour, commodification, and ideology.

\section{(c) Forms of Digital Labour}

The papers included in the third part of this special issue explore the breath of the field by examining a variety of different forms of digital labour including the labour of professional workers in Internet industries, unwaged labour, audience labour, and playbour. In Digital Labour in the New Media Sweatshop Bingqing Xia presents an analysis of the working conditions of professional workers in Chinese Internet industries. Another form of digital labourunwaged labour-is the focus of Brian Brown in contribution 'Will Work For Free': The Biopolitics of Unwaged Digital Labour. He proposes a theoretically nuanced definition of unwaged digital labour that captures main characteristics of unpaid labour in digital capitalism. Brice Nixon in Toward a Political Economy of 'Audience Labour' in the Digital Era addresses a specific form of unwaged labour-the labour of audiences. Drawing on the work of Karl Marx, David Harvey, and Raymond Williams, Nixon discusses the political economy of the audience labour process. Finally, Arwid Lund examines the relation between labour and play in his contribution Playing, Gaming, Working, and Labouring: Framing the Concepts and Relations. Lund contributes to an understanding and critique of playbour by constructing a typology of the concepts of playing, working, gaming, and labouring.

The papers collected in this special issue theorise digital labour as a multifaceted field characterised by exploitation, alienation, precariousness, power, inequality, ideology, and struggle. These problems of digital labour are however not inherent to digital technology as such but result from its inclusion and application in capitalist relations of production.

We can learn from Marx's discussion of the dialectics of machinery for understanding the contradictory potentials of digital technologies today. Marx regarded machinery as a powerful instrument to reduce the working day while highlighting that under capitalism it operates in the opposite way as a means for its infinite extension. He stressed: under capitalism machinery, "the most powerful instrument for reducing labour-time suffers a dialectical inversion and becomes the most unfailing means for turning the whole lifetime of the worker and his family into labour time at capital's disposal for its own valorization" (Marx 1976/1867, 532). 
Taking a Marxian perspective helps to understand technology in a dialectical way: it can be employed to increase the domination and exploitation of workers but at the same time has the potential to alleviate work and reduce socially necessary labour time. Today, almost 150 years after Marx formulated his thoughts on the impact of machinery on labour, digital technologies still confront us with similar contradictions. In many ways they have made our (working) lives easier: they enable fast communication; allow connecting with people around the world; facilitate the storing and reproduction of content and data; provide access to a huge amount of information, etc. At the same time, digital technologies serve as an instrument for the exploitation, surveillance, and control of workers not only within but also way beyond factory and office walls.

Herbert Marcuse highlighted that realising technology's potential to reduce human toil requires radical social change: "If the completion of the technological project involves a break with the prevailing technological rationality, the break in turn depends on the continued existence of the technical base itself. For it is this base which has rendered possible the satisfaction of needs and the reduction of toil-it remains the very base of all forms of human freedom." (Marcuse 1964, 236). As Marcuse argues, the full realization of human freedom depends on technology—but technology without technological rationality, which characterizes capitalist society.

Theorising digital labour, as labour that produces or makes use of digital technologies, can help to understand its problems, limits, potentials, and contradictions. It can therefore highlight the need for social change and inspire political action. However, the act of freeing digital technology from being an instrument for the domination of labour requires to go beyond just interpreting the world and to engage in social struggles that want to change it.

\section{References}

Marcuse, Herbert. 1964. One-Dimensional Man. Studies in the Ideology of Advanced Industrial Society. Boston: Beacon Press

Marx, Karl. 1845/1975. Theses on Feuerbach. In Karl Marx. Early Writings, 421-423. London: Penguin.

Marx, Karl. 1967/1976. Capital, Volume I. London: Penguin.

\section{About the Authors}

\section{Marisol Sandoval}

is a Lecturer at the Department of Culture and Creative Industries at City University London. She is author of From Corporate to Social Media. Critical Perspectives on Corporate Social Responsibility in Media and Communication Industries (Routledge 2014) and editor of the open access journal tripleC Communication, Capitalism and Critique. Currently Marisol is working on a study of potentials and contradictions of worker co-operatives in the cultural sector.

\section{Christian Fuchs}

is professor of social media at the University of Westminster's Communication and Media Research Institute and the Centre for Social Media Research. He is editor of tripleC: Communication, Capitalism \& Critique and author of many publications, including the books Social Media: A Critical Introduction (Sage 2014), Digital Labour and Karl Marx (Routledge 2014), OccupyMedia! The Occupy Movement and Social Media in Crisis Capitalism (Zero Books 2014), Foundations of Critical Media and Information Studies (Routledge 2011), Internet and Society: Social Theory in the Information Age (Routledge 2008). He recently edited together with Marisol Sandoval the collected volume Critique, Social Media and the Information Society (Routledge 2014).

\section{Jernej A. Prodnik}

is a post-doctoral researcher at the Institute of Communication Studies and Journalism (the PolCoRe research group) at the Faculty of Social Sciences, Charles University in Prague (Czech Republic), and a researcher at the Social Communication Research Centre, Faculty of Social Sciences, University of Ljubljana (Slovenia). He defended his PhD in media and communication studies at the University of Ljubljana in 2013 under the title "Political Economy of Communication and Structural Transformations of Capitalism". His principal research interests include critique of political economy (with a focus on 
media and communication) and the wider social context of technological changes and democratic potentials brought about by new technologies. He is a member of the editorial board of Casopis za kritiko znanosti [Journal for the Critique of Science] and international journal for critical studies of media, information and power in capitalist societies tripleC: Communication, Capitalism \& Critique.

\section{Sebastian Sevignani}

is member of the Unified Theory of Information Research Group (UTI) and postdoctoral researcher at the University of Jena's institute of sociology. His dissertation deals with economic and ideological aspects of commodification of privacy in the Internet age (forthcoming with Routledge). Currently, he works on a re-actualisation of the concept of false needs. Further information: http://sevignani.uti.at and http://www.soziologie.uni-jena.de/en/.

\section{Thomas Allmer}

studied media and communication at the University of Salzburg, Austria, and Victoria University, Melbourne, Australia, and completed his $\mathrm{PhD}$ in 2013. He currently is a postdoctoral fellow at the Unified Theory of Information Research Group, Austria. His publications include Towards a Critical Theory of Surveillance in Informational Capitalism (Peter Lang, 2012) and Critical Theory and Social Media: Between Emancipation and Commodification (Routledge, forthcoming). Further information: http://allmer.uti.at. 


\title{
Work and Labour as Metonymy and Metaphor
}

\author{
Olivier Frayssé
}

\author{
Université Paris-Sorbonne, HDEA-TCS, Paris, France, Fraysseo@aol.com, http://www.paris- \\ sorbonne.fr/presentation-5414
}

\begin{abstract}
This paper proposes to use the tools of literary analysis (the reference to subtexts) and of linguistics (metaphor and metonymy) to shed light on the work/labour controversy and, beyond that, to map the galaxy of representations of work/labour through a study of the meanings associated with work/labour in several languages. It aims to provide a set of theoretical tools that can be used to find a common language in order to discuss digital work/labour issues as a subcategory of work/labour issues in general.
\end{abstract}

Keywords: work, labour, metonymy, metaphor, subtext, cognitive linguistics.

Acknowledgement: In addition to the dictionaries mentioned in the bibliography, I have relied on the help of several friends and colleagues who were more familiar than me with the languages I have tried to approach, and to recognized experts in the field of linguistics. In particular, I would like to express my warm thanks to my colleagues Professors Leo Carruthers (Old English), Pierre Cotte (Linguistics), Frédéric Lagrange (Arabic), Laure Troubetzkoy and Stéphane Viellard (Russian) Dr. Eran Fischer (Hebrew), to my doctoral student Medina Niang (Woloff), to my friends Shuai Zhang (Chinese) and Daniel Koechlin (Sanskrit and Gaelic), and to my son David (Lingala and Swahili). Special thanks to my friend and colleague Dr. Barbara Lelan, who, among her many other insights, especially regarding sign languages, has led me along the path to metonymy as fractal compression, and Ursula Huws, who directed me to relevant sources for the vocabulary of digital work and labour, and the reviewers of this paper, who forced me to answer very interesting questions.

\section{Introduction}

There is an ongoing discussion on the difference between work and labour. In the Marxist tradition, the word labour has been associated with alienated and exploited work, a historical category, as opposed to work, an anthropological category, quintessential to the human species (Fuchs and Sevignani 2013). This paper is a modest contribution to the debate, from the angle of linguistics in the broad sense of the word.

Work/labour is an elusive notion in many ways. I am using the phrase work/labour as a provisional notion in order not to take sides at the beginning of the investigation, and also because, in several languages, such as French (travail) or German (Arbeit), there is but one word, so that the speaker needs to use a modifier to convey the precise meaning he/she gives to the word (abstract/concrete, living/dead, etc.) within his/her theoretical framework. When translated into English, the word becomes either work or labour, depending on both the translator's understanding of the original meaning within the original theory, and his or her own theoretical view that has associated either work or labour, possibly with modifiers, with one concept or another. There is plenty of room both for misunderstandings and competing translations. Arbeitsproze $\beta$ is thus translated either by labour process or work process depending on the theoretical views of the translator, and sometimes he/she uses the two indifferently.

There is another difficulty. Whenever one tries to define the notion either some aspects of work/labour are excluded or the definition stretches too far to be useful. Try Marx's often quoted definition of the "labour process" or "work process" (Arbeitsprozeß), depending on the translation and the context as "human action with a view to the production of use-values, appropriation of natural substances to human requirements", "zweckmäßige Tätigkeit zur Herstellung von Gebrauchswerten, Aneignung des Natürlichen für menschliche Bedürfnisse", (Marx 1990, 290, Marx and Engels 1968ff, 24 and 198). It can be stretched to such an extent that most bodily functions such as eating could be included if we consider only tangible use- 
values, while kicking stones along the path, scratching one's beard and every form of play could be added if we included intangible use-values.

A third difficulty comes from the connotations of the words work and labour, which vary depending on the texts, the contexts, and the subtexts. In particular, the words work and labour will be referred to more or less explicitly when using other words, either as particular aspects of work/labour, or by opposition. Talk of drudge, chore, toil, on the one hand, or play, rest, idleness on the other, and a particular aspect of work/labour comes to mind. The words work and labour belong to a galaxy of representations that comprises many words, and a complete vision of the galaxy is required to help us choose the right definition/translation in each instance. The organization of this galaxy enables one to identify the sometimes hidden and unconscious dimensions of the work/labour concept.

This paper proposes to use the tools of literary analysis (the reference to subtexts) and of linguistics (metaphor and metonymy) to shed light on the work/labour controversy and, beyond that, to map the galaxy of representations of work/labour, through a study of the meanings associated with work/labour in several languages, both Indo-European and non-IndoEuropean. The list of languages studied here is reduced, and both my own limited knowledge of the languages and the lack of space to explore further the relevant lexicon in each language restrict the validity of the research findings. What is hoped nevertheless is that the tentative classification that results, which points to anthropological constants and historical variables, can serve as a starting point for a more extensive (in terms of the number of languages covered) and comprehensive (in terms of the number of metonymies used for classification) mapping of this galaxy of representations. But to shed light on the work/labour controversy between Marxists and non-Marxists, and different interpretations of Marx, I would like to start with the subtext of Engels' footnote, Adam Smith's Wealth of Nations.

\section{The importance of Subtexts in the Work/Labour Controversy}

A footnote of Friedrich Engels to the fourth German edition of Marx's Capital claimed that "the English language has the advantage of possessing two separate words for these two different aspects of labour" (Marx 1990, 138n16), so that the former could be used for all productive activities, regardless of their social context, while the latter was associated with what makes these productive activities useful for capital, that is the generation of surplus value.

As a matter of fact, the distinction between work and labour is not germane to English as a lexical distinction, it is "a split that few people can make sense of in the English-speaking world" (Kley 2008, 12). While there is no commonly accepted lexical distinction between the words work and labour, as words, in general, in the English language, there are distinctions arising from the contexts. One cannot substitute "work" for "labour" when speaking of the Labour unions, nor can one say he or she is looking for "labour" when in quest of employment.

There are also distinctions arising from subtexts. A subtext is a text to which another text refers, usually implicitly. Just like contexts, subtexts are often indispensible for us to ascertain the precise meaning of a word for which there exists a variety of lexical definitions. For instance, a sizeable part of the New Testament has the Old Testament as a subtext, and no scientific exegesis can ignore this subtext. The importance of subtexts is particularly relevant to the work/labour controversy between various schools of Marxists and non-Marxists. Since the subtexts are the theoretical works in the English language studied by Marx and Engels, the distinction is a conceptual one, which only some people, interested in theory, will make. The relative plasticity of language enables one to assign precise definitions to words according to one's theoretical preoccupations. For instance, Free-Masons would assign special meanings (as distinct from lexical differences accepted by every speaker of the language) to the words labour, work, and business, according to their own vision of the world, and the place of their philosophy, that glorifies work/labour in all its shapes: "It is one of the most beautiful features of the Masonic Institution, that it teaches not only the necessity, but the nobility of labor. From the time of opening to that of closing, a Lodge is said to be at labor. 
[...] When the lodge is engaged in reading petitions, hearing reports, debating financial matters, etc., it is said to be occupied in business; but when it is engaged in the form and ceremony of initiation into any of the degrees, it is said to be at work" (Mackey 1914, 419).

Is then the Marxist distinction between work and labour, which does not rest on a lexical distinction, a purely arbitrary one, born of Marxian theory and forced into a lexical distinction for adherents of the theory, who then would have to go to great lengths to try to justify the distinction as a lexical one, using etymology for instance? Was Engels ignorant of the absence of any commonly accepted lexical difference, except in particular contexts? Or was there, within the context of the particular lexical field of nascent political economy, a subtext that legitimated the distinction? As a matter of fact, there was, as we can see when we analyze one of the principal subtexts of Marx's work, and Engels's footnote, i.e. the use of the word labour by Adam Smith, with whose work Marx and Engels were very familiar (there are 72 references to Smith in the 1844 manuscripts, versus only 19 for Jean-Baptiste Say for example).

In Wealth of Nations (1999), Smith uses the word work to mean several different things: in Book I, chapter I, "Of the Division of Labour", it means alternatively the productive tasks to be done in a manufacture, ${ }^{2}$ the nature of the tasks performed by individual workers, ${ }^{3}$ the amount produced, ${ }^{4}$ the labour power or capacity of individuals, ${ }^{5}$ the type of work or employment ${ }^{6}$, the product of work, qualitatively and quantitatively. ${ }^{7}$ In a sentence like: "But in consequence of

1 The lexical ambiguity of the words labour and work resists the attempt of the writer to come up with precise conceptual definitions, as one can see under the entry "Business": "Everything that is done in a Masonic Lodge, relating to the initiation of candidates into the several degrees, is called its work or labor; all transactions such as are common to other associations and societies come under the head of business, and they are governed with some peculiar differences by rules of order, as in other societies." (Mackey 1914, 125).

2 "In those great manufactures, on the contrary, which are destined to supply the great wants of the great body of the people, every different branch of the work employs so great a number of workmen that it is impossible to collect them all into the same workhouse" (Smith 1999, 109). In chapter VI, "Of the Component Parts of the Price of Commodities", work, in the plural, is used to mean manufacture, and labour to mean work as a task: "In many great works almost the whole labour of this kind is committed to some principal clerk" (Smith 1999, 152).

3 "What is the work of one man in a rude state of society being generally that of several in an improved one" (Smith 1999, 111). "Secondly, the advantage which is gained by saving the time commonly lost in passing from one sort of work to another is much greater than we should at first view be apt to imagine it" (Smith 1999, 113). "Whoever has been much accustomed to visit such manufactures must frequently have been shown very pretty machines, which were the inventions of such workmen in order to facilitate and quicken their particular part of the work" (Smith, 1999, 114). "In such situations we can scarce expect to find even a smith, a carpenter, or a mason, within less than twenty miles of another of the same trade. The scattered families that live at eight or ten miles distance from the nearest of them must learn to perform themselves a great number of little pieces of work, for which, in more populous countries, they would call in the assistance of those workmen" (Smith 1999, 122). Work in this case is the same as trade or occupation, as in Chapter VIII, "Of the wages of labour", where Smith writes: "In all arts and manufactures the greater part of the workmen stand in need of a master to advance them the materials of their work, and their wages and maintenance till it be completed" (Smith 1999, 168).

4 "This great increase of the quantity of work which, in consequence of the division of labour, the same number of people are capable of performing, is owing to three different circumstances" (Smith 1999, 112). "Each individual becomes more expert in his own peculiar branch, more work is done upon the whole, and the quantity of science is considerably increased by it" (Smith 1999, 115).

5 "Every workman has a great quantity of his own work to dispose of beyond what he himself has occasion for; and every other workman being exactly in the same situation, he is enabled to exchange a great quantity of his own goods for a great quantity, or, what comes to the same thing, for the price of a great quantity of theirs" (Smith 1999, 115).

6 "But without the disposition to truck, barter, and exchange, every man must have procured to himself every necessary and conveniency of life which he wanted. All must have had the same duties to perform, and the same work to do, and there could have been no such difference of employment as could alone give occasion to any great difference of talents" (Smith 1999, 120).

"A country carpenter deals in every sort of work that is made of wood: a country smith in every sort of work that is made of iron" (Smith 1999, 122). "It is impossible there should be such a trade as even that of a nailer in the remote and inland parts of the Highlands of Scotland. Such a workman at the rate of a thousand nails a day, and three hundred working days in the year, will make three hundred thousand nails in the year. But in such a situation it would be impossible to dispose of one thousand, that is, of one day's work in the year" (Smith 1999, 122). This meaning of work as the result of productive activity also appears in chapter VI, Of the Component Parts of the Price of Commodities: "As soon as stock has accumulated in the hands of particular persons, some of them 
the division of labour, the whole of every man's attention comes naturally to be directed towards some one very simple object. It is naturally to be expected, therefore, that some one or other of those who are employed in each particular branch of labour should soon find out easier and readier methods of performing their own particular work, wherever the nature of it admits of such improvement" (Smith 1999, 114), labour and work are almost synonymous, since division of labour consists in parcelling work.

In chapter VII, "Of the Natural and Market Price of Commodities", labour is defined as "work to be done", whereas commodities are "work done"8. In chapter VIII, "Of the Wages of Labour", work means production, and labour means the use of labour power: "Let us suppose, for example, that in the greater part of employments the productive powers of labour had been improved to tenfold, or that a day's labour could produce ten times the quantity of work which it had done originally" (Smith, 1999, 167). But in the same chapter, the wages of "labour" are equated with the price of "work": "The former [wage workers] are disposed to combine in order to raise, the latter [employers] in order to lower the wages of labour. [...] We have no acts of parliament against combining to lower the price of work; but many against combining to raise it" (Smith 1999, 169). And, still in the same chapter, work means waged employment: "A man must always live by his work, and his wages must at least be sufficient to maintain him" (Smith 1999, 170).

The distinction between work and labour becomes sharp when Smith's focus is on labour as the measure of (exchange) value, as in chapter V, "Of the Real and Nominal Price of Commodities, or their Price in Labour, and their Price in Money":

The real price of everything, what everything really costs to the man who wants to acquire it, is the toil and trouble of acquiring it. What everything is really worth to the man who has acquired it, and who wants to dispose of it or exchange it for something else, is the toil and trouble which it can save to himself, and which it can impose upon other people. What is bought with money or with goods is purchased by labour as much as what we acquire by the toil of our own body. That money or those goods indeed save us this toil. They contain the value of a certain quantity of labour which we exchange for what is supposed at the time to contain the value of an equal quantity. Labour was the first price, the original purchase-money that was paid for all things. It was not by gold or by silver, but by labour, that all the wealth of the world was originally purchased; and its value, to those who possess it, and who want to exchange it for some new productions, is precisely equal to the quantity of labour which it can enable them to purchase or command. It is difficult to ascertain the proportion between two different quantities of labour. The time spent in two different sorts of work will not always alone determine this proportion. The different degrees of hardship endured, and of ingenuity exercised, must likewise be taken into account. There may be more labour in an hour's hard work than in two hours' easy business; or in an hour's application to a trade which it cost ten years' labour to learn, than in a month's industry at an ordinary and obvious employment. But it is not easy to find any accurate measure either of hardship or ingenuity. In exchanging, indeed, the different productions of different sorts of labour for one another, some allowance is commonly made for both. It is adjusted, however, not by any accurate measure, but by the haggling and bargaining of the market, according to that sort of rough equality which, though not exact, is sufficient for carrying on the business of common life" (Smith 1999, 134).

What we can see here is that, while the lexical fuzziness persists (mark the presence, in this paragraph, of synonyms like "business", "employment", "industry"), a conceptual distinction is

will naturally employ it in setting to work industrious people, whom they will supply with materials and subsistence, in order to make a profit by the sale of their work, or by what their labour adds to the value of the materials" (Smith 1999, 151).

8 "Such fluctuations affect both the value and the rate either of wages or of profit, according as the market happens to be either overstocked or understocked with commodities or with labour; with work done, or with work to be done" (Smith 1999, 162).

${ }^{9}$ This could be related to the practice of paying craftsmen, as opposed to journeymen, by the piece and not by the day. 
made between (what Marx would call concrete) work producing real things as use values and involving a concrete experience ("hardship") and (what Marx would call abstract) labour generating exchange values, which can be measured ("proportion of quantities"), thanks to the "higgling and bargaining of the market". Here work and labour cannot be used interchangeably. Once labour has thus been opposed to work, Smith is able define capital as "a certain quantity of labour stocked and stored up to be employed", a definition taken up by Marx in the 1844 manuscript. After Smith, English political economists such as Malthus (1814) and Ricardo (1821) used exclusively the word labour in relation to exchange value, whether they disagreed (Malthus) or agreed (Ricardo) with Smith. It is this conceptual distinction that Engels seemed to apprehend as a lexical distinction in English. By forcing these exclusive conceptual meanings into the lexically polysemic English words labour and work for the purpose of clarity, he himself created a subtext for Marxists that separated them from non-Marxists, unwillingly creating an obstacle to discussion. Attempts at distinguishing between labour and work by looking at etymology, identifying labour with something painful and linked with both exploitation and alienation while work would be related to man's quintessence and related to freedom and happy self-expression, cannot adequately account for the original conceptual distinction that we find to be born with Adam Smith: in the above mentioned conceptually decisive passage, it is "work" that is linked with hardship and pain, not "labour". Hardship and pain can only exist in the actual experience of working. While exploitation (extracting surplus from actual work to maximize and appropriate the resulting labour value) does increase the level of hardship involved, while alienation does simultaneously increase (by dehumanizing) and lessen (through ideology) the feeling of hardship, there is an irreducible aspect of pain involved in actual work, which is also associated with a feeling of pleasure, but that is another story. When labour is "purchased" and "commanded", then the "toil and trouble" of work is shifted from employer to employee.

Thus, the study of Marx's subtext helps us clarify the work/labour controversy, by ascertaining its character, i.e. a conceptual distinction between two aspects of work/labour. In the course of that enquiry, we have seen the variety of words used to refer to work/labour in the English language, the richness of their connotations in various contexts, and the polysemic character of the word work itself. Both the number of work/labour related words and the very polysemic of the word work itself invite us to pursue the enquiry in the direction of linguistics. What can we learn from a study of the various words that are used to express work/labour, in English but also in other languages? What does this polysemy tell us about the important dimensions of work/labour for human beings, an importance that is reflected in their nomination of work/labour? To answer these questions, we must turn to cognitive linguistics.

\section{The Contribution of Cognitive Linguistics}

The founding hypothesis of cognitive linguistics, born with Ronald Wayne Langacker (1973), is that "our ordinary conceptual system, in terms of which we both think and act, is fundamentally metaphorical in nature" (Lakoff and Johnson 1980, 3] ${ }^{10}$. In a metaphor, the relationships between elements of a source domain are viewed as similar to those prevailing in the target domain, which enables the speaker to use words from the source domain to describe relationships in the target domain. Lakoff and Johnson's classical example is the metaphor "love is a journey", where "journey" is the source domain and "love" is the "target domain". There are several characteristics of a journey that are similar to those of an amorous relationship (beginning, duration, end, purpose, ups and downs, twists and turns, etc.). This analogy between love and a journey enables people to say, for example, that their relationship is at a crossroads. Jacques Lacan (1957), developing Freud, has related the metaphor

\footnotetext{
${ }^{10}$ This method has fruitfully been applied to contemporary debates on issues directly related to the relationship between digitalized work and property by Steve Larsson in his article on "The Conceptions of Copyright in a Digital Context: A Comparison between French and American File-sharers" (Larsson 2014).
} 
to the unconscious process of condensation (Verdichtung), where two signifiers are superimposed.

When Shakespeare writes of Love's Labour's Lost, or when I say that I'm working on a steak, the realm of work/labour is the source domain of the metaphors and love, or eating, are the target domains. The omnipresence of work/labour in our lives, its centrality, make this particular source domain available for a myriad of metaphors in a host of target domains. The ever-growing commodification of everything makes it necessary for workers to sell their labour power to obtain access to nature's untransformed bounties, such as a fresh breeze of clean air or a drop of pure water, silence or an unspoilt landscape. The appropriation by capitalists of the exchange value generated by activities not designed for the purpose of creating exchange values, as we can see in data mining, give these activities the character of unpaid labour, as prosumers and social network users realize that their activity is profitable to others and start demanding compensation for something they were doing for free, thereby accepting the commoditization of whole aspects of their private lives, which now look like work/labour to them. The exaltation of a hard-working ethos, whether it is salaried work (viz. the stigma attached to unemployment) or working out in a gym point to the internalization of the demand made by capital that every human being maximizes his or her productive effort, whatever the circumstances, and the word work ends up encompassing all human activities that can directly or indirectly be turned into a profit.

All the words denoting labour or work are abstractions, since they put together various activities, which, viewed concretely, bear another name: to dig a hole, cut a piece of metal, fish, hunt, clean, put things in their proper place, write, etc. The question is thus: what do these activities have in common that they should be called work or labour, and from which angle is the similarity perceived? For we know very well that fishing, writing, driving, building a table, etc. can be called either work/labour or play under different circumstances. Harry Cleaver (2002) has suggested that since the work/labour concept was born with modernity and the rise of capitalism as a "capitalist category", we should not use it for previous periods. He certainly has a point, whether we are discussing labour as the all-encompassing word for surplus value generating activities, or work as the all-encompassing word for purposeful human activities in the wake of the Reformation, as Max Weber (2010) has shown. But historicization should be carried further back, since the words work, labour, and their equivalents in all languages did exist before their extended and often metaphorical meanings in modern times.

A genealogical, etymological investigation is certainly in order, with the caveat that some etymologies can be deceptive. For instance, in Russian, the word trud (труд), based on the Indo-European root treudō (v. Sanskrit tard, Latin trudo), meaning painful effort, applies both to hard labour, ascetic pursuits of monks and [...] academic publications (well, not that deceptive in the end!). In the course of that etymological investigation, one question has emerged: what is the cognitive linguistic process that has resulted in the invention of the words that denote work/labour? And the answer is that all the words denoting work/labour are metonymies, often coupled with metaphors.

A metonymy, according to the Oxford English Dictionary, is "a figure of speech which consists in substituting for the name of a thing the name of an attribute of it or of something closely related" (OED Online, 2013). In traditional rhetoric, three types of metonymies are distinguished: whole for part, part for whole, and part for part. In cognitive linguistics, there is an ongoing debate on whether metonymy is a sub-category of the metaphor, or whether it is the opposite pole of the metaphor, but this debate will not be dealt with here. Cognitive linguistics distinguishes between two types of metonymy, source-in-target metonymy, and target-in-source metonymy. Source-in-target metonymy consists in using a word that is a part ("source subdomain") of what one wants to represent ("target domain"): in the phrase "all hands on deck", hands ("source subdomain") are a part of sailors ("target domain") that "stands for" sailors. It involves "domain extension", in the sense that they provide access, from the subdomain, to the full concept or "matrix domain" (Ruiz de Mendoza 2000, 109132). Target-in-source metonymy consist in using the wider domain as source to refer to the subdomain, as in "I'm tying my shoes", while what I am actually tying is a part of my shoes, the laces. In this case, cognitive linguists speak of domain reduction, drawing attention to the 
"salient" feature of what one wants to represent in the context. I tie my laces because I want to use my shoes, so I can use the metonymy, because the focus is on the shoes, not on the laces, which are a means to an end. If my laces are broken, the focus will be on the laces, and I will not be able to use a metonymy by saying "I need new shoes". Lacan (1957) relates the metonymy process with the other fundamental unconscious process that is displacement (Verschiebung), in which the emotional charge of the original signifier is transferred to another signifier, which is made possible by their contiguity.

The French word travail, like the Spanish trabajo comes from Latin trepālium, an instrument of torture. It was formed by metonymy first: the part of the work experience that is the repetition of the pain inflicted on the worker by the work process is the "salient" part (the subdomain that stands for the whole domain); then a metaphorical process occurs, involving analogy: it is an experience similar to torture, and then the metaphor of a torture instrument can be used to name it. In the same way, when going to work to, say, an office job, some French speakers, notably of working class origins, ${ }^{11}$ will jokingly use the phrase "je pars à la mine" ("I'm going to the mine") as the salient part of their prospective workday is hardship (metonymy) and miner's work is the archetype of hardship at work (metaphor). The same French speaker would also say at the same point in the morning "je vais à la boîte chercher de la galette": I'm going to the (slang for) company worksite to get (slang for) money, here stressing the necessity to be employed that is related with proletarian status.

While metaphors such as trepālium are interesting, it is way too late to understand the precise circumstances that led to their use in the first place, and the reasons for the success they met. On the other hand, one can easily reconstruct the original metonymy, which sheds light on the "salient" characteristic of work/labour that was perceived as salient by the speakers. More difficult to find out is in what class of society the metonymy originated with, an avenue of research that is promising, and could be explored by looking at the lexicon of selected subcultures. No Frenchman without connections with the working class would use the mine metaphor. In the end anyway, the whole of society adopts a common stock of metonymies and metaphors to build a national language.

What we propose to do here is to explore and classify the types of metonymies that have made up the lexical field of work/labour in several languages, with the hope of discovering useful conceptual distinctions. Only a small part of the languages spoken by human beings on the planet across the ages are under study, and none is given a complete treatment. In particular, I could not access the languages of so-called "primitive" (i.e. non-literate) societies, which must be of particular interest. There is a lot of work to be done to verify the generalizations I arrived at in this research. Restricted as it was, my field of investigation extended far beyond my linguistic competences, and I am very grateful for the help received from friends and colleagues.

\section{Founding Metonymies and Related Metaphors}

I have found six types of metonymies at the origin of the words denoting work/labour in the languages under study ${ }^{12}$ :

\footnotetext{
${ }^{11}$ The example comes from personal experience with my first father-in-law, who graduated from Lumpenproletariat origins to metallurgy worker to office worker and was a prominent labour activist but still used the lexicon of his original subculture.

12 There might be a seventh one, based on the performance of discrete tasks to which one is compelled by necessity, linked with the precariousness of employment and/or of subsistence. In English, the word job meaning "a piece of work; esp. a small definite piece of work done in the way of one's special occupation or profession" is said by the Oxford English Dictionary to be "of obscure origin: prob. in colloquial use some time before it appeared in literature. Possibly connected with prec., sense 2" [i.e. A cart-load, or what a horse and cart can bring at one time], itself obviously connected to the first sense: "a small compact portion of some substance; a piece, lump; a stump, block; a tassel", possibly originating in Old French gobe, goube a mouthful, lump, etc., possibly from Gaul gob. A similar word is gig, an engagement for a musician. The Chinese 活 huo, to live, living, life, also means work, job, in colloquial Chinese, what "keeps body and soul together" as in the French "gagne-pain" (what serves to earn one's bread), that is what provides the means of life. Associated with 农 nong, farm, 农活 nonghuo
} 
- 4.1. Denoting activity

- 4.2. Denoting effort, difficulty, pain

- 4.3. Denoting the result or product of activity

- 4.4. Denoting torture

- 4.5. Denoting status of workers

- 4.6. Based on one particular activity

\subsection{Denoting Activity}

This is the original metonymy for the origin of the English word "work", a case of target-insource metonymy involving domain reduction, from activity in general to a particular type of activity, work. One of the oldest available occurrences of the word work in Old English, Beowulf 287 B9, circa A.D. 1000, is clearly referring to action, deeds, as opposed to words (praxis or poiesis as opposed to lexis): "gescad witan, worda ond worca, se pe wel penceð.." ("A keen-witted shield-bearer who thinks things out carefully must know the distinction between words and deeds, keep the difference clear") (Chickering, 2006, 64-65).

The root is the proto-Indo-European word Wergom, hence the Indo-Eupanobueg ug -, denoting activity. It resulted in ergon ("̌pyov), organon (öpyavov) in Greek, weorc or worc in Old English, Werk in German, gwreith in Middle Cymric, etc. ${ }^{13}$ Analyzing this passage of Beowulf, Peter Clemoes $(2006,158-161)$ refers this language to the Germanic legal tradition, detailing the obligations of a person to his lord, not only words, but also acts, deeds. Thus even the most abstract form of the concept (action as opposed to inaction, movement as opposed to inertia-as in the definition of (mechanical) work in physics introduced by the French physicist Coriolis in 1826 under the name travail, translated as work in English and Arbeit in German-or activity opposed to repose) was entangled in the web of social relationships when it appeared in English. The original notion of work in English could therefore

means farmwork. Pending further research in other languages, and considering that no verbs are associated, this notion is best left in a footnote for the time being.

${ }^{13}$ Podkorny's entry, p. 988, adapted, with the abbreviations developed and translated into English:

uĝ -2, Lê̂ -

English meaning: to do, work

German meaning: wirken, tun

Deitä : :Lệmilek

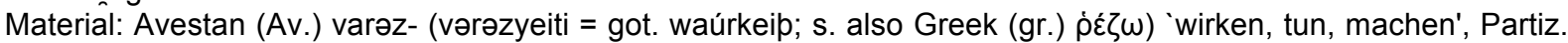
varšta-, varəza- m. 'Wirken, Verrichten von, Tätigkeit' (npers. varz, barz 'Feldarbeit, Ackerbau'), varšti- f. 'Handeln, Tun', varštva- Adj. 'was zu tun ist'; Armeinian (arm.) gorc 'Werk' (mit sekundärem o); Greek (gr). हैpyov,

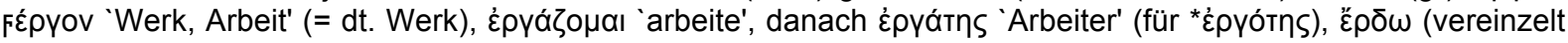

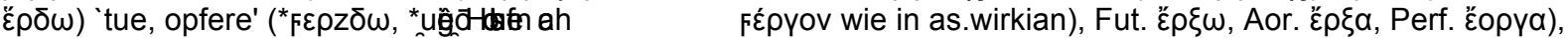

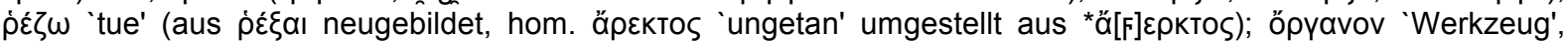

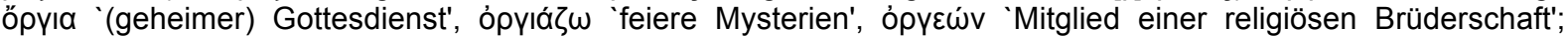

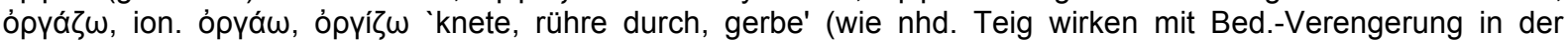
Berufssprache), wozu ćópyn 'Quirl' (wohl redupl. ₹E-Fópyā); alb. rregj 'reinigen', Mediopassiv rregjem 'mühen, streben' (St. E. Mann Lg. 26, 382 f.); Old Breton (abret.) guerg 'efficax', Gallic (gall.) vergo-bretus 'oberste Behörde der Aeduer', auch verco-breto (Pokorny, Vox Romanica 10, 266 f.); Middle Cymric (mcymr.) gwreith 'Tat' (đ̛̣ $\hat{g}$-tu-), 1. Pl. Imper. acymr. guragun, jünger gwnawn usw. (n statt r durch Einfluß von *gnī- 'machen', S. 373), Cornish (corn.) gruen, mbr. gr-(uepr đa r $\hat{g}$ - 'machen'), Lewis-Pedersen S. 336 f.; as. wirkian (Neubildung nach werk), warhta, High old German (ahd.) Frankish (fränk.) wirkan, wirchen, war(a)hta `arbeiten, tätig sein, wirken'; got. waúrkjan (= av. vərəzyeiti), aisl. yrkja, orta, ags. wyrcan, worhte, ahd. (obdt.) wurchen, wor(a)hta 'wirken, tun, machen, bewirken', ahd. gawurht f. 'Tat, Handlung', got. frawaúrhts 'sündig', f. 'Sünde' usw., got. waúrstw n. 'Werk' ('waúrh-stwa-; ähnlich av. varštva-); High old German (ahd.) werc, werah, Anglo-Saxon (as) werk, aisl. werk n. (= ع̋pyov) 'Werk, Tätigkeit, Arbeit' Anglo-Saxon (as.) weorc auch 'Mühsal, Qual', weshalb auch Old Icelandic (aisl.) verkr, Genitive verkjar (m. i-St.) 'Schmerz, Leid' hierhergehören kann; High old German (ahd.) wirken 'nähend, stickend, webend verfertigen' = as. wirkian, ags. wircan, und das davon nicht trennbare High old German (ahd.) werih in der Bed. 'Werg, stuppa', āwirihhi, āwurihhi 'Werg' zeigen Anwendung unserer Wz. auf die

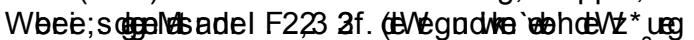

- 'drehen, winden' zuteilen möchte); an nhd. Werg erinnert Cymric (cymr.) cy-warch 'Hanf, Flachs' = Breton (bret.) koarc'h, Olf Breto ( et ) calting. c̀maina; -k- 'drehen'. 
be related with obligation by a third party: there is no reason why the words that are the equivalents of "work/labour" in other languages would not have appeared at the same moment as servitude: fishing, hunting, gathering, cooking, etc., were activities with names of their own, and there was no reason to lump them together under the heading "work" before work was imposed on people by their masters. More on this anon. In Wolof, the word ligeey, to be active, is the same as to be at work, and also employed, and its opposite, tok, which means to sit, is the word used for the status of unemployed.

The notion of activity also predominates in the Russian zanimát'sja (замиав в ), to busy oneself (when studying, doing sports, etc.). In sign language, as a source-in-target metonymy, "activity" is signed in the same way as work (see infra), with only the mouthing differing. In Lingala, mosálá, to work, is the same as to do. In standard Arabic, one of the two words meaning "work" similarly carries primarily the notion of activity, "to do": fa3ala/yaf3alu (ف) / (ف) (ف) $)^{14}$. Interestingly, this word exists only in standard Arabic, the language developed from classical Arabic in the early $19^{\text {th }}$ century, and appears in the various dialects only as variations borrowed from the standard Arabic. As early as the $8^{\text {th }}$ century, the Arab grammarians chose to use it as the root (in its graphic aspect, as opposed to the phonetic aspect) to represent the morphologic schemes of the Arabic language. Thus, the word kātib (الكاتب), writer, is viewed by the grammarians as following the fā3il scheme. One is reminded of Hannah Arendt's remarks on the birth of rhetoric as the substitution of persuasion for violence at the birth of the polis, with the separation of words from deeds (which were coupled in heroic times) (Arendt 1998, 25-26), except that written words are the words involved here. A keenwitted shield-bearer who thinks things out carefully must indeed know the distinction between written and spoken words on the one hand and deeds on the other hand, keep the difference clear, and also see the social relationships that connect them.

\subsection{Denoting Effort, Difficulty, Pain}

In many languages, the notions of effort, difficulty and pain are present in the lexicon of work, and most of the time these salient characteristics are present together in the same words, with only the context pointing to one separate characteristic. Only in Wolof (but there must be other examples) could I find two words for "to work", one of which does imply effort, but not necessarily painful effort. Should this distinction be found to exist in other languages, the present section would need to be divided in two and the typology extended.

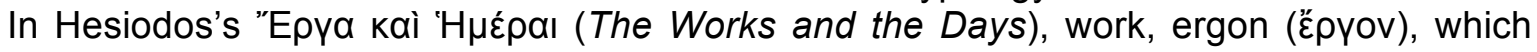
does imply effort, but not necessarily painful effort, is first introduced as a valued activity motivated by envy of another man's wealth. It is part of human nature, and, even in the Golden Age (before Zeus punished Prometheus for stealing the gods' fire by sending them Pandora and her box) men satisfied their needs through work, the difference with the later ages being

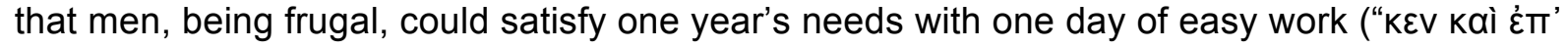

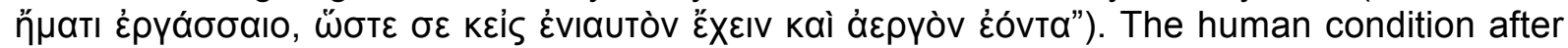
the Golden Age is characterized by the addition of ponos (móvos) painful and difficult work

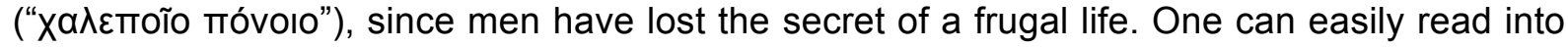
the fable the Marxist concept of surplus work/labour demanded by masters and refer the causes of the existence of surplus work/labour to domination rather than Pandora's myth.

Among the meanings of ponos (móvos), we find hard work, toil, as in toil of battle, trouble, bodily exertion, exercise, stress, trouble, distress, suffering, pain, especially physical-

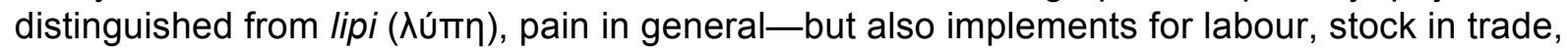
task, business, enterprise, undertaking. While the original metonymy was a target-in-source metonymy involving domain reduction (from the painful aspect of work to hard work in general), it gave rise to the opposite, source-in-target metonymy involving domain extension (from the work experience, seen as painful, to the notion of enterprise, and even anything

\footnotetext{
${ }^{14}$ fa3ala / yaf3alu: he did, he does (there is no infinitive form in Arabic). The scheme is fā3il.
} 


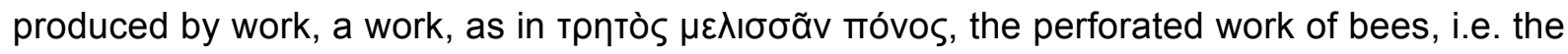
honeycomb in the poetic language of Pindarus.)

The Indo-European root of the Greek word ponos (móvos) is (s)pend, ${ }^{15}$ which seems to relate to the pulling motion that comes with spinning and weaving (from the same the root "spend"). The painful experience of women spinning and weaving seems to have been at the origin of the word. Another painful experience, that of carrying heavy loads, has been at the origin of the Latin word labor (labor, lapsi, lapsus sum) which has given the French labeur and labour (ploughing), the English labour, the Italian lavoro, etc. The meaning of the Latin labor is to slip (as in lapsus, a slip of the tongue), under a heavy burden. The same reference to a heavy burden can be found in the Russian language, which has two words for work: trud (труд), and rabota (работа), close to rabotsva (рабства), meaning slavery (see infra 3.5). Trud (труд) belongs to the group of words denoting pain, together with the Latin trūdō (to thrust, push, shove; to crowd or shove forward; to press on, drive, impel), both rooted in Indo-European tr-eu-d-, to press, to squeeze, like under a heavy burden. ${ }^{16}$ It is the word used

${ }^{15}$ Podkorny's entry, p. 988, adapted, with the abbreviations developed and translated into English: (s)pen(d): to pull; to spin, 'ziehen, spannen' und 'spinnen', indem die zu webenden Fäden zuerst ausgespannt wurden.

General comments: (s)pen- : spē(i)- 'ziehen' = pen- 'füttern': pā- 'Vieh weiden, füttern' \{to graze cattle, to feed\} =

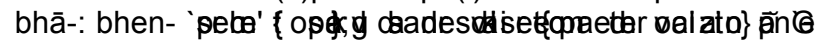
oben $\{$ see above $\}$ S $\{\mathrm{p}\} .788$.

Material: 1. Formen ohne -s-

Armenian (Arm.) hanum, aorist (Aor.) hanay und henum, aorist (Aor.) heni 'weben, zusammennähen'; s. darüber

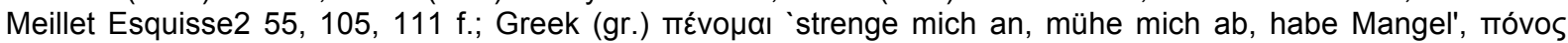

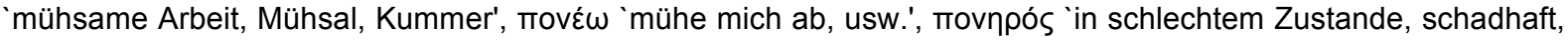

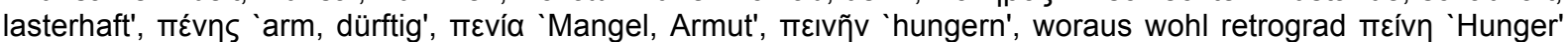

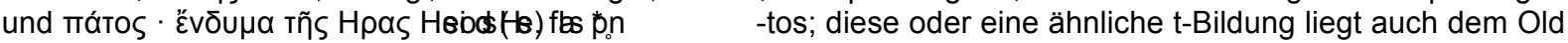
Irish (air.) ēt- 'kleiden' zugrunde; Lituanian (lit.) pinù, pìnti ‘flechten', pántis m. f., Old Prussish (apr.) panto f. 'Fessel', Latvian (lett.) pinu, pît 'flechten', pinekls 'Fessel'; Old Church Slavic (aksl.) pbnǫ, pęti ‘spannen', (ablaut.) opona f. 'Vorhang', ponjava 'Umhang, Kleid', pǫto `Fessel'(serb. püto), wozu Old Russian (a.russ.) prepjátb 'hindern', raspjátb 'kreuzigen', pjatb, pnutb 'mit dem Fußestoßen' und Old Church Slavic (aksl.) pęta 'Ferse' Serbian (serb.) petasati 'mit den Füßen ausschlagen'), Russian (russ.) pjatá, Serbian (serb.) péta, Lituanian (lit.) péntis m. 'ds.; Rücken der Axt, der Sense', Old Prussish (apr.) pentis 'Ferse'; vielleicht Albanian (alb.) pendë, pëndë 'Paar Ochsen; Joch (Ackermaß)' aus einem *pentā ‘*Gespann'; auch penk 'Koppel';

2. Formen mit anlaut. s-: Latin (lat.) sponte `aus eigenem Antrieb, aus freiem Willen', Gothic (got.) Old High German

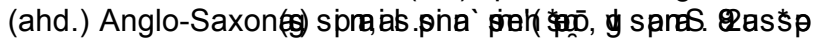
-nuфळ+b

German (ahd.) spinna 'Spinne'; mit einfachem n: Old Icelandic (aisl.) spuni m. 'Gespinst', Anglo-Saxon (ags.) spinel, Old High German (ahd.) spinala (und spinnila) 'Spindel'.

3. Erweiterung (s)pen-d-:

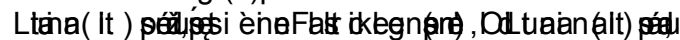

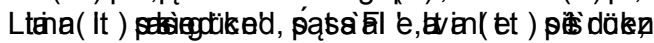

-yti 'spannen', spuôsts 'Fallstrick, Falle', Latvian (lett.) spendele 'Feder an einem Schlosse', spanda 'Strickwerk am Pflug', wie

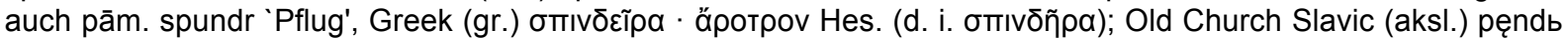
'Spanne', pǫditi 'drängen, treiben' (ursprüngl. etwa 'ein Vieh an gespanntem Strick vorwärtsziehen'); vermutlich auch als 'gespannt hängen', Latin (lat.) pendēo, -ēre 'hangen, herabhangen', pendō, -ěre 'wägen, schätzen, zahlen' (zum Wägen aufhängen), Umbrian (umbr.) ampentu 'impenditō'; ob auch Anglo-Saxon (ags.) finta m. 'Schwanz, Folge'?

${ }^{16}$ Podkorny's entry, p. 1095, adapted, with the abbreviations developed and translated into English: tr-eu-d-

English meaning: to press, push

German meaning: 'quetschen, stoßen, drücken'

General comments: wohl Erw. zu ter-3, tereu- 'reiben'

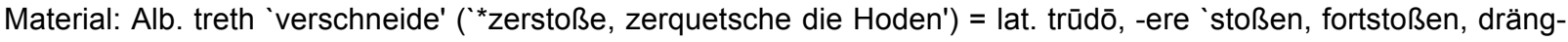
en' (trūdis 'eisenbeschlagene Stange zum Fortstoßen'); mcymr. cythruð 'quälen', godruð 'wild', gorthruð 'Bedrückung' ("-treudo-); mir. trotaid 'streitet' (*truzd- aus *trud-d-), cymr. trythill, drythill 'wollüstig' (daraus mir. treitell 'Liebling'); air. tromm, cymr. trwm 'schwer' ('trudsmo- 'drückend'); got. uspriutan 'beschwerlich fallen', aisl. prjōta 'mangeln', ags. (ā-)ðrēotan unpers. ‘ermüden, überdrüssig werden', ahd. (ar-, bi-)driozan 'bedrängen, belästigen', nhd. verdrießen; aisl. prjōtr 'widerspenstiger Mensch', ahd. urdrioz 'Verdruß'; aisl. praut f. 'Kraftprobe, Bedrängnis', ags. đrēat m. 'Gedränge, Gewalttätigkeit, Drohung', mhd. drōz 'Verdruß, Last, Beschwerde' (= slav. trudъ); ags. đrēat(n)ian 'drängen, quälen, schelten, drohen'; ags. đrīetan 'ermüden (tr.), drängen', aisl. preyta 'Kraft aufwenden, aushalten, ermüden (tr. und intr.)'; aisı. prȳsta, ahd. ðrūstit, ags. geðryscan 'bedrücken', ðrysman 'erdrücken, ersticken', mnd. drussemen 'erdrosseln, erdrücken'; aksl. trudъ 'Mühe', truždǫ, truditi 'beschweren, quälen'. 
by Orthodox monks to translate into Old Slavic the Greek word ponos (móvoऽ), and also the words kopos (ко́тоऽ), pain, here a self-inflicted blow to the chest (in the context of asceti-

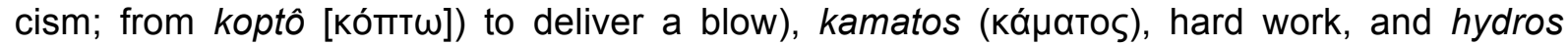

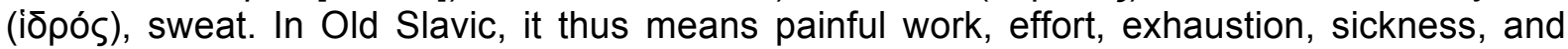
also difficulty. The reference to sweat is also to be found in the original curse, "by the sweat of thy brow", but also in Wolof, where painful work is called niax jariñu, niax meaning sweat, and jariñu meaning useful. Another Wolof word for "to work", daan dole, meaning to use one's strength (dole) puts more stress on the notion of effort than on the related pain. This brings to mind the notion of energy, its physiology, and what Freudians call the psychological economy of the libido.

The adjective formed on trud is used to denote the particularly difficult characteristic of one type of work, as in trudnaja rabota (трудная работа), hard and painful work. But the word becomes dignified when used by monks, extolling their own ascetic and associated intellectual pursuits and later communists, when trudjaschiesja (трудящиеся) became the official word for the supposedly dominant working class. There is even an organization ${ }^{17}$ in Russia entitled "Workers against slavery", Trudjaschiesja protiv rabotsva (Трудящиеся против рабства), under a banner inspired from the French Socialist Party logo. While the original metonymy was a target-in-source metonymy involving domain reduction (from the painful aspect of work to hard work in general), it gave rise to the opposite, source-in-target metonymy involving domain extension (from the work experience, seen as painful, to the notions of cultural production, dignified group).

The painful connotation is present in the standard Arabic 'amal (a) ), which resulted in the Swahili amali, pointing to colonization and slave production in the area.

The sinogram 劳 lao denotes painful work. According to the etymological dictionary Shuōwén Jiězì (說文解字), made available in $121 \mathrm{AD}$, and reflecting the specular turn of mind prevailing under the Hans, the original sinogram, in the xiaozhuan (Small Seal Script (小管, 221-207 BC) is the superposition of 火火 the night light (fire) at the top, $\neg$ the roof in the middle, signifying the hose, and 力 force, in the bottom. Rooted in lao, we find 劳人 laoren (lao + ren [man]) hard worker, 劳力 (lao + li [strength]) to perform manual work, 劳神 laoshen (lao + shen [mind]) to think hard, intellectual fatigue. Associated with 动 dong, denoting movement, we find 脑力劳动 naolilaodong (nao [brain[ + li [strength] + lao + dong), intellectual work, 体力劳动 tillilaodong (ti = body), manual work, but also 劳动法 laodong+fa (law), labour law, and 劳动合同 laodong + hetong (contract), work contract.

The painful characteristic of work has resulted in labour referring to birth giving (travail in French), which is in fact a combination of two source-in target metonymies, one stressing the painful character of both processes, the other one emphasizing, in both cases too, the result of activity (something is produced, see infra 3.3) therefore inviting comparison and leading to the creation of the metaphor, long before it became part of the Marxist feminist notion of reproductive labour as reproducing labour power. It was obviously sourced in the Christian tradition, since there is no such use of the word labour or its equivalent in Hebrew to denote childbearing, although the original curse in Hebrew refers to pain (itstsabon, עיכָּבון both for the woman's experience of childbirth and the man's experience of tilling the ground). The English language has appropriated the French word travail, to designate all sorts of painful experiences, including travel before the days of package tours.

Some degree of pain is unavoidable when "grappling with reality", the working subject is engaged in a struggle against the object that is worked on, hence the metaphor of battle, in the English word toil, which originally meant argument, strife, battle.

\subsection{Denoting the Result or Product of Activity}

\footnotetext{
${ }^{17}$ http://sd-inform.org/biblioteka/antitotalitarizm/trudjaschiesja-protiv-rabstva, accessed January 10, 2014.
} 
In the Latin family of Indo-European languages, the concrete product of work receives names based on the Indo-European root $\mathrm{Op}^{18}$ : Latin opus, French œuvre, Spanish obra, Italian opera, etc. The process that leads to the creation of these works, concrete work, is called operare (Latin, Italian) œuvrer (French, also ouvrage, both the result of work and the work process), obrar (Spanish), etc. And the individuals involved in the process of producing such objects, the workers, are called ouvriers (French), operai (Italian), obreros (Spanish), etc., a particular class of waged workers that produce material goods outside agriculture (but the concept can be extended to agricultural waged work as in the French ouvrier agricole, with a modifier).

A first metonymy projects the result of work onto the work process, another one projects the work process onto the worker, a third one can project it on the end result. The French labourer (to plough), originating in Latin labor (see supra) gives labour, as the activity ("faire les labours" = labourer, to plough) of the laboureur (ploughman) and the result of the activity ("marcher dans les labours", to walk through ploughed fields).

An opposite process can be observed in English, in which the activity gives its name to the result of activity, especially for intellectual work (works of art, good works in the language of religion) or in German (Werk). In Russian, the word trud (труд), denoting painful and difficult work, gives its name to the resulting work of the mind, as in Труды Математического Института имени В. А. Стеклова, Trudy Matematicheskogo Instituta imeni V. A. Steklova, for a mathematical journal, and in Hebrew, where, most interestingly melâ'kâh (מלאכה), free labour (see infra 3.5) also means the result of work, property, thereby anticipating by a few centuries or millennia the Lockean theory of property founded on labour (Locke 2000, 298299)..$^{19}$

\subsection{Denoting Torture}

In the Latin family of Indo-European languages, French (travail), Spanish (trabajo) Provençal (trebalh), Portuguese (trabalho), Italian (travaglio) originate in trepālium (Latin), a torture instrument. The connection points to pain that does not stop, as a consequence of a minute

${ }^{18}$ Podkorny's entry, p. 780, adapted, with the abbreviations developed and translated into English. op-1

English meaning: to work, perform

German meaning; arbeiten, zustande bringen; Ertrag der Arbeit, Reichtum

Derivatives: op-os- 'Werk 'Material: Old Hindic (ai.) ápas- n. 'Werk' (= lat. opus), Avestan (av.) hv-apah- 'gutes Werk (verriheф̆ ấpa - n. 'Werk, religiöse Handlung'; ápnas- n. 'Ertrag, Habe, Besitz', av. afnah-vant- 'reich

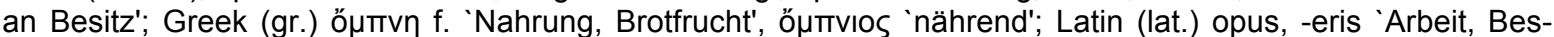
chäftigung, Handlung, Werk', opus est 'es ist nötig' ('`ist Mußarbeit'), wovon operō, -āre 'arbeiten', Oscan (osk.) úpsannam 'operandam', upsatuh sent ('factī sunt'), Perf. upsed 'fecit', uupsens 'fēcērunt', (dehnstufiges Perf. wie in lat. ōdī), Umbrian (umbr.) osatu 'facitō', pälign. upsaseter 'fieret'; lat. ops, opis 'Vermögen, Reichtum, Macht; Hilfe, Beistand', bei Ennius auch 'Bemühung, Dienst', officium 'Pflicht' < *opi-ficium 'Arbeitsverrichtung', Ops 'Göttin des Erntesegens', inops, cōpia ( $\left.{ }^{*} \mathrm{co}-\mathrm{opia}\right)$, opulentus 'reich an Vermögen, mächtig', wohl auch optimus 'der Beste' (eig. 'der Wohlhabendste') ; vielleicht der Name der Oscī, Opscī, 'Отাкоí als 'die Verehrer der Ops' und lat. omnis `all, ganz, jeder' ('op-ni-s); vielleicht air. somme 'reich', domme `arm' (su-, dus-op-simo -);

Anglo-Saxon (ags.) efnan, Old Icelandic (aisl.) efna 'wirken, tun'; dehnstufig Old High German (ahd.) uobo 'Landbauer', uoben 'ins Werk setzen, ausüben, verehren', nhd. üben, ahd. uoba m. PI. 'Feier', Middle High German

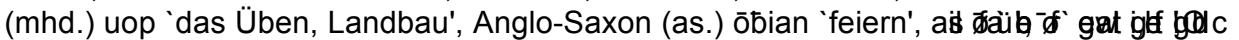
landic (aisl.) efna `ausführen', efni 'Stoff, Zeug für etwas'; über aisl. afl 'Kraft' usw. s. oben S. 52; Hittite (hitt.) happinahb- 'reich machen'.

${ }^{19}$ As expressed in chapter V of the Second Treatise on Civil Government: "44. From all which it is evident, that though the

things of Nature are given in common, man (by being master of himself, and proprietor of his own person, and the actions or Labour of it) had still in himself the great Foundation of Property; and that which made up the great part of what he applied to the support or comfort of his being, when invention and arts had improved the conveniences of life, was perfectly his own, and did not belong in common to others." The bourgeoisie was the only class in the history of Europe that both possessed means of production and performed manual work/labour, hence its special relationship to the notion of work/labour and property. But similar groups of self-employed "free" workers existed before, and could link work and the result of work sub specie of property. 
division of labour that reduces work to a repetition of the same motions for an extended period.

Marx's ideal workday, when I am able "to hunt in the morning, fish in the afternoon, rear cattle in the evening, criticise after dinner, just as I have a mind, without ever becoming hunter, fisherman, shepherd or critic" (Marx and Engels 1845/46, 53), points to a crucial characteristic of work from a eudemonistic perspective: if a change of work is a holiday, then what makes work an especially painful activity is its continuation through an over-extended period of time, the ensuing boredom, and the transformation of pain, which is an unavoidable part of life, into suffering, which is an avoidable part of life. Here, the connection with extra work demanded by masters is also present.

The repetition of the same, even innocuous per se, becomes a torment, as in the "Chinese water torture" where the pain comes from a steady dripping of water droplets on the same part of the body. And the classical image of the Fordist worker, chained to the assembly line so that he/she must repeat the same motion relentlessly, resembles that of the galley slave who does nothing but work the oars. Relentless repetition of the same is what Thanatos (the death impulse identified by Freud) is about. Relentlessness and endlessness are the two things that make hell hellish. Hell is everlasting death, as Paradise is everlasting life.

Another dimension of torture, which applies to the "Chinese water torture" concept too, is excessive focusing on one thing, the concentration involved, which is painful in itself. "In consequence of the division of labour, the whole of every man's attention comes naturally to be directed towards some one very simple object" (Smith 1999, 114).

All in all, man is living through and craving for variety, alternation and diversity. Just as we alternate inhaling and exhaling, chewing and swallowing, sleeping and waking, we need to alternate our activities. Post-Fordist human resources specialists of Internet work, who have understood this, would allow private Internet surfing (cyberloafing) to employees while at their workstation to increase productivity by lowering stress Chen and Lim 2011). This raises the issue of rhythm. Autonomous work (when I choose to work) is set to individual biorhythms, heteronomous work (when I have work imposed on me from outside, whether by necessity or violence) is set to extraneous rhythms: slaves on a galley pulling on the oars to the drum's rhythm, marching soldiers, workers on the assembly-line, Internet slaves desperately trying to catch up with incoming emails are all subject to a form of torture. In moderate doses, the extraneous rhythm energizes, as when dancing to a tune, while it becomes painful and even lethal in excessive doses, as any stimulus.

\subsection{Denoting Status of Workers: Subordination}

Heteronomous work suggests the existence of domination relationships. But, even in the absence of an actual master, the very nature of work involves a certain form of subordination. As a matter of fact, both the goal of the freely chosen work activity, and the specificities of the chosen work material themselves, impose their irreducible strangeness on the worker. When "I'm committed to my work", the goal, the end in view becomes my master, imposes a discipline, and, when I carve wood, I have to obey the dictates of the wood grain at the very moment when I transform nature. "Nature to be commanded must be obeyed" wrote Francis Bacon (Bacon 1960, 39).

In Indo-European languages, the root orbho, ${ }^{20}$ signifying orphan, bereft, servant, slave, weak child, work, has given Arbeit in German and rabota (работа) in Russian. Here, the

\footnotetext{
${ }^{20}$ Podkorny's entry, p. 780, adapted, with the abbreviations developed and translated into English. orbho-

English meaning: orphan; servant; work

German meaning: 'verwaist, Waise'; daraus Armenian (arm.) and Greek (gr.) mit -ano-, Celtic (kelt.) German (germ.) mit io-Ableitung) 'Waisengut = Erbe', wovon 'der Erbe'; 'Waise' = 'kleines Kind, klein, schwach, hilflos' (ai., slav.); 'verwaistes, schutzloses Kind, das fürs Gnadenbrot alle niedrige Arbeit zu verrichten hat, Knecht, Sklave' (slav., arm.), wovon 'Knechtesarbeit'.
} 
salient aspect of work is domination, subordination. A feeling of pity for the orphan, the weakling, resonates. Too bad human beings are subjected to this condition. Aristotle in his Politics (Ross 1957, 1253b; Davis \& Jowett 2008, 31) dreamt that one day slaves would be replaced by machines ${ }^{21}$; so did Marxist Paul Lafargue, who quoted him in his Droit à la Paresse $(1883,38)$. And Czech Karel Čapek invented the word robot (from Czech rob, slave) in his science-fiction play R.U.R. (Rossum's Universal Robots) first staged in Prague in 1921.

The Russian sluzhaschie (служащие), from the verb to serve (служить) is used for employees: hence sluzhba (служба), white-collar work. In the days of Czarist Russia, civil and military servants in the huge bureaucracy would "serve", sluzhit' (служить) rather than work (работаtь), and today's office workers, when at their workstations, are still "in service" na skuzhbe (на службе) rather than "at work" na rabote (на работе). The "noble" meaning of service, in a bureaucracy engaged primarily in intellectual and direction activities is related to feudal relations. It is comparable to the French employé, a placeholder (emploi meaning position). When a large class of waged workers appeared in the United States, the word employé was originally used, borrowed from the French, then it was anglicized into employee. In dialectal Arabic, in the Maghreb and Egypt, khedma (خد) work, is the same as service, rooted in the experience of the Arab conquest.

One of the three words denoting work / labour in Hebrew, avoda (עבוד) is similarly rooted in slavery. In Rabbinic Hebrew, it means divine worship, service (of God, originally performed by the priests in the Temple). It is a metaphor of slavery / service, since the relationship of man to god is similar to the relationship of the slave / servant (eved, עבד) to the master. In modern Hebrew, it is the most commonly used word to mean work, with the verb la'avod (לעבוד), meaning to work, the same verb used by Genesis 2.2 to describe god's work of creation (when he made himself a servant to creation [...]), [...] and gives its name to the Labour Party (Mifleget HaAvoda HaYisraelit ליתהישרא). Investigating the notion of calling (Beruf) in his Protestant Ethic and the Spirit of Capitalism, Max Weber goes at great length to make the point that the notion of calling (Beruf in German), as a service to god in worldly work, is recent, linked with the Reformation ${ }^{22}$, and that there is no equivalent in languages not affected by the Reformation. He tackles the issue of ancient languages in a footnote, where, identifying Hebrew as the only ancient language in which there is a connexion between divine service and work with the word avoda, he remarks: "Admittedly, as Professor Merx informs me, even in ancient times the Hebrew word אל had completely lost any link with the original concept [...]". "Even in ancient times", avoda must have first referred to work/labour as subordinated work, and the technical religious word must have been borrowed from the common language, not the other way round. The other Hebrew word for work / labour, melâ'kâh

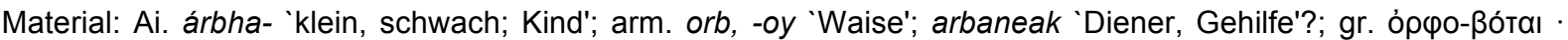

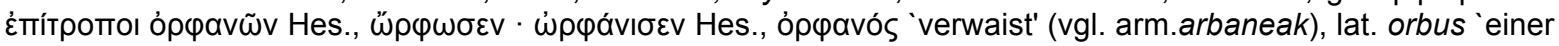
Sache beraubt, verwaist'; Old irish (air.) orb(b)e, orpe m. n. 'der, das Erbe' ( ${ }^{*} o b{ }_{0} \circ \quad-$ ), comarbe 'Miterbe', Gallic (gall.) Orbius MN (dazu das Verbum air. no-m-erpimm 'committo me', ro-eirpset `sie übergaben' usw., vielleicht aus *air-orb-), Gothic (got.) arbi n. 'das Erbe', Old high German (ahd.) arbi, erbi n. ds., Anglo-Saxon (ags.) ierfe, yrfe n. ds. Old Icelandic (aisl.) arfr m. 'das Erbe' ist zu arfi, arfa 'der Erbe, die Erbin' neugebildet), Old Icelandic (aisl.) erfi (run. arbija) n. 'Leichenmahl'; Gothic (got.) arbja, Old Icelandic (aisl.) arfi (f. arfa), Old high German (ahd.) arpeo, erbo 'der Erbe', ags. ierfe n. 'das Erbe'; die germ. Wörter stammen wegen des Folgenden kaum aus dem Keltischen; aus ein intr. Verbum *arbē-iō 'bin verwaistes, zur harten Arbeit verdingtes Kind?' führt man zurück Gothic (got.) arbaips f. 'Mühsal, Arbeit', Old Icelandic (aisl.) erfiði n. ds., as. araちēd f., arbēdi n., AngloSaxon (ags.) earfop f., earfepe n. 'Mühe, Arbeit', ahd. arabeit 'Arbeit' (aisl. erfiðr, ags. earfepe 'beschwerlich'),

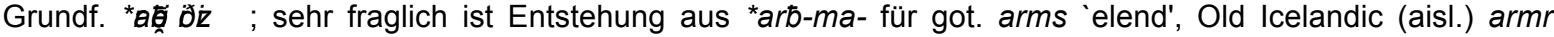
'elend, unglücklich', Old high German (ahd.) as. ar(a)m, Anglo-Saxon (ags.) earm 'arm, dürftig'; Grundbed. wäre etwa 'armes Waisenkind'; abg. rabъ 'Knecht', rabota 'servitus', Cezch (čech.) rob 'Sklave', robe `kleines Kind', Russian (russ.) rebjáta 'Kinder', rebënok 'Kind'; die russ. Formen gehen auf rob-, Old Slavic (urslav.) *orb- zurück (Vasmer brieflich); vielleicht Hititte (hitt.) arpa- 'Ungunst, Mißerfolg'.

21 "For if every instrument could accomplish its own work, obeying or anticipating the will of others, like the statues of Daedalus, or the tripods of Hephaestus, which, says the poet, of their own accord entered the assembly of the gods; if, in like manner, the shuttle would weave and the plectrum touch the lyre without a hand to guide them, chief workmen would not want servants, nor masters slaves."

As a matter of fact, in English, the word starts referring to "a trade" only in 1551, while the word calling to translate the Latin vocation appears with Wycliffe in 1382, according to the Oxford English Dictionary. 
(מלאכה), linked with artisans, handicrafts, is similarly based on the status of the worker, this time not servile: in the Old Testament, it refers to angels, ministers, i.e. employees above the rank of slaves. In Chinese, the word ye 業 (in traditional Chinese), 业 (in simplified Chinese), meaning line of business, industry, occupation, job, employment, school studies, enterprise, also means property, and, in the context of Buddhism, karma, a person's station in life viewed as the result of his/her actions. The Semitic root a.b/v.d., present in avoda, is matched by the Arabic abd (عبودية) which means servant, slave, and, by extension, worshipper as in Abdullah, servant of god.

The relationship between work / labour and slavery is sweetly expressed in the Spanish word for retirement, jubilación, which refers to the Jubilee, the biblical period, every 50 years, when slaves would be free, lands restored to their original owners and debts extinguished.

\subsection{Metonymy Based on the Gestures Involved in One Particular Type of Work}

The Chinese have three words for work/labour. One of them is gong (工). The sinogram (Chinese character) I is a stylised image of a hand-held rammer, an implement used to flatten, compact and stabilize the soil in order to build a house without foundations. Associated with other sinograms, it has given the noun 工作 (gongzuo) meaning work in general, also the verb to work, 工作者 gongzhe (gongzuo + zhe [man]) worker, 工作日 gongri (gongzuo + $r i$ [day]) workday, 工作服 (gongzuo $+f u$ [clothes]) work clothes, 工作证 (gongzuo + zheng [document]) working permit, 工人 gongren (gongzuo + ren [male]) worker, 工会 gonghui, (gongzuo + hui [meeting]) labour union, etc., and, interestingly, 工资 gongzi (gongzuo + zī [ resources, capital, to provide, to supply, to support, money, expense]), wages.

The same process (source-in-target metonymy, domain extension) has been used by the inventors of sign language for the deaf: in French Sign Language ${ }^{23}$, and in American Sign Language, which is derived from the French Sign Language, "the sign for 'work' is made by shaping both hands into 'fist shapes' [...]. With your palms facing downward, use your dominant fist to tap the wrist or the side of your non-dominant fist a few times (Memory Aid: Think of working with a hammer)": ${ }^{24}$

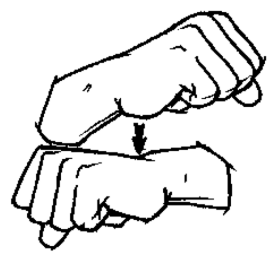

Figure 1: "work" in American Sign Language

This mimics the action of hammering, the repetition shows that there is a purpose to the activity, and the number of repetitions is left to the speaker. There is also a connotation of pain in the mutual shocks that flesh is heir to. The opposed poles of active / passive, "dominant" / subservient, subject / object are at work. The French Sign Language for unemployment ${ }^{25}$ starts very much like "work" with crossed wrists, but the hands move apart and come to rest on the hips.

In British Sign Language ${ }^{26}$ the sign for work is made with "hands held with open palms, prime hand chops down on secondary hand", as in cutting wood or meat, the upper hand functioning as an axe or chopper. "Unemployed" is signed by "Secondary hand held open

\footnotetext{
${ }^{23} \mathrm{http}: / /$ www.Isfdico-injsmetz.fr/recherche-alphabetique.php?mot=563\&lettre=t

24 http://lifeprint.com/asl101/pages-signs/w/work.htm

$25 \mathrm{http}: / /$ www.Isfdico-injsmetz.fr/recherche-alphabetique.php? mot $=920 \&$ lettre $=c \&$ valeur $=100$

26 http://www.britishsignlanguage.com/words/index.php?id=37
} 
with thumb upward. Prime hand brushes top of secondary hand", moving away from where the chopping action can be performed, showing the impossibility of chopping. ${ }^{27}$

If, like German philologists of the $19^{\text {th }}$ century, we were looking for the word work/labour in the "original language", the Ursprache, at the anthropological level, the sign languages might well point us in the right direction for "work" as a specifically human activity: the signs mimic the movement of the flint stone chopper for shaping other flint stones (Leroy-Gourhan 1964, 133-134): it is the original machine tool, the tool to make tools that makes humans human, whereas animals use tools found in nature and do not make them. Whether we agree or not in totality with the theory of The Gestural Origin of Language (Armstrong and Wilcox 2007), it seems that the Tower of Babel was built with cut stones.

\section{Conclusion}

The reasons why we use metonymies are both functional and contextual. Every object that we consider is related in our minds to a particular context and/or a particular structure, which assigns a function to the various elements which can be considered, in the last analysis, as the referents (what is signified) of any given situation. Each referent is seen as part of a whole that transcends it (irrespective of whether we have source-in-target or target-in-source metonymy) and assigns to it a function, a raison d'être (the reason why it is mentioned in the first place) within the larger situation where the referent appears. In the example "I'm tying my shoes", laces only have a function within the structural and functional scenario of a certain kind of shoe. In the case of work/labour metonymies, as in all metonymies, each metonymy is a "fractal compression" (de Oliveira e Paiva and Menezes 2010) of the web of historical and social relationships in which the referents themselves have been entangled.

It is now for us to explore in more detail the way metonymies and metaphors born along the ages from the actual experience of work/labour by our ancestors have been recycled in the Age of the Internet, as an avenue to identify the web of social relationships in which today's work situations that contribute to the existence of the Web, or connect to it in their daily course, are entangled, taking into account the salient dimensions of work/labour that linguistic inquiry has provided for further research.

Unsurprisingly, the nascent vocabulary of Internet work / labour has relied on metaphors, using existing off-line realities as a source domain to name activities in the target domain of "virtual work", which is understandable since it is a new activity which in some respects "looks like" previous activities. The "web", the "net", the "cloud" are metaphors.

But when it comes to work and labour, metonymies are back. Interestingly, writers trying to describe work on the Internet have used metonymies that were not sourced in the domain of work and labour, but in the domain of technology, specifically computer technology. Take "elancer", coined by Helen Wilkinson in 1999 (Barbrook 2006, 96), "cybertariat" (Huws 2001), or "digital labour". The prefixes "e-" and "cyber", or the modifier "digital" are used to stress one salient aspect of Internet work, which is that it depends on computers. Then one particular aspect of computers is used to refer to the world of computers in general, and by extension to the Internet: computers rely on electronics ("-e"), are a complex information system ("cyber"), based on digits ("digital"), etc. Once one of these particular aspects of computer technology has found its way into the current language as a proxy for Internet-related activity, it can be associated with a word referring to work and labour: e + freelancer = elancer, cyber + proletariat: cybertariat, digital + labour. The choice of the work-related element is of course linked with the political stance of the author. Those metonymies point to the dependence of the worker on the technology. Amazon's Mechanical Turk refers to the Turk, a chess-playing automaton that was ultimately discovered to provide a hiding place for an actual chess expert, and provides "artificial artificial intelligence". A metonymy derived from one particular aspect of work on the Internet (and thus analogous to 3.6) would be clickworker.

Most striking is the fact that no new word has emerged from the wealth of "new names" that clog the literature about the Internet to replace "to work" as a verb. This would be further

\footnotetext{
${ }^{27}$ http://www.britishsignlanguage.com/words/index.php?id=75
} 
proof that work on the Internet is definitely not virtual, since virtual is defined by the OED as "not physically existing as such but made by software to appear to do so from the point of view of the program or the user".

\section{References}

Arendt. 1998. The Human Condition. Chicago: University of Chicago Press.

Aristotle. 1957. Aristotle's Politica. Edited by William David Ross. Oxford: Clarendon Press. Available at: http://nlp.perseus.tufts.edu/hopper/

Aristotle. 2008. Davis, Henry William Carless and Benjamin Jowett. Aristotle Politics. New York: Cosimo Classics.

Armstrong, David F. and Sherman E. Wilcox. 2007. The Gestural Origin of Language: Perspectives on Deafness. Oxford: Oxford University Press.

Bacon, Francis. 1960. The New Organon and Related Writings. Edited by Fulton H. Anderson. Indianapolis and New York: Bobbs-Merrill.

Barbrook, Richard. 2006. The Class of the New. Accessed April 2, 2014. http://www.theclassofthenew.net.

Barcelona, Antonio, Réka Benczes and Francisco José Ruiz de Mendoza Ibáñez. 2011. Defining Metonymy in Cognitive Linguistics: Towards a Consensus Biew. Amsterdam: John Benjamins Publishing.

Chen, Don J.Q. and Vivien K.G. Lim. 2011. Impact of Cyberloafing on Psychological Engagement. 71st Academy of Management Meeting. San Antonio, Texas, USA, 12th-16th Aug, 2011.

Chickering, Howell D., Jr. 2006. Beowulf: A Dual-Language Edition. New York: Anchor Books.

Cleaver, Harry. 2002. Work is Still the Central Issue! New Words for New Worlds. In The Labour Debate: An Investigation into the Theory and Reality of Capitalist Work, edited by Ana Dienrstein and Michael Leary, 135-148. Hampshire: Ashgate.

Clemoes, Peter. 2006. Interactions of Thought and Language in Old English Poetry. Cambridge: Cambridge University Press.

de Oliveira e Paiva, Vera and Lúcia Menezes. 2010. A metonímia como processo fractal multimodal. Revista Veredas 14 (1): 7-19.

Dirven, René and Ralf Pörings 2003. Metaphor and Metonymy in Comparison and Contrast. Berlin: Walter de Gruyter.

Fuchs, Christian and Sebastian Sevignani. 2013. What Is Digital Labour? What Is Digital Work? What's Their Difference? And Why Do These Questions Matter For Understanding Social Media? tripleC: Communication, Capitalism \& Critique 11 (2): 237-293.

Fuchs, Christian. 2014. Theoretical Foundations of Studying Digital Labour. London: Routledge.

Gaffiot, Félix. 1934. Dictionnaire illustré Latin/Français. Paris: Hachette.

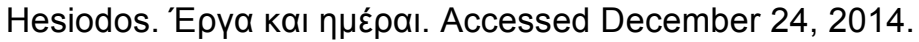
http://el.wikisource.org/wiki/\%CE\%88\%CF\%81\%CE\%B3\%CE\%B1_\%CE\%BA\%CE\%B1\%CE\%B9 \%CE\%B7\%CE\%BC\%CE\%AD\%CF\%81\%CE\%B1\%CE\%B9.

Huws, Ursula. 2001. The Making Of A Cybertariat? Virtual Work In A Real World. Socialist Register 37: 2-23.

Kley, Martin Michael. 2008. All Work and No Play? Labor, Literature and Industrial Modernity in the Weimar Left. Ph.D. Austin: The University of Texas at Austin.

Lacan, Jacques. 1957. L'instance de la lettre dans l'inconscient ou la raison depuis Freud. La Psychanalyse (3): 47-81.

Lafargue, Paul. 1883. Droit à la Paresse. Paris. Available at: http://classiques.uqac.ca/classiques/lafargue paul/droit paresse/droit paresse.html.

Langacker, Ronald Wayne. 1973. Language and its Structure: Some Fundamental Linguistic Concepts. New York: Harcourt Brace Jovanovich.

Lakoff, George and Mark L. Johnson. 1980. Metaphors We Live By. Chicago: University of Chicago.

Leroi-Gourhan, André. 1964. Le geste et la parole. Technique et langage. Paris: Albin Michel.

Liddell, Henry George and Robert Scott. 1940. A Greek-English Lexicon, Revised and Augmented Throughout by Sir Henry Stuart Jones, with the Assistance of Roderick McKenzie. Oxford: Clarendon Press. Available, with other lexicons, at the Perseus Digital Library: http://www.perseus.tufts.edu/hopper/.

Locke, John. 2000. Two Treatises of Government. In Cambridge Texts in the History of Political Thought (Series), edited by Peter Laslett. Cambridge: Cambridge University Press. 
Mackey, Albert. 1914. Encyclopedia of Freemasonry and its Kindred Sciences. New York and London: Masonic History Company.

Madan, Arthur Cornwallis. 1903. A Swahili-English Dictionary. Oxford: Clarendon Press.

Malthus, Thomas Robert. 1814. Observations on the Effects of the Corn Laws, and of a Rise or Fall in the Price of Corn on the Agriculture and General Wealth of the Country. London: J. Johnson and Co. Available at: http://www.gutenberg.org/files/4334/4334-h/4334-h.html.

Marx, Karl and Friedrich Engels. 1845/46. The German Ideology. Amherst, NY: Prometheus Books.

Marx, Karl and Friedrich Engels. 1968ff. Marx Engels Werke. Berlin: Dietz.

Marx, Karl. 1990 The Capital. Translated by Ben Fowkes. London: Penguin Classics.

Oxford English Dictionary. OED Online. December 2013. Oxford: Oxford University Press.

Pokorny, Julius. 1959. Indogermanisches etymologisches Wörterbuch. 2 vols. Bern: Francke. Available at: https://archive.org/details/indogermanisches02pokouoft.

Rey, Alain. 2012. Dire le travail: une histoire d'idées. Forum (2). http://www.revueforum.fr/2012/01/dire-le-travail-une-histoire-d\%E2\%80\%99idees/,

Ricardo, David. 1821. Principles of Political Economy. London: John Murray. Available at http://socserv.mcmaster.ca/econ/ugcm/3ll3/ricardo/Principles.pdf

Ruiz de Mendoza Ibañez, Francisco J. 2000. The Role of Mappings and Domains in Understanding Metonymy. In Metaphor and Metonymy at the Crossroads, edited by Antonio Barcelona, 109-132. Berlin/New York: Mouton de Gruyter, .

Shuōwén Jiězì. C. 100-121. http://www.shuowenjiezi.com/ gives access to the Shuōwén Jiězì (說文解字), the original etymological dictionary, and the later glosses.

Smith, Adam. 1999. The Wealth of Nations, Books I-III, edited by Andrew Skinner. London: Penguin Classics.

The Quranic Arabic Corpus. Available at: http://corpus.quran.com/.

Weber, Max. 2010. Die protestantische Ethik und der Geist des Kapitalismus, Vollständige Ausgabe. Edited by Dirk Kaesler. Munich: C.H. Beck.

Weber, Max. 2002. The Protestant Ethic and the Spirit of Capitalism and other writings, translated and edited by Peter Baer and Gordon C. Wells. Harmondsworh: Penguin.

Wilkinson, Helen. Quoted in Bar, 96.

http://www.helenwilkinson.com/ventures/founding\%20ventures.asp

\section{About the Author}

Olivier Frayssé

A graduate of École Normale Supérieure and Institut d'Études politiques de Paris, Olivier Frayssé is currently a Professor in the English Department of Paris-Sorbonne Université. His lifelong interest, whether as labour activist or researcher, has been work/labour, especially in relation with property, and the connection between work/labour, culture, and society. 


\title{
Digital Workers of the World Unite! A Framework for Critically Theorising and Analysing Digital Labour
}

\author{
Christian Fuchs* and Marisol Sandoval ${ }^{\dagger}$ \\ "University of Westminster, christian.fuchs@uti.at \\ ${ }^{\dagger}$ City University London, marisol.sandoval.1@city.ac.uk
}

\begin{abstract}
The overall task of this paper is to elaborate a typology of the forms of labour that are needed for the production, circulation, and use of digital media. First, we engage with the question what labour is, how it differs from work, which basic dimensions it has and how these dimensions can be used for defining digital labour. Second, we introduce the theoretical notion of the mode of production as analytical tool for conceptualizing digital labour. Modes of production are dialectical units of relations of production and productive forces. Relations of production are the basic social relations that shape the economy. Productive forces are a combination of labour power, objects and instruments of work in a work process, in which new products are created. Third, we have a deeper look at dimensions of the work process and the conditions under which it takes place. We present a typology that identifies dimensions of working conditions. It is a general typology that can be used for the analysis of any production process. Fourth, we apply the typology of working conditions to the realm of digital labour and identify different forms of digital labour and the basic conditions, under which they take place. Finally, we discuss political implications of our analysis and what can be done to overcome bad working conditions that digital workers are facing today.
\end{abstract}

Keywords: critical theory, critical political economy of communication and the media, social theory, digital labour, digital work, digital media, philosophy

Muhanga is an enslaved miner in Kivu (Democratic Republic of Congo). He extracts cassiterite, a mineral that is needed for the manufacturing of laptops and mobile phones: "As you crawl through the tiny hole, using your arms and fingers to scratch, there's not enough space to dig properly and you get badly grazed all over. And then, when you do finally come back out with the cassiterite, the soldiers are waiting to grab it at gunpoint. Which means you have nothing to buy food with. So we're always hungry" (Finnwatch 2007, 20).

The Chinese engineer Lu assembles mobile phones at Foxconn Shenzhen. He reports about overwork and exhaustion: "We produced the first generation iPad. We were busy throughout a 6-month period and had to work on Sundays. We only had a rest day every 13 days. And there was no overtime premium for weekends. Working for 12 hours a day really made me exhausted" (SACOM 2010, 7; for an analysis of Foxconn see also Sandoval 2013).

In Silicon Valley, the Cambodian ICT (information and communications technology) assembler Bopha has been exposed to toxic substances. He highlights: "I talked to my coworkers who felt the same way [that I did] but they never brought it up, out of fear of losing their job" (Pellow and Park 2002, 139).

Mohan, a project manager in the Indian software industry who is in his mid-30s, explains, "Work takes a priority. [...] The area occupied by family and others keeps reducing" (D'Mello and Sahay 2007, 179). Bob, a software engineer at Google explains that, "because of the large amounts of benefits (such as free foods) there seems to be an unsaid rule that employees are expected to work longer hours. Many people work more than 8 hours a day and then will be on email or work for a couple hours at home, at night as well (or on the weekends). It may be hard to perform extremely well with a good work/life balance. Advice to Senior Management-Give engineers more freedom to use $20 \%$ time to work on cool projects without the stress of having to do $120 \%$ work" (data source: www.glassdoor.com). 
Ann, a web designer, writer, and illustrator, offers her services on the freelance market platform People Per Hour that mediates the creation and purchase of products and services that are not remunerated by worked hours, but by a fixed product price. She describes her work:

\begin{abstract}
My design styles are as broad as my client base, from typical hard hitting, sound, clear, and concise business branding, to more stylised and fluid hand drawn or illustrated work. I relish working to a deadline, and although I often work to very specific criteria, some clients are looking for a moment of inspiration, and that's where I excel. I'm always ready for a challenge, and providing the brief is concise and well conceived. I can produce work to a very tight schedule. If you are online, you will see amendments almost immediately! (data source: peopleperhour.com).
\end{abstract}

The working lives of Muhanga, Lu, Bopha, Mohan, Bob, and Ann seem completely different. Muhanga extracts minerals from nature. Lu and Bopha are industrial workers. Mohan, Bob and Ann are information workers creating either software or designs. They work under different conditions, such as slavery, wage labour, or freelancing. Yet they have in common that their labour is in different ways related to the production and use of digital technologies and that ICT companies profit from it. In this paper we discuss the commonalities and differences of the working lives of workers like these by identifying different dimensions of digital labour.

Section 1 introduces a cultural-materialist perspective on theorising digital labour. Section 2 discusses the relevance of Marx's concept of the mode of production for the analysis of digital labour. Section 3 introduces a typology of the dimensions of working conditions. Section 4 based on the preceding sections presents a digital labour analysis toolbox. Finally, we draw some conclusions in section 5 .

\title{
1. (Digital) Work and Labour: A Cultural-Materialist Perspective
}

The digital labour debate has in a first phase focused mainly on understanding the value creation mechanisms on corporate social media such as Facebook, YouTube, and Twitter. Authors have for example discussed the usefulness of Karl Marx's labour theory of value (Fuchs 2010, Arvidsson and Colleoni 2012, Fuchs 2012b, Scholz 2013), how the notion of alienation shall be used in the context of digital labour (Andrejevic 2012, Fisher 2012), or if and how Dallas Smythe's concept of audience labour can be used for understanding digital labour (for an overview discussion see Fuchs 2012a). The book Social Media: A Critical Introduction (Fuchs 2014b) provides a general introduction to many of these issues. The general task has been to understand and conceptualise a situation in which users under realtime and far-reaching conditions of commercial surveillance create a data commodity that is sold to advertising clients. This involved a discussion of the question of who exactly creates the value that manifests itself in social media corporations' profits. But going beyond these initial debates, studying digital labour requires paying attention to digital labour in all its forms.

In approaching a definition of digital labour one can learn from debates on how to define cultural and communication labour.

\subsection{Defining Cultural Labour}

There exists a latent debate between Vincent Mosco and David Hesmondhalgh about how to define cultural and communication work and where to draw the boundaries. According to Hesmondhalgh cultural industries "deal primarily with the industrial production and circulation of texts" (Hesmondhalgh 2013, 16). Thus cultural industries include broadcasting, film, music, print and electronic publishing, video and computer games, advertising, marketing and public relations, and web design. Cultural labour is therefore according to this understanding all labour conducted in these industries. Cultural labour deals "primarily with the industrial production and circulation of texts" (Hesmondhalgh 2013, 17). Following this definition Hesmondhalgh describes cultural work as "the work of symbol creators" (Hesmondhalgh 2013, 20). 
Vincent Mosco and Catherine McKercher argue for a much broader definition of communication work, including "anyone in the chain of producing and distributing knowledge products" (Mosco and McKercher 2009, 25). In the case of the book industry, this definition includes not only writers but, equally, librarians and also printers.

Hesmondhalgh's definition of cultural industries and cultural work focuses on content production. Such a definition tends to exclude digital media, ICT hardware, software, and Internet phenomena such as social media and search engines. It thereby makes the judgment that content industries are more important than digital media industries. It is idealistic in that it focuses on the production of ideas and excludes the fact that these ideas can only be communicated based on the use of physical devices, computers, software, and the Internet. For Hesmondhalgh $(2013,19)$ software engineers for example are no cultural workers because he considers their work activity as "functional" and its outcomes not as text with social meaning. Software engineering is highly creative: it is not just about creating a piece of code that serves specific purposes, but also about writing the code by devising algorithms, which poses logical challenges for the engineers. Robert L. Glass (2006) argues that software engineering is a complex form of problem solving that requires a high level of creativity that he terms software creativity. Software is semantic in multiple ways: a) when its code is executed, each line of the code is interpreted by the computer which results in specific operations; b) when using a software application online or offline our brains constantly interpret the presented information; c) software not only supports cognition, but also communication and collaboration and therefore helps humans create and reproduce social meaning. Software engineers are not just digital workers. They are also cultural workers.

Hesmondhalgh opposes Mosco's and McKercher's broad definition of cultural work because "such a broad conception risks eliminating the specific importance of culture, of mediated communication, and of the content of communication products" (Hesmondhalgh and Baker 2011, 60). Our view is that there are many advantages of a broad definition as:

1. it avoids "cultural idealism" (Williams 1977, 19) that ignores the materiality of culture,

2. it can take into account the connectedness of technology and content, and

3. it recognizes the importance of the global division of labour, the exploitation of labour in developing countries, slavery and other bloody forms of labour and thereby avoids the Western-centric parochialism of cultural idealism.

Probably most importantly, a broad conception of cultural work can inform political solidarity: "A more heterogeneous vision of the knowledge-work category points to another type of politics, one predicated on questions about whether knowledge workers can unite across occupational or national boundaries, whether they can maintain their new-found solidarity, and what they should do with it" (Mosco and McKercher 2009, 26).

Likewise, Eli Noam opposes the separation of hardware and content producers and argues for a broad definition of the information industry: "Are the physical components of media part of the information sector? Yes. Without transmitters and receivers a radio station is an abstraction. Without PCs, routers, and servers there is no Internet" (Noam 2009, 46). Noam argues for a materialist unity of content and hardware producers in the category of the information industry.

While some definitions of creative work and creative industries are input- and occupationfocused (Caves 2000, Cunningham 2005, Hartley 2005), the broad notion of cultural work we are proposing focuses on industry and output. Input- and output-oriented definitions of cultural work/industries reflect a distinction that already Fritz Machlup (1962) and Daniel Bell (1974) used in their classical studies of the information economy: the one between occupational and industry definitions of knowledge work. Our approach differs both from inputoriented definitions and narrow output-oriented definitions.

We argue that cultural workers should be seen as what Marx termed Gesamtarbeiter. Marx describes this figure of the collective worker (Gesamtarbeiter) in the Grundrisse where he discusses labour as communal or combined labour (Marx 1857/1858, 470). This idea was also taken up in Capital, Volume 1, where he defines the collective worker as "a collective 
labourer, i.e. a combination of workers" (Marx 1867, 644), and argues that labour is productive if it is part of the combined labour force: "In order to work productively, it is no longer necessary for the individual himself to put his hand to the object; it is sufficient for him to be an organ of the collective labourer, and to perform any one of its subordinate functions" (ibid.). The collective worker is an "aggregate worker" whose "combined activity results materially in an aggregate product" (ibid., 1040). The "activity of this aggregate labour-power" is "the immediate production of surplus-value, the immediate conversion of this latter into capital" (ibid.).

The question of how to define cultural and eventually also digital labour has to do with the more general question of how to understand culture. It therefore makes sense to pay some attention to the works of one of the most profound cultural theorists: Raymond Williams.

\subsection{Cultural Materialism}

In his early works, Raymond Williams was trying to understand working-class culture in contrast to bourgeois culture, which illustrates his genuinely socialist position and interest in culture. But although Williams stresses the focus on totality, i.e. culture as "the way of life as a whole" (Williams 1958, 281) and "a general social process" (Williams 1958, 282), he in his early works tended to categorically separate culture and the economy: "even if the economic element is determining, it determines a whole way of life" (Williams 1958, 281). This notion of determination implies that the two realms of the economy and culture are connected, but that in the first instance they are also separate.

Later, in Marxism and Literature, Raymond Williams questioned Marxism's historical tendency to see culture as "dependent, secondary, 'superstructural': a realm of 'mere' ideas, beliefs, arts, customs, determined by the basic material history" (Williams 1977, 19). He discusses various concepts that Marxist theories have used for conceptualising the relationship of the economy and culture: determination, reflection, reproduction, mediation, homology. $\mathrm{He}$ argues that these concepts all assume a relationship between the economy and culture that to a varying degree is shaped by causal determination or mutual causality. But all of them would share the assumption of "the separation of 'culture' from material social life" (Williams $1977,19)$ that Williams $(1977,59)$ considers to be "idealist". In Williams view the problem with these approaches is not that they are too economistic and materialist but quite on the contrary that they are not "materialist enough" (Williams 1977, 92).

Williams $(1977,78)$ argues that Marx opposed the "separation of 'areas' of thought and activity". Production would be distinct from "consumption, distribution, and exchange" as well as from social relations (Williams 1977, 91). Productive forces would be "all and any of the means of the production and reproduction of real life", including the production of social knowledge and co-operation (Williams 1977, 91). Politics and culture would be realms of material production: ruling classes would produce castles, palaces, churches, prisons, workhouses, schools, weapons, a controlled press, etc. (Williams 1977, 93). Therefore Williams highlights the "material character of the production of a social and political order" and describes the concept of the superstructure an evasion (Williams 1977, 93). Here, Williams reflects Gramsci's insight that "popular beliefs" and "similar ideas are themselves material forces" (Gramsci 1988, 215).

Raymond Williams $(1977,111)$ formulates as an important postulate of Cultural Materialism that "[c]ultural work and activity are not [...] a superstructure" because people would use physical resources for leisure, entertainment, and art. Combining Williams' assumptions that cultural work is material and economic and that the physical and ideational activities underlying the existence of culture are interconnected means that culture is a totality that connects all physical and ideational production processes that are connected and required for the existence of culture. Put in simpler terms this means that for Williams the piano maker, the composer, and the piano player all are cultural workers.

Williams $(1977,139)$ concludes that Cultural Materialism needs to see "the complex unity of the elements" required for the existence of culture: ideas, institutions, formations, distribution, technology, audiences, forms of communication and interpretation, worldviews (138f). A 
sign system would involve the social relations that produce it, the institutions in which it is formed and its role as a cultural technology (Williams 1977, 140). In order to avoid the "real danger of separating human thought, imagination and concepts from 'men's material lifeprocess'" (Williams 1989, 203), one needs to focus on the "totality of human activity" (Williams 1989,203$)$ when discussing culture: We "have to emphasise cultural practice as from the beginning social and material" (Williams 1989, 206). The "productive forces of "mental labour' have, in themselves, an inescapable material and thus social history" (William 1989, 211). Marx expressed the basic assumption of Cultural Materialism well by stressing that the "production of ideas, of conceptions, of consciousness, is at first directly interwoven with the material activity and the material intercourse of men" (Marx and Engels 1845/46, 42). The production of ideas is therefore the "language of real life" (Marx and Engels 1845/46, 42). "Men are the producers of their conceptions, ideas, etc., that is, real, active men, as they are conditioned by a definite development of their productive forces and of the intercourse corresponding to these, up to its furthest forms" (Marx and Engels 1845/46, 42). Thinking and communicating for Marx are processes of production that are embedded into humans' everyday life and work. Human beings produce their own capacities and realities of thinking and communication in work and social relations.

In his later works, Williams stressed that it is particularly the emergence of an information economy in which information, communication, and audiences are sold as commodities that requires rethinking the separation of the economy and culture and to see culture as material. "[l]nformation processes [...] have become a qualitative part of economic organization" (Williams 1981, 231). "Thus a major part of the whole modern labour process must be defined in terms which are not easily theoretically separable from the traditional 'cultural' activities. [...] so many more workers are involved in the direct operations and activations of these systems that there are quite new social and social-class complexities" (Williams 1981, 232).

As information is an important aspect of economic production in information societies, the culture concept cannot be confined to popular culture, entertainment, works of arts, and the production of meaning through the consumption of goods, but needs to be extended to the realm of economic production and value creation. Cultural labour is a crucial concept in this context.

\subsection{A Materialist Notion of Cultural Labour}

Inspired by Raymond Williams' cultural materialism, it is feasible to argue for a broad understanding of cultural and digital labour that transcends the cultural idealism of the early digital labour debate and some positions within the cultural industries school. On the one hand Williams refutes the separation of culture and the economy as well as base and superstructure. On the other hand he maintains that culture, as a signifying system, is a distinct system of society. How can we make sense of these claims that at first sight seem to be mutually exclusive? If one thinks dialectically, then a concept of culture as material and necessarily economic and at the same time distinct from the economy is feasible: culture and politics are dialectical sublations (Aufhebung) of the economy. In Hegelian philosophy sublation means that a system or phenomenon is preserved, eliminated, and lifted up. Culture is not the same as the economy, it is more than the sum of various acts of labour, it has emergent qualitiesit communicates meanings in society-that cannot be found in the economy alone. But at the same time, the economy is preserved in culture: culture is not independent from labour, production and physicality, but requires and incorporates all of them.

Wolfgang Hofkirchner has introduced stage models as a way for philosophically conceptualizing the logic connections between different levels of organization. In a stage model, "one step taken by a system in question-that produces a layer-depends on the stage taken prior to that but cannot be reversed! [...] layers-that are produced by steps-build upon layers below them but cannot be reduced to them!" (Hofkirchner 2013, 123f). Emergence is the foundational principle of a stage model (Hofkirchner 2013, 115): a specific level of organization of matter has emergent qualities so that the systems organized on this level are more than the sum of their parts, to which they cannot be reduced. An organization level has new 
qualities that are grounded in the underlying systems and levels that are preserved on the upper level and through synergies produce new qualities of the upper level. In the language of dialectical philosophy this means that the emergent quality of an organization level is a sublation (Aufhebung) of the underlying level.

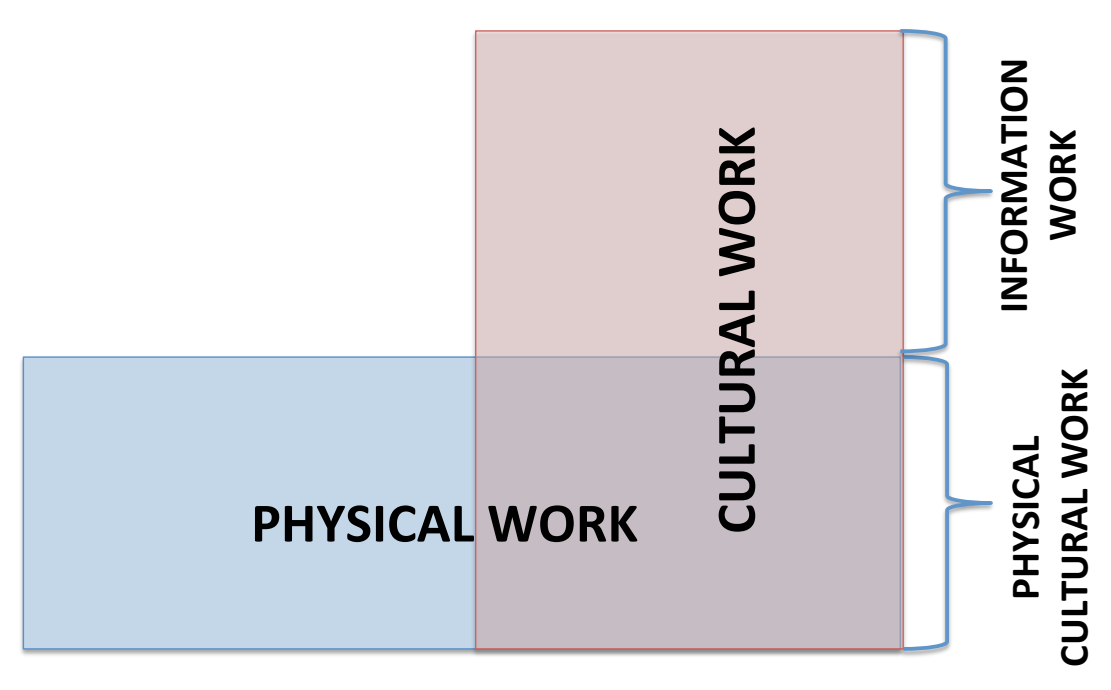

Figure 1: A stage model of cultural work

Using a stage model allows us to identify and relate different levels of cultural and digital work (see figure 1). Cultural work is a term that encompasses organisational levels of work that are at the same time distinct and dialectically connected: cultural work has an emergent quality, namely information work that creates content, that is based on and grounded in physical cultural work, which creates information technologies through agricultural and industrial work processes. Physical work takes place inside and outside of culture: it creates information technologies and its components (cultural physical work) as well as other products (non-cultural physical work) that do not primarily have symbolic functions in society (such as cars, tooth brushes or cups). Cars, toothbrushes, or cups do not primarily have the role of informing others or communicating with others, but rather help humans achieve the tasks of transport, cleanliness and nutrition. Culture and information work however feedback on these products and create symbolic meanings used by companies for marketing them. Cultural work is a unity of physical cultural work and information work that interact with each other, are connected and at the same time distinct.

The production of meaning, social norms, morals, and the communication of meanings, norms, and morals are work processes: they create cultural use-values. Culture requires on the one hand human creativity for creating cultural content and on the other hand specific forms and media for storage and communication. Work that creates information and communication through language is specific for work conducted in the cultural system: informational and communication work. For having social effects in society, information, and communication are organized (stored, processed, transported, analysed, transformed, created) with the help of information and communication technologies, such as computers, TV, radio, newspapers, books, recorded films, recorded music, language, etc. These technologies are produced by physical cultural work. Culture encompasses a) physical and informational work that create cultural technologies (information and communication technologies) and b) information work that creates information and communication.

These two types of work act together in order to produce and reproduce culture. Meanings and judgements are emergent qualities of culture that are created by informational work, they take on relative autonomy that has effects inside but also outside the economic system. This means that specific forms of work create culture, but culture cannot be reduced to the economy-it has emergent qualities. 
Following Williams, communication is the "passing of ideas, information, and attitudes from person to person", whereas communications means the "institutions and forms in which ideas, information, and attitudes are transmitted and received" (Williams 1962, 9). Information and communication are meaning-making activities created by informational work. Physical cultural work creates communications as institutions and forms that organize the creation and passing of information in social processes.

Marx identified two forms of information work: The first results in cultural goods that "exist separately from the producer, i.e. they can circulate in the interval between production and consumption as commodities, e.g. books, paintings and all products of art as distinct from the artistic achievement of the practising artist". In the second, "the product is not separable from the act of producing" (Marx 1867, 1047f). The first requires a form, institution or technology that stores and transports information, as in the case of computer-mediated communication, the second uses language as main medium (e.g. theatre). The first requires physical cultural work for organizing storage, organization, and transport of information; the second is possible based only on information work.

Given the notion of cultural labour and a cultural-materialist framework inspired by Raymond Williams, we can next ask the question what is specific about the digital mode of cultural labour.

\subsection{Digital Work and Digital Labour}

The realm of digital media is a specific subsystem of the cultural industries and of cultural labour. Digital labour is a specific form of cultural labour that has to do with the production and productive consumption of digital media. There are other forms of cultural labour that are non-digital. Think for example of a classical music or rock concert. But these forms of live entertainment that are specific types of cultural labour also do not exist independently from the digital realm: Artists publish their recordings in digital format on iTunes, Spotify, and similar online platforms. Fans bring their mobile phones for taking pictures and recording concert excerpts that they share on social media platforms. There is little cultural labour that is fully independent from the digital realm today. The notion of digital work and digital labour wants to signify those forms of cultural labour that contribute to the existence of digital technologies and digital content. It is a specific form of cultural labour. Figure 2 applies the stage model of cultural work (see figure 1 above) to digital work.

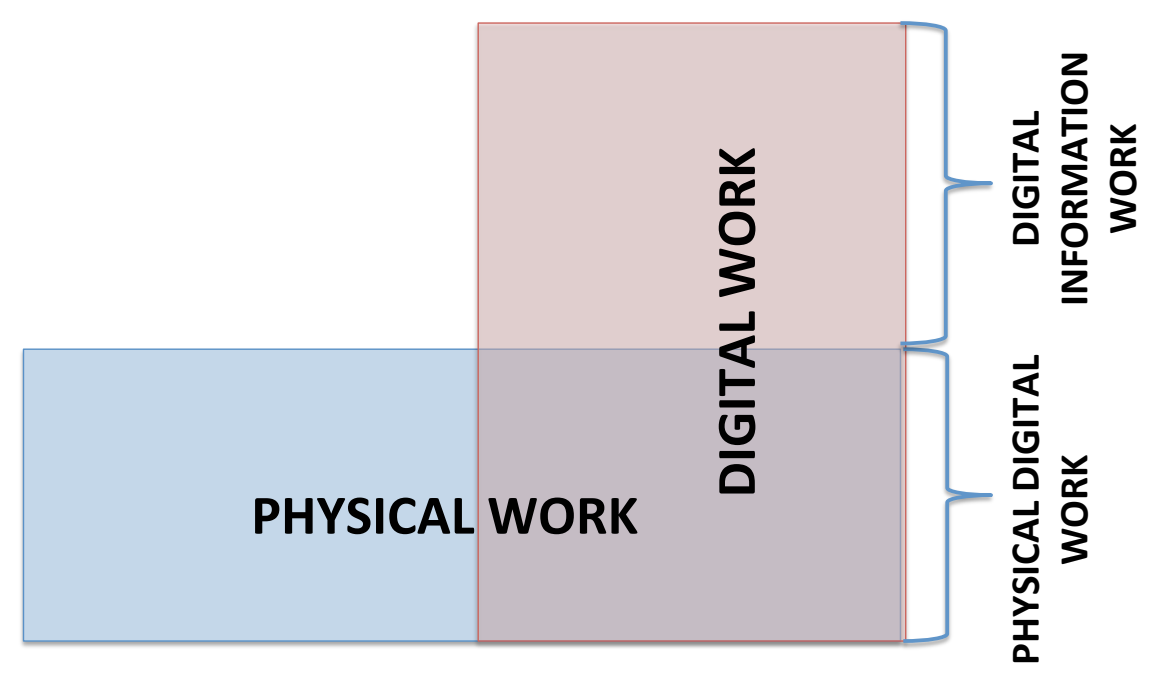

Figure 2: A stage model of digital work

If culture were merely symbolic, mind, spirit, "immaterial", superstructural, informational, a world of ideas, then digital labour as expression of culture clearly would exclude the concrete 
works of mining and hardware assemblage that are required for producing digital media. Williams' Cultural Materialism, contrary to the position of Cultural Idealism, makes it possible to argue that digital labour includes both the creation of physical products and information that are required for the production and usage of digital technologies. Some digital workers create hardware, others hardware components, minerals, software or content that are all objectified in or the outcome of the application of digital technologies. Some workers, e.g. miners, not just contribute to the emergence of digital media, but to different products. If one knows the mines' sales, then it is possible to determine to which extent the performed labour is digital or other labour.

In order to illustrate this point that culture is material, we now want return in greater detail to a passage where Marx reflects about the work of making and playing the piano. Marx wrote:

\begin{abstract}
Productive labour is only that which produces capital. Is it not crazy, asks e.g. (or at least something similar) $\mathrm{Mr}$ Senior, that the piano maker is a productive worker, but not the piano player, although obviously the piano would be absurd without the piano player? But this is exactly the case. The piano maker reproduces capital; the pianist only exchanges his labour for revenue. But doesn't the pianist produce music and satisfy our musical ear, does he not even to a certain extent produce the latter? He does indeed: his labour produces something; but that does not make it productive labour in the economic sense; no more than the labour of the madman who produces delusions is productive. Labour becomes productive only by producing its own opposite (Marx 1857/58, 305).
\end{abstract}

Williams remarks that today, other than in Marx's time, "the production of music (and not just its instruments) is an important branch of capitalist production" (Williams 1977, 93).

If the economy and culture are two separate realms, then building the piano is work and part of the economy and playing it is not work, but culture. Marx leaves however no doubt that playing the piano produces a use-value that satisfies human ears and is therefore a form of work. As a consequence, the production of music must just like the production of the piano be an economic activity. Williams $(1977,94)$ stresses that cultural materialism means to see the material character of art, ideas, aesthetics and ideology and that when considering piano making and piano playing it is important to discover and describe "relations between all these practices" and to not assume "that only some of them are material".

Apart from the piano maker and the piano player there is also the composer of music. All three forms of work are needed and necessarily related in order to guarantee the existence of piano music. Fixing one of these three productive activities categorically as culture and excluding the others from it limits the concept of culture and does not see that one cannot exist without the other. Along with this separation come political assessments of the separated entities. A frequent procedure is to include the work of the composer and player and to exclude the work of the piano maker. Cultural elitists then argue that only the composer and player are truly creative, whereas vulgar materialists hold that only the piano maker can be a productive worker because he works with his hands and produces an artefact. Both judgments are isolationist and politically problematic.

Taking the example of piano music and transferring it to digital media, we find correspondences: Just like we find piano makers, music composers and piano players in the music industry, we find labour involved in hardware production (makers), content and software production (composers) and productive users (prosumers, players, play labour) in the world of digital labour. In the realm of digital labour, we have to emphasize that practices are "from the beginning social and material" (Williams 1989, 206).

There is a difference if piano makers, players and music composers do so just as a hobby or for creating commodities that are sold on the market. This distinction can be explored based on Marx's distinction between work (Werktätigkeit) and labour (Arbeit): Brigitte Weingart (1997) describes the origins of the terms work in English and Arbeit and Werk in German: In German, the word Arbeit comes from the Germanic term arba, which meant slave. The English term work comes from the Middle English term weorc. It was a fusion of the Old English terms wyrcan (creating) and wircan (to affect something). So to work means 
to create something that brings about some changes in society. Weorc is related to the German terms Werk and werken. Both work in English and Werk in German were derived from the Indo-European term uerg (doing, acting). Werken in German is a term still used today for creating something. Its origins are quite opposed to the origins of the term Arbeit. The result of the process of werken is called Werk. Both werken and Werk have the connotative meaning of being creative. Both terms have an inherent connotation of artistic creation. Arendt (1958, 80f) confirms the etymological distinction between ergazesthai (Greek)/facere, fabricari (Latin)/work (English)/werken (German)/ouvrer (French) and ponein (Greek)/laborare (Latin)/labour (English)/arbeiten (German)/travailler (French).

Raymond Williams $(1983,176-179)$ argues that the word "labour" comes from the French word labor and the Latin term laborem and appeared in the English language first around 1300. It was associated with hard work, pain and trouble. In the 18th century, it would have attained the meaning of work under capitalist conditions that stands in a class relationship with capital. The term "work" comes from the Old English word weorc and is the "most general word for doing something" (ibid., 334). In capitalism the term on the one hand has, according to Williams (ibid., 334-337), acquired the same meaning as labour-a paid job-but would have in contrast also kept its original broader meaning. In order to be able to differentiate the dual historical and essential character of work, it is feasible to make a semantic differentiation between labour and work.

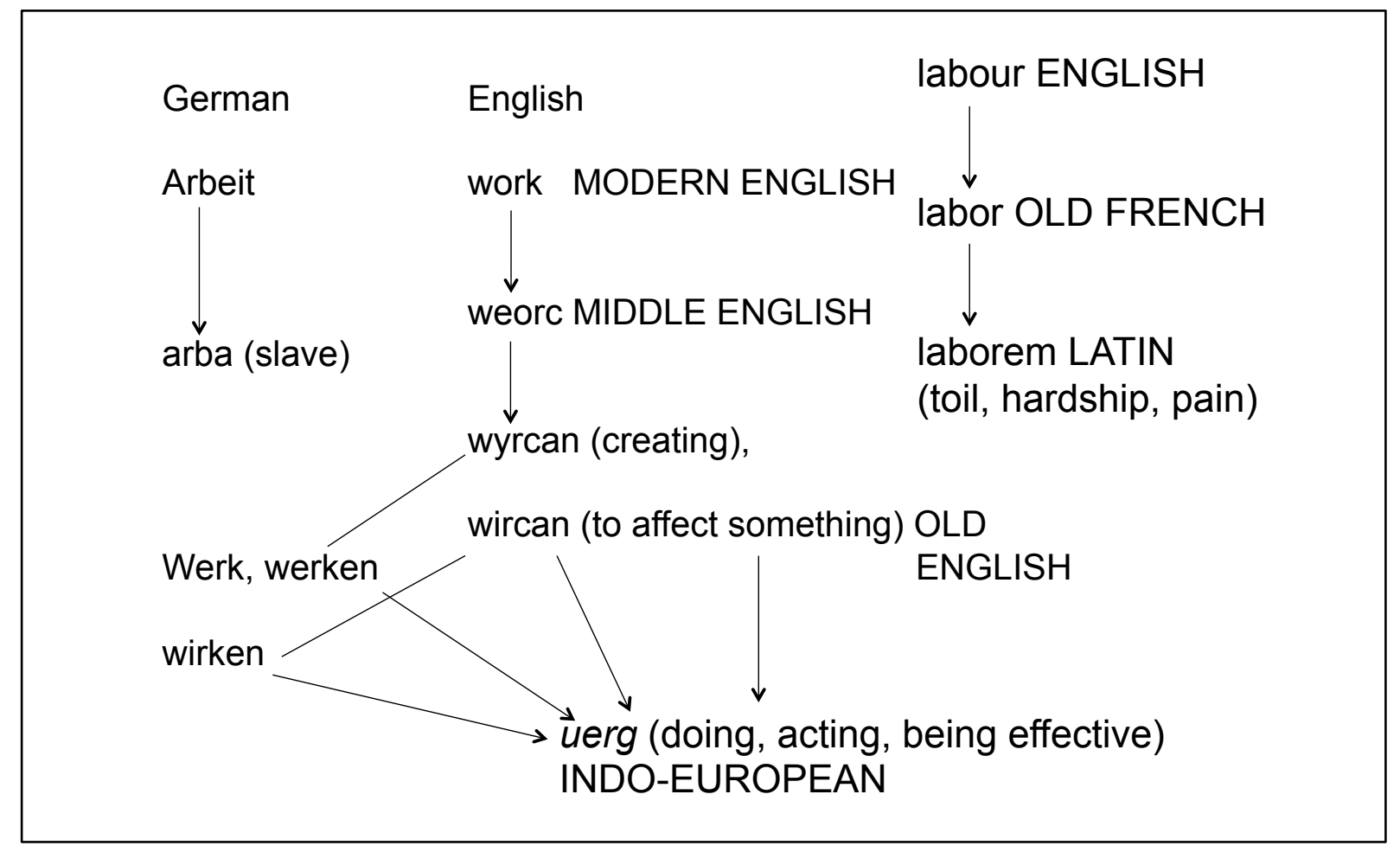

Figure 3: The etymology of the terms work, labour and Arbeit

The meaning and usage of words develops historically and may reflect the structures and changes of society, culture and the economy. Given that we find an etymological distinction between the general aspects of productive human activities and the specific characteristics that reflect the realities of class societies, it makes sense to categorically distinguish between the anthropological dimension of human creative and productive activities that result in usevalues that satisfy human needs and the historical dimension that describes how these activities are embedded into class relations (Fuchs 2014a). A model of the general work process is visualized in figure 4 . 


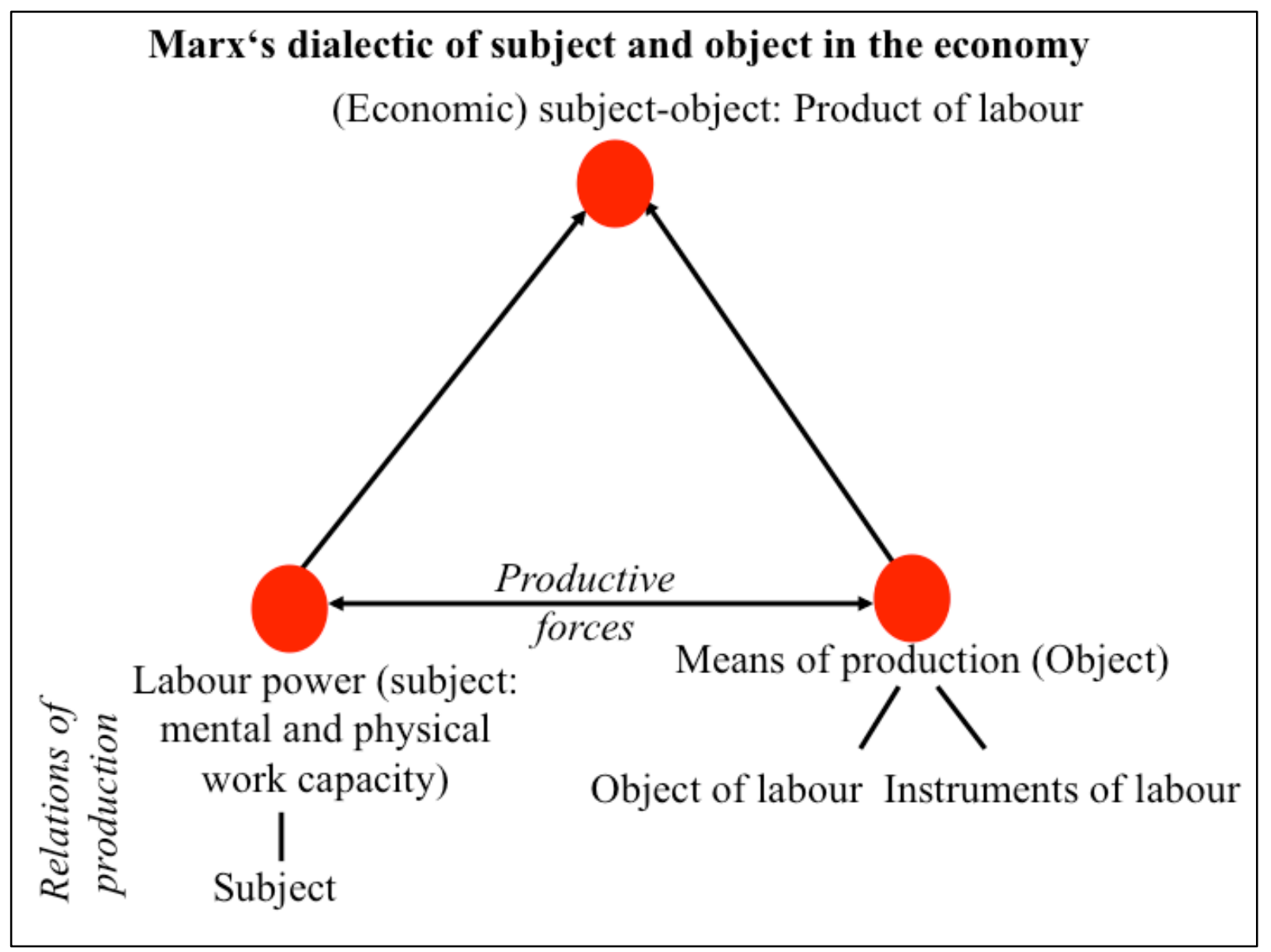

Figure 4: The general work process

Human subjects have labour power. Their labour in the work process interacts with the means of production (object). The means of production consist of the object of labour (resources, raw materials) and the instruments of labour (technology). In the work process, humans transform an object (nature, culture) by making use of their labour power with the help of instruments of labour. The result is a product that unites the objectified labour of the subject with the objective materials s/he works on. Work becomes objectified in a product and the object is as a result transformed into a use value that serves human needs. The productive forces are a system, in which subjective productive forces (human labour power) make use of technical productive forces (part of the objective productive forces) in order to transform parts of the nature/culture so that a product emerges.

The general work process is an anthropological model of work under all historical conditions. The connection of the human subject to other subjects in figure 4 indicates that work is normally not conducted individually, but in relations with others. A society could hardly exist based on isolated people trying to sustain themselves independently. It requires economic relations in the form of co-operation and a social organization of production, distribution and consumption. This means that work takes place under specific historical social relations of production. There are different possibilities for the organization of the relations of production. In general the term labour points towards the organization of labour under class relations, i.e. power relationships that determine that any or some of the elements in the work process are not controlled by the workers themselves, but by a group of economic controllers. Labour designates specific organization forms of work, in which the human subject does not control his/her labour power (she is compelled to work for others) and/or there is a lack of control of the objects of labour and/or the instruments of labour and/or the products of labour.

Karl Marx pinpoints this lack of control by the term alienation and understands the unity of these forms of alienation as exploitation of labour: "The material on which it [labour] works is 
alien material; the instrument is likewise an alien instrument; its labour appears as a mere accessory to their substance and hence objectifies itself in things not belonging to it. Indeed, living labour itself appears as alien vis-à-vis living labour capacity, whose labour it is, whose own life's expression it is, for it has been surrendered to capital in exchange for objectified labour, for the product of labour itself. [...] labour capacity's own labour is as alien to it - and it really is, as regards its direction etc.-as are material and instrument. Which is why the product then appears to it as a combination of alien material, alien instrument and alien labour-as alien property" (Marx 1857/58, 462). Figure 5 visualizes potential dimensions of the labour process as alienated work process.

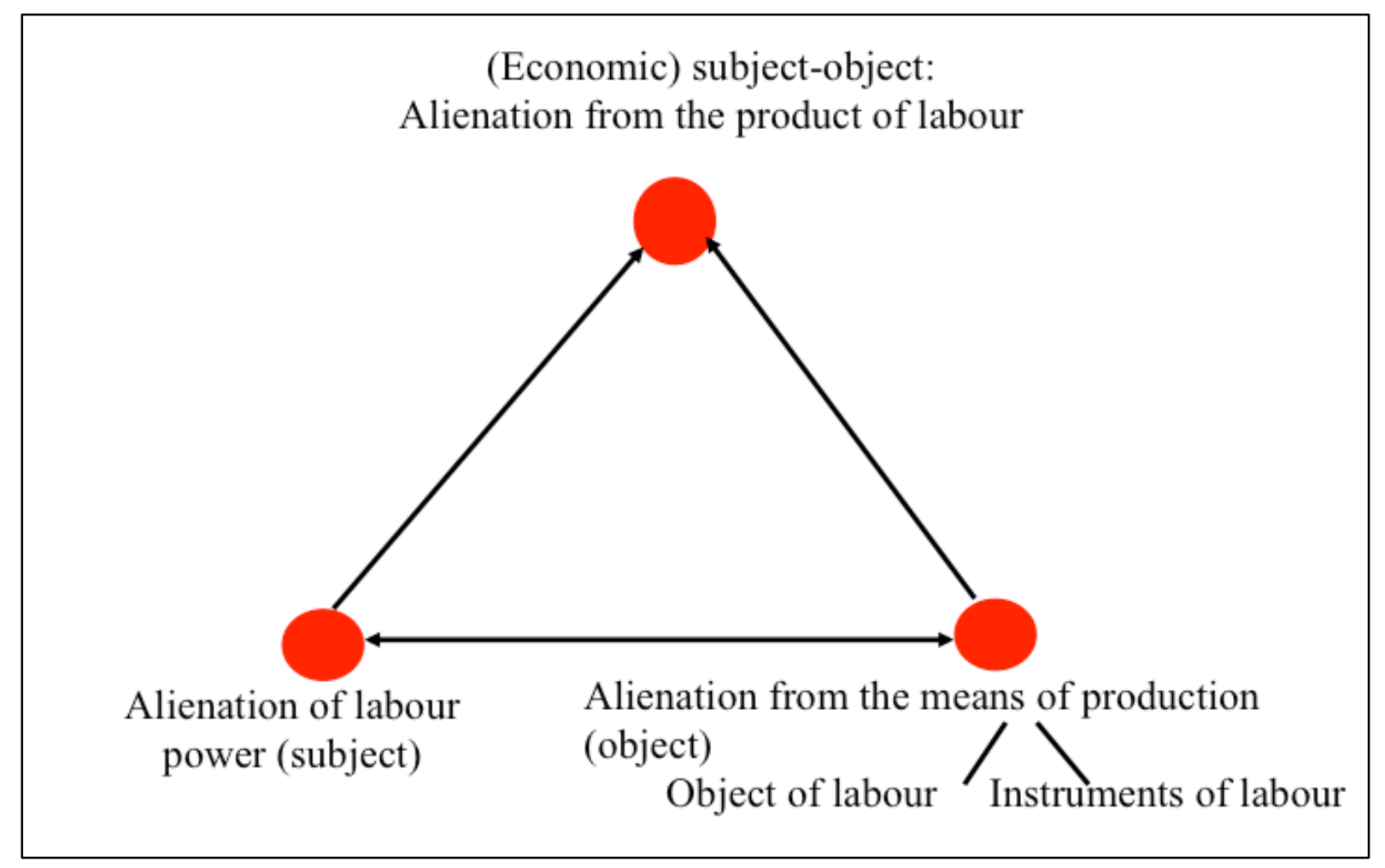

Figure 5: Labour as alienated work process

Given these preliminary assumptions about the work-labour distinction and cultural materialism, one can provide a definition of digital work and digital labour:

Digital work is a specific form of work that makes use of the body, mind or machines or a combination of all or some of these elements as an instrument of work in order to organize nature, resources extracted from nature, or culture and human experiences, in such a way that digital media are produced and used. The products of digital work are depending on the type of work: minerals, components, digital media tools or digitally mediated symbolic representations, social relations, artefacts, social systems and communities. Digital work includes all activities that create use-values that are objectified in digital media technologies, contents and products generated by applying digital media" (Fuchs 2014a, 352).

Digital labour is alienated digital work: it is alienated from itself, from the instruments and objects of labour and from the products of labour. Alienation is alienation of the subject from itself (labour-power is put to use for and is controlled by capital), alienation from the object (the objects of labour and the instruments of labour) and the subject-object (the products of labour). Digital work and digital labour are broad categories that involve all activities in the production of digital media technologies and contents. This means that in the capitalist media industry, different forms of alienation and exploitation can be encountered. Examples are slave workers in mineral extraction, Taylorist hardware assemblers, 
software engineers, professional online content creators (e.g. online journalists), call centre agents and social media prosumers" (Fuchs 2014a, 351f).

Work and labour are not isolated individual activities, but take place as part of social relations and larger modes of how the economy is organised. The concepts of digital work and digital labour need therefore to be related to a concept that can describe the organisational structure of the economy. One such concept is Marx's notion of the mode of production.

\section{Digital Labour and Modes of Production}

Michael Porter (1985) introduced the notion of the value chain that he defined as "a collection of activities that are performed to design, produce, market, deliver and support its product" (Porter 1985: 36). The term value chain has become a popular category for analysing the organisation of capital, which is indicated by the circumstance that 11682 articles indexed in the academic database Business Source Premier use the term in their abstract (accessed on May 21,2013$)$. The term has also been used in mainstream media economics for analysing the value chains of traditional media and ICTs (see Zerdick et al. 2000: 126-135). The problem of the mainstream use of the concept of the value chain is that it focuses on the stages in commodity production and tends to neglect aspects of working conditions and class relations. Also critical scholars have used the notion of the global value chain (see for example: Huws 2008, Huws and Dahlmann 2010).

An alternative concept that was introduced by critical studies is the notion of the new international division of labour (NIDL):

The development of the world economy has increasingly created conditions (forcing the development of the new international division of labour) in which the survival of more and more companies can only be assured through the relocation of production to new industrial sites, where labour-power is cheap to buy, abundant and well-disciplined; in short, through the transnational reorganization of production (Fröbel, Heinrichs and Kreye 1981, 15).

A further development is that "commodity production is being increasingly subdivided into fragments which can be assigned to whichever part of the world can provide the most profitable combination of capital and labour" (Fröbel, Heinrichs and Kreye 1981, 14). In critical media and cultural studies, Miller et al. (2004) have used this concept for explaining the international division of cultural labour (NICL). The concept of the NIDL has the advantage that it stresses the class relationship between capital and labour and how in processes of class struggle capital tries to increase profits by decreasing its overall wage costs via the global diffusion of the production process. It is also a concept that encompasses workers' struggles against the negative effects of capitalist restructuring.

The approach taken in this paper stands in the Marxist tradition that stresses class contradictions in the analysis of globalisation. It explores how the notion of the mode of production can be connected to the concept of the new international division of labour. The notion of the mode of production stresses a dialectical interconnection of on the one hand class relationships (relations of production) and on the other hand the forms of organisation of capital, labour and technology (productive forces). The class relationship is a social relationship that determines who owns private property and has the power to make others produce surplusvalue that they do not own and that is appropriated by private property owners. Class relationships involve an owning class and a non-owing class: the non-owning class is compelled to produce surplus value that is appropriated by the owning class.

The relations of production determine the property relations (who owns which share (full, some, none) of labour power, the means of production, products of labour), the mode of allocation and distribution of goods, the mode of coercion used for defending property relations and the division of labour. Class relationships are forms of organization of the relations of production, in which a dominant class controls the modes of ownership, distribution and coercion for exploiting a subordinated class. In a classless society human control ownership and distribution in common. 
Every economy produces a certain amount of goods per year. Specific resources are invested and there is a specific output. If there is no contraction of the economy due to a crisis, then a surplus product is created, i.e. an excess over the initial resources. The property relations determine who owns the economy's initial resources and surplus. Table 2 (see further below) distinguishes modes of production (patriarchy, slavery, feudalism, capitalism, communism) based on various modes of ownership, i.e. property relations.

The mode of allocation and distribution defines how products are distributed and allocated: In a communist society, each person gets whatever s/he requires to survive and satisfy human needs. In class societies, distribution is organized in the form of exchange: exchange means that one product is exchanged for another. If you have nothing to exchange because you own nothing, then you cannot get hold of other goods and services, except those that are not exchanged, but provided for free. There are different forms how exchange can be organized: general exchange, exchange for exchange-value ( $x$ commodity $A=y$ commodity $B$ ), exchange for maximum exchange-value, exchange for capital accumulation.

The mode of coercion takes on the form of physical violence (overseers, security forces, military), structural violence (markets, institutionalised wage labour contracts, legal protection of private property, etc) and cultural violence (ideologies that present the existing order as the best possible or only possible order and try to defer the causes of societal problems by scapegoating). In a free society no mode of coercion is needed.

The division of labour defines who conducts which activities in the household, the economy, politics and culture. Historically there has been a gender division of labour, a division between mental and physical work, a division into many different functions conducted by specialists and an international division of labour that is due to the globalization of production. Marx in contrast imagined a society of generalists that overcomes the divisions of labour so that society is based on well-rounded universally active humans (Marx 1867, 334-335). Marx $(1857 / 58,238)$ says that in class society "labour will create alien property and property will command alien labour". The historical alternative is a communist society and mode of production, in which class relationships are dissolved and the surplus product and private property are owned and controlled in common.

The relations of production are dialectically connected to the system of the productive forces (see figure 3 in section 1 of this paper): human subjects have labour power that in the labour process interacts with the means of production (object). The means of production consist of the object of labour (natural resources, raw materials) and the instruments of labour (technology). In the labour process, humans transform the object of labour (nature, culture) by making use of their labour power with the help of instruments of labour. The result is a product of labour, which is a Hegelian subject-object, or, as Marx says, a product, in which labour has become bound up in its object: labour is objectified in the product and the object is as a result transformed into a use value that serves human needs. The productive forces are a system, in which subjective productive forces (human labour power) make use of technical productive forces (part of the objective productive forces) in order to transform parts of the natural productive forces (which are also part of the objective productive forces) so that a labour product emerges. One goal of the development of the system of productive forces is to increase the productivity of labour, i.e. the output (amount of products) that labour generates per unit of time. Marx $(1867,431)$ spoke in this context of the development of the productive forces. Another goal of the development of the productive forces can be the enhancement of human self-development by reducing necessary labour time and hard work (toil).

In Capital, Marx (1867) makes a threefold distinction between labour-power, the object of labour and the instruments of labour: "The simple elements of the labour process are (1) purposeful activity, (2) the object on which that work is performed, and (3) the instruments of that work" (284). Marx's discussion of the production process can be presented in a systematic way by using Hegel's concept of the dialectic of subject and object. Hegel (1991) has spoken of a dialectical relation of subject and object: the existence of a producing subject is based on an external objective environment that enables and constrains (i.e. conditions) human existence. Human activities can transform the external (social, cultural, economic, politi- 
cal, natural) environment. As a result of the interaction of subject and object, new reality is created-Hegel terms the result of this interaction "subject-object". Figure 5 shows that $\mathrm{He}-$ gel's notion of subject, object, and subject-object form a dialectical triangle.

Hegel (1991) characterizes the "subjective concept" as formal notion (\$162), a finite determination of understanding a general notion (§162), "altogether concrete" (§164). He defines "the subject" as "the posited unseparatedness of the moments in their distinction" (§164). Hegel characterizes objectivity as totality (§193),"external objectivity"(§208),"external to an other" (§193), "the objective world in general" that "falls apart inwardly into [an] undetermined manifoldness" (§193), "immediate being" (§194), "indifference vis-à-vis the distinction" (§194), "realisation of purpose" (§194), "purposive activity" (§206) and "the means" (§206).The Idea is "the Subject-Object" (§162), absolute Truth (§162), the unity of the subjective and the objective (\$212), "the absolute unity of Concept and objectivity" (§213), "the Subject-Object" understood as "the unity of the ideal and the real, of the finite and the infinite, of the soul and the body" (\$214). Hegel also says that the "Idea is essentially process" (\$215). Marx applied Hegel's dialectic of subject and object on a more concrete level to the economy in order to explain how the process of economic production works as an interconnection of a subject (labour power) and an object (objects and instruments) so that a subject-object (product) emerges (see figure 6).

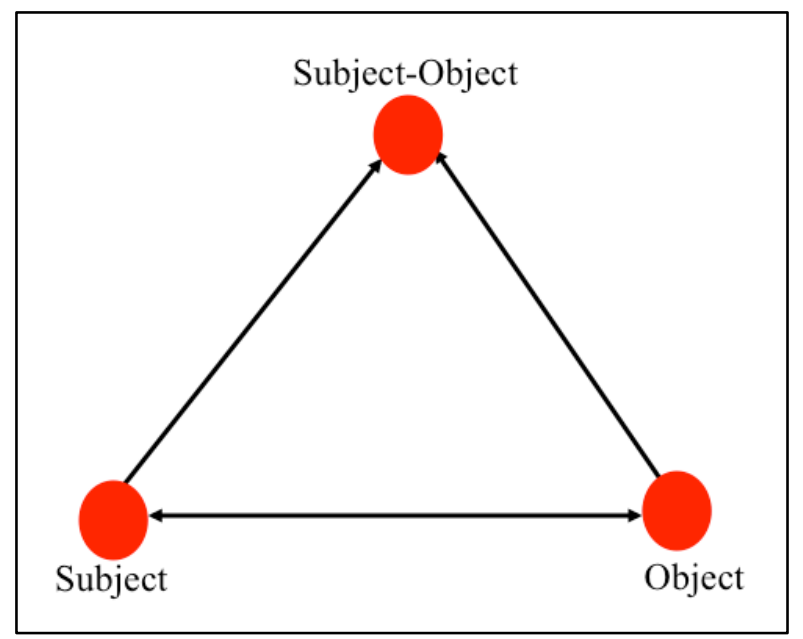

Figure 6: Hegel's dialectic of subject and object

The instruments of work can be the human brain and body, mechanical tools and complex machine systems. They also include specific organizations of space-time, i.e. locations of production that are operated at specific time periods. The most important aspect of time is the necessary work time that depends on the level of productivity. It is the work time that is needed per year for guaranteeing the survival of a society. The objects and products of work can be natural, industrial or informational resources or a combination thereof.

The productive forces are a system of production that creates use-values. There are different modes of organization of the productive forces, such as agricultural productive forces, industrial productive forces and informational productive forces. Table 1 gives an overview. 


\begin{tabular}{|l|l|l|l|}
\hline Mode & Instruments of work & Objects of work & $\begin{array}{l}\text { Products of } \\
\text { work }\end{array}$ \\
\hline $\begin{array}{l}\text { Agricultural productive } \\
\text { forces }\end{array}$ & $\begin{array}{l}\text { Body, brain, tools, } \\
\text { machines }\end{array}$ & Nature & Basic products \\
\hline $\begin{array}{l}\text { Industrial productive } \\
\text { forces }\end{array}$ & $\begin{array}{l}\text { Body, brain, tools, } \\
\text { machines }\end{array}$ & $\begin{array}{l}\text { Basic products, in- } \\
\text { dustrial products }\end{array}$ & $\begin{array}{l}\text { Industrial prod- } \\
\text { ucts }\end{array}$ \\
\hline $\begin{array}{l}\text { Informational produc- } \\
\text { tive forces }\end{array}$ & $\begin{array}{l}\text { Body, brain, tools, } \\
\text { machines }\end{array}$ & Experiences, ideas & $\begin{array}{l}\text { Informational } \\
\text { products }\end{array}$ \\
\hline
\end{tabular}

Table 1. Three Modes of Organization of the Productive Forces

Figure 7 shows dimensions of the relations of production and the productive forces.

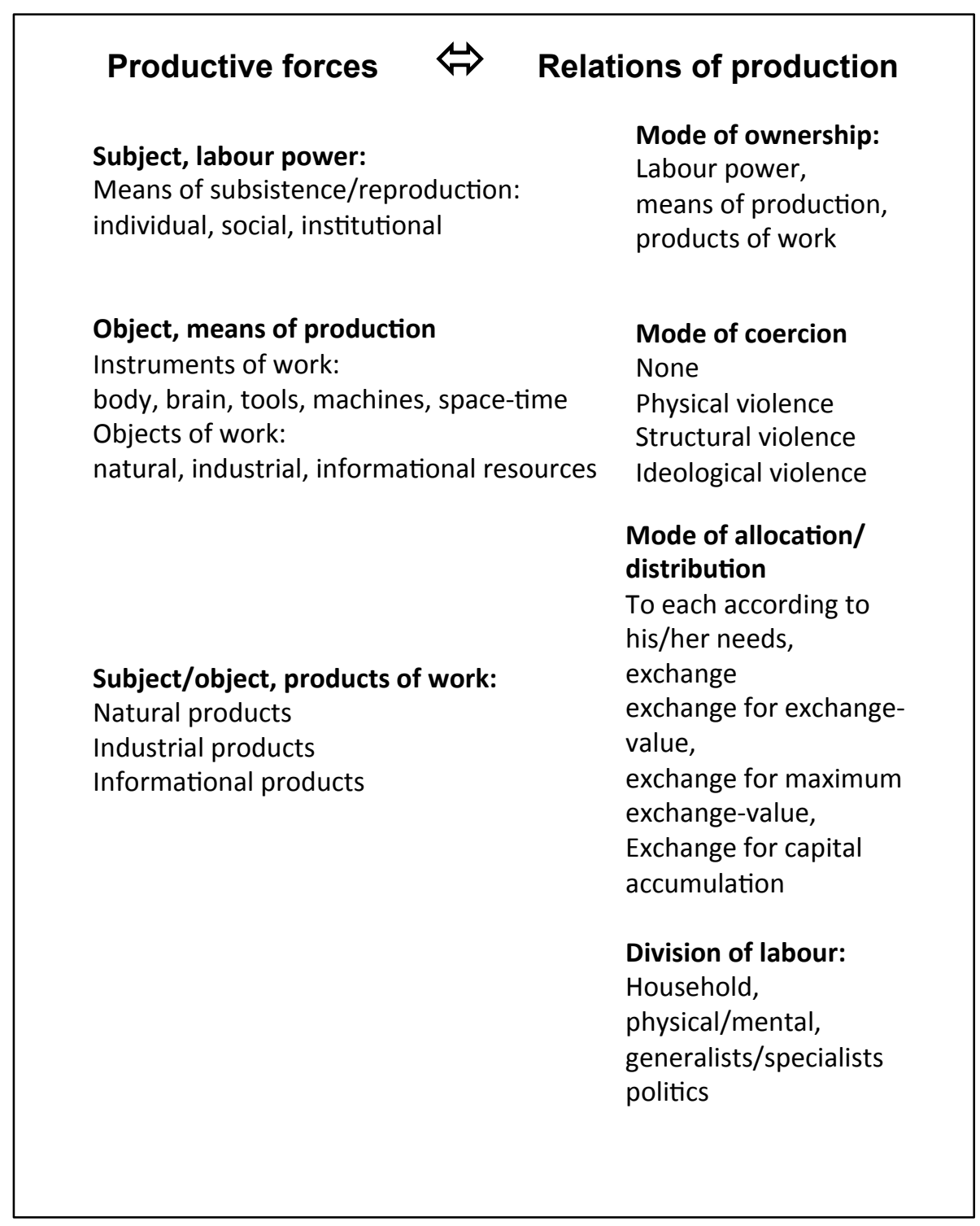

Figure 7: Dimensions of the Productive Forces and the Relations of Production 
Classical slavery, serfdom and wage labour are three important historical forms of class relations that are at the heart of specific modes of production (Engels 1884). Marx and Engels argue that private property and slavery have their origin in the family: The first historical form of private property can be found in the patriarchal family (Marx and Engels 1845/46, 52). The family is a mode of production, in which labour power is no commodity, but organised by personal and emotional relationships that result in commitment that includes family work that is unremunerated and produces affects, social relations and the reproduction of the human mind and body. It can therefore also be called reproductive work.

A wage worker's labour power has a price, its wage, whereas a slave's labour power does not have a price-it is not a commodity. However, the slave him-/herself has a price, which means that its entire human body and mind can be sold as a commodity from one slave owner to another, who then commands the entire life time of the slave (Marx, 1857/58: 288289). The slave in both ancient slavery and feudalism is treated like a thing and has the status of a thing (Marx 1857/58, 464-465).

In the Grundrisse's section "Forms which precede capitalist production" (Marx 1857/58, 471-514) as well as in the German Ideology's section "Feuerbach: Opposition of the materialist and idealist outlooks" (Marx and Engels 1845/46), Marx discusses the following modes of production:

1. The tribal community based on the patriarchal family;

2. Ancient communal property in cities (Rome, Greece);

3. Feudal production in the countryside;

4. Capitalism.

Table 2 provides a classification of modes of production based on the dominant forms of ownership (self-control, partly self-control and partly alien control, full alien control)

\begin{tabular}{|l|l|l|l|}
\hline & $\begin{array}{l}\text { Owner of labour } \\
\text { power }\end{array}$ & $\begin{array}{l}\text { Owner of the means } \\
\text { of production }\end{array}$ & $\begin{array}{l}\text { Owner of the prod- } \\
\text { ucts of work }\end{array}$ \\
\hline Patriarchy & Patriarch & Patriarch & Family \\
\hline Slavery & Slavemaster & Slavemaster & Slavemaster \\
\hline Feudalism & $\begin{array}{l}\text { Partly self-control, } \\
\text { partly lord }\end{array}$ & $\begin{array}{l}\text { Partly self-control, } \\
\text { partly lord }\end{array}$ & $\begin{array}{l}\text { Partly self-control, } \\
\text { partly lord }\end{array}$ \\
\hline Capitalism & Worker & Capitalist & Capitalist \\
\hline Communism & Self & All & $\begin{array}{l}\text { Partly all, partly indi- } \\
\text { vidual }\end{array}$ \\
\hline
\end{tabular}

Table 2: The main forms of ownership in various modes of production

But how are modes of production related to each other? In a historical way, where they supersede each other, or in a historical-logical way within a specific social formation that sublates older formations but encompasses older modes of production into itself? Jairus Banaji (2011) argues that Stalinism and vulgar Marxism have conceptualised the notion of the mode of production based on the assumption that a specific mode contains only one specific historical form of labour and surplus-value appropriation and eliminates previous modes so that history develops in the form of a linear evolution: slavery $\rightarrow$ feudalism $\rightarrow$ capitalism $\rightarrow$ communism. So for example Althusser and Balibar (1970) argue that the historical development of society is non-dialectical and does not involve sublations, but rather transitions "from one mode of production to another" (Althusser and Balibar 1970, 307) so that one mode succeeds the other. This concept of history is one of the reasons why E.P. Thompson (1978, 131) has characterized Althusser's approach as "Stalinism at the level of theory". The Stalinist "metaphysical-scholastic formalism" (Banaji 2011, 61) has been reproduced in liberal theory's assumption that there is an evolutionary historical development from the agricultural society to the industrial society to the information society so that each stage eliminates the previous one (as argued by: Bell 1974; Toffler 1980), which shows that in the realm of theory 
some liberals of today share in their theory elements of Stalinism. According to Banaji, capitalism often intensified feudal or semi feudal production relations. In parts of Europe and outside, feudalism would have only developed as a "commodity-producing enterprise" (Banaji $2011,88)$. In the Islamic world capitalism would have developed without slavery and feudalism (Banaji 2011, 6).

Banaji advances in contrast to formalist interpretations a complex reading of Marx's theory, in which a mode of production is "capable of subsuming often much earlier forms" (Banaji 2011,1 ), "similar forms of labour-use can be found in very different modes of production" (6), capitalism is "working through a multiplicity of forms of exploitation" (145) and is a combined form of development (358) that integrates "diverse forms of exploitation and ways of organising labour in its drive to produce surplus value" (359).

A mode of production is a unity of productive forces and relations of production (Marx and Engels 1845/46, 91). If these modes are based on classes as their relations of production, then they have specific contradictions that can via class struggles result in the sublation (Aufhebung) of one mode of production and the emergence of a new one. The emergence of a new mode of production does not necessarily abolish, but rather sublate (aufheben) older modes of production. This means that history is for Marx a dialectical process precisely in Hegel's threefold meaning of the term Aufhebung (sublation): 1) uplifting, 2) elimination, 3) preservation: 1) There are new qualities of the economy, 2) the dominance of an older mode of production vanishes, 3 ) but this older mode continues to exist in the new mode in a specific form and relation to the new mode. The rise of e.g. capitalism however did not bring an end to patriarchy, but the latter continued to exist in such a way that a specific household economy emerged that fulfils the role of the reproduction of modern labour power. A sublation can be more or less fundamental. A transition from capitalism to communism requires a fundamental elimination of capitalism, the question is however if this is immediately possible. Elimination and preservation can take place to differing degrees. A sublation is also no linear progression. It is always possible that relations that resemble earlier modes of organization are created.

Capitalism is at the level of the relations of production organised around relations between capital owners on the one side and paid/unpaid labour and the unemployed on the other side. On the level of the productive forces, it has developed from industrial to informational productive forces. The informational productive forces do not eliminate, but sublate (aufheben) other productive forces (Adorno 1968/2003, Fuchs 2014a, chapter 5): in order for informational products to exist a lot of physical production is needed, which includes agricultural production, mining and industrial production. The emergence of informational capitalism has not virtualised production or made it weightless or immaterial, but is grounded in physical production (Huws 1999, Maxwell and Miller 2012). Whereas capitalism is a mode of production, the terms agricultural society, industrial society and information society characterise specific forms of the organisation of the productive forces (Adorno 1968/2003; Fuchs 2014a, chapter 5).

The new international division of labour (NIDL) organises the labour process in space and time in such a way that specific components of the overall commodity are produced in specific spaces in the global economy and are reassembled in order to form a coherent whole that is sold as a commodity. It thereby can command labour on the whole globe and during the whole day. The approach taken by the authors of this paper advocates a broad understanding of digital labour based on an industry rather than an occupation definition in order to stress the commonality of exploitation, capital as the common enemy of a broad range of workers and the need to globalize and network struggles in order to overcome the rule of capitalism. Some of the workers described in this article are not just exploited by digital media capital, but also and sometimes simultaneously by other forms of capital. It is then a matter of degree to which extent these forms of labour are digital labour and simultaneously other forms of labour. If we imagine a company with job rotation so that each worker on average assembles laptops for $50 \%$ of his/her work time and cars for the other half of the time, a worker in this factory is a digital worker for $50 \%$. S/he is however an industrial worker for $100 \%$ because the content of both manufacturing activities is the industrial assemblage of 
components into commodities. The different forms of digital labour are connected in an international division of digital labour (IDDL), in which all labour necessary for the existence, usage and application of digital media is "disconnected, isolated [...], carried on side by side" and ossified "into a systematic division" (Marx 1867, 456).

Given a model of the mode of production, the question arises how one can best analyze the working conditions in a specific company, industry or sector of the economy when conducting a labour process and class analysis. Which dimensions of labour have to be taken into account in such an analysis? The next section will address this question.

\section{A Typology of the Dimensions of Working Conditions}

A suitable starting point for a systematic model of different dimensions of working conditions is the circuit of capital accumulation as Karl Marx described it $(1867,248-253 ; 1885,109)$. According to Marx, capital accumulation in a first stage requires the investment of capital in order to buy what is necessary for producing commodities, the productive forces: labour time of workers ( $L$ or variable capital) on the one hand, and working equipment like machines and raw materials (MoP or constant capital) on the other hand (Marx 1885/1992, 110). Thus, money $(\mathrm{M})$ is used in order to buy labour power as well as machines and resources as commodities $(C)$ that then in a second stage enter the labour process and produce $(P)$ a new commodity (C') (Marx 1885, 118). This new commodity $\left(C^{\prime}\right)$ contains more value than the sum of its parts, i.e. surplus value. This surplus value needs to be realized and turned into more money (M') by selling the commodity in the market (Marx 1885/1992, 125). The circuit of capital accumulation can thus be described with the following formula:

$$
M \rightarrow C \ldots P \ldots C^{\prime} \rightarrow M^{\prime}(\operatorname{Marx} 1885,110)
$$

According to Marx, surplus value can only be generated due to the specific qualities of labour-power as a commodity. Marx argued that labour power is the only commodity "whose use-value possesses the peculiar property of being a source of value, whose actual consumption is therefore itself an objectification of labour, hence a creation of value" (Marx 1867, 270).

Labour is thus essential to the process of capital accumulation. The model in figure 7 takes the labour process as its point of departure for identifying different dimensions that shape working conditions (Sandoval 2013). The purpose of this model is to provide comprehensive guidelines that can be applied for systematically studying working conditions in different sectors (for a systematic study of corporate irresponsibility of working and production conditions in 8 companies in the media industries see Sandoval 2014). 


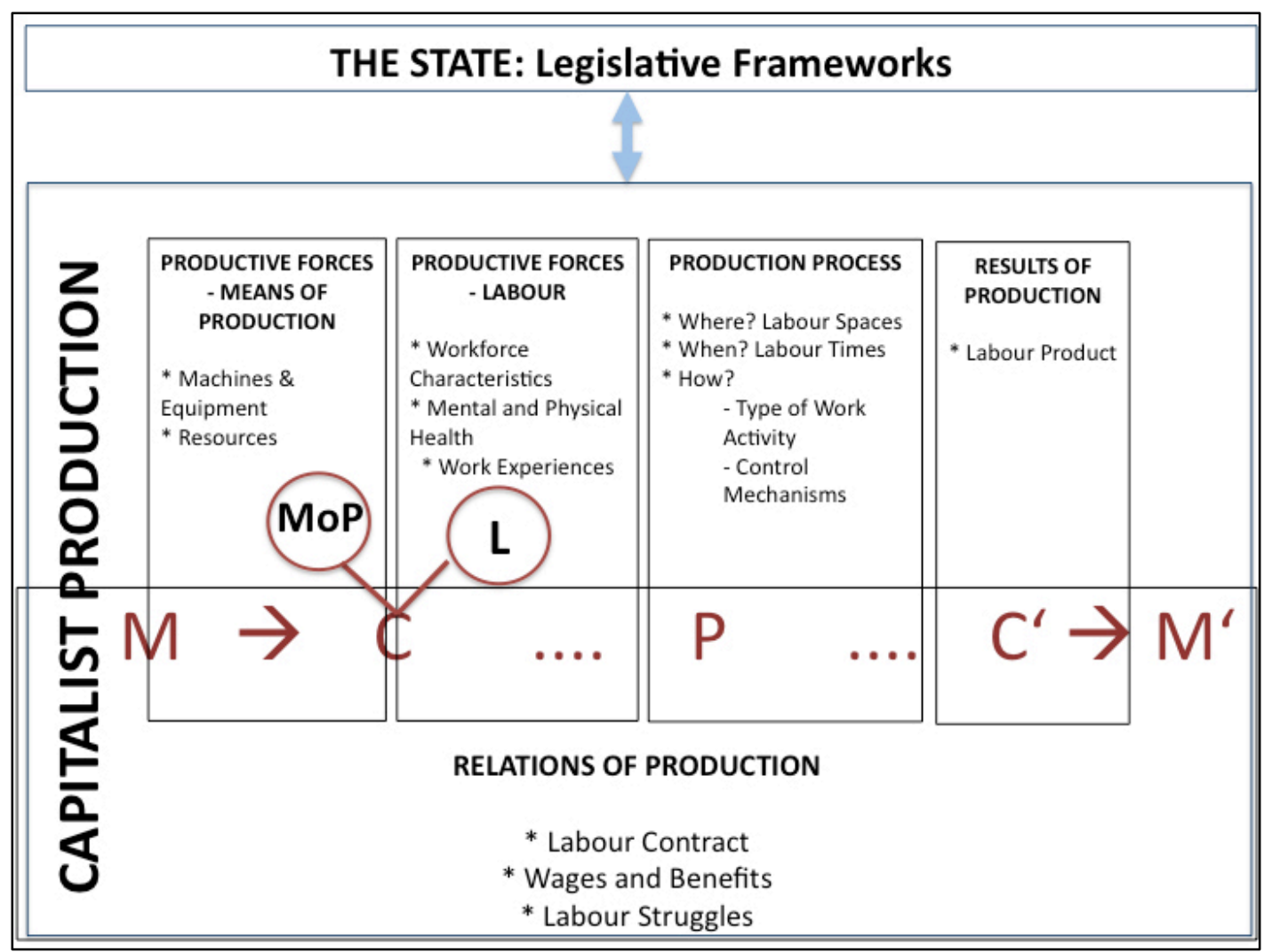

Figure 8: Dimensions of working conditions

The model pictured in figure 8 identifies five areas that shape working conditions throughout the capital accumulation process: means of production, labour, relations of production, the production process and the outcome of production. Furthermore this model includes the state's impact on working conditions through labour legislation:

- Productive Forces-Means of Production: Means of production include machines and equipment on the one hand and resources that are needed for production on the other hand. The question whether workers operate big machines, work at the assembly line, use mobile devices such as laptops, handle potentially hazardous substances, use high-tech equipment, traditional tools or no technology at all etc. shapes the experience of work and has a strong impact on work processes and working conditions.

- Productive Forces-Labour: The subjects of the labour process are workers themselves. One dimension that impacts work in a certain sector is the question how the workforce is composed in terms of gender, ethnic background, age, education levels etc. Another question concerns worker health and safety and how it is affected by the means of production, the relations of production, the labour process, and labour law. Apart from outside impacts on the worker, an important factor is how workers themselves experience their working conditions.

- Relations of Production: Within capitalist relations of production, capitalists buy labour power as a commodity. Thereby a relation between capital and labour is established. The purchase of labour power is expressed through wages. Wages are the primary means of subsistence for workers and the reason why they enter a wage labour relation. The level of wages thus is a central element of working conditions. Labour contracts specify the conditions under which capital and labour enter this relation, including working hours, wages, work roles and responsibilities etc. The content of this contract is subject to negotiations and often struggles between capital and labour. The relation between capital and labour is thus established through a wage relation and formally enacted by a labour contract that is 
subject to negotiations and struggles. These three dimensions of the relation between capital and labour set the framework for the capitalist labour process.

- Production process: Assessing working conditions, furthermore, requires looking at the specifics of the actual production process. A first factor in this context is its spatial location. Whether it is attached to a certain place or is location independent, whether it takes place in a factory, an office building, or outdoors etc. are important questions. A second factor relates to the temporal dimension of work. Relevant questions concern the amount of regular working hours and overtime, work rhythms, the flexibility or rigidness of working hours, the relation between work time and free time etc. Finally working conditions are essentially shaped by how the production process is executed. This includes on the one hand the question which types of work activity are performed. The activities can range from intellectual work, to physical work, to service work, from skilled to unskilled work, from creative work to monotonous and standardized work tasks, etc. On the other hand another aspect of the production process is how it is controlled and managed. Different management styles can range from strict control of worker behaviour and the labour process to high degrees of autonomy, self-management or participatory management etc. Space, time, activity and control are essential qualities of the production process and therefore need to be considered when studying working conditions.

- Product: Throughout the production process workers put their time, effort and energy into producing a certain product. This actual outcome of production and how it relates back to the worker thus needs to be considered for understanding work in a certain sector.

- The state: Finally the state has an impact on working conditions through enacting labour laws that regulate minimum wages, maximum working hours, social security, safety standards etc.

Table 3 summarizes the dimensions of working conditions that we described above.

\begin{tabular}{|c|c|c|}
\hline \multirow[t]{2}{*}{$\begin{array}{l}\text { Productive forces - Means of } \\
\text { production }\end{array}$} & Machines and equipment & $\begin{array}{l}\text { Which technology is being } \\
\text { used during the production } \\
\text { process? }\end{array}$ \\
\hline & Resources & $\begin{array}{l}\text { What resources are used } \\
\text { during the production pro- } \\
\text { cess? }\end{array}$ \\
\hline \multirow[t]{3}{*}{ Productive forces - Labour } & Workforce characteristics & $\begin{array}{l}\text { What are important charac- } \\
\text { teristics of the workforce for } \\
\text { example in terms of age, } \\
\text { gender, ethnic background } \\
\text { etc? }\end{array}$ \\
\hline & Mental and physical health & $\begin{array}{l}\text { How do the employed means } \\
\text { of production and the labour } \\
\text { process impact mental and } \\
\text { physical health of workers? }\end{array}$ \\
\hline & Work experiences & $\begin{array}{l}\text { How do workers experience } \\
\text { their working conditions? }\end{array}$ \\
\hline \multirow[t]{3}{*}{ Relations of production } & Labour contracts & $\begin{array}{l}\text { Which type of contracts do } \\
\text { workers receive, what do } \\
\text { they regulate? }\end{array}$ \\
\hline & Wages and benefits & $\begin{array}{l}\text { How high/low are wage lev- } \\
\text { els and what are other mate- } \\
\text { rial benefits for workers? }\end{array}$ \\
\hline & Labour struggles & $\begin{array}{l}\text { How do workers organize } \\
\text { and engage in negotiations } \\
\text { with capital and what is the } \\
\text { role of worker protests? }\end{array}$ \\
\hline
\end{tabular}




\begin{tabular}{|l|l|l|}
\hline Production process & Labour spaces & $\begin{array}{l}\text { Where does the production } \\
\text { process take place? }\end{array}$ \\
\cline { 3 - 3 } & Labour times & $\begin{array}{l}\text { How many working hours are } \\
\text { common within a certain } \\
\text { sector, how are they en- } \\
\text { forced and how is the rela- } \\
\text { tionship between work and } \\
\text { free time? }\end{array}$ \\
\cline { 3 - 4 } & Work activity & $\begin{array}{l}\text { Which type of mental and/or } \\
\text { physical activity are workers } \\
\text { performing? }\end{array}$ \\
\cline { 3 - 4 } & Control mechanism & $\begin{array}{l}\text { Which type of mechanisms } \\
\text { are in place that control the } \\
\text { behaviour of workers? }\end{array}$ \\
\hline Results of production & $\begin{array}{l}\text { Which kinds of products or } \\
\text { services are being pro- } \\
\text { duced? }\end{array}$ \\
\hline The state & Labour product & $\begin{array}{l}\text { Which regulations regarding } \\
\text { minimum wages, maximum } \\
\text { working hours, safety, social } \\
\text { security etc are in place and } \\
\text { how are they enforced? }\end{array}$ \\
\hline
\end{tabular}

Table 3: Dimensions of working conditions

Given an identification of dimensions of working conditions, we can next bring this typology together with aspects of digital labour.

\section{The Conditions of Digital Labour}

In section 1, we introduced a cultural-materialist model of cultural work (figure 1) that distinguishes between physical cultural work and information work. Figure 8 is an application of this model to the realm of digital labour: digital labour is a special form of cultural work that results in the production and use of digital media. It distinguishes three forms of digital labour that represent different modes of the organisation of the productive forces: agricultural digital labour, industrial digital labour, informational digital labour. They are articulations of the three organisation forms of the productive forces that we identified in table 1: agricultural, industrial and informational productive forces. Agricultural and industrial digital work/labour are forms of physical cultural work/labour in the context of digital media. Informational digital work/labour is an expression of information work in the realm of digital media production.

Figure 9 shows a model of the major production processes that are involved in digital labour. Each production step/labour process involves human subjects (S) using technologies/instruments of labour $(\mathrm{T})$ on objects of labour $(\mathrm{O})$ so that a product emerges. The very foundation of digital labour is an agricultural labour cycle in which miners extract minerals. These minerals enter the next production process as objects so that processors based on them in physical labour processes create ICT components. These components enter the next labour cycle as objects: assemblage workers build digital media technologies and take ICT components as inputs. Processors and assemblers are industrial workers involved in digital production. The outcome of such labour are digital media technologies that enter various forms of information work as tools for the production, distribution, circulation, prosumption, and consumption of diverse types of information. 


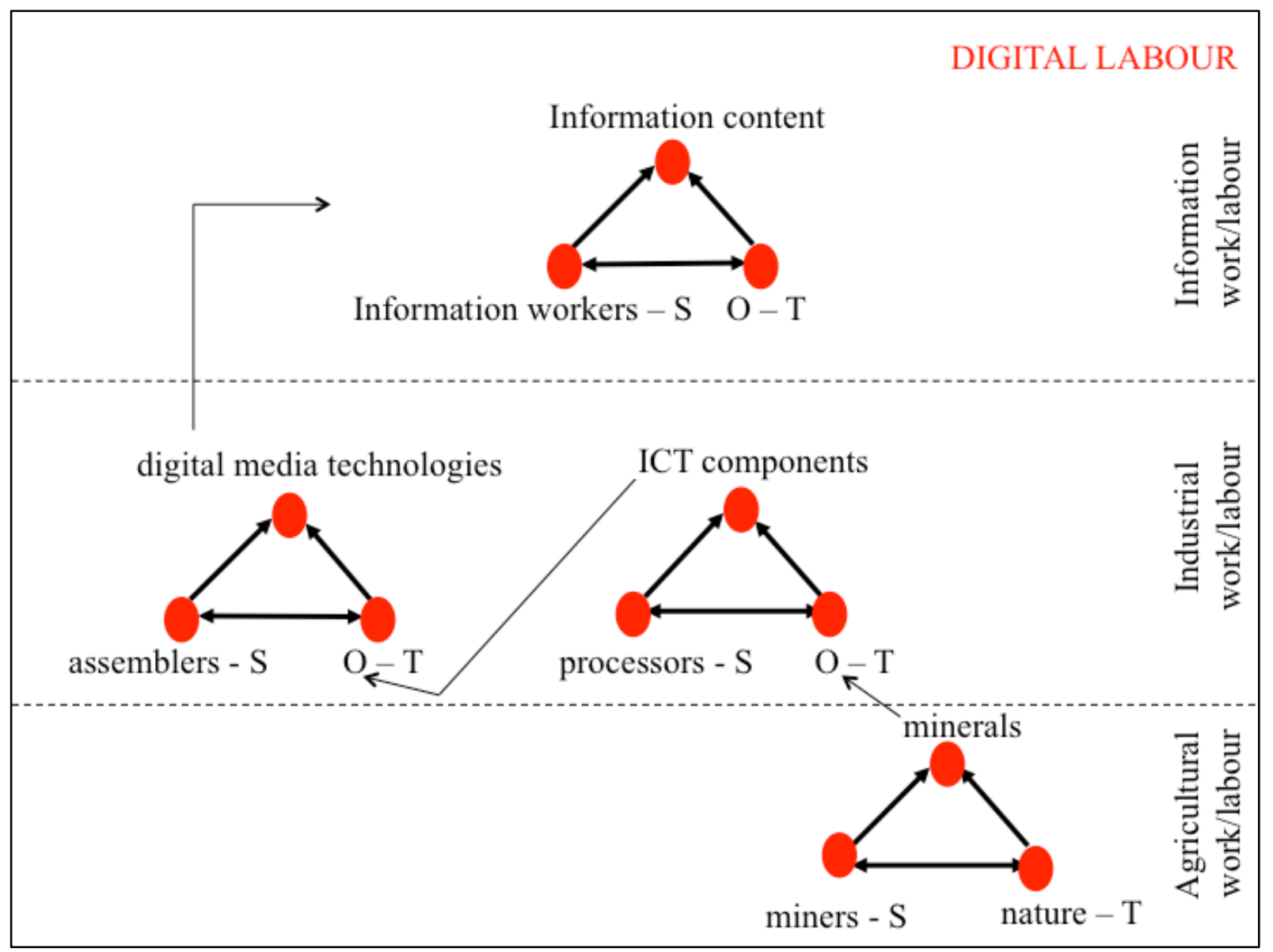

Figure 9: The complex network of cycles of digital labour

"Digital labour" is not a term that only describes the production of digital content. We rather use the term in a more general sense for the whole mode of digital production that contains a network of agricultural, industrial and informational forms of work that enables the existence and usage of digital media. The subjects involved in the digital mode of production (S) miners, processors, assemblers, information workers and related workers-stand in specific relations of production that are either class relations or non-class relations. So what we designate as $S$ in figure 8 is actually a relationship $S_{1}-S_{2}$ between different subjects or subject groups. In contemporary capitalist society, most of these digital relations of production tend to be shaped by wage labour, slave labour, unpaid labour, precarious labour, and freelance labour.

In section 2, we introduced a model of the work process in general (figures 4, 6, 7; tables $1,2)$. Section 3 presented a model for the analysis of capitalist working conditions in capitalism (table 3, figure 8). How are these two models connected? The first one is more general and presents typologies for all modes of production (patriarchy, slavery, feudalism, capitalism, communism) and productive forces (agricultural, industrial, informational). The second model shown in figure 8 and table 3 shows dimension of labour within the capitalist mode of production. Table 4 shows how elements in model 1 (figure 4 ) correspond to elements in model 2 (figure 8 , table 3 ). 


\begin{tabular}{|c|c|c|}
\hline \multicolumn{2}{|l|}{ MODEL 2 (figure 8, table 3) } & MODEL 1 (figure 4) \\
\hline \multirow{2}{*}{$\begin{array}{l}\text { Productive forces - Means } \\
\text { of production }\end{array}$} & Machines and equipment & Object: Instruments of labour \\
\hline & Resources & Object: Object of labour \\
\hline \multirow[t]{3}{*}{ Productive forces - Labour } & Workforce characteristics & Subject \\
\hline & Mental and physical health & Subject \\
\hline & Work experiences & Subject \\
\hline \multirow[t]{3}{*}{ Relations of production } & Labour contracts & $\begin{array}{l}\text { Subject-subject relation- } \\
\text { ships: Relations of produc- } \\
\text { tion }\end{array}$ \\
\hline & Wages and benefits & $\begin{array}{l}\text { Subject-subject relation- } \\
\text { ships: Relations of produc- } \\
\text { tion }\end{array}$ \\
\hline & Labour struggles & $\begin{array}{l}\text { Subject-subject relation- } \\
\text { ships: Relations of produc- } \\
\text { tion }\end{array}$ \\
\hline \multirow[t]{4}{*}{ Production process } & Labour spaces & Object: Instruments of labour \\
\hline & Labour times & $\begin{array}{l}\text { Subject-subject relation- } \\
\text { ships: Relations of produc- } \\
\text { tion }\end{array}$ \\
\hline & Work activity & Subject \\
\hline & Control mechanism & $\begin{array}{l}\text { Subject-subject relation- } \\
\text { ships: Relations of produc- } \\
\text { tion }\end{array}$ \\
\hline Results of production & Labour product & $\begin{array}{l}\text { Subject-object: Products of } \\
\text { labour }\end{array}$ \\
\hline The state & Labour law & $\begin{array}{l}\text { Subject-subject relation- } \\
\text { ships: Relations of produc- } \\
\text { tion }\end{array}$ \\
\hline
\end{tabular}

Table 4: Dimensions of working conditions

We have developed a systematic digital labour analysis toolkit that helps asking systematic questions about the involved labour processes. It can be applied to agricultural, industrial and informational digital labour and combinations of these forms of work. Table 5 presents the digital labour analysis toolkit that is based on the more general model introduced in table 3. 


\begin{tabular}{|c|c|c|c|}
\hline \multirow[t]{2}{*}{$\begin{array}{l}\text { Productive } \\
\text { forces - } \\
\text { Means of } \\
\text { production }\end{array}$} & $\begin{array}{l}\text { Machines and } \\
\text { equipment }\end{array}$ & $\begin{array}{l}\text { What technologies or } \\
\text { combinations thereof are } \\
\text { being used during the ag- } \\
\text { ricultural, industrial and } \\
\text { informational production } \\
\text { process that create digital } \\
\text { media and contents? }\end{array}$ & $\begin{array}{l}\text { a) non-digital machines } \\
\text { b) digital machines } \\
\text { c) human brain } \\
\text { d) human hands }\end{array}$ \\
\hline & Resources & $\begin{array}{l}\text { What resources or combi- } \\
\text { nations thereof are used } \\
\text { during the agricultural, } \\
\text { industrial and information- } \\
\text { al production processes of } \\
\text { digital media and content? }\end{array}$ & $\begin{array}{l}\text { a) physical resources: natural } \\
\text { resources } \\
\text { b) digital and mediated da- } \\
\text { ta/information } \\
\text { c) human ideas } \\
\text { d) physical resources: indus- } \\
\text { trial resource }\end{array}$ \\
\hline \multirow[t]{3}{*}{$\begin{array}{l}\text { Productive } \\
\text { forces - La- } \\
\text { bour }\end{array}$} & $\begin{array}{l}\text { Workforce } \\
\text { characteristics }\end{array}$ & $\begin{array}{l}\text { What are important char- } \\
\text { acteristics of the workforce } \\
\text { in agricultural, industrial } \\
\text { and informational digital } \\
\text { labour (for example in } \\
\text { terms of age, gender, eth- } \\
\text { nic background etc)? }\end{array}$ & $\begin{array}{l}\text { a) class } \\
\text { b) gender } \\
\text { b) age } \\
\text { c) ethnicity } \\
\text { d) abilities } \\
\text { e) education, } \\
\text { etc. }\end{array}$ \\
\hline & $\begin{array}{l}\text { Mental and } \\
\text { physical health }\end{array}$ & $\begin{array}{l}\text { How do the employed } \\
\text { means of production and } \\
\text { the labour process impact } \\
\text { mental and physical health } \\
\text { of agricultural, industrial } \\
\text { and informational digital } \\
\text { workers? }\end{array}$ & $\begin{array}{l}\text { a) mental health } \\
\text { b) physical health }\end{array}$ \\
\hline & $\begin{array}{l}\text { Work experi- } \\
\text { ences }\end{array}$ & $\begin{array}{l}\text { How do agricultural, indus- } \\
\text { trial and informational digi- } \\
\text { tal workers experience } \\
\text { their working conditions? }\end{array}$ & \\
\hline \multirow[t]{2}{*}{$\begin{array}{l}\text { Relations of } \\
\text { production }\end{array}$} & $\begin{array}{l}\text { Labour con- } \\
\text { tracts }\end{array}$ & $\begin{array}{l}\text { Are there labour contracts } \\
\text { or not? In the case, where } \\
\text { there are labour contracts: } \\
\text { Which type of contracts do } \\
\text { digital workers receive, } \\
\text { what do they regulate? }\end{array}$ & $\begin{array}{l}\text { a) no contract, } \\
\text { b) written/oral contract, } \\
\text { c) part-time or full-time em- } \\
\text { ployment contract, } \\
\text { d) permanent or temporary } \\
\text { employment contract, } \\
\text { e) employment or service } \\
\text { contract, } \\
\text { f) freelancer or employee, } \\
\text { etc. }\end{array}$ \\
\hline & $\begin{array}{l}\text { Wages and } \\
\text { benefits }\end{array}$ & $\begin{array}{l}\text { Are there wages and spe- } \\
\text { cific benefits digital work- } \\
\text { ers enjoy or not? In case, } \\
\text { where there are wages } \\
\text { and benefits: How } \\
\text { high/low are wage levels } \\
\text { and what are other mate- } \\
\text { rial benefits for digital }\end{array}$ & $\begin{array}{l}\text { a) wage level } \\
\text { b) included/excluded health } \\
\text { benefits } \\
\text { c) included/excluded retire- } \\
\text { ment insurance } \\
\text { (state/private/company/mixed } \\
\text { insurance) } \\
\text { d) included/excluded unem- }\end{array}$ \\
\hline
\end{tabular}




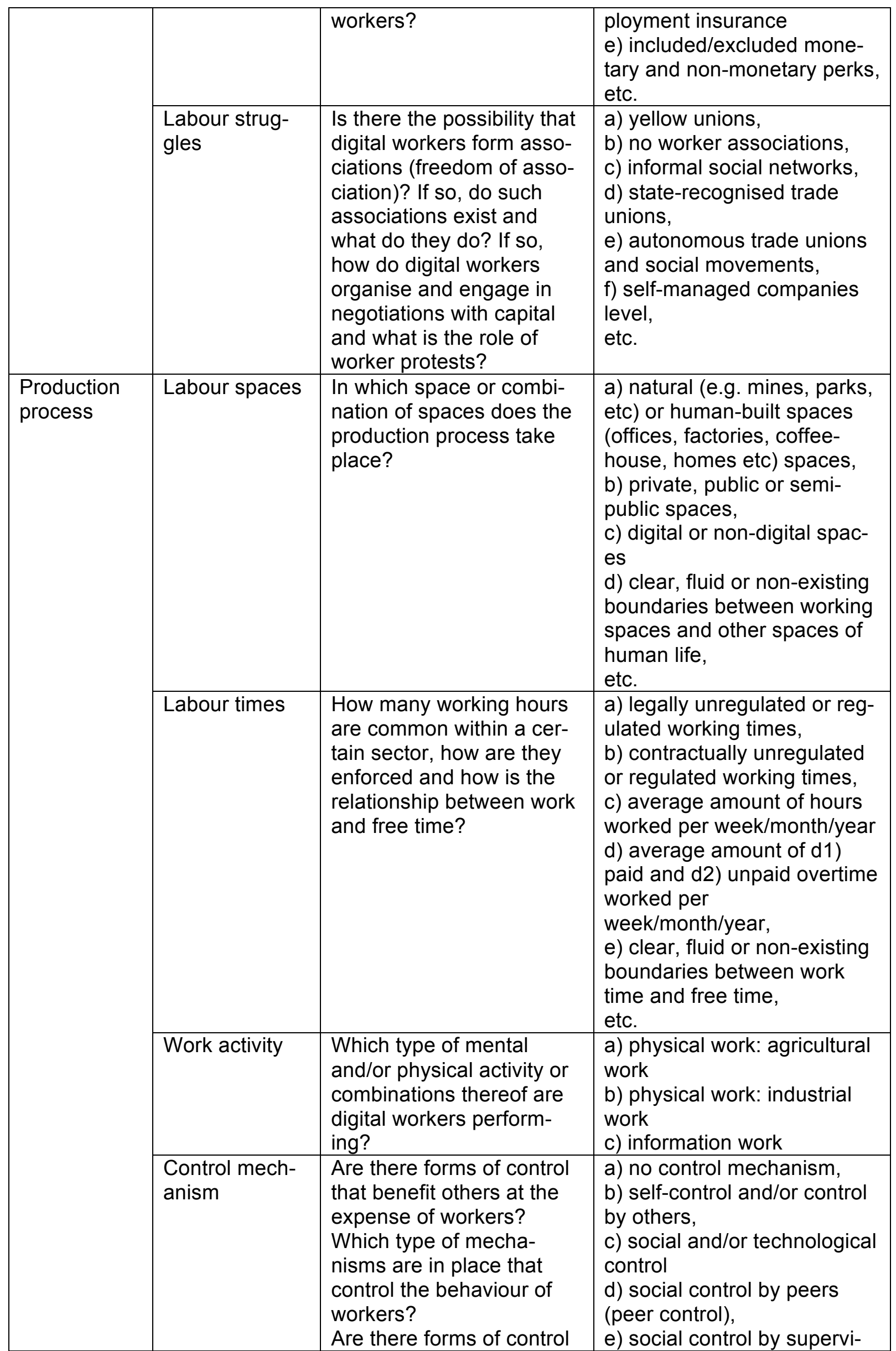




\begin{tabular}{|c|c|c|c|}
\hline & & $\begin{array}{l}\text { that control the control- } \\
\text { lers? }\end{array}$ & $\begin{array}{l}\text { sors and mangers, } \\
\text { f) digital or non-digital tech- } \\
\text { nological control, } \\
\text { g) surveillance of applicants, } \\
\text { workplace, workforce, output, } \\
\text { activities, property, consum- } \\
\text { ers, prosumers, competitors, } \\
\text { h) controls that are inherent } \\
\text { to production technologies, } \\
\text { controls that are external (i.e. } \\
\text { separate control technolo- } \\
\text { gies), } \\
\text { g) forms of counter-control } \\
\text { (corporate watchdogs, work- } \\
\text { place inspectors, }\end{array}$ \\
\hline $\begin{array}{l}\text { Results of } \\
\text { production }\end{array}$ & Labour product & $\begin{array}{l}\text { Which kinds of products or } \\
\text { services does digital la- } \\
\text { bour produce? }\end{array}$ & $\begin{array}{l}\text { a) digital or non-digital prod- } \\
\text { ucts, } \\
\text { b) online or offline products, } \\
\text { c) physical (agricultural, in- } \\
\text { dustrial) and/or informational } \\
\text { and/or social (-service) prod- } \\
\text { uct, etc. }\end{array}$ \\
\hline The state & $\begin{array}{l}\text { Labour legisla- } \\
\text { tion }\end{array}$ & $\begin{array}{l}\text { Are there state laws that } \\
\text { regulate work? Which reg- } \\
\text { ulations regarding mini- } \\
\text { mum wages, maximum } \\
\text { working hours, safety, so- } \\
\text { cial security etc are in } \\
\text { place and how are they } \\
\text { enforced? }\end{array}$ & $\begin{array}{l}\text { a) Regulation and enforce- } \\
\text { ment of work and service } \\
\text { contracts, legal dispute reso- } \\
\text { lution } \\
\text { b) Wage legislation: } \\
\text { wage protections, minimum } \\
\text { wage regulation, etc, } \\
\text { c) Work time legislation: } \\
\text { standard working times, max- } \\
\text { imum working hours, over- } \\
\text { time regulations, } \\
\text { annual leave, sabbatical } \\
\text { leave, on-the-job-training } \\
\text { times and further education, } \\
\text { flexible working, termination } \\
\text { of employment (protection } \\
\text { from unfair dismissal, redun- } \\
\text { dancy payments, } \\
\text { etc), etc. } \\
\text { d) Health and safety legisla- } \\
\text { tion: } \\
\text { work space regulations, work } \\
\text { equipment and resources } \\
\text { regulations, dangerous sub- } \\
\text { stances, protective gear, etc. } \\
\text { e) Social security benefit } \\
\text { legislation: } \\
\text { parental leave, unemploy- } \\
\text { ment, pension, health care, } \\
\text { etc. } \\
\text { f) Employee representation } \\
\text { and freedom of association, }\end{array}$ \\
\hline
\end{tabular}




\begin{tabular}{|l|l|l|}
\hline & & g) Taxation: \\
& & $\begin{array}{l}\text { corporation tax, income and } \\
\text { wage tax, etc; } \\
\text { etc. }\end{array}$ \\
\hline
\end{tabular}

Table 5: Digital labour analysis toolkit

Case studies and the analysis of digital labour shows that digital labour is a global network of various forms of labour that represent various interlinked modes of production and various levels of organization of the productive forces (Fuchs 2014a). Examples are African slave workers who mine minerals that are used for the production of digital media components, Tayloristic ICT hardware assemblers working under Taylorist and hazardous conditions in toxic workplaces, highly paid and highly stressed software engineers and knowledge professionals, precarious digital media freelancers, Taylorised call centre workers, unpaid social media prosumers creating personal data commodities for social media corporations etc (Fuchs 2014a). These working conditions reflect various modes of production such as slavery, patriarchy and capitalism, various forms of the organization of the capitalist mode of production (Fordist/Taylorist labour, post-Fordist labour, etc) different organization forms of the productive forces and the labour conducted in it (agricultural labour, industrial labour, informational labour).

Jairus Banaji's (2011) stresses that Marx's theory of the mode of production shows that "capitalist relations of production are compatible with a wide variety of forms of labour, from chattel-slavery, sharecropping, or the domination of casual labour-markets, top the coerced wage-labour peculiar to colonial regimes and, of course, 'free' wage-labour" (Banaji 2011, 359). Banaji's concept of the mode of production matters for understanding the digital media economy because in this economy a variety of modes of production and organisations of the productive forces (=variations within a specific mode of production) are articulated, including slavery in mineral extraction, military forms of Taylorist industrialism in hardware assemblage, an informational organisation of the productive forces of capitalism that articulates a highly paid knowledge labour aristocracy, precarious service workers as well as imperialistically exploited knowledge workers in developing countries; industrial recycling and management of e-waste as well as highly hazardous informal physical e-waste labour (Fuchs 2014a).

Digital media are information technologies. So although they are created by physical, agricultural and scientific development work, they are used and applied as tools of cognition, communication and collaboration and therefore have a crucial cultural dimension of usage, work and labour (Fuchs 2014b).

The upper level of information work in figure 8 is an important dimension of digital labour. It contains those digital workers who create digital content. They are digital content/information workers. Table 6 presents a typology for classifying digital information work. The table identifies 8 specific dimensions of digital information work. These eight dimensions are elements of the capital accumulation process in the digital content industry: There is 1) a human subject engaging in work, 2) a capitalist looking for making profits, 3) a contractual economic relationship, 4) technologies as instruments/means of production, 5) resources as means/objects of production, 6 ) the output of production (the product), 7 ) the distribution of products, 8 ) the consumption of products. 


\begin{tabular}{|c|c|c|c|c|c|c|c|}
\hline $\begin{array}{l}\text { Infor- } \\
\text { mation } \\
\text { worker's } \\
\text { job- } \\
\text { seeking } \\
\text { strategy }\end{array}$ & $\begin{array}{l}\text { Employ- } \\
\text { er, con- } \\
\text { tractor }\end{array}$ & $\begin{array}{l}\text { Relations } \\
\text { of pro- } \\
\text { duction }\end{array}$ & $\begin{array}{l}\text { Technolo- } \\
\text { gy }\end{array}$ & Objects & $\begin{array}{l}\text { Prod- } \\
\text { ucts }\end{array}$ & $\begin{array}{l}\text { Distri- } \\
\text { bution }\end{array}$ & $\begin{array}{l}\text { Con- } \\
\text { sump- } \\
\text { tion }\end{array}$ \\
\hline 1 online & 1 online & 1 online & 1 brain & 1 digital & 1 digital & 1 online & 1 digita \\
\hline \multirow[t]{3}{*}{2 offline } & 2 offline & 2 offline & $\begin{array}{l}2 \text { brain }+ \\
\text { digital } \\
\text { technolo- } \\
\text { gies }\end{array}$ & $\begin{array}{l}2 \text { non- } \\
\text { digital }\end{array}$ & $\begin{array}{l}2 \text { non- } \\
\text { digital }\end{array}$ & 2 offline & $\begin{array}{l}2 \text { non- } \\
\text { digital }\end{array}$ \\
\hline & & 3 blended & $\begin{array}{l}3 \text { brain }+ \\
\text { non-digital } \\
\text { technolo- } \\
\text { gies }\end{array}$ & $\begin{array}{l}3 \text { blend- } \\
\text { ed }\end{array}$ & $\begin{array}{l}3 \text { blend- } \\
\text { ed }\end{array}$ & & \\
\hline & & & $\begin{array}{l}4 \text { brain }+ \\
\text { digital } \\
\text { technolo- } \\
\text { gies }+ \text { non- } \\
\text { digital } \\
\text { technolo- } \\
\text { gies }\end{array}$ & & & & \\
\hline
\end{tabular}

Table 6: A typology of the digitalisation of information labour

Digital information labour can take on different forms. A first important dimension is how information workers find jobs, projects or employment. The information worker can have an online profile/website/blog etc or not in order to find work. Also the employer/contractor can have an online profile/website/blog etc or not. It is of course likely that those workers and employers who present themselves online and look for economic relations online do so also offline. They then fall into the category " 1 online". The distinction here wants to draw a separation line between those who use the Internet for establishing economic relations and those who do not. The relationship between the two can be established and maintained primarily online (e.g. via platforms such as Amazon Mechanical Turk, oDesk or PeoplePerHour), offline or in a blended way. The technologies used for production always involve the brain because we talk about information work. But in addition also digital tools and/or non-digital tools can be used as means of production. The objects on which the labour is conducted can either be entirely digital, non-digital or both digital and non-digital. The created products can be digital, non-digital or a mix of both. Their distribution and consumption can take place online or offline. This means that there are 8 dimensions of digital information labour that can have various characteristics. The number of logical forms of digital information labour can be calculated by multiplying various binominal coefficients: 


$$
\begin{aligned}
& \left(\begin{array}{l}
2 \\
1
\end{array}\right) *\left(\begin{array}{l}
2 \\
1
\end{array}\right) *\left(\begin{array}{l}
3 \\
1
\end{array}\right) *\left(\begin{array}{l}
4 \\
1
\end{array}\right) *\left(\begin{array}{l}
3 \\
1
\end{array}\right) *\left(\begin{array}{l}
3 \\
1
\end{array}\right) *\left(\begin{array}{l}
2 \\
1
\end{array}\right) *\left(\begin{array}{l}
2 \\
1
\end{array}\right) \\
& \left(\begin{array}{l}
n \\
k
\end{array}\right)=\frac{n !}{k ! *(n-k) !} \\
& \left(\begin{array}{l}
2 \\
1
\end{array}\right)=\frac{2 !}{1 ! * 1 !}=2 \\
& \left(\begin{array}{l}
3 \\
1
\end{array}\right)=\frac{3 !}{1 ! * 2 !}=3 \\
& \left(\begin{array}{l}
4 \\
1
\end{array}\right)=\frac{4 !}{1 ! * 3 !}=4
\end{aligned}
$$

$2 * 2 * 3 * 4 * 3 * 3 * 2 * 2=1728$

So from a purely logical point of view, there are 1728 different possible forms of digital information labour. Which of them occur in actual reality or are in a logically feasible manner included into the category of digital information labour is an empirical and theoretical question. These 1728 possibilities represent the productive forces of digital information work that are embedded into and interact with specific relations of production. Annex A presents a full list of all 1728 logically possible forms of digital information labour.

It is a theoretical question if all of these forms of labour are digital labour or if only those that satisfy a minimum number of characteristics that are digital should be considered as digital labour. Or should all activities characterised by the typology that contain at least one dimension that is digital be considered as forms of digital labour? The typology shows in any case that it is possible to observe and with this typology characterise the digitalisation or informatisation of various dimensions of work, such as the way people look for jobs and employment, employers' search for labour power, the relations of production, the technological means of production, the used resources, the created products, forms of distribution, and forms of consumption. Rudi Schmiede (1996) uses the term informatisation of work for describing how information technologies shape the work process. He does not limit the term to the computerisation of work, but mentions other information technologies, such as the postal service, the telegraph, double bookkeeping, books of accounts, or file card systems (Schmiede 1996, 122). The computerisation or digitalisation of work is one specific form of the informatisation of work: digital media technologies shape various aspects of different forms of work. Schmiede says the fact that computer technologies enable the networking of information has resulted in a form of abstract societalisation (Vergesellschaftung) in capitalism: all forms of work could in principle be shaped and influenced by the networked computer so that "the informatisation of societal work opens access for the measure of value and valorisation to each individual work that is integrated into an in principle global information context" (Schmiede 1996, 125, translation from German ${ }^{1}$ ). The typology in table 6 describes various dimensions of the digitalisation or networked computerisation of labour. It is a theoretical question which of these forms of labour should be termed digital information labour and which ones should not.

Let us consider an example: a blogger who generates postings for a newspaper's website and works from home. She conducts her work primarily online, i.e. she blogs on the Internet, and her employer's presence for her is its newspaper website. The communication between the blogger and the newspaper's online editor takes place primarily online, but from time to time there are real life meetings in order to discuss the newspaper's online strategy. So the production relationship has a blended character. The blogger uses her brain and digital tech-

\footnotetext{
1 „Allgemein gesagt, eröffnet die Informatisierung der gesellschaftlichen Arbeit dem Wert- und Verwertungsmaßstab den Zugriff auf jede einzelne Arbeit, die in einen prinzipiell globalen Inforamtionszusammenhang eingegliedert ist".
} 
nologies such as a laptop connected to the Internet and a blogging platform, so the used technologies are a human brain and digital technologies. The objects of work are the blogger's experiences, opinions and thoughts (non-digital information) and other online documents to which she links (digital), so the objects of work are blended. The product is a digital text that is distributed and consumed online in digital format. Using the typology in table 4, we can characterize the blogger's work as an example of digital information work version number 11323111 . This characterisation of digital information work makes use of eight symbolic positions: each describes one dimension of digital information work according to table 6. Each dimension's expression is defined according to the codes in table 6 . The typology in table 6 describes various dimensions of the digitalisation or networked computerisation of labour. It is a theoretical question which of these forms of labour should be termed digital information labour and which ones should not.

\section{Conclusion}

In this paper, we have introduced a cultural-materialist approach for theorising digital labour. Many approaches are idealist in that they define concepts such as digital labour, virtual work, online work, cyberwork, immaterial labour, knowledge labour, creative work, cultural labour, communicative labour, information(al) work, digital craft, service work, prosumption, consumption work, audience labour, playbour, etc., only as an externalisation of human ideas that are objectified in contents and thereby neglect that this labour is based on and only possible because there is a global division of labour, in which many different forms of labour are conducted under specific modes of production. We have used Raymond Williams' framework of cultural materialism for arguing that we should overcome digital idealism and analyse digital labour based the framework of digital materialism.

We have introduced specific concepts for a digital materialist theory of digital labour: cultural work, physical cultural work, information work, modes of production, productive forces, relations of production, digital work, digital labour, physical digital work/labour (agricultural digital work/labour, industrial digital work/labour), informational digital work/labour. Furthermore we have suggested a digital labour analysis toolbox that distinguishes elements of digital labour processes and can be used as framework for the concrete empirical analysis of specific forms of digital work/labour. Conducting such analyses often faces the problem of what the elements of analysis are. We argue for avoiding particularistic analyses that focus only on single elements of single production processes and for conducting holistic analyses that focus on the totality of elements and networks that determine and shape digital labour. The toolkit allows analysing the totality of elements of elements of digital labour processes. Digital labour analysis should also look at how one specific form of digital labour that is analysed is connected to and articulated with other forms of digital labour that express certain organisational forms of the productive forces and the relations of production.

The world of digital media is shaped by a complex global articulation of various modes of production that together constitute the capitalist mode of creating and using digital media. The digital tools that we use for writing, reading, communicating, uploading, browsing, collaborating, chatting, befriending, or liking are embedded into a world of exploitation. Yet most of us cannot and do not want to imagine a world without digital media. So the alternative is not digital Luddism, but political praxis.

Digital labour analysis can only interpret the digital media world; the point is to change it. Change can only be good change if it is informed change. Critical theory can inform potential and actual struggles for a better world. Everyday working realities of different people and in different parts of the world look so heterogeneous, different and unconnected that it is often difficult to see what they have in common. Digital labour theory and digital labour analysis can help to identify and make visible the common and different experiences of suffering and enjoyment, pleasure and pain, security and insecurity, alienation and appropriation, exploitation and resistance, creativity and toil. It is in this respect a digital sociology of critique. But it is at the same time also a political philosophy, a critical digital sociology that helps identifying and clarifying foundations and germ forms of a better future and grounding judgements about 
what is good and bad in the context of digital media. Digital labour theory and analysis therefore takes on the role of a critical sociology of critique that is both at once a critical sociology and a sociology of critique (Boltanski and Honneth 2009). It analyses the reality of life under digital capitalism, contributes intellectually to questioning this mode of human existence in order to show that there is and to help realise life beyond capitalism.

\section{References}

Adorno, Theodor W. 1968/2003. Late Capitalism or Industrial Society? The Fundamental Question of the Present Structure of Society. In Can One Live After Auschwitz?, edited by Rolf Tiedemann, 111-125. Stanford: Stanford University Press.

Althusser, Louis and Étienne Balibar. 1970. Reading Capital. London: NLB.

Andrejevic, Mark. 2012. Exploitation in the Data Mine. In Internet and Surveillance. The Challenges of Web 2.0 and Social Media, edited by Christian Fuchs, Kees Boersma, Anders Albrechtslund and Marisol Sandoval, 71-88. New York: Routledge.

Arendt, Hannah. 1958. The Human Condition. Chicago: University of Chicago Press.

Arvidsson, Adam and Eleanor Colleoni. 2012. Value in Informational Capitalism and on the Internet. The Information Society 28 (3): 135-150.

Banaji, Jairus. 2011. Theory as History. Essays on Modes of Production and Exploitation. Chicago: Haymarket Books.

Bell, Daniel. 1974. The Coming of Post-Industrial Society. London: Heinemann.

Boltanski, Luc and Axel Honneth. 2009. Soziologie der Kritik oder Kritische Theorie? In Was ist Kritik?, edited by Rahel Jaeggi and Tilo Wesche, 81-114. Frankfurt am Main: Suhrkamp.

Caves, Richard E. 2000. Creative Industries. Cambridge: Harvard University Press.

Cunningham, Stuart. 2005. Creative Enterprises. In Creative Industries, edited by John Hartley, 282298. Malden: Blackwell.

D'Mello, Marisa, and Sundeep Sahay. 2007. "I am a Kind of Nomad Where I Have to Go Places and Places" . . . Understanding Mobility, Place and Identity in Global Software Work from India. Information and Organization 17 (3): 162-192.

Engels, Friedrich. 1884. The Origin of the Family, Private Property and the State. Accessed November 10, 2013. http://www.marxists.org/archive/marx/works/1884/origin-family/

Finnwatch. 2007. Connecting Components, Dividing Communities: Tin Production for Consumer Electronics in the DR Congo and Indonesia. makelTfair Report: http://germanwatch.org/corp/it-tin.pdf (accessed August 9, 2013).

Fisher, Eran. 2012. How Less Alienation Creates More Exploitation. tripleC: Communication, Capitalism \& Critique 10 (2): 171-183.

Fröbel, Folker, Jürgen Heinrichs and Otto Kreye. 1981. The New International Division of Labour. Cambridge: Cambridge University Press.

Fuchs, Christian. 2010. Labor in Informational Capitalism and on the Internet. The Information Society 26 (3): 179-196.

Fuchs, Christian. 2012a. Dallas Smythe Today. The Audience Commodity, the Digital Labour Debate, Marxist Political Economy and Critical Theory. Prolegomena to a Digital Labour Theory of Value. tripleC: Capitalism, Communication \& Critique 10 (2): 692-740.

Fuchs, Christian. 2012b. With or Without Marx? With or Without capitalism? A Rejoinder to Adam Arvidsson and Eleanor Colleoni. tripleC: Communication, Capitalism \& Critique: Journal for a Global Sustainable Information Society 10 (2): 633-645.

Fuchs, Christian. 2014a. Digital Labour and Karl Marx. New York: Routledge.

Fuchs, Christian. 2014b. Social Media: A Critical Introduction. London: Sage.

Glass, Robert L. 2006. Software Creativity 2.0. Atlanta, GA: developer. ${ }^{*}$ Books.

Gramsci, Antonio. 1988. The Antonio Gramsci Reader. In Selected Writings 1916-1935, edited by David Forgacs. London: Lawrence and Wishart.

Hartley, John. 2005. Creative Industries. In Creative Industries, 62-76, edited by John Hartley. Malden: Blackwell.

Hegel, Georg Wilhelm Friedrich. 1991. The Encyclopaedia Logic. Indianapolis, IN: Hackett.

Hesmondhalgh, David. 2013. The Cultural Industries. London: Sage. Third edition.

Hesmondhalgh, David and Sarah Baker. 2011. Creative Labour. Media Work in Three Cultural Industries. London: Routledge. 
Hofkirchner, Wolfgang. 2013. Emergent Information. A Unified Theory of Information Framework. Singapore: World Scientific.

Huws, Ursula. 1999. Material World. The Myth of the Weightless Economy. Socialist Register 35: 2955.

Huws, Ursula. 2008. Break or Weld? Trade Union Responses to Global Value Chain Restructuring. Work Organisation, Labour and Globalisation 2 (1): 1-10.

Huws, Ursula and Simone Dahlmann. 2010. Global Restructuring of Value Chains and Class Issues. In Interrogating the New Economy. Restructuring Work in the 21st Century, edited by Norene J. Pupo and Mark P. Thomas, 65-91. Toronto: University of Toronto Press.

Machlup, Fritz. 1962. The Production and Distribution of Knowledge in the United States. Princeton: Princeton University Press.

Marx, Karl. 1857/58. Grundrisse. London: Penguin.

Marx, Karl. 1867. Capital Vol I. London: Penguin.

Marx Karl 1885/1992. Capital Vol II. London: Penguin.

Marx, Karl and Friedrich Engels. 1845/46. The German Ideology. Amherst: Prometheus Books.

Maxwell, Richard and Toby Miller. 2012. Greening the Media. Oxford: Oxford University Press.

Miller, Toby, Nitin Govil, John McMurria, Richard Maxwell and Ting Wang. 2004. Global Hollywood 2. London: British Film Institute.

Mosco, Vincent and Catherine McKercher. 2009. The Laboring of Communication. Will Knowledge Workers of the World Unite? Lanham, MD: Lexington Books.

Noam, Eli. 2009. Media Ownership and Concentration in America. Oxford: Oxford University Press.

Porter, Michael. 1985. Competitive Advantage. Creating and Sustaining Superior Performance. New York: Free Press.

Sandoval, Marisol. 2013. Foxconned Labour as the Dark Side of the Information Age: Working Conditions at Apple's Contract Manufacturers in China. tripleC: Communication, Capitalism \& Critique 11 (2): 318-347.

Sandoval, Marisol. 2014. From Corporate to Social Media. Critical Perspectives on Corporate Social Responsibility in Media and Communication Industries. New York: Routledge.

Schmiede, Rudi. 1996. Informatisierung und gesellschaftliche Arbeit - Strukturveränderungen von Arbeit und Gesellschaft. In Virtuelle Arbeitswelten. Arbeit, Produktion und Subjekt in der „Informationsgesellschaft", 107-128. Berlin: edition sigma.

Scholz, Trebor, ed. 2013. Digital Labor. The Internet as Playground and Factory. New York: Routledge.

Students \& Scholars against Corporate Misbehaviour (SACOM). 2010. Workers as Machines: Military Management in Foxconn. Accessed August 9, 2013. http://sacom.hk/wpcontent/uploads/2010/11/reporton-foxconn-workers-as-machines sacom.pdf

Pellow, David N. and Lisa Sun-Hee Park. 2002. The Silicon Valley of Dreams: Environmental Injustice, Immigrant Workers, and the High-Tech Global Economy. NewYork: NewYork University Press.

Thompson, Edward P. 1978. The Poverty of Theory and Other Essays. New York: Monthly Review Press.

Toffler, Alvin. 1980. The Third Wave. New York: Bantam.

Weingart, Brigitte. 1997. Arbeit - ein Wort mit langer Geschichte. Accessed November 10, 2013. http://www.ethikprojekte.ch/texte/arbeit.htm

Williams, Raymond. 1958. Culture \& Society, 1780-1950. New York: Columbia University Press.

Williams, Raymond. 1962. Communications. Harmondsworth: Penguin.

Williams, Raymond. 1977. Marxism and Literature. Oxford: Oxford University Press.

Williams, Raymond. 1981. The Sociology of Culture. Chicago, IL: University of Chicago Press.

Williams, Raymond. 1983. Keywords. New York: Oxford University Press.

Williams, Raymond. 1989. What I Came to Say. London: Hutchinson Radius.

Zerdick, Axel et al. 2000. E-conomics. Strategies for the Digital Marketplace. Berlin: Springer.

\section{About the Authors}

Christian Fuchs

Christian Fuchs is professor of social media at the University of Westminster and co-editor of tripleC:

Communication, Capitalism \& Critique. http://fuchs.uti.at. 
Marisol Sandoval

Marisol Sandoval is a lecturer at City University London's Department of Culture and Creative Industries and co-editor of tripleC: Communication, Capitalism \& Critique. 
Annex A: 1728 Logically Possible Forms of Digital Information Labour

\begin{tabular}{|c|c|c|c|c|c|c|c|c|}
\hline$\#$ & $\begin{array}{l}\text { Information } \\
\text { worker }\end{array}$ & $\begin{array}{l}\text { Employer, } \\
\text { contractor }\end{array}$ & $\begin{array}{l}\text { Relationship } \\
\text { of production }\end{array}$ & Technology & Objects & Products & $\begin{array}{l}\text { Distri- } \\
\text { bution }\end{array}$ & $\begin{array}{l}\text { Con- } \\
\text { sumption }\end{array}$ \\
\hline 1 & Online & Online & Online & Brain + digital & Digital & Digital & Online & Online \\
\hline 2 & Online & Online & Online & Brain + digital & Digital & Digital & Online & Offline \\
\hline 3 & Online & Online & Online & Brain + digital & Digital & Digital & Offline & Online \\
\hline 4 & Online & Online & Online & Brain + digital & Digital & Digital & Offline & Offline \\
\hline 5 & Online & Online & Online & Brain + digital & Digital & Non-digital & Online & Online \\
\hline 6 & Online & Online & Online & Brain + digital & Digital & Non-digital & Online & Offline \\
\hline 7 & Online & Online & Online & Brain + digital & Digital & Non-digital & Offline & Online \\
\hline 8 & Online & Online & Online & Brain + digital & Digital & Non-digital & Offline & Offline \\
\hline 9 & Online & Online & Online & Brain + digital & Digital & Blended & Online & Online \\
\hline 10 & Online & Online & Online & Brain + digital & Digital & Blended & Online & Offline \\
\hline 11 & Online & Online & Online & Brain + digital & Digital & Blended & Offline & Online \\
\hline 12 & Online & Online & Online & Brain + digital & Digital & Blended & Offline & Offline \\
\hline 13 & Online & Online & Online & Brain + digital & $\begin{array}{l}\text { Non- } \\
\text { digital }\end{array}$ & Digital & Online & Online \\
\hline 14 & Online & Online & Online & Brain + digital & $\begin{array}{l}\text { Non- } \\
\text { digital }\end{array}$ & Digital & Online & Offline \\
\hline 15 & Online & Online & Online & Brain + digital & $\begin{array}{l}\text { Non- } \\
\text { digital }\end{array}$ & Digital & Offline & Online \\
\hline 16 & Online & Online & Online & Brain + digital & $\begin{array}{l}\text { Non- } \\
\text { digital }\end{array}$ & Digital & Offline & Offline \\
\hline 17 & Online & Online & Online & Brain + digital & $\begin{array}{l}\text { Non- } \\
\text { digital }\end{array}$ & Non-digital & Online & Online \\
\hline 18 & Online & Online & Online & Brain + digital & $\begin{array}{l}\text { Non- } \\
\text { digital }\end{array}$ & Non-digital & Online & Offline \\
\hline 19 & Online & Online & Online & Brain + digital & $\begin{array}{l}\text { Non- } \\
\text { digital }\end{array}$ & Non-digital & Offline & Online \\
\hline 20 & Online & Online & Online & Brain + digital & $\begin{array}{l}\text { Non- } \\
\text { digital }\end{array}$ & Non-digital & Offline & Offline \\
\hline 21 & Online & Online & Online & Brain + digital & $\begin{array}{l}\text { Non- } \\
\text { digital }\end{array}$ & Blended & Online & Online \\
\hline 22 & Online & Online & Online & Brain + digital & $\begin{array}{l}\text { Non- } \\
\text { digital }\end{array}$ & Blended & Online & Offline \\
\hline 23 & Online & Online & Online & Brain + digital & $\begin{array}{l}\text { Non- } \\
\text { digital }\end{array}$ & Blended & Offline & Online \\
\hline 24 & Online & Online & Online & Brain + digital & $\begin{array}{l}\text { Non- } \\
\text { digital }\end{array}$ & Blended & Offline & Offline \\
\hline 25 & Online & Online & Online & Brain + digital & $\begin{array}{l}\text { Blend- } \\
\text { ed }\end{array}$ & Digital & Online & Online \\
\hline 26 & Online & Online & Online & Brain + digital & $\begin{array}{l}\text { Blend- } \\
\text { ed }\end{array}$ & Digital & Online & Offline \\
\hline 27 & Online & Online & Online & Brain + digital & $\begin{array}{l}\text { Blend- } \\
\text { ed }\end{array}$ & Digital & Offline & Online \\
\hline 28 & Online & Online & Online & Brain + digital & $\begin{array}{l}\text { Blend- } \\
\text { ed }\end{array}$ & Digital & Offline & Offline \\
\hline 29 & Online & Online & Online & Brain + digital & $\begin{array}{l}\text { Blend- } \\
\text { ed }\end{array}$ & Non-digital & Online & Online \\
\hline 30 & Online & Online & Online & Brain + digital & $\begin{array}{l}\text { Blend- } \\
\text { ed }\end{array}$ & Non-digital & Online & Offline \\
\hline 31 & Online & Online & Online & Brain + digital & $\begin{array}{l}\text { Blend- } \\
\text { ed }\end{array}$ & Non-digital & Offline & Online \\
\hline 32 & Online & Online & Online & Brain + digital & $\begin{array}{l}\text { Blend- } \\
\text { ed }\end{array}$ & Non-digital & Offline & Offline \\
\hline 33 & Online & Online & Online & Brain + digital & $\begin{array}{l}\text { Blend- } \\
\text { ed }\end{array}$ & Blended & Online & Online \\
\hline 34 & Online & Online & Online & Brain + digital & $\begin{array}{l}\text { Blend- } \\
\text { ed }\end{array}$ & Blended & Online & Offline \\
\hline 35 & Online & Online & Online & Brain + digital & $\begin{array}{l}\text { Blend- } \\
\text { ed }\end{array}$ & Blended & Offline & Online \\
\hline 36 & Online & Online & Online & Brain + digital & $\begin{array}{l}\text { Blend- } \\
\text { ed }\end{array}$ & Blended & Offline & Offline \\
\hline 37 & Online & Online & Online & Brain & Digital & Digital & Online & Online \\
\hline
\end{tabular}




\begin{tabular}{|c|c|c|c|c|c|c|c|c|}
\hline 38 & Online & Online & Online & Brain & Digital & Digital & Online & Offline \\
\hline 39 & Online & Online & Online & Brain & Digital & Digital & Offline & Online \\
\hline 40 & Online & Online & Online & Brain & Digital & Digital & Offline & Offline \\
\hline 41 & Online & Online & Online & Brain & Digital & Non-digital & Online & Online \\
\hline 42 & Online & Online & Online & Brain & Digital & Non-digital & Online & Offline \\
\hline 43 & Online & Online & Online & Brain & Digital & Non-digital & Offline & Online \\
\hline 44 & Online & Online & Online & Brain & Digital & Non-digital & Offline & Offline \\
\hline 45 & Online & Online & Online & Brain & Digital & Blended & Online & Online \\
\hline 46 & Online & Online & Online & Brain & Digital & Blended & Online & Offline \\
\hline 47 & Online & Online & Online & Brain & Digital & Blended & Offline & Online \\
\hline 48 & Online & Online & Online & Brain & Digital & Blended & Offline & Offline \\
\hline 49 & Online & Online & Online & Brain & $\begin{array}{l}\text { Non- } \\
\text { digital }\end{array}$ & Digital & Online & Online \\
\hline 50 & Online & Online & Online & Brain & $\begin{array}{l}\text { Non- } \\
\text { digital }\end{array}$ & Digital & Online & Offline \\
\hline 51 & Online & Online & Online & Brain & $\begin{array}{l}\text { Non- } \\
\text { digital }\end{array}$ & Digital & Offline & Online \\
\hline 52 & Online & Online & Online & Brain & $\begin{array}{l}\text { Non- } \\
\text { digital }\end{array}$ & Digital & Offline & Offline \\
\hline 53 & Online & Online & Online & Brain & $\begin{array}{l}\text { Non- } \\
\text { digital }\end{array}$ & Non-digital & Online & Online \\
\hline 54 & Online & Online & Online & Brain & $\begin{array}{l}\text { Non- } \\
\text { digital }\end{array}$ & Non-digital & Online & Offline \\
\hline 55 & Online & Online & Online & Brain & $\begin{array}{l}\text { Non- } \\
\text { digital }\end{array}$ & Non-digital & Offline & Online \\
\hline 56 & Online & Online & Online & Brain & $\begin{array}{l}\text { Non- } \\
\text { digital }\end{array}$ & Non-digital & Offline & Offline \\
\hline 57 & Online & Online & Online & Brain & $\begin{array}{l}\text { Non- } \\
\text { digital }\end{array}$ & Blended & Online & Online \\
\hline 58 & Online & Online & Online & Brain & $\begin{array}{l}\text { Non- } \\
\text { digital }\end{array}$ & Blended & Online & Offline \\
\hline 59 & Online & Online & Online & Brain & $\begin{array}{l}\text { Non- } \\
\text { digital }\end{array}$ & Blended & Offline & Online \\
\hline 60 & Online & Online & Online & Brain & $\begin{array}{l}\text { Non- } \\
\text { digital }\end{array}$ & Blended & Offline & Offline \\
\hline 61 & Online & Online & Online & Brain & $\begin{array}{l}\text { Blend- } \\
\text { ed }\end{array}$ & Digital & Online & Online \\
\hline 62 & Online & Online & Online & Brain & $\begin{array}{l}\text { Blend- } \\
\text { ed }\end{array}$ & Digital & Online & Offline \\
\hline 63 & Online & Online & Online & Brain & $\begin{array}{l}\text { Blend- } \\
\text { ed }\end{array}$ & Digital & Offline & Online \\
\hline 64 & Online & Online & Online & Brain & $\begin{array}{l}\text { Blend- } \\
\text { ed }\end{array}$ & Digital & Offline & Offline \\
\hline 65 & Online & Online & Online & Brain & $\begin{array}{l}\text { Blend- } \\
\text { ed }\end{array}$ & Non-digital & Online & Online \\
\hline 66 & Online & Online & Online & Brain & $\begin{array}{l}\text { Blend- } \\
\text { ed }\end{array}$ & Non-digital & Online & Offline \\
\hline 67 & Online & Online & Online & Brain & $\begin{array}{l}\text { Blend- } \\
\text { ed }\end{array}$ & Non-digital & Offline & Online \\
\hline 68 & Online & Online & Online & Brain & $\begin{array}{l}\text { Blend- } \\
\text { ed }\end{array}$ & Non-digital & Offline & Offline \\
\hline 69 & Online & Online & Online & Brain & $\begin{array}{l}\text { Blend- } \\
\text { ed }\end{array}$ & Blended & Online & Online \\
\hline 70 & Online & Online & Online & Brain & $\begin{array}{l}\text { Blend- } \\
\text { ed }\end{array}$ & Blended & Online & Offline \\
\hline 71 & Online & Online & Online & Brain & $\begin{array}{l}\text { Blend- } \\
\text { ed }\end{array}$ & Blended & Offline & Online \\
\hline 72 & Online & Online & Online & Brain & $\begin{array}{l}\text { Blend- } \\
\text { ed }\end{array}$ & Blended & Offline & Offline \\
\hline 73 & Online & Online & Online & Brain $+d+n-d$ & Digital & Digital & Online & Online \\
\hline 74 & Online & Online & Online & Brain $+d+n-d$ & Digital & Digital & Online & Offline \\
\hline 75 & Online & Online & Online & Brain $+d+n-d$ & Digital & Digital & Offline & Online \\
\hline 76 & Online & Online & Online & Brain $+d+n-d$ & Digital & Digital & Offline & Offline \\
\hline 77 & Online & Online & Online & Brain $+d+n-d$ & Digital & Non-digital & Online & Online \\
\hline 78 & Online & Online & Online & Brain $+d+n-d$ & Digital & Non-digital & Online & Offline \\
\hline
\end{tabular}




\begin{tabular}{|c|c|c|c|c|c|c|c|c|}
\hline 79 & Online & Online & Online & Brain $+d+n-d$ & Digital & Non-digital & Offline & Online \\
\hline 80 & Online & Online & Online & Brain $+d+n-d$ & Digital & Non-digital & Offline & Offline \\
\hline 81 & Online & Online & Online & Brain $+d+n-d$ & Digital & Blended & Online & Online \\
\hline 82 & Online & Online & Online & Brain $+d+n-d$ & Digital & Blended & Online & Offline \\
\hline 83 & Online & Online & Online & Brain $+d+n-d$ & Digital & Blended & Offline & Online \\
\hline 84 & Online & Online & Online & Brain $+d+n-d$ & Digital & Blended & Offline & Offline \\
\hline 85 & Online & Online & Online & Brain $+d+n-d$ & $\begin{array}{l}\text { Non- } \\
\text { digital }\end{array}$ & Digital & Online & Online \\
\hline 86 & Online & Online & Online & Brain $+d+n-d$ & $\begin{array}{l}\text { Non- } \\
\text { digital }\end{array}$ & Digital & Online & Offline \\
\hline 87 & Online & Online & Online & Brain $+d+n-d$ & $\begin{array}{l}\text { Non- } \\
\text { digital }\end{array}$ & Digital & Offline & Online \\
\hline 88 & Online & Online & Online & Brain $+d+n-d$ & $\begin{array}{l}\text { Non- } \\
\text { digital }\end{array}$ & Digital & Offline & Offline \\
\hline 89 & Online & Online & Online & Brain $+d+n-d$ & $\begin{array}{l}\text { Non- } \\
\text { digital }\end{array}$ & Non-digital & Online & Online \\
\hline 90 & Online & Online & Online & Brain $+d+n-d$ & $\begin{array}{l}\text { Non- } \\
\text { digital }\end{array}$ & Non-digital & Online & Offline \\
\hline 91 & Online & Online & Online & Brain $+d+n-d$ & $\begin{array}{l}\text { Non- } \\
\text { digital }\end{array}$ & Non-digital & Offline & Online \\
\hline 92 & Online & Online & Online & Brain $+d+n-d$ & $\begin{array}{l}\text { Non- } \\
\text { digital }\end{array}$ & Non-digital & Offline & Offline \\
\hline 93 & Online & Online & Online & Brain $+d+n-d$ & $\begin{array}{l}\text { Non- } \\
\text { digital }\end{array}$ & Blended & Online & Online \\
\hline 94 & Online & Online & Online & Brain $+d+n-d$ & $\begin{array}{l}\text { Non- } \\
\text { digital }\end{array}$ & Blended & Online & Offline \\
\hline 95 & Online & Online & Online & Brain $+d+n-d$ & $\begin{array}{l}\text { Non- } \\
\text { digital }\end{array}$ & Blended & Offline & Online \\
\hline 96 & Online & Online & Online & Brain $+d+n-d$ & $\begin{array}{l}\text { Non- } \\
\text { digital }\end{array}$ & Blended & Offline & Offline \\
\hline 97 & Online & Online & Online & Brain $+d+n-d$ & $\begin{array}{l}\text { Blend- } \\
\text { ed }\end{array}$ & Digital & Online & Online \\
\hline 98 & Online & Online & Online & Brain $+d+n-d$ & $\begin{array}{l}\text { Blend- } \\
\text { ed }\end{array}$ & Digital & Online & Offline \\
\hline 99 & Online & Online & Online & Brain $+d+n-d$ & $\begin{array}{l}\text { Blend- } \\
\text { ed }\end{array}$ & Digital & Offline & Online \\
\hline 100 & Online & Online & Online & Brain $+d+n-d$ & $\begin{array}{l}\text { Blend- } \\
\text { ed }\end{array}$ & Digital & Offline & Offline \\
\hline 101 & Online & Online & Online & Brain $+d+n-d$ & $\begin{array}{l}\text { Blend- } \\
\text { ed }\end{array}$ & Non-digital & Online & Online \\
\hline 102 & Online & Online & Online & Brain $+d+n-d$ & $\begin{array}{l}\text { Blend- } \\
\text { ed }\end{array}$ & Non-digital & Online & Offline \\
\hline 103 & Online & Online & Online & Brain $+d+n-d$ & $\begin{array}{l}\text { Blend- } \\
\text { ed }\end{array}$ & Non-digital & Offline & Online \\
\hline 104 & Online & Online & Online & Brain $+d+n-d$ & $\begin{array}{l}\text { Blend- } \\
\text { ed }\end{array}$ & Non-digital & Offline & Offline \\
\hline 105 & Online & Online & Online & Brain $+d+n-d$ & $\begin{array}{l}\text { Blend- } \\
\text { ed }\end{array}$ & Blended & Online & Online \\
\hline 106 & Online & Online & Online & Brain $+d+n-d$ & $\begin{array}{l}\text { Blend- } \\
\text { ed }\end{array}$ & Blended & Online & Offline \\
\hline 107 & Online & Online & Online & Brain $+d+n-d$ & $\begin{array}{l}\text { Blend- } \\
\text { ed }\end{array}$ & Blended & Offline & Online \\
\hline 108 & Online & Online & Online & Brain $+d+n-d$ & $\begin{array}{l}\text { Blend- } \\
\text { ed }\end{array}$ & Blended & Offline & Offline \\
\hline 109 & Online & Online & Online & Brain + n-d & Digital & Digital & Online & Online \\
\hline 110 & Online & Online & Online & Brain + n-d & Digital & Digital & Online & Offline \\
\hline 111 & Online & Online & Online & Brain + n-d & Digital & Digital & Offline & Online \\
\hline 112 & Online & Online & Online & Brain + n-d & Digital & Digital & Offline & Offline \\
\hline 113 & Online & Online & Online & Brain + n-d & Digital & Non-digital & Online & Online \\
\hline 114 & Online & Online & Online & Brain + n-d & Digital & Non-digital & Online & Offline \\
\hline 115 & Online & Online & Online & Brain + n-d & Digital & Non-digital & Offline & Online \\
\hline 116 & Online & Online & Online & Brain + n-d & Digital & Non-digital & Offline & Offline \\
\hline 117 & Online & Online & Online & Brain + n-d & Digital & Blended & Online & Online \\
\hline 118 & Online & Online & Online & Brain + n-d & Digital & Blended & Online & Offline \\
\hline 119 & Online & Online & Online & Brain $+n-d$ & Digital & Blended & Offline & Online \\
\hline
\end{tabular}




\begin{tabular}{|c|c|c|c|c|c|c|c|c|}
\hline 120 & Online & Online & Online & Brain + n-d & Digital & Blended & Offline & Offline \\
\hline 121 & Online & Online & Online & Brain + n-d & $\begin{array}{l}\text { Non- } \\
\text { digital }\end{array}$ & Digital & Online & Online \\
\hline 122 & Online & Online & Online & Brain + n-d & $\begin{array}{l}\text { Non- } \\
\text { digital }\end{array}$ & Digital & Online & Offline \\
\hline 123 & Online & Online & Online & Brain + n-d & $\begin{array}{l}\text { Non- } \\
\text { digital }\end{array}$ & Digital & Offline & Online \\
\hline 124 & Online & Online & Online & Brain + n-d & $\begin{array}{l}\text { Non- } \\
\text { digital }\end{array}$ & Digital & Offline & Offline \\
\hline 125 & Online & Online & Online & Brain + n-d & $\begin{array}{l}\text { Non- } \\
\text { digital }\end{array}$ & Non-digital & Online & Online \\
\hline 126 & Online & Online & Online & Brain + n-d & $\begin{array}{l}\text { Non- } \\
\text { digital }\end{array}$ & Non-digital & Online & Offline \\
\hline 127 & Online & Online & Online & Brain + n-d & $\begin{array}{l}\text { Non- } \\
\text { digital }\end{array}$ & Non-digital & Offline & Online \\
\hline 128 & Online & Online & Online & Brain $+n-d$ & $\begin{array}{l}\text { Non- } \\
\text { digital }\end{array}$ & Non-digital & Offline & Offline \\
\hline 129 & Online & Online & Online & Brain + n-d & $\begin{array}{l}\text { Non- } \\
\text { digital }\end{array}$ & Blended & Online & Online \\
\hline 130 & Online & Online & Online & Brain + n-d & $\begin{array}{l}\text { Non- } \\
\text { digital }\end{array}$ & Blended & Online & Offline \\
\hline 131 & Online & Online & Online & Brain + n-d & $\begin{array}{l}\text { Non- } \\
\text { digital }\end{array}$ & Blended & Offline & Online \\
\hline 132 & Online & Online & Online & Brain $+n-d$ & $\begin{array}{l}\text { Non- } \\
\text { digital }\end{array}$ & Blended & Offline & Offline \\
\hline 133 & Online & Online & Online & Brain + n-d & $\begin{array}{l}\text { Blend- } \\
\text { ed }\end{array}$ & Digital & Online & Online \\
\hline 134 & Online & Online & Online & Brain + n-d & $\begin{array}{l}\text { Blend- } \\
\text { ed }\end{array}$ & Digital & Online & Offline \\
\hline 135 & Online & Online & Online & Brain + n-d & $\begin{array}{l}\text { Blend- } \\
\text { ed }\end{array}$ & Digital & Offline & Online \\
\hline 136 & Online & Online & Online & Brain $+n-d$ & $\begin{array}{l}\text { Blend- } \\
\text { ed }\end{array}$ & Digital & Offline & Offline \\
\hline 137 & Online & Online & Online & Brain + n-d & $\begin{array}{l}\text { Blend- } \\
\text { ed }\end{array}$ & Non-digital & Online & Online \\
\hline 138 & Online & Online & Online & Brain + n-d & $\begin{array}{l}\text { Blend- } \\
\text { ed }\end{array}$ & Non-digital & Online & Offline \\
\hline 139 & Online & Online & Online & Brain + n-d & $\begin{array}{l}\text { Blend- } \\
\text { ed }\end{array}$ & Non-digital & Offline & Online \\
\hline 140 & Online & Online & Online & Brain + n-d & $\begin{array}{l}\text { Blend- } \\
\text { ed }\end{array}$ & Non-digital & Offline & Offline \\
\hline 141 & Online & Online & Online & Brain + n-d & $\begin{array}{l}\text { Blend- } \\
\text { ed }\end{array}$ & Blended & Online & Online \\
\hline 142 & Online & Online & Online & Brain + n-d & $\begin{array}{l}\text { Blend- } \\
\text { ed }\end{array}$ & Blended & Online & Offline \\
\hline 143 & Online & Online & Online & Brain + n-d & $\begin{array}{l}\text { Blend- } \\
\text { ed }\end{array}$ & Blended & Offline & Online \\
\hline 144 & Online & Online & Online & Brain + n-d & $\begin{array}{l}\text { Blend- } \\
\text { ed }\end{array}$ & Blended & Offline & Offline \\
\hline 145 & Online & Online & Offline & Brain + digital & Digital & Digital & Online & Online \\
\hline 146 & Online & Online & Offline & Brain + digital & Digital & Digital & Online & Offline \\
\hline 147 & Online & Online & Offline & Brain + digital & Digital & Digital & Offline & Online \\
\hline 148 & Online & Online & Offline & Brain + digital & Digital & Digital & Offline & Offline \\
\hline 149 & Online & Online & Offline & Brain + digital & Digital & Non-digital & Online & Online \\
\hline 150 & Online & Online & Offline & Brain + digital & Digital & Non-digital & Online & Offline \\
\hline 151 & Online & Online & Offline & Brain + digital & Digital & Non-digital & Offline & Online \\
\hline 152 & Online & Online & Offline & Brain + digital & Digital & Non-digital & Offline & Offline \\
\hline 153 & Online & Online & Offline & Brain + digital & Digital & Blended & Online & Online \\
\hline 154 & Online & Online & Offline & Brain + digital & Digital & Blended & Online & Offline \\
\hline 155 & Online & Online & Offline & Brain + digital & Digital & Blended & Offline & Online \\
\hline 156 & Online & Online & Offline & Brain + digital & Digital & Blended & Offline & Offline \\
\hline 157 & Online & Online & Offline & Brain + digital & $\begin{array}{l}\text { Non- } \\
\text { digital }\end{array}$ & Digital & Online & Online \\
\hline 158 & Online & Online & Offline & Brain + digital & $\begin{array}{l}\text { Non- } \\
\text { digital }\end{array}$ & Digital & Online & Offline \\
\hline
\end{tabular}




\begin{tabular}{|c|c|c|c|c|c|c|c|c|}
\hline 159 & Online & Online & Offline & Brain + digital & $\begin{array}{l}\text { Non- } \\
\text { digital }\end{array}$ & Digital & Offline & Online \\
\hline 160 & Online & Online & Offline & Brain + digital & $\begin{array}{l}\text { Non- } \\
\text { digital }\end{array}$ & Digital & Offline & Offline \\
\hline 161 & Online & Online & Offline & Brain + digital & $\begin{array}{l}\text { Non- } \\
\text { digital }\end{array}$ & Non-digital & Online & Online \\
\hline 162 & Online & Online & Offline & Brain + digital & $\begin{array}{l}\text { Non- } \\
\text { digital }\end{array}$ & Non-digital & Online & Offline \\
\hline 163 & Online & Online & Offline & Brain + digital & $\begin{array}{l}\text { Non- } \\
\text { digital }\end{array}$ & Non-digital & Offline & Online \\
\hline 164 & Online & Online & Offline & Brain + digital & $\begin{array}{l}\text { Non- } \\
\text { digital }\end{array}$ & Non-digital & Offline & Offline \\
\hline 165 & Online & Online & Offline & Brain + digital & $\begin{array}{l}\text { Non- } \\
\text { digital }\end{array}$ & Blended & Online & Online \\
\hline 166 & Online & Online & Offline & Brain + digital & $\begin{array}{l}\text { Non- } \\
\text { digital }\end{array}$ & Blended & Online & Offline \\
\hline 167 & Online & Online & Offline & Brain + digital & $\begin{array}{l}\text { Non- } \\
\text { digital }\end{array}$ & Blended & Offline & Online \\
\hline 168 & Online & Online & Offline & Brain + digital & $\begin{array}{l}\text { Non- } \\
\text { digital }\end{array}$ & Blended & Offline & Offline \\
\hline 169 & Online & Online & Offline & Brain + digital & $\begin{array}{l}\text { Blend- } \\
\text { ed }\end{array}$ & Digital & Online & Online \\
\hline 170 & Online & Online & Offline & Brain + digital & $\begin{array}{l}\text { Blend- } \\
\text { ed }\end{array}$ & Digital & Online & Offline \\
\hline 171 & Online & Online & Offline & Brain + digital & $\begin{array}{l}\text { Blend- } \\
\text { ed }\end{array}$ & Digital & Offline & Online \\
\hline 172 & Online & Online & Offline & Brain + digital & $\begin{array}{l}\text { Blend- } \\
\text { ed }\end{array}$ & Digital & Offline & Offline \\
\hline 173 & Online & Online & Offline & Brain + digital & $\begin{array}{l}\text { Blend- } \\
\text { ed }\end{array}$ & Non-digital & Online & Online \\
\hline 174 & Online & Online & Offline & Brain + digital & $\begin{array}{l}\text { Blend- } \\
\text { ed }\end{array}$ & Non-digital & Online & Offline \\
\hline 175 & Online & Online & Offline & Brain + digital & $\begin{array}{l}\text { Blend- } \\
\text { ed }\end{array}$ & Non-digital & Offline & Online \\
\hline 176 & Online & Online & Offline & Brain + digital & $\begin{array}{l}\text { Blend- } \\
\text { ed }\end{array}$ & Non-digital & Offline & Offline \\
\hline 177 & Online & Online & Offline & Brain + digital & $\begin{array}{l}\text { Blend- } \\
\text { ed }\end{array}$ & Blended & Online & Online \\
\hline 178 & Online & Online & Offline & Brain + digital & $\begin{array}{l}\text { Blend- } \\
\text { ed }\end{array}$ & Blended & Online & Offline \\
\hline 179 & Online & Online & Offline & Brain + digital & $\begin{array}{l}\text { Blend- } \\
\text { ed }\end{array}$ & Blended & Offline & Online \\
\hline 180 & Online & Online & Offline & Brain + digital & $\begin{array}{l}\text { Blend- } \\
\text { ed }\end{array}$ & Blended & Offline & Offline \\
\hline 181 & Online & Online & Offline & Brain & Digital & Digital & Online & Online \\
\hline 182 & Online & Online & Offline & Brain & Digital & Digital & Online & Offline \\
\hline 183 & Online & Online & Offline & Brain & Digital & Digital & Offline & Online \\
\hline 184 & Online & \begin{tabular}{|l|} 
Online \\
\end{tabular} & Offline & Brain & Digital & Digital & Offline & Offline \\
\hline 185 & Online & Online & Offline & Brain & Digital & Non-digital & Online & Online \\
\hline 186 & Online & Online & Offline & Brain & Digital & Non-digital & Online & Offline \\
\hline 187 & Online & Online & Offline & Brain & Digital & Non-digital & Offline & Online \\
\hline 188 & Online & Online & Offline & Brain & Digital & Non-digital & Offline & Offline \\
\hline 189 & Online & Online & Offline & Brain & Digital & Blended & Online & Online \\
\hline 190 & Online & Online & Offline & Brain & Digital & Blended & Online & Offline \\
\hline 191 & Online & Online & Offline & Brain & Digital & Blended & Offline & Online \\
\hline 192 & Online & Online & Offline & Brain & Digital & Blended & Offline & Offline \\
\hline 193 & Online & Online & Offline & Brain & $\begin{array}{l}\text { Non- } \\
\text { digital }\end{array}$ & Digital & Online & Online \\
\hline 194 & Online & Online & Offline & Brain & $\begin{array}{l}\text { Non- } \\
\text { digital } \\
\end{array}$ & Digital & Online & Offline \\
\hline 195 & Online & Online & Offline & Brain & $\begin{array}{l}\text { Non- } \\
\text { digital }\end{array}$ & Digital & Offline & Online \\
\hline 196 & Online & Online & Offline & Brain & $\begin{array}{l}\text { Non- } \\
\text { digital }\end{array}$ & Digital & Offline & Offline \\
\hline
\end{tabular}




\begin{tabular}{|c|c|c|c|c|c|c|c|c|}
\hline 197 & Online & Online & Offline & Brain & $\begin{array}{l}\text { Non- } \\
\text { digital }\end{array}$ & Non-digital & Online & Online \\
\hline 198 & Online & Online & Offline & Brain & $\begin{array}{l}\text { Non- } \\
\text { digital }\end{array}$ & Non-digital & Online & Offline \\
\hline 199 & Online & Online & Offline & Brain & $\begin{array}{l}\text { Non- } \\
\text { digital }\end{array}$ & Non-digital & Offline & Online \\
\hline 200 & Online & Online & Offline & Brain & $\begin{array}{l}\text { Non- } \\
\text { digital }\end{array}$ & Non-digital & Offline & Offline \\
\hline 201 & Online & Online & Offline & Brain & $\begin{array}{l}\text { Non- } \\
\text { digital }\end{array}$ & Blended & Online & Online \\
\hline 202 & Online & Online & Offline & Brain & $\begin{array}{l}\text { Non- } \\
\text { digital }\end{array}$ & Blended & Online & Offline \\
\hline 203 & Online & Online & Offline & Brain & $\begin{array}{l}\text { Non- } \\
\text { digital }\end{array}$ & Blended & Offline & Online \\
\hline 204 & Online & Online & Offline & Brain & $\begin{array}{l}\text { Non- } \\
\text { digital }\end{array}$ & Blended & Offline & Offline \\
\hline 205 & Online & Online & Offline & Brain & $\begin{array}{l}\text { Blend- } \\
\text { ed }\end{array}$ & Digital & Online & Online \\
\hline 206 & Online & Online & Offline & Brain & $\begin{array}{l}\text { Blend- } \\
\text { ed }\end{array}$ & Digital & Online & Offline \\
\hline 207 & Online & Online & Offline & Brain & $\begin{array}{l}\text { Blend- } \\
\text { ed }\end{array}$ & Digital & Offline & Online \\
\hline 208 & Online & Online & Offline & Brain & $\begin{array}{l}\text { Blend- } \\
\text { ed }\end{array}$ & Digital & Offline & Offline \\
\hline 209 & Online & Online & Offline & Brain & $\begin{array}{l}\text { Blend- } \\
\text { ed }\end{array}$ & Non-digital & Online & Online \\
\hline 210 & Online & Online & Offline & Brain & $\begin{array}{l}\text { Blend- } \\
\text { ed }\end{array}$ & Non-digital & Online & Offline \\
\hline 211 & Online & Online & Offline & Brain & $\begin{array}{l}\text { Blend- } \\
\text { ed }\end{array}$ & Non-digital & Offline & Online \\
\hline 212 & Online & Online & Offline & Brain & $\begin{array}{l}\text { Blend- } \\
\text { ed }\end{array}$ & Non-digital & Offline & Offline \\
\hline 213 & Online & Online & Offline & Brain & $\begin{array}{l}\text { Blend- } \\
\text { ed }\end{array}$ & Blended & Online & Online \\
\hline 214 & Online & Online & Offline & Brain & $\begin{array}{l}\text { Blend- } \\
\text { ed }\end{array}$ & Blended & Online & Offline \\
\hline 215 & Online & Online & Offline & Brain & $\begin{array}{l}\text { Blend- } \\
\text { ed }\end{array}$ & Blended & Offline & Online \\
\hline 216 & Online & Online & Offline & Brain & $\begin{array}{l}\text { Blend- } \\
\text { ed }\end{array}$ & Blended & Offline & Offline \\
\hline 217 & Online & Online & Offline & Brain $+d+n-d$ & Digital & Digital & Online & Online \\
\hline 218 & Online & Online & Offline & Brain $+d+n-d$ & Digital & Digital & Online & Offline \\
\hline 219 & Online & Online & Offline & Brain $+d+n-d$ & Digital & Digital & Offline & Online \\
\hline 220 & Online & Online & Offline & Brain $+d+n-d$ & Digital & Digital & Offline & Offline \\
\hline 221 & Online & Online & Offline & Brain $+d+n-d$ & Digital & Non-digital & Online & Online \\
\hline 222 & Online & Online & Offline & Brain $+d+n-d$ & Digital & Non-digital & Online & Offline \\
\hline 223 & Online & Online & Offline & Brain $+d+n-d$ & Digital & Non-digital & Offline & Online \\
\hline 224 & Online & Online & Offline & Brain $+d+n-d$ & Digital & Non-digital & Offline & Offline \\
\hline 225 & Online & Online & Offline & Brain $+d+n-d$ & Digital & Blended & Online & Online \\
\hline 226 & Online & Online & Offline & Brain $+d+n-d$ & Digital & Blended & Online & Offline \\
\hline 227 & Online & Online & Offline & Brain + d + n-d & Digital & Blended & Offline & Online \\
\hline 228 & Online & Online & Offline & Brain + d + n-d & Digital & Blended & Offline & Offline \\
\hline 229 & Online & Online & Offline & Brain $+d+n-d$ & $\begin{array}{l}\text { Non- } \\
\text { digital }\end{array}$ & Digital & Online & Online \\
\hline 230 & Online & Online & Offline & Brain $+d+n-d$ & $\begin{array}{l}\text { Non- } \\
\text { digital }\end{array}$ & Digital & Online & Offline \\
\hline 231 & Online & Online & Offline & Brain $+d+n-d$ & $\begin{array}{l}\text { Non- } \\
\text { digital }\end{array}$ & Digital & Offline & Online \\
\hline 232 & Online & Online & Offline & Brain $+d+n-d$ & $\begin{array}{l}\text { Non- } \\
\text { digital }\end{array}$ & Digital & Offline & Offline \\
\hline 233 & Online & Online & Offline & Brain $+d+n-d$ & $\begin{array}{l}\text { Non- } \\
\text { digital }\end{array}$ & Non-digital & Online & Online \\
\hline 234 & Online & Online & Offline & Brain $+d+n-d$ & $\begin{array}{l}\text { Non- } \\
\text { digital }\end{array}$ & Non-digital & Online & Offline \\
\hline
\end{tabular}




\begin{tabular}{|c|c|c|c|c|c|c|c|c|}
\hline 235 & Online & Online & Offline & Brain $+d+n-d$ & $\begin{array}{l}\text { Non- } \\
\text { digital }\end{array}$ & Non-digital & Offline & Online \\
\hline 236 & Online & Online & Offline & Brain $+d+n-d$ & $\begin{array}{l}\text { Non- } \\
\text { digital }\end{array}$ & Non-digital & Offline & Offline \\
\hline 237 & Online & Online & Offline & Brain $+d+n-d$ & $\begin{array}{l}\text { Non- } \\
\text { digital }\end{array}$ & Blended & Online & Online \\
\hline 238 & Online & Online & Offline & Brain $+d+n-d$ & $\begin{array}{l}\text { Non- } \\
\text { digital }\end{array}$ & Blended & Online & Offline \\
\hline 239 & Online & Online & Offline & Brain $+d+n-d$ & $\begin{array}{l}\text { Non- } \\
\text { digital }\end{array}$ & Blended & Offline & Online \\
\hline 240 & Online & Online & Offline & Brain $+d+n-d$ & $\begin{array}{l}\text { Non- } \\
\text { digital }\end{array}$ & Blended & Offline & Offline \\
\hline 241 & Online & Online & Offline & Brain $+d+n-d$ & $\begin{array}{l}\text { Blend- } \\
\text { ed }\end{array}$ & Digital & Online & Online \\
\hline 242 & Online & Online & Offline & Brain $+d+n-d$ & $\begin{array}{l}\text { Blend- } \\
\text { ed }\end{array}$ & Digital & Online & Offline \\
\hline 243 & Online & Online & Offline & Brain $+d+n-d$ & $\begin{array}{l}\text { Blend- } \\
\text { ed }\end{array}$ & Digital & Offline & Online \\
\hline 244 & Online & Online & Offline & Brain $+d+n-d$ & $\begin{array}{l}\text { Blend- } \\
\text { ed }\end{array}$ & Digital & Offline & Offline \\
\hline 245 & Online & Online & Offline & Brain $+d+n-d$ & $\begin{array}{l}\text { Blend- } \\
\text { ed }\end{array}$ & Non-digital & Online & Online \\
\hline 246 & Online & Online & Offline & Brain $+d+n-d$ & $\begin{array}{l}\text { Blend- } \\
\text { ed }\end{array}$ & Non-digital & Online & Offline \\
\hline 247 & Online & Online & Offline & Brain $+d+n-d$ & $\begin{array}{l}\text { Blend- } \\
\text { ed }\end{array}$ & Non-digital & Offline & Online \\
\hline 248 & Online & Online & Offline & Brain $+d+n-d$ & $\begin{array}{l}\text { Blend- } \\
\text { ed }\end{array}$ & Non-digital & Offline & Offline \\
\hline 249 & Online & Online & Offline & Brain $+d+n-d$ & $\begin{array}{l}\text { Blend- } \\
\text { ed }\end{array}$ & Blended & Online & Online \\
\hline 250 & Online & Online & Offline & Brain $+d+n-d$ & $\begin{array}{l}\text { Blend- } \\
\text { ed }\end{array}$ & Blended & Online & Offline \\
\hline 251 & Online & Online & Offline & Brain $+d+n-d$ & $\begin{array}{l}\text { Blend- } \\
\text { ed }\end{array}$ & Blended & Offline & Online \\
\hline 252 & Online & Online & Offline & Brain $+d+n-d$ & $\begin{array}{l}\text { Blend- } \\
\text { ed }\end{array}$ & Blended & Offline & Offline \\
\hline 253 & Online & Online & Offline & Brain $+n-d$ & Digital & Digital & Online & Online \\
\hline 254 & Online & Online & Offline & Brain $+n-d$ & Digital & Digital & Online & Offline \\
\hline 255 & Online & Online & Offline & Brain $+n-d$ & Digital & Digital & Offline & Online \\
\hline 256 & Online & Online & Offline & Brain $+n-d$ & Digital & Digital & Offline & Offline \\
\hline 257 & Online & Online & Offline & Brain + n-d & Digital & Non-digital & Online & Online \\
\hline 258 & Online & Online & Offline & Brain + n-d & Digital & Non-digital & Online & Offline \\
\hline 259 & Online & Online & Offline & Brain $+n-d$ & Digital & Non-digital & Offline & Online \\
\hline 260 & Online & Online & Offline & Brain $+n-d$ & Digital & Non-digital & Offline & Offline \\
\hline 261 & Online & Online & Offline & Brain + n-d & Digital & Blended & Online & Online \\
\hline 262 & Online & Online & Offline & Brain + n-d & Digital & Blended & Online & Offline \\
\hline 263 & Online & Online & Offline & Brain $+n-d$ & Digital & Blended & Offline & Online \\
\hline 264 & Online & Online & Offline & Brain $+n-d$ & Digital & Blended & Offline & Offline \\
\hline 265 & Online & Online & Offline & Brain + n-d & $\begin{array}{l}\text { Non- } \\
\text { digital }\end{array}$ & Digital & Online & Online \\
\hline 266 & Online & Online & Offline & Brain $+n-d$ & $\begin{array}{l}\text { Non- } \\
\text { digital }\end{array}$ & Digital & Online & Offline \\
\hline 267 & Online & Online & Offline & Brain $+n-d$ & $\begin{array}{l}\text { Non- } \\
\text { digital }\end{array}$ & Digital & Offline & Online \\
\hline 268 & Online & Online & Offline & Brain + n-d & $\begin{array}{l}\text { Non- } \\
\text { digital }\end{array}$ & Digital & Offline & Offline \\
\hline 269 & Online & Online & Offline & Brain + n-d & $\begin{array}{l}\text { Non- } \\
\text { digital }\end{array}$ & Non-digital & Online & Online \\
\hline 270 & Online & Online & Offline & Brain + n-d & $\begin{array}{l}\text { Non- } \\
\text { digital }\end{array}$ & Non-digital & Online & Offline \\
\hline 271 & Online & Online & Offline & Brain + n-d & $\begin{array}{l}\text { Non- } \\
\text { digital }\end{array}$ & Non-digital & Offline & Online \\
\hline 272 & Online & Online & Offline & Brain $+n-d$ & $\begin{array}{l}\text { Non- } \\
\text { digital }\end{array}$ & Non-digital & Offline & Offline \\
\hline
\end{tabular}




\begin{tabular}{|c|c|c|c|c|c|c|c|c|}
\hline 273 & Online & Online & Offline & Brain + n-d & $\begin{array}{l}\text { Non- } \\
\text { digital }\end{array}$ & Blended & Online & Online \\
\hline 274 & Online & Online & Offline & Brain + n-d & $\begin{array}{l}\text { Non- } \\
\text { digital }\end{array}$ & Blended & Online & Offline \\
\hline 275 & Online & Online & Offline & Brain + n-d & $\begin{array}{l}\text { Non- } \\
\text { digital }\end{array}$ & Blended & Offline & Online \\
\hline 276 & Online & Online & Offline & Brain + n-d & $\begin{array}{l}\text { Non- } \\
\text { digital }\end{array}$ & Blended & Offline & Offline \\
\hline 277 & Online & Online & Offline & Brain + n-d & $\begin{array}{l}\text { Blend- } \\
\text { ed }\end{array}$ & Digital & Online & Online \\
\hline 278 & Online & Online & Offline & Brain + n-d & $\begin{array}{l}\text { Blend- } \\
\text { ed }\end{array}$ & Digital & Online & Offline \\
\hline 279 & Online & Online & Offline & Brain + n-d & $\begin{array}{l}\text { Blend- } \\
\text { ed }\end{array}$ & Digital & Offline & Online \\
\hline 280 & Online & Online & Offline & Brain + n-d & $\begin{array}{l}\text { Blend- } \\
\text { ed }\end{array}$ & Digital & Offline & Offline \\
\hline 281 & Online & Online & Offline & Brain + n-d & $\begin{array}{l}\text { Blend- } \\
\text { ed }\end{array}$ & Non-digital & Online & Online \\
\hline 282 & Online & Online & Offline & Brain + n-d & $\begin{array}{l}\text { Blend- } \\
\text { ed }\end{array}$ & Non-digital & Online & Offline \\
\hline 283 & Online & Online & Offline & Brain + n-d & $\begin{array}{l}\text { Blend- } \\
\text { ed }\end{array}$ & Non-digital & Offline & Online \\
\hline 284 & Online & Online & Offline & Brain + n-d & $\begin{array}{l}\text { Blend- } \\
\text { ed }\end{array}$ & Non-digital & Offline & Offline \\
\hline 285 & Online & Online & Offline & Brain + n-d & $\begin{array}{l}\text { Blend- } \\
\text { ed }\end{array}$ & Blended & Online & Online \\
\hline 286 & Online & Online & Offline & Brain + n-d & $\begin{array}{l}\text { Blend- } \\
\text { ed }\end{array}$ & Blended & Online & Offline \\
\hline 287 & Online & Online & Offline & Brain + n-d & $\begin{array}{l}\text { Blend- } \\
\text { ed }\end{array}$ & Blended & Offline & Online \\
\hline 288 & Online & Online & Offline & Brain + n-d & $\begin{array}{l}\text { Blend- } \\
\text { ed }\end{array}$ & Blended & Offline & Offline \\
\hline 289 & Online & Online & Blended & Brain + digital & Digital & Digital & Online & Online \\
\hline 290 & Online & Online & Blended & Brain + digital & Digital & Digital & Online & Offline \\
\hline 291 & Online & Online & Blended & Brain + digital & Digital & Digital & Offline & Online \\
\hline 292 & Online & Online & Blended & Brain + digital & Digital & Digital & Offline & Offline \\
\hline 293 & Online & Online & Blended & Brain + digital & Digital & Non-digital & Online & Online \\
\hline 294 & Online & Online & Blended & Brain + digital & Digital & Non-digital & Online & Offline \\
\hline 295 & Online & Online & Blended & Brain + digital & Digital & Non-digital & Offline & Online \\
\hline 296 & Online & Online & Blended & Brain + digital & Digital & Non-digital & Offline & Offline \\
\hline 297 & Online & Online & Blended & Brain + digital & Digital & Blended & Online & Online \\
\hline 298 & Online & Online & Blended & Brain + digital & Digital & Blended & Online & Offline \\
\hline 299 & Online & Online & Blended & Brain + digital & Digital & Blended & Offline & Online \\
\hline 300 & Online & Online & Blended & Brain + digital & Digital & Blended & Offline & Offline \\
\hline 301 & Online & Online & Blended & Brain + digital & $\begin{array}{l}\text { Non- } \\
\text { digital }\end{array}$ & Digital & Online & Online \\
\hline 302 & Online & Online & Blended & Brain + digital & $\begin{array}{l}\text { Non- } \\
\text { digital }\end{array}$ & Digital & Online & Offline \\
\hline 303 & Online & Online & Blended & Brain + digital & $\begin{array}{l}\text { Non- } \\
\text { digital }\end{array}$ & Digital & Offline & Online \\
\hline 304 & Online & Online & Blended & Brain + digital & $\begin{array}{l}\text { Non- } \\
\text { digital }\end{array}$ & Digital & Offline & Offline \\
\hline 305 & Online & Online & Blended & Brain + digital & $\begin{array}{l}\text { Non- } \\
\text { digital }\end{array}$ & Non-digital & Online & Online \\
\hline 306 & Online & Online & Blended & Brain + digital & $\begin{array}{l}\text { Non- } \\
\text { diqital }\end{array}$ & Non-digital & Online & Offline \\
\hline 307 & Online & Online & Blended & Brain + digital & $\begin{array}{l}\text { Non- } \\
\text { digital }\end{array}$ & Non-digital & Offline & Online \\
\hline 308 & Online & Online & Blended & Brain + digital & $\begin{array}{l}\text { Non- } \\
\text { diqital }\end{array}$ & Non-digital & Offline & Offline \\
\hline 309 & Online & Online & Blended & Brain + digital & $\begin{array}{l}\text { Non- } \\
\text { digital }\end{array}$ & Blended & Online & Online \\
\hline 310 & Online & Online & Blended & Brain + digital & $\begin{array}{l}\text { Non- } \\
\text { digital }\end{array}$ & Blended & Online & Offline \\
\hline
\end{tabular}




\begin{tabular}{|c|c|c|c|c|c|c|c|c|}
\hline 311 & Online & Online & Blended & Brain + digital & $\begin{array}{l}\text { Non- } \\
\text { digital }\end{array}$ & Blended & Offline & Online \\
\hline 312 & Online & Online & Blended & Brain + digital & $\begin{array}{l}\text { Non- } \\
\text { digital }\end{array}$ & Blended & Offline & Offline \\
\hline 313 & Online & Online & Blended & Brain + digital & $\begin{array}{l}\text { Blend- } \\
\text { ed }\end{array}$ & Digital & Online & Online \\
\hline 314 & Online & Online & Blended & Brain + digital & $\begin{array}{l}\text { Blend- } \\
\text { ed }\end{array}$ & Digital & Online & Offline \\
\hline 315 & Online & Online & Blended & Brain + digital & $\begin{array}{l}\text { Blend- } \\
\text { ed }\end{array}$ & Digital & Offline & Online \\
\hline 316 & Online & Online & Blended & Brain + digital & $\begin{array}{l}\text { Blend- } \\
\text { ed }\end{array}$ & Digital & Offline & Offline \\
\hline 317 & Online & Online & Blended & Brain + digital & $\begin{array}{l}\text { Blend- } \\
\text { ed }\end{array}$ & Non-digital & Online & Online \\
\hline 318 & Online & Online & Blended & Brain + digital & $\begin{array}{l}\text { Blend- } \\
\text { ed }\end{array}$ & Non-digital & Online & Offline \\
\hline 319 & Online & Online & Blended & Brain + digital & $\begin{array}{l}\text { Blend- } \\
\text { ed }\end{array}$ & Non-digital & Offline & Online \\
\hline 320 & Online & Online & Blended & Brain + digital & $\begin{array}{l}\text { Blend- } \\
\text { ed }\end{array}$ & Non-digital & Offline & Offline \\
\hline 321 & Online & Online & Blended & Brain + digital & $\begin{array}{l}\text { Blend- } \\
\text { ed }\end{array}$ & Blended & Online & Online \\
\hline 322 & Online & Online & Blended & Brain + digital & $\begin{array}{l}\text { Blend- } \\
\text { ed }\end{array}$ & Blended & Online & Offline \\
\hline 323 & Online & Online & Blended & Brain + digital & $\begin{array}{l}\text { Blend- } \\
\text { ed }\end{array}$ & Blended & Offline & Online \\
\hline 324 & Online & Online & Blended & Brain + digital & $\begin{array}{l}\text { Blend- } \\
\text { ed }\end{array}$ & Blended & Offline & Offline \\
\hline 325 & Online & Online & Blended & Brain & Digital & Digital & Online & Online \\
\hline 326 & Online & Online & Blended & Brain & Digital & Digital & Online & Offline \\
\hline 327 & Online & Online & Blended & Brain & Digital & Digital & Offline & Online \\
\hline 328 & Online & Online & Blended & Brain & Digital & Digital & Offline & Offline \\
\hline 329 & Online & Online & Blended & Brain & Digital & Non-digital & Online & Online \\
\hline 330 & Online & Online & Blended & Brain & Digital & Non-digital & Online & Offline \\
\hline 331 & Online & Online & Blended & Brain & Digital & Non-digital & Offline & Online \\
\hline 332 & Online & Online & Blended & Brain & Digital & Non-digital & Offline & Offline \\
\hline 333 & Online & Online & Blended & Brain & Digital & Blended & Online & Online \\
\hline 334 & Online & Online & Blended & Brain & Digital & Blended & Online & Offline \\
\hline 335 & Online & Online & Blended & Brain & Digital & Blended & Offline & Online \\
\hline 336 & Online & Online & Blended & Brain & Digital & Blended & Offline & Offline \\
\hline 337 & Online & Online & Blended & Brain & $\begin{array}{l}\text { Non- } \\
\text { digital }\end{array}$ & Digital & Online & Online \\
\hline 338 & Online & Online & Blended & Brain & $\begin{array}{l}\text { Non- } \\
\text { digital }\end{array}$ & Digital & Online & Offline \\
\hline 339 & Online & Online & Blended & Brain & $\begin{array}{l}\text { Non- } \\
\text { digital }\end{array}$ & Digital & Offline & Online \\
\hline 340 & Online & Online & Blended & Brain & $\begin{array}{l}\text { Non- } \\
\text { digital }\end{array}$ & Digital & Offline & Offline \\
\hline 341 & Online & Online & Blended & Brain & $\begin{array}{l}\text { Non- } \\
\text { digital }\end{array}$ & Non-digital & Online & Online \\
\hline 342 & Online & Online & Blended & Brain & $\begin{array}{l}\text { Non- } \\
\text { digital }\end{array}$ & Non-digital & Online & Offline \\
\hline 343 & Online & Online & Blended & Brain & $\begin{array}{l}\text { Non- } \\
\text { digital }\end{array}$ & Non-digital & Offline & Online \\
\hline 344 & Online & Online & Blended & Brain & $\begin{array}{l}\text { Non- } \\
\text { diqital }\end{array}$ & Non-digital & Offline & Offline \\
\hline 345 & Online & Online & Blended & Brain & $\begin{array}{l}\text { Non- } \\
\text { digital }\end{array}$ & Blended & Online & Online \\
\hline 346 & Online & Online & Blended & Brain & $\begin{array}{l}\text { Non- } \\
\text { digital }\end{array}$ & Blended & Online & Offline \\
\hline 347 & Online & Online & Blended & Brain & $\begin{array}{l}\text { Non- } \\
\text { diqital }\end{array}$ & Blended & Offline & Online \\
\hline 348 & Online & Online & Blended & Brain & $\begin{array}{l}\text { Non- } \\
\text { digital }\end{array}$ & Blended & Offline & Offline \\
\hline
\end{tabular}




\begin{tabular}{|c|c|c|c|c|c|c|c|c|}
\hline 349 & Online & Online & Blended & Brain & $\begin{array}{l}\text { Blend- } \\
\text { ed }\end{array}$ & Digital & Online & Online \\
\hline 350 & Online & Online & Blended & Brain & $\begin{array}{l}\text { Blend- } \\
\text { ed }\end{array}$ & Digital & Online & Offline \\
\hline 351 & Online & Online & Blended & Brain & $\begin{array}{l}\text { Blend- } \\
\text { ed }\end{array}$ & Digital & Offline & Online \\
\hline 352 & Online & Online & Blended & Brain & $\begin{array}{l}\text { Blend- } \\
\text { ed }\end{array}$ & Digital & Offline & Offline \\
\hline 353 & Online & Online & Blended & Brain & $\begin{array}{l}\text { Blend- } \\
\text { ed }\end{array}$ & Non-digital & Online & Online \\
\hline 354 & Online & Online & Blended & Brain & $\begin{array}{l}\text { Blend- } \\
\text { ed }\end{array}$ & Non-digital & Online & Offline \\
\hline 355 & Online & Online & Blended & Brain & $\begin{array}{l}\text { Blend- } \\
\text { ed }\end{array}$ & Non-digital & Offline & Online \\
\hline 356 & Online & Online & Blended & Brain & $\begin{array}{l}\text { Blend- } \\
\text { ed }\end{array}$ & Non-digital & Offline & Offline \\
\hline 357 & Online & Online & Blended & Brain & $\begin{array}{l}\text { Blend- } \\
\text { ed }\end{array}$ & Blended & Online & Online \\
\hline 358 & Online & Online & Blended & Brain & $\begin{array}{l}\text { Blend- } \\
\text { ed }\end{array}$ & Blended & Online & Offline \\
\hline 359 & Online & Online & Blended & Brain & $\begin{array}{l}\text { Blend- } \\
\text { ed }\end{array}$ & Blended & Offline & Online \\
\hline 360 & Online & Online & Blended & Brain & $\begin{array}{l}\text { Blend- } \\
\text { ed }\end{array}$ & Blended & Offline & Offline \\
\hline 361 & Online & Online & Blended & Brain $+d+n-d$ & Digital & Digital & Online & Online \\
\hline 362 & Online & Online & Blended & Brain $+d+n-d$ & Digital & Digital & Online & Offline \\
\hline 363 & Online & Online & Blended & Brain $+d+n-d$ & Digital & Digital & Offline & Online \\
\hline 364 & Online & Online & Blended & Brain $+d+n-d$ & Digital & Digital & Offline & Offline \\
\hline 365 & Online & Online & Blended & Brain + d + n-d & Digital & Non-digital & Online & Online \\
\hline 366 & Online & Online & Blended & Brain $+d+n-d$ & Digital & Non-digital & Online & Offline \\
\hline 367 & Online & Online & Blended & Brain $+d+n-d$ & Digital & Non-digital & Offline & Online \\
\hline 368 & Online & Online & Blended & Brain $+d+n-d$ & Digital & Non-digital & Offline & Offline \\
\hline 369 & Online & Online & Blended & Brain $+d+n-d$ & Digital & Blended & Online & Online \\
\hline 370 & Online & Online & Blended & Brain $+d+n-d$ & Digital & Blended & Online & Offline \\
\hline 371 & Online & Online & Blended & Brain $+d+n-d$ & Digital & Blended & Offline & Online \\
\hline 372 & Online & Online & Blended & Brain $+d+n-d$ & Digital & Blended & Offline & Offline \\
\hline 373 & Online & Online & Blended & Brain $+d+n-d$ & $\begin{array}{l}\text { Non- } \\
\text { digital }\end{array}$ & Digital & Online & Online \\
\hline 374 & Online & Online & Blended & Brain $+d+n-d$ & $\begin{array}{l}\text { Non- } \\
\text { digital }\end{array}$ & Digital & Online & Offline \\
\hline 375 & Online & Online & Blended & Brain $+d+n-d$ & $\begin{array}{l}\text { Non- } \\
\text { digital }\end{array}$ & Digital & Offline & Online \\
\hline 376 & Online & Online & Blended & Brain $+d+n-d$ & $\begin{array}{l}\text { Non- } \\
\text { digital }\end{array}$ & Digital & Offline & Offline \\
\hline 377 & Online & Online & Blended & Brain + d + n-d & $\begin{array}{l}\text { Non- } \\
\text { digital }\end{array}$ & Non-digital & Online & Online \\
\hline 378 & Online & Online & Blended & Brain + d + n-d & $\begin{array}{l}\text { Non- } \\
\text { digital }\end{array}$ & Non-digital & Online & Offline \\
\hline 379 & Online & Online & Blended & Brain $+d+n-d$ & $\begin{array}{l}\text { Non- } \\
\text { digital }\end{array}$ & Non-digital & Offline & Online \\
\hline 380 & Online & Online & Blended & Brain $+d+n-d$ & $\begin{array}{l}\text { Non- } \\
\text { digital }\end{array}$ & Non-digital & Offline & Offline \\
\hline 381 & Online & Online & Blended & Brain + d + n-d & $\begin{array}{l}\text { Non- } \\
\text { digital }\end{array}$ & Blended & Online & Online \\
\hline 382 & Online & Online & Blended & Brain + d + n-d & $\begin{array}{l}\text { Non- } \\
\text { digital }\end{array}$ & Blended & Online & Offline \\
\hline 383 & Online & Online & Blended & Brain $+d+n-d$ & $\begin{array}{l}\text { Non- } \\
\text { digital }\end{array}$ & Blended & Offline & Online \\
\hline 384 & Online & Online & Blended & Brain + d + n-d & $\begin{array}{l}\text { Non- } \\
\text { digital }\end{array}$ & Blended & Offline & Offline \\
\hline 385 & Online & Online & Blended & Brain + d + n-d & $\begin{array}{l}\text { Blend- } \\
\text { ed }\end{array}$ & Digital & Online & Online \\
\hline 386 & Online & Online & Blended & Brain + d + n-d & $\begin{array}{l}\text { Blend- } \\
\text { ed }\end{array}$ & Digital & Online & Offline \\
\hline
\end{tabular}




\begin{tabular}{|c|c|c|c|c|c|c|c|c|}
\hline 387 & Online & Online & Blended & Brain $+d+n-d$ & $\begin{array}{l}\text { Blend- } \\
\text { ed }\end{array}$ & Digital & Offline & Online \\
\hline 388 & Online & Online & Blended & Brain $+d+n-d$ & $\begin{array}{l}\text { Blend- } \\
\text { ed }\end{array}$ & Digital & Offline & Offline \\
\hline 389 & Online & Online & Blended & Brain $+d+n-d$ & $\begin{array}{l}\text { Blend- } \\
\text { ed }\end{array}$ & Non-digital & Online & Online \\
\hline 390 & Online & Online & Blended & Brain $+d+n-d$ & $\begin{array}{l}\text { Blend- } \\
\text { ed }\end{array}$ & Non-digital & Online & Offline \\
\hline 391 & Online & Online & Blended & Brain $+d+n-d$ & $\begin{array}{l}\text { Blend- } \\
\text { ed }\end{array}$ & Non-digital & Offline & Online \\
\hline 392 & Online & Online & Blended & Brain $+d+n-d$ & $\begin{array}{l}\text { Blend- } \\
\text { ed }\end{array}$ & Non-digital & Offline & Offline \\
\hline 393 & Online & Online & Blended & Brain $+d+n-d$ & $\begin{array}{l}\text { Blend- } \\
\text { ed }\end{array}$ & Blended & Online & Online \\
\hline 394 & Online & Online & Blended & Brain $+d+n-d$ & $\begin{array}{l}\text { Blend- } \\
\text { ed }\end{array}$ & Blended & Online & Offline \\
\hline 395 & Online & Online & Blended & Brain $+d+n-d$ & $\begin{array}{l}\text { Blend- } \\
\text { ed }\end{array}$ & Blended & Offline & Online \\
\hline 396 & Online & Online & Blended & Brain $+d+n-d$ & $\begin{array}{l}\text { Blend- } \\
\text { ed }\end{array}$ & Blended & Offline & Offline \\
\hline 397 & Online & Online & Blended & Brain $+n-d$ & Digital & Digital & Online & Online \\
\hline 398 & Online & Online & Blended & Brain $+n-d$ & Digital & Digital & Online & Offline \\
\hline 399 & Online & Online & Blended & Brain $+n-d$ & Digital & Digital & Offline & Online \\
\hline 400 & Online & Online & Blended & Brain + n-d & Digital & Digital & Offline & Offline \\
\hline 401 & Online & Online & Blended & Brain $+n-d$ & Digital & Non-digital & Online & Online \\
\hline 402 & Online & Online & Blended & Brain $+n-d$ & Digital & Non-digital & Online & Offline \\
\hline 403 & Online & Online & Blended & Brain $+n-d$ & Digital & Non-digital & Offline & Online \\
\hline 404 & Online & Online & Blended & Brain $+n-d$ & Digital & Non-digital & Offline & Offline \\
\hline 405 & Online & Online & Blended & Brain $+n-d$ & Digital & Blended & Online & Online \\
\hline 406 & Online & Online & Blended & Brain $+n-d$ & Digital & Blended & Online & Offline \\
\hline 407 & Online & Online & Blended & Brain + n-d & Digital & Blended & Offline & Online \\
\hline 408 & Online & Online & Blended & Brain $+n-d$ & Digital & Blended & Offline & Offline \\
\hline 409 & Online & Online & Blended & Brain + n-d & $\begin{array}{l}\text { Non- } \\
\text { digital }\end{array}$ & Digital & Online & Online \\
\hline 410 & Online & Online & Blended & Brain $+n-d$ & $\begin{array}{l}\text { Non- } \\
\text { digital }\end{array}$ & Digital & Online & Offline \\
\hline 411 & Online & Online & Blended & Brain $+n-d$ & $\begin{array}{l}\text { Non- } \\
\text { digital }\end{array}$ & Digital & Offline & Online \\
\hline 412 & Online & Online & Blended & Brain + n-d & $\begin{array}{l}\text { Non- } \\
\text { digital }\end{array}$ & Digital & Offline & Offline \\
\hline 413 & Online & Online & Blended & Brain $+n-d$ & $\begin{array}{l}\text { Non- } \\
\text { digital }\end{array}$ & Non-digital & Online & Online \\
\hline 414 & Online & Online & Blended & Brain $+n-d$ & $\begin{array}{l}\text { Non- } \\
\text { diqital }\end{array}$ & Non-digital & Online & Offline \\
\hline 415 & Online & Online & Blended & Brain $+n-d$ & $\begin{array}{l}\text { Non- } \\
\text { digital }\end{array}$ & Non-digital & Offline & Online \\
\hline 416 & Online & Online & Blended & Brain + n-d & $\begin{array}{l}\text { Non- } \\
\text { digital }\end{array}$ & Non-digital & Offline & Offline \\
\hline 417 & Online & Online & Blended & Brain + n-d & $\begin{array}{l}\text { Non- } \\
\text { digital }\end{array}$ & Blended & Online & Online \\
\hline 418 & Online & Online & Blended & Brain $+n-d$ & $\begin{array}{l}\text { Non- } \\
\text { digital }\end{array}$ & Blended & Online & Offline \\
\hline 419 & Online & Online & Blended & Brain + n-d & $\begin{array}{l}\text { Non- } \\
\text { digital }\end{array}$ & Blended & Offline & Online \\
\hline 420 & Online & Online & Blended & Brain + n-d & $\begin{array}{l}\text { Non- } \\
\text { digital }\end{array}$ & Blended & Offline & Offline \\
\hline 421 & Online & Online & Blended & Brain $+n-d$ & $\begin{array}{l}\text { Blend- } \\
\text { ed }\end{array}$ & Digital & Online & Online \\
\hline 422 & Online & Online & Blended & Brain + n-d & $\begin{array}{l}\text { Blend- } \\
\text { ed }\end{array}$ & Digital & Online & Offline \\
\hline 423 & Online & Online & Blended & Brain + n-d & $\begin{array}{l}\text { Blend- } \\
\text { ed }\end{array}$ & Digital & Offline & Online \\
\hline 424 & Online & Online & Blended & Brain $+n-d$ & $\begin{array}{l}\text { Blend- } \\
\text { ed }\end{array}$ & Digital & Offline & Offline \\
\hline
\end{tabular}




\begin{tabular}{|c|c|c|c|c|c|c|c|c|}
\hline 425 & Online & Online & Blended & Brain + n-d & $\begin{array}{l}\text { Blend- } \\
\text { ed }\end{array}$ & Non-digital & Online & Online \\
\hline 426 & Online & Online & Blended & Brain + n-d & $\begin{array}{l}\text { Blend- } \\
\text { ed }\end{array}$ & Non-digital & Online & Offline \\
\hline 427 & Online & Online & Blended & Brain + n-d & $\begin{array}{l}\text { Blend- } \\
\text { ed }\end{array}$ & Non-digital & Offline & Online \\
\hline 428 & Online & Online & Blended & Brain + n-d & $\begin{array}{l}\text { Blend- } \\
\text { ed }\end{array}$ & Non-digital & Offline & Offline \\
\hline 429 & Online & Online & Blended & Brain + n-d & $\begin{array}{l}\text { Blend- } \\
\text { ed }\end{array}$ & Blended & Online & Online \\
\hline 430 & Online & Online & Blended & Brain + n-d & $\begin{array}{l}\text { Blend- } \\
\text { ed }\end{array}$ & Blended & Online & Offline \\
\hline 431 & Online & Online & Blended & Brain + n-d & $\begin{array}{l}\text { Blend- } \\
\text { ed }\end{array}$ & Blended & Offline & Online \\
\hline 432 & Online & Online & Blended & Brain + n-d & $\begin{array}{l}\text { Blend- } \\
\text { ed }\end{array}$ & Blended & Offline & Offline \\
\hline 433 & Online & Offline & Online & Brain + digital & Digital & Digital & Online & Online \\
\hline 434 & Online & Offline & Online & Brain + digital & Digital & Digital & Online & Offline \\
\hline 435 & Online & Offline & Online & Brain + digital & Digital & Digital & Offline & Online \\
\hline 436 & Online & Offline & Online & Brain + digital & Digital & Digital & Offline & Offline \\
\hline 437 & Online & Offline & Online & Brain + digital & Digital & Non-digital & Online & Online \\
\hline 438 & Online & Offline & Online & Brain + digital & Digital & Non-digital & Online & Offline \\
\hline 439 & Online & Offline & Online & Brain + digital & Digital & Non-digital & Offline & Online \\
\hline 440 & Online & Offline & Online & Brain + digital & Digital & Non-digital & Offline & Offline \\
\hline 441 & Online & Offline & Online & Brain + digital & Digital & Blended & Online & Online \\
\hline 442 & Online & Offline & Online & Brain + digital & Digital & Blended & Online & Offline \\
\hline 443 & Online & Offline & Online & Brain + digital & Digital & Blended & Offline & Online \\
\hline 444 & Online & Offline & Online & Brain + digital & Digital & Blended & Offline & Offline \\
\hline 445 & Online & Offline & Online & Brain + digital & $\begin{array}{l}\text { Non- } \\
\text { digital }\end{array}$ & Digital & Online & Online \\
\hline 446 & Online & Offline & Online & Brain + digital & $\begin{array}{l}\text { Non- } \\
\text { digital }\end{array}$ & Digital & Online & Offline \\
\hline 447 & Online & Offline & Online & Brain + digital & $\begin{array}{l}\text { Non- } \\
\text { digital }\end{array}$ & Digital & Offline & Online \\
\hline 448 & Online & Offline & Online & Brain + digital & $\begin{array}{l}\text { Non- } \\
\text { digital }\end{array}$ & Digital & Offline & Offline \\
\hline 449 & Online & Offline & Online & Brain + digital & $\begin{array}{l}\text { Non- } \\
\text { digital }\end{array}$ & Non-digital & Online & Online \\
\hline 450 & Online & Offline & Online & Brain + digital & $\begin{array}{l}\text { Non- } \\
\text { digital }\end{array}$ & Non-digital & Online & Offline \\
\hline 451 & Online & Offline & Online & Brain + digital & $\begin{array}{l}\text { Non- } \\
\text { digital }\end{array}$ & Non-digital & Offline & Online \\
\hline 452 & Online & Offline & Online & Brain + digital & $\begin{array}{l}\text { Non- } \\
\text { digital }\end{array}$ & Non-digital & Offline & Offline \\
\hline 453 & Online & Offline & Online & Brain + digital & $\begin{array}{l}\text { Non- } \\
\text { digital }\end{array}$ & Blended & Online & Online \\
\hline 454 & Online & Offline & Online & Brain + digital & $\begin{array}{l}\text { Non- } \\
\text { digital }\end{array}$ & Blended & Online & Offline \\
\hline 455 & Online & Offline & Online & Brain + digital & $\begin{array}{l}\text { Non- } \\
\text { digital }\end{array}$ & Blended & Offline & Online \\
\hline 456 & Online & Offline & Online & Brain + digital & $\begin{array}{l}\text { Non- } \\
\text { digital }\end{array}$ & Blended & Offline & Offline \\
\hline 457 & Online & Offline & Online & Brain + digital & $\begin{array}{l}\text { Blend- } \\
\text { ed }\end{array}$ & Digital & Online & Online \\
\hline 458 & Online & Offline & Online & Brain + digital & $\begin{array}{l}\text { Blend- } \\
\text { ed }\end{array}$ & Digital & Online & Offline \\
\hline 459 & Online & Offline & Online & Brain + digital & $\begin{array}{l}\text { Blend- } \\
\text { ed }\end{array}$ & Digital & Offline & Online \\
\hline 460 & Online & Offline & Online & Brain + digital & $\begin{array}{l}\text { Blend- } \\
\text { ed }\end{array}$ & Digital & Offline & Offline \\
\hline 461 & Online & Offline & Online & Brain + digital & $\begin{array}{l}\text { Blend- } \\
\text { ed }\end{array}$ & Non-digital & Online & Online \\
\hline 462 & Online & Offline & Online & Brain + digital & $\begin{array}{l}\text { Blend- } \\
\text { ed }\end{array}$ & Non-digital & Online & Offline \\
\hline
\end{tabular}




\begin{tabular}{|c|c|c|c|c|c|c|c|c|}
\hline 463 & Online & Offline & Online & Brain + digital & $\begin{array}{l}\text { Blend- } \\
\text { ed }\end{array}$ & Non-digital & Offline & Online \\
\hline 464 & Online & Offline & Online & Brain + digital & $\begin{array}{l}\text { Blend- } \\
\text { ed }\end{array}$ & Non-digital & Offline & Offline \\
\hline 465 & Online & Offline & Online & Brain + digital & $\begin{array}{l}\text { Blend- } \\
\text { ed }\end{array}$ & Blended & Online & Online \\
\hline 466 & Online & Offline & Online & Brain + digital & $\begin{array}{l}\text { Blend- } \\
\text { ed }\end{array}$ & Blended & Online & Offline \\
\hline 467 & Online & Offline & Online & Brain + digital & $\begin{array}{l}\text { Blend- } \\
\text { ed }\end{array}$ & Blended & Offline & Online \\
\hline 468 & Online & Offline & Online & Brain + digital & $\begin{array}{l}\text { Blend- } \\
\text { ed }\end{array}$ & Blended & Offline & Offline \\
\hline 469 & Online & Offline & Online & Brain & Digital & Digital & Online & Online \\
\hline 470 & Online & Offline & Online & Brain & Digital & Digital & Online & Offline \\
\hline 471 & Online & Offline & Online & Brain & Digital & Digital & Offline & Online \\
\hline 472 & Online & Offline & Online & Brain & Digital & Digital & Offline & Offline \\
\hline 473 & Online & Offline & Online & Brain & Digital & Non-digital & Online & Online \\
\hline 474 & Online & Offline & Online & Brain & Digital & Non-digital & Online & Offline \\
\hline 475 & Online & Offline & Online & Brain & Digital & Non-digital & Offline & Online \\
\hline 476 & Online & Offline & Online & Brain & Digital & Non-digital & Offline & Offline \\
\hline 477 & Online & Offline & Online & Brain & Digital & Blended & Online & Online \\
\hline 478 & Online & Offline & Online & Brain & Digital & Blended & Online & Offline \\
\hline 479 & Online & Offline & Online & Brain & Digital & Blended & Offline & Online \\
\hline 480 & Online & Offline & Online & Brain & Digital & Blended & Offline & Offline \\
\hline 481 & Online & Offline & Online & Brain & $\begin{array}{l}\text { Non- } \\
\text { digital }\end{array}$ & Digital & Online & Online \\
\hline 482 & Online & Offline & Online & Brain & $\begin{array}{l}\text { Non- } \\
\text { digital }\end{array}$ & Digital & Online & Offline \\
\hline 483 & Online & Offline & Online & Brain & $\begin{array}{l}\text { Non- } \\
\text { digital }\end{array}$ & Digital & Offline & Online \\
\hline 484 & Online & Offline & Online & Brain & $\begin{array}{l}\text { Non- } \\
\text { digital }\end{array}$ & Digital & Offline & Offline \\
\hline 485 & Online & Offline & Online & Brain & $\begin{array}{l}\text { Non- } \\
\text { digital }\end{array}$ & Non-digital & Online & Online \\
\hline 486 & Online & Offline & Online & Brain & $\begin{array}{l}\text { Non- } \\
\text { digital }\end{array}$ & Non-digital & Online & Offline \\
\hline 487 & Online & Offline & Online & Brain & $\begin{array}{l}\text { Non- } \\
\text { digital }\end{array}$ & Non-digital & Offline & Online \\
\hline 488 & Online & Offline & Online & Brain & $\begin{array}{l}\text { Non- } \\
\text { digital }\end{array}$ & Non-digital & Offline & Offline \\
\hline 489 & Online & Offline & Online & Brain & $\begin{array}{l}\text { Non- } \\
\text { digital }\end{array}$ & Blended & Online & Online \\
\hline 490 & Online & Offline & Online & Brain & $\begin{array}{l}\text { Non- } \\
\text { diqital }\end{array}$ & Blended & Online & Offline \\
\hline 491 & Online & Offline & Online & Brain & $\begin{array}{l}\text { Non- } \\
\text { digital }\end{array}$ & Blended & Offline & Online \\
\hline 492 & Online & Offline & Online & Brain & $\begin{array}{l}\text { Non- } \\
\text { digital }\end{array}$ & Blended & Offline & Offline \\
\hline 493 & Online & Offline & Online & Brain & $\begin{array}{l}\text { Blend- } \\
\text { ed }\end{array}$ & Digital & Online & Online \\
\hline 494 & Online & Offline & Online & Brain & $\begin{array}{l}\text { Blend- } \\
\text { ed }\end{array}$ & Digital & Online & Offline \\
\hline 495 & Online & Offline & Online & Brain & $\begin{array}{l}\text { Blend- } \\
\text { ed }\end{array}$ & Digital & Offline & Online \\
\hline 496 & Online & Offline & Online & Brain & $\begin{array}{l}\text { Blend- } \\
\text { ed }\end{array}$ & Digital & Offline & Offline \\
\hline 497 & Online & Offline & Online & Brain & $\begin{array}{l}\text { Blend- } \\
\text { ed }\end{array}$ & Non-digital & Online & Online \\
\hline 498 & Online & Offline & Online & Brain & $\begin{array}{l}\text { Blend- } \\
\text { ed }\end{array}$ & Non-digital & Online & Offline \\
\hline 499 & Online & Offline & Online & Brain & $\begin{array}{l}\text { Blend- } \\
\text { ed }\end{array}$ & Non-digital & Offline & Online \\
\hline 500 & Online & Offline & Online & Brain & $\begin{array}{l}\text { Blend- } \\
\text { ed }\end{array}$ & Non-digital & Offline & Offline \\
\hline
\end{tabular}




\begin{tabular}{|c|c|c|c|c|c|c|c|c|}
\hline 501 & Online & Offline & Online & Brain & $\begin{array}{l}\text { Blend- } \\
\text { ed }\end{array}$ & Blended & Online & Online \\
\hline 502 & Online & Offline & Online & Brain & $\begin{array}{l}\text { Blend- } \\
\text { ed }\end{array}$ & Blended & Online & Offline \\
\hline 503 & Online & Offline & Online & Brain & $\begin{array}{l}\text { Blend- } \\
\text { ed }\end{array}$ & Blended & Offline & Online \\
\hline 504 & Online & Offline & Online & Brain & $\begin{array}{l}\text { Blend- } \\
\text { ed }\end{array}$ & Blended & Offline & Offline \\
\hline 505 & Online & Offline & Online & Brain $+d+n-d$ & Digital & Digital & Online & Online \\
\hline 506 & Online & Offline & Online & Brain $+d+n-d$ & Digital & Digital & Online & Offline \\
\hline 507 & Online & Offline & Online & Brain $+d+n-d$ & Digital & Digital & Offline & Online \\
\hline 508 & Online & Offline & Online & Brain $+d+n-d$ & Digital & Digital & Offline & Offline \\
\hline 509 & Online & Offline & Online & Brain $+d+n-d$ & Digital & Non-digital & Online & Online \\
\hline 510 & Online & Offline & Online & Brain $+d+n-d$ & Digital & Non-digital & Online & Offline \\
\hline 511 & Online & Offline & Online & Brain $+d+n-d$ & Digital & Non-digital & Offline & Online \\
\hline 512 & Online & Offline & Online & Brain $+d+n-d$ & Digital & Non-digital & Offline & Offline \\
\hline 513 & Online & Offline & Online & Brain $+d+n-d$ & Digital & Blended & Online & Online \\
\hline 514 & Online & Offline & Online & Brain $+d+n-d$ & Digital & Blended & Online & Offline \\
\hline 515 & Online & Offline & Online & Brain $+d+n-d$ & Digital & Blended & Offline & Online \\
\hline 516 & Online & Offline & Online & Brain $+d+n-d$ & Digital & Blended & Offline & Offline \\
\hline 517 & Online & Offline & Online & Brain $+d+n-d$ & $\begin{array}{l}\text { Non- } \\
\text { digital }\end{array}$ & Digital & Online & Online \\
\hline 518 & Online & Offline & Online & Brain $+d+n-d$ & $\begin{array}{l}\text { Non- } \\
\text { digital }\end{array}$ & Digital & Online & Offline \\
\hline 519 & Online & Offline & Online & Brain $+d+n-d$ & $\begin{array}{l}\text { Non- } \\
\text { digital }\end{array}$ & Digital & Offline & Online \\
\hline 520 & Online & Offline & Online & Brain $+d+n-d$ & $\begin{array}{l}\text { Non- } \\
\text { digital }\end{array}$ & Digital & Offline & Offline \\
\hline 521 & Online & Offline & Online & Brain $+d+n-d$ & $\begin{array}{l}\text { Non- } \\
\text { digital }\end{array}$ & Non-digital & Online & Online \\
\hline 522 & Online & Offline & Online & Brain $+d+n-d$ & $\begin{array}{l}\text { Non- } \\
\text { digital }\end{array}$ & Non-digital & Online & Offline \\
\hline 523 & Online & Offline & Online & Brain $+d+n-d$ & $\begin{array}{l}\text { Non- } \\
\text { digital }\end{array}$ & Non-digital & Offline & Online \\
\hline 524 & Online & Offline & Online & Brain $+d+n-d$ & $\begin{array}{l}\text { Non- } \\
\text { digital }\end{array}$ & Non-digital & Offline & Offline \\
\hline 525 & Online & Offline & Online & Brain $+d+n-d$ & $\begin{array}{l}\text { Non- } \\
\text { digital }\end{array}$ & Blended & Online & Online \\
\hline 526 & Online & Offline & Online & Brain $+d+n-d$ & $\begin{array}{l}\text { Non- } \\
\text { digital }\end{array}$ & Blended & Online & Offline \\
\hline 527 & Online & Offline & Online & Brain $+d+n-d$ & $\begin{array}{l}\text { Non- } \\
\text { digital }\end{array}$ & Blended & Offline & Online \\
\hline 528 & Online & Offline & Online & Brain $+d+n-d$ & $\begin{array}{l}\text { Non- } \\
\text { digital }\end{array}$ & Blended & Offline & Offline \\
\hline 529 & Online & Offline & Online & Brain + d + n-d & $\begin{array}{l}\text { Blend- } \\
\text { ed }\end{array}$ & Digital & Online & Online \\
\hline 530 & Online & Offline & Online & Brain $+d+n-d$ & $\begin{array}{l}\text { Blend- } \\
\text { ed }\end{array}$ & Digital & Online & Offline \\
\hline 531 & Online & Offline & Online & Brain $+d+n-d$ & $\begin{array}{l}\text { Blend- } \\
\text { ed }\end{array}$ & Digital & Offline & Online \\
\hline 532 & Online & Offline & Online & Brain $+d+n-d$ & $\begin{array}{l}\text { Blend- } \\
\text { ed }\end{array}$ & Digital & Offline & Offline \\
\hline 533 & Online & Offline & Online & Brain $+d+n-d$ & $\begin{array}{l}\text { Blend- } \\
\text { ed }\end{array}$ & Non-digital & Online & Online \\
\hline 534 & Online & Offline & Online & Brain $+d+n-d$ & $\begin{array}{l}\text { Blend- } \\
\text { ed }\end{array}$ & Non-digital & Online & Offline \\
\hline 535 & Online & Offline & Online & Brain $+d+n-d$ & $\begin{array}{l}\text { Blend- } \\
\text { ed }\end{array}$ & Non-digital & Offline & Online \\
\hline 536 & Online & Offline & Online & Brain $+d+n-d$ & $\begin{array}{l}\text { Blend- } \\
\text { ed }\end{array}$ & Non-digital & Offline & Offline \\
\hline 537 & Online & Offline & Online & Brain $+d+n-d$ & $\begin{array}{l}\text { Blend- } \\
\text { ed }\end{array}$ & Blended & Online & Online \\
\hline 538 & Online & Offline & Online & Brain $+d+n-d$ & $\begin{array}{l}\text { Blend- } \\
\text { ed }\end{array}$ & Blended & Online & Offline \\
\hline
\end{tabular}




\begin{tabular}{|c|c|c|c|c|c|c|c|c|}
\hline 539 & Online & Offline & Online & Brain $+d+n-d$ & $\begin{array}{l}\text { Blend- } \\
\text { ed }\end{array}$ & Blended & Offline & Online \\
\hline 540 & Online & Offline & Online & Brain $+d+n-d$ & $\begin{array}{l}\text { Blend- } \\
\text { ed }\end{array}$ & Blended & Offline & Offline \\
\hline 541 & Online & Offline & Online & Brain + n-d & Digital & Digital & Online & Online \\
\hline 542 & Online & Offline & Online & Brain + n-d & Digital & Digital & Online & Offline \\
\hline 543 & Online & Offline & Online & Brain + n-d & Digital & Digital & Offline & Online \\
\hline 544 & Online & Offline & Online & Brain + n-d & Digital & Digital & Offline & Offline \\
\hline 545 & Online & Offline & Online & Brain + n-d & Digital & Non-digital & Online & Online \\
\hline 546 & Online & Offline & Online & Brain + n-d & Digital & Non-digital & Online & Offline \\
\hline 547 & Online & Offline & Online & Brain + n-d & Digital & Non-digital & Offline & Online \\
\hline 548 & Online & Offline & Online & Brain + n-d & Digital & Non-digital & Offline & Offline \\
\hline 549 & Online & Offline & Online & Brain + n-d & Digital & Blended & Online & Online \\
\hline 550 & Online & Offline & Online & Brain + n-d & Digital & Blended & Online & Offline \\
\hline 551 & Online & Offline & Online & Brain $+n-d$ & Digital & Blended & Offline & Online \\
\hline 552 & Online & Offline & Online & Brain + n-d & Digital & Blended & Offline & Offline \\
\hline 553 & Online & Offline & Online & Brain + n-d & $\begin{array}{l}\text { Non- } \\
\text { digital }\end{array}$ & Digital & Online & Online \\
\hline 554 & Online & Offline & Online & Brain + n-d & $\begin{array}{l}\text { Non- } \\
\text { digital }\end{array}$ & Digital & Online & Offline \\
\hline 555 & Online & Offline & Online & Brain + n-d & $\begin{array}{l}\text { Non- } \\
\text { digital }\end{array}$ & Digital & Offline & Online \\
\hline 556 & Online & Offline & Online & Brain + n-d & $\begin{array}{l}\text { Non- } \\
\text { digital }\end{array}$ & Digital & Offline & Offline \\
\hline 557 & Online & Offline & Online & Brain + n-d & $\begin{array}{l}\text { Non- } \\
\text { digital }\end{array}$ & Non-digital & Online & Online \\
\hline 558 & Online & Offline & Online & Brain + n-d & $\begin{array}{l}\text { Non- } \\
\text { digital }\end{array}$ & Non-digital & Online & Offline \\
\hline 559 & Online & Offline & Online & Brain + n-d & $\begin{array}{l}\text { Non- } \\
\text { digital }\end{array}$ & Non-digital & Offline & Online \\
\hline 560 & Online & Offline & Online & Brain + n-d & $\begin{array}{l}\text { Non- } \\
\text { digital }\end{array}$ & Non-digital & Offline & Offline \\
\hline 561 & Online & Offline & Online & Brain + n-d & $\begin{array}{l}\text { Non- } \\
\text { digital }\end{array}$ & Blended & Online & Online \\
\hline 562 & Online & Offline & Online & Brain + n-d & $\begin{array}{l}\text { Non- } \\
\text { digital }\end{array}$ & Blended & Online & Offline \\
\hline 563 & Online & Offline & Online & Brain + n-d & $\begin{array}{l}\text { Non- } \\
\text { digital }\end{array}$ & Blended & Offline & Online \\
\hline 564 & Online & Offline & Online & Brain + n-d & $\begin{array}{l}\text { Non- } \\
\text { digital }\end{array}$ & Blended & Offline & Offline \\
\hline 565 & Online & Offline & Online & Brain + n-d & $\begin{array}{l}\text { Blend- } \\
\text { ed }\end{array}$ & Digital & Online & Online \\
\hline 566 & Online & Offline & Online & Brain + n-d & $\begin{array}{l}\text { Blend- } \\
\text { ed }\end{array}$ & Digital & Online & Offline \\
\hline 567 & Online & Offline & Online & Brain + n-d & $\begin{array}{l}\text { Blend- } \\
\text { ed }\end{array}$ & Digital & Offline & Online \\
\hline 568 & Online & Offline & Online & Brain + n-d & $\begin{array}{l}\text { Blend- } \\
\text { ed }\end{array}$ & Digital & Offline & Offline \\
\hline 569 & Online & Offline & Online & Brain + n-d & $\begin{array}{l}\text { Blend- } \\
\text { ed }\end{array}$ & Non-digital & Online & Online \\
\hline 570 & Online & Offline & Online & Brain + n-d & $\begin{array}{l}\text { Blend- } \\
\text { ed }\end{array}$ & Non-digital & Online & Offline \\
\hline 571 & Online & Offline & Online & Brain + n-d & $\begin{array}{l}\text { Blend- } \\
\text { ed }\end{array}$ & Non-digital & Offline & Online \\
\hline 572 & Online & Offline & Online & Brain + n-d & $\begin{array}{l}\text { Blend- } \\
\text { ed }\end{array}$ & Non-digital & Offline & Offline \\
\hline 573 & Online & Offline & Online & Brain + n-d & $\begin{array}{l}\text { Blend- } \\
\text { ed }\end{array}$ & Blended & Online & Online \\
\hline 574 & Online & Offline & Online & Brain + n-d & $\begin{array}{l}\text { Blend- } \\
\text { ed }\end{array}$ & Blended & Online & Offline \\
\hline 575 & Online & Offline & Online & Brain + n-d & $\begin{array}{l}\text { Blend- } \\
\text { ed }\end{array}$ & Blended & Offline & Online \\
\hline 576 & Online & Offline & Online & Brain + n-d & $\begin{array}{l}\text { Blend- } \\
\text { ed }\end{array}$ & Blended & Offline & Offline \\
\hline 577 & Online & Offline & Offline & Brain + digital & Digital & Digital & Online & Online \\
\hline
\end{tabular}




\begin{tabular}{|c|c|c|c|c|c|c|c|c|}
\hline 578 & Online & Offline & Offline & Brain + digital & Digital & Digital & Online & Offline \\
\hline 579 & Online & Offline & Offline & Brain + digital & Digital & Digital & Offline & Online \\
\hline 580 & Online & Offline & Offline & Brain + digital & Digital & Digital & Offline & Offline \\
\hline 581 & Online & Offline & Offline & Brain + digital & Digital & Non-digital & Online & Online \\
\hline 582 & Online & Offline & Offline & Brain + digital & Digital & Non-digital & Online & Offline \\
\hline 583 & Online & Offline & Offline & Brain + digital & Digital & Non-digital & Offline & Online \\
\hline 584 & Online & Offline & Offline & Brain + digital & Digital & Non-digital & Offline & Offline \\
\hline 585 & Online & Offline & Offline & Brain + digital & Digital & Blended & Online & Online \\
\hline 586 & Online & Offline & Offline & Brain + digital & Digital & Blended & Online & Offline \\
\hline 587 & Online & Offline & Offline & Brain + digital & Digital & Blended & Offline & Online \\
\hline 588 & Online & Offline & Offline & Brain + digital & Digital & Blended & Offline & Offline \\
\hline 589 & Online & Offline & Offline & Brain + digital & $\begin{array}{l}\text { Non- } \\
\text { digital }\end{array}$ & Digital & Online & Online \\
\hline 590 & Online & Offline & Offline & Brain + digital & $\begin{array}{l}\text { Non- } \\
\text { digital }\end{array}$ & Digital & Online & Offline \\
\hline 591 & Online & Offline & Offline & Brain + digital & $\begin{array}{l}\text { Non- } \\
\text { digital }\end{array}$ & Digital & Offline & Online \\
\hline 592 & Online & Offline & Offline & Brain + digital & $\begin{array}{l}\text { Non- } \\
\text { digital }\end{array}$ & Digital & Offline & Offline \\
\hline 593 & Online & Offline & Offline & Brain + digital & $\begin{array}{l}\text { Non- } \\
\text { digital }\end{array}$ & Non-digital & Online & Online \\
\hline 594 & Online & Offline & Offline & Brain + digital & $\begin{array}{l}\text { Non- } \\
\text { digital }\end{array}$ & Non-digital & Online & Offline \\
\hline 595 & Online & Offline & Offline & Brain + digital & $\begin{array}{l}\text { Non- } \\
\text { digital }\end{array}$ & Non-digital & Offline & Online \\
\hline 596 & Online & Offline & Offline & Brain + digital & $\begin{array}{l}\text { Non- } \\
\text { digital }\end{array}$ & Non-digital & Offline & Offline \\
\hline 597 & Online & Offline & Offline & Brain + digital & $\begin{array}{l}\text { Non- } \\
\text { digital }\end{array}$ & Blended & Online & Online \\
\hline 598 & Online & Offline & Offline & Brain + digital & $\begin{array}{l}\text { Non- } \\
\text { digital }\end{array}$ & Blended & Online & Offline \\
\hline 599 & Online & Offline & Offline & Brain + digital & $\begin{array}{l}\text { Non- } \\
\text { digital }\end{array}$ & Blended & Offline & Online \\
\hline 600 & Online & Offline & Offline & Brain + digital & $\begin{array}{l}\text { Non- } \\
\text { digital }\end{array}$ & Blended & Offline & Offline \\
\hline 601 & Online & Offline & Offline & Brain + digital & $\begin{array}{l}\text { Blend- } \\
\text { ed }\end{array}$ & Digital & Online & Online \\
\hline 602 & Online & Offline & Offline & Brain + digital & $\begin{array}{l}\text { Blend- } \\
\text { ed }\end{array}$ & Digital & Online & Offline \\
\hline 603 & Online & Offline & Offline & Brain + digital & $\begin{array}{l}\text { Blend- } \\
\text { ed }\end{array}$ & Digital & Offline & Online \\
\hline 604 & Online & Offline & Offline & Brain + digital & $\begin{array}{l}\text { Blend- } \\
\text { ed }\end{array}$ & Digital & Offline & Offline \\
\hline 605 & Online & Offline & Offline & Brain + digital & $\begin{array}{l}\text { Blend- } \\
\text { ed }\end{array}$ & Non-digital & Online & Online \\
\hline 606 & Online & Offline & Offline & Brain + digital & $\begin{array}{l}\text { Blend- } \\
\text { ed }\end{array}$ & Non-digital & Online & Offline \\
\hline 607 & Online & Offline & Offline & Brain + digital & $\begin{array}{l}\text { Blend- } \\
\text { ed }\end{array}$ & Non-digital & Offline & Online \\
\hline 608 & Online & Offline & Offline & Brain + digital & $\begin{array}{l}\text { Blend- } \\
\text { ed }\end{array}$ & Non-digital & Offline & Offline \\
\hline 609 & Online & Offline & Offline & Brain + digital & $\begin{array}{l}\text { Blend- } \\
\text { ed }\end{array}$ & Blended & Online & Online \\
\hline 610 & Online & Offline & Offline & Brain + digital & $\begin{array}{l}\text { Blend- } \\
\text { ed }\end{array}$ & Blended & Online & Offline \\
\hline 611 & Online & Offline & Offline & Brain + digital & $\begin{array}{l}\text { Blend- } \\
\text { ed }\end{array}$ & Blended & Offline & Online \\
\hline 612 & Online & Offline & Offline & Brain + digital & $\begin{array}{l}\text { Blend- } \\
\text { ed }\end{array}$ & Blended & Offline & Offline \\
\hline 613 & Online & Offline & Offline & Brain & Digital & Digital & Online & Online \\
\hline 614 & Online & Offline & Offline & Brain & Digital & Digital & Online & Offline \\
\hline 615 & Online & Offline & Offline & Brain & Digital & Digital & Offline & Online \\
\hline 616 & Online & Offline & Offline & Brain & Digital & Digital & Offline & Offline \\
\hline 617 & Online & Offline & Offline & Brain & Digital & Non-digital & Online & Online \\
\hline 618 & Online & Offline & Offline & Brain & Digital & Non-digital & Online & Offline \\
\hline
\end{tabular}




\begin{tabular}{|c|c|c|c|c|c|c|c|c|}
\hline 619 & Online & Offline & Offline & Brain & Digital & Non-digital & Offline & Online \\
\hline 620 & Online & Offline & Offline & Brain & Digital & Non-digital & Offline & Offline \\
\hline 621 & Online & Offline & Offline & Brain & Digital & Blended & Online & Online \\
\hline 622 & Online & Offline & Offline & Brain & Digital & Blended & Online & Offline \\
\hline 623 & Online & Offline & Offline & Brain & Digital & Blended & Offline & Online \\
\hline 624 & Online & Offline & Offline & Brain & Digital & Blended & Offline & Offline \\
\hline 625 & Online & Offline & Offline & Brain & $\begin{array}{l}\text { Non- } \\
\text { digital }\end{array}$ & Digital & Online & Online \\
\hline 626 & Online & Offline & Offline & Brain & $\begin{array}{l}\text { Non- } \\
\text { digital }\end{array}$ & Digital & Online & Offline \\
\hline 627 & Online & Offline & Offline & Brain & $\begin{array}{l}\text { Non- } \\
\text { digital }\end{array}$ & Digital & Offline & Online \\
\hline 628 & Online & Offline & Offline & Brain & $\begin{array}{l}\text { Non- } \\
\text { digital }\end{array}$ & Digital & Offline & Offline \\
\hline 629 & Online & Offline & Offline & Brain & $\begin{array}{l}\text { Non- } \\
\text { digital }\end{array}$ & Non-digital & Online & Online \\
\hline 630 & Online & Offline & Offline & Brain & $\begin{array}{l}\text { Non- } \\
\text { digital }\end{array}$ & Non-digital & Online & Offline \\
\hline 631 & Online & Offline & Offline & Brain & $\begin{array}{l}\text { Non- } \\
\text { digital }\end{array}$ & Non-digital & Offline & Online \\
\hline 632 & Online & Offline & Offline & Brain & $\begin{array}{l}\text { Non- } \\
\text { digital }\end{array}$ & Non-digital & Offline & Offline \\
\hline 633 & Online & Offline & Offline & Brain & $\begin{array}{l}\text { Non- } \\
\text { digital }\end{array}$ & Blended & Online & Online \\
\hline 634 & Online & Offline & Offline & Brain & $\begin{array}{l}\text { Non- } \\
\text { digital }\end{array}$ & Blended & Online & Offline \\
\hline 635 & Online & Offline & Offline & Brain & $\begin{array}{l}\text { Non- } \\
\text { digital }\end{array}$ & Blended & Offline & Online \\
\hline 636 & Online & Offline & Offline & Brain & $\begin{array}{l}\text { Non- } \\
\text { digital }\end{array}$ & Blended & Offline & Offline \\
\hline 637 & Online & Offline & Offline & Brain & $\begin{array}{l}\text { Blend- } \\
\text { ed }\end{array}$ & Digital & Online & Online \\
\hline 638 & Online & Offline & Offline & Brain & $\begin{array}{l}\text { Blend- } \\
\text { ed }\end{array}$ & Digital & Online & Offline \\
\hline 639 & Online & Offline & Offline & Brain & $\begin{array}{l}\text { Blend- } \\
\text { ed }\end{array}$ & Digital & Offline & Online \\
\hline 640 & Online & Offline & Offline & Brain & $\begin{array}{l}\text { Blend- } \\
\text { ed }\end{array}$ & Digital & Offline & Offline \\
\hline 641 & Online & Offline & Offline & Brain & $\begin{array}{l}\text { Blend- } \\
\text { ed }\end{array}$ & Non-digital & Online & Online \\
\hline 642 & Online & Offline & Offline & Brain & $\begin{array}{l}\text { Blend- } \\
\text { ed }\end{array}$ & Non-digital & Online & Offline \\
\hline 643 & Online & Offline & Offline & Brain & $\begin{array}{l}\text { Blend- } \\
\text { ed }\end{array}$ & Non-digital & Offline & Online \\
\hline 644 & Online & Offline & Offline & Brain & $\begin{array}{l}\text { Blend- } \\
\text { ed }\end{array}$ & Non-digital & Offline & Offline \\
\hline 645 & Online & Offline & Offline & Brain & $\begin{array}{l}\text { Blend- } \\
\text { ed }\end{array}$ & Blended & Online & Online \\
\hline 646 & Online & Offline & Offline & Brain & $\begin{array}{l}\text { Blend- } \\
\text { ed }\end{array}$ & Blended & Online & Offline \\
\hline 647 & Online & Offline & Offline & Brain & $\begin{array}{l}\text { Blend- } \\
\text { ed }\end{array}$ & Blended & Offline & Online \\
\hline 648 & Online & Offline & Offline & Brain & $\begin{array}{l}\text { Blend- } \\
\text { ed }\end{array}$ & Blended & Offline & Offline \\
\hline 649 & Online & Offline & Offline & Brain $+d+n-d$ & Digital & Digital & Online & Online \\
\hline 650 & Online & Offline & Offline & Brain $+d+n-d$ & Digital & Digital & Online & Offline \\
\hline 651 & Online & Offline & Offline & Brain $+d+n-d$ & Digital & Digital & Offline & Online \\
\hline 652 & Online & Offline & Offline & Brain $+d+n-d$ & Digital & Digital & Offline & Offline \\
\hline 653 & Online & Offline & Offline & Brain $+d+n-d$ & Digital & Non-digital & Online & Online \\
\hline 654 & Online & Offline & Offline & Brain $+d+n-d$ & Digital & Non-digital & Online & Offline \\
\hline 655 & Online & Offline & Offline & Brain $+d+n-d$ & Digital & Non-digital & Offline & Online \\
\hline 656 & Online & Offline & Offline & Brain $+d+n-d$ & Digital & Non-digital & Offline & Offline \\
\hline 657 & Online & Offline & Offline & Brain $+d+n-d$ & Digital & Blended & Online & Online \\
\hline 658 & Online & Offline & Offline & Brain $+d+n-d$ & Digital & Blended & Online & Offline \\
\hline 659 & Online & Offline & Offline & Brain $+d+n-d$ & Digital & Blended & Offline & Online \\
\hline
\end{tabular}




\begin{tabular}{|c|c|c|c|c|c|c|c|c|}
\hline 660 & Online & Offline & Offline & Brain $+d+n-d$ & Digital & Blended & Offline & Offline \\
\hline 661 & Online & Offline & Offline & Brain $+d+n-d$ & $\begin{array}{l}\text { Non- } \\
\text { digital }\end{array}$ & Digital & Online & Online \\
\hline 662 & Online & Offline & Offline & Brain $+d+n-d$ & $\begin{array}{l}\text { Non- } \\
\text { digital }\end{array}$ & Digital & Online & Offline \\
\hline 663 & Online & Offline & Offline & Brain + d + n-d & $\begin{array}{l}\text { Non- } \\
\text { digital }\end{array}$ & Digital & Offline & Online \\
\hline 664 & Online & Offline & Offline & Brain $+d+n-d$ & $\begin{array}{l}\text { Non- } \\
\text { digital }\end{array}$ & Digital & Offline & Offline \\
\hline 665 & Online & Offline & Offline & Brain $+d+n-d$ & $\begin{array}{l}\text { Non- } \\
\text { digital }\end{array}$ & Non-digital & Online & Online \\
\hline 666 & Online & Offline & Offline & Brain $+d+n-d$ & $\begin{array}{l}\text { Non- } \\
\text { digital }\end{array}$ & Non-digital & Online & Offline \\
\hline 667 & Online & Offline & Offline & Brain $+d+n-d$ & $\begin{array}{l}\text { Non- } \\
\text { digital }\end{array}$ & Non-digital & Offline & Online \\
\hline 668 & Online & Offline & Offline & Brain $+d+n-d$ & $\begin{array}{l}\text { Non- } \\
\text { digital }\end{array}$ & Non-digital & Offline & Offline \\
\hline 669 & Online & Offline & Offline & Brain $+d+n-d$ & $\begin{array}{l}\text { Non- } \\
\text { digital }\end{array}$ & Blended & Online & Online \\
\hline 670 & Online & Offline & Offline & Brain $+d+n-d$ & $\begin{array}{l}\text { Non- } \\
\text { digital }\end{array}$ & Blended & Online & Offline \\
\hline 671 & Online & Offline & Offline & Brain $+d+n-d$ & $\begin{array}{l}\text { Non- } \\
\text { digital }\end{array}$ & Blended & Offline & Online \\
\hline 672 & Online & Offline & Offline & Brain $+d+n-d$ & $\begin{array}{l}\text { Non- } \\
\text { digital }\end{array}$ & Blended & Offline & Offline \\
\hline 673 & Online & Offline & Offline & Brain $+d+n-d$ & $\begin{array}{l}\text { Blend- } \\
\text { ed }\end{array}$ & Digital & Online & Online \\
\hline 674 & Online & Offline & Offline & Brain $+d+n-d$ & $\begin{array}{l}\text { Blend- } \\
\text { ed }\end{array}$ & Digital & Online & Offline \\
\hline 675 & Online & Offline & Offline & Brain $+d+n-d$ & $\begin{array}{l}\text { Blend- } \\
\text { ed }\end{array}$ & Digital & Offline & Online \\
\hline 676 & Online & Offline & Offline & Brain $+d+n-d$ & $\begin{array}{l}\text { Blend- } \\
\text { ed }\end{array}$ & Digital & Offline & Offline \\
\hline 677 & Online & Offline & Offline & Brain $+d+n-d$ & $\begin{array}{l}\text { Blend- } \\
\text { ed }\end{array}$ & Non-digital & Online & Online \\
\hline 678 & Online & Offline & Offline & Brain $+d+n-d$ & $\begin{array}{l}\text { Blend- } \\
\text { ed }\end{array}$ & Non-digital & Online & Offline \\
\hline 679 & Online & Offline & Offline & Brain $+d+n-d$ & $\begin{array}{l}\text { Blend- } \\
\text { ed }\end{array}$ & Non-digital & Offline & Online \\
\hline 680 & Online & Offline & Offline & Brain $+d+n-d$ & $\begin{array}{l}\text { Blend- } \\
\text { ed }\end{array}$ & Non-digital & Offline & Offline \\
\hline 681 & Online & Offline & Offline & Brain $+d+n-d$ & $\begin{array}{l}\text { Blend- } \\
\text { ed }\end{array}$ & Blended & Online & Online \\
\hline 682 & Online & Offline & Offline & Brain $+d+n-d$ & $\begin{array}{l}\text { Blend- } \\
\text { ed }\end{array}$ & Blended & Online & Offline \\
\hline 683 & Online & Offline & Offline & Brain $+d+n-d$ & $\begin{array}{l}\text { Blend- } \\
\text { ed }\end{array}$ & Blended & Offline & Online \\
\hline 684 & Online & Offline & Offline & Brain $+d+n-d$ & $\begin{array}{l}\text { Blend- } \\
\text { ed }\end{array}$ & Blended & Offline & Offline \\
\hline 685 & Online & Offline & Offline & Brain + n-d & Digital & Digital & Online & Online \\
\hline 686 & Online & Offline & Offline & Brain + n-d & Digital & Digital & Online & Offline \\
\hline 687 & Online & Offline & Offline & Brain + n-d & Digital & Digital & Offline & Online \\
\hline 688 & Online & Offline & Offline & Brain + n-d & Digital & Digital & Offline & Offline \\
\hline 689 & Online & Offline & Offline & Brain + n-d & Digital & Non-digital & Online & Online \\
\hline 690 & Online & Offline & Offline & Brain + n-d & Digital & Non-digital & Online & Offline \\
\hline 691 & Online & Offline & Offline & Brain + n-d & Digital & Non-digital & Offline & Online \\
\hline 692 & Online & Offline & Offline & Brain + n-d & Digital & Non-digital & Offline & Offline \\
\hline 693 & Online & Offline & Offline & Brain + n-d & Digital & Blended & Online & Online \\
\hline 694 & Online & Offline & Offline & Brain + n-d & Digital & Blended & Online & Offline \\
\hline 695 & Online & Offline & Offline & Brain + n-d & Digital & Blended & Offline & Online \\
\hline 696 & Online & Offline & Offline & Brain + n-d & Digital & Blended & Offline & Offline \\
\hline 697 & Online & Offline & Offline & Brain + n-d & $\begin{array}{l}\text { Non- } \\
\text { digital }\end{array}$ & Digital & Online & Online \\
\hline 698 & Online & Offline & Offline & Brain + n-d & $\begin{array}{l}\text { Non- } \\
\text { digital }\end{array}$ & Digital & Online & Offline \\
\hline
\end{tabular}




\begin{tabular}{|c|c|c|c|c|c|c|c|c|}
\hline 699 & Online & Offline & Offline & Brain + n-d & $\begin{array}{l}\text { Non- } \\
\text { digital }\end{array}$ & Digital & Offline & Online \\
\hline 700 & Online & Offline & Offline & Brain + n-d & $\begin{array}{l}\text { Non- } \\
\text { digital }\end{array}$ & Digital & Offline & Offline \\
\hline 701 & Online & Offline & Offline & Brain + n-d & $\begin{array}{l}\text { Non- } \\
\text { digital }\end{array}$ & Non-digital & Online & Online \\
\hline 702 & Online & Offline & Offline & Brain + n-d & $\begin{array}{l}\text { Non- } \\
\text { digital }\end{array}$ & Non-digital & Online & Offline \\
\hline 703 & Online & Offline & Offline & Brain + n-d & $\begin{array}{l}\text { Non- } \\
\text { digital }\end{array}$ & Non-digital & Offline & Online \\
\hline 704 & Online & Offline & Offline & Brain + n-d & $\begin{array}{l}\text { Non- } \\
\text { digital }\end{array}$ & Non-digital & Offline & Offline \\
\hline 705 & Online & Offline & Offline & Brain + n-d & $\begin{array}{l}\text { Non- } \\
\text { digital }\end{array}$ & Blended & Online & Online \\
\hline 706 & Online & Offline & Offline & Brain + n-d & $\begin{array}{l}\text { Non- } \\
\text { digital }\end{array}$ & Blended & Online & Offline \\
\hline 707 & Online & Offline & Offline & Brain + n-d & $\begin{array}{l}\text { Non- } \\
\text { digital }\end{array}$ & Blended & Offline & Online \\
\hline 708 & Online & Offline & Offline & Brain + n-d & $\begin{array}{l}\text { Non- } \\
\text { digital }\end{array}$ & Blended & Offline & Offline \\
\hline 709 & Online & Offline & Offline & Brain + n-d & $\begin{array}{l}\text { Blend- } \\
\text { ed }\end{array}$ & Digital & Online & Online \\
\hline 710 & Online & Offline & Offline & Brain + n-d & $\begin{array}{l}\text { Blend- } \\
\text { ed }\end{array}$ & Digital & Online & Offline \\
\hline 711 & Online & Offline & Offline & Brain + n-d & $\begin{array}{l}\text { Blend- } \\
\text { ed }\end{array}$ & Digital & Offline & Online \\
\hline 712 & Online & Offline & Offline & Brain + n-d & $\begin{array}{l}\text { Blend- } \\
\text { ed }\end{array}$ & Digital & Offline & Offline \\
\hline 713 & Online & Offline & Offline & Brain $+n-d$ & $\begin{array}{l}\text { Blend- } \\
\text { ed }\end{array}$ & Non-digital & Online & Online \\
\hline 714 & Online & Offline & Offline & Brain $+n-d$ & $\begin{array}{l}\text { Blend- } \\
\text { ed }\end{array}$ & Non-digital & Online & Offline \\
\hline 715 & Online & Offline & Offline & Brain $+n-d$ & $\begin{array}{l}\text { Blend- } \\
\text { ed }\end{array}$ & Non-digital & Offline & Online \\
\hline 716 & Online & Offline & Offline & Brain + n-d & $\begin{array}{l}\text { Blend- } \\
\text { ed }\end{array}$ & Non-digital & Offline & Offline \\
\hline 717 & Online & Offline & Offline & Brain $+n-d$ & $\begin{array}{l}\text { Blend- } \\
\text { ed }\end{array}$ & Blended & Online & Online \\
\hline 718 & Online & Offline & Offline & Brain $+n-d$ & $\begin{array}{l}\text { Blend- } \\
\text { ed }\end{array}$ & Blended & Online & Offline \\
\hline 719 & Online & Offline & Offline & Brain + n-d & $\begin{array}{l}\text { Blend- } \\
\text { ed }\end{array}$ & Blended & Offline & Online \\
\hline 720 & Online & Offline & Offline & Brain + n-d & $\begin{array}{l}\text { Blend- } \\
\text { ed }\end{array}$ & Blended & Offline & Offline \\
\hline 721 & Online & Offline & Blended & Brain + digital & Digital & Digital & Online & Online \\
\hline 722 & Online & Offline & Blended & Brain + digital & Digital & Digital & Online & Offline \\
\hline 723 & Online & Offline & Blended & Brain + digital & Digital & Digital & Offline & Online \\
\hline 724 & Online & Offline & Blended & Brain + digital & Digital & Digital & Offline & Offline \\
\hline 725 & Online & Offline & Blended & Brain + digital & Digital & Non-digital & Online & Online \\
\hline 726 & Online & Offline & Blended & Brain + digital & Digital & Non-digital & Online & Offline \\
\hline 727 & Online & Offline & Blended & Brain + digital & Digital & Non-digital & Offline & Online \\
\hline 728 & Online & Offline & Blended & Brain + digital & Digital & Non-digital & Offline & Offline \\
\hline 729 & Online & Offline & Blended & Brain + digital & Digital & Blended & Online & Online \\
\hline 730 & Online & Offline & Blended & Brain + digital & Digital & Blended & Online & Offline \\
\hline 731 & Online & Offline & Blended & Brain + digital & Digital & Blended & Offline & Online \\
\hline 732 & Online & Offline & Blended & Brain + digital & Digital & Blended & Offline & Offline \\
\hline 733 & Online & Offline & Blended & Brain + digital & $\begin{array}{l}\text { Non- } \\
\text { digital }\end{array}$ & Digital & Online & Online \\
\hline 734 & Online & Offline & Blended & Brain + digital & $\begin{array}{l}\text { Non- } \\
\text { digital }\end{array}$ & Digital & Online & Offline \\
\hline 735 & Online & Offline & Blended & Brain + digital & $\begin{array}{l}\text { Non- } \\
\text { digital }\end{array}$ & Digital & Offline & Online \\
\hline 736 & Online & Offline & Blended & Brain + digital & $\begin{array}{l}\text { Non- } \\
\text { digital }\end{array}$ & Digital & Offline & Offline \\
\hline
\end{tabular}




\begin{tabular}{|c|c|c|c|c|c|c|c|c|}
\hline 737 & Online & Offline & Blended & Brain + digital & $\begin{array}{l}\text { Non- } \\
\text { digital }\end{array}$ & Non-digital & Online & Online \\
\hline 738 & Online & Offline & Blended & Brain + digital & $\begin{array}{l}\text { Non- } \\
\text { digital }\end{array}$ & Non-digital & Online & Offline \\
\hline 739 & Online & Offline & Blended & Brain + digital & $\begin{array}{l}\text { Non- } \\
\text { digital }\end{array}$ & Non-digital & Offline & Online \\
\hline 740 & Online & Offline & Blended & Brain + digital & $\begin{array}{l}\text { Non- } \\
\text { digital }\end{array}$ & Non-digital & Offline & Offline \\
\hline 741 & Online & Offline & Blended & Brain + digital & $\begin{array}{l}\text { Non- } \\
\text { digital }\end{array}$ & Blended & Online & Online \\
\hline 742 & Online & Offline & Blended & Brain + digital & $\begin{array}{l}\text { Non- } \\
\text { digital }\end{array}$ & Blended & Online & Offline \\
\hline 743 & Online & Offline & Blended & Brain + digital & $\begin{array}{l}\text { Non- } \\
\text { digital }\end{array}$ & Blended & Offline & Online \\
\hline 744 & Online & Offline & Blended & Brain + digital & $\begin{array}{l}\text { Non- } \\
\text { digital }\end{array}$ & Blended & Offline & Offline \\
\hline 745 & Online & Offline & Blended & Brain + digital & $\begin{array}{l}\text { Blend- } \\
\text { ed }\end{array}$ & Digital & Online & Online \\
\hline 746 & Online & Offline & Blended & Brain + digital & $\begin{array}{l}\text { Blend- } \\
\text { ed }\end{array}$ & Digital & Online & Offline \\
\hline 747 & Online & Offline & Blended & Brain + digital & $\begin{array}{l}\text { Blend- } \\
\text { ed }\end{array}$ & Digital & Offline & Online \\
\hline 748 & Online & Offline & Blended & Brain + digital & $\begin{array}{l}\text { Blend- } \\
\text { ed }\end{array}$ & Digital & Offline & Offline \\
\hline 749 & Online & Offline & Blended & Brain + digital & $\begin{array}{l}\text { Blend- } \\
\text { ed }\end{array}$ & Non-digital & Online & Online \\
\hline 750 & Online & Offline & Blended & Brain + digital & $\begin{array}{l}\text { Blend- } \\
\text { ed }\end{array}$ & Non-digital & Online & Offline \\
\hline 751 & Online & Offline & Blended & Brain + digital & $\begin{array}{l}\text { Blend- } \\
\text { ed }\end{array}$ & Non-digital & Offline & Online \\
\hline 752 & Online & Offline & Blended & Brain + digital & $\begin{array}{l}\text { Blend- } \\
\text { ed }\end{array}$ & Non-digital & Offline & Offline \\
\hline 753 & Online & Offline & Blended & Brain + digital & $\begin{array}{l}\text { Blend- } \\
\text { ed }\end{array}$ & Blended & Online & Online \\
\hline 754 & Online & Offline & Blended & Brain + digital & $\begin{array}{l}\text { Blend- } \\
\text { ed }\end{array}$ & Blended & Online & Offline \\
\hline 755 & Online & Offline & Blended & Brain + digital & $\begin{array}{l}\text { Blend- } \\
\text { ed }\end{array}$ & Blended & Offline & Online \\
\hline 756 & Online & Offline & Blended & Brain + digital & $\begin{array}{l}\text { Blend- } \\
\text { ed }\end{array}$ & Blended & Offline & Offline \\
\hline 757 & Online & Offline & Blended & Brain & Digital & Digital & Online & Online \\
\hline 758 & Online & Offline & Blended & Brain & Digital & Digital & Online & Offline \\
\hline 759 & Online & Offline & Blended & Brain & Digital & Digital & Offline & Online \\
\hline 760 & Online & Offline & Blended & Brain & Digital & Digital & Offline & Offline \\
\hline 761 & Online & Offline & Blended & Brain & Digital & Non-digital & Online & Online \\
\hline 762 & Online & Offline & Blended & Brain & Digital & Non-digital & Online & Offline \\
\hline 763 & Online & Offline & Blended & Brain & Digital & Non-digital & Offline & Online \\
\hline 764 & Online & Offline & Blended & Brain & Digital & Non-digital & Offline & Offline \\
\hline 765 & Online & Offline & Blended & Brain & Digital & Blended & Online & Online \\
\hline 766 & Online & Offline & Blended & Brain & Digital & Blended & Online & Offline \\
\hline 767 & Online & Offline & Blended & Brain & Digital & Blended & Offline & Online \\
\hline 768 & Online & Offline & Blended & Brain & Digital & Blended & Offline & Offline \\
\hline 769 & Online & Offline & Blended & Brain & $\begin{array}{l}\text { Non- } \\
\text { digital }\end{array}$ & Digital & Online & Online \\
\hline 770 & Online & Offline & Blended & Brain & $\begin{array}{l}\text { Non- } \\
\text { digital }\end{array}$ & Digital & Online & Offline \\
\hline 771 & Online & Offline & Blended & Brain & $\begin{array}{l}\text { Non- } \\
\text { digital }\end{array}$ & Digital & Offline & Online \\
\hline 772 & Online & Offline & Blended & Brain & $\begin{array}{l}\text { Non- } \\
\text { digital }\end{array}$ & Digital & Offline & Offline \\
\hline 773 & Online & Offline & Blended & Brain & $\begin{array}{l}\text { Non- } \\
\text { digital }\end{array}$ & Non-digital & Online & Online \\
\hline 774 & Online & Offline & Blended & Brain & $\begin{array}{l}\text { Non- } \\
\text { digital }\end{array}$ & Non-digital & Online & Offline \\
\hline
\end{tabular}




\begin{tabular}{|c|c|c|c|c|c|c|c|c|}
\hline 775 & Online & Offline & Blended & Brain & $\begin{array}{l}\text { Non- } \\
\text { digital }\end{array}$ & Non-digital & Offline & Online \\
\hline 776 & Online & Offline & Blended & Brain & $\begin{array}{l}\text { Non- } \\
\text { digital }\end{array}$ & Non-digital & Offline & Offline \\
\hline 777 & Online & Offline & Blended & Brain & $\begin{array}{l}\text { Non- } \\
\text { digital }\end{array}$ & Blended & Online & Online \\
\hline 778 & Online & Offline & Blended & Brain & $\begin{array}{l}\text { Non- } \\
\text { digital }\end{array}$ & Blended & Online & Offline \\
\hline 779 & Online & Offline & Blended & Brain & $\begin{array}{l}\text { Non- } \\
\text { digital }\end{array}$ & Blended & Offline & Online \\
\hline 780 & Online & Offline & Blended & Brain & $\begin{array}{l}\text { Non- } \\
\text { digital }\end{array}$ & Blended & Offline & Offline \\
\hline 781 & Online & Offline & Blended & Brain & $\begin{array}{l}\text { Blend- } \\
\text { ed }\end{array}$ & Digital & Online & Online \\
\hline 782 & Online & Offline & Blended & Brain & $\begin{array}{l}\text { Blend- } \\
\text { ed }\end{array}$ & Digital & Online & Offline \\
\hline 783 & Online & Offline & Blended & Brain & $\begin{array}{l}\text { Blend- } \\
\text { ed }\end{array}$ & Digital & Offline & Online \\
\hline 784 & Online & Offline & Blended & Brain & $\begin{array}{l}\text { Blend- } \\
\text { ed }\end{array}$ & Digital & Offline & Offline \\
\hline 785 & Online & Offline & Blended & Brain & $\begin{array}{l}\text { Blend- } \\
\text { ed }\end{array}$ & Non-digital & Online & Online \\
\hline 786 & Online & Offline & Blended & Brain & $\begin{array}{l}\text { Blend- } \\
\text { ed }\end{array}$ & Non-digital & Online & Offline \\
\hline 787 & Online & Offline & Blended & Brain & $\begin{array}{l}\text { Blend- } \\
\text { ed }\end{array}$ & Non-digital & Offline & Online \\
\hline 788 & Online & Offline & Blended & Brain & $\begin{array}{l}\text { Blend- } \\
\text { ed }\end{array}$ & Non-digital & Offline & Offline \\
\hline 789 & Online & Offline & Blended & Brain & $\begin{array}{l}\text { Blend- } \\
\text { ed }\end{array}$ & Blended & Online & Online \\
\hline 790 & Online & Offline & Blended & Brain & $\begin{array}{l}\text { Blend- } \\
\text { ed }\end{array}$ & Blended & Online & Offline \\
\hline 791 & Online & Offline & Blended & Brain & $\begin{array}{l}\text { Blend- } \\
\text { ed }\end{array}$ & Blended & Offline & Online \\
\hline 792 & Online & Offline & Blended & Brain & $\begin{array}{l}\text { Blend- } \\
\text { ed }\end{array}$ & Blended & Offline & Offline \\
\hline 793 & Online & Offline & Blended & Brain $+d+n-d$ & Digital & Digital & Online & Online \\
\hline 794 & Online & Offline & Blended & Brain $+d+n-d$ & Digital & Digital & Online & Offline \\
\hline 795 & Online & Offline & Blended & Brain $+d+n-d$ & Digital & Digital & Offline & Online \\
\hline 796 & Online & Offline & Blended & Brain $+d+n-d$ & Digital & Digital & Offline & Offline \\
\hline 797 & Online & Offline & Blended & Brain $+d+n-d$ & Digital & Non-digital & Online & Online \\
\hline 798 & Online & Offline & Blended & Brain $+d+n-d$ & Digital & Non-digital & Online & Offline \\
\hline 799 & Online & Offline & Blended & Brain $+d+n-d$ & Digital & Non-digital & Offline & Online \\
\hline 800 & Online & Offline & Blended & Brain $+d+n-d$ & Digital & Non-digital & Offline & Offline \\
\hline 801 & Online & Offline & Blended & Brain $+d+n-d$ & Digital & Blended & Online & Online \\
\hline 802 & Online & Offline & Blended & Brain $+d+n-d$ & Digital & Blended & Online & Offline \\
\hline 803 & Online & Offline & Blended & Brain $+d+n-d$ & Digital & Blended & Offline & Online \\
\hline 804 & Online & Offline & Blended & Brain $+d+n-d$ & Digital & Blended & Offline & Offline \\
\hline 805 & Online & Offline & Blended & Brain $+d+n-d$ & $\begin{array}{l}\text { Non- } \\
\text { digital }\end{array}$ & Digital & Online & Online \\
\hline 806 & Online & Offline & Blended & Brain $+d+n-d$ & $\begin{array}{l}\text { Non- } \\
\text { digital }\end{array}$ & Digital & Online & Offline \\
\hline 807 & Online & Offline & Blended & Brain $+d+n-d$ & $\begin{array}{l}\text { Non- } \\
\text { digital }\end{array}$ & Digital & Offline & Online \\
\hline 808 & Online & Offline & Blended & Brain $+d+n-d$ & $\begin{array}{l}\text { Non- } \\
\text { digital }\end{array}$ & Digital & Offline & Offline \\
\hline 809 & Online & Offline & Blended & Brain $+d+n-d$ & $\begin{array}{l}\text { Non- } \\
\text { digital }\end{array}$ & Non-digital & Online & Online \\
\hline 810 & Online & Offline & Blended & Brain $+d+n-d$ & $\begin{array}{l}\text { Non- } \\
\text { digital }\end{array}$ & Non-digital & Online & Offline \\
\hline 811 & Online & Offline & Blended & Brain $+d+n-d$ & $\begin{array}{l}\text { Non- } \\
\text { digital }\end{array}$ & Non-digital & Offline & Online \\
\hline 812 & Online & Offline & Blended & Brain $+d+n-d$ & $\begin{array}{l}\text { Non- } \\
\text { digital }\end{array}$ & Non-digital & Offline & Offline \\
\hline
\end{tabular}




\begin{tabular}{|c|c|c|c|c|c|c|c|c|}
\hline 813 & Online & Offline & Blended & Brain $+d+n-d$ & $\begin{array}{l}\text { Non- } \\
\text { digital }\end{array}$ & Blended & Online & Online \\
\hline 814 & Online & Offline & Blended & Brain $+d+n-d$ & $\begin{array}{l}\text { Non- } \\
\text { digital }\end{array}$ & Blended & Online & Offline \\
\hline 815 & Online & Offline & Blended & Brain $+d+n-d$ & $\begin{array}{l}\text { Non- } \\
\text { digital }\end{array}$ & Blended & Offline & Online \\
\hline 816 & Online & Offline & Blended & Brain $+d+n-d$ & $\begin{array}{l}\text { Non- } \\
\text { digital }\end{array}$ & Blended & Offline & Offline \\
\hline 817 & Online & Offline & Blended & Brain $+d+n-d$ & $\begin{array}{l}\text { Blend- } \\
\text { ed }\end{array}$ & Digital & Online & Online \\
\hline 818 & Online & Offline & Blended & Brain $+d+n-d$ & $\begin{array}{l}\text { Blend- } \\
\text { ed }\end{array}$ & Digital & Online & Offline \\
\hline 819 & Online & Offline & Blended & Brain $+d+n-d$ & $\begin{array}{l}\text { Blend- } \\
\text { ed }\end{array}$ & Digital & Offline & Online \\
\hline 820 & Online & Offline & Blended & Brain $+d+n-d$ & $\begin{array}{l}\text { Blend- } \\
\text { ed }\end{array}$ & Digital & Offline & Offline \\
\hline 821 & Online & Offline & Blended & Brain $+d+n-d$ & $\begin{array}{l}\text { Blend- } \\
\text { ed }\end{array}$ & Non-digital & Online & Online \\
\hline 822 & Online & Offline & Blended & Brain $+d+n-d$ & $\begin{array}{l}\text { Blend- } \\
\text { ed }\end{array}$ & Non-digital & Online & Offline \\
\hline 823 & Online & Offline & Blended & Brain $+d+n-d$ & $\begin{array}{l}\text { Blend- } \\
\text { ed }\end{array}$ & Non-digital & Offline & Online \\
\hline 824 & Online & Offline & Blended & Brain $+d+n-d$ & $\begin{array}{l}\text { Blend- } \\
\text { ed }\end{array}$ & Non-digital & Offline & Offline \\
\hline 825 & Online & Offline & Blended & Brain $+d+n-d$ & $\begin{array}{l}\text { Blend- } \\
\text { ed }\end{array}$ & Blended & Online & Online \\
\hline 826 & Online & Offline & Blended & Brain $+d+n-d$ & $\begin{array}{l}\text { Blend- } \\
\text { ed }\end{array}$ & Blended & Online & Offline \\
\hline 827 & Online & Offline & Blended & Brain $+d+n-d$ & $\begin{array}{l}\text { Blend- } \\
\text { ed }\end{array}$ & Blended & Offline & Online \\
\hline 828 & Online & Offline & Blended & Brain $+d+n-d$ & $\begin{array}{l}\text { Blend- } \\
\text { ed }\end{array}$ & Blended & Offline & Offline \\
\hline 829 & Online & Offline & Blended & Brain + n-d & Digital & Digital & Online & Online \\
\hline 830 & Online & Offline & Blended & Brain + n-d & Digital & Digital & Online & Offline \\
\hline 831 & Online & Offline & Blended & Brain + n-d & Digital & Digital & Offline & Online \\
\hline 832 & Online & Offline & Blended & Brain + n-d & Digital & Digital & Offline & Offline \\
\hline 833 & Online & Offline & Blended & Brain + n-d & Digital & Non-digital & Online & Online \\
\hline 834 & Online & Offline & Blended & Brain + n-d & Digital & Non-digital & Online & Offline \\
\hline 835 & Online & Offline & Blended & Brain + n-d & Digital & Non-digital & Offline & Online \\
\hline 836 & Online & Offline & Blended & Brain + n-d & Digital & Non-digital & Offline & Offline \\
\hline 837 & Online & Offline & Blended & Brain + n-d & Digital & Blended & Online & Online \\
\hline 838 & Online & Offline & Blended & Brain + n-d & Digital & Blended & Online & Offline \\
\hline 839 & Online & Offline & Blended & Brain + n-d & Digital & Blended & Offline & Online \\
\hline 840 & Online & Offline & Blended & Brain + n-d & Digital & Blended & Offline & Offline \\
\hline 841 & Online & Offline & Blended & Brain + n-d & $\begin{array}{l}\text { Non- } \\
\text { digital }\end{array}$ & Digital & Online & Online \\
\hline 842 & Online & Offline & Blended & Brain + n-d & $\begin{array}{l}\text { Non- } \\
\text { digital }\end{array}$ & Digital & Online & Offline \\
\hline 843 & Online & Offline & Blended & Brain + n-d & $\begin{array}{l}\text { Non- } \\
\text { digital }\end{array}$ & Digital & Offline & Online \\
\hline 844 & Online & Offline & Blended & Brain + n-d & $\begin{array}{l}\text { Non- } \\
\text { digital }\end{array}$ & Digital & Offline & Offline \\
\hline 845 & Online & Offline & Blended & Brain + n-d & $\begin{array}{l}\text { Non- } \\
\text { digital }\end{array}$ & Non-digital & Online & Online \\
\hline 846 & Online & Offline & Blended & Brain + n-d & $\begin{array}{l}\text { Non- } \\
\text { digital }\end{array}$ & Non-digital & Online & Offline \\
\hline 847 & Online & Offline & Blended & Brain + n-d & $\begin{array}{l}\text { Non- } \\
\text { digital }\end{array}$ & Non-digital & Offline & Online \\
\hline 848 & Online & Offline & Blended & Brain + n-d & $\begin{array}{l}\text { Non- } \\
\text { digital }\end{array}$ & Non-digital & Offline & Offline \\
\hline 849 & Online & Offline & Blended & Brain + n-d & $\begin{array}{l}\text { Non- } \\
\text { digital }\end{array}$ & Blended & Online & Online \\
\hline 850 & Online & Offline & Blended & Brain + n-d & $\begin{array}{l}\text { Non- } \\
\text { digital }\end{array}$ & Blended & Online & Offline \\
\hline
\end{tabular}




\begin{tabular}{|c|c|c|c|c|c|c|c|c|}
\hline 851 & Online & Offline & Blended & Brain + n-d & $\begin{array}{l}\text { Non- } \\
\text { digital }\end{array}$ & Blended & Offline & Online \\
\hline 852 & Online & Offline & Blended & Brain + n-d & $\begin{array}{l}\text { Non- } \\
\text { digital }\end{array}$ & Blended & Offline & Offline \\
\hline 853 & Online & Offline & Blended & Brain $+n-d$ & $\begin{array}{l}\text { Blend- } \\
\text { ed }\end{array}$ & Digital & Online & Online \\
\hline 854 & Online & Offline & Blended & Brain + n-d & $\begin{array}{l}\text { Blend- } \\
\text { ed }\end{array}$ & Digital & Online & Offline \\
\hline 855 & Online & Offline & Blended & Brain + n-d & $\begin{array}{l}\text { Blend- } \\
\text { ed }\end{array}$ & Digital & Offline & Online \\
\hline 856 & Online & Offline & Blended & Brain + n-d & $\begin{array}{l}\text { Blend- } \\
\text { ed }\end{array}$ & Digital & Offline & Offline \\
\hline 857 & Online & Offline & Blended & Brain $+n-d$ & $\begin{array}{l}\text { Blend- } \\
\text { ed }\end{array}$ & Non-digital & Online & Online \\
\hline 858 & Online & Offline & Blended & Brain + n-d & $\begin{array}{l}\text { Blend- } \\
\text { ed }\end{array}$ & Non-digital & Online & Offline \\
\hline 859 & Online & Offline & Blended & Brain + n-d & $\begin{array}{l}\text { Blend- } \\
\text { ed }\end{array}$ & Non-digital & Offline & Online \\
\hline 860 & Online & Offline & Blended & Brain + n-d & $\begin{array}{l}\text { Blend- } \\
\text { ed }\end{array}$ & Non-digital & Offline & Offline \\
\hline 861 & Online & Offline & Blended & Brain $+n-d$ & $\begin{array}{l}\text { Blend- } \\
\text { ed }\end{array}$ & Blended & Online & Online \\
\hline 862 & Online & Offline & Blended & Brain $+n-d$ & $\begin{array}{l}\text { Blend- } \\
\text { ed }\end{array}$ & Blended & Online & Offline \\
\hline 863 & Online & Offline & Blended & Brain + n-d & $\begin{array}{l}\text { Blend- } \\
\text { ed }\end{array}$ & Blended & Offline & Online \\
\hline 864 & Online & Offline & Blended & Brain + n-d & $\begin{array}{l}\text { Blend- } \\
\text { ed }\end{array}$ & Blended & Offline & Offline \\
\hline 865 & Offline & Online & Online & Brain + digital & Digital & Digital & Online & Online \\
\hline 866 & Offline & Online & Online & Brain + digital & Digital & Digital & Online & Offline \\
\hline 867 & Offline & Online & Online & Brain + digital & Digital & Digital & Offline & Online \\
\hline 868 & Offline & Online & Online & Brain + digital & Digital & Digital & Offline & Offline \\
\hline 869 & Offline & Online & Online & Brain + digital & Digital & Non-digital & Online & Online \\
\hline 870 & Offline & Online & Online & Brain + digital & Digital & Non-digital & Online & Offline \\
\hline 871 & Offline & Online & Online & Brain + digital & Digital & Non-digital & Offline & Online \\
\hline 872 & Offline & Online & Online & Brain + digital & Digital & Non-digital & Offline & Offline \\
\hline 873 & Offline & Online & Online & Brain + digital & Digital & Blended & Online & Online \\
\hline 874 & Offline & Online & Online & Brain + digital & Digital & Blended & Online & Offline \\
\hline 875 & Offline & Online & Online & Brain + digital & Digital & Blended & Offline & Online \\
\hline 876 & Offline & Online & Online & Brain + digital & Digital & Blended & Offline & Offline \\
\hline 877 & Offline & Online & Online & Brain + digital & $\begin{array}{l}\text { Non- } \\
\text { digital }\end{array}$ & Digital & Online & Online \\
\hline 878 & Offline & Online & Online & Brain + digital & $\begin{array}{l}\text { Non- } \\
\text { digital }\end{array}$ & Digital & Online & Offline \\
\hline 879 & Offline & Online & Online & Brain + digital & $\begin{array}{l}\text { Non- } \\
\text { digital }\end{array}$ & Digital & Offline & Online \\
\hline 880 & Offline & Online & Online & Brain + digital & $\begin{array}{l}\text { Non- } \\
\text { digital }\end{array}$ & Digital & Offline & Offline \\
\hline 881 & Offline & Online & Online & Brain + digital & $\begin{array}{l}\text { Non- } \\
\text { digital }\end{array}$ & Non-digital & Online & Online \\
\hline 882 & Offline & Online & Online & Brain + digital & $\begin{array}{l}\text { Non- } \\
\text { digital }\end{array}$ & Non-digital & Online & Offline \\
\hline 883 & Offline & Online & Online & Brain + digital & $\begin{array}{l}\text { Non- } \\
\text { digital }\end{array}$ & Non-digital & Offline & Online \\
\hline 884 & Offline & Online & Online & Brain + digital & $\begin{array}{l}\text { Non- } \\
\text { digital }\end{array}$ & Non-digital & Offline & Offline \\
\hline 885 & Offline & Online & Online & Brain + digital & $\begin{array}{l}\text { Non- } \\
\text { digital }\end{array}$ & Blended & Online & Online \\
\hline 886 & Offline & Online & Online & Brain + digital & $\begin{array}{l}\text { Non- } \\
\text { digital }\end{array}$ & Blended & Online & Offline \\
\hline 887 & Offline & Online & Online & Brain + digital & $\begin{array}{l}\text { Non- } \\
\text { digital }\end{array}$ & Blended & Offline & Online \\
\hline 888 & Offline & Online & Online & Brain + digital & $\begin{array}{l}\text { Non- } \\
\text { digital }\end{array}$ & Blended & Offline & Offline \\
\hline
\end{tabular}




\begin{tabular}{|c|c|c|c|c|c|c|c|c|}
\hline 889 & Offline & Online & Online & Brain + digital & $\begin{array}{l}\text { Blend- } \\
\text { ed }\end{array}$ & Digital & Online & Online \\
\hline 890 & Offline & Online & Online & Brain + digital & $\begin{array}{l}\text { Blend- } \\
\text { ed }\end{array}$ & Digital & Online & Offline \\
\hline 891 & Offline & Online & Online & Brain + digital & $\begin{array}{l}\text { Blend- } \\
\text { ed }\end{array}$ & Digital & Offline & Online \\
\hline 892 & Offline & Online & Online & Brain + digital & $\begin{array}{l}\text { Blend- } \\
\text { ed }\end{array}$ & Digital & Offline & Offline \\
\hline 893 & Offline & Online & Online & Brain + digital & $\begin{array}{l}\text { Blend- } \\
\text { ed }\end{array}$ & Non-digital & Online & Online \\
\hline 894 & Offline & Online & Online & Brain + digital & $\begin{array}{l}\text { Blend- } \\
\text { ed }\end{array}$ & Non-digital & Online & Offline \\
\hline 895 & Offline & Online & Online & Brain + digital & $\begin{array}{l}\text { Blend- } \\
\text { ed }\end{array}$ & Non-digital & Offline & Online \\
\hline 896 & Offline & Online & Online & Brain + digital & $\begin{array}{l}\text { Blend- } \\
\text { ed }\end{array}$ & Non-digital & Offline & Offline \\
\hline 897 & Offline & Online & Online & Brain + digital & $\begin{array}{l}\text { Blend- } \\
\text { ed }\end{array}$ & Blended & Online & Online \\
\hline 898 & Offline & Online & Online & Brain + digital & $\begin{array}{l}\text { Blend- } \\
\text { ed }\end{array}$ & Blended & Online & Offline \\
\hline 899 & Offline & Online & Online & Brain + digital & $\begin{array}{l}\text { Blend- } \\
\text { ed }\end{array}$ & Blended & Offline & Online \\
\hline 900 & Offline & Online & Online & Brain + digital & $\begin{array}{l}\text { Blend- } \\
\text { ed }\end{array}$ & Blended & Offline & Offline \\
\hline 901 & Offline & Online & Online & Brain & Digital & Digital & Online & Online \\
\hline 902 & Offline & Online & Online & Brain & Digital & Digital & Online & Offline \\
\hline 903 & Offline & Online & Online & Brain & Digital & Digital & Offline & Online \\
\hline 904 & Offline & Online & Online & Brain & Digital & Digital & Offline & Offline \\
\hline 905 & Offline & Online & Online & Brain & Digital & Non-digital & Online & Online \\
\hline 906 & Offline & Online & Online & Brain & Digital & Non-digital & Online & Offline \\
\hline 907 & Offline & Online & Online & Brain & Digital & Non-digital & Offline & Online \\
\hline 908 & Offline & Online & Online & Brain & Digital & Non-digital & Offline & Offline \\
\hline 909 & Offline & Online & Online & Brain & Digital & Blended & Online & Online \\
\hline 910 & Offline & Online & Online & Brain & Digital & Blended & Online & Offline \\
\hline 911 & Offline & Online & Online & Brain & Digital & Blended & Offline & Online \\
\hline 912 & Offline & Online & Online & Brain & Digital & Blended & Offline & Offline \\
\hline 913 & Offline & Online & Online & Brain & $\begin{array}{l}\text { Non- } \\
\text { digital }\end{array}$ & Digital & Online & Online \\
\hline 914 & Offline & Online & Online & Brain & $\begin{array}{l}\text { Non- } \\
\text { digital }\end{array}$ & Digital & Online & Offline \\
\hline 915 & Offline & Online & Online & Brain & $\begin{array}{l}\text { Non- } \\
\text { digital }\end{array}$ & Digital & Offline & Online \\
\hline 916 & Offline & Online & Online & Brain & $\begin{array}{l}\text { Non- } \\
\text { digital }\end{array}$ & Digital & Offline & Offline \\
\hline 917 & Offline & Online & Online & Brain & $\begin{array}{l}\text { Non- } \\
\text { digital }\end{array}$ & Non-digital & Online & Online \\
\hline 918 & Offline & Online & Online & Brain & $\begin{array}{l}\text { Non- } \\
\text { digital }\end{array}$ & Non-digital & Online & Offline \\
\hline 919 & Offline & Online & Online & Brain & $\begin{array}{l}\text { Non- } \\
\text { digital }\end{array}$ & Non-digital & Offline & Online \\
\hline 920 & Offline & Online & Online & Brain & $\begin{array}{l}\text { Non- } \\
\text { digital }\end{array}$ & Non-digital & Offline & Offline \\
\hline 921 & Offline & Online & Online & Brain & $\begin{array}{l}\text { Non- } \\
\text { digital }\end{array}$ & Blended & Online & Online \\
\hline 922 & Offline & Online & Online & Brain & $\begin{array}{l}\text { Non- } \\
\text { digital }\end{array}$ & Blended & Online & Offline \\
\hline 923 & Offline & Online & Online & Brain & $\begin{array}{l}\text { Non- } \\
\text { digital }\end{array}$ & Blended & Offline & Online \\
\hline 924 & Offline & Online & Online & Brain & $\begin{array}{l}\text { Non- } \\
\text { digital }\end{array}$ & Blended & Offline & Offline \\
\hline 925 & Offline & Online & Online & Brain & $\begin{array}{l}\text { Blend- } \\
\text { ed }\end{array}$ & Digital & Online & Online \\
\hline 926 & Offline & Online & Online & Brain & $\begin{array}{l}\text { Blend- } \\
\text { ed }\end{array}$ & Digital & Online & Offline \\
\hline
\end{tabular}




\begin{tabular}{|c|c|c|c|c|c|c|c|c|}
\hline 927 & Offline & Online & Online & Brain & $\begin{array}{l}\text { Blend- } \\
\text { ed }\end{array}$ & Digital & Offline & Online \\
\hline 928 & Offline & Online & Online & Brain & $\begin{array}{l}\text { Blend- } \\
\text { ed }\end{array}$ & Digital & Offline & Offline \\
\hline 929 & Offline & Online & Online & Brain & $\begin{array}{l}\text { Blend- } \\
\text { ed }\end{array}$ & Non-digital & Online & Online \\
\hline 930 & Offline & Online & Online & Brain & $\begin{array}{l}\text { Blend- } \\
\text { ed }\end{array}$ & Non-digital & Online & Offline \\
\hline 931 & Offline & Online & Online & Brain & $\begin{array}{l}\text { Blend- } \\
\text { ed }\end{array}$ & Non-digital & Offline & Online \\
\hline 932 & Offline & Online & Online & Brain & $\begin{array}{l}\text { Blend- } \\
\text { ed }\end{array}$ & Non-digital & Offline & Offline \\
\hline 933 & Offline & Online & Online & Brain & $\begin{array}{l}\text { Blend- } \\
\text { ed }\end{array}$ & Blended & Online & Online \\
\hline 934 & Offline & Online & Online & Brain & $\begin{array}{l}\text { Blend- } \\
\text { ed }\end{array}$ & Blended & Online & Offline \\
\hline 935 & Offline & Online & Online & Brain & $\begin{array}{l}\text { Blend- } \\
\text { ed }\end{array}$ & Blended & Offline & Online \\
\hline 936 & Offline & Online & Online & Brain & $\begin{array}{l}\text { Blend- } \\
\text { ed }\end{array}$ & Blended & Offline & Offline \\
\hline 937 & Offline & Online & Online & Brain $+d+n-d$ & Digital & Digital & Online & Online \\
\hline 938 & Offline & Online & Online & Brain $+d+n-d$ & Digital & Digital & Online & Offline \\
\hline 939 & Offline & Online & Online & Brain $+d+n-d$ & Digital & Digital & Offline & Online \\
\hline 940 & Offline & Online & Online & Brain $+d+n-d$ & Digital & Digital & Offline & Offline \\
\hline 941 & Offline & Online & Online & Brain $+d+n-d$ & Digital & Non-digital & Online & Online \\
\hline 942 & Offline & Online & Online & Brain $+d+n-d$ & Digital & Non-digital & Online & Offline \\
\hline 943 & Offline & Online & Online & Brain $+d+n-d$ & Digital & Non-digital & Offline & Online \\
\hline 944 & Offline & Online & Online & Brain $+d+n-d$ & Digital & Non-digital & Offline & Offline \\
\hline 945 & Offline & Online & Online & Brain $+d+n-d$ & Digital & Blended & Online & Online \\
\hline 946 & Offline & Online & Online & Brain $+d+n-d$ & Digital & Blended & Online & Offline \\
\hline 947 & Offline & Online & Online & Brain $+d+n-d$ & Digital & Blended & Offline & Online \\
\hline 948 & Offline & Online & Online & Brain $+d+n-d$ & Digital & Blended & Offline & Offline \\
\hline 949 & Offline & Online & Online & Brain $+d+n-d$ & $\begin{array}{l}\text { Non- } \\
\text { digital }\end{array}$ & Digital & Online & Online \\
\hline 950 & Offline & Online & Online & Brain $+d+n-d$ & $\begin{array}{l}\text { Non- } \\
\text { digital }\end{array}$ & Digital & Online & Offline \\
\hline 951 & Offline & Online & Online & Brain $+d+n-d$ & $\begin{array}{l}\text { Non- } \\
\text { digital }\end{array}$ & Digital & Offline & Online \\
\hline 952 & Offline & Online & Online & Brain $+d+n-d$ & $\begin{array}{l}\text { Non- } \\
\text { digital }\end{array}$ & Digital & Offline & Offline \\
\hline 953 & Offline & Online & Online & Brain $+d+n-d$ & $\begin{array}{l}\text { Non- } \\
\text { digital }\end{array}$ & Non-digital & Online & Online \\
\hline 954 & Offline & Online & Online & Brain $+d+n-d$ & $\begin{array}{l}\text { Non- } \\
\text { diqital }\end{array}$ & Non-digital & Online & Offline \\
\hline 955 & Offline & Online & Online & Brain $+d+n-d$ & $\begin{array}{l}\text { Non- } \\
\text { digital }\end{array}$ & Non-digital & Offline & Online \\
\hline 956 & Offline & Online & Online & Brain $+d+n-d$ & $\begin{array}{l}\text { Non- } \\
\text { diqital }\end{array}$ & Non-digital & Offline & Offline \\
\hline 957 & Offline & Online & Online & Brain $+d+n-d$ & $\begin{array}{l}\text { Non- } \\
\text { diqital }\end{array}$ & Blended & Online & Online \\
\hline 958 & Offline & Online & Online & Brain $+d+n-d$ & $\begin{array}{l}\text { Non- } \\
\text { diqital }\end{array}$ & Blended & Online & Offline \\
\hline 959 & Offline & Online & Online & Brain $+d+n-d$ & $\begin{array}{l}\text { Non- } \\
\text { digital }\end{array}$ & Blended & Offline & Online \\
\hline 960 & Offline & Online & Online & Brain $+d+n-d$ & $\begin{array}{l}\text { Non- } \\
\text { diqital }\end{array}$ & Blended & Offline & Offline \\
\hline 961 & Offline & Online & Online & Brain $+d+n-d$ & $\begin{array}{l}\text { Blend- } \\
\text { ed }\end{array}$ & Digital & Online & Online \\
\hline 962 & Offline & Online & Online & Brain $+d+n-d$ & $\begin{array}{l}\text { Blend- } \\
\text { ed }\end{array}$ & Digital & Online & Offline \\
\hline 963 & Offline & Online & Online & Brain $+d+n-d$ & $\begin{array}{l}\text { Blend- } \\
\text { ed }\end{array}$ & Digital & Offline & Online \\
\hline 964 & Offline & Online & Online & Brain $+d+n-d$ & $\begin{array}{l}\text { Blend- } \\
\text { ed }\end{array}$ & Digital & Offline & Offline \\
\hline
\end{tabular}




\begin{tabular}{|c|c|c|c|c|c|c|c|c|}
\hline 965 & Offline & Online & Online & Brain $+d+n-d$ & $\begin{array}{l}\text { Blend- } \\
\text { ed }\end{array}$ & Non-digital & Online & Online \\
\hline 966 & Offline & Online & Online & Brain $+d+n-d$ & $\begin{array}{l}\text { Blend- } \\
\text { ed }\end{array}$ & Non-digital & Online & Offline \\
\hline 967 & Offline & Online & Online & Brain $+d+n-d$ & $\begin{array}{l}\text { Blend- } \\
\text { ed }\end{array}$ & Non-digital & Offline & Online \\
\hline 968 & Offline & Online & Online & Brain $+d+n-d$ & $\begin{array}{l}\text { Blend- } \\
\text { ed }\end{array}$ & Non-digital & Offline & Offline \\
\hline 969 & Offline & Online & Online & Brain $+d+n-d$ & $\begin{array}{l}\text { Blend- } \\
\text { ed }\end{array}$ & Blended & Online & Online \\
\hline 970 & Offline & Online & Online & Brain $+d+n-d$ & $\begin{array}{l}\text { Blend- } \\
\text { ed }\end{array}$ & Blended & Online & Offline \\
\hline 971 & Offline & Online & Online & Brain $+d+n-d$ & $\begin{array}{l}\text { Blend- } \\
\text { ed }\end{array}$ & Blended & Offline & Online \\
\hline 972 & Offline & Online & Online & Brain $+d+n-d$ & $\begin{array}{l}\text { Blend- } \\
\text { ed }\end{array}$ & Blended & Offline & Offline \\
\hline 973 & Offline & Online & Online & Brain + n-d & Digital & Digital & Online & Online \\
\hline 974 & Offline & Online & Online & Brain + n-d & Digital & Digital & Online & Offline \\
\hline 975 & Offline & Online & Online & Brain + n-d & Digital & Digital & Offline & Online \\
\hline 976 & Offline & Online & Online & Brain + n-d & Digital & Digital & Offline & Offline \\
\hline 977 & Offline & Online & Online & Brain + n-d & Digital & Non-digital & Online & Online \\
\hline 978 & Offline & Online & Online & Brain + n-d & Digital & Non-digital & Online & Offline \\
\hline 979 & Offline & Online & Online & Brain + n-d & Digital & Non-digital & Offline & Online \\
\hline 980 & Offline & Online & Online & Brain + n-d & Digital & Non-digital & Offline & Offline \\
\hline 981 & Offline & Online & Online & Brain + n-d & Digital & Blended & Online & Online \\
\hline 982 & Offline & Online & Online & Brain + n-d & Digital & Blended & Online & Offline \\
\hline 983 & Offline & Online & Online & Brain + n-d & Digital & Blended & Offline & Online \\
\hline 984 & Offline & Online & Online & Brain + n-d & Digital & Blended & Offline & Offline \\
\hline 985 & Offline & Online & Online & Brain + n-d & $\begin{array}{l}\text { Non- } \\
\text { digital }\end{array}$ & Digital & Online & Online \\
\hline 986 & Offline & Online & Online & Brain + n-d & $\begin{array}{l}\text { Non- } \\
\text { digital }\end{array}$ & Digital & Online & Offline \\
\hline 987 & Offline & Online & Online & Brain + n-d & $\begin{array}{l}\text { Non- } \\
\text { digital }\end{array}$ & Digital & Offline & Online \\
\hline 988 & Offline & Online & Online & Brain + n-d & $\begin{array}{l}\text { Non- } \\
\text { digital }\end{array}$ & Digital & Offline & Offline \\
\hline 989 & Offline & Online & Online & Brain + n-d & $\begin{array}{l}\text { Non- } \\
\text { digital }\end{array}$ & Non-digital & Online & Online \\
\hline 990 & Offline & Online & Online & Brain + n-d & $\begin{array}{l}\text { Non- } \\
\text { digital }\end{array}$ & Non-digital & Online & Offline \\
\hline 991 & Offline & Online & Online & Brain + n-d & $\begin{array}{l}\text { Non- } \\
\text { digital }\end{array}$ & Non-digital & Offline & Online \\
\hline 992 & Offline & Online & Online & Brain + n-d & $\begin{array}{l}\text { Non- } \\
\text { digital }\end{array}$ & Non-digital & Offline & Offline \\
\hline 993 & Offline & Online & Online & Brain + n-d & $\begin{array}{l}\text { Non- } \\
\text { digital }\end{array}$ & Blended & Online & Online \\
\hline 994 & Offline & Online & Online & Brain + n-d & $\begin{array}{l}\text { Non- } \\
\text { digital }\end{array}$ & Blended & Online & Offline \\
\hline 995 & Offline & Online & Online & Brain + n-d & $\begin{array}{l}\text { Non- } \\
\text { digital }\end{array}$ & Blended & Offline & Online \\
\hline 996 & Offline & Online & Online & Brain + n-d & $\begin{array}{l}\text { Non- } \\
\text { digital }\end{array}$ & Blended & Offline & Offline \\
\hline 997 & Offline & Online & Online & Brain + n-d & $\begin{array}{l}\text { Blend- } \\
\text { ed }\end{array}$ & Digital & Online & Online \\
\hline 998 & Offline & Online & Online & Brain + n-d & $\begin{array}{l}\text { Blend- } \\
\text { ed }\end{array}$ & Digital & Online & Offline \\
\hline 999 & Offline & Online & Online & Brain + n-d & $\begin{array}{l}\text { Blend- } \\
\text { ed }\end{array}$ & Digital & Offline & Online \\
\hline 1000 & Offline & Online & Online & Brain + n-d & $\begin{array}{l}\text { Blend- } \\
\text { ed }\end{array}$ & Digital & Offline & Offline \\
\hline 1001 & Offline & Online & Online & Brain + n-d & $\begin{array}{l}\text { Blend- } \\
\text { ed }\end{array}$ & Non-digital & Online & Online \\
\hline 1002 & Offline & Online & Online & Brain + n-d & $\begin{array}{l}\text { Blend- } \\
\text { ed }\end{array}$ & Non-digital & Online & Offline \\
\hline
\end{tabular}




\begin{tabular}{|c|c|c|c|c|c|c|c|c|}
\hline 1003 & Offline & Online & Online & Brain + n-d & $\begin{array}{l}\text { Blend- } \\
\text { ed }\end{array}$ & Non-digital & Offline & Online \\
\hline 1004 & Offline & Online & Online & Brain $+n-d$ & $\begin{array}{l}\text { Blend- } \\
\text { ed }\end{array}$ & Non-digital & Offline & Offline \\
\hline 1005 & Offline & Online & Online & Brain $+n-d$ & $\begin{array}{l}\text { Blend- } \\
\text { ed }\end{array}$ & Blended & Online & Online \\
\hline 1006 & Offline & Online & Online & Brain + n-d & $\begin{array}{l}\text { Blend- } \\
\text { ed }\end{array}$ & Blended & Online & Offline \\
\hline 1007 & Offline & Online & Online & Brain $+n-d$ & $\begin{array}{l}\text { Blend- } \\
\text { ed }\end{array}$ & Blended & Offline & Online \\
\hline 1008 & Offline & Online & Online & Brain + n-d & $\begin{array}{l}\text { Blend- } \\
\text { ed }\end{array}$ & Blended & Offline & Offline \\
\hline 1009 & Offline & Online & Offline & Brain + digital & Digital & Digital & Online & Online \\
\hline 1010 & Offline & Online & Offline & Brain + digital & Digital & Digital & Online & Offline \\
\hline 1011 & Offline & Online & Offline & Brain + digital & Digital & Digital & Offline & Online \\
\hline 1012 & Offline & Online & Offline & Brain + digital & Digital & Digital & Offline & Offline \\
\hline 1013 & Offline & Online & Offline & Brain + digital & Digital & Non-digital & Online & Online \\
\hline 1014 & Offline & Online & Offline & Brain + digital & Digital & Non-digital & Online & Offline \\
\hline 1015 & Offline & Online & Offline & Brain + digital & Digital & Non-digital & Offline & Online \\
\hline 1016 & Offline & Online & Offline & Brain + digital & Digital & Non-digital & Offline & Offline \\
\hline 1017 & Offline & Online & Offline & Brain + digital & Digital & Blended & Online & Online \\
\hline 1018 & Offline & Online & Offline & Brain + digital & Digital & Blended & Online & Offline \\
\hline 1019 & Offline & Online & Offline & Brain + digital & Digital & Blended & Offline & Online \\
\hline 1020 & Offline & Online & Offline & Brain + digital & Digital & Blended & Offline & Offline \\
\hline 1021 & Offline & Online & Offline & Brain + digital & $\begin{array}{l}\text { Non- } \\
\text { digital }\end{array}$ & Digital & Online & Online \\
\hline 1022 & Offline & Online & Offline & Brain + digital & $\begin{array}{l}\text { Non- } \\
\text { digital }\end{array}$ & Digital & Online & Offline \\
\hline 1023 & Offline & Online & Offline & Brain + digital & $\begin{array}{l}\text { Non- } \\
\text { digital }\end{array}$ & Digital & Offline & Online \\
\hline 1024 & Offline & Online & Offline & Brain + digital & $\begin{array}{l}\text { Non- } \\
\text { digital }\end{array}$ & Digital & Offline & Offline \\
\hline 1025 & Offline & Online & Offline & Brain + digital & $\begin{array}{l}\text { Non- } \\
\text { digital }\end{array}$ & Non-digital & Online & Online \\
\hline 1026 & Offline & Online & Offline & Brain + digital & $\begin{array}{l}\text { Non- } \\
\text { digital }\end{array}$ & Non-digital & Online & Offline \\
\hline 1027 & Offline & Online & Offline & Brain + digital & $\begin{array}{l}\text { Non- } \\
\text { digital }\end{array}$ & Non-digital & Offline & Online \\
\hline 1028 & Offline & Online & Offline & Brain + digital & $\begin{array}{l}\text { Non- } \\
\text { digital }\end{array}$ & Non-digital & Offline & Offline \\
\hline 1029 & Offline & Online & Offline & Brain + digital & $\begin{array}{l}\text { Non- } \\
\text { digital }\end{array}$ & Blended & Online & Online \\
\hline 1030 & Offline & Online & Offline & Brain + digital & $\begin{array}{l}\text { Non- } \\
\text { digital }\end{array}$ & Blended & Online & Offline \\
\hline 1031 & Offline & Online & Offline & Brain + digital & $\begin{array}{l}\text { Non- } \\
\text { digital }\end{array}$ & Blended & Offline & Online \\
\hline 1032 & Offline & Online & Offline & Brain + digital & $\begin{array}{l}\text { Non- } \\
\text { digital }\end{array}$ & Blended & Offline & Offline \\
\hline 1033 & Offline & Online & Offline & Brain + digital & $\begin{array}{l}\text { Blend- } \\
\text { ed }\end{array}$ & Digital & Online & Online \\
\hline 1034 & Offline & Online & Offline & Brain + digital & $\begin{array}{l}\text { Blend- } \\
\text { ed }\end{array}$ & Digital & Online & Offline \\
\hline 1035 & Offline & Online & Offline & Brain + digital & $\begin{array}{l}\text { Blend- } \\
\text { ed }\end{array}$ & Digital & Offline & Online \\
\hline 1036 & Offline & Online & Offline & Brain + digital & $\begin{array}{l}\text { Blend- } \\
\text { ed }\end{array}$ & Digital & Offline & Offline \\
\hline 1037 & Offline & Online & Offline & Brain + digital & $\begin{array}{l}\text { Blend- } \\
\text { ed }\end{array}$ & Non-digital & Online & Online \\
\hline 1038 & Offline & Online & Offline & Brain + digital & $\begin{array}{l}\text { Blend- } \\
\text { ed }\end{array}$ & Non-digital & Online & Offline \\
\hline 1039 & Offline & Online & Offline & Brain + digital & $\begin{array}{l}\text { Blend- } \\
\text { ed }\end{array}$ & Non-digital & Offline & Online \\
\hline 1040 & Offline & Online & Offline & Brain + digital & $\begin{array}{l}\text { Blend- } \\
\text { ed }\end{array}$ & Non-digital & Offline & Offline \\
\hline
\end{tabular}




\begin{tabular}{|c|c|c|c|c|c|c|c|c|}
\hline 1041 & Offline & Online & Offline & Brain + digital & $\begin{array}{l}\text { Blend- } \\
\text { ed }\end{array}$ & Blended & Online & Online \\
\hline 1042 & Offline & Online & Offline & Brain + digital & $\begin{array}{l}\text { Blend- } \\
\text { ed }\end{array}$ & Blended & Online & Offline \\
\hline 1043 & Offline & Online & Offline & Brain + digital & $\begin{array}{l}\text { Blend- } \\
\text { ed }\end{array}$ & Blended & Offline & Online \\
\hline 1044 & Offline & Online & Offline & Brain + digital & $\begin{array}{l}\text { Blend- } \\
\text { ed }\end{array}$ & Blended & Offline & Offline \\
\hline 1045 & Offline & Online & Offline & Brain & Digital & Digital & Online & Online \\
\hline 1046 & Offline & Online & Offline & Brain & Digital & Digital & Online & Offline \\
\hline 1047 & Offline & Online & Offline & Brain & Digital & Digital & Offline & Online \\
\hline 1048 & Offline & Online & Offline & Brain & Digital & Digital & Offline & Offline \\
\hline 1049 & Offline & Online & Offline & Brain & Digital & Non-digital & Online & Online \\
\hline 1050 & Offline & Online & Offline & Brain & Digital & Non-digital & Online & Offline \\
\hline 1051 & Offline & Online & Offline & Brain & Digital & Non-digital & Offline & Online \\
\hline 1052 & Offline & Online & Offline & Brain & Digital & Non-digital & Offline & Offline \\
\hline 1053 & Offline & Online & Offline & Brain & Digital & Blended & Online & Online \\
\hline 1054 & Offline & Online & Offline & Brain & Digital & Blended & Online & Offline \\
\hline 1055 & Offline & Online & Offline & Brain & Digital & Blended & Offline & Online \\
\hline 1056 & Offline & Online & Offline & Brain & Digital & Blended & Offline & Offline \\
\hline 1057 & Offline & Online & Offline & Brain & $\begin{array}{l}\text { Non- } \\
\text { digital }\end{array}$ & Digital & Online & Online \\
\hline 1058 & Offline & Online & Offline & Brain & $\begin{array}{l}\text { Non- } \\
\text { digital }\end{array}$ & Digital & Online & Offline \\
\hline 1059 & Offline & Online & Offline & Brain & $\begin{array}{l}\text { Non- } \\
\text { digital }\end{array}$ & Digital & Offline & Online \\
\hline 1060 & Offline & Online & Offline & Brain & $\begin{array}{l}\text { Non- } \\
\text { digital }\end{array}$ & Digital & Offline & Offline \\
\hline 1061 & Offline & Online & Offline & Brain & $\begin{array}{l}\text { Non- } \\
\text { digital }\end{array}$ & Non-digital & Online & Online \\
\hline 1062 & Offline & Online & Offline & Brain & $\begin{array}{l}\text { Non- } \\
\text { digital }\end{array}$ & Non-digital & Online & Offline \\
\hline 1063 & Offline & Online & Offline & Brain & $\begin{array}{l}\text { Non- } \\
\text { digital }\end{array}$ & Non-digital & Offline & Online \\
\hline 1064 & Offline & Online & Offline & Brain & $\begin{array}{l}\text { Non- } \\
\text { digital }\end{array}$ & Non-digital & Offline & Offline \\
\hline 1065 & Offline & Online & Offline & Brain & $\begin{array}{l}\text { Non- } \\
\text { digital }\end{array}$ & Blended & Online & Online \\
\hline 1066 & Offline & Online & Offline & Brain & $\begin{array}{l}\text { Non- } \\
\text { digital }\end{array}$ & Blended & Online & Offline \\
\hline 1067 & Offline & Online & Offline & Brain & $\begin{array}{l}\text { Non- } \\
\text { digital }\end{array}$ & Blended & Offline & Online \\
\hline 1068 & Offline & Online & Offline & Brain & $\begin{array}{l}\text { Non- } \\
\text { digital }\end{array}$ & Blended & Offline & Offline \\
\hline 1069 & Offline & Online & Offline & Brain & $\begin{array}{l}\text { Blend- } \\
\text { ed }\end{array}$ & Digital & Online & Online \\
\hline 1070 & Offline & Online & Offline & Brain & $\begin{array}{l}\text { Blend- } \\
\text { ed }\end{array}$ & Digital & Online & Offline \\
\hline 1071 & Offline & Online & Offline & Brain & $\begin{array}{l}\text { Blend- } \\
\text { ed }\end{array}$ & Digital & Offline & Online \\
\hline 1072 & Offline & Online & Offline & Brain & $\begin{array}{l}\text { Blend- } \\
\text { ed }\end{array}$ & Digital & Offline & Offline \\
\hline 1073 & Offline & Online & Offline & Brain & $\begin{array}{l}\text { Blend- } \\
\text { ed }\end{array}$ & Non-digital & Online & Online \\
\hline 1074 & Offline & Online & Offline & Brain & $\begin{array}{l}\text { Blend- } \\
\text { ed }\end{array}$ & Non-digital & Online & Offline \\
\hline 1075 & Offline & Online & Offline & Brain & $\begin{array}{l}\text { Blend- } \\
\text { ed }\end{array}$ & Non-digital & Offline & Online \\
\hline 1076 & Offline & Online & Offline & Brain & $\begin{array}{l}\text { Blend- } \\
\text { ed }\end{array}$ & Non-digital & Offline & Offline \\
\hline 1077 & Offline & Online & Offline & Brain & $\begin{array}{l}\text { Blend- } \\
\text { ed }\end{array}$ & Blended & Online & Online \\
\hline 1078 & Offline & Online & Offline & Brain & $\begin{array}{l}\text { Blend- } \\
\text { ed }\end{array}$ & Blended & Online & Offline \\
\hline
\end{tabular}




\begin{tabular}{|c|c|c|c|c|c|c|c|c|}
\hline 1079 & Offline & Online & Offline & Brain & $\begin{array}{l}\text { Blend- } \\
\text { ed }\end{array}$ & Blended & Offline & Online \\
\hline 1080 & Offline & Online & Offline & Brain & $\begin{array}{l}\text { Blend- } \\
\text { ed }\end{array}$ & Blended & Offline & Offline \\
\hline 1081 & Offline & Online & Offline & Brain $+d+n-d$ & Digital & Digital & Online & Online \\
\hline 1082 & Offline & Online & Offline & Brain $+d+n-d$ & Digital & Digital & Online & Offline \\
\hline 1083 & Offline & Online & Offline & Brain $+d+n-d$ & Digital & Digital & Offline & Online \\
\hline 1084 & Offline & Online & Offline & Brain $+d+n-d$ & Digital & Digital & Offline & Offline \\
\hline 1085 & Offline & Online & Offline & Brain $+d+n-d$ & Digital & Non-digital & Online & Online \\
\hline 1086 & Offline & Online & Offline & Brain $+d+n-d$ & Digital & Non-digital & Online & Offline \\
\hline 1087 & Offline & Online & Offline & Brain $+d+n-d$ & Digital & Non-digital & Offline & Online \\
\hline 1088 & Offline & Online & Offline & Brain $+d+n-d$ & Digital & Non-digital & Offline & Offline \\
\hline 1089 & Offline & Online & Offline & Brain $+d+n-d$ & Digital & Blended & Online & Online \\
\hline 1090 & Offline & Online & Offline & Brain $+d+n-d$ & Digital & Blended & Online & Offline \\
\hline 1091 & Offline & Online & Offline & Brain $+d+n-d$ & Digital & Blended & Offline & Online \\
\hline 1092 & Offline & Online & Offline & Brain $+d+n-d$ & Digital & Blended & Offline & Offline \\
\hline 1093 & Offline & Online & Offline & Brain $+d+n-d$ & $\begin{array}{l}\text { Non- } \\
\text { digital }\end{array}$ & Digital & Online & Online \\
\hline 1094 & Offline & Online & Offline & Brain + d + n-d & $\begin{array}{l}\text { Non- } \\
\text { digital }\end{array}$ & Digital & Online & Offline \\
\hline 1095 & Offline & Online & Offline & Brain $+d+n-d$ & $\begin{array}{l}\text { Non- } \\
\text { digital }\end{array}$ & Digital & Offline & Online \\
\hline 1096 & Offline & Online & Offline & Brain $+d+n-d$ & $\begin{array}{l}\text { Non- } \\
\text { digital }\end{array}$ & Digital & Offline & Offline \\
\hline 1097 & Offline & Online & Offline & Brain + d + n-d & $\begin{array}{l}\text { Non- } \\
\text { digital }\end{array}$ & Non-digital & Online & Online \\
\hline 1098 & Offline & Online & Offline & Brain + d + n-d & $\begin{array}{l}\text { Non- } \\
\text { digital }\end{array}$ & Non-digital & Online & Offline \\
\hline 1099 & Offline & Online & Offline & Brain $+d+n-d$ & $\begin{array}{l}\text { Non- } \\
\text { digital }\end{array}$ & Non-digital & Offline & Online \\
\hline 1100 & Offline & Online & Offline & Brain $+d+n-d$ & $\begin{array}{l}\text { Non- } \\
\text { digital }\end{array}$ & Non-digital & Offline & Offline \\
\hline 1101 & Offline & Online & Offline & Brain + d + n-d & $\begin{array}{l}\text { Non- } \\
\text { digital }\end{array}$ & Blended & Online & Online \\
\hline 1102 & Offline & Online & Offline & Brain $+d+n-d$ & $\begin{array}{l}\text { Non- } \\
\text { digital }\end{array}$ & Blended & Online & Offline \\
\hline 1103 & Offline & Online & Offline & Brain $+d+n-d$ & $\begin{array}{l}\text { Non- } \\
\text { digital }\end{array}$ & Blended & Offline & Online \\
\hline 1104 & Offline & Online & Offline & Brain + d + n-d & $\begin{array}{l}\text { Non- } \\
\text { digital }\end{array}$ & Blended & Offline & Offline \\
\hline 1105 & Offline & Online & Offline & Brain $+d+n-d$ & $\begin{array}{l}\text { Blend- } \\
\text { ed }\end{array}$ & Digital & Online & Online \\
\hline 1106 & Offline & Online & Offline & Brain $+d+n-d$ & $\begin{array}{l}\text { Blend- } \\
\text { ed }\end{array}$ & Digital & Online & Offline \\
\hline 1107 & Offline & Online & Offline & Brain + d + n-d & $\begin{array}{l}\text { Blend- } \\
\text { ed }\end{array}$ & Digital & Offline & Online \\
\hline 1108 & Offline & Online & Offline & Brain + d + n-d & $\begin{array}{l}\text { Blend- } \\
\text { ed }\end{array}$ & Digital & Offline & Offline \\
\hline 1109 & Offline & Online & Offline & Brain + d + n-d & $\begin{array}{l}\text { Blend- } \\
\text { ed }\end{array}$ & Non-digital & Online & Online \\
\hline 1110 & Offline & Online & Offline & Brain $+d+n-d$ & $\begin{array}{l}\text { Blend- } \\
\text { ed }\end{array}$ & Non-digital & Online & Offline \\
\hline 1111 & Offline & Online & Offline & Brain + d + n-d & $\begin{array}{l}\text { Blend- } \\
\text { ed }\end{array}$ & Non-digital & Offline & Online \\
\hline 1112 & Offline & Online & Offline & Brain + d + n-d & $\begin{array}{l}\text { Blend- } \\
\text { ed }\end{array}$ & Non-digital & Offline & Offline \\
\hline 1113 & Offline & Online & Offline & Brain $+d+n-d$ & $\begin{array}{l}\text { Blend- } \\
\text { ed }\end{array}$ & Blended & Online & Online \\
\hline 1114 & Offline & Online & Offline & Brain + d + n-d & $\begin{array}{l}\text { Blend- } \\
\text { ed }\end{array}$ & Blended & Online & Offline \\
\hline 1115 & Offline & Online & Offline & Brain $+d+n-d$ & Blend- & Blended & Offline & Online \\
\hline 1116 & Offline & Online & Offline & Brain $+d+n-d$ & $\begin{array}{l}\text { Blend- } \\
\text { ed }\end{array}$ & Blended & Offline & Offline \\
\hline 1117 & Offline & Online & Offline & Brain $+n-d$ & Digital & Digital & Online & Online \\
\hline
\end{tabular}




\begin{tabular}{|c|c|c|c|c|c|c|c|c|}
\hline 1118 & Offline & Online & Offline & Brain + n-d & Digital & Digital & Online & Offline \\
\hline 1119 & Offline & Online & Offline & Brain + n-d & Digital & Digital & Offline & Online \\
\hline 1120 & Offline & Online & Offline & Brain + n-d & Digital & Digital & Offline & Offline \\
\hline 1121 & Offline & Online & Offline & Brain + n-d & Digital & Non-digital & Online & Online \\
\hline 1122 & Offline & Online & Offline & Brain + n-d & Digital & Non-digital & Online & Offline \\
\hline 1123 & Offline & Online & Offline & Brain + n-d & Digital & Non-digital & Offline & Online \\
\hline 1124 & Offline & Online & Offline & Brain + n-d & Digital & Non-digital & Offline & Offline \\
\hline 1125 & Offline & Online & Offline & Brain + n-d & Digital & Blended & Online & Online \\
\hline 1126 & Offline & Online & Offline & Brain + n-d & Digital & Blended & Online & Offline \\
\hline 1127 & Offline & Online & Offline & Brain + n-d & Digital & Blended & Offline & Online \\
\hline 1128 & Offline & Online & Offline & Brain + n-d & Digital & Blended & Offline & Offline \\
\hline 1129 & Offline & Online & Offline & Brain + n-d & $\begin{array}{l}\text { Non- } \\
\text { digital }\end{array}$ & Digital & Online & Online \\
\hline 1130 & Offline & Online & Offline & Brain + n-d & $\begin{array}{l}\text { Non- } \\
\text { digital }\end{array}$ & Digital & Online & Offline \\
\hline 1131 & Offline & Online & Offline & Brain $+n-d$ & $\begin{array}{l}\text { Non- } \\
\text { digital }\end{array}$ & Digital & Offline & Online \\
\hline 1132 & Offline & Online & Offline & Brain $+n-d$ & $\begin{array}{l}\text { Non- } \\
\text { digital }\end{array}$ & Digital & Offline & Offline \\
\hline 1133 & Offline & Online & Offline & Brain + n-d & $\begin{array}{l}\text { Non- } \\
\text { digital }\end{array}$ & Non-digital & Online & Online \\
\hline 1134 & Offline & Online & Offline & Brain + n-d & $\begin{array}{l}\text { Non- } \\
\text { digital }\end{array}$ & Non-digital & Online & Offline \\
\hline 1135 & Offline & Online & Offline & Brain + n-d & $\begin{array}{l}\text { Non- } \\
\text { digital }\end{array}$ & Non-digital & Offline & Online \\
\hline 1136 & Offline & Online & Offline & Brain + n-d & $\begin{array}{l}\text { Non- } \\
\text { digital }\end{array}$ & Non-digital & Offline & Offline \\
\hline 1137 & Offline & Online & Offline & Brain + n-d & $\begin{array}{l}\text { Non- } \\
\text { digital }\end{array}$ & Blended & Online & Online \\
\hline 1138 & Offline & Online & Offline & Brain + n-d & $\begin{array}{l}\text { Non- } \\
\text { digital }\end{array}$ & Blended & Online & Offline \\
\hline 1139 & Offline & Online & Offline & Brain + n-d & $\begin{array}{l}\text { Non- } \\
\text { digital }\end{array}$ & Blended & Offline & Online \\
\hline 1140 & Offline & Online & Offline & Brain + n-d & $\begin{array}{l}\text { Non- } \\
\text { digital }\end{array}$ & Blended & Offline & Offline \\
\hline 1141 & Offline & Online & Offline & Brain + n-d & $\begin{array}{l}\text { Blend- } \\
\text { ed }\end{array}$ & Digital & Online & Online \\
\hline 1142 & Offline & Online & Offline & Brain + n-d & $\begin{array}{l}\text { Blend- } \\
\text { ed }\end{array}$ & Digital & Online & Offline \\
\hline 1143 & Offline & Online & Offline & Brain + n-d & $\begin{array}{l}\text { Blend- } \\
\text { ed }\end{array}$ & Digital & Offline & Online \\
\hline 1144 & Offline & Online & Offline & Brain + n-d & $\begin{array}{l}\text { Blend- } \\
\text { ed }\end{array}$ & Digital & Offline & Offline \\
\hline 1145 & Offline & Online & Offline & Brain + n-d & $\begin{array}{l}\text { Blend- } \\
\text { ed }\end{array}$ & Non-digital & Online & Online \\
\hline 1146 & Offline & Online & Offline & Brain + n-d & $\begin{array}{l}\text { Blend- } \\
\text { ed }\end{array}$ & Non-digital & Online & Offline \\
\hline 1147 & Offline & Online & Offline & Brain + n-d & $\begin{array}{l}\text { Blend- } \\
\text { ed }\end{array}$ & Non-digital & Offline & Online \\
\hline 1148 & Offline & Online & Offline & Brain + n-d & $\begin{array}{l}\text { Blend- } \\
\text { ed }\end{array}$ & Non-digital & Offline & Offline \\
\hline 1149 & Offline & Online & Offline & Brain + n-d & $\begin{array}{l}\text { Blend- } \\
\text { ed }\end{array}$ & Blended & Online & Online \\
\hline 1150 & Offline & Online & Offline & Brain + n-d & $\begin{array}{l}\text { Blend- } \\
\text { ed }\end{array}$ & Blended & Online & Offline \\
\hline 1151 & Offline & Online & Offline & Brain + n-d & $\begin{array}{l}\text { Blend- } \\
\text { ed }\end{array}$ & Blended & Offline & Online \\
\hline 1152 & Offline & Online & Offline & Brain + n-d & $\begin{array}{l}\text { Blend- } \\
\text { ed }\end{array}$ & Blended & Offline & Offline \\
\hline 1153 & Offline & Online & Blended & Brain + digital & Digital & Digital & Online & Online \\
\hline 1154 & Offline & Online & Blended & Brain + digital & Digital & Digital & Online & Offline \\
\hline 1155 & Offline & Online & Blended & Brain + digital & Digital & Digital & Offline & Online \\
\hline 1156 & Offline & Online & Blended & Brain + digital & Digital & Digital & Offline & Offline \\
\hline 1157 & Offline & Online & Blended & Brain + digital & Digital & Non-digital & Online & Online \\
\hline 1158 & Offline & Online & Blended & Brain + digital & Digital & Non-digital & Online & Offline \\
\hline
\end{tabular}




\begin{tabular}{|c|c|c|c|c|c|c|c|c|}
\hline 1159 & Offline & Online & Blended & Brain + digital & Digital & Non-digital & Offline & Online \\
\hline 1160 & Offline & Online & Blended & Brain + digital & Digital & Non-digital & Offline & Offline \\
\hline 1161 & Offline & Online & Blended & Brain + digital & Digital & Blended & Online & Online \\
\hline 1162 & Offline & Online & Blended & Brain + digital & Digital & Blended & Online & Offline \\
\hline 1163 & Offline & Online & Blended & Brain + digital & Digital & Blended & Offline & Online \\
\hline 1164 & Offline & Online & Blended & Brain + digital & Digital & Blended & Offline & Offline \\
\hline 1165 & Offline & Online & Blended & Brain + digital & $\begin{array}{l}\text { Non- } \\
\text { digital }\end{array}$ & Digital & Online & Online \\
\hline 1166 & Offline & Online & Blended & Brain + digital & $\begin{array}{l}\text { Non- } \\
\text { digital }\end{array}$ & Digital & Online & Offline \\
\hline 1167 & Offline & Online & Blended & Brain + digital & $\begin{array}{l}\text { Non- } \\
\text { digital }\end{array}$ & Digital & Offline & Online \\
\hline 1168 & Offline & Online & Blended & Brain + digital & $\begin{array}{l}\text { Non- } \\
\text { digital }\end{array}$ & Digital & Offline & Offline \\
\hline 1169 & Offline & Online & Blended & Brain + digital & $\begin{array}{l}\text { Non- } \\
\text { digital }\end{array}$ & Non-digital & Online & Online \\
\hline 1170 & Offline & Online & Blended & Brain + digital & $\begin{array}{l}\text { Non- } \\
\text { digital }\end{array}$ & Non-digital & Online & Offline \\
\hline 1171 & Offline & Online & Blended & Brain + digital & $\begin{array}{l}\text { Non- } \\
\text { digital }\end{array}$ & Non-digital & Offline & Online \\
\hline 1172 & Offline & Online & Blended & Brain + digital & $\begin{array}{l}\text { Non- } \\
\text { digital }\end{array}$ & Non-digital & Offline & Offline \\
\hline 1173 & Offline & Online & Blended & Brain + digital & $\begin{array}{l}\text { Non- } \\
\text { digital }\end{array}$ & Blended & Online & Online \\
\hline 1174 & Offline & Online & Blended & Brain + digital & $\begin{array}{l}\text { Non- } \\
\text { digital }\end{array}$ & Blended & Online & Offline \\
\hline 1175 & Offline & Online & Blended & Brain + digital & $\begin{array}{l}\text { Non- } \\
\text { digital }\end{array}$ & Blended & Offline & Online \\
\hline 1176 & Offline & Online & Blended & Brain + digital & $\begin{array}{l}\text { Non- } \\
\text { digital }\end{array}$ & Blended & Offline & Offline \\
\hline 1177 & Offline & Online & Blended & Brain + digital & $\begin{array}{l}\text { Blend- } \\
\text { ed }\end{array}$ & Digital & Online & Online \\
\hline 1178 & Offline & Online & Blended & Brain + digital & $\begin{array}{l}\text { Blend- } \\
\text { ed }\end{array}$ & Digital & Online & Offline \\
\hline 1179 & Offline & Online & Blended & Brain + digital & $\begin{array}{l}\text { Blend- } \\
\text { ed }\end{array}$ & Digital & Offline & Online \\
\hline 1180 & Offline & Online & Blended & Brain + digital & $\begin{array}{l}\text { Blend- } \\
\text { ed }\end{array}$ & Digital & Offline & Offline \\
\hline 1181 & Offline & Online & Blended & Brain + digital & $\begin{array}{l}\text { Blend- } \\
\text { ed }\end{array}$ & Non-digital & Online & Online \\
\hline 1182 & Offline & Online & Blended & Brain + digital & $\begin{array}{l}\text { Blend- } \\
\text { ed }\end{array}$ & Non-digital & Online & Offline \\
\hline 1183 & Offline & Online & Blended & Brain + digital & $\begin{array}{l}\text { Blend- } \\
\text { ed }\end{array}$ & Non-digital & Offline & Online \\
\hline 1184 & Offline & Online & Blended & Brain + digital & $\begin{array}{l}\text { Blend- } \\
\text { ed }\end{array}$ & Non-digital & Offline & Offline \\
\hline 1185 & Offline & Online & Blended & Brain + digital & $\begin{array}{l}\text { Blend- } \\
\text { ed }\end{array}$ & Blended & Online & Online \\
\hline 1186 & Offline & Online & Blended & Brain + digital & $\begin{array}{l}\text { Blend- } \\
\text { ed }\end{array}$ & Blended & Online & Offline \\
\hline 1187 & Offline & Online & Blended & Brain + digital & $\begin{array}{l}\text { Blend- } \\
\text { ed }\end{array}$ & Blended & Offline & Online \\
\hline 1188 & Offline & Online & Blended & Brain + digital & $\begin{array}{l}\text { Blend- } \\
\text { ed }\end{array}$ & Blended & Offline & Offline \\
\hline 1189 & Offline & Online & Blended & Brain & Digital & Digital & Online & Online \\
\hline 1190 & Offline & Online & Blended & Brain & Digital & Digital & Online & Offline \\
\hline 1191 & Offline & Online & Blended & Brain & Digital & Digital & Offline & Online \\
\hline 1192 & Offline & Online & Blended & Brain & Digital & Digital & Offline & Offline \\
\hline 1193 & Offline & Online & Blended & Brain & Digital & Non-digital & Online & Online \\
\hline 1194 & Offline & Online & Blended & Brain & Digital & Non-digital & Online & Offline \\
\hline 1195 & Offline & Online & Blended & Brain & Digital & Non-digital & Offline & Online \\
\hline 1196 & Offline & Online & Blended & Brain & Digital & Non-digital & Offline & Offline \\
\hline 1197 & Offline & Online & Blended & Brain & Digital & Blended & Online & Online \\
\hline 1198 & Offline & Online & Blended & Brain & Digital & Blended & Online & Offline \\
\hline 1199 & Offline & Online & Blended & Brain & Digital & Blended & Offline & Online \\
\hline
\end{tabular}




\begin{tabular}{|c|c|c|c|c|c|c|c|c|}
\hline 1200 & Offline & Online & Blended & Brain & Digital & Blended & Offline & Offline \\
\hline 1201 & Offline & Online & Blended & Brain & $\begin{array}{l}\text { Non- } \\
\text { digital }\end{array}$ & Digital & Online & Online \\
\hline 1202 & Offline & Online & Blended & Brain & $\begin{array}{l}\text { Non- } \\
\text { digital }\end{array}$ & Digital & Online & Offline \\
\hline 1203 & Offline & Online & Blended & Brain & $\begin{array}{l}\text { Non- } \\
\text { digital }\end{array}$ & Digital & Offline & Online \\
\hline 1204 & Offline & Online & Blended & Brain & $\begin{array}{l}\text { Non- } \\
\text { digital }\end{array}$ & Digital & Offline & Offline \\
\hline 1205 & Offline & Online & Blended & Brain & $\begin{array}{l}\text { Non- } \\
\text { digital }\end{array}$ & Non-digital & Online & Online \\
\hline 1206 & Offline & Online & Blended & Brain & $\begin{array}{l}\text { Non- } \\
\text { digital }\end{array}$ & Non-digital & Online & Offline \\
\hline 1207 & Offline & Online & Blended & Brain & $\begin{array}{l}\text { Non- } \\
\text { digital }\end{array}$ & Non-digital & Offline & Online \\
\hline 1208 & Offline & Online & Blended & Brain & $\begin{array}{l}\text { Non- } \\
\text { digital }\end{array}$ & Non-digital & Offline & Offline \\
\hline 1209 & Offline & Online & Blended & Brain & $\begin{array}{l}\text { Non- } \\
\text { digital }\end{array}$ & Blended & Online & Online \\
\hline 1210 & Offline & Online & Blended & Brain & $\begin{array}{l}\text { Non- } \\
\text { digital }\end{array}$ & Blended & Online & Offline \\
\hline 1211 & Offline & Online & Blended & Brain & $\begin{array}{l}\text { Non- } \\
\text { digital }\end{array}$ & Blended & Offline & Online \\
\hline 1212 & Offline & Online & Blended & Brain & $\begin{array}{l}\text { Non- } \\
\text { digital }\end{array}$ & Blended & Offline & Offline \\
\hline 1213 & Offline & Online & Blended & Brain & $\begin{array}{l}\text { Blend- } \\
\text { ed }\end{array}$ & Digital & Online & Online \\
\hline 1214 & Offline & Online & Blended & Brain & $\begin{array}{l}\text { Blend- } \\
\text { ed }\end{array}$ & Digital & Online & Offline \\
\hline 1215 & Offline & Online & Blended & Brain & $\begin{array}{l}\text { Blend- } \\
\text { ed }\end{array}$ & Digital & Offline & Online \\
\hline 1216 & Offline & Online & Blended & Brain & $\begin{array}{l}\text { Blend- } \\
\text { ed }\end{array}$ & Digital & Offline & Offline \\
\hline 1217 & Offline & Online & Blended & Brain & $\begin{array}{l}\text { Blend- } \\
\text { ed }\end{array}$ & Non-digital & Online & Online \\
\hline 1218 & Offline & Online & Blended & Brain & $\begin{array}{l}\text { Blend- } \\
\text { ed }\end{array}$ & Non-digital & Online & Offline \\
\hline 1219 & Offline & Online & Blended & Brain & $\begin{array}{l}\text { Blend- } \\
\text { ed }\end{array}$ & Non-digital & Offline & Online \\
\hline 1220 & Offline & Online & Blended & Brain & $\begin{array}{l}\text { Blend- } \\
\text { ed }\end{array}$ & Non-digital & Offline & Offline \\
\hline 1221 & Offline & Online & Blended & Brain & $\begin{array}{l}\text { Blend- } \\
\text { ed }\end{array}$ & Blended & Online & Online \\
\hline 1222 & Offline & Online & Blended & Brain & $\begin{array}{l}\text { Blend- } \\
\text { ed }\end{array}$ & Blended & Online & Offline \\
\hline 1223 & Offline & Online & Blended & Brain & $\begin{array}{l}\text { Blend- } \\
\text { ed }\end{array}$ & Blended & Offline & Online \\
\hline 1224 & Offline & Online & Blended & Brain & $\begin{array}{l}\text { Blend- } \\
\text { ed }\end{array}$ & Blended & Offline & Offline \\
\hline 1225 & Offline & Online & Blended & Brain $+d+n-d$ & Digital & Digital & Online & Online \\
\hline 1226 & Offline & Online & Blended & Brain $+d+n-d$ & Digital & Digital & Online & Offline \\
\hline 1227 & Offline & Online & Blended & Brain $+d+n-d$ & Digital & Digital & Offline & Online \\
\hline 1228 & Offline & Online & Blended & Brain $+d+n-d$ & Digital & Digital & Offline & Offline \\
\hline 1229 & Offline & Online & Blended & Brain $+d+n-d$ & Digital & Non-digital & Online & Online \\
\hline 1230 & Offline & Online & Blended & Brain $+d+n-d$ & Digital & Non-digital & Online & Offline \\
\hline 1231 & Offline & Online & Blended & Brain $+d+n-d$ & Digital & Non-digital & Offline & Online \\
\hline 1232 & Offline & Online & Blended & Brain $+d+n-d$ & Digital & Non-digital & Offline & Offline \\
\hline 1233 & Offline & Online & Blended & Brain $+d+n-d$ & Digital & Blended & Online & Online \\
\hline 1234 & Offline & Online & Blended & Brain $+d+n-d$ & Digital & Blended & Online & Offline \\
\hline 1235 & Offline & Online & Blended & Brain $+d+n-d$ & Digital & Blended & Offline & Online \\
\hline 1236 & Offline & Online & Blended & Brain + d + n-d & Digital & Blended & Offline & Offline \\
\hline 1237 & Offline & Online & Blended & Brain $+d+n-d$ & $\begin{array}{l}\text { Non- } \\
\text { digital }\end{array}$ & Digital & Online & Online \\
\hline 1238 & Offline & Online & Blended & Brain $+d+n-d$ & $\begin{array}{l}\text { Non- } \\
\text { digital }\end{array}$ & Digital & Online & Offline \\
\hline
\end{tabular}




\begin{tabular}{|c|c|c|c|c|c|c|c|c|}
\hline 1239 & Offline & Online & Blended & Brain $+d+n-d$ & $\begin{array}{l}\text { Non- } \\
\text { digital }\end{array}$ & Digital & Offline & Online \\
\hline 1240 & Offline & Online & Blended & Brain $+d+n-d$ & $\begin{array}{l}\text { Non- } \\
\text { digital }\end{array}$ & Digital & Offline & Offline \\
\hline 1241 & Offline & Online & Blended & Brain $+d+n-d$ & $\begin{array}{l}\text { Non- } \\
\text { digital }\end{array}$ & Non-digital & Online & Online \\
\hline 1242 & Offline & Online & Blended & Brain $+d+n-d$ & $\begin{array}{l}\text { Non- } \\
\text { digital }\end{array}$ & Non-digital & Online & Offline \\
\hline 1243 & Offline & Online & Blended & Brain $+d+n-d$ & $\begin{array}{l}\text { Non- } \\
\text { digital }\end{array}$ & Non-digital & Offline & Online \\
\hline 1244 & Offline & Online & Blended & Brain $+d+n-d$ & $\begin{array}{l}\text { Non- } \\
\text { digital }\end{array}$ & Non-digital & Offline & Offline \\
\hline 1245 & Offline & Online & Blended & Brain $+d+n-d$ & $\begin{array}{l}\text { Non- } \\
\text { digital }\end{array}$ & Blended & Online & Online \\
\hline 1246 & Offline & Online & Blended & Brain $+d+n-d$ & $\begin{array}{l}\text { Non- } \\
\text { digital }\end{array}$ & Blended & Online & Offline \\
\hline 1247 & Offline & Online & Blended & Brain $+d+n-d$ & $\begin{array}{l}\text { Non- } \\
\text { digital }\end{array}$ & Blended & Offline & Online \\
\hline 1248 & Offline & Online & Blended & Brain $+d+n-d$ & $\begin{array}{l}\text { Non- } \\
\text { digital }\end{array}$ & Blended & Offline & Offline \\
\hline 1249 & Offline & Online & Blended & Brain $+d+n-d$ & $\begin{array}{l}\text { Blend- } \\
\text { ed }\end{array}$ & Digital & Online & Online \\
\hline 1250 & Offline & Online & Blended & Brain $+d+n-d$ & $\begin{array}{l}\text { Blend- } \\
\text { ed }\end{array}$ & Digital & Online & Offline \\
\hline 1251 & Offline & Online & Blended & Brain $+d+n-d$ & $\begin{array}{l}\text { Blend- } \\
\text { ed }\end{array}$ & Digital & Offline & Online \\
\hline 1252 & Offline & Online & Blended & Brain $+d+n-d$ & $\begin{array}{l}\text { Blend- } \\
\text { ed }\end{array}$ & Digital & Offline & Offline \\
\hline 1253 & Offline & Online & Blended & Brain $+d+n-d$ & $\begin{array}{l}\text { Blend- } \\
\text { ed }\end{array}$ & Non-digital & Online & Online \\
\hline 1254 & Offline & Online & Blended & Brain $+d+n-d$ & $\begin{array}{l}\text { Blend- } \\
\text { ed }\end{array}$ & Non-digital & Online & Offline \\
\hline 1255 & Offline & Online & Blended & Brain $+d+n-d$ & $\begin{array}{l}\text { Blend- } \\
\text { ed }\end{array}$ & Non-digital & Offline & Online \\
\hline 1256 & Offline & Online & Blended & Brain $+d+n-d$ & $\begin{array}{l}\text { Blend- } \\
\text { ed }\end{array}$ & Non-digital & Offline & Offline \\
\hline 1257 & Offline & Online & Blended & Brain $+d+n-d$ & $\begin{array}{l}\text { Blend- } \\
\text { ed }\end{array}$ & Blended & Online & Online \\
\hline 1258 & Offline & Online & Blended & Brain $+d+n-d$ & $\begin{array}{l}\text { Blend- } \\
\text { ed }\end{array}$ & Blended & Online & Offline \\
\hline 1259 & Offline & Online & Blended & Brain $+d+n-d$ & $\begin{array}{l}\text { Blend- } \\
\text { ed }\end{array}$ & Blended & Offline & Online \\
\hline 1260 & Offline & Online & Blended & Brain $+d+n-d$ & $\begin{array}{l}\text { Blend- } \\
\text { ed }\end{array}$ & Blended & Offline & Offline \\
\hline 1261 & Offline & Online & Blended & Brain $+n-d$ & Digital & Digital & Online & Online \\
\hline 1262 & Offline & Online & Blended & Brain $+n-d$ & Digital & Digital & Online & Offline \\
\hline 1263 & Offline & Online & Blended & Brain + n-d & Digital & Digital & Offline & Online \\
\hline 1264 & Offline & Online & Blended & Brain + n-d & Digital & Digital & Offline & Offline \\
\hline 1265 & Offline & Online & Blended & Brain + n-d & Digital & Non-digital & Online & Online \\
\hline 1266 & Offline & Online & Blended & Brain + n-d & Digital & Non-digital & Online & Offline \\
\hline 1267 & Offline & Online & Blended & Brain + n-d & Digital & Non-digital & Offline & Online \\
\hline 1268 & Offline & Online & Blended & Brain + n-d & Digital & Non-digital & Offline & Offline \\
\hline 1269 & Offline & Online & Blended & Brain + n-d & Digital & Blended & Online & Online \\
\hline 1270 & Offline & Online & Blended & Brain + n-d & Digital & Blended & Online & Offline \\
\hline 1271 & Offline & Online & Blended & Brain + n-d & Digital & Blended & Offline & Online \\
\hline 1272 & Offline & Online & Blended & Brain + n-d & Digital & Blended & Offline & Offline \\
\hline 1273 & Offline & Online & Blended & Brain $+n-d$ & $\begin{array}{l}\text { Non- } \\
\text { digital }\end{array}$ & Digital & Online & Online \\
\hline 1274 & Offline & Online & Blended & Brain + n-d & $\begin{array}{l}\text { Non- } \\
\text { digital }\end{array}$ & Digital & Online & Offline \\
\hline 1275 & Offline & Online & Blended & Brain + n-d & $\begin{array}{l}\text { Non- } \\
\text { digital }\end{array}$ & Digital & Offline & Online \\
\hline 1276 & Offline & Online & Blended & Brain + n-d & $\begin{array}{l}\text { Non- } \\
\text { digital }\end{array}$ & Digital & Offline & Offline \\
\hline
\end{tabular}




\begin{tabular}{|c|c|c|c|c|c|c|c|c|}
\hline 1277 & Offline & Online & Blended & Brain + n-d & $\begin{array}{l}\text { Non- } \\
\text { digital }\end{array}$ & Non-digital & Online & Online \\
\hline 1278 & Offline & Online & Blended & Brain + n-d & $\begin{array}{l}\text { Non- } \\
\text { digital }\end{array}$ & Non-digital & Online & Offline \\
\hline 1279 & Offline & Online & Blended & Brain + n-d & $\begin{array}{l}\text { Non- } \\
\text { digital }\end{array}$ & Non-digital & Offline & Online \\
\hline 1280 & Offline & Online & Blended & Brain + n-d & $\begin{array}{l}\text { Non- } \\
\text { digital }\end{array}$ & Non-digital & Offline & Offline \\
\hline 1281 & Offline & Online & Blended & Brain + n-d & $\begin{array}{l}\text { Non- } \\
\text { digital }\end{array}$ & Blended & Online & Online \\
\hline 1282 & Offline & Online & Blended & Brain + n-d & $\begin{array}{l}\text { Non- } \\
\text { digital }\end{array}$ & Blended & Online & Offline \\
\hline 1283 & Offline & Online & Blended & Brain + n-d & $\begin{array}{l}\text { Non- } \\
\text { digital }\end{array}$ & Blended & Offline & Online \\
\hline 1284 & Offline & Online & Blended & Brain + n-d & $\begin{array}{l}\text { Non- } \\
\text { digital }\end{array}$ & Blended & Offline & Offline \\
\hline 1285 & Offline & Online & Blended & Brain + n-d & $\begin{array}{l}\text { Blend- } \\
\text { ed }\end{array}$ & Digital & Online & Online \\
\hline 1286 & Offline & Online & Blended & Brain + n-d & $\begin{array}{l}\text { Blend- } \\
\text { ed }\end{array}$ & Digital & Online & Offline \\
\hline 1287 & Offline & Online & Blended & Brain + n-d & $\begin{array}{l}\text { Blend- } \\
\text { ed }\end{array}$ & Digital & Offline & Online \\
\hline 1288 & Offline & Online & Blended & Brain + n-d & $\begin{array}{l}\text { Blend- } \\
\text { ed }\end{array}$ & Digital & Offline & Offline \\
\hline 1289 & Offline & Online & Blended & Brain + n-d & $\begin{array}{l}\text { Blend- } \\
\text { ed }\end{array}$ & Non-digital & Online & Online \\
\hline 1290 & Offline & Online & Blended & Brain + n-d & $\begin{array}{l}\text { Blend- } \\
\text { ed }\end{array}$ & Non-digital & Online & Offline \\
\hline 1291 & Offline & Online & Blended & Brain + n-d & $\begin{array}{l}\text { Blend- } \\
\text { ed }\end{array}$ & Non-digital & Offline & Online \\
\hline 1292 & Offline & Online & Blended & Brain + n-d & $\begin{array}{l}\text { Blend- } \\
\text { ed }\end{array}$ & Non-digital & Offline & Offline \\
\hline \multirow[t]{4}{*}{1293} & Offline & Online & Blended & Brain + n-d & $\begin{array}{l}\text { Blend- } \\
\text { ed }\end{array}$ & Blended & Online & Online \\
\hline & Offline & Online & Blended & Brain + n-d & $\begin{array}{l}\text { Blend- } \\
\text { ed }\end{array}$ & Blended & Online & Offline \\
\hline & Offline & Online & Blended & Brain + n-d & $\begin{array}{l}\text { Blend- } \\
\text { ed }\end{array}$ & Blended & Offline & Online \\
\hline & Offline & Online & Blended & Brain + n-d & $\begin{array}{l}\text { Blend- } \\
\text { ed }\end{array}$ & Blended & Offline & Offline \\
\hline \multicolumn{9}{|l|}{1293} \\
\hline \multicolumn{9}{|l|}{1294} \\
\hline \multicolumn{9}{|l|}{1295} \\
\hline \multicolumn{9}{|l|}{1296} \\
\hline 1297 & Offline & Offline & Online & Brain + digital & Digital & Digital & Online & Online \\
\hline 1298 & Offline & Offline & Online & Brain + digital & Digital & Digital & Online & Offline \\
\hline 1299 & Offline & Offline & Online & Brain + digital & Digital & Digital & Offline & Online \\
\hline 1300 & Offline & Offline & Online & Brain + digital & Digital & Digital & Offline & Offline \\
\hline 1301 & Offline & Offline & Online & Brain + digital & Digital & Non-digital & Online & Online \\
\hline 1302 & Offline & Offline & Online & Brain + digital & Digital & Non-digital & Online & Offline \\
\hline 1303 & Offline & Offline & Online & Brain + digital & Digital & Non-digital & Offline & Online \\
\hline 1304 & Offline & Offline & Online & Brain + digital & Digital & Non-digital & Offline & Offline \\
\hline 1305 & Offline & Offline & Online & Brain + digital & Digital & Blended & Online & Online \\
\hline 1306 & Offline & Offline & Online & Brain + digital & Digital & Blended & Online & Offline \\
\hline 1307 & Offline & Offline & Online & Brain + digital & Digital & Blended & Offline & Online \\
\hline 1308 & Offline & Offline & Online & Brain + digital & Digital & Blended & Offline & Offline \\
\hline 1309 & Offline & Offline & Online & Brain + digital & $\begin{array}{l}\text { Non- } \\
\text { digital }\end{array}$ & Digital & Online & Online \\
\hline 1310 & Offline & Offline & Online & Brain + digital & $\begin{array}{l}\text { Non- } \\
\text { digital }\end{array}$ & Digital & Online & Offline \\
\hline 1311 & Offline & Offline & Online & Brain + digital & $\begin{array}{l}\text { Non- } \\
\text { digital }\end{array}$ & Digital & Offline & Online \\
\hline 1312 & Offline & Offline & Online & Brain + digital & $\begin{array}{l}\text { Non- } \\
\text { digital }\end{array}$ & Digital & Offline & Offline \\
\hline
\end{tabular}




\begin{tabular}{|c|c|c|c|c|c|c|c|c|}
\hline 1313 & Offline & Offline & Online & Brain + digital & $\begin{array}{l}\text { Non- } \\
\text { digital }\end{array}$ & Non-digital & Online & Online \\
\hline 1314 & Offline & Offline & Online & Brain + digital & $\begin{array}{l}\text { Non- } \\
\text { digital }\end{array}$ & Non-digital & Online & Offline \\
\hline 1315 & Offline & Offline & Online & Brain + digital & $\begin{array}{l}\text { Non- } \\
\text { digital }\end{array}$ & Non-digital & Offline & Online \\
\hline 1316 & Offline & Offline & Online & Brain + digital & $\begin{array}{l}\text { Non- } \\
\text { digital }\end{array}$ & Non-digital & Offline & Offline \\
\hline 1317 & Offline & Offline & Online & Brain + digital & $\begin{array}{l}\text { Non- } \\
\text { digital }\end{array}$ & Blended & Online & Online \\
\hline 1318 & Offline & Offline & Online & Brain + digital & $\begin{array}{l}\text { Non- } \\
\text { digital }\end{array}$ & Blended & Online & Offline \\
\hline 1319 & Offline & Offline & Online & Brain + digital & $\begin{array}{l}\text { Non- } \\
\text { digital }\end{array}$ & Blended & Offline & Online \\
\hline 1320 & Offline & Offline & Online & Brain + digital & $\begin{array}{l}\text { Non- } \\
\text { digital }\end{array}$ & Blended & Offline & Offline \\
\hline 1321 & Offline & Offline & Online & Brain + digital & $\begin{array}{l}\text { Blend- } \\
\text { ed }\end{array}$ & Digital & Online & Online \\
\hline 1322 & Offline & Offline & Online & Brain + digital & $\begin{array}{l}\text { Blend- } \\
\text { ed }\end{array}$ & Digital & Online & Offline \\
\hline 1323 & Offline & Offline & Online & Brain + digital & $\begin{array}{l}\text { Blend- } \\
\text { ed }\end{array}$ & Digital & Offline & Online \\
\hline 1324 & Offline & Offline & Online & Brain + digital & $\begin{array}{l}\text { Blend- } \\
\text { ed }\end{array}$ & Digital & Offline & Offline \\
\hline 1325 & Offline & Offline & Online & Brain + digital & $\begin{array}{l}\text { Blend- } \\
\text { ed }\end{array}$ & Non-digital & Online & Online \\
\hline 1326 & Offline & Offline & Online & Brain + digital & $\begin{array}{l}\text { Blend- } \\
\text { ed }\end{array}$ & Non-digital & Online & Offline \\
\hline 1327 & Offline & Offline & Online & Brain + digital & $\begin{array}{l}\text { Blend- } \\
\text { ed }\end{array}$ & Non-digital & Offline & Online \\
\hline 1328 & Offline & Offline & Online & Brain + digital & $\begin{array}{l}\text { Blend- } \\
\text { ed }\end{array}$ & Non-digital & Offline & Offline \\
\hline 1329 & Offline & Offline & Online & Brain + digital & $\begin{array}{l}\text { Blend- } \\
\text { ed }\end{array}$ & Blended & Online & Online \\
\hline 1330 & Offline & Offline & Online & Brain + digital & $\begin{array}{l}\text { Blend- } \\
\text { ed }\end{array}$ & Blended & Online & Offline \\
\hline 1331 & Offline & Offline & Online & Brain + digital & $\begin{array}{l}\text { Blend- } \\
\text { ed }\end{array}$ & Blended & Offline & Online \\
\hline 1332 & Offline & Offline & Online & Brain + digital & $\begin{array}{l}\text { Blend- } \\
\text { ed }\end{array}$ & Blended & Offline & Offline \\
\hline 1333 & Offline & Offline & Online & Brain & Digital & Digital & Online & Online \\
\hline 1334 & Offline & Offline & Online & Brain & Digital & Digital & Online & Offline \\
\hline 1335 & Offline & Offline & Online & Brain & Digital & Digital & Offline & Online \\
\hline 1336 & Offline & Offline & Online & Brain & Digital & Digital & Offline & Offline \\
\hline 1337 & Offline & Offline & Online & Brain & Digital & Non-digital & Online & Online \\
\hline 1338 & Offline & Offline & Online & Brain & Digital & Non-digital & Online & Offline \\
\hline 1339 & Offline & Offline & Online & Brain & Digital & Non-digital & Offline & Online \\
\hline 1340 & Offline & Offline & Online & Brain & Digital & Non-digital & Offline & Offline \\
\hline 1341 & Offline & Offline & Online & Brain & Digital & Blended & Online & Online \\
\hline 1342 & Offline & Offline & Online & Brain & Digital & Blended & Online & Offline \\
\hline 1343 & Offline & Offline & Online & Brain & Digital & Blended & Offline & Online \\
\hline 1344 & Offline & Offline & Online & Brain & Digital & Blended & Offline & Offline \\
\hline 1345 & Offline & Offline & Online & Brain & $\begin{array}{l}\text { Non- } \\
\text { digital }\end{array}$ & Digital & Online & Online \\
\hline 1346 & Offline & Offline & Online & Brain & $\begin{array}{l}\text { Non- } \\
\text { digital }\end{array}$ & Digital & Online & Offline \\
\hline 1347 & Offline & Offline & Online & Brain & $\begin{array}{l}\text { Non- } \\
\text { digital }\end{array}$ & Digital & Offline & Online \\
\hline 1348 & Offline & Offline & Online & Brain & $\begin{array}{l}\text { Non- } \\
\text { digital }\end{array}$ & Digital & Offline & Offline \\
\hline 1349 & Offline & Offline & Online & Brain & $\begin{array}{l}\text { Non- } \\
\text { digital }\end{array}$ & Non-digital & Online & Online \\
\hline 1350 & Offline & Offline & Online & Brain & $\begin{array}{l}\text { Non- } \\
\text { digital }\end{array}$ & Non-digital & Online & Offline \\
\hline
\end{tabular}




\begin{tabular}{|c|c|c|c|c|c|c|c|c|}
\hline 1351 & Offline & Offline & Online & Brain & $\begin{array}{l}\text { Non- } \\
\text { digital }\end{array}$ & Non-digital & Offline & Online \\
\hline 1352 & Offline & Offline & Online & Brain & $\begin{array}{l}\text { Non- } \\
\text { digital }\end{array}$ & Non-digital & Offline & Offline \\
\hline 1353 & Offline & Offline & Online & Brain & $\begin{array}{l}\text { Non- } \\
\text { digital }\end{array}$ & Blended & Online & Online \\
\hline 1354 & Offline & Offline & Online & Brain & $\begin{array}{l}\text { Non- } \\
\text { digital }\end{array}$ & Blended & Online & Offline \\
\hline 1355 & Offline & Offline & Online & Brain & $\begin{array}{l}\text { Non- } \\
\text { digital }\end{array}$ & Blended & Offline & Online \\
\hline 1356 & Offline & Offline & Online & Brain & $\begin{array}{l}\text { Non- } \\
\text { digital }\end{array}$ & Blended & Offline & Offline \\
\hline 1357 & Offline & Offline & Online & Brain & $\begin{array}{l}\text { Blend- } \\
\text { ed }\end{array}$ & Digital & Online & Online \\
\hline 1358 & Offline & Offline & Online & Brain & $\begin{array}{l}\text { Blend- } \\
\text { ed }\end{array}$ & Digital & Online & Offline \\
\hline 1359 & Offline & Offline & Online & Brain & $\begin{array}{l}\text { Blend- } \\
\text { ed }\end{array}$ & Digital & Offline & Online \\
\hline 1360 & Offline & Offline & Online & Brain & $\begin{array}{l}\text { Blend- } \\
\text { ed }\end{array}$ & Digital & Offline & Offline \\
\hline 1361 & Offline & Offline & Online & Brain & $\begin{array}{l}\text { Blend- } \\
\text { ed }\end{array}$ & Non-digital & Online & Online \\
\hline 1362 & Offline & Offline & Online & Brain & $\begin{array}{l}\text { Blend- } \\
\text { ed }\end{array}$ & Non-digital & Online & Offline \\
\hline 1363 & Offline & Offline & Online & Brain & $\begin{array}{l}\text { Blend- } \\
\text { ed }\end{array}$ & Non-digital & Offline & Online \\
\hline 1364 & Offline & Offline & Online & Brain & $\begin{array}{l}\text { Blend- } \\
\text { ed }\end{array}$ & Non-digital & Offline & Offline \\
\hline 1365 & Offline & Offline & Online & Brain & $\begin{array}{l}\text { Blend- } \\
\text { ed }\end{array}$ & Blended & Online & Online \\
\hline 1366 & Offline & Offline & Online & Brain & $\begin{array}{l}\text { Blend- } \\
\text { ed }\end{array}$ & Blended & Online & Offline \\
\hline 1367 & Offline & Offline & Online & Brain & $\begin{array}{l}\text { Blend- } \\
\text { ed }\end{array}$ & Blended & Offline & Online \\
\hline 1368 & Offline & Offline & Online & Brain & $\begin{array}{l}\text { Blend- } \\
\text { ed }\end{array}$ & Blended & Offline & Offline \\
\hline 1369 & Offline & Offline & Online & Brain $+d+n-d$ & Digital & Digital & Online & Online \\
\hline 1370 & Offline & Offline & Online & Brain $+d+n-d$ & Digital & Digital & Online & Offline \\
\hline 1371 & Offline & Offline & Online & Brain $+d+n-d$ & Digital & Digital & Offline & Online \\
\hline 1372 & Offline & Offline & Online & Brain + d + n-d & Digital & Digital & Offline & Offline \\
\hline 1373 & Offline & Offline & Online & Brain $+d+n-d$ & Digital & Non-digital & Online & Online \\
\hline 1374 & Offline & Offline & Online & Brain $+d+n-d$ & Digital & Non-digital & Online & Offline \\
\hline 1375 & Offline & Offline & Online & Brain $+d+n-d$ & Digital & Non-digital & Offline & Online \\
\hline 1376 & Offline & Offline & Online & Brain $+d+n-d$ & Digital & Non-digital & Offline & Offline \\
\hline 1377 & Offline & Offline & Online & Brain $+d+n-d$ & Digital & Blended & Online & Online \\
\hline 1378 & Offline & Offline & Online & Brain $+d+n-d$ & Digital & Blended & Online & Offline \\
\hline 1379 & Offline & Offline & Online & Brain $+d+n-d$ & Digital & Blended & Offline & Online \\
\hline 1380 & Offline & Offline & Online & Brain $+d+n-d$ & Digital & Blended & Offline & Offline \\
\hline 1381 & Offline & Offline & Online & Brain $+d+n-d$ & $\begin{array}{l}\text { Non- } \\
\text { digital }\end{array}$ & Digital & Online & Online \\
\hline 1382 & Offline & Offline & Online & Brain $+d+n-d$ & $\begin{array}{l}\text { Non- } \\
\text { digital }\end{array}$ & Digital & Online & Offline \\
\hline 1383 & Offline & Offline & Online & Brain $+d+n-d$ & $\begin{array}{l}\text { Non- } \\
\text { digital }\end{array}$ & Digital & Offline & Online \\
\hline 1384 & Offline & Offline & Online & Brain $+d+n-d$ & $\begin{array}{l}\text { Non- } \\
\text { digital }\end{array}$ & Digital & Offline & Offline \\
\hline 1385 & Offline & Offline & Online & Brain $+d+n-d$ & $\begin{array}{l}\text { Non- } \\
\text { digital }\end{array}$ & Non-digital & Online & Online \\
\hline 1386 & Offline & Offline & Online & Brain $+d+n-d$ & $\begin{array}{l}\text { Non- } \\
\text { digital }\end{array}$ & Non-digital & Online & Offline \\
\hline 1387 & Offline & Offline & Online & Brain $+d+n-d$ & $\begin{array}{l}\text { Non- } \\
\text { digital }\end{array}$ & Non-digital & Offline & Online \\
\hline 1388 & Offline & Offline & Online & Brain $+d+n-d$ & $\begin{array}{l}\text { Non- } \\
\text { digital }\end{array}$ & Non-digital & Offline & Offline \\
\hline
\end{tabular}




\begin{tabular}{|c|c|c|c|c|c|c|c|c|}
\hline 1389 & Offline & Offline & Online & Brain + d + n-d & $\begin{array}{l}\text { Non- } \\
\text { digital }\end{array}$ & Blended & Online & Online \\
\hline 1390 & Offline & Offline & Online & Brain $+d+n-d$ & $\begin{array}{l}\text { Non- } \\
\text { digital }\end{array}$ & Blended & Online & Offline \\
\hline 1391 & Offline & Offline & Online & Brain $+d+n-d$ & $\begin{array}{l}\text { Non- } \\
\text { digital }\end{array}$ & Blended & Offline & Online \\
\hline 1392 & Offline & Offline & Online & Brain $+d+n-d$ & $\begin{array}{l}\text { Non- } \\
\text { digital }\end{array}$ & Blended & Offline & Offline \\
\hline 1393 & Offline & Offline & Online & Brain $+d+n-d$ & $\begin{array}{l}\text { Blend- } \\
\text { ed }\end{array}$ & Digital & Online & Online \\
\hline 1394 & Offline & Offline & Online & Brain $+d+n-d$ & $\begin{array}{l}\text { Blend- } \\
\text { ed }\end{array}$ & Digital & Online & Offline \\
\hline 1395 & Offline & Offline & Online & Brain $+d+n-d$ & $\begin{array}{l}\text { Blend- } \\
\text { ed }\end{array}$ & Digital & Offline & Online \\
\hline 1396 & Offline & Offline & Online & Brain $+d+n-d$ & $\begin{array}{l}\text { Blend- } \\
\text { ed }\end{array}$ & Digital & Offline & Offline \\
\hline 1397 & Offline & Offline & Online & Brain $+d+n-d$ & $\begin{array}{l}\text { Blend- } \\
\text { ed }\end{array}$ & Non-digital & Online & Online \\
\hline 1398 & Offline & Offline & Online & Brain $+d+n-d$ & $\begin{array}{l}\text { Blend- } \\
\text { ed }\end{array}$ & Non-digital & Online & Offline \\
\hline 1399 & Offline & Offline & Online & Brain $+d+n-d$ & $\begin{array}{l}\text { Blend- } \\
\text { ed }\end{array}$ & Non-digital & Offline & Online \\
\hline 1400 & Offline & Offline & Online & Brain $+d+n-d$ & $\begin{array}{l}\text { Blend- } \\
\text { ed }\end{array}$ & Non-digital & Offline & Offline \\
\hline 1401 & Offline & Offline & Online & Brain $+d+n-d$ & $\begin{array}{l}\text { Blend- } \\
\text { ed }\end{array}$ & Blended & Online & Online \\
\hline 1402 & Offline & Offline & Online & Brain $+d+n-d$ & $\begin{array}{l}\text { Blend- } \\
\text { ed }\end{array}$ & Blended & Online & Offline \\
\hline 1403 & Offline & Offline & Online & Brain $+d+n-d$ & $\begin{array}{l}\text { Blend- } \\
\text { ed }\end{array}$ & Blended & Offline & Online \\
\hline 1404 & Offline & Offline & Online & Brain $+d+n-d$ & $\begin{array}{l}\text { Blend- } \\
\text { ed }\end{array}$ & Blended & Offline & Offline \\
\hline 1405 & Offline & Offline & Online & Brain + n-d & Digital & Digital & Online & Online \\
\hline 1406 & Offline & Offline & Online & Brain $+n-d$ & Digital & Digital & Online & Offline \\
\hline 1407 & Offline & Offline & Online & Brain + n-d & Digital & Digital & Offline & Online \\
\hline 1408 & Offline & Offline & Online & Brain $+n-d$ & Digital & Digital & Offline & Offline \\
\hline 1409 & Offline & Offline & Online & Brain $+n-d$ & Digital & Non-digital & Online & Online \\
\hline 1410 & Offline & Offline & Online & Brain + n-d & Digital & Non-digital & Online & Offline \\
\hline 1411 & Offline & Offline & Online & Brain + n-d & Digital & Non-digital & Offline & Online \\
\hline 1412 & Offline & Offline & Online & Brain $+n-d$ & Digital & Non-digital & Offline & Offline \\
\hline 1413 & Offline & Offline & Online & Brain + n-d & Digital & Blended & Online & Online \\
\hline 1414 & Offline & Offline & Online & Brain $+n-d$ & Digital & Blended & Online & Offline \\
\hline 1415 & Offline & Offline & Online & Brain $+n-d$ & Digital & Blended & Offline & Online \\
\hline 1416 & Offline & Offline & Online & Brain $+n-d$ & Digital & Blended & Offline & Offline \\
\hline 1417 & Offline & Offline & Online & Brain + n-d & $\begin{array}{l}\text { Non- } \\
\text { digital }\end{array}$ & Digital & Online & Online \\
\hline 1418 & Offline & Offline & Online & Brain + n-d & $\begin{array}{l}\text { Non- } \\
\text { digital }\end{array}$ & Digital & Online & Offline \\
\hline 1419 & Offline & Offline & Online & Brain + n-d & $\begin{array}{l}\text { Non- } \\
\text { digital }\end{array}$ & Digital & Offline & Online \\
\hline 1420 & Offline & Offline & Online & Brain + n-d & $\begin{array}{l}\text { Non- } \\
\text { digital }\end{array}$ & Digital & Offline & Offline \\
\hline 1421 & Offline & Offline & Online & Brain + n-d & $\begin{array}{l}\text { Non- } \\
\text { digital }\end{array}$ & Non-digital & Online & Online \\
\hline 1422 & Offline & Offline & Online & Brain + n-d & $\begin{array}{l}\text { Non- } \\
\text { digital }\end{array}$ & Non-digital & Online & Offline \\
\hline 1423 & Offline & Offline & Online & Brain + n-d & $\begin{array}{l}\text { Non- } \\
\text { digital }\end{array}$ & Non-digital & Offline & Online \\
\hline 1424 & Offline & Offline & Online & Brain + n-d & $\begin{array}{l}\text { Non- } \\
\text { digital }\end{array}$ & Non-digital & Offline & Offline \\
\hline 1425 & Offline & Offline & Online & Brain + n-d & $\begin{array}{l}\text { Non- } \\
\text { digital }\end{array}$ & Blended & Online & Online \\
\hline 1426 & Offline & Offline & Online & Brain + n-d & $\begin{array}{l}\text { Non- } \\
\text { digital }\end{array}$ & Blended & Online & Offline \\
\hline
\end{tabular}




\begin{tabular}{|c|c|c|c|c|c|c|c|c|}
\hline 1427 & Offline & Offline & Online & Brain + n-d & $\begin{array}{l}\text { Non- } \\
\text { digital }\end{array}$ & Blended & Offline & Online \\
\hline 1428 & Offline & Offline & Online & Brain + n-d & $\begin{array}{l}\text { Non- } \\
\text { digital }\end{array}$ & Blended & Offline & Offline \\
\hline 1429 & Offline & Offline & Online & Brain + n-d & $\begin{array}{l}\text { Blend- } \\
\text { ed }\end{array}$ & Digital & Online & Online \\
\hline 1430 & Offline & Offline & Online & Brain + n-d & $\begin{array}{l}\text { Blend- } \\
\text { ed }\end{array}$ & Digital & Online & Offline \\
\hline 1431 & Offline & Offline & Online & Brain + n-d & $\begin{array}{l}\text { Blend- } \\
\text { ed }\end{array}$ & Digital & Offline & Online \\
\hline 1432 & Offline & Offline & Online & Brain + n-d & $\begin{array}{l}\text { Blend- } \\
\text { ed }\end{array}$ & Digital & Offline & Offline \\
\hline 1433 & Offline & Offline & Online & Brain + n-d & $\begin{array}{l}\text { Blend- } \\
\text { ed }\end{array}$ & Non-digital & Online & Online \\
\hline 1434 & Offline & Offline & Online & Brain + n-d & $\begin{array}{l}\text { Blend- } \\
\text { ed }\end{array}$ & Non-digital & Online & Offline \\
\hline 1435 & Offline & Offline & Online & Brain + n-d & $\begin{array}{l}\text { Blend- } \\
\text { ed }\end{array}$ & Non-digital & Offline & Online \\
\hline 1436 & Offline & Offline & Online & Brain + n-d & $\begin{array}{l}\text { Blend- } \\
\text { ed }\end{array}$ & Non-digital & Offline & Offline \\
\hline 1437 & Offline & Offline & Online & Brain + n-d & $\begin{array}{l}\text { Blend- } \\
\text { ed }\end{array}$ & Blended & Online & Online \\
\hline 1438 & Offline & Offline & Online & Brain + n-d & $\begin{array}{l}\text { Blend- } \\
\text { ed }\end{array}$ & Blended & Online & Offline \\
\hline 1439 & Offline & Offline & Online & Brain + n-d & $\begin{array}{l}\text { Blend- } \\
\text { ed }\end{array}$ & Blended & Offline & Online \\
\hline 1440 & Offline & Offline & Online & Brain + n-d & $\begin{array}{l}\text { Blend- } \\
\text { ed }\end{array}$ & Blended & Offline & Offline \\
\hline 1441 & Offline & Offline & Offline & Brain + digital & Digital & Digital & Online & Online \\
\hline 1442 & Offline & Offline & Offline & Brain + digital & Digital & Digital & Online & Offline \\
\hline 1443 & Offline & Offline & Offline & Brain + digital & Digital & Digital & Offline & Online \\
\hline 1444 & Offline & Offline & Offline & Brain + digital & Digital & Digital & Offline & Offline \\
\hline 1445 & Offline & Offline & Offline & Brain + digital & Digital & Non-digital & Online & Online \\
\hline 1446 & Offline & Offline & Offline & Brain + digital & Digital & Non-digital & Online & Offline \\
\hline 1447 & Offline & Offline & Offline & Brain + digital & Digital & Non-digital & Offline & Online \\
\hline 1448 & Offline & Offline & Offline & Brain + digital & Digital & Non-digital & Offline & Offline \\
\hline 1449 & Offline & Offline & Offline & Brain + digital & Digital & Blended & Online & Online \\
\hline 1450 & Offline & Offline & Offline & Brain + digital & Digital & Blended & Online & Offline \\
\hline 1451 & Offline & Offline & Offline & Brain + digital & Digital & Blended & Offline & Online \\
\hline 1452 & Offline & Offline & Offline & Brain + digital & Digital & Blended & Offline & Offline \\
\hline 1453 & Offline & Offline & Offline & Brain + digital & $\begin{array}{l}\text { Non- } \\
\text { digital }\end{array}$ & Digital & Online & Online \\
\hline 1454 & Offline & Offline & Offline & Brain + digital & $\begin{array}{l}\text { Non- } \\
\text { digital }\end{array}$ & Digital & Online & Offline \\
\hline 1455 & Offline & Offline & Offline & Brain + digital & $\begin{array}{l}\text { Non- } \\
\text { digital }\end{array}$ & Digital & Offline & Online \\
\hline 1456 & Offline & Offline & Offline & Brain + digital & $\begin{array}{l}\text { Non- } \\
\text { digital }\end{array}$ & Digital & Offline & Offline \\
\hline 1457 & Offline & Offline & Offline & Brain + digital & $\begin{array}{l}\text { Non- } \\
\text { digital }\end{array}$ & Non-digital & Online & Online \\
\hline 1458 & Offline & Offline & Offline & Brain + digital & $\begin{array}{l}\text { Non- } \\
\text { digital }\end{array}$ & Non-digital & Online & Offline \\
\hline 1459 & Offline & Offline & Offline & Brain + digital & $\begin{array}{l}\text { Non- } \\
\text { digital }\end{array}$ & Non-digital & Offline & Online \\
\hline 1460 & Offline & Offline & Offline & Brain + digital & $\begin{array}{l}\text { Non- } \\
\text { digital }\end{array}$ & Non-digital & Offline & Offline \\
\hline 1461 & Offline & Offline & Offline & Brain + digital & $\begin{array}{l}\text { Non- } \\
\text { digital }\end{array}$ & Blended & Online & Online \\
\hline 1462 & Offline & Offline & Offline & Brain + digital & $\begin{array}{l}\text { Non- } \\
\text { digital }\end{array}$ & Blended & Online & Offline \\
\hline 1463 & Offline & Offline & Offline & Brain + digital & $\begin{array}{l}\text { Non- } \\
\text { digital }\end{array}$ & Blended & Offline & Online \\
\hline 1464 & Offline & Offline & Offline & Brain + digital & $\begin{array}{l}\text { Non- } \\
\text { digital }\end{array}$ & Blended & Offline & Offline \\
\hline
\end{tabular}




\begin{tabular}{|c|c|c|c|c|c|c|c|c|}
\hline 1465 & Offline & Offline & Offline & Brain + digital & $\begin{array}{l}\text { Blend- } \\
\text { ed }\end{array}$ & Digital & Online & Online \\
\hline 1466 & Offline & Offline & Offline & Brain + digital & $\begin{array}{l}\text { Blend- } \\
\text { ed }\end{array}$ & Digital & Online & Offline \\
\hline 1467 & Offline & Offline & Offline & Brain + digital & $\begin{array}{l}\text { Blend- } \\
\text { ed }\end{array}$ & Digital & Offline & Online \\
\hline 1468 & Offline & Offline & Offline & Brain + digital & $\begin{array}{l}\text { Blend- } \\
\text { ed }\end{array}$ & Digital & Offline & Offline \\
\hline 1469 & Offline & Offline & Offline & Brain + digital & $\begin{array}{l}\text { Blend- } \\
\text { ed }\end{array}$ & Non-digital & Online & Online \\
\hline 1470 & Offline & Offline & Offline & Brain + digital & $\begin{array}{l}\text { Blend- } \\
\text { ed }\end{array}$ & Non-digital & Online & Offline \\
\hline 1471 & Offline & Offline & Offline & Brain + digital & $\begin{array}{l}\text { Blend- } \\
\text { ed }\end{array}$ & Non-digital & Offline & Online \\
\hline 1472 & Offline & Offline & Offline & Brain + digital & $\begin{array}{l}\text { Blend- } \\
\text { ed }\end{array}$ & Non-digital & Offline & Offline \\
\hline 1473 & Offline & Offline & Offline & Brain + digital & $\begin{array}{l}\text { Blend- } \\
\text { ed }\end{array}$ & Blended & Online & Online \\
\hline 1474 & Offline & Offline & Offline & Brain + digital & $\begin{array}{l}\text { Blend- } \\
\text { ed }\end{array}$ & Blended & Online & Offline \\
\hline 1475 & Offline & Offline & Offline & Brain + digital & $\begin{array}{l}\text { Blend- } \\
\text { ed }\end{array}$ & Blended & Offline & Online \\
\hline 1476 & Offline & Offline & Offline & Brain + digital & $\begin{array}{l}\text { Blend- } \\
\text { ed }\end{array}$ & Blended & Offline & Offline \\
\hline 1477 & Offline & Offline & Offline & Brain & Digital & Digital & Online & Online \\
\hline 1478 & Offline & Offline & Offline & Brain & Digital & Digital & Online & Offline \\
\hline 1479 & Offline & Offline & Offline & Brain & Digital & Digital & Offline & Online \\
\hline 1480 & Offline & Offline & Offline & Brain & Digital & Digital & Offline & Offline \\
\hline 1481 & Offline & Offline & Offline & Brain & Digital & Non-digital & Online & Online \\
\hline 1482 & Offline & Offline & Offline & Brain & Digital & Non-digital & Online & Offline \\
\hline 1483 & Offline & Offline & Offline & Brain & Digital & Non-digital & Offline & Online \\
\hline 1484 & Offline & Offline & Offline & Brain & Digital & Non-digital & Offline & Offline \\
\hline 1485 & Offline & Offline & Offline & Brain & Digital & Blended & Online & Online \\
\hline 1486 & Offline & Offline & Offline & Brain & Digital & Blended & Online & Offline \\
\hline 1487 & Offline & Offline & Offline & Brain & Digital & Blended & Offline & Online \\
\hline 1488 & Offline & Offline & Offline & Brain & Digital & Blended & Offline & Offline \\
\hline 1489 & Offline & Offline & Offline & Brain & $\begin{array}{l}\text { Non- } \\
\text { diaital }\end{array}$ & Digital & Online & Online \\
\hline 1490 & Offline & Offline & Offline & Brain & $\begin{array}{l}\text { Non- } \\
\text { digital }\end{array}$ & Digital & Online & Offline \\
\hline 1491 & Offline & Offline & Offline & Brain & $\begin{array}{l}\text { Non- } \\
\text { diaital }\end{array}$ & Digital & Offline & Online \\
\hline 1492 & Offline & Offline & Offline & Brain & $\begin{array}{l}\text { Non- } \\
\text { diaital }\end{array}$ & Digital & Offline & Offline \\
\hline 1493 & Offline & Offline & Offline & Brain & $\begin{array}{l}\text { Non- } \\
\text { digital }\end{array}$ & Non-digital & Online & Online \\
\hline 1494 & Offline & Offline & Offline & Brain & $\begin{array}{l}\text { Non- } \\
\text { diaital }\end{array}$ & Non-digital & Online & Offline \\
\hline 1495 & Offline & Offline & Offline & Brain & $\begin{array}{l}\text { Non- } \\
\text { digital }\end{array}$ & Non-digital & Offline & Online \\
\hline 1496 & Offline & Offline & Offline & Brain & $\begin{array}{l}\text { Non- } \\
\text { diaital }\end{array}$ & Non-digital & Offline & Offline \\
\hline 1497 & Offline & Offline & Offline & Brain & $\begin{array}{l}\text { Non- } \\
\text { digital }\end{array}$ & Blended & Online & Online \\
\hline 1498 & Offline & Offline & Offline & Brain & $\begin{array}{l}\text { Non- } \\
\text { digital }\end{array}$ & Blended & Online & Offline \\
\hline 1499 & Offline & Offline & Offline & Brain & $\begin{array}{l}\text { Non- } \\
\text { digital }\end{array}$ & Blended & Offline & Online \\
\hline 1500 & Offline & Offline & Offline & Brain & $\begin{array}{l}\text { Non- } \\
\text { digital }\end{array}$ & Blended & Offline & Offline \\
\hline 1501 & Offline & Offline & Offline & Brain & $\begin{array}{l}\text { Blend- } \\
\text { ed }\end{array}$ & Digital & Online & Online \\
\hline 1502 & Offline & Offline & Offline & Brain & $\begin{array}{l}\text { Blend- } \\
\text { ed }\end{array}$ & Digital & Online & Offline \\
\hline
\end{tabular}




\begin{tabular}{|c|c|c|c|c|c|c|c|c|}
\hline 1503 & Offline & Offline & Offline & Brain & $\begin{array}{l}\text { Blend- } \\
\text { ed }\end{array}$ & Digital & Offline & Online \\
\hline 1504 & Offline & Offline & Offline & Brain & $\begin{array}{l}\text { Blend- } \\
\text { ed }\end{array}$ & Digital & Offline & Offline \\
\hline 1505 & Offline & Offline & Offline & Brain & $\begin{array}{l}\text { Blend- } \\
\text { ed }\end{array}$ & Non-digital & Online & Online \\
\hline 1506 & Offline & Offline & Offline & Brain & $\begin{array}{l}\text { Blend- } \\
\text { ed }\end{array}$ & Non-digital & Online & Offline \\
\hline 1507 & Offline & Offline & Offline & Brain & $\begin{array}{l}\text { Blend- } \\
\text { ed }\end{array}$ & Non-digital & Offline & Online \\
\hline 1508 & Offline & Offline & Offline & Brain & $\begin{array}{l}\text { Blend- } \\
\text { ed }\end{array}$ & Non-digital & Offline & Offline \\
\hline 1509 & Offline & Offline & Offline & Brain & $\begin{array}{l}\text { Blend- } \\
\text { ed }\end{array}$ & Blended & Online & Online \\
\hline 1510 & Offline & Offline & Offline & Brain & $\begin{array}{l}\text { Blend- } \\
\text { ed }\end{array}$ & Blended & Online & Offline \\
\hline 1511 & Offline & Offline & Offline & Brain & $\begin{array}{l}\text { Blend- } \\
\text { ed }\end{array}$ & Blended & Offline & Online \\
\hline 1512 & Offline & Offline & Offline & Brain & $\begin{array}{l}\text { Blend- } \\
\text { ed }\end{array}$ & Blended & Offline & Offline \\
\hline 1513 & Offline & Offline & Offline & Brain $+d+n-d$ & Digital & Digital & Online & Online \\
\hline 1514 & Offline & Offline & Offline & Brain +d + n-d & Digital & Digital & Online & Offline \\
\hline 1515 & Offline & Offline & Offline & Brain $+d+n-d$ & Digital & Digital & Offline & Online \\
\hline 1516 & Offline & Offline & Offline & Brain $+d+n-d$ & Digital & Digital & Offline & Offline \\
\hline 1517 & Offline & Offline & Offline & Brain + d + n-d & Digital & Non-digital & Online & Online \\
\hline 1518 & Offline & Offline & Offline & Brain $+d+n-d$ & Digital & Non-digital & Online & Offline \\
\hline 1519 & Offline & Offline & Offline & Brain $+d+n-d$ & Digital & Non-digital & Offline & Online \\
\hline 1520 & Offline & Offline & Offline & Brain + d + n-d & Digital & Non-digital & Offline & Offline \\
\hline 1521 & Offline & Offline & Offline & Brain + d + n-d & Digital & Blended & Online & Online \\
\hline 1522 & Offline & Offline & Offline & Brain $+d+n-d$ & Digital & Blended & Online & Offline \\
\hline 1523 & Offline & Offline & Offline & Brain + d + n-d & Digital & Blended & Offline & Online \\
\hline 1524 & Offline & Offline & Offline & Brain $+d+n-d$ & Digital & Blended & Offline & Offline \\
\hline 1525 & Offline & Offline & Offline & Brain $+d+n-d$ & $\begin{array}{l}\text { Non- } \\
\text { digital }\end{array}$ & Digital & Online & Online \\
\hline 1526 & Offline & Offline & Offline & Brain $+d+n-d$ & $\begin{array}{l}\text { Non- } \\
\text { digital }\end{array}$ & Digital & Online & Offline \\
\hline 1527 & Offline & Offline & Offline & Brain $+d+n-d$ & $\begin{array}{l}\text { Non- } \\
\text { digital }\end{array}$ & Digital & Offline & Online \\
\hline 1528 & Offline & Offline & Offline & Brain $+d+n-d$ & $\begin{array}{l}\text { Non- } \\
\text { digital }\end{array}$ & Digital & Offline & Offline \\
\hline 1529 & Offline & Offline & Offline & Brain $+d+n-d$ & $\begin{array}{l}\text { Non- } \\
\text { digital }\end{array}$ & Non-digital & Online & Online \\
\hline 1530 & Offline & Offline & Offline & Brain $+d+n-d$ & $\begin{array}{l}\text { Non- } \\
\text { digital }\end{array}$ & Non-digital & Online & Offline \\
\hline 1531 & Offline & Offline & Offline & Brain $+d+n-d$ & $\begin{array}{l}\text { Non- } \\
\text { diqital }\end{array}$ & Non-digital & Offline & Online \\
\hline 1532 & Offline & Offline & Offline & Brain $+d+n-d$ & $\begin{array}{l}\text { Non- } \\
\text { digital }\end{array}$ & Non-digital & Offline & Offline \\
\hline 1533 & Offline & Offline & Offline & Brain $+d+n-d$ & $\begin{array}{l}\text { Non- } \\
\text { digital }\end{array}$ & Blended & Online & Online \\
\hline 1534 & Offline & Offline & Offline & Brain $+d+n-d$ & $\begin{array}{l}\text { Non- } \\
\text { digital }\end{array}$ & Blended & Online & Offline \\
\hline 1535 & Offline & Offline & Offline & Brain + d + n-d & $\begin{array}{l}\text { Non- } \\
\text { digital }\end{array}$ & Blended & Offline & Online \\
\hline 1536 & Offline & Offline & Offline & Brain + d + n-d & $\begin{array}{l}\text { Non- } \\
\text { diqital }\end{array}$ & Blended & Offline & Offline \\
\hline 1537 & Offline & Offline & Offline & Brain $+d+n-d$ & $\begin{array}{l}\text { Blend- } \\
\text { ed }\end{array}$ & Digital & Online & Online \\
\hline 1538 & Offline & Offline & Offline & Brain $+d+n-d$ & $\begin{array}{l}\text { Blend- } \\
\text { ed }\end{array}$ & Digital & Online & Offline \\
\hline 1539 & Offline & Offline & Offline & Brain + d + n-d & $\begin{array}{l}\text { Blend- } \\
\text { ed }\end{array}$ & Digital & Offline & Online \\
\hline 1540 & Offline & Offline & Offline & Brain + d + n-d & $\begin{array}{l}\text { Blend- } \\
\text { ed }\end{array}$ & Digital & Offline & Offline \\
\hline
\end{tabular}




\begin{tabular}{|c|c|c|c|c|c|c|c|c|}
\hline 1541 & Offline & Offline & Offline & Brain $+d+n-d$ & $\begin{array}{l}\text { Blend- } \\
\text { ed }\end{array}$ & Non-digital & Online & Online \\
\hline 1542 & Offline & Offline & Offline & Brain $+d+n-d$ & $\begin{array}{l}\text { Blend- } \\
\text { ed }\end{array}$ & Non-digital & Online & Offline \\
\hline 1543 & Offline & Offline & Offline & Brain $+d+n-d$ & $\begin{array}{l}\text { Blend- } \\
\text { ed }\end{array}$ & Non-digital & Offline & Online \\
\hline 1544 & Offline & Offline & Offline & Brain $+d+n-d$ & $\begin{array}{l}\text { Blend- } \\
\text { ed }\end{array}$ & Non-digital & Offline & Offline \\
\hline 1545 & Offline & Offline & Offline & Brain $+d+n-d$ & $\begin{array}{l}\text { Blend- } \\
\text { ed }\end{array}$ & Blended & Online & Online \\
\hline 1546 & Offline & Offline & Offline & Brain $+d+n-d$ & $\begin{array}{l}\text { Blend- } \\
\text { ed }\end{array}$ & Blended & Online & Offline \\
\hline 1547 & Offline & Offline & Offline & Brain $+d+n-d$ & $\begin{array}{l}\text { Blend- } \\
\text { ed }\end{array}$ & Blended & Offline & Online \\
\hline 1548 & Offline & Offline & Offline & Brain $+d+n-d$ & $\begin{array}{l}\text { Blend- } \\
\text { ed }\end{array}$ & Blended & Offline & Offline \\
\hline 1549 & Offline & Offline & Offline & Brain + n-d & Digital & Digital & Online & Online \\
\hline 1550 & Offline & Offline & Offline & Brain $+n-d$ & Digital & Digital & Online & Offline \\
\hline 1551 & Offline & Offline & Offline & Brain + n-d & Digital & Digital & Offline & Online \\
\hline 1552 & Offline & Offline & Offline & Brain $+n-d$ & Digital & Digital & Offline & Offline \\
\hline 1553 & Offline & Offline & Offline & Brain $+n-d$ & Digital & Non-digital & Online & Online \\
\hline 1554 & Offline & Offline & Offline & Brain $+n-d$ & Digital & Non-digital & Online & Offline \\
\hline 1555 & Offline & Offline & Offline & Brain $+n-d$ & Digital & Non-digital & Offline & Online \\
\hline 1556 & Offline & Offline & Offline & Brain $+n-d$ & Digital & Non-digital & Offline & Offline \\
\hline 1557 & Offline & Offline & Offline & Brain $+n-d$ & Digital & Blended & Online & Online \\
\hline 1558 & Offline & Offline & Offline & Brain + n-d & Digital & Blended & Online & Offline \\
\hline 1559 & Offline & Offline & Offline & Brain $+n-d$ & Digital & Blended & Offline & Online \\
\hline 1560 & Offline & Offline & Offline & Brain + n-d & Digital & Blended & Offline & Offline \\
\hline 1561 & Offline & Offline & Offline & Brain + n-d & $\begin{array}{l}\text { Non- } \\
\text { digital }\end{array}$ & Digital & Online & Online \\
\hline 1562 & Offline & Offline & Offline & Brain + n-d & $\begin{array}{l}\text { Non- } \\
\text { digital }\end{array}$ & Digital & Online & Offline \\
\hline 1563 & Offline & Offline & Offline & Brain + n-d & $\begin{array}{l}\text { Non- } \\
\text { digital }\end{array}$ & Digital & Offline & Online \\
\hline 1564 & Offline & Offline & Offline & Brain $+n-d$ & $\begin{array}{l}\text { Non- } \\
\text { digital }\end{array}$ & Digital & Offline & Offline \\
\hline 1565 & Offline & Offline & Offline & Brain $+n-d$ & $\begin{array}{l}\text { Non- } \\
\text { digital }\end{array}$ & Non-digital & Online & Online \\
\hline 1566 & Offline & Offline & Offline & Brain + n-d & $\begin{array}{l}\text { Non- } \\
\text { digital }\end{array}$ & Non-digital & Online & Offline \\
\hline 1567 & Offline & Offline & Offline & Brain $+n-d$ & $\begin{array}{l}\text { Non- } \\
\text { digital }\end{array}$ & Non-digital & Offline & Online \\
\hline 1568 & Offline & Offline & Offline & Brain $+n-d$ & $\begin{array}{l}\text { Non- } \\
\text { digital }\end{array}$ & Non-digital & Offline & Offline \\
\hline 1569 & Offline & Offline & Offline & Brain $+n-d$ & $\begin{array}{l}\text { Non- } \\
\text { digital }\end{array}$ & Blended & Online & Online \\
\hline 1570 & Offline & Offline & Offline & Brain + n-d & $\begin{array}{l}\text { Non- } \\
\text { digital }\end{array}$ & Blended & Online & Offline \\
\hline 1571 & Offline & Offline & Offline & Brain $+n-d$ & $\begin{array}{l}\text { Non- } \\
\text { digital }\end{array}$ & Blended & Offline & Online \\
\hline 1572 & Offline & Offline & Offline & Brain $+n-d$ & $\begin{array}{l}\text { Non- } \\
\text { digital }\end{array}$ & Blended & Offline & Offline \\
\hline 1573 & Offline & Offline & Offline & Brain + n-d & $\begin{array}{l}\text { Blend- } \\
\text { ed }\end{array}$ & Digital & Online & Online \\
\hline 1574 & Offline & Offline & Offline & Brain + n-d & $\begin{array}{l}\text { Blend- } \\
\text { ed }\end{array}$ & Digital & Online & Offline \\
\hline 1575 & Offline & Offline & Offline & Brain $+n-d$ & $\begin{array}{l}\text { Blend- } \\
\text { ed }\end{array}$ & Digital & Offline & Online \\
\hline 1576 & Offline & Offline & Offline & Brain + n-d & $\begin{array}{l}\text { Blend- } \\
\text { ed }\end{array}$ & Digital & Offline & Offline \\
\hline 1577 & Offline & Offline & Offline & Brain + n-d & $\begin{array}{l}\text { Blend- } \\
\text { ed }\end{array}$ & Non-digital & Online & Online \\
\hline 1578 & Offline & Offline & Offline & Brain $+n-d$ & $\begin{array}{l}\text { Blend- } \\
\text { ed }\end{array}$ & Non-digital & Online & Offline \\
\hline
\end{tabular}




\begin{tabular}{|c|c|c|c|c|c|c|c|c|}
\hline 1579 & Offline & Offline & Offline & Brain + n-d & $\begin{array}{l}\text { Blend- } \\
\text { ed }\end{array}$ & Non-digital & Offline & Online \\
\hline 1580 & Offline & Offline & Offline & Brain + n-d & $\begin{array}{l}\text { Blend- } \\
\text { ed }\end{array}$ & Non-digital & Offline & Offline \\
\hline 1581 & Offline & Offline & Offline & Brain + n-d & $\begin{array}{l}\text { Blend- } \\
\text { ed }\end{array}$ & Blended & Online & Online \\
\hline 1582 & Offline & Offline & Offline & Brain + n-d & $\begin{array}{l}\text { Blend- } \\
\text { ed }\end{array}$ & Blended & Online & Offline \\
\hline 1583 & Offline & Offline & Offline & Brain + n-d & $\begin{array}{l}\text { Blend- } \\
\text { ed }\end{array}$ & Blended & Offline & Online \\
\hline 1584 & Offline & Offline & Offline & Brain + n-d & $\begin{array}{l}\text { Blend- } \\
\text { ed }\end{array}$ & Blended & Offline & Offline \\
\hline 1585 & Offline & Offline & Blended & Brain + digital & Digital & Digital & Online & Online \\
\hline 1586 & Offline & Offline & Blended & Brain + digital & Digital & Digital & Online & Offline \\
\hline 1587 & Offline & Offline & Blended & Brain + digital & Digital & Digital & Offline & Online \\
\hline 1588 & Offline & Offline & Blended & Brain + digital & Digital & Digital & Offline & Offline \\
\hline 1589 & Offline & Offline & Blended & Brain + digital & Digital & Non-digital & Online & Online \\
\hline 1590 & Offline & Offline & Blended & Brain + digital & Digital & Non-digital & Online & Offline \\
\hline 1591 & Offline & Offline & Blended & Brain + digital & Digital & Non-digital & Offline & Online \\
\hline 1592 & Offline & Offline & Blended & Brain + digital & Digital & Non-digital & Offline & Offline \\
\hline 1593 & Offline & Offline & Blended & Brain + digital & Digital & Blended & Online & Online \\
\hline 1594 & Offline & Offline & Blended & Brain + digital & Digital & Blended & Online & Offline \\
\hline 1595 & Offline & Offline & Blended & Brain + digital & Digital & Blended & Offline & Online \\
\hline 1596 & Offline & Offline & Blended & Brain + digital & Digital & Blended & Offline & Offline \\
\hline 1597 & Offline & Offline & Blended & Brain + digital & $\begin{array}{l}\text { Non- } \\
\text { digital }\end{array}$ & Digital & Online & Online \\
\hline 1598 & Offline & Offline & Blended & Brain + digital & $\begin{array}{l}\text { Non- } \\
\text { digital }\end{array}$ & Digital & Online & Offline \\
\hline 1599 & Offline & Offline & Blended & Brain + digital & $\begin{array}{l}\text { Non- } \\
\text { digital }\end{array}$ & Digital & Offline & Online \\
\hline 1600 & Offline & Offline & Blended & Brain + digital & $\begin{array}{l}\text { Non- } \\
\text { digital }\end{array}$ & Digital & Offline & Offline \\
\hline 1601 & Offline & Offline & Blended & Brain + digital & $\begin{array}{l}\text { Non- } \\
\text { digital }\end{array}$ & Non-digital & Online & Online \\
\hline 1602 & Offline & Offline & Blended & Brain + digital & $\begin{array}{l}\text { Non- } \\
\text { digital }\end{array}$ & Non-digital & Online & Offline \\
\hline 1603 & Offline & Offline & Blended & Brain + digital & $\begin{array}{l}\text { Non- } \\
\text { digital }\end{array}$ & Non-digital & Offline & Online \\
\hline 1604 & Offline & Offline & Blended & Brain + digital & $\begin{array}{l}\text { Non- } \\
\text { digital }\end{array}$ & Non-digital & Offline & Offline \\
\hline 1605 & Offline & Offline & Blended & Brain + digital & $\begin{array}{l}\text { Non- } \\
\text { digital }\end{array}$ & Blended & Online & Online \\
\hline 1606 & Offline & Offline & Blended & Brain + digital & $\begin{array}{l}\text { Non- } \\
\text { digital }\end{array}$ & Blended & Online & Offline \\
\hline 1607 & Offline & Offline & Blended & Brain + digital & $\begin{array}{l}\text { Non- } \\
\text { digital }\end{array}$ & Blended & Offline & Online \\
\hline 1608 & Offline & Offline & Blended & Brain + digital & $\begin{array}{l}\text { Non- } \\
\text { digital }\end{array}$ & Blended & Offline & Offline \\
\hline 1609 & Offline & Offline & Blended & Brain + digital & $\begin{array}{l}\text { Blend- } \\
\text { ed }\end{array}$ & Digital & Online & Online \\
\hline 1610 & Offline & Offline & Blended & Brain + digital & $\begin{array}{l}\text { Blend- } \\
\text { ed }\end{array}$ & Digital & Online & Offline \\
\hline 1611 & Offline & Offline & Blended & Brain + digital & $\begin{array}{l}\text { Blend- } \\
\text { ed }\end{array}$ & Digital & Offline & Online \\
\hline 1612 & Offline & Offline & Blended & Brain + digital & $\begin{array}{l}\text { Blend- } \\
\text { ed }\end{array}$ & Digital & Offline & Offline \\
\hline 1613 & Offline & Offline & Blended & Brain + digital & $\begin{array}{l}\text { Blend- } \\
\text { ed }\end{array}$ & Non-digital & Online & Online \\
\hline 1614 & Offline & Offline & Blended & Brain + digital & $\begin{array}{l}\text { Blend- } \\
\text { ed }\end{array}$ & Non-digital & Online & Offline \\
\hline 1615 & Offline & Offline & Blended & Brain + digital & $\begin{array}{l}\text { Blend- } \\
\text { ed }\end{array}$ & Non-digital & Offline & Online \\
\hline 1616 & Offline & Offline & Blended & Brain + digital & $\begin{array}{l}\text { Blend- } \\
\text { ed }\end{array}$ & Non-digital & Offline & Offline \\
\hline
\end{tabular}




\begin{tabular}{|c|c|c|c|c|c|c|c|c|}
\hline 1617 & Offline & Offline & Blended & Brain + digital & $\begin{array}{l}\text { Blend- } \\
\text { ed }\end{array}$ & Blended & Online & Online \\
\hline 1618 & Offline & Offline & Blended & Brain + digital & $\begin{array}{l}\text { Blend- } \\
\text { ed }\end{array}$ & Blended & Online & Offline \\
\hline 1619 & Offline & Offline & Blended & Brain + digital & $\begin{array}{l}\text { Blend- } \\
\text { ed }\end{array}$ & Blended & Offline & Online \\
\hline 1620 & Offline & Offline & Blended & Brain + digital & $\begin{array}{l}\text { Blend- } \\
\text { ed }\end{array}$ & Blended & Offline & Offline \\
\hline 1621 & Offline & Offline & Blended & Brain & Digital & Digital & Online & Online \\
\hline 1622 & Offline & Offline & Blended & Brain & Digital & Digital & Online & Offline \\
\hline 1623 & Offline & Offline & Blended & Brain & Digital & Digital & Offline & Online \\
\hline 1624 & Offline & Offline & Blended & Brain & Digital & Digital & Offline & Offline \\
\hline 1625 & Offline & Offline & Blended & Brain & Digital & Non-digital & Online & Online \\
\hline 1626 & Offline & Offline & Blended & Brain & Digital & Non-digital & Online & Offline \\
\hline 1627 & Offline & Offline & Blended & Brain & Digital & Non-digital & Offline & Online \\
\hline 1628 & Offline & Offline & Blended & Brain & Digital & Non-digital & Offline & Offline \\
\hline 1629 & Offline & Offline & Blended & Brain & Digital & Blended & Online & Online \\
\hline 1630 & Offline & Offline & Blended & Brain & Digital & Blended & Online & Offline \\
\hline 1631 & Offline & Offline & Blended & Brain & Digital & Blended & Offline & Online \\
\hline 1632 & Offline & Offline & Blended & Brain & Digital & Blended & Offline & Offline \\
\hline 1633 & Offline & Offline & Blended & Brain & $\begin{array}{l}\text { Non- } \\
\text { digital }\end{array}$ & Digital & Online & Online \\
\hline 1634 & Offline & Offline & Blended & Brain & $\begin{array}{l}\text { Non- } \\
\text { digital }\end{array}$ & Digital & Online & Offline \\
\hline 1635 & Offline & Offline & Blended & Brain & $\begin{array}{l}\text { Non- } \\
\text { digital }\end{array}$ & Digital & Offline & Online \\
\hline 1636 & Offline & Offline & Blended & Brain & $\begin{array}{l}\text { Non- } \\
\text { digital }\end{array}$ & Digital & Offline & Offline \\
\hline 1637 & Offline & Offline & Blended & Brain & $\begin{array}{l}\text { Non- } \\
\text { digital }\end{array}$ & Non-digital & Online & Online \\
\hline 1638 & Offline & Offline & Blended & Brain & $\begin{array}{l}\text { Non- } \\
\text { digital }\end{array}$ & Non-digital & Online & Offline \\
\hline 1639 & Offline & Offline & Blended & Brain & $\begin{array}{l}\text { Non- } \\
\text { digital }\end{array}$ & Non-digital & Offline & Online \\
\hline 1640 & Offline & Offline & Blended & Brain & $\begin{array}{l}\text { Non- } \\
\text { digital }\end{array}$ & Non-digital & Offline & Offline \\
\hline 1641 & Offline & Offline & Blended & Brain & $\begin{array}{l}\text { Non- } \\
\text { digital }\end{array}$ & Blended & Online & Online \\
\hline 1642 & Offline & Offline & Blended & Brain & $\begin{array}{l}\text { Non- } \\
\text { digital }\end{array}$ & Blended & Online & Offline \\
\hline 1643 & Offline & Offline & Blended & Brain & $\begin{array}{l}\text { Non- } \\
\text { digital }\end{array}$ & Blended & Offline & Online \\
\hline 1644 & Offline & Offline & Blended & Brain & $\begin{array}{l}\text { Non- } \\
\text { diaital }\end{array}$ & Blended & Offline & Offline \\
\hline 1645 & Offline & Offline & Blended & Brain & $\begin{array}{l}\text { Blend- } \\
\text { ed }\end{array}$ & Digital & Online & Online \\
\hline 1646 & Offline & Offline & Blended & Brain & $\begin{array}{l}\text { Blend- } \\
\text { ed }\end{array}$ & Digital & Online & Offline \\
\hline 1647 & Offline & Offline & Blended & Brain & $\begin{array}{l}\text { Blend- } \\
\text { ed }\end{array}$ & Digital & Offline & Online \\
\hline 1648 & Offline & Offline & Blended & Brain & $\begin{array}{l}\text { Blend- } \\
\text { ed }\end{array}$ & Digital & Offline & Offline \\
\hline 1649 & Offline & Offline & Blended & Brain & $\begin{array}{l}\text { Blend- } \\
\text { ed }\end{array}$ & Non-digital & Online & Online \\
\hline 1650 & Offline & Offline & Blended & Brain & $\begin{array}{l}\text { Blend- } \\
\text { ed }\end{array}$ & Non-digital & Online & Offline \\
\hline 1651 & Offline & Offline & Blended & Brain & $\begin{array}{l}\text { Blend- } \\
\text { ed }\end{array}$ & Non-digital & Offline & Online \\
\hline 1652 & Offline & Offline & Blended & Brain & $\begin{array}{l}\text { Blend- } \\
\text { ed }\end{array}$ & Non-digital & Offline & Offline \\
\hline 1653 & Offline & Offline & Blended & Brain & $\begin{array}{l}\text { Blend- } \\
\text { ed }\end{array}$ & Blended & Online & Online \\
\hline 1654 & Offline & Offline & Blended & Brain & $\begin{array}{l}\text { Blend- } \\
\text { ed }\end{array}$ & Blended & Online & Offline \\
\hline
\end{tabular}




\begin{tabular}{|c|c|c|c|c|c|c|c|c|}
\hline 1655 & Offline & Offline & Blended & Brain & $\begin{array}{l}\text { Blend- } \\
\text { ed }\end{array}$ & Blended & Offline & Online \\
\hline 1656 & Offline & Offline & Blended & Brain & $\begin{array}{l}\text { Blend- } \\
\text { ed }\end{array}$ & Blended & Offline & Offline \\
\hline 1657 & Offline & Offline & Blended & Brain $+d+n-d$ & Digital & Digital & Online & Online \\
\hline 1658 & Offline & Offline & Blended & Brain $+d+n-d$ & Digital & Digital & Online & Offline \\
\hline 1659 & Offline & Offline & Blended & Brain $+d+n-d$ & Digital & Digital & Offline & Online \\
\hline 1660 & Offline & Offline & Blended & Brain $+d+n-d$ & Digital & Digital & Offline & Offline \\
\hline 1661 & Offline & Offline & Blended & Brain $+d+n-d$ & Digital & Non-digital & Online & Online \\
\hline 1662 & Offline & Offline & Blended & Brain $+d+n-d$ & Digital & Non-digital & Online & Offline \\
\hline 1663 & Offline & Offline & Blended & Brain $+d+n-d$ & Digital & Non-digital & Offline & Online \\
\hline 1664 & Offline & Offline & Blended & Brain $+d+n-d$ & Digital & Non-digital & Offline & Offline \\
\hline 1665 & Offline & Offline & Blended & Brain $+d+n-d$ & Digital & Blended & Online & Online \\
\hline 1666 & Offline & Offline & Blended & Brain $+d+n-d$ & Digital & Blended & Online & Offline \\
\hline 1667 & Offline & Offline & Blended & Brain $+d+n-d$ & Digital & Blended & Offline & Online \\
\hline 1668 & Offline & Offline & Blended & Brain $+d+n-d$ & Digital & Blended & Offline & Offline \\
\hline 1669 & Offline & Offline & Blended & Brain $+d+n-d$ & $\begin{array}{l}\text { Non- } \\
\text { digital }\end{array}$ & Digital & Online & Online \\
\hline 1670 & Offline & Offline & Blended & Brain $+d+n-d$ & $\begin{array}{l}\text { Non- } \\
\text { digital }\end{array}$ & Digital & Online & Offline \\
\hline 1671 & Offline & Offline & Blended & Brain $+d+n-d$ & $\begin{array}{l}\text { Non- } \\
\text { digital }\end{array}$ & Digital & Offline & Online \\
\hline 1672 & Offline & Offline & Blended & Brain $+d+n-d$ & $\begin{array}{l}\text { Non- } \\
\text { digital }\end{array}$ & Digital & Offline & Offline \\
\hline 1673 & Offline & Offline & Blended & Brain $+d+n-d$ & $\begin{array}{l}\text { Non- } \\
\text { digital }\end{array}$ & Non-digital & Online & Online \\
\hline 1674 & Offline & Offline & Blended & Brain $+d+n-d$ & $\begin{array}{l}\text { Non- } \\
\text { digital }\end{array}$ & Non-digital & Online & Offline \\
\hline 1675 & Offline & Offline & Blended & Brain $+d+n-d$ & $\begin{array}{l}\text { Non- } \\
\text { digital }\end{array}$ & Non-digital & Offline & Online \\
\hline 1676 & Offline & Offline & Blended & Brain $+d+n-d$ & $\begin{array}{l}\text { Non- } \\
\text { digital }\end{array}$ & Non-digital & Offline & Offline \\
\hline 1677 & Offline & Offline & Blended & Brain $+d+n-d$ & $\begin{array}{l}\text { Non- } \\
\text { digital }\end{array}$ & Blended & Online & Online \\
\hline 1678 & Offline & Offline & Blended & Brain $+d+n-d$ & $\begin{array}{l}\text { Non- } \\
\text { digital }\end{array}$ & Blended & Online & Offline \\
\hline 1679 & Offline & Offline & Blended & Brain $+d+n-d$ & $\begin{array}{l}\text { Non- } \\
\text { digital }\end{array}$ & Blended & Offline & Online \\
\hline 1680 & Offline & Offline & Blended & Brain $+d+n-d$ & $\begin{array}{l}\text { Non- } \\
\text { digital }\end{array}$ & Blended & Offline & Offline \\
\hline 1681 & Offline & Offline & Blended & Brain $+d+n-d$ & $\begin{array}{l}\text { Blend- } \\
\text { ed }\end{array}$ & Digital & Online & Online \\
\hline 1682 & Offline & Offline & Blended & Brain $+d+n-d$ & $\begin{array}{l}\text { Blend- } \\
\text { ed }\end{array}$ & Digital & Online & Offline \\
\hline 1683 & Offline & Offline & Blended & Brain $+d+n-d$ & $\begin{array}{l}\text { Blend- } \\
\text { ed }\end{array}$ & Digital & Offline & Online \\
\hline 1684 & Offline & Offline & Blended & Brain $+d+n-d$ & $\begin{array}{l}\text { Blend- } \\
\text { ed }\end{array}$ & Digital & Offline & Offline \\
\hline 1685 & Offline & Offline & Blended & Brain $+d+n-d$ & $\begin{array}{l}\text { Blend- } \\
\text { ed }\end{array}$ & Non-digital & Online & Online \\
\hline 1686 & Offline & Offline & Blended & Brain $+d+n-d$ & $\begin{array}{l}\text { Blend- } \\
\text { ed }\end{array}$ & Non-digital & Online & Offline \\
\hline 1687 & Offline & Offline & Blended & Brain $+d+n-d$ & $\begin{array}{l}\text { Blend- } \\
\text { ed }\end{array}$ & Non-digital & Offline & Online \\
\hline 1688 & Offline & Offline & Blended & Brain $+d+n-d$ & $\begin{array}{l}\text { Blend- } \\
\text { ed }\end{array}$ & Non-digital & Offline & Offline \\
\hline 1689 & Offline & Offline & Blended & Brain $+d+n-d$ & $\begin{array}{l}\text { Blend- } \\
\text { ed }\end{array}$ & Blended & Online & Online \\
\hline 1690 & Offline & Offline & Blended & Brain $+d+n-d$ & $\begin{array}{l}\text { Blend- } \\
\text { ed }\end{array}$ & Blended & Online & Offline \\
\hline 1691 & Offline & Offline & Blended & Brain $+d+n-d$ & $\begin{array}{l}\text { Blend- } \\
\text { ed }\end{array}$ & Blended & Offline & Online \\
\hline 1692 & Offline & Offline & Blended & Brain $+d+n-d$ & $\begin{array}{l}\text { Blend- } \\
\text { ed }\end{array}$ & Blended & Offline & Offline \\
\hline 1693 & Offline & Offline & Blended & Brain $+n-d$ & Digital & Digital & Online & Online \\
\hline
\end{tabular}




\begin{tabular}{|c|c|c|c|c|c|c|c|c|}
\hline 1694 & Offline & Offline & Blended & Brain $+n-d$ & Digital & Digital & Online & Offline \\
\hline 1695 & Offline & Offline & Blended & Brain $+n-d$ & Digital & Digital & Offline & Online \\
\hline 1696 & Offline & Offline & Blended & Brain $+n-d$ & Digital & Digital & Offline & Offline \\
\hline 1697 & Offline & Offline & Blended & Brain $+n-d$ & Digital & Non-digital & Online & Online \\
\hline 1698 & Offline & Offline & Blended & Brain $+n-d$ & Digital & Non-digital & Online & Offline \\
\hline 1699 & Offline & Offline & Blended & Brain $+n-d$ & Digital & Non-digital & Offline & Online \\
\hline 1700 & Offline & Offline & Blended & Brain $+n-d$ & Digital & Non-digital & Offline & Offline \\
\hline 1701 & Offline & Offline & Blended & Brain + n-d & Digital & Blended & Online & Online \\
\hline 1702 & Offline & Offline & Blended & Brain $+n-d$ & Digital & Blended & Online & Offline \\
\hline 1703 & Offline & Offline & Blended & Brain $+n-d$ & Digital & Blended & Offline & Online \\
\hline 1704 & Offline & Offline & Blended & Brain $+n-d$ & Digital & Blended & Offline & Offline \\
\hline 1705 & Offline & Offline & Blended & Brain + n-d & $\begin{array}{l}\text { Non- } \\
\text { digital }\end{array}$ & Digital & Online & Online \\
\hline 1706 & Offline & Offline & Blended & Brain $+n-d$ & $\begin{array}{l}\text { Non- } \\
\text { digital }\end{array}$ & Digital & Online & Offline \\
\hline 1707 & Offline & Offline & Blended & Brain $+n-d$ & $\begin{array}{l}\text { Non- } \\
\text { digital }\end{array}$ & Digital & Offline & Online \\
\hline 1708 & Offline & Offline & Blended & Brain $+n-d$ & $\begin{array}{l}\text { Non- } \\
\text { digital }\end{array}$ & Digital & Offline & Offline \\
\hline 1709 & Offline & Offline & Blended & Brain + n-d & $\begin{array}{l}\text { Non- } \\
\text { digital }\end{array}$ & Non-digital & Online & Online \\
\hline 1710 & Offline & Offline & Blended & Brain + n-d & $\begin{array}{l}\text { Non- } \\
\text { digital }\end{array}$ & Non-digital & Online & Offline \\
\hline 1711 & Offline & Offline & Blended & Brain + n-d & $\begin{array}{l}\text { Non- } \\
\text { digital }\end{array}$ & Non-digital & Offline & Online \\
\hline 1712 & Offline & Offline & Blended & Brain + n-d & $\begin{array}{l}\text { Non- } \\
\text { digital }\end{array}$ & Non-digital & Offline & Offline \\
\hline 1713 & Offline & Offline & Blended & Brain + n-d & $\begin{array}{l}\text { Non- } \\
\text { digital }\end{array}$ & Blended & Online & Online \\
\hline 1714 & Offline & Offline & Blended & Brain + n-d & $\begin{array}{l}\text { Non- } \\
\text { digital }\end{array}$ & Blended & Online & Offline \\
\hline 1715 & Offline & Offline & Blended & Brain $+n-d$ & $\begin{array}{l}\text { Non- } \\
\text { digital }\end{array}$ & Blended & Offline & Online \\
\hline 1716 & Offline & Offline & Blended & Brain $+n-d$ & $\begin{array}{l}\text { Non- } \\
\text { digital }\end{array}$ & Blended & Offline & Offline \\
\hline 1717 & Offline & Offline & Blended & Brain $+n-d$ & $\begin{array}{l}\text { Blend- } \\
\text { ed }\end{array}$ & Digital & Online & Online \\
\hline 1718 & Offline & Offline & Blended & Brain $+n-d$ & $\begin{array}{l}\text { Blend- } \\
\text { ed }\end{array}$ & Digital & Online & Offline \\
\hline 1719 & Offline & Offline & Blended & Brain + n-d & $\begin{array}{l}\text { Blend- } \\
\text { ed }\end{array}$ & Digital & Offline & Online \\
\hline 1720 & Offline & Offline & Blended & Brain $+n-d$ & $\begin{array}{l}\text { Blend- } \\
\text { ed }\end{array}$ & Digital & Offline & Offline \\
\hline 1721 & Offline & Offline & Blended & Brain + n-d & $\begin{array}{l}\text { Blend- } \\
\text { ed }\end{array}$ & Non-digital & Online & Online \\
\hline 1722 & Offline & Offline & Blended & Brain $+n-d$ & $\begin{array}{l}\text { Blend- } \\
\text { ed }\end{array}$ & Non-digital & Online & Offline \\
\hline 1723 & Offline & Offline & Blended & Brain + n-d & $\begin{array}{l}\text { Blend- } \\
\text { ed }\end{array}$ & Non-digital & Offline & Online \\
\hline 1724 & Offline & Offline & Blended & Brain + n-d & $\begin{array}{l}\text { Blend- } \\
\text { ed }\end{array}$ & Non-digital & Offline & Offline \\
\hline 1725 & Offline & Offline & Blended & Brain $+n-d$ & $\begin{array}{l}\text { Blend- } \\
\text { ed }\end{array}$ & Blended & Online & Online \\
\hline 1726 & Offline & Offline & Blended & Brain + n-d & $\begin{array}{l}\text { Blend- } \\
\text { ed }\end{array}$ & Blended & Online & Offline \\
\hline 1727 & Offline & Offline & Blended & Brain $+n-d$ & $\begin{array}{l}\text { Blend- } \\
\text { ed }\end{array}$ & Blended & Offline & Online \\
\hline 1728 & Offline & Offline & Blended & Brain + n-d & $\begin{array}{l}\text { Blend- } \\
\text { ed }\end{array}$ & Blended & Offline & Offline \\
\hline
\end{tabular}




\title{
Circuits of Labour: A Labour Theory of the iPhone Era
}

\author{
Jack Linchuan Qiu*, Melissa Gregg ${ }^{\dagger}$ and Kate Crawford ${ }^{\ddagger}$ \\ "The Chinese University of Hong Kong, Hong Kong, China, jacklqiu@gmail.com \\ ${ }^{\dagger}$ Intel Labs, USA, melissa.gregg@intel.com \\ ${ }^{\ddagger}$ Microsoft Research and MIT Center for Civic Media, NYC, USA, kate@katecrawford.net
}

\begin{abstract}
This paper questions the binary of material and immaterial labour in the information era. Instead, we propose a "circuits of labour" model, a holistic framework that helps connect various concepts and traditions in the study of labour and ICT (information and communication technology). Inspired by du Gay et al's "circuit of culture", we argue conventional frameworks need to be synthesized and updated to reflect fundamental changes and persisting issues of labor in our contemporary era, of which the iPhone is emblematic. On the one hand, our model consists of formal circuits, in which hierarchical domination is imposed by capital over the body of labour. On the other hand, it consists of informal circuits where relationships are defined communally between embodied practices and social and communicative capital. The informal and formal circuits of labour are "short-circuited" by survival labour and 'playbour', meaning either circuit may absorb productive energy from the other. This article then uses the case of Foxconn, the world's largest electronic manufacturer that also produces iPhones, to illustrate the usefulness of the "circuits of labour" model. We finally discuss the broader implications and questions for future research.
\end{abstract}

Keywords: labour, iPhone, circuit of culture, Foxconn.

Acknowledgement: This paper is a result from "the iPhone Workshop" organized by Jean Burgess, Larissa Hjorth and Ingrid Richardson in July 2009 at Queensland University of Technology, Australia. We would like to thank Carl DiSalvo and Maggie Leung for graph design and Dennis Leung for research assistance. We are also indebted to two anonymous tripleC reviewers, who provided excellent critique.

\section{Introduction}

The last fifteen years have seen an expansion of studies on the relationship between labour and the information and communication technologies (ICTs). While there has been a distinct "turn to labour" in ICT research (Fortunati 2007; Huws 2003; Wajcman 2008), the scholarship remains disconnected and fragmented across disciplines, methodological approaches and national particularities. New conceptual frameworks have been applied to the forms of labour in ICTs, from "free labour" and "playbour" (Terranova 2000; Kücklich 2005) to a focus on "networked" and "creative" labour (Brouillette 2009; Fuchs 2010; Xiang 2007). However, such terms have tended to focus on forms of paid and unpaid information work. Less attention has been paid to blue collar, "gray collar" (Qiu 2010), and "survival circuit" workers (Sassen 2004) also known as "generic labour" (Castells 1998).

In this project, we seek to bring together these threads of research into a single holistic framework which we call "circuits of labour". We take inspiration from a defining moment in the field of cultural studies, which took the arrival of the Sony Walkman as an iconic and rupturing event in the study of contemporary media and society (Du Gay et al. 1997). Doing Cultural Studies: The Story of the Sony Walkman introduced a "circuit of culture" model to illustrate the mutually linked considerations necessary to produce rich, veritable and convincing empirical and theoretical data. Following in this tradition, we propose to engage the iPhone as both a historic signifier and a rich empirical source for theory building, in order for us to better grasp persisting issues of labor, as well as their characteristics and adaptations, within the interconnected "circuits of labour". 
The iPhone is not just a smartphone. Rather, it "represents a distinctive moment, both in the very short history of mobile media and in the much longer history of cultural technologies" (Hjorth et al. 2012, 1). We take it as symbolic of a set of practices, both personal and industrial, in the contemporary era. This is not to aggrandize the iPhone's commercial success as "the most popular Apple product by far" (Beech and Jiang 2012, 32), or to champion "digital capitalism" (Schiller 2000). Nor do we intend to reject the device as simply a means of exploitation (Chan and Pun 2010), a "cult" (Belk and Tumblat 2005), or another techno-cultural "myth" (Mosco 2004; Turner 2004). Instead, we begin by taking the iPhone as representative of "a key moment of metastasis, when an already intimate, popularized technology expanded to encompass a host of media forms" (Crawford 2012, 219). We analyze the iPhone as a device that is used for various forms of communication, care-work, and micro-organization as well as a product that draws on multiple forms of labour in order to function and thrive.

While du Gay et al. used the term "circuit" to refer to a set of connected ideas, another common meaning of a circuit is "[t]he course traversed by an electric current between the two poles of a battery; the path of a voltaic current" (Oxford English Dictionary 1989, 229). Taking these notions together, our account of the circuits involved in the assembly, distribution and consumption of the iPhone will address some notable limitations in current theories of labor in media and communication studies today. Our "circuits of labour" theory reads the iPhone as (a) a vertical circuit of capital accumulation, (b) a horizontal circuit of social networking and body politics, and (c) the links, or short-circuits, between (a) and (b). Metaphorically speaking, the iPhone is our "battery", whose two poles are capital and the body, respectively. But this is more than a simple circuit. Instead, the "circuits of labour" is conceptualized as an "integrated circuit", a circuit comprised of many separate components.

This article elucidates this "circuits of labour" mode of understanding, why we need it, how to use it. In order to do so, we conduct a review of the significant literature on labour and ICTs, and outline how our approach can be used to reflect on and draw connections among the research currents. To illustrate our thinking, and critique it, we draw on data and fieldwork observations from the world's largest electronics manufacturer that produces most Apple products-including the iPhone-Foxconn (Chan and Pun 2010; Qiu 2012; Sandoval 2013). For more than two years, participatory action research has been conducted in China following a startling spate of employee suicides at Foxconn in 2010. Finally, we discuss the research results and their broader implications, given the global campaign against labour abuse at Foxconn (SACOM 2010; 2011).

In sum, the iPhone acts as a symbol for the amalgam of labor issues we tackle. This symbol is tangible yet powerful: it comes from far away, but becomes something intimate in our daily work and life (Gregg 2011). It expands conventional modes of exploitation, but also stands for alternative developments of the future. It draws on mythic imaginaries of constant connection and individual control, while silently extracting data about the user: sometimes with their knowledge and consent, sometimes without (Crawford 2012). For good or for bad, its enormous centrality in our era makes it an appropriate metaphor for the "circuits of labour" that surround us, subsume us, and substantiate us.

\section{Labour and ICT: An Overview}

An important change in recent years is the outpouring of labour studies conducted in the context of, or in relation to, ICTs. This should be fairly clear to readers of this tripleC special issue. The trend has become particularly notable since the global financial crisis of 2008-2009, probably because, for too long, "labor remains a blind spot of western communication studies" (McKercher and Mosco 2006, 493). In cultural studies and sociology we have also observed a similar "turn to labor" among leading ICT scholars (Fortunati 2007; Huws 2003; Wajcman 2008).

What, then, is labour? What are the kinds of labour issues being examined in this growing body of literature? What questions can now emerge, after labour meets ICTs?

First, there are studies of new media workers or "digital labour", whose delineation is above all technological. This is essentially about wage labour in ICT-based "new economy" 
environments, either in the particular sectors of so-called new media, Internet or digital industries (Heeks 2008; Neff et al. 2005; Xiang 2007), or in contexts where ICTs have disseminated in the larger labour market, at work and beyond (DiMaggio and Bonikowski 2008; Freeman 2002; Gregg 2011). The diffusion of ICTs for work and the importance of the IT industry for contemporary economy make it imperative to study labor relations in Internet companies (eg, Mayer-Ahuja and Wolf 2007), media organizations (eg, Deuze 2007), as well as other emerging sectors of work, including Indian software programmers (Xiang, 2007), Chinese "gold farmers (producers of virtual goods and services for online gamers)" (Heeks 2008), and Amazon Mechanical Turk workers (Irani 2013).

Despite sectoral and national difference, these studies all examine wage labour, how their work affects and is affected by ICTs, with increasing flexibility, and what are subsequent changes in their income and bargaining power. But which sector is completely untouched by ICTs today? Are there real differences between employees who work directly with computers and those who do not? While many studies find ICTs do contribute to improving welfare and upward mobility (eg, DiMaggio and Bonikowski 2008), others show that the rise of digital "piece work" is part of a broader shift toward outsourcing and casualization, hence weakening the labor market positioning of employees overall (eg, Deuze 2007; Xiang 2007).

A second thread of research goes from the celebratory "creative class" (Florida 2002) to the more critical "knowledge workers" (McKercher and Mosco 2006; Mosco and McKercher 2009) and "immaterial labour" (Lazzarato 1996; Fortunati 2007). Here, labour is defined, not by technologies or wages, but by its immateriality, meaning the symbolic, informational, or affective products and services generated through a wide range of work processes. While Florida and his followers argue the new "creative class" represents a higher level of modernity at work, others see "immaterial labor" as the deepening of capitalist domination from workplace to everyday life, from the corporeal to the cerebral (Brouillette 2009).

Does it really make sense to separate material labour from the immaterial, and to assume the former belongs to the Industrial Era, the latter the Information Era? Are the "creative" or "knowledge" workers a new labor aristocracy? How does this type of labour relate to capital? These are some of the gaps that exist in the current literature.

One tradition of theorizing "immaterial labour" began with Lazarrato (1996) and was further developed by Hardt and Negri $(2000 ; 2004)$, as well as Terranova in her essay 'Free Labor: Producing Culture for the Digital Economy' (2000). This notion has been very influential among critical studies of user-generated content (UGC) in recent years (Fuchs 2010; Paasonen 2010; Suhr 2009). In studies of online games, Kücklich (2005) introduces the idea of "precarious playbour", or the forms of unpaid labour (such as computer game modification or "modding"), which are done by players and are cost-free from the perspective of online game companies. Kücklich points to how certain activities are veiled by the perception that they are just "leisure activities" while they are simultaneously part of a wider economy of industrial innovation and value-generation.

This tradition is, however, criticized by post-Marxian scholars such as Arvidsson and Colleoni (2012), who argue against applying Marxist "labour theory of value" to online production practices because doing so would underestimate the importance of affect-based labor and financialization. For Hardt and Negri, "affective labour" and "caring labour" constitute a subset of "immaterial labour" $(2000,8)$. But feminist scholars contend this conceptualization is too limiting in that "the domestic sphere and more extensively the sphere of social reproduction" are more important bases for the growth of "immaterial labour", from the very beginning of its conceptual roots in Italian academia and activism (Fortunati 2007, 139).

Yet another line of research is about volunteer labour, now also adopting digital tools in social enterprises (Fish and Srinivasan 2012), online activist campaigns (Tatarchevskiy, 2011), nonprofit organizations (Mook et al. 2007), and "commons-based peer production" (Benkler and Nissenbaum 2006). Here, the labour at stake is not defined by its subordination to capital or power in the domestic sphere. Rather, individuals and communities expand existing gift economies, and create new ones online and off, with the hope of forming alternative power structures in global and local civil societies. One step further into the more radical leftist direction, we get to the much older ideals of digital anarcho-communism (Barbrook 
1997) and "cyber-Marx" (Dyer-Witheford 1999) that perceive the struggle over ICTs as the ultimate battle through which labor will be emancipated.

Finally, one can define labor by its relationship to the network society, whether workers are included as "self-programmable labor" or excluded and "downgraded" into "generic labour" (Castells 1998). This is a dichotomous model. On one side, there are bankers, realtors, famous artists, those well-paid, including self-employed, workers who do projects by themselves, although they face high risk and insecurity typical of "work in the new capitalism" (Sennett 1999). On the other side, there are nannies, janitors, sex-workers, and those dispossessed and underemployed struggling to make ends meet in the "survival circuits" (Sassen 2004) of this same era. If those in the former category exercise and enjoy more social and cultural capital relative to the latter, in each case workers "program", train and coax bodies, minds and performances to match the affective bearing of physically and emotionally demanding work.

The binary conception of networked labor has been complicated in recent years by studies such as Mayer (2011), which spans Brazil and the US. In the context of China, Zhao and Duffy echo Langman's notion of "internetworked social movements" (2005) and maintain that the institutional constraints over China's labor, from migrant workers and laid-off workers to low-level media workers, have been increasingly "short-circuited" (2007). Members of the "generic labour" category, although still in "survival circuits", have started to inter-connect, with each other and with other concerned citizens in China and beyond, through public intellectuals, NGOs, and transnational networking. This trend has accelerated since the global economic crisis (Hong 2010). Meanwhile, Qiu demonstrates that the phenomenal diffusion of "working-class ICTs" in Chinese cities has led to the rise of the "information have-less", a new lower-middle stratum in the country's network society, generating a new class of "network labor" (2009; 2012), ie, "a materializing pillar of the network society, parallel to the emergence of the network enterprise and the network state, globally and regionally" (2010, 81).

\section{The Need to Connect}

The above review shows that the overall picture of ICT-related labor studies consists of scattered pieces of knowledge, strung together by quite different approaches, dominated by loose fragments at national and local levels, disconnected from each other and from global contexts. There are many dividing cleavages: material or immaterial labour, affective or mechanized labour, networked or isolated labour, wage labour or "free" "volunteer" labour, creative or not-so-creative labour, suppressed/suppressing labour or liberated/liberating labour. It is a growing body of research, some with more classic Marxian lineage than others, often conducted by graduate students and emerging scholars. How shall we make sense of all these concepts and arguments, not only individually, but also in a way that enriches ICT research as well as labour studies as a whole? Indeed, if all the studies above are taken as products of "intellectual labour", one may well lament its lack of labour solidarity.

The "circuits of labour" model we propose is designed to connect conceptual developments at the crossroads between labor and ICTs, and to do so without discounting the significance of the corporeal and affective dimensions of the formal market sphere for labour. The impasse in attempts to theorize so-called "immaterial" labour stems from a largely Western notion of separate public and private spheres for labour, which removed waged labour from the home, and feminized many aspects of reproductive and domestic labour in the process. Our model seeks to disrupt this binary just as its circuit logic enables a systematic interrogation of the empirical case centered on the iPhone and Foxconn.

The metaphor of the circuit has long been present in scholarship that takes a network approach to labour, especially when this involves dispossessed and disadvantaged workers (Sassen 2004; Zhao and Duffy 2007). By contrast, du Gay et al's "circuit of culture" (1997) draws on a formulation first outlined by Johnson (1986) in the essay 'What is Cultural Studies 
Anyway? ${ }^{11}$ The model connects five moments of cultural practice: representation, identity, production, consumption, and regulation. The five moments are organized in a circle, in which cultural practices can relocate from any one moment to any other. This is a parsimonious and prominent framework. But if applied to issues of labor and ICTs, it would be too general, free flowing, even idealistic, to capture either entrenched power inequalities or possibilities of change.

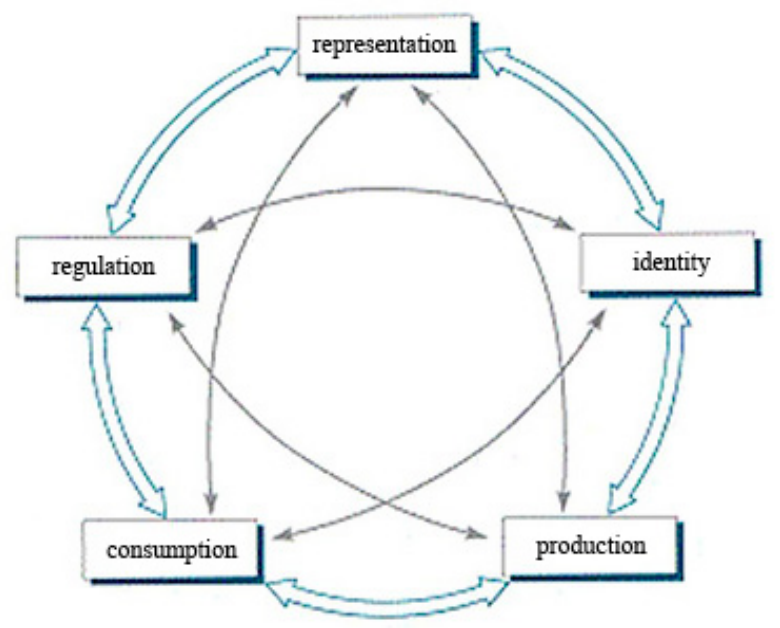

Figure 1: du Gay et al's circuit of culture (1997)

In the two examples-the Sony Walkman, an iconic device of the 1980s, and the iPhone, an icon of the early $21^{\text {st }}$ century-the "cultural circuit" depicts only one circle of events. Suppose the circuit of mainstream culture is problematic, how can one escape and start an alternative circuit? On the other hand, by calling it a "circuit of culture", there is the covert assumption that cultural labor deserves more scholarly attention vis-à-vis material labour. One of our goals is to bridge this gap between immaterial culture and material manufacture, in a similar move to Mayer's analysis of the New Television Economy (2011).

Much has changed since the neoliberal turn of the 1980s in both the culture of personal electronics and its manufacture. Consumption has been expanded; national regulation corroded; representation more infiltrated by corporate marketing; identity more contested; material production outsourced to countries like China and India (Castells 1998; Dyer-Witheford 1999; Gregg 2011; McKerner and Mosco 2006; Qiu 2009; Schiller 2005). A subsequent trend is the spatial separation between production and consumption in the global commodity chain, whereas in terms of content, a rhetoric of "prosumption" has also emerged especially with the diffusion of personal, portable devices. Typifying both trends is the iPhone, a flagship Apple product.

Apple Inc. is the "the largest U.S. company ever, measured by stock-market value" (Browning et al. 2012). At the end of 2011, Apple earns 53\% of its total revenue, and $67 \%$ of its gross profit, from the iPhone alone (Ray 2012). This is the best of times for capital. Is it the worst of times for labour? Following our description of the "circuits of labour", we posit the iPhone era is characterized by a remarkable regime of domination that exacerbates social inequality. At the same time, however, we note that the iPhone-and similar ICT productsalso stimulate and enable alternative developments toward change.

\footnotetext{
${ }^{1}$ Thanks to an anonymous TripleC reviewer, who points out Johnson grounded this essay in a reading of Marx's Grundrisse (1973), although du Gay et al (1997) made no reference to Marx. The analytical focus of their "circuit of culture" model is also clearly placed on audiences, rather than political economy structures, a pattern that is not uncommon in cultural studies.
} 


\section{The Circuits of Labour}

\subsection{Formal Circuit of Labour}

While many emphasize the "immaterial" and therefore, "revolutionary" and "weightless", aspects of the IT industry (Coyle 1998; Negroponte 1996), structural continuities can be clearly observed if we examine its formal industrial system and compare it with the history of Fordism. Despite the new spatial and temporal characteristics of "flexible accumulation", capital continues to dominate labour, which is centrally expressed through the persistent and fundamental subjugation of labourer's body into the hierarchical circuit of capitalist production and consumption.

At stake here is the entirety of the formalized IT economy, including hardware and software, electronic manufacturing, marketing, sales, services, and the processing of e-waste (Maxwell and Miller 2012). While some of these work processes may be more virtual than others, all of them necessarily rely on physical labour from hardware manufacture to infrastructure construction, from the transportation of parts and products to repair and e-waste processing.

Even the least material forms of labor input-for instance, by employees of software or advertising companies - have a physical dimension: they have to position their bodies in front of the computers and/or telephones, and expect to remain so for certain hours of the day. It is for this reason that software engineers from Hyderabad, India, called their wage labor system "body shopping" (Xiang 2007).

The body is the key, to be subjugated and subsumed, on this decentralized, globalized, and deceptively "immaterialized" shop floor of the IT industry. What is extracted from these bodies and their bodily movements (or non-movements) is, however, the same thing: surplus value, measured by time, and sometimes affect as well (Arvidsoon and Golleoni 2012; Fuchs 2010).

The essence of labour input remains time. Labour power is most formidable if it reclaims its control over time. If we use this classic labour theory of Marx's (1973) Capital, we can say labor politics has always been immaterial in a fundamental sense from the very beginning of modern capitalism. The iPhone era of the global IT industry does not challenge the established power structure of capital dominance in this regard. What we see is the reverse, as surplus value continues to be extracted and labour continues to have little agency regarding production and redistribution. The logic of capital domination has even been strengthened with the rise of financial capitalism and global outsourcing, both contributing greatly to the shaping of the IT industry today. Traditional trade unions and public authorities are left behind in their limited, usually local or national, sphere of governance. The IT industry facilitates the flight of capital. In so doing, it becomes a stronghold of new capitalism itself (Ross 2006; Schiller 2005). 


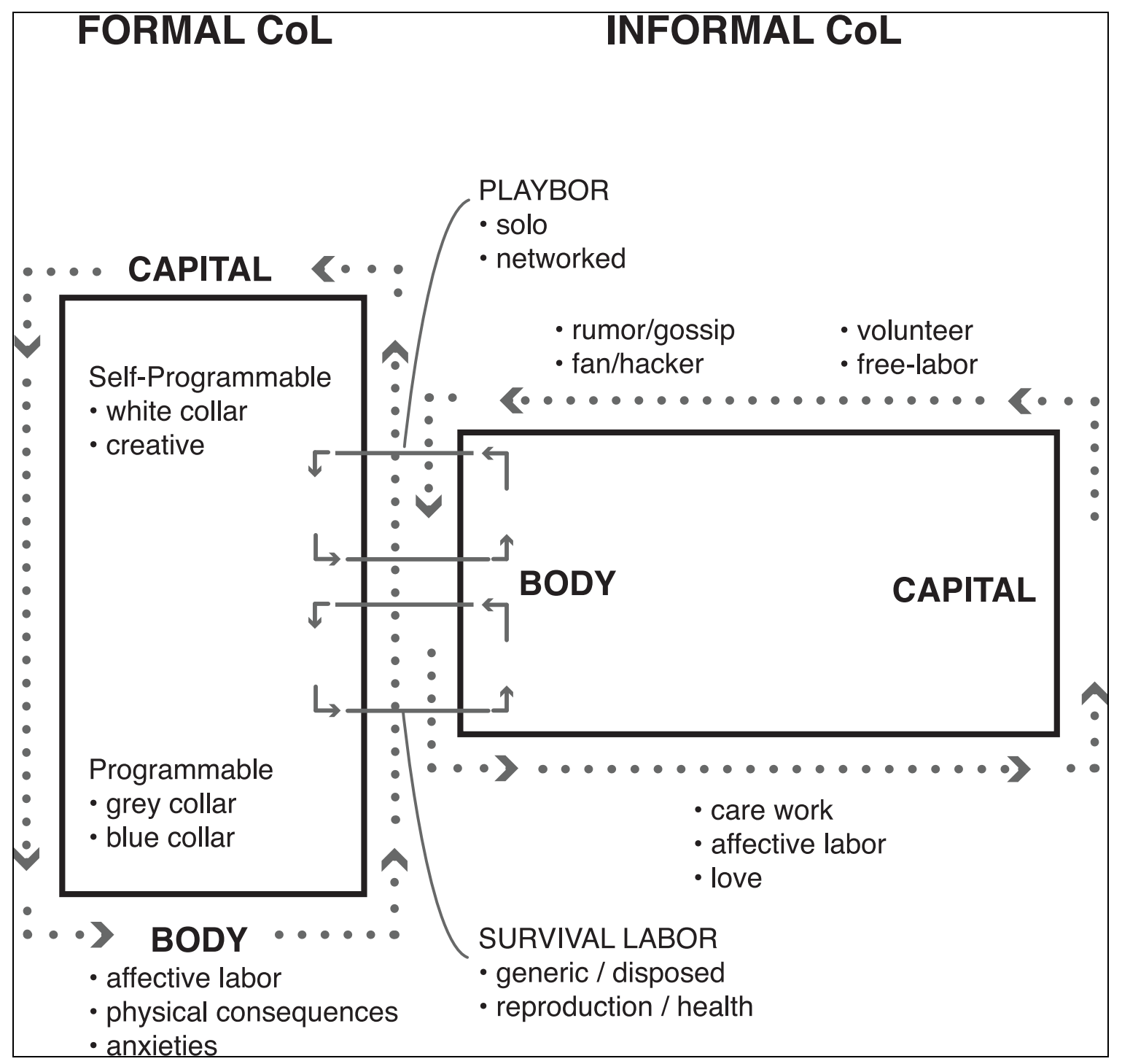

Figure 2: The formal and informal circuits of labor. To the left, the body and capital form a top-down circuit and a class-based hierarchy of constraint. To the right, the worker draws on internal resources and self-made networks to develop new avenues for pleasure, survival and resistance.

The hierarchical circuit of labour on the left of the diagram is not entirely new, although its specific expressions reflect the overall structural transformation of global capitalism as well as the peculiarities of the IT industry. The top-down domination of capital in formal labour processes-in this case protected and locked down by contracts, intellectual property rights regimes, and education/credentialing systems-is expressed through the increasing polarization and internal stratification of labor itself. Some of these are durable patterns, for example, the differentiation of labour aristocrats from ordinary members of the working class, of white collars from blue collars, and of self-programmable labor from generic labour (Castells 1998). Yet there are also new patterns with a particular spatial spin. While in the West, including Japan and the Asian "tiger" economies of South Korea, Taiwan, Hong Kong, and Singapore, the contradiction between well-paid self-programmable labour and disposable generic labor has become more acute, middle-rank jobs in the global IT industry have moved to the more globalized regions of the developing world, most decisively China and India. This has given rise to a new category of the "information have-less" (ie, people caught in between the haves and the have-nots of the digital divide), which constitutes the social basis for "programmable labor": people who perform "simplified tasks in the new information industry" (Qiu 2009, 9). 
Examples for such programmable labour, also known as "gray collars" in the Chinese context, include software testers, low-end graphic designers, quality control personnel, and database input personnel. They are called "gray collars" because allegedly their company uniforms are often gray in color and their work, while often performed using computers, is repetitive and labor-intensive in nature (Qiu, 2010). Applying the notion of gray collars to India, it would include such positions as lower-rank software engineers and call center personnel. The term "programmable" highlights the ruthlessness and inhumanity of the labor process, determined in classic Taylorist fashion, which still constitutes much of the production process in this iPhone era, as in Foxconn.

In our illustration of the formal and hierarchical circuit, white-collar self-programmable workers are positioned higher than gray collars, who in turn enjoy more benefits than blue collars. Generic labour may enter the formal circuit when demand exceeds supply at the very bottom of the system, although they can also enter the rank of survival labor that acts as a reserve army, whose existence supposedly helps discipline those who remain in the formal economy. Indeed, white collars can also be ejected from the formal circuit at critical moments like the dot-com crash of 2000-2001. Hence, even these presumably more "treasured" workers of the self-programmable rank are not necessarily better off than gray collars and blue collars in terms of their sense of risk, level of stress, and social isolation (Sennett 1999; Gregg 2011). The pervasiveness of such a disempowerment effect from the white collars to generic labor betrays the hierarchical nature of this formal circuit of labor, hidden behind the rosy camouflage of the New Economy rhetoric. After all, the logic of capital comes from the top down, imposing control across all labor strata-or so is its aim-so that surplus value can be ensured to flow from the bottom up, enabling the next round of accumulation and continued subsumption of body politics in its profitable circuit of labour.

\subsection{Informal Circuit of Labour}

The limitations of this "formal" circuit of labour have long been evident, not least due to substantial feminist critiques of the 1970 s and $80 \mathrm{~s}^{2}$ The more integrated, global economy of the present calls for still further theoretical refinement. Recent work on the international trade in surrogacy, clinical trial testing and cell harvesting (eg, Cooper and Waldby 2014) shows how the body can be used productively for profit-often by women and others suffering minority status in particular contexts. This remains the case for other "informal" or dubiously regulated sectors of the market economy, such as prostitution, which has a long history of association with "white-collar" as much as other "formal" labour categories (Allison 1994).

For our purposes, the informal circuits of labour attached to the iPhone produce a lateral circuit between social or communicative capital on one hand and the "creative" or "reproductive" body on the other (see Figure 2). On the user/consumption side, the communicative capacity of the device provides new avenues for the gendered experience of care work, which now not only includes the actual provision of companionship but new social and psychological dependencies. The full extent of women's affective labour, which in the domestic sphere includes "affect, care, love, education, socialization, communication, information, entertainment, organization, planning, coordination, logistics" (Fortunati 2007, 144), finds new coherence through the iPhone. For working women, regardless of their position in the hierarchy of the formal circuits of labour, the iPhone annihilates prior separation between the paid and unpaid labour obligation. Spatial distinctions dissolve in a loop of competing communicative demands which Gregg (2011) describes as "presence bleed". The affective labour of the intimate device is a feature of work in cognitive capitalism.

If women's growing centrality to the formal and informal economy makes their experiences representative (Morini 2007; McRobbie 2010), what remains to be acknowledged are the forms of capital such workers accrue through sometimes highly gendered expertise (Hakim 2010). The gradual feminization of the workplace through increased participation is mirrored

\footnotetext{
${ }^{2}$ If political movements often drove recognition of these inadequacies-for instance, in the "wages for housework" campaigns in the UK and Italy (see Oakley, 1974; Frederici 2012)—they also offer a precedent for the scholaractivist collaborations we will see in the recent investigations of Foxconn.
} 
in management strategies inviting employees to express their feelings in the workplace for the benefit of the company and to ensure smooth collegiality amongst team members (Illouz 2007; Boltanski and Chiappello 2005). Meanwhile women's competence in genres of "talk" whether in the attempt to generate good feeling through "deep acting" (Hochschild 1983) or the more volatile terrain of gossip and rumour offer further resources that we will explore shortly.

The informal circuits also encompass the variety of volunteer, gift, and pirate exchanges online that are enabled by online connectivity. Whether it is the fan labour contributing to the improvement of a cherished media text (Andrejevic 2008) or the "free labour" (Terranova 2000) of app design, testing and development, the iPhone has protracted the start-up company boom beyond the optimistic peak of social media and Web 2.0 to the data-fuelled appjams and hackathons of today (Gregg and DiSalvo 2013). In other areas, the host of torrent sites distributing locked-down proprietary content in textual, audio, and visual form are the online equivalent of the trade in "fake", "bandit", and "pirate" products filling a similar and substantial market need (Ho 2010).

From China and Vietnam to Turkey and sub-Saharan Africa, fake iPhones, a popular type of "bandit phone" or "shanzhaiji", are an extreme case of network labour subverting the conservative capitalistic logic-a display of bottom-up creativity, which may be channelled back into the formal system, for example, via "licensed" bandit phone manufacturers (Wallis and Qiu 2012). One should not be surprised that these inexpensive bandit phones played an important role in spreading rumours, and even hate speech, that led to ethnic riots in various developing regions of the world, from China to the Middle East, showing how "mere" talk (communication) can lead to material bodies assembling in action (Qiu 2009). A more recent expression of this informal communication circuit is the Yue Yuen shoe factory strike that involved more than 30,000 smartphone-equipped workers in Guangdong and Jiangxi (Kaiman, 2014).

\subsection{Short Circuits}

The formal and informal circuits as we have depicted them are not insulated from each other. While structural forces of "informalization" in the social, economic, and regulatory realms push labour processes from the formal circuits to the informal ones (Sassen 1998), online and real-world communities as well as civil society networks also build collective identity, even solidarity, using tools made through formal circuits. There are two main "short circuits". One is "survival labour" located in what Sassen terms "survival circuits" (2004), where dispossessed members of the working class become re-connected with each other and with other concerned citizens by joining alternative networks, regionally or transnationally.

On the other hand, there is "playbour", whose original form is found among computer gamers, either individually or networked as hacker/geek groups, which is actively exploited by corporations as free labour (Kücklich 2005). This also includes other networks such as fan culture (Jenkins 2006), or unwitting forms of value extraction, such as iPhone users whose location data was tracked by Apple without their knowledge (Crawford 2012), all providing crucial resources drawn from the informal circuits to allow the formal circuits to continue to expand and evolve (see also Lobato et al. 2011).

This is, however, describing only one type of flow-of capital, body, and creativitybetween the formal and the informal. While formal circuits of labour can short-circuit the informal for the benefit of the former, the reverse also happens when informal circuits draw material and immaterial resources from the formal for the building of alternative, even progressive networks, as the following case of Foxconn factory workers demonstrates.

\section{5. iPhone and Foxconn}

Just as Ford and the Model $\mathrm{T}$ automobile have become emblematic of the Fordist era, we argue Apple can be seen as an emblem of contemporary capitalist world order and the $\mathrm{iPh}$ one as a prototype for labour processes in the twenty-first century. The particular labor formations have distinctive globalized and networked characteristics as we have shown in our 
illustration of circuits of labour. In this sense, Apple is a typical global network enterprise with the employees in its headquarters in Cupertino, California, being white-collar selfprogrammable labour, and the Foxconn assembly-line workers in China - and increasingly elsewhere (Andrijasevic et al. 2013)—being grey-collar programmable labour and blue-collar labour.

It is critical to pay close attention to the material aspect of ICT production processes, despite the popular depiction of the "weightless" immaterial ICT industry (Sandoval 2013). Foxconn, the world's largest Apple subcontractor, once had more than 200,000 workers in one facility in Longhua, a factory district of Shenzhen in south China also known as "iPod City" (Webster 2006). In a few years, the Longhua factory grew to about a 400,000 population (Pun et al. 2011) and the total number of Foxconn employees in China exceeded 1 million in 2012 (Markoff 2012). Foxconn has a notorious "military-style" management system, which abused workers and caused at least 17 workers attempting suicide in the first eight months of 2010 (Chan and Pun 2010), an unprecedented tragedy in the history of electronics manufacture.

Terry Guo, Foxconn owner and CEO once publicly stated, "as human beings are also animals, to manage one million animals gives me a headache" (Markoff 2012). Calling workers "animals" is a candid reflection that the factory only values the bodily input of its labour force, not other aspects of its humanity. Yet in the larger industrial system of iPhone production, although Foxconn accounts for the bulk of employees-mostly grey-collar, programmable labour, but also some self-programmable and generic labour-it is, nonetheless a colossal "body" that serves the "brain", ie, Apple's R\&D and marketing branches, and transnational capital (Chan and Pun 2010). Foxconn achieves its goals by controlling workers' bodies, at work and off duty, physically and mentally, through formal contractual and managerial measures that brew an "anti-social" culture. This culture atomizes workers through an extreme version of corporate biopolitics (Pun et al. 2011).

Suicide was but one way the formal circuit of labour at Foxconn spins workers off as survival labour, which also happens daily through work injuries and long overtime that hurts workers' health or results in more injuries at work. The most common injury is workers' fingers being cut or crushed by machinery. According to local labour activists, Foxconn was once responsible for about half of all finger-related work injuries in key hospitals of Shenzhen's factory zones in Longhua and Guanlan. To contextualize this datum, in Shenzhen and the surrounding Pearl River Delta of south China, "factory workers lose or break about 40,000 fingers on the job each year" (Barboza 2008). Although only some of these work injuries occurred in IT-industry plants like iPod City, electronic manufacturing is particularly hazardous for hand injuries: workers often have to work very long hours with heavy machinery that moulds and cuts metal parts, factory owners and managers often prioritize productivity over safety, and electronics products like the iPhone have been the fastest growing Chinese exports in recent years.

Foxconn resolved many of the injury or suicide cases through extra-legal means, including several cases that we followed closely (Pun et al. 2011; Qiu 2012). Since 2010, it has also used large number of "student interns", including child labour, to generate more profit by evading China's labour contract law (Mozur 2012a), thus offering yet another illustration for the informalization process: formal circuits cannot be sustained for long without tapping into informal circuits.

This is true for not only Foxconn but also Apple, whose extraordinarily high profit margin cannot be maintained without the fan culture known as "Apple cult" (Beech and Jiang 2012; Belk and Tumblat 2005). That is, in addition to material manufacturing, the sales and technical-support personnel for the world's fast growing IT industry also belong to the rank of programmable labour and grey collars, and those sweeping the floor in shopping malls or moving iPhone boxes belong to blue collars and generic labour.

But Apple certainly did not invent outsourcing and offshoring. The global commodity chain and the unequal international division of labour existed long before the iPhone. What Apple adds significantly to the existing model is iTunes, a further utilization of technical means to lock down content and applications within a given iPhone. There are cycles of boom and bust 
in the iTunes app market, despite the oft-told success stories of "playbour" game designers earning fortunes through the App Store (Farrell 2009). But unlike the gold rushes of the past, Apple's iTunes authorization, distribution, and charging system is not only massive in scale and diversity but also much more controlled through technical and legal codes that limit how content flows, and under what revenue sharing schemes. For example, consider those $\mathrm{iPh}-$ one game testers whose job consists of spending many hours a day concentrating on the operation of apps for the device. Although they don't risk losing or breaking their fingers, they face working lives filled with repetition and low wages along with the assembly-line workers in the iPod City.

Growing awareness of the massive exploitation behind the iPhone, especially at Foxconn, has triggered civil society groups, labour activists, creative designers, and concerned citizens to come together regionally and transnationally to establish alternative networks and their own informal circuits of labour. Following the Foxconn suicides in 2010, more than 60 students and scholars from 20 universities in Hong Kong, Taiwan, and Mainland China formed a volunteer investigation team to study Foxconn's working conditions and provide support for suicide survivors and their families (Pun et al. 2011). This group then connected with other NGOs, citizen reporters, media organizations, and individual workers in and outside Foxconn.

The means of connection include face-to-face and small-group contact, but increasingly they rely on digital social networks of blogs, micro-blogs (Weibo), QQ (Chinese online instant-messenger popular among workers), online forums, mobile phones, Internet video, and most recently their own Chinese-language web portal, www.iLabour.org. There is no formal process for people to join this new anti-sweatshop campaign. Rewards are social, not monetary. Embodied experience is still essential for the fieldwork, including several students who volunteered to work inside Foxconn as "interns" for periods up to one month. Their physical presence was essential to the social and communicative capital that circulates in the volunteer community. In so doing, the informal circuits keep expanding. As Foxconn moves its production facility into China's hinterlands, this informal circuit also starts to include more active members from the inland regions.

This is a transborder movement that goes beyond China's mainland. The 20-university consortium, for example, was first initiated in Taiwan in June 2010, when Pun Ngai, a professor from Hong Kong Polytechnic University was visiting. Several Taiwanese universities and research institutes became the first to join the consortium partly because Foxconn is a Taiwanese company. During the joint investigation that has been carried out since July 2010, Scholars and Students Against Corporate Misbehavior (SACOM, http://sacom.hk), a labour NGO consisting of mostly Hong Kong college students, became a key regional hub of coordination (Sandoval 2013). This was due to Hong Kong's strategic location adjacent to Mainland China and its tolerance of grassroots labour organizing, which is still severely oppressed in the mainland. SACOM also connects the consortium with the global anti-sweatshop movement, working closely with international NGOs such as Good Electronics (http://goodelectronics.org/) and makelTfair (http://makeitfair.org/en).

Particularly notable is the case of Tian $\mathrm{Yu}$, who survived her jump from a Foxconn dormitory in March 2010 and has been receiving care from campaign volunteers since July 2010. Tian Yu's paralyzed body is an illustration of what we term "survival labour": acquiring social and cultural capital from the informal circuit of labour, she became the face of the antiFoxconn movement, mobilizing further awareness and activism. Figure 3 is the cover image of the Pun et al. book, showing Tian Yu in her wheelchair. She also lent her voice to the short film Deconstructing Foxconn (https://vimeo.com/17558439). Much more than a passive recipient of affective labour, Tian Yu and her family in rural Hubei also provide their own input, materially and immaterially. Since early 2011, they have been making handcrafts that are sold online as well as offline fair-trade stores and promoted through Weibo, China's Twitterlike services (Qiu 2012).

Another transnational development is PhoneStory, a smart phone game produced by Molleindustria, a radical game designer. This is an educational game satirically addressing four issues in the hierarchical circuits: child labour used in extracting Coltan in Congo, Foxconn 
suicides in China, consumerism among iPhone users, and hazardous e-waste processing in Bangladesh. Originally the game was designed for gamers using either Apple's App Store or Android Market (ie, Google Play), but within hours it was censored by App Store (Dredge 2011). However, the Android Market sales still went well and Molleindustria was able to donate $\$ 6000$ to Tian Yu via SACOM. Figure 4 shows the "business model" of this alternative gaming circuit, which has been successful, socially and commercially, by tapping in to Apple/iPhone fandom.

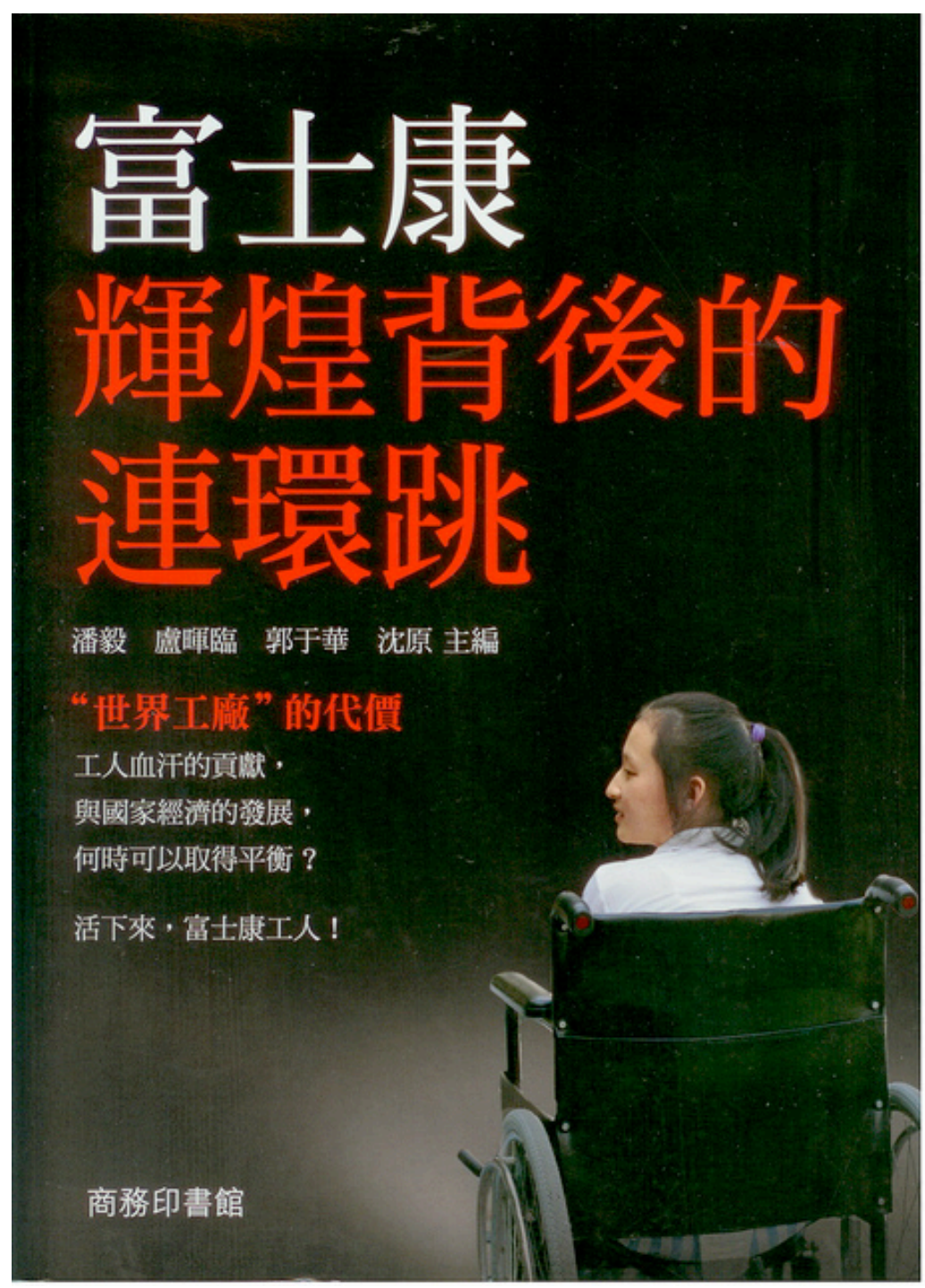

Figure 3: The Pun et al. (2011) book showing Tian Yu, a Foxconn survivor, in her wheel chair 


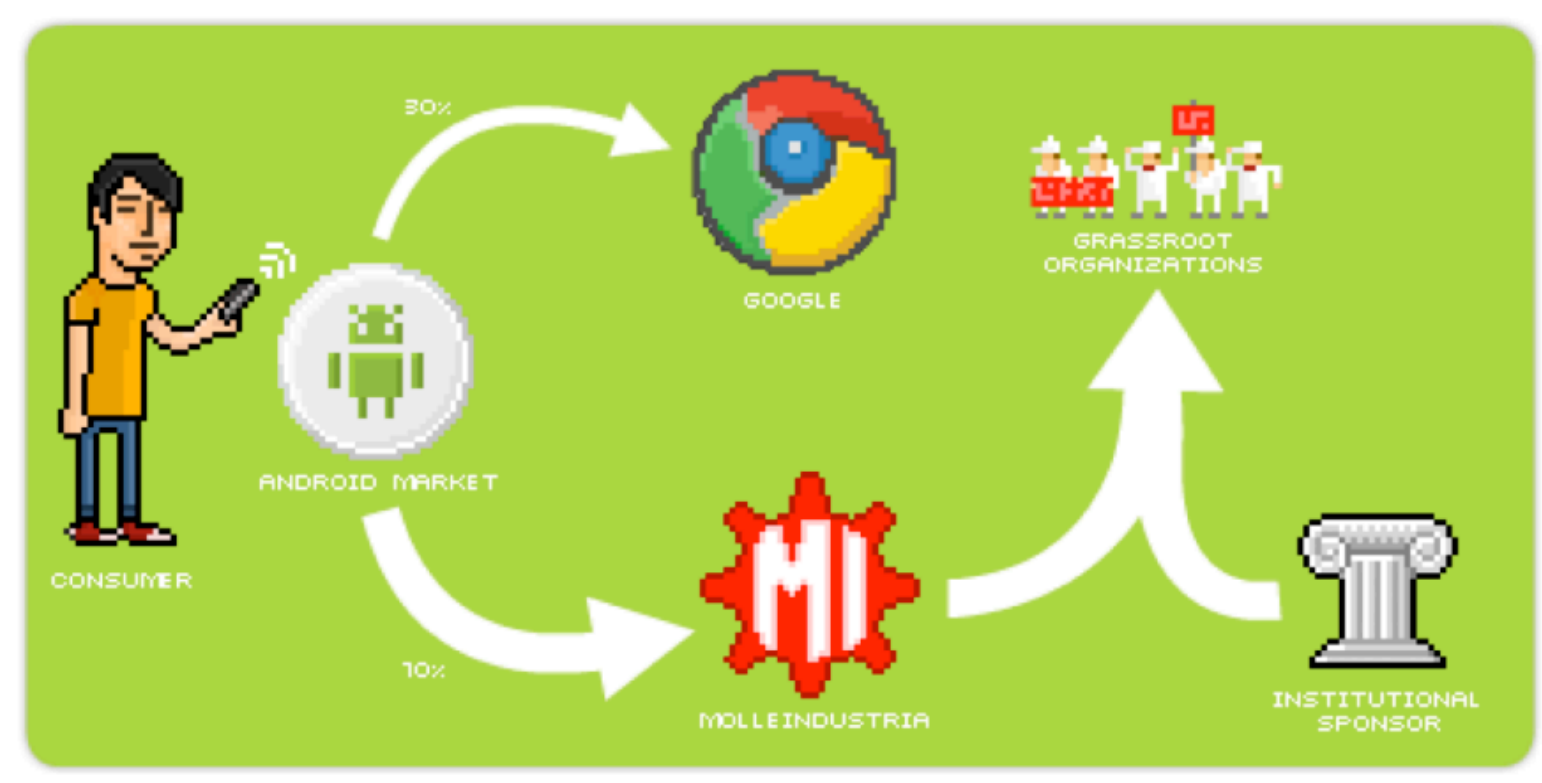

Source: http://www.phonestory.org/

Figure 4: The business model of Molleindustria, a group of radical game designers who created the educational game PhoneStory to address in part the Foxconn tragedies

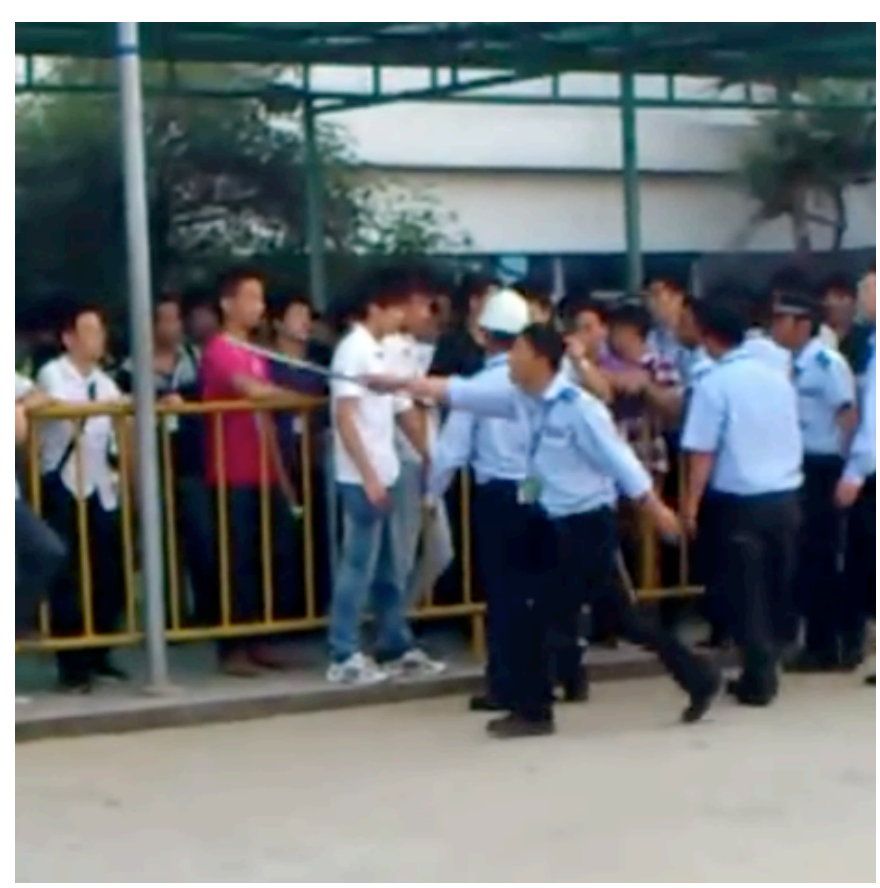

Source: Authors' collection

Figure 5: Mobile phone video image of security guards beating and threatening workers at one Foxconn gate 
Finally, a growing trend since 2011 is the proliferation of user-generated content (UGC), especially in the form of mobile-phone images captured and shared by Foxconn workers themselves. This differs from the conventional pattern of NGOs, activists, or students speaking out for workers, or helping workers to disseminate content (Chan and Pun 2010). As a result, there are many more "raw" images from Foxconn workers. There is a growing genre of Internet videos showing Foxconn security guards beating up or threatening workers who dare to disobey.

Figure 5 shows a screen capture from a 4-minute video, which generated not only anger but also rumour about yet another clash at a Foxconn factory gate in June 2011. This is a moment of playbour short-circuit, where informal, online popular culture can be manipulated to serve the formal circuit of labour, cultivating fear among workers. This is Foxconn's version of the "happy slapper", when violent assaults are recorded on camera phones and shared online. However, not all these attempts to use the informal to serve the formal ended up being successful. For instance, during the worker uprising at Foxconn's Taiyuan plant in September 2012, police and guards reportedly targeted workers who tried to record the event with their mobile phones (Mozur 2012b), showing that the short circuits move in both directions and Foxconn was significantly concerned about the consequences of these circulating video "rumours".

\section{Concluding Remarks}

"Circuits of labour" is a holistic framework that helps connect various concepts and traditions in the study of labour and ICTs, from communication and cultural studies to political economy and feminist traditions. More than a synthesis of existing conceptual frameworks, the circuits of labour model offers a closer interrogation of the interplay between labor and ICTs in our contemporary era, recognizing the inventive and capacious work of the body under capital. Through a focus on the iPhone and Foxconn, our analysis brings different approaches - material or immaterial labour, affective or mechanized labour, networked or atomized labour, wage labour or "free" "volunteer" labour-into productive relationship. In the process, our decision to write collaboratively across countries, institutions, and companies also intends to model the new conversations that are emerging as much as they are necessary to address the complexity of labour relations and mutual interdependence in the "Asian Century". Media studies needs better measures to reflect the shifts taking place in the geopolitics of production and consumption, which now involves the coordination and surveillance of vast corporate logistics enterprises that escape any one national perspective. The "circuits of labour" model is just one attempt to reflect the significance of these changes, and will hopefully generate many more.

\section{References}

Allison, Anne. 1994. Nightwork: Sexuality, Pleasure, and Corporate Masculinity in a Tokyo Hostess Club. Chicago: University of Chicago Press.

Andrejevic, Mark. 2008. Watching Television without Pity: The Productivity of Online Fans. Television \& New Media 9 (1): 24-46.

Andrijasevic, Rutvica, Devi Sacchetto and Nuran Gülenç. 2013. The Fox at Europe's Door: Foxconn in Turkey. Open Democracy. Accessed May 5, 2014.

Arvidsson, Adam and Elanor Colleoni. 2012. Value in Informational Capitalism and on the Internet. The Information Society 28 (3): 135-150.

Barboza, David. 2008. In Many Chinese Factories, Loss of Fingers and Low Pay. New York Times.. Accessed April 7, 2014, from http://www.nytimes.com/2008/01/05/business/worldbusiness/05sweatshop.html?pagewanted=all\& $r=0$

Barbrook, Richard. 1997. The Digital Economy. Nettime.org. Accessed August 20, 2012. http://www.nettime.org/Lists-Archives/nettime-l-9706/msg00143.html

Beech, Hannah and Chengcheng Jiang. 2012. The Cult of Apple in China. Time. July 2, 2012: 30-35. 
Belk, Russell W. and Gülnur Tumbat. 2005. The Cult of Macintosh. Consumption, Markets \& Culture 8 (3): 205-217.

Benkler, Yochai and Helen Nissenbaum. 2006. Commons-Based Peer Production and Virtue. Journal of Political Philosophy 14 (4): 394-419.

Boltanski, Luc and Eve Chiapello. 2005. The New Spirit of Capitalism. London; New York: Verso.

Brouillette, Sarah. 2009. Creative Labor. Mediations 24 (2):140-149.

Browning, E.S., Steven Russolillo and Jessica Vascellaro. 2012. Apple Now Biggest-Ever U.S. Company. Wall Street Journal. Accessed April 7, 2014.

http://online.wsj.com/news/articles/SB10000872396390443855804577601773524745182

Castells, Manuel. 1998. The End of Millennium. Oxford, UK: Blackwell.

Chan, Jenny and Ngai Pun. 2010. Suicide as Protest for the New Generation of Chinese Migrant Workers: Foxconn, Global Capital, and the State. The Asian Pacific Journal: Japan Focus. Accessed August 16, 2012, from http://www.japanfocus.org/-Ngai-Pun/3408

Cooper, Melinda and Catherine Waldby. 2014. Clinical Labor: Tissue Donors and Research Subjects in the Global Bioeconomy. Durham: Duke University Press.

Coyle, Diane. 1998. The Weightless World: Strategies for Managing the Digital Economy. Cambridge, MA: The MIT Press.

Crawford, Kate. 2012. Four Ways of Listening with an iPhone. In Studying Mobile Media: Cultural Technologies, Mobile Communication, and the iPhone, edited by Larissa Hjorth, Jean Burgess, and Ingrid Richardson, 213-228. London: Routledge.

Deuze, Mark. 2007. Media Work. Cambridge, UK: Polity.

DiMaggio, Paul, and Bart Bonikowski. 2008. Make Money Surfing the Web: The Impact of Internet Use on the Earnings of U.S. Workers. American Sociological Review 73 (2): 227-250.

Dredge, Stuart. 2011. Apple Bans Satirical IPhone Game Phone Story from Its App Store. The Guardian. Accessed April 7, 2014. http://www.guardian.co.uk/technology/appsblog/2011/sep/14/apple-phone-story-rejection

Du Gay, Paul, Stuart Hall, Linda James, Hugh Mackay and Keith Negus. 1997. Doing Cultural Studies: he Story of the Sony Walkman. London: Sage.

Dyer-Witheford, Nick. 1999. Cyber-Marx: Cycles and Circuits of Struggle in High-Technology Capitalism. Urbana; Chicago: University of Illinois Press.

Farrell, Michael B. 2009. One Billion IPhone Apps Downloaded. But How Many are Worth It? The Christian Science Monitor. Accessed April 7, 2014, from http://www.csmonitor.com/Innovation/Tech-Culture/2009/0423/one-billion-iphone-apps-but-howmany-are-worth-downloading

Fish, Adam and Ramesh Srinivasan. 2012. Digital Labor is the New Killer App. New Media \& Society 14 (1): 137-152.

Florida, Richard. 2002. The Rise of the Creative Class. New York: Basic Books.

Fortunati, Leopoldina. 2007. Immaterial Labor and Its Machinization. Ephemera: Theory \& Politics Organization 7(1): 139-157.

Frederici, Silvia. 2002. Revolution at Point Zero: Housework, Reproduction, and Feminist Struggle. PM Press.

Freeman, Richard B. 2002. The Labour Market in the New Information Economy. Oxford Review of Economic Policy 18 (3): 288-305.

Fuchs, Christian. 2010. Labor in Informational Capitalism and on the Internet. The Information Society 26 (3): 179-196.

Gregg, Melissa. 2011. Work's Intimacy. Cambridge, UK: Polity.

Gregg, Melissa and Carl DiSalvo. 2013. The Trouble With White Hats. The New Inquiry. Accessed May 6, 2014. http://thenewinquiry.com/essays/the-trouble-with-white-hats/

Hakim, Catherine. 2010. Erotic Capital. European Sociological Review 26 (5): 499-518.

Hardt Michael and Antonio Negri. 2000. Empire. Cambridge, MA: Harvard University Press.

Hardt Michael and Antonio Negri. 2004. Multitude. New York: Penguin Press.

Heeks, Richard. 2008. Current Analysis and Future Research Agenda on "Gold Farming": Real-World Production in Developing Countries for the Virtual Economies of Online Games. Development Informatics Working Paper Series (32). Manchester: Institute for Development Policy and Management. Accessed August 19, 2012. http://www.sed.manchester.ac.uk/idpm/dig

Hjorth, Larissa, Jean Burgess, Ingrid Richardson, edits. 2012. Studying Mobile Media: Cultural Technologies, Mobile Communication, and the iPhone. London: Routledge.

Ho, Josephine. 2010. ShanZhai: Economic/Cultural Production through the Cracks of Globalization. Plenary presentation, ACS Crossroads: 2010 Cultural Studies Conference, Hong Kong, June 1721. 
Hochschild, Arlie Russell. 1983. The Managed Heart: Commercialization of Human Feeling. Berkeley: University of California Press.

Hong, Yu. 2010. Will Chinese ICT Workers Unite? New Signs of Change in the Aftermath of the Global Economic Crisis. Work, Organization, Labor and Globalization 4 (2): 60-82.

Huws, Ursula. 2003. The Making of a Cybertariat: Virtual Work in a Real World. London: The Merlin Press.

Illouz, Eva. 2007. The Rise of Homo Sentimentalis. In Cold Intimacies: The Making of Emotional Capitalism, 1-39. London: Polity Press.

Irani, Lilly. 2013. The Cultural Work of Microwork. New Media and Society (Preprint). DOI: $10.1177 / 1461444813511926$.

Jenkins, Henry. 2006. Convergence Culture: Where Old and New Media Collide. New York: NYU Press.

Johnson, Richard. 1986. What Is Cultural Studies Anyway? Social Text 16 (Winter): 38-80.

Kaiman, Jonathan. 2014. Strike Spreads at Chinese Supplier to Adidas and Nike. The Guardian. Accessed May 12, 2014. http://www.theguardian.com/world/2014/apr/22/strike-spreads-chinesesupplier-adidas-nike-yeu-yuen-factory-jiangxi-guangdong

Kücklich, Julian. 2005. Precarious Playbor: Modders and the Digital Games Industry. The Fibreculture Journal 5. Accessed April 7, 2014. http://www.journal.fibreculture.org/issue5/kucklich.html

Langman, Lauren. 2005. From Virtual Public Spheres to Global Justice: A Critical Theory of Internetworked Social Movements. Sociological Theory 23 (1): 42-74.

Lazzarato, Maurizio. 1996. Immaterial Labor. In Radical Thought in Italy: A Potential Politics, edited by Paolo Virno and Michael Hardt, 132-146. Minneapolis, MN: University of Minnesota Press.

Lobato, Ramon, Julian Thomas and Dan Hunter. 2011. Histories of User-Generated Content: Between Formal and Informal Media Economies. International Journal of Communication (5): 899-914.

Markoff, John. 2012. Skilled Work, Without the Worker. The New York Times. Accessed April 7, 2014. http://www.nytimes.com/2012/08/19/business/new-wave-of-adept-robots-is-changing-globalindustry.html?pagewanted=all

Marx, Karl. 1973. The Grundrisse. Translated by Martin Nicolaus. London: Penguin Books.

Mayer, Vicki. 2011. Below the Line: Producers and Production Studies in the New Television Economy. Durham, NC: Duke University Press.

Mayer-Ahuja, Nicole and Harald Wolf. 2007. Beyond the Hype: Working in the German Internet Industry. Critical Sociology 33 (1/2): 73-99.

Maxwell, Richard, and Toby Miller. 2012. Greening the Media. New York: Oxford University Press.

McKerner, Catherine and Vincent Mosco, edited. 2006. Special issue on the laboring of communication. Canadian Journal of Communication 31 (3).

McRobbie, Angela. 2010. Reflections on Feminism, Immaterial Labour and the Post-Fordist Regime. New Formations 70: 60-76.

Mook, Laurie, Femida Handy and Jack Quarter. 2007. Reporting volunteer labor at the organizational level: A study of Canadian nonprofits. Voluntas: International Journal of Voluntary and Nonprofit Organizations 18 (1): 55-71.

Morini, Cristina. 2007. The Feminization of Labour in Cognitive Capitalism. Feminist Review 87: 40-59.

Mosco, Vincent. 2004. The Digital Sublime: Myth, Power, and Cyberspace. Cambridge, MA: MIT Press.

Mosco, Vincent and Catherine McKercher. 2009. The Laboring of Communication: Will Knowledge Workers of the World Unite? Lanham, MD: Lexington Books.

Mozur, Paul. 2012. Foxconn Factory in China Used 14-year-old Workers. The Wall Street Journal. Accessed April 7, 2014.

http://online.wsj.com/news/articles/SB20000872396390443675404578060422448515346

Mozur, Paul. 2012. Factory Riot Spotlights Breaking Point in China. The Wall Street Journal. Accessed April 7, 2014. http://live.wsj.com/video/factory-riot-spotlights-breaking-point-inchina/C1A831DB-6D09-4431-888A-6524B2BEE2C0.html

Negroponte, Nicholas.1996. Being Digital. New York: Vintage.

Neff, Gina, Wissinger, Elizabeth and Sharon Zukin. 2005. Entrepreneurial Labor among Cultural Producers: "Cool" Jobs in "Hot" Industries. Social Semiotics 15 (3): 307-334.

Oakley, Ann. 1974. The Sociology of Housework. London: Martin Robertson.

Oxford English Dictionary. 1989. 2nd ed. Oxford: Clarendon Press. Vol. III.

Paasonen, Susanna. 2010. Labors of Love: Netporn, Web 2.0 and the Meanings of Amateurism. New Media \& Society 12 (8): 1297-1312.

Pun, Ngai., Huilin Lu, Yuhua Guo, and Yuan Shen, edits. 2011. Suicides behind the Glory of Foxconn. Hong Kong: Commercial Press. (in Chinese) 
Qiu, Jack Linchuan. 2009. Working-Class Network Society. Cambridge, MA: MIT Press.

Qiu, Jack Linchuan. 2010. Network Labor and Non-Elite Knowledge Workers in China. Work, Organization, Labor \& Globalization 4 (2): 80-95.

Qiu, Jack Linchuan. 2012. "Network Labor: Beyond the Shadow of Foxconn," in Studying Mobile Media: Cultural Technologies, Mobile Communication, and the iPhone, edited by Larissa Hjorth, Jean Burgess, and Ingrid Richardson, 173-189. London: Routledge.

Ray, T. 2012. Cook's Earnings Day: The iPhone is the Apple story. Barron's Online. Accessed May 12, 2014.

http://online.barrons.com/news/articles/SB50001424052748704895604577178941793216900

Ross, Andrew. 2006. Fast Boat to China: Corporate Flight and the Consequences of Free Trade. New York: Pantheon Books.

SACOM (Students and Scholars Against Corporate Misbehavior). 2010. Workers as Machines: Military Management in Foxcon. SACOM. Accessed August 21, 2012. http://sacom.hk/wpcontent/uploads/2010/11/report-on-foxconn-workers-as-machines sacom.pdf

SACOM (Students and Scholars Against Corporate Misbehavior). 2011. Foxconn and Apple Fail to Fulfill Promises: Predicaments of Workers after the Suicides. SACOM. Accessed August 21, 2012. http://sacom.hk/wp-content/uploads/2011/05/2011-05-06_foxconn-and-apple-fail-to-fulfillpromises1.pdf

Sandoval, Marisol. 2013. Foxconned Labor as the Dark Side of the Information Age: Working Conditions at Apple's Contract Manufacturers in China. TripleC_Communication, Capitalism \& Critique 11 (2): 318-347. Accessed May 12, 2014. http://www.triple-c.at

Sassen, Saskia. 1998. Globalization and Its Discontents. New York: The New Press.

Sassen, Saskia. 2004. Global Cities and Survival Circuits. In Global Woman, edited by Barbara Ehrenreich and Arlie Russell Hochschild, 254-274. New York: Holt Paperbacks.

Schiller, Dan. 2005. Poles of Market Growth? Open Questions about China, Information and the World Economy. Global Media and Communication 1 (1): 79-103.

Sennett, Richard. 1999. The Corrosion of Character. New York: W.W. Norton Co.

Suhr, H.C. 2009. Underpinning the Paradoxes in the Artistic Fields of MySpace: The Problematization of Values and Popularity in Convergence Culture. New Media \& Society 11 (1\&2): 179-198.

Tatarchevskiy, Tatiana. 2011. The 'Popular' Culture of Internet Activism. New Media \& Society 13 (2): 297-313.

Terranova, Tiziana. 2000. Free Labor: Producing Culture for the Digital Economy. Social Text 18 (2): 33-58.

Turner, Fred. 2004. From Counterculture to Cyberculture. Chicago, IL: University of Chicago Press.

Wajcman, Judy. 2008. Life in the Fast Lane? Towards a Sociology of Technology and Time. British Journal of Sociology 59 (1): 59-77.

Wallis, Cara and Jack Linchuan Qiu. 2012. Shanzhaiji and the Transformation of the Local Mediascape in Shenzhen. In Mapping Media in China: Region, Province, Locality, edited by Wanning Sun and Je Chio, 109-125. London: Routledge.

Webster, Nick. 2006. Welcome to iPod City: The 'Robot' Workers on 15-hour Days. The Mirror.. Accessed April 7, 2014. http://app.vlex.com/\#/vid/welcome-ipod-robot-workers-15-hour-71021814

Xiang, Biao. 2007. Global "Body Shopping": An Indian Labor System in the Information Technology Industry. Princeton, NJ: Princeton University Press.

Zhao, Yuezhi and Rob Duffy. 2007. Short-Circuited? The Communication of Labor Struggles in China. In Knowledge Workers in the Information Society, edited by Catherine McKercher and Vincent Mosco, 229-247. Lanham, MD: Lexington Books.

\section{About the Author}

\section{Jack Linchuan Qiu}

Jack Linchuan Qiu is associate professor at the School of Journalism and Communication, the Chinese University of Hong Kong. He is the author of World Factory in An Information Era 信息时代的世 界工厂 (Guangxi Normal University Press, 2013) and Working-Class Network Society (MIT Press, 2009).

\section{Melissa Gregg}

Melissa Gregg is Principal Engineer in User Experience Research at Intel Labs, USA. She is author of Work's Intimacy (Polity 2011), Cultural Studies' Affective Voices (2006), and co-editor of The Affect Theory Reader (Duke UP 2010). 
Kate Crawford

Kate Crawford is Principal Researcher at Microsoft Research in NYC, a Visiting Professor at MIT's Center for Civic Media, and a Senior Fellow at NYU's Information Law Institute. Her most recent work focuses on the politics and ethics of big data. 


\title{
Concepts of Digital Labour: Schelling's Naturphilosophie
}

\author{
Kevin Mitchell
}

\author{
Trent University, Peterborough, Canada, kevinmitchell@trentu.ca
}

\begin{abstract}
This paper uses F.W.J. Schelling's Naturphilsophie as a point of departure for theorizing the concept of digital labour. Beginning with Marx's distinction between fulfilling and unfulfilling labour, it is argued that the former is labour immanent to, and in line with, Schelling's notion of Nature as process and ungrounded ground, while unfulfilling labour externalizes Nature and attempts to use it against itself in the service of capital and the establishment of what I call a state-of-power. Schelling's The Ages of the World is re-interpreted by exchanging Schelling's notion of immaterial spirituality for digital virtuality, whereby digital labour is viewed as a consequence of previous forms of world historical developments in their entire contingency. While digital virtuality is in fact materialist in terms of both the labour that activates it and the substrate that sustains it, the materiality of the digital is often overlooked in favour of an implicit anti-materialist stance that works to disconnect the digital labourer from their online activity, and precludes the critical self-awareness necessary for an acknowledgement of their "playful" online activity as labour. The paper ends with an analysis of Mark Zuckerberg's ideational attempt to "re-wire" the world via Facebook's digital infrastructure, which begins (and/or attempts) to set the conditions of possibility for inter-personal interaction, and explores the possibilities for resistance available within Foucault's concept of the care of the self.
\end{abstract}

Keywords: Digital Labour, Immaterial Work, Marx, F.W.J. Schelling, Digital Virtuality, Social Media, Mark Zuckerberg, State-of-Power

\section{Introduction}

In the Grundrisse, Marx notes that there is an inherently contradictory status of labour: On one hand there is "repulsive [...] external forced labour," which is contrary to the labourer's own nature. On the other hand, there is "attractive work [...] which contributes to the individual's self-realization" (Marx 1986, 59-60). While externally forced labour utilizes labour power in a way that alienates the labourer from the wares of his or her work, attractive work "appears in the production process not in a merely natural, spontaneous form, but as an activity regulating all the forces of nature" $(60)$. While in both instances the status of the labourer is in an ambiguous position with relation to nature (a "merely natural, spontaneous form," and "regulating the forces of nature"), the labourer is in both cases (externally forced and internally attractive) considered to be working through the forces of nature-not on it. Taking this a step further, whether negative or positive work, Marx's labourer works immanently in and as nature, and may even be said to work through nature in such a way that the labouring activity coincides with the creative activity of nature itself.

In a similar way Schelling states that: "To philosophize about nature means to create nature [...] to heave it out of the dead mechanism to which it seems predisposed, to quicken it with freedom and to set it into its own free development-to philosophize about nature means, in other words, to tear yourself away from the common view which discerns in nature only what happens-and which, at most, views the act as a factum, not the action itself in its acting" (Schelling 2004, 14-15). Schelling considers the activity of nature to be its defining characteristic. In his Naturphilosophie, nature is immanent to the process of its productive development such that it could be said that it is nothing other than this process. Instead of remaining passively contemplative, the active labour of philosophy for Schelling is an attempt to match the relentless process of nature, which is ceaselessly active and creatively productive. Philosophizing thus takes place alongside the activity of nature, rather than in a purely contemplative (and externally alienating) form. Schelling implies that philosophizing about 
nature is a form of work-akin to Marx's "attractive work"1_-since both physical and philosophical work is conducive to "the individual's self-realization" if they are in line with, and take their impulse from, the creative force(s) of nature. This distinction also resonates with the Aristotelian distinction between "poísesis (the creation of works from nature) and praxis (selfdetermined action)" (Fuchs and Sevignani 2013, 238). While this conceptual distinction mirrors the civilization from which it arose (Fuchs and Sevignani 2013), it also, crucially, disconnects self-determined action from its immediate relation to nature. Contrary to this view, for both Marx and Schelling alike, there is a form of self-determined work that coincides with nature. For Marx, "attractive work" leads to the "self-realization" of the worker. For Schelling, authentic work participates in the process of nature's productive activity; since the worker is part of the very same nature of this activity, the worker participates in (and thereby embodies) his/her own nature. This participatory embodiment is at the opposite end of the spectrum from the alienated form of labour where the worker, while ontologically inseparable from $\mathrm{Na}$ ture's role in the labour process, is nevertheless disconnected from this same Nature in terms of the result (capitalistic accumulation), and the relationship between the worker and his/her own working body. As will be explained in detail below, the alienation that arises as a result of the state-of-power, ossifying Nature's productivity and using it against itself, results in both a social alienation (man from man in the form of an overall state of securitization) as well as man-Nature and Nature from itself. When Nature is reified, and thereby treated as object, Nature's inherent productivity becomes disconnected from itself, and accumulated by one part at the expense of another. This disconnection implies a crisis and a resultant tendency toward a form of resolution in terms of digital virtuality.

Following Christian Fuchs (2013), I will argue that there is indeed a difference between labour and work, and that one viable place to study this difference is found in Schelling's Naturphilosophie when read in conjunction with Marx's theorization, and taxonomy, of different types of labour and their historic conditions of possibility. Briefly, Fuchs and Sevignani point out that there is only one word for work/labour in German: Arbeit. Following the English language's two terms (work and labour), Engels distinguishes between work (which is qualitative and produces use-value) and labour (which is quantitative and produces exchangevalue) (Fuchs and Sevignani 2013, 240). I will consider the implications of this distinction, in the context of immaterial labour, and what may be called the proletariatization of the digital, or the progressive co-opting of Internet activity in the service of capital. I believe this analysis can contribute to the development of a conceptual framework in which these new types of labour may be studied, categorized and understood. I will also indicate some of the advantages of using Schelling to compliment a Marxist understanding of digital labour.

\subsection{Against Essentialism}

Contrary to Aristotelian essentialism, which holds that things have an inherent structure informing them as to what they are, Marx follows Hegel in emphasizing the active and changing status of entities by emphasizing their developmental character. According to this view, entities are not finished once and for all in terms of what they can be, but are the result of historical circumstances operating as their conditions of possibility. Implicated in this analysis is the view that historical phenomena are incomplete, and therefore, are inherently capable of becoming other than they are when moulded in different contexts, temporalities and spaces/placements. This view problematizes the doctrine of identity, which holds that entities have a unitary continuity across temporal instants, and against this, emphasizes difference, contingency, and contradiction. Not only are they emphasized, it is argued that these qualities are inherent to nature and result from the fundamental contingency of reality-a contingency considered by Hegel to be a sign of their vitality. Things are alive when they are capa-

\footnotetext{
${ }^{1}$ This is not to imply that Marx held that philosophizing was a form of work, his famous move to turn Hegel on his head is a move in the opposite direction, since he views philosophy as merely interpreting the world. Schelling on the other hand holds that philosophy is an active creation of nature since it is a manifestation of a higher potency, immanently operating on it as it operates on itself.
} 
ble of change (whether autonomously brought upon by themselves, or externally, by another force acting upon them).

According to this variant of anti-essentialist Hegelianism, to be alive is to be in contradiction. Italian Marxist philosopher Lucio Colletti explains the difference between the doctrine of identity and dialectical materialism thus: "As against contradiction, identity is merely the determination of the simple immediate, of dead being; but contradiction is the root of all movement and vitality" (Colletti 1979, 21). Remarking upon the Hegelian precedents of dialectical materialism, Colletti argues that for Hegel and Marx alike, the material world is not an array of static entities, as in the Aristotelian worldview, but an inter-active network of incomplete entities in disjunctive relation with each other. The inter-active nature of materiality is considered by early dialectical materialists to be the main ingredient for the auto-poietic selfmovement of Nature, and historically situated entities. In this view, the essence of a thing is determined not by what it is, but by what it becomes through its interaction with other entities, in a situation of mutually implicating development.

The analysis of digital labour in this paper highlights the vital process of nature as a constituent presupposition for the infrastructure of the digital, ${ }^{2}$ especially as the former relates to the German Idealist precedents of the Marxist tradition. From this perspective, the two types of labour identified above (externally forced and internally attractive) may be considered to be either dead or alive labour: Repulsive, externally forced labour is dead because it treats both the labour process and the labourer as finished products, while attractive work acknowledges the open-ended nature of the labour process and its inherent potential for selffulfillment through work. In both cases the main question to ask is whether the work philosophizes alongside nature, thereby contributing to the creation of nature itself, or whether it attempts to dominate it, thereby determining it as a dead and static object cut off from the vitality of life. As we shall see, Schelling's Naturphilosophie is helpful because it offers a novel conceptualization of labour and work, whereby it is grounded in the process of nature (instead of treating it as objective material to be worked over and transformed), as well as the conceptual infrastructure for a re-worked notion of natural production.

However, how could dead labour exist within a theory of labour such as Schelling's, which sees labour as immanently grounded in nature such that there is no separation between worker and nature? This contradiction is resolved when it is recalled that labour is dead only as part of a second-order operation which treats it as a product (abstracted from the production process) and detached from nature's immediate activity, thereby using it against itself (section 1.1.1.). In this way it could be argued that Schelling's distinction between authentic and dead labour is consistent with Engel's formulation of the distinction between qualitative work grounded in the necessities of nature that produces use-values, and quantitative labour, which is leveraged in the service of exchange-value capitalistic accumulation (the objectification of living work into dead labour). In both cases the natural form of work, which meets the requirements for the sustenance of life, becomes subverted from this course and re-directed in the service of the accumulation of capital, i.e. the divorce of energy (labour and time) from life. As we will see, a state of power secures this accumulation and uses it against nature, thereby disconnecting nature from itself in a version of what Adorno called the "domination" of nature (Adorno 2005).

Since "dead" labour seems to imply a disconnection of the labourer and nature, even though they are both fundamentally part of the same substrate, this disconnection is possible by means of reverse process that moves against nature by treating it as finished product and not active production. Within the terms of Schelling's philosophy, while work is immanent to nature (and to philosophize about nature is to create it), the instrumental rationality of semiocapitalism is not philosophy, and therefore treats nature and labourer as resources, or means rather than ends facilitated by algorithmic infrastructure put in place to harness the virtual version of the general intellect and oversee its valorisation. While there is no real separation

\footnotetext{
${ }^{2}$ What I mean by this is that the digital is related to, and to a certain degree, is actually reliant on materiality for its existence. This differs from the sometimes held assumption that digital computing is "clean" because it somehow exists in a "cloud" that does not require an extensive network of mass energy-consuming computers to house it.
} 
between the two (nature and work/labour), there is a virtual disconnect made possible by the condensation of a state of power and "rewiring" (section 2.1.).

In conjunction with Naturphilosophie's emphasis on process, Marx argues that "nothing can result at the end of a process that did not occur at its beginning as a prerequisite and condition" (Marx 1986, 78). In other words, process is cumulative since it presupposes what has come before in order for what comes later to be possible. As he puts it, "the result must contain all the elements of the process" (Marx 1986). The process of capitalist production follows this pattern as well, since it preserves what exists before in the form of raw material to be utilized and commoditized. In opposition to the Aristotelian notion of the self-identity of objects, for Marx, objects, ideas and socio-cultural realities are not self-subsistent entities, but are manifestations of their historical conditions. Since these conditions are indeed historical, they are contingent, and as such, objects, ideas and socio-cultural realities are capable of being actualized otherwise.

There are three aspects to Marx's emphasis on process that should be kept in mind. First, process is cumulative; there is a relative continuity of accumulation moving from the past (what happened prior) toward the future (what happens in and as the result). Second, elements that are influential at the beginning may have their effect felt throughout the process, and they continually impact the result. There is therefore a form of archive occurring in the process. Third, since process is cumulative and archiving, it is also holistic (it is more than the sum of its parts) and transcendental (the archive acts as the condition of possibility for what emerges as its result). As such, any historical entity must be considered in these terms, individually, and in conjunction with each other. Accordingly, a product is an actualization of the material conditions of possibility that have gone into making it possible, including the contradictory forces that played a role in its origination and which has come to act as its ground.

While it is true that Schelling may be read as a philosopher of substance, albeit one that is dynamic and ceaselessly changing, it is not clear what the advantages of this interpretation are. Although it is evident that he never abandoned his conception of a divine principle presenting itself through all levels of nature's manifestation (inorganic, organic, and intelligent), his work is better interpreted as one whose main concern is that of freedom, and the question of how to it is possible to reconcile a non-essential conception of nature with that of an ungrounded system. As Heidegger points out in his lectures on Schelling, at first glance it appears contradictory to pursue a system of freedom (Heidegger 1985, 22). Schelling wanted to find an ontological and philosophical ("scientific") definition of freedom, one that emerged out of a rigorous and systematic analysis of the world in all its connections. It thus required him to clarify the reason for his anti-essentialist stance by looking at the productivity of nature itself without treating it as object, and without dissecting it (only) in terms of the empirical method (although it played a role in his Naturphilosophie). As such, it was necessary to disagree with his long-admired friend Fichte, whose own subjective idealism began with the subject, and then went on to derive nature therefrom. Exchanging the primacy of the subject for that of nature, Schelling's Naturphilosophie derives the Fichtean transcendental subject from nature itself, putting nature in the position of the condition of possibility for later variants of the post-Kantian idealist theme. Therefore, for Schelling, substance takes a back seat to existence, and as the old existentialist adage put it: Existence precedes essence. There is a difference between substance and essence: While essence has to do with what a thing is in terms of its mode of being, substance indicates the underlying substrate of a thing (including its matter). However, if the essence of substance is its ungroundedness, and therefore its inherent freedom (both in-itself, and that to which it gives rise), nothing is added to the argument by stating that Schelling is a thinker of a (non-substantial) substance. However, if we say that there is such an infinitely creative universal substance, this universal substance would be defined by its existence, insofar as it comes to "know" itself in its existential mode as ceaselessly productive nature. "It is not me who knows, but only the Universe knows within me [...] This one knowledge however that knows is at the same time what is truly known" (Zimmermann 2005, 36-37). The divine Lebenskraft is immanent to the movement of nature in its self-overcoming, and auto-poietic organization. 


\subsubsection{Schelling's Ungrund_-Ungrounded Ground'}

Throughout his continual attempts to philosophize about, and thereby create nature, Schelling characterized nature in a variety of ways. In First Outline of a System for the Philosophy of Nature (Schelling 2004) for example, he variously characterizes nature as having an "absolutely productive character" (Schelling 2004,5 ) and "reproducing itself anew in each successive phase" (Schelling 2004, xix). He also depicts it as "struggling against everything individual" (Schelling 2004, 6), and as "unconditioned", such that "every individual is a particular expression of it" (Schelling 2004, 13). What these descriptions of nature have in common is that they all portray nature as primary (in relation to "products") and undifferentiated; or, what amounts to the same thing, a manifestation of pure difference, since it is that which gives rise to all individual differences: "Nature exists nowhere as product; all individual productions in Nature are merely apparent products" (Schelling 2004, 16).

Nature, according to Schelling, is also absolute activity. Since all activity is productive in some way (action takes place in relation to an environment presupposed for the action), nature's activity also leads to the production of products. In this sense, to be a product is to be produced by nature's ceaseless activity. Schelling relates the notion of absolute activity to infinity: nature's productive activity has neither beginning nor end. While nature's productive activity terminates in finite products, nature itself does not thereby stop its onslaught of production. Even products that have a veneer of being finished or complete continue to partake in nature's ceaseless activity, and are therefore only ever apparently finished products. ${ }^{3}$ Although products are also in a continual state of internal strife, they are nonetheless finite, and demarcated as limited in relation to the infinite activity of nature. However, since the infinite activity of nature resides even in its products, "every product that now appears fixed in $\mathrm{Na}$ ture would exist only for a moment, gripped in continuous evolution, always changeable, appearing only to fade away again" (Schelling 2004, 18). Products are temporary lapses.

Schelling provides the following example to illustrate the way the underlying forces of nature operate productively: "A stream flows in a straight line forward as long as it encounters no resistance. Where there is resistance-a whirlpool forms. Every original product of nature is such a whirlpool" (Schelling 2004, 18). It is through the activity of nature that products are able to participate in the activity of nature, and it is through work that the human being-as a product of nature in the midst of other products-strives to preserve itself as a formal entity in the midst of nature's ceaseless productive activity. By participating in this activity in its own way, the human is able to limit itself from the infinite activity by producing boundaries (between itself as organism) and an environment. It is through this striving toward preservation-to perpetuate the lapse-that the human cultural world is produced. Since to philosophize about nature is to create nature, it is through work that the human labours to create its own nature as a manifestation of Nature. It is in this context that Schelling's conception of human activity is consistent with Marx's two types of work (externally forced labour and attractive work).

Schelling also differentiates between authentic and dead work. Work that operates on behalf of nature is authentic because it is aware of itself as being an archived manifestation of the process of nature as its condition of possibility. "However, as soon as I separate myself, and with me everything ideal, from nature, nothing remains to me but a dead object, and I cease to comprehend how a life outside me could be possible." Just as for Marx externally forced labour alienates the worker from both the process and the result of his or her labour, Schelling's notion of dead work involves separation; and, similar to Marx's theory of the commodity (and the related notion of reification), Schelling says that "nothing remains to me but a dead object," presumably detached from the transcendental process from which it arose. Work that detaches itself from the immanent activity of nature and instead attempts to control nature and worker alike is dead work. Since nature is characterized as being productively and in process for both Schelling and Marx in their own ways, we may make a critical distinction between work as a continuation of Nature's productive activity by man, and labour

\footnotetext{
${ }^{3}$ We may differentiate this from Aristotle's conception of generation and corruption where the life of particular beings goes through a pre-determined process of attempted actualization of their potential and inevitable decline.
} 
as a form of work, which is discontinuous and detached from nature's productive activity. Attractive work is attractive because it is synthetic and it melds with Nature's activity; external work is disjunctive in regards to nature's productive activity, and is thus alienated and alienating-alienated from nature's process and alienating for the worker. According to Marx, while alienated work is "repulsive" and "external" to the inherent creative process of nature, "attractive work" is not only a "spontaneous form" of labour, but is also "an activity regulating all the forces of nature" (Marx 1986, 60). Attractive work acts as a form of mediation between infinite nature and finite man, where man's labour is productive of culture (the production of a human world set up -as a lapse in relation to nature's activity - to preserve man as an entity) and finally, history, which acts as a bridge between the traditionally transcendent god and finite animality.

Hegel famously considers culture to be a "second nature," the latter being nothing but the product of man's work on nature and translated into history. Culture, as second nature, is thus a transformation of the infinite process of nature into an anthropomorphic product. Through this human work-activity, man imposes the preservation of a particular form on the infinite process of nature, and thereby imparts a lapse in part of nature's process, such that a small part of the process hardens into a structural form, or a state-of-power - which achieves a transformation of productive force into its institutionalization. Nature operates on the basis of force(s), while power is a cultural institutionalization of force reminiscent of man's active attempt to preserve itself. "Force is not to be confused with power. Power is the domestication of force. Force in its wild state arrives from outside to break constraints and open new vistas. Power builds walls" (Massumi 1992, 6).

It is in the form of the state-of-power (the translation of force into a static state of power) that capital is born, since the preservation of a particular static state uses nature's force against itself (Wirth 2000, xxiii) in a way that, instead of allowing it to open up new possibilities and ways of organizing work in the service of the "self-realization" of nature in the form of man (Marx 1986, 59), it actually contributes to its cancellation by detaching Nature's inherent excess and accumulating it in the form of (private) wealth. It is only in the form of culture that this form of accumulation is possible, since, on the level of Nature, there is nothing but ceaseless activity that exceeds the measure of any particular state. Through this counterprocess-which attempts to capture nature and render it static-that "the superabundant energies of the earth have been restricted by the exclusive investments of a particular world" (Wirth, xxiii). ${ }^{4}$ It is only through man, who translates nature into culture through the imposition of his own measure (as part of the creative process of history), that nature is capable of being falsely stored, and used against itself, in the service of the exploitation of one part of its activity against another. It is only through culture that nature is made into an object, and used as an infinite means to a finite end. By saying that Nature is infinite, this does not imply that there are an unlimited number of resources on earth to be exploited and used for the ends of capitalist accumulation. From the perspective of man, there is indeed an end in sight; but from the perspective of Schelling's Nature, which is absolutely indifferent to man, Nature is infinite and does not end with man.

While it can be argued that all species translate the infinite process of nature into a manifestation of their own measure (for example, through the perceptual apparatus used to mediate the contact of their organized bodies with their immediate surroundings), Schelling (and the German Idealists more generally including Marx) put the human in a privileged position in relation to other organisms because of this entity's unique capacity of the intellect to reflect nature back on itself, and thereby attain knowledge, or an image of nature. This is not to say that nature is not reflective of itself apart from man's intellect, but it is only through man that nature reflects itself in the form of a species that also imposes measure in the form of culture and history. It is through the capacity of reflection that man reflects one part of nature (subject) into/onto another part (object), and thereby uses one part against another part. Furthermore, it is through the work of culture, and the related building of institutions, that se-

\footnotetext{
${ }^{4} \mathrm{I}$ am using the term "state" here in all its ambiguity to refer both to the "state of being" (which implies a rendering static of flux) and the "political state" (which also implies a rendering static of flux).
} 
cond-nature becomes semi-autonomous and structured in a way capable of reproducing itself. It is thus that as an organization, the social organism of labour builds a repetition of itself based on the preserved power of Nature's institutionalized force in a way that attempts to match (or mirror) the process of Nature on an anthropocentric scale. Through knowledge (the image of Nature's reflection into subject-object form) nature becomes an object, and as an object, it is reflected back onto the labouring subject. By means of this reflection, the labouring subject becomes part of the objectified version of nature, and one part of nature is thereby used against another part. In other words, nature becomes an object when knowledge's reflection produces a form of objectified production that encapsulates workers as extensions of itself (i.e. objects). What this means is that through the institutionalization of force into a state-of-power, nature becomes unnatural production, and as a result, a particular form of production becomes objectified. In conformity with the other aspects of Nature's process the objectified result of the institutionalization of force is preserved in the form of capital claimed (and owned) by interested parties produced by the institutionalization of force, and coming to fill positions in the institutionalized framework made possible by culture's vocation as second nature in the service of history.

From Marx's perspective, the owners of production own the perpetuation of exploited labour, which is an object that perpetually produces more objects. The infinite process of Nature is transformed into an object-a particular form of cultural activity that becomes a social organism - and thereby comes to organize labour in such a way that preserves the excess of Nature's infinite movement in the form of power, wealth and capital.

\section{Knowledge}

What is particularly disturbing about this narrative is that knowledge is at the root of Nature's transformation into second nature, and from the latter, into a state-of-power and capital accumulation, since it is only through reflection that Nature is used against itself to produce institutional frameworks of repressive labour. This does not of course mean that all knowledge is complicit within the process of objectification and the institutionalization of force characteristic of capitalistic accumulation. Analogous to attractive work, there is a form of knowledge that is resistant to its appropriation by a state-of-power. Just as work aligned with Nature's process is conducive to self-fulfillment, knowledge that actualizes force and resists its accumulation by power is creative, as is evident in Schelling's creative philosophizing of Nature, and an intention of his Naturphilosophie project to address and explicate.

One implication of this is that the two types of labour are productive of two types of culture: communal culture and repressive culture. While communal culture is based on the premise that the fruits of work are shared, since all is a result of Nature's infinite process, repressive culture institutionalizes the fruits of labour in a way that subverts their distribution. It is thus only in attractive work that there could be relative freedom with respect to the parameters of this work, and the wares contributing to the production of a culture mimicking Nature's own flourishing, while externally forced labour leads to a culture of artificial scarcity and an extraordinary amount of resources spent on the base preservation of a particular state-of-power.

While Marx most clearly articulates a distinction between the two types of labourattractive work and repulsive labour-in the Grundrisse, particularly,in "The Fragment on Machines" it becomes clear what the implications for this distinction are for us today. In this section he argues that capital develops to a point where it begins to operate in such a way that it produces-and sustains-the conditions for its continued production in the form of fixed capital. The process of capitalist production, which, as noted earlier, is cumulative, archival and holistic, becomes united with the means of labour (i.e. workers) that actualize it. The means of labour are institutional frameworks for channeling force into accumulated power, and, according to the Marx of these pages of the Grundrisse, the means of labour pass through a series of developmental stages until they finally culminate in what he calls "an automatic system of machinery" (Marx 1993, 692). While he predominantly had factory equipment in mind, it is evident that the worker comes to inhabit the same infrastructural ground of 
labour that the machine actualizes to such a degree that it could be said that the worker's biological rhythms become entirely subsumed by the machinic apparatus-including the worker's intellect itself.

This process is reminiscent of the virtual infrastructure put in place by social media giants Facebook and Twitter. In both cases the machine (or virtual infrastructure) is not a tool utilized by the worker (which would act as a prosthesis requiring a certain level of skill to be utilized appropriately) but an abstract ground indifferent to the presence of the worker, besides their mere presence and "playful" activity. Since the worker is a "material quality of the means of labour" it is as though the machine takes the place of the condition of possibility for the existence of the worker, and is thereby "transformed into an existence adequate to fixed capital and to capital as such" (Marx 1993). While fixed capital-in the form of the machinic means of production and the virtual apparatus-becomes an indiscernible component of the labour process, or means of production, the worker becomes something of an extension of the machine or the virtual environment, since in both cases she merely provides the impetus, or the soul, for the actualization of movement.

Marx characterizes the machine as an automaton "consisting of numerous mechanical and intellectual organs, so that the workers themselves are cast merely as its conscious linkages" (Marx 1993). It is important to note that the workers are conscious linkages, and not merely physico-mechanical cogs in the machine: even in Marx's time the machine required a base level of conscious reflexivity for the perpetuation of the machine's activity to be sustained. This is why he considered capitalism to be vampiric, namely it requires the use of "living labour" to animate "dead labour" (Ross 2013, 29).

Analogous to this is the vast online infrastructure that requires countless hours of the "hive mind" (Lanier 2006) to work anonymously (and uncompensated) to sustain novelty to preserve the interest of its users, to maintain its basic functionality, and to further its cohabitation with the production and consumption of information (Terranova 2012, 33) It is thus at this stage of reflection that the production of nature as object, and the subsequent appropriation by the object of the subject in the cultural exploitation of one part of nature by another, that a secondary form of production arises as an alternative to nature's, one that, instead of enhancing life by producing new arrangements of being, produces dead objects made possible by the institutionalization of force as state-of-power. Detached from the ungrounded ground of Schelling's Nature, capitalistic second nature uses nature against itself by exploiting the reflective capacity of consciousness and knowledge to produce objects devoid of life. Power is appropriated to sustain a particular cultural-political arrangement of being at the expense of others, and the digital labour economy is a reflection of this at a later stage of development beyond the archetype of the machine Marx so presciently theorized under the heading of automaton. The natural activity of the worker, in the form of the average user of the Internet, is skimmed off-and monetized-and sold back to the worker/user in an objectified form. This objectified form, often called in-formation, is then utilized by the worker/user in a way that, in turn, informs the ways in which she is actualized, which is then fed back into this same system. As a result of this ceaseless reflexivity, the feedback loop becomes an ontological concept: It is knowledge's reflectivity in an objectified form, where activity is channeled in such a way that the worker/user recognizes themselves (or their "personal identity") in what is reflected back (fed back through the loop) at them, such that they becomes the ultimate ideological subjects, or absolute objects-"intellectual organs" (Terranova 2012). The worker/user's body becomes a platform for the transcription of force into power through the medium of information exchange (the body is rendered invisible, or ceases to exist outside of its registration into the amorphous abstraction of information). All that is left is an indifferent flow of information, its capture, and its feed-back into a system of capitalistic 'prosumption' where the worker doubles as a consumer who simultaneously consumes herself while she is consumed by the system.

In The Ages of the World Schelling speaks about degrees of power. Not only is there state-of-power, which I defined above as an institutionalization of productive force into state power, but, similar to Marx's notion of the "means of labour," for Schelling, nature's power also moves through certain stages. According to Schelling's doctrine of the three potencies, 
"every higher potency is an archetype of a lower" (Schelling 2000, 57). As he goes on to say, the existence of the higher requires the lower potency's unfolding of the "seed enclosed within it" (Schelling 2000). While not dialectical in the same way as Hegel's Geist, there is a certain movement theorized in this text from Schelling's middle period. For our purposes, we will extend Schelling's analysis to include digital labour as its culmination. The first potency is nature itself; the second is reflective consciousness; and the third is digital virtuality. While there is a vital force, or "world soul," moving through all three levels, it is only at the third level, that Schelling considers it to be at the level of what he calls the "God-head," which is a manifestation of the vital force itself. In an analogous way, it could be argued that it is on the level of virtual digitality that Nature manifests itself as such, in a fully reflective way. Since the movement goes from materiality to consciousness to immateriality, ${ }^{5}$ it is on the third immaterial level that both nature and consciousness are reflected back onto themselves in their "spiritual" form. "Hence, this heavenly, soul-like essence, which was concealed and asleep until now, first awakes with the appearance of the crisis in nature" (Schelling 2000). The crisis in nature is nature's being used against itself in the form of capitalist accumulation. On one side, this spiritual world-soul manifests itself as the "spiritual" aspect of Marx's commodity fetishism; on the other side, it is concretized as the network society of digital production, made manifest in and through its immaterial infrastructure or virtual world.

There are three phases in this re-interpretation of Schelling's theory of the potencies: Nature-materiality, culture-consciousness, and digital-immateriality. Characterized by ceaseless becoming, Nature's process produces entities-species, oceans, trees, and rocks. However, according to the second potency, human consciousness reflects nature in such a way that it is capable of being used against itself as part of a secondary process of manufacturing a second nature (culture and history). The intellect operates by taking things apart and analyzing them according to their component parts, thereby (as distinct from what Colletti considers understanding's vocation of locating the whole in the particular) reifying them into the form of objects; as culture becomes institutionalized into a state-of-power, man becomes an object amongst other objects. However, it is at the level of the third potency, when both Nature and Consciousness become overwhelmed by the excess of nature's force (which can never be entirely captured by the state-of-power) that the other two potencies (Naturemateriality and Culture-consciousness) become consolidated into the service of a third power that goes on to spiritualize both by reducing them to an image mediated and sustained by the flow of information. And it is in this third (digital) form-which happens to be when materiality is at its most immaterial-that Nature takes its revenge. The ceaseless process of force overwhelms man by bringing him into the guise of the third potency, which alienates consciousness from itself, and as the gods have done before, renders man impotent. Process continues to happen without man, and man falls into the abyss of a consciousness that is not his own-one that uses him against himself on a metaphysical level in a similar way that nature was used against itself at an earlier developmental stage.

\subsection{Immaterial Labour?}

At the level of immaterial labour nature is translated twice over, from nature into second nature, and from second nature into an image. Guy Debord had a glimpse of this when, in 1967-thinking predominantly about the impact of television-he wrote that "the image detaches itself from every aspect of life and fuses into a common stream in which the unity of this life can no longer be reestablished" (Debord 1967,6). Although in some passages he risks falling into a romantic idealization and yearning for a former "life" untarnished by technological mediation, the articulation of the image as a detached reflection of life lending an impression of itself as more real than reality itself, speaks to us today.

What, however, can be said to be produced immaterially? In contemporary social media the individual is removed from the product of their labour, since those who own the platform (Facebook, Twitter, Songza) also own the rights to the content and the trace of the activity of the user. However, from the Schelling perspective I am utilizing here, Nature does not stop

\footnotetext{
${ }^{5}$ For the purposes of this essay I will consider immateriality to be interchangeable with digital virtuality.
} 
being operative on the immaterial level. Since process occurs in all three potencies, digital virtuality is a productive activity that operates whether it is self-consciously noticed or not. So while it is free to sign up and use the service, the cost is the appropriation of the results of the productive activity in terms of its monetization, which is again detached from its potential as life-actualizing attractive work, and is further transformed into institutionalized power. However, it is obvious that immaterial labour is different from traditional forms of labour. No longer is anything actually tangibly produced. What is being produced is the vast infrastructure of an institutionalized version of the Internet, where every move is mapped and translated into information, which is in turn fed into a system of monetization and fed back into the "free" activity of individuals, only this time a little more rigid. What is produced is a vast database of information which takes the form of an archive of the individual's "personality" but which boils down to a series of "likes" and comments regarding quasi-political issues, tastes and patterns of consumption. Even the production and uploading of a digital photograph carries the traces of previous forms of "real life" interaction, but only as they are translated into a virtual image of what came before.

However, even (and especially) on the digital level there is ceaseless activity - people are constantly updating their profile, clicking on articles, ordering books, watching movies, or even just checking the weather. All of this constant activity translates into information, as both the trace and the condition of possibility for the activity itself, since information also contributes to the infrastructural form of online activity and the coding that produces the rules of the game. What then is information in this context? Against the traditional theory of information developed by Claude Shannon in his seminal 1948 article "A Mathematical Theory of Communication," (Shannon 1948) where he theorized information in terms of a message being transmitted in a relatively linear fashion in the sender-receiver paradigm of transmission, the concept of information relevant for our discussion of digital labour is qualitative and intensive.

Since information in-forms (produces forms of possible action or moves in the game) it contributes to the constitution of the subject operating in relation to the objectified conditions of its existence; it is pre-perceptual and operative on what Gilbert Simondon calls the level of the "pre-individual" (Combes 2013, 3). It is not yet actual because it makes actualities themselves possible. Information is a by-product of activity but it also constitutes activity by operating on the transcendental level. Schelling's theory of magnetism, conceived in terms of the spiritualization of matter as part of the vital impetus of Nature finds its counterpart in information. Information is a remnant of online activity, harnessed and used in the service of capital through its being captured from above by the state-of-power and used to in-form online activity. As information begins to in-form, by providing a form to an otherwise open system, it slowly begins to re-orient the future in terms of the in-formed structure of the past. Information derives from previous activity, it is a fundamentally retrospective by-product of process; when it is used by capital to in-form the future, it orientates the future in terms of the past thereby stifling any potential the past carries with it and the future could unfold. While the process of digital activity takes place endlessly, the result of the process (analogous to Nature's ceaseless activity in the first potency) is detached from its original impetus and used against itself, or against those subjects who become objects through the alienating power of the mechanisms of state capture. This third stage of development - digital virtuality - is considered immaterial because it is hyper-material; that is, since the walls it builds are purely formal, or what Marx called "ideological," they are impenetrable to force. While power is a derivative of force, the sedimentation of power puts in place an infrastructural arrangement that disallows force to actualize its de-territorializing potential. While force is wild-without constraint or formal arrangement-its institutionalization colonizes it by folding it back on itself. While power folds back, force folds forward. (This is similar to Kierkegaard's paradoxical notion of a repetition of the future.) Since it is operative on the level of Schelling's third potency, it finds nothing in its way, and as a result, it operates unaffected by anything other than itself.

History, which is always the history of culture, or second nature, is the result of the rendering static (or state-like) of the continual process-becoming of nature as a reaction to na- 
ture's continual process. For the state to fortify itself, particular contingent arrangements must be captured and preserved in their self-identity from the onslaught of nature's becoming in order to preserve them as they are, and to thereby lend the semblance of continuity and necessity to that which is discontinuous and contingent.

In more concrete terms we may say that the two types of workattractive and repulsiveare marked by the difference between, on the one hand, work that is in tandem with Nature's process, and which remains open to change and the becoming of life, and on the other hand, work that is not in tandem with Nature's process, and which seeks to use it against itself to thereby consolidate it through the mechanisms of objectifation and exploitation discussed above. Digital labour is not good or bad in itself, but, like the two types of work, it depends on the way it is utilized, since, according to our definition inspired by Schelling's theory of the potencies, the digital is a continuation of nature's productive activity by other means. Unfortunately the open-ended capacity of the Internet is becoming, like the physical resources of nature before it, privatized and monetized. This means that the result of the process of online activity is being captured from above by the same mechanisms operating to secure the state-of-power, and thereby used against those who consider themselves subjects or persons-the progenitors of the activity. To further complicate this picture, while the third stage is identified with the immateriality of the image, the image has now reoriented and recouped its material component. Through the use of mobile communication devices (or "smart" phones), the everyday life of embodied subjectivity has become mediated by information and constructed in terms of an image, not for itself, but for the other (capital). Location services on mobile devices map and track the coordinates of the device's user. By transfering data the owner is inadvertently "checking-in," and now that police forces have been given the authority to remotely de-activate camera funcioning on iOS devices in protest situations (Whittaker 2012), there is no longer any real difference between online and offline activities-all is activated, unified, and encapsulated in the web of virtual digitality.

While the spiritualization of matter in Schelling's magnetism was thought to animate and breathe life into the lower rung of existence, information plays this role on the level of digital virtuality, but in the opposite direction: instead of contributing to the production of novelty, information produces death. Just as for Hegel the stasis of being in identitarian thinking is akin to death, information, when utilized in the service of capital and turned against the prospect of change, alienates the process of production from the product and thereby safeguards the contemporary conditions of exploitable online activity by skimming off (or "valorizing") the excess of nature's product and preserving it in the form of wealth. While the constant law of nature for Schelling is change, the law of second nature, when colonized by capital, is the drive toward stasis by any means. Of course, as is well known, the cost of this drive toward stasis is war when nature's excess manifests itself in what Freud termed "the return of the repressed". The equivalent of the return in the digital realm is the slower but no less deadly-process of safeguarding all aspects of life under the guise of the password. To be digitized is to be amenable to the either/or logic of binary oppositionality (" 1 " or "0"), and all life, when transformed into the reflected image of digital virtuality, is castrated, cut off from its transformative potential, and rendered immobile. The era of the cyber-attack is upon us, where the equivalent of war takes place as the destruction-or pillaging-of information, and the "return of the repressed" takes on a digitally in-formed visage.

So what is being produced? In a digital labour economy it is information that is being produced. Information is a by-product of the process of work; work is the result of organized human activities operating on the basis of the attainment of a desired result. When all aspects of life become productive in this sense, we enter an economy not unlike that pointed out by the Italian autonomists in their notion of the social factory where every aspect of life is directly or indirectly linked to the production of capital. Through an emphasis on the collective aspect of labour-as seen in Marx's theory of machination in the "Fragment"-what they refer to as "knowledge" is produced as a by-product of the living interaction between people. As Terranova explains, for Autonomist Paulo Virno "Mass intellectuality - as an ensemble, as a social body - is the repository of the indivisible knowledges of living subjects and their linguistic cooperation [...] knowledge must come into being as the direct interaction of the 
labour force'" (Virno quoted in Terranova 2012, 45). Knowledge, which is made possible by the reflective capacity of consciousness, is the systematic use of information for a particular purpose. To say that there is a knowledge economy means that information is at the centre of the production process; that is, after a certain stage of development, and beyond the machination of the labour process of the "Fragment", of main importance is not the fact of production, but what may be termed the quiddity of production-the qualitative impetus or immaterial force by means of which production is capable of taking place. It is through the living interaction between people (not necessarily in an overtly labour-intensive environment) that production in the third potency of digital virtuality is made possible. By means of the "vampirical" operation of capital operating on this level, a dead object is produced. This dead object consists of the immaterial "stuff" of living interaction, and when captured from above and transformed into the state of information, it is detached from the living embodied engagement of interaction, and thereby monetized and used against the participants of interaction. The monetization of everyday life is complete when the most insubstantial, private, and even dignified aspects of human life, which can be compared to the arcane gestures analyzed by Adorno in Minima Moralia, are sold back to the living ground out of which they originated as something to be desired, sought after, and which are no longer available without a cost-labour-time and money (Adorno 2005). Without it even being noticed, force, which is part of the free ground of Schelling's nature-and a constitutive component of the human being -is translated into power, institutionalized, and transformed into the buttressing of a system of domination that, while advertised as free to use (e.g., Facebook), happens to gradually determine the ways in which interaction is conceivable. When all of social interaction becomes imaginable only in terms of what is available through this virtual infrastructure, human life becomes completely contained within the structure of capital, Marx's attractive work becomes attractive only in name and as advertised, and the dead object of living interaction is sold back to consume the consumer. Virtual infrastructure encapsulates all aspects of life by transforming them to the logic of its own capacity, i.e. the image, and when it accomplishes this, what Zuckerberg calls the "rewiring of social interaction" (Zuckerberg 2012) is complete because it becomes, not only an external attempt to capture an underlying living web of interaction, but the conditions of possibility for interaction itself. As he himself puts it: "By helping people form these connections, we hope to rewire the way people spread and consume information. We think the world's information infrastructure should resemble the social graph-a network built from the bottom up or peer-to-peer...We also believe that giving people control over what they share is a fundamental principle of this rewiring...our goal is to help this rewiring accelerate" (Zuckerberg 2012). It is up to the many unpaid labourers to forge the connections and highlight the networks - including the conformity of the very form that their interaction takes-that "accelerates" the process of rewiring that is necessary for the total integration of all aspects of living interaction (including affective, unconscious, gestural and imaginative components), and renders embodied engagement with a world a byproduct of what is made possible by the accomplished re-wiring. It gets to a point where the valorization of every aspect of life becomes indistinguishable from the contours of possible action, of relating with each other and imagining possibilities. What Kant called the transcendental conditions of possibility for experience, upon the totalized re-wiring Zuckerberg seeks, becomes identical with the results of the re-wiring; that is, the result of re-wiring would require an updated version of the Critique of Pure Reason as The Critique of Pure Wiring. The map and the territory become integrated as a digitized, monetized, and cognitively reduced program that conditions all aspects of human life, experience, and imaginaries.

Schelling's development of the notion of potencies, especially in Die Weltalter, is already beyond the basic framework of dialectical historico-materialist thinking while complementing it in a unique way. However, the Schellingian notion of potencies are undertheorized and therefore deserving of further scholarly and theoretical attention including their implications for a reconceptualization of digital labour. Schelling's potencies are a decidedly non-Hegelian concept in that they do not dialectically resolve themselves into a higher unity, but, instead, presuppose each other in a way that is not reducible to the previous terms. While they are part of a process of development, the larger framework of 
Schelling's "absolute idealism" sets them immanently within nature as the ungrounded ground for the free development of the spiritualization of matter and the materiality of spirit. Schelling works within a quasi-miraculous framework that sees the potencies abruptly emerge from the previous states in a way that indicates a rupture rather than a continuity. While the Hegelian dialectic may be considered a gradual process of development, mediated by a labourious "tarrying with the negative," Schelling's potencies seem to jump right out of their previous manifestations into a qualitatively different (and higher) form of organizational existence. This conception of development, when used to conceptualize digital labour, provides a propadeautic to Marxist understandings of digital labour that locate it in the realm of the "general intellect." While pre-digital labour required an embodied intellect oerating in unison with machines of production-including a pooling of abstract resources working in anonymous collaborative conditions of dispersed intellectual participatory-involvement-the phenomenon of digital labour requires a different ontological framework altogether since, when when idealist philosophy's Geist is made to stand for the digitial as it is here, it is seen as qualitatively different from other forms of labour, including those available to Marx's direct observation, when he theorized the general intellect as a condition of machinic embodiment.

I think that further attention may be paid to this area of Schelling's philosophy in order to tease out the further implications of its difference from Hegelian-Marxist variants of dialectical materialism for the understanding of digital labour. Important in this regard will be the untranslated Das älteste Systemprogramm des deutschen Idealismus. While its precise authorship is under dispute (it is written in Hegel's handwriting but reads more like Schelling's philosophy) it is reflective of the early formative years of Schelling, Hegel, and Hölderlin when they were theology students (and room mates) at the Tübinger Stift, as well as indicates the development of their engagement with Ficthe's thought as he attempted to grapple with Kant. Schelling considered Hegel's philosophy to be "negative" (as opposed to his own "positive" variant) because he thought it was enamoured with the systematization of a negativity of thought (negation being a central Hegelian concept). It also did not do justice to nature, treating it only as a secondary product used by spirit to recognize itself in the material form, but does not treat nature as generative in its own right. In this way Hegel's philosophy of nature is part of the well-rehearsed tradition of Aristotelianism that treated nature as the passive recipient of the active form or idea. By contrast, Schelling considered nature to be the predecessor for the development of mind and subjectivity, although the two may be said to be temporally identical and only logically sequential. Schelling's identity philosophy accounts for the unity between thought and nature by making thought a moment of nature (albeit its highest manifestation), while Hegel treats nature as the negative moment of thought's development.

By treating the digital as Schelling treated spirit (as I am doing here)-as a quasi-divine essence, or force, which does not transcendend the materiality of nature, but which is constitutively immanent to the development of nature itself-the relationship between digital and non-digital forms of labour and the ways in which the former offer both a break from, and a continuity of the latter. One contradiction, then, between Schelling and Marxist conceptions of development is seen through the notion of potencies. Whereas Marx was heavily influenced by the Hegelian dialectic which plods along negatively through a succession of negations, Schelling's potencies work more like ruptures that bring out more complex manifestations while still remaining immanet to the earlier manifestations. These earlier manifestatiosn are preserved, and they exist alongside the higher potencies. This is similar to the Hegelian Aufhebung (which simultaneously cancels and preserves) minus the negating component of sublation. One thing Marxists can learn from Schelling's philosophy is the way that nature incorporates different levels of complexity as different potencies of the same substance. Early disagreement, and eventual break with Hegel indicates a more fundamental disagreement about the status of nature. While for Hegel work is an external operation leading to a dialectical development through negation and Aufhebung (as demonstrated in through the famous master-slave analysis in the Phenomenology of Spirit), Schelling's work is immanent to the process of nature itself, since it does not transcend nature, and intellectual or spiritual/digital work is material work by other means since it too 
participates in and as nature but only as a higher potency. While "higher" seems to imply better, this is not the case; both participate in the Lebenskraft of nature's vitality, albeit in a more organized fashion.

\begin{tabular}{|c|c|c|}
\hline Concept & Marx & Schelling \\
\hline Dead Labour & $\begin{array}{l}\text { Repulsive external- } \\
\text { ly forced, exploita- } \\
\text { tive labour; ex- } \\
\text { change-value ori- } \\
\text { ented. }\end{array}$ & $\begin{array}{c}\text { Nature used } \\
\text { against itself and } \\
\text { treated merely as } \\
\text { product (capital } \\
\text { accumulation) and } \\
\text { not process. }\end{array}$ \\
\hline Living Labour & $\begin{array}{l}\text { Attractive work; } \\
\text { leads to self- } \\
\text { actualization. Use } \\
\text { value oriented. }\end{array}$ & $\begin{array}{l}\text { Work done in rela- } \\
\text { tion to nature's } \\
\text { inherent productivi- } \\
\text { ty, which "creates" } \\
\text { alongside nature } \\
\text { and contributes to } \\
\text { the creation of na- } \\
\text { ture itself. }\end{array}$ \\
\hline State-of-power & $\begin{array}{l}\text { Capital accumula- } \\
\text { tion. E.g., the own- } \\
\text { ership of the infra- } \\
\text { structure of the } \\
\text { means of produc- } \\
\text { tion. The State. }\end{array}$ & $\begin{array}{l}\text { Domination of na- } \\
\text { ture by using its } \\
\text { productivity to op- } \\
\text { press by securing } \\
\text { resources and in- } \\
\text { frastructure to } \\
\text { deaden alternative } \\
\text { potentials. }\end{array}$ \\
\hline Force/power & $\begin{array}{l}\text { Revolutionary po- } \\
\text { tential; general } \\
\text { intellect/ldeology, } \\
\text { capitalistic systems } \\
\text { of domination. }\end{array}$ & $\begin{array}{l}\text { Chaotic energies of } \\
\text { the ungrounded } \\
\text { ground as nature's } \\
\text { process/ Institu- } \\
\text { tionalized force } \\
\text { (stymying process } \\
\text { into product). }\end{array}$ \\
\hline Capital & $\begin{array}{c}\text { Surplus labour in } \\
\text { the service of ex- } \\
\text { change-value. }\end{array}$ & $\begin{array}{c}\text { Nature as accumu- } \\
\text { lated product and } \\
\text { limited/controlled } \\
\text { process. }\end{array}$ \\
\hline
\end{tabular}

Table: Conceptual Comparison of the Framework of Marx's and Schelling's theory of labour 


\section{2. "What is to be done?"}

Lenin's famous question stands for an entire attitudinal disposition, and worldview of praxis. Within Marxist ontology there is a dialectical relationship between the individual and its conditions for existence (Marx 1986, 54). The conditions of existence simultaneously function on the immediate level of actually existing labour relations, class conflict, economic factors, etc., and on the mediating level of the transcendental conditions of possibility for the existence of the individual and the related possibilities of being. Theorists such as Althusser and Foucault look at the processes of subjectivation that result from the interplay between force and power in their social manifestation. Of particular interest in this regard is Foucault's later work, which emphasized the role of the constitution of selfhood in the displacement and rearticulation of power in ways that attempt to avoid the immediate consumption of living interaction by disciplinary regimes and capital. Through various programs of the "care of the self" - which Foucault performs a genealogical analysis of in History of Sexuality Vol. 3-he illustrates the ways in which power is displaced, externalized and rendered impenetrable to the internal constitution of individual subjectivity when structured in terms of alternative disciplinary regimes of iterated embodiment. In other words, according to the late Foucault it is possible to disengage from institutionalized frameworks of power by building a micro-institution of self-hood that does not completely rely on the immanent arrangement of the alreadyestablished regime of power relations (states-of-power), but takes its nourishment from the sublimity of nature's force, which cannot be readily incorporated into the transcendental framework of the already-existing system. Through the facilitation of selfhood, power is transformed back into force, and force is by definition inarticulable and therefore incapable of falling into the statics of state-of-power. By forging a Foucaultian "self," the immateriality of digital virtuality rubs up against the materiality of existence in a way that renders it incapable of incorporating this alternative form of constitutional arrangement into the categories of the same, despite the accelerated terms of the "re-wiring" already accomplished by the likes of Zuckerberg.

In one sense a self is a distinct nodal point in the already-established network, but, by virtue of its alterity (its inability to be completely incorporated), its modality is not totalizable, and therefore, it is incapable of being recognized and incorporated into the network in place. In Kantian terms, the Foucaultian self is of the sublime component of nature that cannot be subsumed by the available categories. The sublime is Nature's revenge as it pierces through the hubris of second nature's use of nature against itself, and the impetus for the revolutionary fervor that arises, not as a product of the re-wired environment, but despite it. While culture-consciousness and digital-immateriality are other than nature-materiality, like Schelling's three potencies on which they are modeled, they are immanent to each other. So while nature can be thought of as culture's constitutive outside, it is outside only insofar as the inside is dependent on it for its existence. We may say that, following S.J. McGrath's The Dark Ground of Spirit: Schelling and the Unconscious (2012), that nature is culture's unconscious, and that the return of the repressed is the return of Schelling's Nature itself, which is both productive and destructive. The return of Nature (making itself felt in the most minor power outage) renders digital-immateriality obsolete, incapable of sustaining itself, and making clear that Nature is deeper than information can conceive, and that, in fact, it is abyssal, or what Schelling calls "the abyss of freedom".

While an individual is only what it is in relation to its history and material conditions, selfhood is akin to what Deleuze and Guattari called an assemblage, and in this case, it is an assemblage of the history and material conditions for the individual, only re-organized around the centre of its propensity for activity. More concretely, the conditions Marx has in mind in terms of the historical and material conditions for the individual are those of exchange value and the commodity form, which are themselves abstractions of the relations of production. In an exchange economy, like the products and medium through which exchange occurs (i.e. money), individuals come to be reduced to objects for themselves and others. Against this process of objectification, the Foucaultian self's modality of construction taps into the resources of Nature's pure force and (re-)organizes itself around the virtual potential contained 
in and as a constitutive part of these forces. In the capitalist organization of production, the individual is valued according to the calculus determining the value of her labour. The price put on the individual's labour is reflective of the commodification of the individual-the determination of its value by market forces-and its alienation, since it is now split between itself and its recognized exchange value. Exchange value does not develop according to the individual's natural abilities, which they would if these had recognizable intrinsic value, but rather, according to the wage determined by the competition between the owners of production. The move to externalize the labourer from the products of her labour-and from herself-is part of the repulsive labour Marx diagnoses as inhibiting the self-fulfillment of the one who performs the labour. Digital labour often carries with it the imagined belief that it is immaterial, and thus disconnected from the embodied activity of work. This alienation directly results from the move toward objectification, and the reduction of the labourer to its status as individual reflected as a "re-wired" image of virtual-immateriality. Alternatively, the Foucaultian self clogs up these networks of power by constructing an aggregate of these relations in a way that defies the logic of the system by tapping into the pure force of nature, thereby becoming other to the system, even as it remains a constitutive node in it. Just as Schelling says that to philosophize about nature is to create it, similarly, to philosophize about the digital is to forge the contours of a self which works on behalf of nature, and introduces the constitutive outside (or culture's unconscious) into the very conditions of the digital image, thus exposing it to its inherent potential for auto-poietic destruction of itself by itself.

\section{References}

Adorno, Theodor. 2005. Minima Moralia: Reflections on a Damaged Life. Translated by E.F.N. Jepcott. London: Verso.

Colletti, Lucio. 1979. Marxism and Hegel. Translated by Lawrence Garner. London: New Left Books.

Combes, Muriel. 2013. Gilbert Simondon and the Philosophy of the Transindividual. Translated by Thomas LaMarre. Cambridge: MIT Press.

Debord, Guy. Society of the Spectacle. Translated by Black \& Red on Marxists.org. Accessed on November 21, 2013. http://www.marxists.org/reference/archive/debord/society.htm

Deleuze, Gilles and Félix Guattari. 1987. A Thousand Plateaus. Translated by Brian Massumi. Minneapolis and London: University of Minnesota Press.

Foucault, Michel. 1986. The History of Sexuality Volume 3: The Care of the Self. Translated by Robert Hurley. New York: Random House.

Fuchs, Christian and Sebastian Sevignani. 2013. What is Digital Labour? What is Digital Work? What's their Difference? And why do these Questions Matter for Understanding Social Media? tripleC-Cognition, Communication, Co-operation: Open Access Journal for a Global Sustainable Information Society 11 (2): 237-293.

Heidegger, Martin. 1985. Schelling's Treatise on the Essence of Human Freedom. Translated by Joan Stambaugh. Athens, Ohio: Ohio University Press.

Lanier, Jaron. 2006. Digital Maoism: The Hazards of the New Online Collectivism. Edge. Accessed January 9, 2014. http://www.edge.org/conversation/digital-maoism-the-hazards-of-the-new-onlinecollectivism.

Marx, Karl. 1986. From the Grundrisse In Karl Marx: A Reader, edited by Jon Elster. Cambridge: Cambridge University Press, 29-62.

Marx, Karl. 1993. Grundrisse. Translated by Martin Nicolaus. London: Penguin Classics. Reprint Edition.

Massumi, Brian. 1992. A User's Guide to Capitalism and Schizophrenia. Cambridge: MIT Press.

McGrath, S.J. 2012. The Dark Ground of Spirit: Schelling and the Unconscious. New York: Routledge.

Ross, Andrew. 2013. In Search of a Lost Paycheck. In Digital Labour: The Internet as Playground and Factory, edited by Trebor Scholz, 13-32. New York: Routledge.

Schelling, F.W.J. 2000. The Ages of the World. Translated by Jason Wirth. New York: State University of New York Press.

Schelling, F.W.J. 2004. First Outline of a System of Philosophy of Nature. Translated by Keith Peterson. New York: State University of New York Press.

Schelling, F.W.J. 1976. Werke: Historische-kritische Ausgabe. Translated by Keith R. Peterson. Edited by Hans Michael Baumgartner, Wilhelm G. Jacobs, and Hermann Krings. Stuttgart: FromannHolzboog. 
Shannon, Claude. 1948. A Mathematical Theory of Communication. The Bell System Technical Journal 27: 379-423, 623-656.

Terranova, Tiziana. 2012. Free Labour. In Digital Labour: The Internet as Playground and Factory, edited by Trebor Scholz, 33-57. New York: Routledge.

Whittaker, Zack. 2012. Apple Patent Could Remotely Disable Protesters' Phone Cameras. Accessed November 13, 2013. http://www.zdnet.com/apple-patent-could-remotely-disable-protesters-phonecameras-7000003640/.

Wirth, Jason. 2000. Introduction. In Ages of the World, translated by Jason Wirth. New York: State University of New York Press.

Zimmermann, Rainer E. 2005. Otherland Revisited: Philosophical Implications of Artificial Worlds. In Towards Otherland: Languages of Science and Languages Beyond, edited by

Rainer E. Zimmermann and Vladimir G. Budanov, 29-44. Kassel: Kassel University Press.

Zuckerberg, Mark quoted in DeBord, Matthew. 2012. Facbook Files for IPO, Zuckerberg Outlines his Vision for 'Rewiring' Us. The Breakdown. Accessed November 14, 2013.

http://www.scpr.org/blogs/economy/2012/02/01/4494/facebook-files-ipo-zuckerberg-outlines-hisvision- $/$.

\section{About the Author}

Kevin Mitchell

Received his PhD from Trent University in Cultural Studies in May 2014. His dissertation was on the influence of the Kantian transcendental turn for contemporary theory and culture with reference to the so-called concussion crisis in American football, South Park's problematization of the discourse surrounding this crisis, and the literary output of novelist and poet Charles Bukowski. Kevin believes that a Cultural Studies without philosophy is blind, and a philosophy without a socio-cultural component is empty. 


\title{
Digital Labour and the Use-value of Human Work. On the Importance of Labouring Capacity for Understanding Digital Capitalism ${ }^{1}$
}

\author{
Sabine Pfeiffer
}

University of Hohenheim and ISF Munich, Germany, www.sabine-pfeiffer.de

\begin{abstract}
On the face of its virtual and immaterial appearance, digital labour often is seen as a phenomenon of abstract work. Contrary to that common understanding, in Marx's theory the abstraction of labour derives from its historical development into a commodity, splitting human work as all commodities into use-value and exchange-value. Thus the process of abstraction is of economical logic, and not to be explained or characterized by the virtual and immaterial quality that is typical for the means and objects of digital labour. In his early work Marx differentiates between living labouring capacity (Arbeitsvermögen) as the use-value of human work and labour power as its objectified form to be exchanged. In the tradition of Marx's Grundrisse Oskar Negt and Alexander Kluge in Geschichte und Eigensinn pointed to the dialectical relationship between the use-value and exchange-value of labour, revealing how labour on its use-value side "contains and reproduces capacities and energies that exceed its realisation in/as commodity", extending the model of labour power "to a whole range of physiological, sexual, social, and national relations" (Negt and Kluge 1993a, xxxiii). While these qualitative and material as well as corporeal aspects of human work are still visible in industrial production processes, they seem to be vanished in virtual work environments. But, digital capitalism not only opens up exploitation to higher levels and new forms (e.g. Fuchs 2012; Scholz 2012), the relevance of human work and its use-value for capitalism becomes more concealed to the same degree as it becomes more significant. The article develops an analytical conception relying on Marx's dialectical distinction between the use-value (labouring capacity) and the exchange-value (labour power), and transforming it into an operationalized model that could be and has been successfully used for empirical studies of digital labour. Labouring capacity has three levels of phenomena: subjectifying corporeal working action, material means and objects of work (even in virtual environments), and the socially and physically experienceable face of globalised work organisation. This analytical concept of labouring capacity (Arbeitsvermögen; Pfeiffer 2004) is especially helpful to reveal the dialectics in digital work and its sources of value creation (Pfeiffer 2013). The article unfolds the theoretical foundations of the concept, and elaborates its potential to analyse digital labour.
\end{abstract}

Keywords: digital work, labouring capacity, dialectic, subjectifying work action, corporeality, and materiality.

Acknowledgement: The conceptual work on this paper is originated in the context of the COST Action 'Dynamics of Virtual Work' (IS1202) and was continued in the research project 'RAKOON_Innovation by active collaboration in open organisations' (Ref. no. 01FK13045); jointly funded by the German Federal Ministry for Education and Research (BMBF) and the European Social Fund (ESF) and supervised by the German Aerospace Center (DLR). Thanks to both anonymous reviewers and their critical thus supportive thoughts that helped to strengthen my argumentation and to improve this paper.

\section{Introduction}

The debate on digital labour/digital work seems to have reached a new all-time high as it is recently discussed broadly in social sciences (cf. Scholz 2012; Fuchs and Sevignani 2013; Huws 2014). Despite minor differences in the lines of argumentation, we see a consensus

\footnotetext{
${ }^{1}$ The term digital capitalism used heuristically here does not refer to one sphere of production, e.g. ICT, nor does it claim that all material resources or forms of labour and production are of no economical importance. For a typology on definitions of digital or informational society see Fuchs (2013) or Schmiede (2006), fostering the conclusion that we are confronted with a "unity of diversity of capitalism(s)" today. Schiller, who coined the term digital capitalism in adversary to the then popular New Economy emphasis (2000), stresses that capital stays at the "center of the political economy even as the market system was restructured to accept a profitable informationintensive orientation" (Schiller 2011).
} 
that work and labour are analytical terms of sustaining importance. Human labour remains the actual source of value creation in the Internet economy. The vivid debate though, points to a peculiar movement: while labour at its core is becoming more important as a source of value creation, on the manifestation level it seems to be fading from view (Pfeiffer 2013, 30): "Human labour remains quantitatively and qualitatively relevant for value creation in the Internet economy: On the one hand, its commodification takes on expanded forms; whilst on the other, more options open up for labour input beyond employment and exchange relationships or to use its results as commons."

Fuchs and Sevignani $(2013,237)$ stress the importance of Marxian labour theory and argue that the difference between labour and work still matters. For them labour is rendered as the valorisation dimension of digital work. The authors revisit the economically driven process of abstractification that Marx developed as the most important characteristic for all capitalistic labour. Fuchs and Sevignani deserve the credit for demonstrating how vital Marx's concept of work and labour still is: how work as an essential category is necessary labour, producing use-value in a concrete labour process; and, how many layers of abstraction are necessary to transform it into abstract labour as commodity, with a quantifiable exchangevalue $(2013,248)$. Thus far, there seems to be no need for analysing digital work differently than Marx would have done it, and in that respect I totally agree with Fuchs and Sevignani (and many others as well): The double character of work/labour that Marx unfolded is "alive and kicking", be capitalism digital or not. There is no need for re-inventing well serving analytical categories if we want to understand the economic function and movements that are specific for capitalism; there may are new options for and means of exploitation in a digital and therefore more globally organized capitalism, but the underlying logic seems steadfast.

Fuchs and Sevignani also look into digital work, which they understand, mostly as bare of material manifestations, be it body or be it objects. The digital quality in their definition comes out of a special form of organizing experiences, derived from brain, speech, and digital media. Following their further line of argumentation reveals three "dialectically connected" dimensions: cognitive work, communicative work, and co-operative work (Fuchs and Sevignani $2013,252)$. The subjects of these three working dimensions are single humans or groups of humans, their objects of work are experiences, thoughts or meaning; and their instruments of work are described as brain, or brain with mouth and ears; or a brain with mouth, ears and body. Finally, the products of work in their presented table also show relations ordered in an upward manner: cognitive work transforms experiences into thoughts, communicative work produces meaning out of thoughts, and in turn these meanings can be shared in co-operative work (Fuchs and Sevignani 2013, 252). The provided heuristic on digital work helps me to clarify the dimensions I think should be analytically elaborated further, and to specify the questions in due course that have to be asked and should be answered.

First of all, and again, I agree with Fuchs and Sevignani upon the feeling that work in digital times needs to be re-considered, although its use-value producing quality has not changed. Besides that consensus, the fact alone that some means, objects and processes of labour and/or work are more virtualized, digitised, or informatised than a couple of years before, does not convincingly justify to reinvent work as an analytical category. A pinch of unease creeps in here, that is best expressed by Ursula Huws, who criticised the emerging consensus "that the world as we know it is becoming quite dematerialised (or, as Marx put it, 'all that is solid melts into air')", and it became "taken for granted that 'knowledge' is the only source of value, $[\ldots]$ and any assertion of the physical claims of the human body in the hereand-now is hopelessly old-fashioned" (Huws 1999, 30). In that sense, I will follow Ursula Huws on her path into what she named the challenge of "re-embody cyberspace", making "visible the material components of this virtual world" $(1999,30)$.

In its core, this objective is tantamount to a dialectical endeavour. Hence, the second chapter challenges the too often to be seen approach of a dualism that is labelled as dialectic. As an example the concept of Scott Lash is critically discussed, which claims that in times of informationalisation, dualism comes to its end. This notion stands for common debates that non-analytically propose all theoretical terms of the past to be obsolete in the face of the shining brand new phenomenon of the Web. No better could the aim of the second chapter 
be characterized than in the words of Lefebvre: "But to define 'the new' by sifting out everything that distinguishes it from the old is not as easy as the dogmatists with their lack of dialectic used to believe. Our era is truly an era of transition; everything about it is transitory, everything, right down to men and their lives. The informed observer will be as struck as much by similarities as by differences, as much by the unity within contradiction as by contradiction itself. The one must not eliminate the other" (Lefebvre 1991, 50).

The main object of this paper which will be outlined in the third chapter is to develop an analytical conception relying on Marx's dialectical distinction between the use-value (labouring capacity) and the exchange-value (labour power) side of human labour, and transforming it into an operationalized model that could be and has been successfully used for empirical studies of digital labour. As we will see, labouring capacity has three levels of phenomena: subjectifying corporeal work action, material means and objects of work (even in virtual environments), and the socially and physically experienceable face of globalised work organisation. The third chapter will unfold the theoretical foundations of the concept and elaborate its potential to analyse digital labour, bringing materiality and the corporeal back in. The fourth chapter will summarize the key conclusions of this paper and offer some prospects on the potential of the concept of labouring capacity for empirical studies on digital work.

\section{Re-visiting Digital Work, Abstractification and Dialectics}

As the Internet continues to develop, we are exposed to a continuing stream of proclamations of novelty and socio-theoretical diagnoses of the times, from a variety of provenances, each based on the notion that the Web has introduced fundamental changes to existing paradigms. These concepts include the "informatisation of production" in the Age of Empire (Hardt and Negri 2000, 280-303), the new "terms of the economic transitions" beyond capital (Hardt and Negri 2009, 263-311), the notion of the "new economy" in Network Society (Castells $2000,77-100$ ), or the current thesis of a "new social operating system" in Networked by Rainie and Wellman 2012).

In contrast with prominent said positively tinged interpretations of the Web, other authors point to an expanded commodification. In these approaches, mostly from a critical perspective, the Web is clearly tagged as a place where interactive networking between people becomes a new object of commercial exploitation (Abelson et al. 2008, 110-11). Users are not primarily of interest as customers but themselves become merchandise (Fuchs 2011) and community experiences on the Web turn into an object of exploitation and source of capital accumulation (Fuchs 2012). These analyses reveal the fact that the core of many web driven business models is "to get everyone to work for free"; namely, all that sharing has a "dark side [...] that hits labour hardest" (Caldwell 2009, 161).

Elsewhere (Pfeiffer 2013) I tried to show how a web based economy needs an exact distinction between value creation understood as the actual process of producing new values; that is, values that do not exist prior to their production process, before the application of human labour. Value realisation, on the other hand, is the process by which the product that originated as part of value generation is valorised and successfully exchanged in the market for money or other value. In other words, it is about actually realising the already-generated value in the marketplace. This very abstract and generalised distinction will suffice for now. It is not new in the tradition of the economic labour theory of value. It is in this way, in particular, that the Marxist explanation of the economy understands value generation primarily as a material production process, in the course of which an equally material product is created by the exertion of human labour capacity. In this tradition, value realisation is conceived as a one-time, isolated act of exchange: as the transfer of goods from production to distribution. These definitions are ideal-typical, analytical characterisations, which Marx used in order to lay bare the nature of these processes in his political economy. Only in this way could he pursue his objective: to work out the importance of human labour as the crucial source of value added and hence of value creation. Ideal types and analytical distinctions, however, can never-and this applies to all schools of thought - be equated with empirical reality. The Marxian analysis pursued the nature of processes, intent on looking beyond manifestations 
(and their empirical variety). However, in applying Marxist theory the multiplicity of empirical manifestations has too often and for much too long been reduced to phenomena that are as close as possible to their intrinsic ideal type. The (mistaken) conclusion that has often suggested itself is that value can only be produced with material products and through industrial production, because added value is so impressively explained by them. In the same way, the importance of other areas of value generation has been underestimated for a long time, just like the value realisation processes, although there, too, human labour has also always engaged in creating value. Starting from this basic analytical distinction, I do not adopt the constantly recurring differentiation between production and information, which appears so frequently in the works cited above. The emphasis solely on the interactive or the cooperative is equally unconvincing, because it is precisely such stylisation of the importance of cooperation in work and cooperation as work a-historically as a new phenomena that makes it difficult for the Web-induced changes in the relationship between cooperation and work to be grasped analytically.

So far, both perspectives so to speak lack dialectical grounding for an in-depth critique. Here, too, it is a matter of deliberately focusing on the structurally concealed areas and qualities and to embark on a search for the inherent other in the everyday. This perspective is neither an end in itself nor a retreat into empirical minutiae, but rather the expression of a decidedly critical perspective. A critical and policy-relevant perspective will not stop on the level of analysing social conditions and their current variations of economically driven strategies. The critical (and consequently the dialectical) view describes not only the aims and functioning of what it wants to critique but looks for the "wrench in the machinery": It seeks out the places where processes do not run seamlessly, where they encounter resistance, and where subversive dealings with them develop; it looks for what escapes the subsuming logic per se; it searches for the inherent limits on the processes themselves, and it is therefore always on the lookout for starting points for alteration and opposition.

Lash (2002) declares the dualistic relationship between use-value and exchange-value to be obsolete, citing the increase in the immaterial thanks to digitisation. This does not appear to be convincing, for two reasons. First, capitalism, especially in its highly industrialised phase, alongside material goods has always produced the immaterial in the form of services, works of the intellect, information and entire systems of cultural scientification practices. Secondly, it remains unclear-because unsubstantiated-why Lash posits dissolution of this dualism for the information society: "But the logic of informationalisation is altogether different. Unlike the logic of commodification it is not dualist, but immanentist. It explodes and partly marginalise the exchange value/use value couple" (Lash 2002, 9).

Lash does not clarify how and why digitisation in particular can cause the dissolution of use-value from exchange-value; instead, he describes manifestations of this dissolving process: Namely, everything is now allegedly "dis-embedded"-actors, people, non-humans, and networks, cultural and material objects and, above all, also information. This analytical vagueness could be overcome by not conceiving the relationship between exchange-value and use-value as dualistic, but rather as dialectical-as laid out by Marx. It is worthwhile to differentiate clearly between dualism and dialectics. A dualism comprises two sets of facts or characteristics of a clearly and succinctly different nature but which are not opposed as such (e.g. fish and meat). Dialectics, in contrast, distinguishes dialectical unity from logical identity, dialectical from logical difference, and dialectical contradiction from logical contradiction.

Lefebvre explains that by work and leisure: their relation is "not a simple one: the two words are at one and the same time united and contradictory (therefore their relation is dialectical)" (1991, 29). As Joachim Israel develops (1979), dialectics comprises dualistic concepts; opposing elements within a totality are fundamental to dialectical thinking. The relationship between the opposing elements is therefore of an internal nature, a relationship Marx points out in his description of the separated unity commodities are constructed of: "So far two aspects of the commodity-use-value and exchange-value-have been examined, but each one separately. The commodity, however, is the direct unity of use-value and ex- 
change-value, and at the same time it is a commodity only in relation to other commodities" (Marx 1859b, 269-92). ${ }^{2}$

In contrast to a dualistic understanding, use-value and exchange-value indeed do not simply confront each other as oppositional, but simultaneously condition each other and combine inseparably in goods. In order to test the relevance of the dialectical method, in what follows I will assume an unbroken dialectical (not dualistically misunderstood) relationship between use-value and exchange-value and apply it-entirely in the vein of Lash's informationalisation-to information and knowledge. Beforehand, it will pay to reread the "inventor" of this dialectical relationship: While exchange-value, being a quantitative relationship, expresses a proportion (quantitative ratio) that makes the most varied use-values exchangeable, use-value itself encompasses the qualitative, the usefulness of a good: "The utility of a thing makes it a use value. But this utility is not a thing of air. Being limited by the physical properties of the commodity, it has no existence apart from that commodity. A commodity, such as iron, corn, or a diamond, is therefore, so far as it is a material thing, a use value, something useful. This property of a commodity is independent of the amount of labour required to appropriate its useful qualities. When treating of use value, we always assume to be dealing with definite quantities, such as dozens of watches, yards of linen, or tons of iron. The use values of commodities furnish the material for a special study, that of the commercial knowledge of commodities. Use values become a reality only by use or consumption: they also constitute the substance of all wealth, whatever may be the social form of that wealth. In the form of society we are about to consider, they are, in addition, the material depositories of exchange value" (Marx 1887c, 45-51).

If the decisive factor in use-value is its usefulness, which, moreover, is realised exclusively through appropriative use by humans, nothing argues against also applying this definition to goods that are not substantial-material. Thus, the use-value of a book is extracted only by reading it, of an image editing software only with specific use-i.e. in actually manipulating a digital image - and of a computer game by playing it. Still, we might be tempted to agree with Lash-"tons of iron" has such a seductively obsolete industrial society ring to it.

Every good potentially has use-value. At a given point in time, any good can have a certain use for a particular purpose, independent of whether the commodity body, to use Marx's term, is represented materially or immaterially. Realising the use-value that is potentially embedded in a good requires the expenditure of human effort: The use-value must be handled or processed, used up or used, consumed-ultimately, it must be appropriated in some form. It follows that the use-value is something that inheres potentially in every substantial representation of a thing but that it is realised only during the process of appropriation. This assertion is completely transferrable without alteration to digitised products also. The (seeming) non-substantiality of an operating system or a software application is not as immaterial as some authors would have it. Software is software is software. And this it is in a very specific manner; for example, as optimised for a certain type of processor, able to run on a specific operating system, etc. Software is software; it is not a car, nor a shirt, coffee pot or book. It harbours within it a particular purpose sphere, a potential usefulness that constitutes its usevalue. The use-value of a word processor is exploited in writing with it-however much I might exert myself, as a user I have as much chance of teasing out a 3D animation from a word processor as from a scripting language like HTML or a programming language not designed for 3D functionalities. In other words, nothing has changed in the fundamental Marxian assertion about use-value if we apply the concept to seemingly immaterial products-this is also why I do not call them immaterial but abstract-substantial. It holds true for both product types that the substantial-material and the abstract-substantial commodity bodies both register a potential use-value-so, for example, the possibility of riding on a motorcycle is its potential use-value or the possibility of writing texts is a word processor's potential use-value. Both commodity bodies have in common that the respective potential use-value cannot arbi-

\footnotetext{
${ }^{2}$ All citations of Marx in this article are to be found in the online version of the English MECW (Marx Engels Collected Works; http://www.marxists.org/archive/marx/works/cw/). Bibliographical details are each identified by the MECW volume, the specific work and/or chapter, the corresponding range of (offline) pages, and the according link.
} 
trarily be abstracted from the substantial-material or abstract-substantial conditions of the commodity body. Thus, we may indeed misuse both motorcycle and word processor but only within the framework of specific, given implementations that materialise very well in some fashion: "One and the same use-value can be used in various ways. But the extent of its possible application is limited by its existence as an object with distinct properties. It is, moreover, determined not only qualitatively but also quantitatively" (Marx 1859b, 269-92).

In addition, it applies equally to both commodity bodies that their potential use-value realizes itself exclusively in the framework of their use or appropriation. Hence, with respect to use-value, no difference of a fundamental nature can be detected between abstractsubstantial and substantial-material products. ${ }^{3}$ The relevance of use-value is salvaged into informational capitalism. Nevertheless, this does not necessarily prove yet that the usevalue/exchange-value relationship remains as intact dialectically. After all, compared with industrial society, a fundamental difference emerges concerning the production of commodity bodies carrying the respective use-values: While the potential use-values that are bound to substantial-material commodity bodies cannot be reproduced, but instead always require the production of a new commodity body, in the case of abstract-substantial commodity bodies the potential use-value is indeed reproducible, since only the data media holding the actual "immaterial" commodity body must be produced and no longer the commodity body itself. In this regard, the dialectical relationship between use-value and exchange-value must in fact receive renewed scrutiny.

Digital capitalism goes hand in hand with an increase in the quantitative importance of abstract-substantial versus substantial-material commodity bodies. Paradoxically, with the increasing exchange-value compatibility of the commodity bodies, their potential use-values become more conspicuous and important. We can trace this very clearly in the software example: The initial one-time production process (of programming) results in an abstractsubstantial commodity body (the code) that (through compilation and duplicability) turns out be especially exchange-value-compatible, because an increment in the goods to be produced for market does not require a further programming production process, just a download capability. This apparently total decoupling of use-value production from goods production at first glance looks like a capitalistic "wet dream": Make a one-time investment in the means of production and in live workers for the software programming production process; next, with a minimal investment in a duplication process, reproduce the use-value as much as desired and then realize an exchange-value in the market that far exceeds the cost of reproduction every time. In fact, this reproducibility of potential use-value can be interpreted as a new direction of movement within the dialectical relationship between use-value and exchange-value. However, it does not lead, as Lash asserts, to a dissolution or marginalisation of this relationship. Instead,_-and this is something that can only be understood dialectically-the seeming triumph of exchange-value as dominant leads to its opposite: Precisely this is what brings the importance of live work and the use-value side of their products and appropriative processes into sharper relief. The debates during the Open Source movement, involving demands for publishing the source codes or criticism of software patenting, exactly refer to this dialectical movement. Or, as Schmiede points out: "informatisation" should not be understood as "a linear tendency but a contradictory in itself" (2006: 334).

\footnotetext{
${ }^{3}$ Software products for example always have a substantial and physical-material basis, not only because material resources are necessarily applied to produce or to use them. The central point I want to highlight here is that the notion of substantiality addresses the material and bodily quality that immanently resides in all objects and instruments of work and in all human work they derive from. The distinction between physical and intellectual work neglects that even working processes of highly abstract thinking are embedded in human corporeality and in its ability of coping with and acting in a material environment. Marx would be the last to ignore that immanent material aspect as he emphatically analysed how abstractification driven by economic dynamics of capitalism conceals this material foundation of the human species.
} 


\section{Labouring Capacity as an Analytical Framework for Digital Work}

\subsection{On the Origins of a Dually Neglected Category}

Labouring capacity, as we will see can be conceptualized as the use-value side of labour, existent both, beyond paid labour and within. This qualitative core of human labour is dually neglected: First of all capitalism ineluctably disdains though uses and relies on this noncommodity side of human work. Secondly, in critical theory labouring capacity is often overseen and dismissed as romantic ideas of a younger Marx who overcame this weakness in his more elaborated and analysis of the Capital. As debates in Marxism lack a tradition of theorising this dialectical counterpart of labour power, it seems necessary to begin the unfolding of this concept by a clarification on its theoretical origins and semantic notions-an endeavour that unavoidably has to start with the terms work and labour. As mentioned above, Fuchs and Sevignani $(2013,239-49)$ already elaborated the distinction between both with special respect to the terms digital work vs. Digital labour, these debate here will just be briefly touched, and just to the extent that is necessary for understanding the argumentation to follow.

In Marxism usually labour is defined as "the capacity to do useful work which adds value to commodities" (Bottomore et al. 1998, 265). Adolph Douai, a pioneer German-American Marxist sees labour as the "opposite of capital", as "working force employed by capitalists and exploited by them", and therefore as "the enemy of human dignity and self-development" while he defines work as "human activity for the purpose of useful production" (Douai 1887, 1). Hannah Arendt defines labour as the never-ending necessity of producing consumable products, satisfying biological and physiological needs and fulfilling sustenance of life. In her view, not capitalism but the "industrial revolution has replaced all workmanship with labour, and the result has been that the things of the modern world have become labour products whose natural fate is to be consumed, instead of work products which are there to be used" (Arendt 1998, 124). Opposed to Marx's view the use-value of goods here seems to be attached to the realm of their origin, be it work (applying action resulting in work products to be used) or be it labour (fabricating labour products to be consumed). Similar to Arendt's distinctions, more current theories distinguish between work that is connected to art and creativity on one hand and mere production necessity on the other. In his book on gift, originally published in the late 1970s and actually re-discovered in the web-inspired debate on commons and a sharing economy, Lewis Hyde (Hyde 2007) discusses the notions of labour and work, but in a slightly different way: While welding on an assembly line, washing dishes, computing taxes or walking rounds in a psychiatric clinic is considered to be work, writing a poem, raising a child, developing a new calculus or resolving neurosis, and even all forms of inventions are seen as labours (Hyde 2007, 63-64). The line of distinction drawn here is that "there are labours that do not pay because they, or the ends to which they are directed, require built-in constraints on profiteering, exploitation, and-more subtly-the application of comparative value with which the market is by nature at ease. There are two points here, one having to do with the nature of work, the other with the commitment of the worker" (Hyde 2007, 138).

Although Arendt's and Hyde's definitions of work and labour do differ in perspective and wording, they share one central point: claiming there is a natural or deeper source of human working or labouring activity that somehow cannot be reached by market's logic and is evident in its untouched purity only beyond mere necessity needs, that is creative and artful or deeply caring activity. Both views try to save the not-market driven core of human work from the consuming logic of markets, exploitation or industrially organized forms of work. This is explicitly (with Arendt) or implicitly (with Hyde) opposed to a-as will be shown in due course-misunderstanding of Marx's theory of labour: To decipher contradictions in empirically real formations of capitalistic work, critical labour theory immanently needs a dialectical vis-à-vis to labour power even within capitalistic labour, not only as mere transcendental idea.

As he lays out in his earlier work, Marx defines labour in the sense of general life activity and human productivity. For him labour is the "living, form-giving fire; it is the transitoriness of 
things, their temporality, as their formation by living time" (Marx 1857, 333-400). Marx describes labour as man's interaction with nature as a process through which both, nature and man are changed and changing, a process of adopting and expressing the species, the "production of his active species-life" (Marx 1844, First Manuscript, Estranged Labour, XXIV) Opposed to that, work is the historically form of labour in capitalism, limited, distorted and deeply alienated. In the Grundrisse Marx uses the term of living labour capacity as a vis-à-vis to value and to labour (Marx 1857, 451-500) ${ }^{4}$ while in the first volume of the Capital we find the term of living labour-power (Marx 1887).

Initially let me introduce one major contribution to my line of thought, which is the idea of a "political economy of labour power" the German social philosopher Oskar Negt and film producer Alexander Kluge outline in their 1981 published three volumes titled Geschichte und Eigensinn. While their earlier book Öffentlichkeit und Erfahrung (Public Sphere and Experience) was translated in English in 1993 (Negt and Kluge 1993a), unfortunately their main work Geschichte und Eigensinn is not available in English. Jameson (1988) deserves the credit to have pointedly summarized the essentials of both works and thus opened it up to an international readership. As a German-American he also helps us to comprehend the notions of the authors' central concepts which are not easily transferred into another language starting with the "untranslatable word Eigensinn" and suggesting to render it with "self-will" (Jameson 1988, 158). Coping with the ideas of Negt and Kluge offers much more challenge than just that of an adequate translation. As Jameson highlights, the "originality" and "utopian effort" of both authors lies in producing a "discursive space of a new type", addressing the necessity of creating a terminology, a kind of new "public language" that corresponds to "forms and experiences" that do not yet exist (Jameson 1988, 157). This is especially true for the term that plays the main role in my line of argumentation here: the German word Arbeitsvermögen, to which Negt and Kluge are referring in the first volume of Geschichte und Eigensinn (1993b, 87-220), and which is translated in Public Sphere and Experience as living labour power, while Jameson sticks mostly to the term labour capacity.

One of the main issues of Negt's and Kluge's book is-as Miriam Hansen puts it in her forward-"whether and to what extent experience is dis/organized from 'above'-by the exclusionary standards of high culture or in the interest of property-or from 'below,' by the experiencing subjects themselves, on the basis of their context of living" (Negt and Kluge 1993a, xxxi).

Although each worker has "his own experiences", the "horizon of these experiences" forms a unity of the "context of living" (Lebenszusammenhang): "This context embraces both the ladder of production of this worker's commodity and use-value characteristics (socialization, the psychic structure of the individual, school, the acquisition of professional knowledge, leisure, mass media) as well as an element inseparable from this, namely, his induction into the production process. It is via this unified context, which he 'experiences' publicly and privately, that he absorbs 'society as a whole', the totality of the context of mystification" (Negt and Kluge 1993a, 6). The use-value side addressed here is what my argumentation will point to. In a footnote the authors exemplify how relevant the sensual side of the context of living is, how the "sensual presence" of this totality Negt and Kluge define as the public sphere of production "is internalized by human beings" (1993a, 13).

The mode for this internalisation process in the sense of Negt and Kluge is experience, both the result and the actor of this process is what they call "living labour power" and in German define as Arbeitsvermögen. Living labour power is formed by primary socialization in the family, and over the whole life course, it can "neither be generated nor sustained without detours, without a qualitative intensification of biographical stages of development" (Negt and Kluge 1993a, 21, original emphasis).

One central claim of the authors is that experience and living labour power is genuinely qualitative-although applied to and developed by experiencing objective structures and their-more or less-material representations. Experiences can "become commodities", they

\footnotetext{
${ }^{4}$ The two major English translations of the Grundrisse by Penguin (Marx 1993, 161) and the MECW cited above both use the term of living labour capacity.
} 
can be "reduced to a common denominator", they can be generalized - but they still "are produced as qualitative moments" and "lack[ ] the commensurability of commodity relations" (Negt and Kluge 1993a, 44, original emphasis). While the dialectical relationship between labour power and living labour shines through here, a footnote guides us deeper into the core of the authors argumentation: Therefore, "[...] labour power is, on the one hand, merely an object as the object of relations of production, while, on the other, it is simultaneously a subject in that it is living labour. Its subject quality becomes an object by way of its being subsumed beneath the power relations of the bourgeois public sphere" (Negt and Kluge 1993a, 59 , original emphasis).

Negt and Kluge focus on how societal change is thinkable given the alienated and alienating totality of capitalistic society. Although their argumentation following that direction is as actual as ever especially in our so called networked society, my main concern aims more to the empirical phenomenon of how living labour and labour power inevitably intertwine at the work site and what contradictions derive from that. Negt and Kluge offer some inspirations here that need to be elaborated in due course. For example as they stress how "labour power found within an individual" in advanced capitalist industries is "simultaneously mobilized and disqualified" (Negt and Kluge 1993a, 170, original emphasis), how there would be no use "for individuals whose behaviour is reduced to mere reactions", and how therefore capitalism "cannot avoid dirtying its hands with human beings". As Negt and Kluge consider this to be an immanently vulnerable point of capitalism and a source of its "extreme instability" (1993a, 185-86), I want to follow that path and explore how this dialectical drama between labour power and living labour capacity could be analytically framed, and-on that conceptional basis-be studied empirically.

For Negt and Kluge workers will have to gain consciousness about the use-value side of their labour power "which like every use-value is submerged without a trace in the quantitative exchange categories of capitalism. The specific nature of this kind of commodity has consisted in the fact that beneath the cloak of things lay a relation between human beings, that beneath the quantifying crust there was a qualitative, living core" (Negt and Kluge 1993a, 255-56). From my social scientist's point, an admittedly humble contribution is to lay bare this qualitative side, to excavate this living core in real world working environments. What Negt and Kluge claim to be a coming to mind the worker has to reach, could qualify as a guideline too for empirical research in sociology of work: to "recognize the machinery [the worker] encounters as what it is in reality, namely, objectified dead labour" and to "be able to perceive the contradiction between labour power as a commodity and [...] living labour (Negt and Kluge 1993a, 256).

In a similar approach to Negt and Kluge, other authors also pay attention towards the qualitative side of labour, referring to Marx earlier works, and, again, have to tackle finding the right expressions. Gulli (2006) directly addresses potentially arising complications by his synonymous usage of living labour and labour, but thus clarifies: "It must then be said immediately that the word 'living', which apparently qualifies 'labour,' is such a qualifier only from a grammatical, not an ontological, point of view. 'Living,' in the expression of 'living labour,' does not address the 'how' or 'which' of labour, but it is the most essential constituent of its 'what,' of its substance." Accordingly Gulli $(2006,1-2)$ asks: "In fact, how could labour be 'not living'?" Gulli also works with further synonyms like "capacity to work" or "capacity to labour" (e.g. 2006, 96 and 130). For Negri $(1991,68)$ terms like "capacity to work" are too "undifferentiated" as for him the worker's "capacity for labour" is a "creative force" that, although "subsumed by capital under the appearance of an equal exchange relation" (Negri 1991, 79), stays a "subjective power (potenza)" (Negri 1991, 70). The elaborations of both Gulli and Negri try to grasp the dialectical relation between the exchange-value and the use-value side of human labour also in terms of subjectivity and objectivity: Negri discovers with Marx a "dialectical development of an exceptional intensity" and argues against a mere natural or humanist misunderstanding that reduces use-value to a "residue or an appendage of capitalist development" (Negri 1991, 70). In contradiction to that Negri claims that the power of living labour, the potenza is irreducible $(1991,133)$, and Gulli emphasizes the interwoven contradiction in pointing out that this subjectivity is "in itself an objective capacity" $(2006,17)$. 
Beyond the differences in language-starting with the problem that German uses Arbeit for both labour and work - the discussion reveals major problems in finding appropriate terms: Negt and Kluge stressed the fact, that a quality is addressed that points towards a societal sphere that per se could not be of existence in capitalism. Gulli and Negri are coping with the verbal limitations one experience in the attempt of describing deeply dialectical relations. All said authors elaborate on the heritage of Karl Marx who thankfully initiates all those thoughts in his earlier writings thus stubbornly refuses to clearly distinguish between both sides of human labour throughout his oeuvre. Only in Grundrisse Marx explicitly uses the term labouring capacity, describing it as "a resource in the bodiliness of the worker" that "contained in his mere vitality", the "possibility of value-positing activity", and a "creative power" surrendered to capital by the worker (Marx 1857, 322-23). And still, while the Grundrisse since their introduction by Marcuse again and again have been considered a key to "an understanding of Capital and of Marx's theoretical project as a whole" (Musto 20080, i) the term of labouring capacity seems to be widely neglected in current theoretical debates. Maybe that has to do with the as often found as often opposed diagnosis that Marx in his earlier writings "drew on a romantic tradition" (Lash 1984, 28). Romantic or not, Lash elaborates aspects of the Marx concept of labouring capacity that have not been as clearly stated by all other hitherto cited authors: According to Lash, Marx speaks "of a series of species capacities or powers which correspond to human needs. He did not, as did rationalists and the mature Hegel, privilege the cognitive capacity; nor did he, like Kant, privilege the moral capacity. But he spoke, as well, of aesthetic, sexual, and labouring capacities, and a capacity of interpersonal relations. The labouring-capacity became labour-power in his later work. But although labour power is a force of production-and hence is attributed theoretical primacy-no doctrinal primacy of the labouring capacity is, therefore, entailed" (Lash 1984, 28-29). Lefebvre cautions against an over- else underestimation of Marx's early writings as these would "contain great riches, but riches still confused, riches half mined and scarcely exploited" (Lefebvre 1991, 80). By revitalising the notion of labouring capacity, Negt and Kluge began just that, in spelling out labouring capacity as an analytical term apt for empirical research of today's digital work, the next chapter follows that path.

Even Engels was more than concerned with the challenge of translating Marx's work appropriately. His earnest worriedness is expressed in a short text titled "How not to Translate Marx". Unfortunately the English translation (Engels 1885) of this text is extremely shortened and lacks all the clarifying examples Engels quotes, scornfully roasting one Mr. Broadhouse who-in Engels' eyes-epically failed in translating Marx: "Marx is one of the most vigorous and concise writers of the age. To render him adequately, a man must be a master, not only of German, but of English too. [...] Powerful German requires powerful English to render it; the best resources of the language have to be drawn upon; new-coined German terms require the coining of corresponding new terms in English" (Engels 1885). The German original text (Engels 1975, 229-237) also refers to one central question that touches the category that is essential for my argumentation here: living labour or living labour capacity. As one major example for a twist from German sense to English nonsense ("Verdrehung von deutschem Sinn in englischen Unsinn"), Engels talks about the dual character of work, unquestionably one of Marx's most excellent discoveries. While German knows one word (Arbeit), English distinguishes between work and labour, and Engels clearly opts to use this distinction consequently: work as specific producer ("Erzeugerin von Gebrauchswert") of use-value versus labour as general producer of value ("Erzeugerin von Wert"; concrete work vs. abstract labour; technical work versus economic labour. As my intention is just to reveal the importance of the use-value side of work within alienated labour, on first glance it seems more than evident that, in following Engels' advise, I should stick to the notion of working capacity. But there are at least two aspects that persuade me to use labouring capacity instead: First of all, working capacity is an established technical term in physics, and there is no need to further interdisciplinary misunderstandings without good reasons. Second of all and of much more importance, the English version of Marx's earlier works-especially the Grundrisse-do use labouring capacity or living labour capacity, and Marcuse's introduction to said Marx's early works (Marcuse 1973) explicitly discusses labour (not work) beyond its 
economic reductions. Following this traditional path, labouring capacity stays my term of choice. The reason for that is not to fall for a Hegelian trap, that could misleadingly interpret labouring capacity for work as such, meaning as work in its ontological and anthropological quality. Labouring capacity as we will see later, on one side is deeply connected to that underlying genuine human capability, but its phenomenon labouring capacity appears in a historically concrete form, derived from as evenly historically forms of the division of labour. What will be conceptualized in this paper and what will be made empirically evident therefore is the qualitative, use-value side of labour, the manifestation/appearance of work not its being/essence.

\subsection{The Dialectical Drama and its Stage Setting: Three Levels for Analysing Digital Work}

If sociological analysis is to stop summoning up the ever-recurring and long-known or to get off the roller coaster of technology euphoria and disappointment, it is necessary to clear up analytically which qualitative changes accompany the digitisation of work. Sketching such an analytical concept starts out quite trivially with the question of what digitisation impacts, to which the answer at first sounds equally trivial: the societal organisation of work, on the one hand, and, on the other, the subject doing the work.

Like every other commodity, human labour, as soon as it appears as a commodity, also turns out to be "a very queer thing, abounding in metaphysical subtleties and theological niceties" (Marx 1887, 81-94). For, even as labouring capacity is made feasible initially by labour power's commodification, it also mutates under its influence into "something transcendent" (Marx 1887, 81-94); translated from the German version, the meaning is closer to "a sensual transcendental thing". Just as the dialectical pairing of use-value and exchangevalue forms the dualistic character of goods, labouring capacity and labour power are the dialectical expressions of the dualistic character of the subjects that offer their labour power in the marketplace. This dialectical juxtaposition of labouring capacity and labour power connects it to the "political economy of labour power" (Negt and Kluge 1993b, 87-220): Namely, through the contrast of subjective production of labour power on the one hand and its objectification as a function of the wage labour process on the other.

The dialectical bundle of labour power and labouring capacity within the subject can only be severed analytically, not empirically. Empirical capture of both sides will therefore always run up against boundary areas, grey zones and shoals. Capturing as precisely as possible both the dialectical motion of the two toward each other as well as labouring capacity and labour power individually requires two things: an analytically exact distinction between the concepts and their collective investigation empirically. One-sided focus on labour power negates the qualitative aspects of human labour that it conceals. Single-minded focus on the labour capacity category distorts the view of the aspects compatible with exchange-value. A critical perspective will only emerge from looking at both of them together as well as at the dialectical relationship between them.

Labouring capacity is at once process and product. As the latter, it has invariably assumed form in the subject as well as outside it, and in this sense it has assumed material form. To the labouring capacity belong all qualities that systematically elude an objectification, while the capacity and potential, which can be objectified and formalised and hence prove to be amenable, at least potentially, to a power or control grasp of capitalistic exploitation, can be assigned to labour power after the successful transformation-but then and only then. Only when the dividing line is laid alongside objectiviability and formalisability does what is genuinely autonomous in labouring capacity remain visible. This is not about the distinction between what was already formalised and objectified and what is yet to go through this process - for example, as a result of future digitisation phases.

Labouring capacity and labour power are two fundamental aspects, each undergoing historically conditioned change and each behaving in a dialectical relationship to each other that can only be separated clearly into quantitative and qualitative components analytically. Analytical separation and empirical focus of this dialectical process are what first makes it possi- 
ble to expose the contradictions that run through society and the individual subject respectively. Out of the inherent qualitative increment of labouring capacity with respect to labour power and, conversely, the quantitative, formalising dominance of labour power with respect to labouring capacity emerges a dialectical movement that is characteristic of a capitalistically constituted society. As a result, labour power is always the abstraction of labouring capacity, which, however, always eludes complete abstraction. But both are in motion: The formalisability and abstractability-that is, the imprinting potential of the process that transforms labouring capacity into labour power-grow in line with the development of productive forces, while simultaneously labouring capacity differentiates itself qualitatively, spreads, partially dies off, but permanently renews on a new level.

Hence, on the subject level labouring capacity and labour power in their inherent dialectical entanglement are determined to be the key levels for the analysis of digital work. An indispensable precondition for empirical analysis on the subject level is the-for the subject, palpable-form determination in which its labour power and its labouring capacity find application, in which qualitative capacity forms and expends itself on the one hand, and, on the other, is quantitatively appropriated, transformed and economised; in short, the relevant formation of the social organisation of labour or the organisation of labour in its broadest sense. This is not just the stage on which the permanent "dialectical drama" (Lefebvre 1991, 169) between labouring capacity and labour power plays out in the subject, but, at the same time, it also forms the structural framework within which social relationships and their interaction with artefacts represent themselves.

The organisation of work to which the subject is tied is the slice of world most likely to be definable and nameable by the subject for appropriation during the work process and within which the specific structural conditions of permanent labour capacity are formed and partly transformed into labour power. Labour power's transformation, use and exploitation, just like the formation, expenditure and sedimentation of labouring capacity, cannot be conceived of independently of the stage setting that surrounds them and, so to speak, of the stage equipment to be utilised. The analysis of what is happening on the stage itself, narrowly focused on the protagonists labour power and labouring capacity, remains incomplete, unless the preformation parameters surrounding them-which they are ultimately also responsible for staging, i.e. for designing-are included in the analytical view. The subject-oriented analysis of informatised work thus includes in an integrative manner the organisation of work as a third level of analysis, in addition to the dual consideration of the labouring capacity and labour power dialectical pair. While the categories of labour power and labour organisation are, so to speak, a proven stock in trade of labour sociological analysis, rediscovery of labouring capacity as a vital and viable category of contemporary labour sociological analysis calls for a deeper look.

When labour power is interpreted as the subject's commoditised side, then structurally the subject alone cannot form its dialectical pole, which must move instead on the same level as its dialectical counterpart and therefore is situated in the labouring capacity. If we interpret the phenomena that are commonly taken as a subjectification of work, even as a result of a specific, historically achieved degree of complexity of the productive forces, which in turn yields an increased requirement for appropriation by the working subjects, then this signifies qualitatively a new need for a strengthened expenditure and exploitation but also the new formation of labour capacity. Particularly with digital work, which, to a special degree, requires the appropriation of a conglomeration of substantial, abstract and socially objectified, highly-complex work, labouring capacity therefore moves into the gravitational centre of the analysis as the qualitative, use-value side expression of human labour and as appropriation to have assumed form in the subject. Labouring capacity as appropriation that has assumed form is only observable in the context of the appropriation concept itself. Appropriation is always appropriation of world and thus not only of substantial-material (or also immaterial) objects and instruments of labour, but of an ensemble of social relationships, i.e., also affiliations, organisational forms, communication behaviours, etc. The qualitative and social essence of work is displayed in labouring capacity; in contrast, labour power is always already an abstraction from this process. Labouring capacity encompasses all the capabilities that 
are needed for the confrontation with world-i.e. for appropriation processes in the broadest sense-and that renew, convert, and transform in its course: The comprehensive forming and application of the senses, living working knowledge with its objectifiable (but not yet objectified) and non-objectifiable shares of experiential knowledge and, finally, capabilities of the situational concretising application of theoretically-grounded knowledge or of theoretically-grounded procedures and methods.

The more complex the world that is to be appropriated, i.e. the more dead, objectified labour, the more complexity of social affiliations and relationships are in it, the more expenditure and formation of labouring capacity is required in the appropriation process. But capital needs this qualitative aspect of living labour all the more: Human labour as producer of incremental value foremost had and has a quantitative meaning; the meaning of human labour as precondition for appropriation of highly developed productive forces is, and increasingly becomes, a qualitative one. That the organic composition of capital leads to a relative increase in dead labour not only has quantitative economic consequences, but also subjectrelated and socially qualitative ones: It yields an increasing necessity of appropriative activities (i.e. expenditure/formation of labouring capacity) for mastery in dealing with more complex, accumulated dead labour, because an increment in accumulated, objectified labour no longer is just an increment of machinery, but even more an increment in complexity and abstraction levels-a process to which digitisation has made and will continue to make a decisive contribution.

"A machine which does not serve the purposes of labour, is useless. In addition, it falls a prey to the destructive influence of natural forces. Iron rusts and wood rots. Yarn with which we neither weave nor knit, is cotton wasted" (Marx 1887, 187-96). And, we should add, digitised knowledge that is not utilised in real time obsolesces. The objects that Marx enumerated can be replaced without difficulty by more current, increasingly more relevant-hence in part also less substantial-materially represented-means of production. Software that is not updated loses compatibility or capability for running on suitable hardware but the rest of the quote points to the undisputed relevance of the core point to be made: "Living labour must seize upon these things and rouse them from their death-sleep, change them from mere possible use-values into real and effective ones. Bathed in the fire of labour, appropriated as part and parcel of labour's organism, and, as it were, made alive for the performance of their functions in the process, they are in truth consumed, but consumed with a purpose, as elementary constituents of new use-values, of new products, ever ready as means of subsistence for individual consumption, or as means of production for some new labour-process" (Marx 1887, 187-96). The artefacts, i.e. the means of production and products, may have changed their outward forms in the course of digitisation, but the function of living labour in calling them into existence has lost none of its relevance-quite the contrary.

\subsection{Corporeality and Substantiality in the Era of Digitisation}

From the concept of labouring capacity developed up to this point inevitably emerge the phenomenal levels that concretise this concept, for labouring capacity always forms and expends itself in relation to an object of labour as well as in handling certain labour means; and is not, after all, a specific form of labour action and of the utilised and the emerging, living labour knowledge nothing more than the outward form of that which was designated as the formation and expenditure of labouring capacity? Labour object, labour means and labour action-here, in their deliberately chosen relation to the analysis level of labouring capacity, in each case with special emphasis on their respective qualitative use-value sided aspectstherefore also form the necessary triad on the empirical phenomenal level of informatised work.

The understanding posited here of labour object and labour means and the specific interpretation of labour action aims at their respective qualitative, use-value sided aspects as seen from the perspective of labour capacity referred to at the start. With that, they are assumed generally to have substantiality or corporeality, even when labour object and labour means do not in reality present themselves as substantial-material and when labour action 
cannot be grasped corporeally in the traditional sense of physical-manual work. After all, the division into mental and physical work is not just real, from a categorical point of view the expression of the dominance of exchange-value over the use-value side of human labour is concealed by labouring capacity. The analytical separation of labour object and labour means also draws on Marx: "An instrument of labour is a thing, or a complex of things, which the labourer interposes between himself and the subject of his labour, and which serves as the conductor of his activity. He makes use of the mechanical, physical, and chemical properties of some substances in order to make other substances subservient to his aims. Leaving out of consideration such ready-made means of subsistence as fruits, in gathering which a man's own limbs serve as the instruments of his labour, the first thing of which the labourer possesses himself is not the subject of labour but its instrument" (Marx 1887, 187-96).

If we imagine electronic and information technological characteristics added to the mechanical, physical and chemical characteristics referred to in the quote from Marx, it not only highlights the unbroken relevance of this distinction. It also becomes clear in what follows why we can tie to the Marxian distinction between the instruments of labour and the actual object of labour and why a clear analytical division between instruments of labour and object of labour achieves heightened significance, particularly with an increasing degree of digitisation.

A concept of object that clings to sensory experience, and thus, in the final analysis, to a somehow generated material substantiality, seems-particularly in the context of the current discourses on digital work-at first glance to be obsolete. However, the actual labour object as empirical phenomenal level of labouring capacity possesses neither compellingly materialsubstantial characteristics nor is it entirely free of them. Although both have a materialsubstantial core (see above), empirically we find material-objectified labour (physical-material products of all kinds) as well as immaterial-objectified labour (e.g. ideas, inventions, software, program code). The actual object that digital labour relates to can for one have product traits in the sense of material- or immaterial-objectified labour. However, it can also relate to the same degree to processes of labour objectification, which, in turn, can once again have a material (production processes, installation procedures, etc.) or immaterial (e.g. high-level project management) character. Ultimately, the actual object of labour can also consist of processes that relate to corporeal subjects (e.g. a hairdresser's customer) or non-corporeal subjects (e.g. in B2B services), and immaterial processes can certainly exhibit a social objectified character.

Digitisation can have the most varied effects on the actual object of labour-or none whatsoever. To determine this with exactitude is always the task of the analytically sharp empirical eye. Thus, the labour object can be displaced by digitisation, or the degree of being able to experience it with the senses can change. Usually a reduced ability to experience can be verified though the additional digitisation levels; however, particularly in the development of future technologies, expansions of experience are conceivable. The actual object of labour is empirically not immediately obvious and is not susceptible to being derived causally from a particular form of activity or a formal labouring task. For one thing, it is constructed subjectively and sensorial re-contextualised, but in so doing does not arbitrarily abstract from the "substantiality" of the work object, however it may present itself. It can be product related objectified labour as easily as a process of objectification. In the abstract, it is materialised and objectified, but concretely it can also prove to be immaterial.

Any distinction between industrial and digital capitalism that refers only to the material or to the non-material resources that seem to be the foundation of value generation as soon as human labour is applied comes in short handed. Immaterial work objects, be it software code or a concept for change-management, often conceal their material and substantial core, e.g. a specific use in "real life" that this software code will make possible for a specific group of users, or the implementation of team work for a specific group of workers in a given factory. On the contrary, there is no and never has been any material work object that derives only from physical work and material resources and lacks any mental planning or intellectual foundations or could not be used or be the bases for immaterial purposes. The core difference between industrial and digital capitalism much more lies in the questions that have to 
be asked: With new forms of digital production and consumption, with the increasing importance of digitalised objects and instruments of work, and with the decline-at least in some national economies-of physical and industrial labour, with all these phenomena we see and explore empirically: Do we find new modes of exploitation? Do we find realms newly or further occupied by economic logic? And, are there new contradictions arising, even of a new quality? Given we answer these questions of and on with a resolutely or at least hesitant Yes, we have some evidence to call capitalism digital. As capitalism, and all other thinkable forms of economic systems that is, will never be digital in whole, but will always have to include agricultural and industrial spheres of production and consumption, critical social sciences also have to search for new or strengthening contradictions between these three spheres, and also have to explore how digitalisation is infused to and by that changes the modes of agrarian and industrial production. The concept of labouring capacity is meant to support the analysis of the ambivalent and heterogeneous varieties of phenomena we observe at today's work place. As the analytical dimensions of instruments and objects of work could not be fully understood without digging into the substantial core of human labour, empirically observable as work action, the next chapter follows that direction.

\subsection{The Missing Link: Subjectifying Work Action}

In whatever manner the instruments and objects of labour concretise themself, they most likely be empirically determined if we follow their targeted direction, i.e. with reference to the labour action directed toward them. Marx's concept of sensuality (Marx 1844, 326-348) is linked in a very specific, intentional way with the human senses and their practical application, because, according to Marx, only "with all his senses" does the human affirm itself in the objective world (Marx 1844, 293-306) and "eye and ear" are the "organs which take man away from his individuality and make him the mirror and echo of the universe [...]" (Marx 1842, 132-181). The human senses and their practical application in sensory activity, according to Marx, perform a specific function in appropriating the world, and therefore it seems especially obvious on the phenomenal level of labour action to choose a concept that not only sheds light on the use-value aspect of labour action but also pays special attention to the senses: the concept of subjectifying work action (Böhle 1994, 209). Skills of this kind have been receiving neither practical nor systematic consideration and are regarded as tacit skills in most cases. The negation reaches further and hints to one important dilemma: "Although the criteria identified with the model of skilled work stress responsibility, individual initiative and creativity, they largely eliminate subjective factors such as emotions, sensations or impressions derived from personal experience. These may have significance for individual motivation and subjective satisfaction, but for the 'correct' dealing with technical working material and an efficient, goal-oriented mastering of working demands they are perceived as disruptive and the cause of errors" (Böhle 1994, 209).

In German industrial sociology since the Eighties the concept of subjectifying work action has been trying to define categories that describe and study empirically this overseen qualities of human labour, qualities of experiential, tacit and informal skills that are so difficult to define. One of the first studies conducted on basis of said concept revealed that skills and qualifications based on empirical knowledge and individual capabilities, such as a feeling for machines and materials, continue to play an important part in a digitized work environment, empirically shown first by work with computer-controlled machines (Böhle and Milkau 1988).

This proves to be particularly compatible with the empirical capture of labouring capacity because, like it, it persists in an idiosyncratic, non-formalisable moment. In this acknowledgment of resistance is revealed, besides the strong emphasis on sensory activity, an additional chance to bridge the empirical concepts of subjectifying work action and the analytical concept of labouring capacity. It emerges clearly why subjectifying work action can be interpreted as a phenomenal level of labour capacity-and, correspondingly, objectifying work action as a phenomenal level of the labour power analysis level. Thus, there already exists in the subject-oriented sociology of labour a conceptually and empirically oft-validated concept that can be adapted for analysing the empirical phenomena of labouring capacity. 
The concept understands experience neither as basically inferior to theoretical, scientifically grounded knowledge, nor sees it as a quality to be completely replaced by the latter. Subjectifying work action is of great significance as an autonomous form of action and knowledge both for planning and practical action as well as for creative, innovative processes that form the basis for coping with unforeseen circumstances (Pfeiffer 1999). But an understanding of experience in this sense merely as a set of experiences, which have been acquired, is not sufficient. The perspective of having experiences also refers to a given moment and situation and to specific action methods used to tackle concrete situations. The concept includes four dimensions of human work action (Böhle 1994, 209): sensorial perception, thought, action and relationship.

All four dimensions do appear as objectifying and as subjectifying. Both modes have different qualities, and they are entangled in each other in every work-related action. They meet the criteria that are considered to be a relationship of dialectical quality: objectifying and subjectifying modes of action are internally contrary to each other on all four dimensions, thus they are deeply intertwined. Therefore the objectifying dimension covers what we usually associate with work in terms of exact and objective perception, logical thinking, rational and sequential procedures, all combined with an unemotional matter-of-fact relationship with the environment. Subjectifying work-related action, by contrast, is a simultaneous and complex sensory perception that takes place via all senses and body movements and is not detached from subjective feeling. It goes beyond an orientation to precise and clear-cut features towards handling more diffuse and stratified sources of information. While a distinction between these four aspects is possible in theory, in the reality of work-related action they are not separable. Analytically the subjectifying dimensions can be described and thus empirically collected as:

- Physical and sensorial perception;

- Associative and intuitive thought;

- Intuitive and dialogical action;

- And an empathetic relationship towards one's environment (persons, tools, and technical systems) or: the objects and means of work (Böhle and Milkau 1988, 239-40).

In the most recent sociological discussions this layer of working action is considered to play an increasing role in all kind of high-tech production. As automation and digitisation produce a growth of complexity and insofar further uncertainties and imponderables employees have to cope with in their everyday working life (Böhle 2011). These phenomena, not to be determined precisely, call for skills, abilities and a kind of knowledge, which can at least partially be characterized as experiential or subjectifying. Subjective experiences and feelings - such as intuitive action, sensory perception, associative, and intuitive thought, forming an interactive context-are acknowledged as an important basis for mastering complex high technology. While first studies focused on the quality of subjectifying work action in material work sites like production in manufacturing or the automotive and chemical industry, more recent research reveal the occurrence and importance of subjectifying work action within more digitized and so called immaterial work environments, e.g. in service work (Böhle 2013), in virtual forms of information-broking (Pfeiffer 1999), innovation work (Pfeiffer et al. 2010a), or assembly work in highly computerised Toyota Productions Systems (Pfeiffer et al. 2010b). The design of work processes and organisation, the construction of machines and their control units, the usability of interfaces and even concepts for qualification and models of learning mostly focus on the objectifying modes of work action. Although subjectifying work action qualities are widely "ignored and neglected" (Pfeiffer 1999), it is "not denied that experiential knowledge has practical uses. However, in the objectifying model, experiential knowledge is viewed as something that basically can be improved, enlarged and ultimately replaced by scientifically grounded knowledge. Thus experiential knowledge is seen only as a preliminary step for theoretical, scientifically grounded knowledge. In this context experiential knowledge is equated with 'everyday knowledge' or 'rules of thumb"' (Böhle 1994, 209). The table below combines all aspects and dimensions of labouring capacity, giving a comprised overview. 


\begin{tabular}{|c|c|c|c|}
\hline \multicolumn{3}{|c|}{ Digitalisation takes effect on... } & \\
\hline \multirow{2}{*}{ Societal division of labour } & \multicolumn{2}{|c|}{ Working subject } & \\
\hline & \multicolumn{2}{|c|}{ Exchange-value side Use-value side } & \\
\hline Organisation of work & Labour power & Labouring capacity & $\begin{array}{l}\text { Analytical } \\
\text { dimensions }\end{array}$ \\
\hline \multicolumn{3}{|c|}{ Object of work / instruments of work - (immaterial) materiality } & \multirow{2}{*}{$\begin{array}{l}\text { Empirical } \\
\text { dimensions }\end{array}$} \\
\hline \multicolumn{3}{|c|}{ Work action - (non physical) corporeality } & \\
\hline \multirow[t]{2}{*}{$\begin{array}{l}\text { - Company organisation } \\
\text { - Internal and inter-firm } \\
\text { cooperation } \\
\text { - Local / global } \\
\text { - Value chain formations } \\
\text { - Production systems } \\
\text { - Re-engineering } \\
\ldots\end{array}$} & $\begin{array}{l}\text { - Formal qualifications } \\
\text { - Performance/control } \\
\text { - Employment } \\
\text { - Subsumption } \\
\text { - Objectifying work action } \\
\text { - } \ldots\end{array}$ & $\begin{array}{l}\text { - Experiential skills } \\
\text { - Individual modes of } \\
\text { action } \\
\text { - Capabilities and experi- } \\
\text { ence } \\
\text { - Autonomy } \\
\text { - Subjectifying work action } \\
\text { - Sediment of biographical } \\
\text { - experiences } \\
\text { - Non-objectifiable genu- } \\
\text { ine living knowledge } \\
\text { - ... }\end{array}$ & $\begin{array}{l}\text { Empirical } \\
\text { manifestations }\end{array}$ \\
\hline & $\begin{array}{l}\text { Formalised, objectified } \\
\text { scientific-based knowledge }\end{array}$ & $\begin{array}{l}\text { Objectifiable parts of living } \\
\text { knowledge }\end{array}$ & $\begin{array}{l}\text { Transformative } \\
\text { dynamics within } \\
\text { the subject }\end{array}$ \\
\hline
\end{tabular}

Table 1: Analytical concept of labouring capacity

\section{Conclusion: Labouring Capacity in Digital Times}

Humans develop labouring capacity constantly through their life course, applying subjectifying action to all environments they encounter and embracing all dimensions of reality in their every-day life within and beyond what we today call the work place. Hence, labouring capacity is not only historically concrete but always more than a unique situation, a single task or a specific job demands to adopt. This fact and the embodied and immanent qualitative nature of labouring capacity makes it a potentially interminable well for creativity, out of the ordinary solutions, and ad hoc improvisation-a truly genuine potential of our species-being, not at all reserved for the so-called creative class (Florida 2012). Labouring capacity could be seen as a human potential that capitalism relies on without understanding it, exploits it without being able to quantify, and neglects even when economic logic thrives to unfold it (Pfeiffer 2014).

The specific nature of subjectifying abilities, and the deeply embodied thus dynamically adopting core of labouring capacity make this ability not only resistant to automation but also specifically relevant for coping with complexities that arise with every new level of automation. We see these developments not only in environments of material production (Böhle 1994 and 2011; Pfeiffer et al. 2010b), but also in mere digital forms of automation (Pfeiffer 1999). Recently we see an arising debate on what I would call automation 4.0, discussing new ICT usage scenarios and their potential for changing work, economy and society for the better or the worse. Intelligent algorithms combined with embedded systems, cloud availability and Big Data challenge our perspective on the human-machine interaction and the question whether we face a new division of labour (Levy and Murnane 2005) beyond today's state of the art computation (Brynjolfsson and Hitt 2000; Brynjolfsson and McAfee 2014). From the perspective of labouring capacity there is no doubt that new forms of human-machine interaction will arise in the years to come; there will be new levels and a not known before quality of digital automation. But we will see new phenomena of the good old productivity paradox (Brynjolfsson 1993; Aral et al. 2012). And, we will also see accordingly developed labouring capacity, necessarily applied and expressed in this highly complex environment of more and 
more layers of digitally objectified labour. Labouring capacity is so much an indispensable quality the more realms of everyday life are object to economical abstractification. Hence in the light of future developments of automation 4.0 labouring capacity more than ever will prove its analytical worth for critical analysis of digital capitalism.

When labouring capacity is taken to the utmost and the potential of this capacity is always more than is ever demanded of the individual in a particular stage of life and historical setting, the change of perspective toward what Negt and Kluge call the abandoned and marginal that is not yet filled up with analytical work alone is a critique (Negt and Kluge 1993b, 93). Lefebvre argues congenially:

Everyday life, in a sense residual, defined by 'what is left over' after all distinct, superior,
specialized, structured activities have been singled out by analysis, must be defined as a
totality. Considered in their specialization and their technicality, superior activities leave a
'technical vacuum' between one another, which is filled up by everyday life. Everyday life
is profoundly related to all activities, and encompasses them with all their differences and
their conflicts; it is their meeting place, their bond, their common ground. And it is in eve-
ryday life that the sum total of relations which make the human-and every human be-
ing-a whole takes its shape and its form. In it are expressed and fulfilled those relations
which bring into play the totality of the real, albeit in a certain manner which is always par-
tial and incomplete: friendship, comradeship, love, the need to communicate, play, etc.
The substance of everyday life- 'human raw material' in its simplicity and richness-
pierces through all alienation and establishes 'disalienation'. If we take the words 'human
nature' dialectically and in their full meaning, we may say that the critique of everyday life
studies human nature in its concreteness (Lefebvre 1991, 97).

While Lefebvre here aims at everyday life beyond capitalistic labour but nonetheless determined by its societal texture (e.g. leisure time, city life), Negt and Kluge (1993) in Geschichte und Eigensinn plead for an analytical acknowledgement of the non-commoditised qualities within capitalistic labour. While labouring capacity can be understood as the theoretical dimension to analytically embrace these dialectically contrary qualities, subjectifying work action serves as the appropriate concept to grasp its phenomenon empirically.

In attempting to develop labouring capacity as dialectical counterpart to labour power inherent in the subject as a key analytical level for digitised work, I hope to have contributed something to the critical perspective on digitized work. Beyond this, labouring capacity, through the empirical phenomenal levels introduced, is an empirically recordable and operationable category which, through its conceptually designed look at the living and not fully subsumable 'other,' opens sociological analysis up again to an emancipation-oriented perspective geared toward manoeuvring autonomy. In the more general version of labouring capacity developed here, which does not limit its emergence to a specific social sphere beyond paid labour, it should become feasible simultaneously to dock the concept of labouring capacity as a current, operationable category for research within and outside paid labour. Even if the analytical concept presented here is deliberately limited to the labour sociological perspective and hence to the capture of (digitised) labour, the labour capacity category could, through the use-value oriented empirical phenomenal level, prove itself in application both to the employment as well as the private sphere-but even more so in the grey area of various boundary-dissolving processes shifting back and forth between these poles. There is no one labouring capacity, there is instead an infinite number of manifestations of labouring capacity. From the labour sociological viewpoint, labouring capacity appears as the category that makes the state and evolution of work quantitatively capturable. The (re)discovery of labouring capacity therefore readily suggests itself as an operationable and hence useful analytical category for social research, particularly in an era when work is undergoing dynamic qualitative change by digitisation. 


\section{References}

Abelson, Hal, Ken Ledeen, and Harry Lewis. 2008. Blown to Bits: Your Life, Liberty, and Happiness After the Digital Explosion. Upper Saddle River: Addison Wesley.

Aral, Sinan, Erik Brynjolfsson, and Marshall van Alstyne. 2012. Information, Technology and Information Worker Productivity. Information Systems Research Journal (3). Accessed April 4, 2014 http://archive.nyu.edu/bitstream/2451/27758/2/CPP-10-06.pdf

Arendt, Hannah. 1998. The Human Condition. Chicago River: University of Chicago Press.

Böhle, Fritz. 1994. Relevance of Experience-based Work in Modern Processes. Al \& Society. Journal of Human Centered Systems and Machine Intelligence 8 (3): 207-215.

Böhle, Fritz. 2013. 'Subjectifying Action' as a Specific Mode of Working With Customers. In Customers At Work-New Perspectives on Interactive Service Work, edited by Wolfgang Dunkel and Frank Kleemann, 149-74. Basingstoke Yorkork: Palgrave Macmillanago Press.

Böhle, Fritz and Brigitte Milkau. 1988. Computerised Manufacturing and Empirical Knowledge. Al \& Society. Journal of Human Centered Systems and Machine Intelligence 2: 235-243.

Böhle, Fritz. 2011. Management of Uncertainty. A Blind Spot in the Promotion of Innovations. In Enabling Innovation. Innovative Capability-German and International Views, edited by Sabina Jeschke, Ingrid Isenhardt, Frank Hees and Sven Tantrow, 17-30. Berlin, Heidelberg: Springer.

Bottomore, Tom, Laurence Harris, V. G. Kiernan and Ralph Miliband. 1998. A Dictionary of Marxist Thought. Oxford, Malden: Blackwell.

Brynjolfsson, Erik. 1993. The Productivity Paradox of Information. Communications of the Association for Computing Manufacturing 36 (12): 67-77.

Brynjolfsson, Erik and Lorin M. Hitt. 2000. Beyond Computation: Information Technology, Organizational Transformation and Business Performance. Journal of Economic Perspectives 14 (4): 23-48.

Brynjolfsson, Erik and Andrew McAfee. 2014. The Second Machine Age: Work, Progress, and Prosperity in a Time of Brilliant Technologies. New York, London: W. W. Norton \& Co.

Caldwell, John T. 2009. Hive-sourcing is the New Out-sourcing: Studying Old (industrial) Labor Habits in New (consumer) Labor Clothes. Cinema Journal 49 (1): 160-167.

Castells, Manuelegri, Antonio. 2000. The Rise of the Network Society: Economy, Society and Culture. Chichester: Wiley-Blackwell.

Douai, Adolph. 1887. Labor and Work: Labor is the Enemy of Human Dignity. Workmen's Advocatecetions and Society 3 (17): 1-2.

Engels, Friedrich. 1885. How Not to Translate Marx. Accessed January 4, 2014. http://www.marxists.org/archive/marx/works/1885/11/translating-marx.htm

Engels, Friedrich. 1975. Wie man Marx nicht übersetzen sollte. MEW Bd. 21. Acessed January 4, 2014. http://www.mlwerke.de/me/me21/me21_229.htm

Florida, Richard. 2012. The Rise of the Creative Class Revisited. Philadelphia: Basic.

Fuchs, Christian 2011. The Contemporary World Wide Web: Social Medium or New Space of Accumulation? In The Political Economies of Media. the Transformation of the Global Media Industries, edited by Dwayn Winseck and Da Yong Jin. London: Bloomsbury Publishing PLC, 201-220.

Fuchs, Christian. 2013. Capitalism or Information Society? The Fundamental Question of the Present Structure of Society. European Journal of Social Theory 16 (4): 413-434.

Fuchs, Christianh. 2012. Dallas Smythe Today - the Audience Commodity, the Digital Labour Debate, Marxist Political Economy and Critical Theory. Prolegomena to a Digital Labour Theory of Value. tripleC. Journal for a Global Sustainable Information Society 10 (2): 692-740.

Fuchs, Christian and Sebastian Sevignani. 2013. What is Digital Labour? What is Digital Work? What's Their Difference? and Why Do These Questions Matter for Understanding Social Media? Digital Labour Theory of Value. tripleC. Journal for a Global Sustainable Information Society 11 (2): 237-293.

Fuchs, Christian. 2012. Class and the Exploitation on the Internet. In Digital Labor: the Internet as Playground and Factory, edited by Trebor Scholz and Da Yong Jin, 211-224. New York, Oxon: Routledge.

Gulli, Bruno. 2006. Labor of Fire: the Ontology of Labor Between Economy and Culture Socialism. Philadelphia: Temple University Press.

Hardt, Michael and Antonio Negri. 2000. Empire. Cambridge: Harvard University Press.

Hardt, Michael and Antonio Negri. 2009. Commonwealth. Cambridge: Harvard University Press.

Huws, Ursula. 1999. Material World: the Myth of the Weightless Economy. Socialist Register 35: 2955. 
Huws, Ursula. 2014. The Underpinnings of Class in the Digital Age: Living, Labour and Value. Socialist Register 50 (1).

Hyde, Lewis. 2007. The Gift: Creativity and the Artist in the Modern World. New York, Toronto: Vintage.

Israel, Joachim. 1979. Language of Dialectics and the Dialectics of Language. Copenhagen: Munksgaard.

Jameson, Frederic. 1988. On Negt and Kluge. October 46: 151-177.

Lash, Scott. 2002. Critique of Information. London, Thousands Oaks, New Delhi: Sage.

Lash, Scott. 1984. The Militant Worker. Class and Radicalism in France and America. Cranbury, NJ: Associated University Press.

Lefebvre, Henri. 1991. Critique of Everyday Life. Vol. 1. London, New York: Verso.

Levy, Frank and Richard J. Murnane (2005). The New Division of Labor: How Computers are Creating the Next Job Market. Princeton, Oxfordshire: Russell Sage.

Marcuse, Herbert. 1973. On the Philosophical Foundation of the Concept of Labor in Economics. Telos (16): 9-37.

Marx, Karl. 1842. Proceedings of the Sixth Rhine Province Assembly. First Article. Debates on Freedom of the Press and Publication of the Proceedings of the Assembly of the Estates. MECW Vol. 1. Accessed January 4, 2014. http://www.marxists.org/archive/marx/works/cw/volume01/index.htm

Marx, Karl. 1844. Economic and Philosophical Manuscripts of 1844. MECW Vol. 3. Accessed January 4, 2014. http://www.marxists.org/archive/marx/works/cw/volume03/index.htm

Marx, Karl. 1845. Theses on Feuerbach. MECW Vol. 5. Accessed January 4, 2014. http://www.marxists.org/archive/marx/works/1845/theses/original.htm

Marx, Karl. 1857. (Grundrisse) Outlines of the Critique of Political Economy. Accessed January 4, 2014. http://www.marxists.org/archive/marx/works/1857/grundrisse/

Marx, Karl. 1859. Economic Manuscripts of 1857-58 (Second Part of the Grundrisse and the Contribution to the Critique of Political Economy 1859), MECW Vol. 29. Accessed January 4, 2014. http://www.marxists.org/archive/marx/works/cw/volume29/index.htm

Marx, Karl. 1887. Capital I. A Critique of Political Economy. MECW Vol. 35. Accessed January 4, 2014. http://www.marxists.org/archive/marx/works/cw/volume29/index.htm

Marx, Karl. 1993. Grundrisse. Outlines of the Critique of Political Economy. London, New York: Penguin.

Musto, Marcello. 2008. Karl Marx's Grundrisse: Foundations of the Critique of Political Economy 150 Years Later. London, New York: Routledge.

Negri, Antonio. 1991. Marx Beyond Marx: Lessons on the Grundrisse. New York, London: Autonomedia.

Negt, Oskar and Alexander Kluge. 1993a. Public Sphere and Experience: Toward an Analysis of the Bourgeois and Proletarian Public Sphere. Minneapolis: University of Minnesota Press.

Negt, Oskar, and Alexander Kluge. 1993b. Geschichte und Eigensinn. Bd. 1: Entstehung der Industriellen Disziplin aus Trennung und Enteignung. Frankfurt: Suhrkamp.

Pfeiffer, Sabine. 1999. Ignored and Neglected-Work in Cybersociety. In Exploring Cybersociety. Social, Political, Economic and Cultural Issues, Vol. 2, edited by John Armitage and Joan Roberts. Newcastle: University of Northumbrian.

Pfeiffer, Sabine. 2004. Arbeitsvermögen. Ein Schlüssel zur Analyse (reflexiver) Informatisierung. Wiesbaden: VS Verlag für Sozialwissenschaften.

Pfeiffer, Sabine. 2013. Web, Value and Labour. Work Organisation, Labour and Globalisation 7 (1): 12-30.

Pfeiffer, Sabine. 2014. Social Media in Organizations: Fostering Creativity and CommunicationChanging Culture in the Process. In Communication, Culture and Creativity: Reframing the Relations of Media, Knowledge, and Innovation in Society by Hubert Knoblauch, Mark Jacobs and René, 247-266. Frankfurt, Berlin, New York: Lang.

Pfeiffer, Sabine, Petra Schütt and Daniela Wühr. 2010a. Innovation, Market, NetworksInterdependencies, Synergies and Contradictions in Technical Innovation Processes. In Markets as Networks, edited by Tanya Chavdarova, Petya Slavova and Svetla Stoeva, 165-180. Sofia: St. Kliment University Press.

Pfeiffer, Sabine, Petra Schütt, and Daniela Wühr. 2010b. Standardization of Production and Development Processes-Blessing or Curse? in Technical Innovation Processes. In Sixteenth International Working Seminar on Production Economics, edited by Robert W. Grubbström and Hans H. Hinterhuber, 411-422. Innsbruck. 
Rainie, Lee and Barry Wellman. 2012. Networked: the New Social Operating System. Cambridge: MIT Press.

Schiller, Dan. 2000. Digital Capitalism: Networking the Global Market System. Cambridge, London: MIT Press.

Schiller, Dan. 2011. Power Under Pressure: Digital Capitalism In Crisis. International Journal of Communication 5: 924-941.

Schmiede, Rudi. 2006. Knowledge, Work and Subject in Informational Capitalism. In Social Informatics-An Information Society for All? edited by Jacques Berleur, Markku I. Nurminen and John Impagliazzo, 333-354. New York: Springer.

Scholz, Trebor. 2012. Digital Labor: The Internet as Playground and Factory. New York: Routledge.

\section{About the Author}

Sabine Pfeiffer

Prof. Dr. habil. Sabine Pfeiffer worked in the automotive and manufacturing industry as a toolmaker and in technical support for CNC-machines and CAD/CAM-systems for years before she studied sociology, psychology, and philosophy at the University of Hagen. In 2003 her doctoral thesis about labouring capacity (Arbeitsvermögen) as a category for the critical analysis of informatisation was marked outstanding. Sabine Pfeiffer worked at the Institute for Social Science Research (ISF Munich) since 1998 as a senior research fellow, and still serves there as a member of the governing board. Since her habilitation on the dialectics of corporeality and materiality in 2009, she held a professorship for innovation at the University for Applied Sciences Munich from 2010 to 2014, when she was appointed to the chair of sociology at the University of Hohenheim. 


\title{
The Ideological Reproduction: (Free) Labouring and (Social) Working within Digital Landscapes
}

\author{
Marco Briziarelli \\ University of New Mexico, Albuquerque, USA, mbriziarelli@unm.edu
}

\begin{abstract}
The present paper explores the role of (neo-)liberal ideology in reproducing digital labour. Drawing on Terranova's concept of "free labour", and Fuchs and Sevignani's distinction between "work" and "labour", the author claims that theorization of Web 2.0 practices requires the exploration of ideology as a material force and a contradictory phenomenon. More specifically, the paper considers the capability of ideology to mediate between the valorization and exploitation of user-generated content and the utopian thrust that re-signifies unpaid labour into apparently engaged practices resonating with liberal ideology. In the last section, the paper takes Facebook as an exemplification of such an ideological synthesis of exploitative and normative aspects.
\end{abstract}

Keywords: Digital Labour, User Generated Content, Free Labour, Ideology, Marx, Facebook

\section{Introduction}

The present paper tries to answer an apparently simple question: granting the applicability of Smythe's "audience labour" analysis (1977) on digital contests (Fuchs 2010, 2012), what is it that drives web 2.0 prod-users to labour for free, to be exploited and to be treated as a commodity sold to the advertising industry? Why do they accept to generate content that instead of enriching the "general intellect" becomes appropriated for the personal gain of few? Is it a question of "false consciousness" or Žižek's "ideological cynicism"?

I intend to respond to these questions by exploring the reproductive function of ideology in Web 2.0 media practices. I claim that the theorization of digital labour requires the exploration of a multifaceted and contradictory ideological phenomenon. While producing content for the Web, digital labour produces (and simultaneously being a product of) a particular ideology that informs the web 2.0 user's consciousness. Such a worldview is at the same organic to the valorization and exploitation of user-generated content and propelled by a utopic thrust that resonates with the liberal understanding of sociability.

Pushing against a tendency in media studies to privilege an idealist understanding of ideology, this essay examines it as a material force that reproduces labour by extrapolating from "necessity", an apparent "liberty" of production. I will describe such a process by drawing on Terranova's notion of "free labour", Fuchs and Sevignani's (2013) distinction between "labour" and "work", and Bloch's (1986) understanding of ideology. In my view, such a combination of dystopic and utopic elements reflexes the capability of this specific ideology to conciliate the aspect of unpaid "labouring" and the apparently emancipatory "social working" aspect of web 2.0 platforms.

In order to advance my argument, I structure this paper in three main sections: the first one concerning digital labour; the second one exploring the ideological dimension of digital labour; and the third one that uses Facebook as an exemplification of such ideological dynamics. After an introduction to digital labour and a review of some of the central issues around it, the paper discusses how ideology has been conceptualized in media studies. Drawing on Bloch, I argue that making sense of ideology requires consistently maintaining a position in between material and symbolic levels, mystification and demystification, and dystopia and utopia. Finally, the paper looks at Facebook's practices as an exemplification of such a dynamic. In fact, some Facebook users, while exploited in the unpaid production of content for this platform, also follow a liberal normative thrust that frames Facebook's usage as a form of social work towards a better society. 


\section{Labour in the Context of Informational Capitalism}

In recent times, several scholars have tried to understand the new media environment through the perspective of what Castells (2000) defines as "the process of capitalist restructuring undertaken since the 1980s", otherwise called "informational capitalism" (18). Similarly to the notion of "post-industrial society" (Bell 1973), "information society" (Webster 2002), informational capitalism refers to the increasing prominence of information and communication within capitalism under conditions of globalization and rapid technological development.

In such a new configuration, the worker becomes increasingly involved in the tasks of handling, distributing, and creating knowledge (Mosco and McKercher 2007; Florida 2002). Consequently, labour progressively undertakes relational, cognitive, imaginary and sensemaking, affective, and caring activities (Virno 2004). Such a new productive mode of production tends to overcome the separation "between working-time and lifetime, working place and life place, and production and reproduction" (Morini and Fumagalli 2010, 240).

In the field of media studies, much of the interest about informational capitalism could be explained in relation to the alleged capability of new media to radically redefine the meaning of mass communication and blur the boundaries between the moment of production and moment consumption, the moment of dissemination, and reception of the message. I am particularly interested in the one characterized by a normative liberal thrust. In fact, one of those narratives depicts the mediated communication of Web 2.0 platforms as a much more egalitarian process, in which the audience operates at the same level of the traditional institutional communicator as well as producing more space for individual expression (Napoli, 2008). Such a perspective reverberates with a conceptualization of an (politically and socially) emancipated audience who "does things with media" as opposed to "be[ing] used by media".

In reaction to such narrative, several scholars have scrutinized new media practices from the point of view of power, labour, and value creation (see eg, Arvidsson and Colleoni, 2012; Cohen, 2008; Dyer-Witheford 1999; Fuchs, 2010, 2012; Hesmondhalg, 2010; Margonelli 1999; Napoli, 2008; Petersen 2008; Scholz 2008; Terranova 2004; Willmott 2010; Zwick et al. 2009). Concerned with the political economic of new media tendency to capture value outside the traditional productive process; in this section, I briefly review Fuchs' $(2010,2012)$ contribution, the two sided notion of free labour formulated by Terranova (2004) as well as those concepts that resonate with the utopian and dystopian discourse that, according to Mosco, surrounds modern media.

\subsection{Valorising Digital Labor}

According to Scholz (2012) digital labour has become one of the key concepts in order to both understand the political economic environment of new media and the discussion around it. One of the most critical positions in such a debate states that internet has been incorporated in a dominant corporate model of capital accumulation, which is grounded on the exploitation of unpaid labour of "prod-users," ie, the activity of creating content by users while involved in activaties such as blogging or social networking. I find particularly representative of such a discussion Fuchs' perspective (2010, 2012).

Fuchs (2010) applies Marxian theory of value to examine the unpaid labour of web 2.0 users, which while constituting an important factor of production of value, remains unpaid and exploited. Fuchs' essay pushes against several tendencies. On the one hand, the paper rejects a literature (Bell, 1973; Florida, 2002; Beck, 1992) that "negates the relevance of class analysis in informational society" (180). On the other hand, it reacts against the emancipatory interpretation of free labour, which, as Andrejevic observes (2008), recalls the ideologically loaded media studies notion of "active audience". By contrast, the author considers prod-users as exploited knowledge workers, a class fraction of the multitude (Hardt and Negri 2000), for "in informational capitalism, knowledge has become a productive force, but knowledge is produced not only in corporations in the form of knowledge goods, but also in everyday life" (186). 
According to Fuchs, capital freely consumes the commons (product of natural and societal process such as water or education) freely created by prod-users. Drawing on Smythe's seminal essay (1977), Fuchs argues that users are sold as a commodity to advertisers. However, if in the original analysis of TV practices, the audience only consumed media material, in the case of new media, the audience assumes the role of producing content, being engaged in permanent creative activity, communication, community building, and content production. In conclusion, to paraphrase Jhally and Livant (1986), Fuchs critically examines new media by focusing on what media take out from audience (value) rather than what media are suppose to put into it (messages).

\subsection{Why Prod-Using?}

Why prod-users work for free? What drives them? Arvidsson and Colleoni (2012) would claim that the typical subjectivity operating with web 2.0 media tends to value affective relationship so that Facebook or YouTube users do not really reason in terms of labour but rather in terms of building and enjoying a sense of community. On the other hand, Fuchs (2012) provides another plausible explanation that draws on Bourdieu's political economic analysis of power: if we are to acknowledge the existence and relevance of affective relations then, social, cultural. and symbolic capital become necessities and scarce goods. Therefore, one could say that prod-users are in fact compelled to "work" for new media in order to accumulate social relations (social capital), qualification, education, knowledge (cultural capital), and reputation (symbolic capital) (for instance, frequently job candidates are evaluated based on the their social media activity). From this point of view, affective relations can be effectively understood by Marxian theory of value because the effort to build them and accumulate social capital requires labour time and should be understood in the general framework in which "presumption" activities (Fuchs, 2013) work in the capitalist regime of time.

In my view, both desire of sociability and exploitation should be combined in order to explain the set of motivations that drives prod-users and that requires an understanding of ideology capable of synthetizing what I consider some dualisms characterizing digital labour: coercion and consent, and false and "true" consciousness. Thus, in relation to the classic Marxist perspective according to which exploited subjects "simply don't know," and Žižek's reformulation "they do know but they still do it", I claim that a functionalist understanding of ideology-ie, stressing the instrumental function of ideology in re-producing the dominant relations of productions-must also consider a normative set of motivations that cannot be considered as completely fictitious nor completely cynical and that, in this particular case, resonate with (neo-)liberal ideology.

\section{Ideology as a Material and Mediating Force}

As Garnham (2000), Mosco (2011), and Miller (2011) have noticed, the great majority of communication and media studies have been consistently affected by a chronic dis-interest about labour and material production. In this sense, against a mostly idealist approach to ideology characterizing this field, this paper explores the role of ideology in materially reproducing the conditions of production of digital labour. As I will show later on, ideology is in my view, what mediates two co-existing aspects in web 2.0 activities: what Fuchs and Sevignani would define as "working" and "labouring" and what could be synthetized in Terranova's concept of "free labour".

\subsection{The Idealism of Ideology in Media Studies}

In the last decades, the interest in theorizing the ideological agency of media in both reproducing and transforming a given society has been impelled by the seminal contributions of several Marxist authors. Some of those are Althusser and his theorization of media as Ideological State Apparati (1970), Gramsci's study of cultural hegemony and the study of media as the most dynamic ideological force (1971), Horkheimer and Adorno's conceptualization of media as culture industry that created a pervasive understanding of the world (1970), 
Debord's definition of capitalism as a mediatic spectacle that promotes consumer culture and a highly fetishized representation of social reality (1968), and finally Williams's theorization of cultural materialism (1977), which, building on Gramsci and Goldman, re-positions communication at the core of material production of life.

Such literature should be credited to have "emancipated" media and communication process from the superstructural/epiphenomenal condition. However, as Peck (2006), Artz, Maceck and Cloud (2006) and Aune (2004) claim, most of media research seems to still operate within the idealist assumption that "consciousness determines social being." In many instances, the interest of media scholars for ideology is more focused on the communication process that channels ideology rather than ideology itself. As a result, ideology is almost never linked to labour and material production but reduced to a "result" within the still powerful tradition of mass communication effects.

Exemplary of how media studies tend to interpret ideology is Hall's very influential approach. According to Gunster (2004), whereas Hall initially drew from Althusser's concept of Ideological State Apparati (1970) and Gramsci's concept of cultural hegemony (1971), subsequently his understanding significantly distanced from the other two authors. Hall in fact, passes from an Althusserian understanding of ideology-a highly contradictory living practice that in the last instance reflects the dominant relations of production-to a pluralist approach that describes the confrontation among competing ideologies. Thus, whereas for Althusse, ideology could be distinguished from science, and for Gramsci the ideologically loaded common sense had to be distinguished from a "good common sense", for late Hall, no ideology has the guarantee of becoming dominant and no worldview can definitively raise (good) consciousness.

Thus, "late" Hall's interest is not so much about the relationship between ideology and an alleged objective reality but rather on an ideologically constituted environment that can never been completely escaped. In this context, for Hall, media are involved in "the politics of signification", and "cultural wars" (Davidson Hunter 1991), according to which media produce images of the world that not only give events particular constitutive meaning. As Hall (1982) puts it, "Representation is a very different notion from that of reflection. It implies the active work of selecting and presenting, of structuring and shaping; not merely the transmitting of an already-existing meaning, but the more active labour of making things mean" (64).

As Crocteau (2002) notices, "most media scholars use ideology as a concept to understand representation, focusing on how media texts signify. Therefore, they are in most cases prone to treat ideology in idealist terms, because the key issues regards ideas reflected on texts, images and semiotic codes, their interpretations, their representation, their signification: "For ideological analysis, the key is the fit between the images and words in a specific media text and ways of thinking about, even defining, social and cultural issues" (160). Thus, paradoxically enough, if for a long time Marxist scholarship had been accused of embracing a "crude materialism" that anti-dialectically privileged the material over the mental/symbolic, the economic over the cultural (Godelier 1986), in media studies the opposite seems to be true. That is to say that consciousness is privileged over social being.

However, the well-known reverse of the formula-"social being determines consciousness"-cannot be accepted uncritically or without qualifications. In fact, Marx and Engels did not enter a conversation dominated by the Hegelian "Geist" and Kantian "a priori" forms, to replace absolute idealism with absolute materialism as they argue that "The production of ideas, of conceptions, of consciousness, is at first directly interwoven with the material activity and the material intercourse of men, the language of real life" $(1977,164)$. In other words, Marxian historical materialism does not necessarily treat ideas and consciousness as mere appearance of a "material essence" because they, in turn, contribute to reproducing the social being. Their intervention meant to criticize the tendencies of German contemporary theorist to understand consciousness as process abstracted from the real and material processes of history.

According to Marx (1977), grasping this meant acquiring superior and standpoint that could allow scientific explanation of socials phenomena and even prediction. Conversely, failing to realize such a nature of social reality and therefore abstracting it produces ideology, 
a cognitive mistake: If in all ideology men and their circumstances appear upside down as in a camera obscura, this phenomenon arises just as much from their historical life process as the inversion of objects on the retina does from their physical life process (47). The mistake is not so much about giving credit to a particular ideology or a worldview as opposed to material circumstances, but rather abstracting the former from the latter.

Equally telling is Engels's statement that the mistake of the subject living by an ideology is due to be centred in its own artefact self-referential world. Again, the problem found in it is not necessarily about ideas, because without ideas the architect could not be distinguished from the bee, but about ideas abstracted from real material conditions and relationships: "Ideology is a process accomplished by the so-called thinker consciously indeed but with a false consciousness. The real motives impelling him remain unknown to him; otherwise it would not be an ideological process at all. Hence he imagines false or apparent motives. Because it is a process of thought he derives both its form and its content from pure thought, either his own or his predecessors" (Letter to Mehring 1893, 2).

So, the question then is whether a worldview driven by a historical materialist approach, therefore not abstracted from material conditions, could be considered as a "good" ideology, in other words to become aware? In a renewed passage Marx seems to suggest that, therefore implying an understanding of ideology that frictions with the conceptualization of ideology as false consciousness: The distinction should always be made between the material transformation of the economic conditions of production [...] and the legal, political, religious, aesthetic, or philosophic-in short, ideological-forms in which men become conscious of this conflict and fight it out (Williams, 1985 157).

Williams states (1985) that this more neutral understanding of ideology would characterize some important Marxist scholarship of the last century as it is exemplified by the fact that for Lenin key aspect of struggles translated into the clash between competing ideologies, or for Gramsci exists a common sense as naturalization of hegemony and good common sense. From the perspective that I am trying to define in this paper, ideology is understood as both: it is actually the mediation between awareness and the false consciousness, between theoretical knowledge and empirical experience because as Gramsci claims, the awareness about the historical and materialist nature of social reality takes place in a continuous interaction with a given ideologically driven worldview and historic specific social conditions.

Accordingly, in the historic-specific context of informational capitalism, the worldview I describe combines the Marcuse (1965) and Horkheimer and Adorno (1974) account of how late capitalism fuses labour and leisure time and Mosco (2005), Bloch (1986), and Jameson (1991) cultural diagnostics of the function of utopia. According to such view, digital workers do not only labour for "fun", and the instrumental acquisition of social cultural and symbolic capital (Fuchs, 2012), but also are motivated by the technological utopianism that connects new media with the hope to regenerate civil society, Gemeinschaft, democracy and affective relations. In fact, by generating content, Web 2.0 users also generate subjectivity (Coté and Pybus, 2007) and an ideology that ethically signifies knowledge work as a kind of social work towards a utopic society emancipated by technology.

In order to explain the way I understand ideology I find Eagleton's (1991) taxonomy very useful. The author provides six possible ways to conceptualize ideology, from a more general to a more specific kind of phenomenon: first it could be defined as "general material process of production of ideas" (28); according to the second definition those ideas could pertain to a "socially significant group" (29); according to the third definition, this group may actively aim at the "promotion and legitimation" (29) of such an ideology; according to the fourth such a class project may involve "the complicit of subordinated classes" (30); according to the fifth, such a project can also entail deceptive aspects such as "distortion and dissimulation" (30); finally according to the sixth definition, ideology may be systematically reproduced by arising "from the material structure of society as a whole" (30).

In relation to such a conceptual mapping, my understanding of ideology would try to synthetize different aspects of those definitions: it consists of a class-based project, which aims at a hegemonic ruling in the Gramscian sense, thus implying the effort to combine force and consent (of subordinated classes), as well as trying to symbolically and materially universal- 
izing its world view. However, from a longitudinal perspective, such a worldview cannot be easily tied to a class because it becomes structurally reproduced by all sorts of productive and consumptive practices, as in the case of commodity fetishism. Thus, in my definition, ideology consistently aspires to hegemony, it is the "particular" that aspires to a "general," the "subjective" that aspires to the "objective", the "explicit" that aspires to be "implicit", and finally, the "cultural" that aspires to become "natural."

In relation to digital labour, such a position echoes the Marxian notion of "productive consumption", in the sense that audience produces consciousness but also articulation of ideas by consuming commodified culture (in Nixon, 2012 443). Marx and Engels in German Ideology (2001) maintain that the production of consciousness and the production of ideology are tightly linked to the concrete human activity: the production of ideas, concepts, and consciousness is, first of all, directly interwoven with the material intercourse of man, the language of real life. Conceiving, thinking, the spiritual intercourse of men, appear here as the direct efflux of men's material behavior [...] Consciousness does not determine life: life determines consciousness (35-36).

However, the worker's consciousness and his/her adoption of specific worldviews does not passively reflect "real life" but interacts with it and therefore, cannot be simply reduced to an instrumentalized epiphenomenon of productive logics. Following Bloch's (1986) consideration of the complexity of the ideological phenomenon, I would argue that the ideology of the prod-users is not simply "false consciousness" in the same way as consciousness is not simply "false ideology" because aside from its concealing of exploitation, it comprises a genuine desire of the community for a better society. As Kellner and O'Hara explain (1976), Bloch considers ideology as an ambiguous two-sided phenomenon as it encompasses distortion, mystifications, techniques of manipulation, and domination, but it also incorporates a utopian residue. Thus, the cognitive error of ideology and the cognitive accuracy of consciousness (as opposed to false consciousness) maybe distinct but also are highly intertwined. In relation to my previously provided definition of ideology, it is the aspiration to become holistic that allows ideology to resonate with utopia.

In Part III of his Principle of Hope, Bloch applies his utopian reading to all sorts phenomena associated to ordinary life and popular mediated culture: advertisements, circus, fairy tales, illustrated magazines, the cinema, and the theatre. For instance, Bloch understands all consumer products to take care of the body as means to transcend the material corporality of the body. Just like in daydreaming, ideology is characterized by the tension between past and future. On the one hand, it operates in a pre-existing framework of ideas and practices; on the other hand, when embraced by particular subjects, it becomes a projection for the future, an imagine of what life could be with for instance more money, with a beautiful body, or with a revolution. From this point of view, ideology is for Bloch a very dynamic ensemble of lived relations and concrete possibilities more than is more imaginary in the Lacanian sense than simply deceptive.

Such a process of production of ideology and consciousness generating through the produsers' activity does not take place in vacuum, but is immerged in a pre-existing ensemble of ideas and practices. In other words, it interacts also with previous existing worldviews that contribute to give meaning to both media and their usages. Drawing on Mosco (2005), I see in media practices, both the resurgence of a liberal public sphere ideology that tries to colonize all social experiences (Negt and Kluge, 1993) and the assertion of a technological utopianism that equates hi-tech progress with social progress and that consistently manifests with the introduction of (relatively) new means of communication.

So how does this interaction between "practical consciousness" and practical activity more concretely function? In the next section, I try to clarify that with the idea that ideology mediates between two distinct but interconnected aspects of Web 2.0 activity: unpaid labouring and social working. 


\subsection{Free Labour as Working and Labouring}

By the review just considered, I meant to point out how in media studies ideology tends to be treated in idealist ways. Conversely, I want to approach ideology by the same question Althusser poses in his essay on Ideological State Apparati (1970), as a question about material "re-production of the conditions of production" (1). In the specific case of user-generated content this implies considering how to reproduce that specific subjectivity that drives a Web 2.0 users to accept a condition of unpaid and exploited labour.

I believe that the Terranova's (2004) notion of free labour points to one of those ideological conditions of reproduction of the system of valorization of prod-users, that is a production of the production of consciousness and ideology to understand the consensual reproductive aspects of digital labouring and the production of neoliberal hegemony. In fact, such a framework allows combining the objective element of exploitation and the subjective element of consciousness and ideology.

In the specific context of digital labour, the reproductive function of ideology reveals itself in its capability of conciliating the exploitative and the normative aspect of "free labour". More than a decade ago, Terranova (2000) published an influential essay that tried to make sense of the media practices of Internet users. According to the author, the so-called "net slaves" were exemplar of the so-called digital economy. In relation to idealist tendency previously mentioned in media studies, Terranova (2000) finds in Internet more than ideas, but a place "animated by cultural and technical labor through and through, a continuous production of value that is completely immanent to the flows of the network society at large" (35). The Internet is materially arranged in "out-ternet" "the network of social, cultural, and economic relationships that crisscrosses and exceeds the Internet-surrounds and connects the latter to larger flows of labour, culture, and power" (34).

The objective of Terranova is to map a conceptual and material territory that stands in between production and consumption, exploitation, and a promise of liberations. It is a concept that tried to mediate apparent dualism. In fact, echoing the autonomist Janus faced position, free labour "has something of a double meaning" (Hesmondhalg, 2010, 273). It refers to unpaid work, but, in line with Terranova's explicitly autonomist sympathies, it also refers to the way in which labour cannot be fully controlled, because of capital's continuing and problematic reliance on it. On the other hand, drawing from Hardt, Negri, Lazzarato, and Virno seem also to implicitly believe that immaterial labour can provide the social pre-conditions for a spontaneous form of communism. Thus "free labour" is both captive and (potentially) free.

Terranova tries to make sense of a historic specific reality that presupposes the existence of Internet and the post-fordist capitalist mode of production. However, in my view, her intuition goes further than that and speaks about a dialectical way to conceive human productive activity. In this sense, Fuchs and Sevignani (2013) spell in very clear ways the dialectical aspects of producing. With their investigation on how Marx (and Engels) conceives human practical activity, they suggest the need to distinguish between "labour" and "work". Fuchs and Sevignani's contribution can clarify the two aspects of free labour in the digital environment. They distinguish the two concepts as follows: "Labour is a necessarily alienated form of work, in which humans do not control and own the means and results of production. Work in contrast is a much more general concept common to all societies. It is a process in which humans in social relations make use of technologies in order to transform nature, culture and society in such a way that goods and services are created that satisfy human needs" (240).

The two authors point out how productive work seems to be an inherent characteristic of human beings, which then develop differently according to different social and historical contexts. However, work is not simply the general "anthropological" conceptualization of human productive activity. In relation to how historically labour developed in capitalist societies, work remains as more positive alternative. In fact, if it is true that in the Economic and Philosophic Manuscripts Marx says that one major alienation is both from the worker's own work and own product of work, then work, is understood not only as necessity due to the inherent scarcity that characterizes our material environment, but also as a chance of human expression and signification, a chance to make of the product into signified matter. Instead waged labour 
appears as alienated work, the denial of the latter. Thus, one can find a dialectic unity of alienation and emancipation, which seems to be inherent in how human practical activity historically developed in capitalist societies.

In the concrete social relations that surround digital labour, working and labouring are in fact synthetized: on the one hand, the web 2.0 user is unpaid, alienated from his/her own product and from the work process and becomes a commodity sold to the advertising industry; on the other hand the Web 2.0 user is a social worker that constructs his/her own identify by constructing the social environment around but also is driven by the idea of digital citizenship and the fact the content that he/she produce has a social and political value. Thus, the web 2.0 user is alienated by his/her unpaid labouring but feels emancipated by his/her social working. As I will show in the next section Facebook eloquently exemplifies such a condition.

\section{Face to Face with Facebook}

Since its first formulations, Liberalism has consistently been capable of fusing together a political and an economic project. When, for instance, Milton in his manifesto of 1644 Aeropagitica demanded a "free market of good and ideas" implied that the liberalization of the market and the public sphere were tightly connected. In fact, the link between the two aspects is so profound that both in the public and academic discourse the market frequently becomes metaphor for democracy and democracy becomes a metaphor for the market.

In this sense, communication technology has very often embodied such a link in powerful ways. Facebook is a perfect example, as its rhetoric intermeshes together political and economic arguments. Kushner in a Rolling Stone article applauded Facebook in terms of "citizen" and "consumer" choice (2006): "the long epoch of top-down culture [...] is fading faster than anyone predicted. The more vibrant world is bottom up, powered by the people." As Cohen notices (2008), innovations in information and communication technology tend to be accompanied by the teleological narratives of progress and human perfectibility.

Indeed, Facebook reflects the liberal normative social projects to liberate men through the market, through Smith's public use of instrumental and individualist reason, through technology and communication represent. Those are all facets of the liberal discourse/ideology of modernity (Passerin and Benhabib, 1997). They are at same time ideological fictions but also relevant promises that have historically mobilized people for quite some time now and they still operate through the utopia of media as technological promises of human comprehension and social pacification. The exemplification of such a complex sentiment is provided by one of the most popular Web 2.0 platforms, Facebook, that indeed combines both exploitative and utopic aspects of media.

On the one hand, Facebook outsources and crowd-sources the work of media production through the producer-consumer, a practice of cost reducing that not accidentally coincided with rising layoffs in media industries (Deuze 2007). As Huws notices (2003) the exploitative aspect of unpaid labour in new media comes from a general tendency of capital to offload labour cost onto consumers juts like on the case of self service gasoline stations, ATM, grocery check out which "burden the consumers of unpaid labour" (69). Such a system of exploitation of prod-users has in the case of Facebook exhibited itself rather explicitly as in the case of the program Facebook Diaries (Cohen 2008), which asks Facebook users to send stories and therefore "participate" to a contest that rewards the best stories by broadcasting them on TV.

On the other hand, as Kessler (2007) notices, Zuckerberg, the founder of Facebook, consistently utilizes rhetoric of novelty, social change, and promise of social amelioration through media technology, which, in its clear instrumentality, also resonates with genuine existing democratic sentiments. In the Facebook "manifesto," (2007) the founder describes the "social mission" of the platform as it follows:

Facebook aspires to build the services that give people the power to share and help them once again transform many of our core institutions and industries [...] We believe building tools to help people share can bring a more honest and transparent dialogue around government that could lead to more direct empowerment of people, more accountability 
for officials and better solutions to some of the biggest problems of our time. By giving people the power to share, we are starting to see people make their voices heard on a different scale from what has historically been possible. These voices will increase in number and volume. They cannot be ignored. Over time, we expect governments will become more responsive to issues and concerns rose directly by all their people rather than through intermediaries controlled by a select few (1).

Zuckerberg's rhetorical arguments makes leverage on the technologic utopianism which recovers liberal ideology through the reassertion of the public sphere and Kantian publicity, which is represented in the text by both the use of reason in public and the idea of political power under public scrutiny. Such a rhetoric is also conveyed by a more general narrative of new media, which are often described as "Web 2.0", "social media", "participatory media", "citizen journalism", "user generated content", "user driven innovation", or "social software". Such a civic consciousness ultimately re-articulates in terms of "ethical work" and "ethical citizenship" the (neo-)liberal ideological fiction of a civic society not ruled by the state as external apparatus but by the market as an internal, ethical, self-sufficient, and self-balancing system.

Such a normative thrust is not simply present in the rhetoric of its founder but also in the daily practices of Facebook users. Aside the significant aspect of management impression according which users utilize this platform to construct, negotiate, and reproduce a given identity, many users understand the function of "sharing" links and content as a way to establish a platform for discussion of matter of public interests, to sensitize specific issues. Furthermore, as the study conducted by DeRosa (2013) confirms, people use web 2.0 platform in order to construct a new utopic understanding of citizenship, a digital citizenship that reinterpret democratic deliberation as "e-democracy". As Dean (2009) observes social media are less places enhancing critical debate than platforms devoted to corporate profit. However, as I tried to demonstrate they also work as platforms that provide an ideological rationalization and moralization of "free labour" because they produce the impression of subjective visibility and social and political agency.

Thus, as Bloch recognizes, although often mirroring "how the ruling class wishes the wishes of the weak to be" (1986a: 13), the ideologies in Facebook can also provide a "transition' stage and display a drive towards a better life, and real possibilities to transform societies. In Facebook, Bloch $(2004,40)$ would probably find two different utopia-informed ideological aspects. First, via Facebook users embrace what Bloch defines as "wish-fulfillment" aspects, which consist in the elimination of the roots of human un-happiness such as the "arcana imperii" characteristic of power, the alienation and social solipsism allegedly characterizing modern mass societies. The second utopian aspect Bloch would find operates by "constructing" (40) the utopia; in the case of Facebook, by contributing to re-arrange labour, social organization, civic order, and what Aristotle defined as Politeia.

The apparent openness of Facebook to whatever content or community organization would appear as promise of the reality "not yet" realized. The "anticipatory consciousness" (Bloch, 1986) of Facebook users perceives the unrealized emancipatory potential in Facebook. Indeed, as already mentioned, Facebook is far from being a public sphere for critical thinking but in such an environment the users experience the ideological surplus value of the bourgeois notion of citizenship with its individual rights, civil liberties, and actively engaged autonomy expressed something more than mere legitimation and apologetics for bourgeois institutions and practices.

Thus, what the example of Facebook points out is an ambiguous reality. On the one hand, Facebook can and should be considered as further rationalization, colonization, and commodification of the Lebenswelt. On the other, the fact that our private sphere becomes a commodity that is a sold in a particular market makes that market as a Miltonian liberal arena in which, aside from goods, ideas can, wishful-thinkingly enough, move freely. As a result, the unpaid and exploited labour of generating content also gives the impression of a social working towards a more democratically progressive society. 


\section{Conclusions}

According to Sartre (1976), practical human activity represents the most concrete experience of dialectics. The inherent condition of scarcity of our world, ie, the fact that there is not enough for everybody, stands as a fundamental negation of the human existence, which is in turn negated by way of praxis, our work. Productive praxis expresses for Sartre a statement of human emancipation. However, the negation of the negation, this positive act of human self-liberation contained in praxis, should also be understood in the continuous and inescapable production of its antithesis: an inertia produced by the traces of mine and somebody else praxis that go against my objectives.

In this sense, this paper tried to point how digital labour dialectically contains both the subjective dimension of purposeful and potentially emancipatory work and the objective condition of unpaid labour, generating content valorized for the benefit of others. Thus, the practical experience of digital labour may reproduce in the consciousness and the ideology of the Web 2.0 such a dialectical experience of making things: a never-ending process of subjectivization and objectification, appropriation and alienation, estrangement and partial reconnection, coercion and consent. In fact, what is being produced in the process of generation of content is not just a "update of Facebook status" but also a particular understanding of the world that simultaneously contributes to reproduce the condition of production of content, ie, of valorization and monetization of digital labour, and aspires to realize a social political project such as the liberal one.

Such a consciousness is not plainly false, but is rather contradictory. On the one hand, as Althusser would say, the consciousness generated during the productive consumption of Web 2.0 users "represents the imaginary relationship of individuals to their real conditions of existence" $(1970,52)$ because the moralized mimicry of social working, while generating content, hides dimension exploitation, therefore a level false consciousness. On the other hand, prod-users 'consciousness and ideology seem to draw from existing genuine desires mediated by what Mosco considers as technological utopianism (2005), an (neo-)liberal understanding of knowledge work as social work in post Welfarist context, and the prod-users' rational motivation to accumulate social, cultural and symbolic capital.

To conclude, this paper has certainly not tried to suggest that informational capitalism is "ambiguously bad". Indeed, this mode of production is bad enough as it still functions with the perverse logic of socializing production and privatizing the gain from that. Capitalism must be overcome and the intellectual wealth produced by knowledge workers gathered into commons, become a collective resource. The "moral of the story" of this paper is in many ways a caveat: the subsumption process of capital is strong enough to produce utopic and moralized aspects of it; strong enough to produce aspects of emancipatory work within exploited labor. Thus, as many dialectical narratives do, mine suggests that if are theoretically able to distinguish destructive and constructive aspects in human practical activity, in the concrete historical experience, the desired replacement of alienated labour with liberatory and selfexpressive work may not be as straightforward and linear as we may think.

\section{References}

Althusser, Louis. 1970. Lenin and Philosophy and other essays. NY \& London: Monthly. Andrejevic, Mark. 2008. Watching Television without Pity. Television \& New Media 9 (1): 24-46.

Artz, Lee, Maceck, Stephen and Cloud, Dana (eds). 2006. Communication and Marxism: The Point is to Change it. New York: Peter Lang.

Arvidsson, Adam and Eleanor Colleoni. 2012. Value in Informational Capitalism and on the Internet, The Information Society: An International Journal 28 (3): 135-150.

Aune, James. 2004. Rhetoric and Marxism. Boulder: Westview Press.

Bloch, Ernst. 1986. The Principle of Hope. Cambridge, MA: MIT Press.

Caraway, Brett. 2011. Audience Labor in the New Media Environment: A Marxian Revisiting of the Audience Commodity. Media, Culture \& Society 33 (5): 693-708.

Castells, Manuel. 2000. The Rise of the Network Society. Malden, MA: Blackwell Publishers.

Chomsky, Noam. 1999. Profits over People—Neoliberalism and Global Order. New York: Seven 
Stories Press.

Bell, Daniel. 1973. The Coming of Post-industrial Society. Hammondsworth, England: Penguin.

Coté, Michelle and Jean, Pybus. 2007. Learning to Immaterial Labour 2.0: Facebook and Social Networks Ephemera. Theory and Politics in Organization 7 (1): 88-106, 83.

Cohen, Nicole. 2008. The Valorization of Surveillance: Towards a Political Economy of Facebook. Democratic Communiqué 22 (1): 5-22.

Dean, Jody. 2009. Democracy and Other Neoliberal Fantasies: Communicative Capitalism and Left Politics. Durham: Duke University Press.

De Rosa, Rosanna. 2013. Cittadini Digitali. L'agire Politico al tempo dei Social Media. Napoli, Apogeo.

Deuze, Mark. 2007. Toward an Ethics of the Sociable Web: A Conversation between Trebor Scholz and Mark Deuze. Accessed June 3, 2013. http:// https://mailman.thing.net/pipermail/idc/2007July/002652.html

Dyer-Witheford, Nick. 1999. Cyber-Marx. Cycles and Circuits of Struggle in High-Technology Capitalism. Urbana: University of Illinois Press.

Eagleton, Terry. 1991. Ideology: An Introduction. London: Verso.

Florida, Robert. 2002. The Rise of the Creative Class. New York: Basic Books.

Fuchs, Christian. 2010. Labor in Informational Capitalism and on the Internet. The Information Society: An International Journal 26 (3): 179-196.

Fuchs, Christian. 2012. With or Without Marx? With or Without Capitalism? A Rejoinder to Adam Arvidsson and Eleanor Colleoni. tripleC_Communitcation, Capitalism \& Critique 10 (2): 633-645.

Fuchs, Christian and Sebastian Sevignani. 2013. What is Digital Labour? What is Digital Work? What's their Difference? And why do these Questions Matter for Understanding Social Media? tripleC_Communitcation, Capitalism \& Critique 11 (2): 237-293.

Godelier, Maurice. 1986. Mental and Material. London: Verso.

Gramsci, Antonio. 1971. Selections from the Prison Notebooks. New York: International Publishers.

Gunster, Shane. 2004. Capitalizing on Culture: Critical Theory for Cultural Studies. Toronto: University of Toronto Press.

Habermas, Jürgen. 1991. The Structural Transformation of the Public Sphere: An Inquiry into category of Bourgeois Society. Cambridge, MA: MIT Press.

Hardt, Michael and Antonio Negri. 2000. Empire. Cambridge, MA: Harvard University Press.

Harvey, David. 1989. The Condition of Postmodernity. London: Basil Blackwell.

Hesmondhalg, David. 2010. User-Generated Content, Free Labor and the Cultural Industries. Ephemera 10 (3/4): 267-84.

Horkheimer, Max and Theodor Adorno. 1974. The Dialectics of Enlightenment. London: Verso.

Huws, Ursula. 2003. The Making of a Cybertariat: Virtual Work in a Real World. New York: Monthly Review Press.

Jhally, Sut and Bil Livant. 1986. Watching as Working: The Valorization of Audience Consciousness. Journal of Communication 36 (3): 124-143.

Kellner Douglas and H. O'Hara. 1976. Utopia and Marxism in Ernst Bloch. New German Critique 9: 11-34.

Lazzarato, Maurizio. 1996. Immaterial Labor. In Radical thought in Italy, edited by P. Virno and M. Hardt. Minneapolis: University of Minnesota Press.

Lukacs, Georg. 1971. History and Class Consciousness: Studies in Marxist Dialectics. Cambridge, MA: MIT Press.

Marcuse, Herbert. 1965. Remarks on a Redefinition of Culture. In The Essential Marcuse: Selected Writings of Philosopher and Social Critic Herbert Marcuse, edited by Andrew Feenberg and Wiliam Leiss, 13-31. Boston, MA: Beacon Press.

Margonelli, Luca. 1999. Inside AOL's “Cyber-Sweatshop”. Wired 7 (10). Accessed June 12, 2013. http://archive.wired.com/wired/archive/7.10/volunteers.html

Marx, Karl. 1867. Capital: A Critique of Political Economy. Vol.1. Translated by Ben Fowkes. London: Penguin Classics.

Marx, Karl and Friedrich Engels. 2001. The German Ideology Part One. New York: International Publishers.

McKercher, Catherine and Vincent Mosco, eds. 2006. Special Issue on the Laboring of Communication. Canadian Journal of Communication 31(3).

_ 
Morini, Cristina and Andrea Fumagalli. 2010. Life put to Work: Towards a Life Theory of Value. Ephemera 10 (3/4): 234-52.

Mosco, Vincent. 2005. The Digital Sublime-Myth, Power, and Cyberspace. Cambridge, MA: MIT Press.

Muehlebach, Andrea. 2012. The Moral Neoliberal: Welfare \& Citizenship in Italy. Chicago: Chicago University Press.

Napoli, Paul. 2010. Revisiting "Mass Communication" and the "Work" of the Audience in the New Media Environment. Media, Culture \& Society 32 (3): 505-516.

Negt, Oskar and Andres Kluge. 1993. Bourgeois and Proletarian Public Sphere. Minneapolis: University of Minnesota Press.

Nixon Brice. 2011, Dialectical Method and the Critical Political Economy of Culture. tripleCCommunication, Capitalism \& Critique 10 (2): 439-456.

Passerin, Maurizio \& Seyla, Benhabib, eds. 1997. Habermas and the Unfinished Project of Modernity: Critical Essays on the Philosophical Discourse of Modernity. Cambridge, MA: MIT Press.

Peck, Janice. 2006. Why We Shouldn't Be Bored with the Political Economy versus Cultural Studies Debate? Cultural Critique 64: 92-126.

Peters, John. 1999. Speaking into the Air: A History of the Idea of Communication. IL: University of Chicago Press.

Petersen, Soren. 2008. Loser Generated Content: From Participation to Exploitation. First Monday 13 (3). Accessed June 12, 2013. http://firstmonday.org/article/view/2141/1948

Resnick, Stephen and Richard Wolff. 1987. Knowledge and Class: A Marxian Critique of Political Economy. Chicago: University of Chicago Press.

Scholz, Trebor. 2008. What the MySpace Generation Should Know About Working for Free. Accessed February 12, 2011. http://collectivate.net/the-social-web/

Smythe, Dallas. 1981. On the Audience Commodity and its Work. In Media and Cultural Studies, edited by M. G. Durham, and D. M. Kellner, 230-56. Malden, MA: Blackwell.

Terranova, Tiziana. 2000. Free Labour: Producing Culture for the Digital Economy. Social Text 18 (2): 33-57.

Terranova, Tiziana. 2004. Network Culture: Politics for the Information Age. London: Pluto Press.

Virno, Paolo. 2004. A Grammar of the Multitude. London: Pluto Verso.

Webster, Frank. 2002. Theories of the Information Society. New York: Routledge.

Wennerlind, Carl. 2002. The Labor Theory of Value and the Strategic Role of Alienation. Capital \& Class 26 (2): 1-21.

Williams, Raymond. 1985. Keywords: A Vocabulary of Culture and Society. Revised edition. New York: Oxford University Press.

Wilmott, Hugh. 2010. Creating Value Beyond the Point of Production: Branding, Financialization and Market Capitalization. Organization 17 (5): 517-42.

Žižek, Slavoy. 1989. The Sublime Object of Ideology. London; New York: Verso.

Zompetti, Joseph. 1997. Toward a Gramscian Critical Rhetoric. Western Journal of Communication 61.

Zuckerberg, Mark. 2007. Keynote Speech Delivered at the f8 Conference at the San Francisco Design Centre. Accessed June 6, 2013. https://developers.facebook.com/docs/graphapi/reference/v2.0/video

Zwick, Detlev, Samuel Bonsu and Aaron Darmondy. 2009. Putting Consumers to Work. "Co-creation" and New Marketing Govern-Mentality. Journal of Consumer Culture 8 (2): 163-96.

\section{About the Author}

Marco Briziarelli

is an assistant professor at the University of New Mexico's Communication and Journalism department. He studies and teaches courses on media theory, political economy of media and media and social movements. 


\title{
Alienation and Digital Labour-A Depth-Hermeneutic Inquiry into Online Commodification and the Unconscious
}

\author{
Steffen Krüger ${ }^{*}$ and Jacob Johanssen ${ }^{\dagger}$ \\ "University of Oslo, Norway, steffen.krueger@media.uio.no \\ ${ }^{\dagger}$ University of East London, England, jacob@cyborgsubjects.org
}

\begin{abstract}
At the core of this paper is a psychosocial inquiry into the Marxist concept of alienation and its applications to the field of digital labour. Following a brief review of theoretical works on alienation, it looks into its recent conceptualisations and applications to the study of online social networking sites. Subsequently, the authors offer suggestions on how to extend and render more complex these recent approaches through in-depth analyses of Facebook posts that exemplify how alienation is experienced, articulated, and expressed online. Supporting this perspective, the article draws on Rahel Jaeggi's (2005) reassessment of alienation, as well as the depth-hermeneutic method of "scenic understanding" developed by Alfred Lorenzer (e.g. 1970; 1986).
\end{abstract}

Keywords: Alienation, Psychoanalysis, Alfred Lorenzer, Digital Labour, Depth-Hermeneutics, Psychosocial

\section{Introduction}

In his 2011 article "Surveillance and Alienation in the Online Economy", Mark Andrejevic makes the following observation apropos alienation and the specific form that the concept takes on in online social networking practices.

\begin{abstract}
Each form of intentional user-generated content-a blog post, a Facebook update, a Tweet, is redoubled in the form of 'cybernetic commodities' (Mosco 1989). [...] [W]hile they are created by users, they are not controlled by users, who have little choice over how and when this data is generated and little say in how it is used. In this sense we might describe the generation and use of this data as the alienated or estranged dimension of their activity. To the extent that this information can be used to predict and influence user behaviour it is an activity that returns to users in an unrecognizable form as a means of fulfilling the imperatives of others (Andrejevic 2011, 286, our emphasis).
\end{abstract}

This is an extremely fertile point of departure for thinking about the uses of the concept of alienation in the online sphere. Since our aim in this paper is to contribute to the discussion of alienation within the study of digital labour from a psychoanalytically oriented perspective, Andrejevic's observations here are highly suggestive. He creates a scenario in which alienation arises not simply on the basis of the users' symbolic production on a given corporate platform; rather, he presents the feeding back of the produced and double-used data as a decisive part in the dynamics of alienation. In so doing, he opens up for a complex and sophisticated conception of the relation between a social networking site (SNS) and its users, as well as the potential place of alienation within it.

Indeed, the nuance which Andrejevic adds to his text, namely that it is not simply a product that "returns to users" in disguise, but an activity (Andrejevic 2011, 286) moves his observation yet closer to psychodynamic thinking, where we suspect a strong connection between intra and inter-psychic processes. Thus, a psychoanalytically informed perspective is geared to seek out why-as well as how-we do not recognise that which returns to us users, as well as how we fare under-and suffer from-a relationship in the making of which we do not recognise our own hand. It is questions such as these that we want to put forth in this paper. 
We will approach these questions by presenting in-depth readings of an exemplary choice of users' posts to Facebook's Site Governance Page. For these in-depth readings we will rely on the "depth-hermeneutic approach to cultural analysis", which was developed by the sociologist and psychoanalyst Alfred Lorenzer (1922-2002) throughout the 1970s and 1980s (e.g. Lorenzer and Orban 1987/88). What depth-hermeneutics offers is a theoretical and methodological frame for interpreting concrete articulations of and within a culture from both a psychoanalytic and culture-analytic angle. Depth hermeneutics places the vantage point of our inquiry first and foremost on the subjective, i.e. users' symbolic production itself. The reading of this production as symptomatic-as subjective articulations of the objective societal conditions-will allow us to lay open the specific form of online alienation in the relations between the subjective and its socio-cultural context. How does alienation make itself felt in the production and, ultimately, in the products at the heart of the exploitative structure of online social networks that Andrejevic describes above?

In order to set the stage for our inquiry we will first give a brief overview of the history of the concept of alienation. We will then turn to the contemporary debate on digital labour and the place of alienation therein. The main part of this article will consist of our exemplary analyses of online alienation.

\section{A Brief History of Alienation}

Alienation holds a particularly central place in Marxist theory; after all, it is the category that decisively mediates between a worker's living conditions and the relations of production, between the subject and its objects, or even between the subjective and the objective in the social per se.

It is this in-between position that Marx [1844] (1988) granted alienation that made the concept so extremely popular in the tradition of critical thinking about culture and society. Jon Elster (1986) deems alienation to be "the most important concept" of Marx's normative theory (Elster 1986, 41). "The efficacy of capitalist exploitation," he writes, "rests on its ability to perpetuate the conditions under which it appears as morally legitimate" (ibid, 56). Marcello Musto (2010), in turn, traces the concept's career as the main pillar of the critique of capitalism's efficacy throughout long stretches of the intellectual history of the $20^{\text {th }}$ century. After the publication of Marx's Economic and Philosophic Manuscripts from 1844 in 1932 several Frankfurt School authors, such as Adorno and Horkheimer (1972), Herbert Marcuse (1966), Erich Fromm (1961; 1965), produced Freudo-Marxist readings of alienation (Musto 2010, 83-85). French existentialists, in turn, rendered the concept "much more generic than in Marx's thought", identifying it "with a diffuse discontent of man in society" (ibid, 86-87), and the 1960s, then, literally became "the age of alienation tout court" in which,

authors from various political backgrounds and academic disciplines identified its causes as commodification, overspecialization, anomie, bureaucratization, conformism, consumerism, loss of a sense of self amid new technologies, even personal isolation, apathy, social or ethnic marginalization, and environmental pollution (Musto 2010, 90).

In view of this "craze" it might be understandable why, at the beginning of the $21^{\text {st }}$ century, Rahel Jaeggi (2005) can observe that the term nearly disappeared from all sociophilosophical literature.

Too inflationary was the use of the term alienation in its boom phase, too old-fashioned and outdated its philosophical foundations in the age of the 'postmodern', too questionable its political consequences in the age of "political liberalism"-and probably also too pointless the concern of the critique of alienation under the sway of victorious capitalism (Jaeggi 2005, 11).

However, it was specifically developments in the online sphere, such as the increasingly monopolistic corporate structures on the World Wide Web and the orientation towards the generation of exchange value, that have brought the concept of alienation, together with the full 
set of Marxist terminology, back onto the social scientific agenda. Especially media and communication scholars (Comor 2010; Andrejevic 2011, 2014; Fuchs 2012a; Fisher 2012; Giritli Nygren and Gidlund 2012; Fuchs and Sevignani 2013; Sevignani 2013), have successfully resuscitated Marxist terminology-and specifically alienation-to examine social networking sites more closely.

\title{
3. Alienation Defined
}

There are two texts by Karl Marx that have proven canonical for the current critical understanding of alienation in digital labour. We will start with the chronologically later passage, which can be found in Marx's Grundrisse [1857-61] (1973). Here, Marx writes apropos the "real alien relation [Fremdheit] of the worker to his product":

\begin{abstract}
The material on which it [i.e. living labour capacity; S.K. and J.J.] works is alien material; the instrument is likewise an alien instrument [...]. Indeed, living labour itself appears as alien vis-à-vis living labour capacity, whose labour it is, whose own life's expression it is, for it has been surrendered to capital in exchange for objectified labour, for the product of labour itself (Marx 1857-61 [1973], 394, italics in original).
\end{abstract}

Four dimensions of alienation can be identified in this passage, specifically: (1) alienation from the material of one's labour, (2), alienation from the instruments, (3) alienation from the product(s), and (4) alienation from oneself (i.e. living labour as "alien vis-à-vis living labour capacity"). It is along these lines that Fuchs and Sevignani (2013) detect and list the various variables of alienation on online SNSs. Before turning to their article, however, we would like to introduce the chronologically earlier passage on alienation by Marx, which can be found in the Economic and Philosophic Manuscripts [1844] (1956/2009). Already here Marx identified four dimensions of alienation; yet, his formulations in this text are more straightforwardly directed towards its relational aspects-a difference which ultimately produces different categories as well. Thus, one can sum up Marx's early observations as follows: The worker is alienated (1) in the "relation [...] to the product of labour" and (2) in the "relation [...] to the act of production in the labour process" (Marx 1844 [1956/2009], 30-31); these two alienated relations have a further alienating effect, specifically that of "estranging" the worker (3) from her/himself ${ }^{1}$ as well as (4) from others:

What applies to a man's relation to his work, to the product of his labor and to himself, also holds to a man's relation to the other man, and to the other man's labor and object of labor. [...] The estrangement of man, and in fact every relationship in which man [stands] to himself, is realized and expressed only in the relationship in which a man stands to other men (ibid, 32).

As with Andrejevic's quotation above, Marx's relational focus here meets the psychoanalytic interest of this paper; after all, if users are indeed caught up in an alienating, estranging and reifying relation with a corporate SNS, the foremost task is to describe in detail the characteristics of this relation.

In order to fully savour what the relational focus in Marx's early statements might mean for an inquiry into users' relations to a corporate online SNS it is worth taking a look at Rahel Jaeggi's (2005) social-philosophical approach to alienation. Summing her own take on the concept up in the formula of "a relation of unrelatedness", Jaeggi explains: "alienation does [...] not mean the absence of a relation, but is a relation itself-if only a deficient one" (Jaeggi 2005, 19, our translation). She chooses Marx's passage in the Economic and Philosophic Manuscripts (1844) as her point of departure. This description of alienation, she writes,

already contains both aspects, which according to my thesis are the main points of Marx's discussion of alienation. Firstly, what we find as being thought together here (i.e.

\footnotetext{
${ }^{1}$ Marx here uses the term "species being" which can roughly be defined as the anthropological potential of a human being in a given historical situation (see Marx 1844 [1956/2009], 32).
} 
in the field of tension between appropriation and alienness, S.K. and J.J.) are two problems: that of the 'loss of meaning', of impoverishment and 'meaninglessness of the world', and that of impotence and powerlessness vis-a-vis this world. Secondly, what shows in this central text of Marx's theory of alienation is the specific twist that Marx adds to the problem of the unrelatedness between world and human being: the scandal of alienation is that it is an alienation from what one has done and made oneself. It is our own activities and products, the social institutions and relations that we have created ourselves, which here become an alien power (Jaeggi 2005, 29-30, our translation).

Jaeggi's understanding of the concept is valuable to our approach as she translates Marx's various points into a subjective (but by no means subjectivist) state of being alienated - i.e. the vague and paradoxical feeling of being related to what one experiences as strange and the paralysing meaninglessness and powerlessness as the dominating feelings in this relationship. The particular value of this reading of Marx's early definition of alienation for the field of digital labour lies in the perspective it provides for thinking Andrejevic's observation of the return of one's own activities in disguised form further. Specifically, it allows us to ask how the returning of the users' activities impacts on these very users, their interactions with the social media platform, with each other, and their produce. In this respect, Jaeggi's translations of Marx's formulations into "the inability to identify meaningfully with what one does and with whom one does it with", as well as "the inability to exert control over what one does" (Jaeggi 2005, 30) become touchstones in and for our analysis of online alienation.

Thus equipped, let's take a look at the debate on digital labour and the place of alienation within it, before turning to our own exemplary readings of online alienation.

\section{Alienation in Digital Labour}

The field of digital labour studies has seen an immense rise in popularity; in the past years research has tackled the relationship between users and forms of unpaid or paid digital practices that produce exchange and use value (Terranova 2000; Kücklich 2005; Mosco and McKercher 2007; Burston, Dyer-Witheford and Hearn, 2010, Manzerolle 2010, Comor 2010, Dyer-Witheford 2010; Kang and McAllister 2011; Fuchs 2010, 2012a; Fuchs and Sevignani 2013; Scholz 2013). The argument that has been made by various scholars in the digital labour debate is that many forms of online practices do not appear to be labour but, in fact, contribute significantly to, say, the value of a website or online game. For a number of years, academic writing that does not specifically subscribe to the term "digital labour" but investigates "virtual work" from a critical perspective has also proliferated (e.g. Dyer-Whiteford 2001; Greenhill and Wilson 2006; Hermann 2009; Huws 2009). ${ }^{2}$

In order to provide a common basis upon which to build our argument, we will give a brief description of digital labour as it has been put forth-and defended plausibly - by Marxist oriented researchers, such as Christian Fuchs (e.g. 2012a). This position draws heavily on Dallas Smythe and his political-economic study on media audiences $(1977 ; 1981)$. Smythe argues that what is sold in commercial media is not so much programmes to audiences, but audiences to advertisers. The volte of Smythe's approach is that it is the audiences themselves who are turned into commodities. Advertisers pay television channels for air time in order to be granted access to these audiences. The bigger the audience of a specific programme, the more money a channel can charge for its exposure to other messages. Smythe therefore held that the reception activity of audiences constitutes a form of unpaid labour. If no one would do the labour of watching a TV programme, no company would want to have advertisement placed on it. Watching television thus marks the unequal exchange of a television programme for the audience's time and attention.

Smythe's model becomes particularly relevant when transferred to the Internet and applied to user-generated content (Fuchs 2012a) where the central task of value production is "out/crowdsourced" to the users. In order to emphasise this basic conflation of the spheres of consumption and production on corporate online platforms, Alvin Toffler's term of the

\footnotetext{
${ }^{2}$ We follow Fuchs's and Sevignani's $(2013,240)$ definition of the term "labour" in this paper.
} 
"prosumer" (1980) has been adapted to this context, signifying that the service's user produces goods (which the service owner then commodifies) not only while consuming the service offered but rather: by consuming this service. The products thus produced are "usergenerated data, personal data, social networks and transaction data about [users'] browsing behaviour and communication behaviour on corporate social media" (Fuchs 2012a, 708). Fuchs continues that "[c]orporate social media sell the users' data commodity to advertising clients at a price that is larger than the invested constant and variable capital," making it partly the users and partly the corporations' employees that "create the surplus value contained in this commodity. The difference is that the users are unpaid and therefore-in monetary terms-infinitely exploited" (Fuchs 2012a, 713).

In bold strokes, these are the outlines of the dynamics of digital labour from a political economic perspective. Whereas the dictum of infinite exploitation is, as Fuchs cautions, limited to the monetary dimension (2012a), the point of alienation is decisively more open to subjective interpretations. Returning thus to alienation, Fuchs and Sevignani (2013, 257-259) use the four dimensions of alienation that they found in Marx's Grundrisse (see above) in order to sound out its scope on Facebook, the biggest SNS online. The authors use broad, general categories, such as ownership, ideological coercion, instrumentalisation and commodification of data to outline the functional character of online alienation. Thus, users are alienated from themselves because they are coerced to use Facebook by way of peer pressure and a lack of viable alternatives (Fuchs and Sevignani 2013,257); users are alienated from "the instruments of labour" because they do not own the communication platform itself and because their "brains, hands, mouths, ears and speech, the Internet and platforms" are instrumentalised "for advertising" (ibid, 258); moreover, users are alienated from the "objects of labour" on Facebook-which the authors identify as shared representations of experiences-"through a legally binding agreement" (ibid, 259) that makes it possible for the platform owners to use them for economic purposes; and finally users are alienated from the product of labour due to the peculiar divide between use-value and exchange-value of symbolic produce online: the "use-values that Facebook users create are at the same time commodities that Facebook offers for sale on a market" (ibid).

From our perspective, the problem with such a categorical treatment of alienation is that it merely claims its existence theoretically instead of describing it phenomenologically. Such description would have to take into consideration the relational dimensions of being alienated in the ways outlined above. Fortunately, Fuchs and Sevignani (2013) also provide a measure of such phenomenological description when they introduce their concept of the "inverse fetish character of the social media commodity" $(2013,261)$ and offer an exemplary articulation: "In the world of digital labour," the authors write,

\begin{abstract}
the fetish character of the commodity takes on an inverted form. We can speak of an inverse fetish character of the social media commodity. The commodity character of Facebook data is hidden behind the social use-value of Facebook, i.e. the social relations and functions enabled by platform use. The inverse fetish of Facebook is typically expressed in statements like 'Facebook does not exploit me because I benefit from it by connecting to other users'. The object status of users, i.e. the fact that they serve the profit interests of Facebook, is hidden behind the social networking enabled by Facebook (Fuchs and Sevignani 2013, 261).
\end{abstract}

In a more empirically directed study, Sebastian Sevignani (2013) elaborates along the lines suggested by the concept of "inverse fetishism" (Fuchs and Sevignani 2013, 261). Based on qualitative interviews with 30 students, he identifies "trade-off strategies between privacy needs and perceived user benefits" (Sevignani 2013,332) as potential sites for "prosumer alienation" to become articulated. However, the author abstains from presenting concrete online material, but rather offers summarising interpretations: "The only thing SNS users can do is to limit their information disclosure no matter if they are SNS literate or not" (ibid). It is at that point of the trade-off that Fuchs and Sevignani's ideal example-"Facebook does not exploit me because I benefit from it by connecting to other users"-might be true to a certain, negotiated extent. 
One more contribution needs mentioning before we turn to our depth-hermeneutic inquiry into exemplary cases of online alienation, specifically, that of Eran Fisher (2012). With his wish to highlight "the humanist aspects in Marx's critique of capitalism and distinguish it from his more structural and economic critique" $(2012,173)$, Fisher approximates a notion of alienation that is in line with Jaeggi's and our own (lack of control; lacking ability to relate). The aim of his study, specifically to "highlight the contradictions of capitalism from a humanist viewpoint" (ibid), coincides with the present approach as well. However, the main thesis that Fisher puts forth on this basis seems somewhat too neat. This thesis-the existence of an inverse-proportional relation between exploitation and alienation-is summed up in the title of his paper, "How Less Alienation Creates More Exploitation?" While we find the overall direction of this argument thought provoking, Fisher's simple equation of de-alienation with such suspiciously affirmative categories as "self-expression, authenticity, and relations with others" appears highly problematic. "[l]n order to be de-alienated," Fisher writes, "users must communicate and socialize: they must establish social networks, share information, talk to their friends and read their posts, follow and be followed. By so doing they also exacerbate their exploitation" (Fisher 2012, 179). This simple acceptance of user interaction as de-alienating seems remarkable-especially in the face of the many piercing observations of potentially alienating constellations and affordances on SNSs that Fisher offers otherwise. Amongst these observations are: Facebook's inherent bias towards communication $(2012,175)$, and specifically towards that of the mundane and the emotional (ibid, 177); the "position of forced reflexivity" that users are put into and the subsequent categorising and "rationalising of selfdisclosure" (ibid, 176), the registering and expressing of emotions "almost as they occur", as well as the construction of identity through a "web of 'Likes'" (ibid, 177). It seems to us as though Fisher discards these observations in an attempt to unambiguously side with the users while disregarding the other dimensions of alienation.

Let us briefly recapitulate our theoretical frame. Rahel Jaeggi's reading of Marx's early definition of alienation emphasises the failing act of identification and appropriation on part of the workers, as well as the concurring feelings of meaninglessness and powerlessness. It is this reading that guides our understanding of other researchers' approaches to the question of alienation in digital labour-approaches that are crystallised in concepts such as ideological coercion, instrumentalisation, inverse fetishism, etc.

\section{Scenes of Online Alienation}

Building a bridge to our approach to online alienation via Alfred Lorenzer's method of "scenic understanding" (1970, 139-141), let us take one more look at Rahel Jaeggi's remarks on alienation. When Jaeggi cautions other authors to be economical in their assessments of alienation by writing that, "a life form could be criticised as wrong without there existing the equivalent of a subjective perception of psychological strain" (ibid, 47), such subjective perception as the point of departure for her own narrative approach becomes clear. Depthhermeneutics shares this starting point. In the introduction of On the Foundation of a Materialist Theory of Socialisation (Zur Begründung einer materialistischen Sozialisationstheorie, 1972), one of the central documents of Alfred Lorenzer's project of a materialistpsychoanalytic social science, he writes:

It is the task of psychoanalysis [in social science and cultural studies; S.K.] to render more precise in content those terms that have been abstracted from the analysis of objective societal processes, as for example 'alienation.' This is done through a critical process that departs from the sensually experiencable suffering of the subjects. We have to put into question the 'real' genesis of concrete suffering in order to uncover in these places an ideological veiling of the subjective factor that only Freud began to tear open. ${ }^{3}$ (Lorenzer 1972, 15, our translation)

\footnotetext{
${ }^{3}$ German original: „Psychoanalyse hat die Aufgabe, die aus der Analyse objektiver gesellschaftlicher Prozesse abstrahierten Begriffe wie z.B. ,Entfremdung' inhaltlich zu präzisieren in einem kritischen Verfahren, das von dem sinnlich-erfahrbaren Leiden der Subjekte ausgeht. Es gilt, konkretes Leiden in seiner ,wirklichen' Genese zu hin-
} 
Terms that were once won from concretely analysing societal processes, such as alienation, are to be brought in touch again with equivalent processes in an individual's given historical situation. Yet, these processes are not initially approached from the side of the social conditions, but, first of all, from that of the "sensually experiencable suffering of the subjects" (ibid).

In the following, we would like to present Lorenzer's theory and method by way of a demonstration, rather than explanation; in this way we hope to do justice to Lorenzer's materialist appreciation of a practical encounter with concrete socio-cultural practice (Lorenzer 1972, 9; Bereswill et al 2010), as well as contribute ideas and insights to the present understanding of online alienation. For this task, the question of how to identify and locate concrete articulations of alienation in everyday individual online practice becomes decisive. Therefore, a depth-hermeneutic inquiry into Facebook (to stay with the most common example), would have to depart from those realms of discourse and symbolic interaction where such concrete articulations become clearly perceivable - not only as subjective perceptions, but as perceptions relating to Facebook as the, or an Other in the interaction. What is thus at stake in a depth-hermeneutic inquiry is the relatedness and relation between a societal institution and the individuals interacting with this agency. Arguably, this is the case on the Facebook Site Governance page that the platform introduced in 2009. ${ }^{4}$ Here users are invited to discuss, and in some cases vote on changes in the programming and user policies (Fuchs and Sevignani 2013, 258). Entering into a dialogue with this page, depth hermeneutics applies a mode of "scenic understanding" (Lorenzer 1970) in which the researcher is to use her/his own subjectivity-her/his own experiences and culturally formed expectations-in order to detect within him/herself the irritations arising from her/his relating to the text.

Under standard methodological conditions, then, the task of a depth-hermeneutic inquiry into Facebook would be to detect and gather the irritations resulting from the researcher(s)'s reading of materials such as the comments on the Site Governance website. The selection made in this way is then ordered and systematised not according to cognitive categories, but relational and affective ones, specifically, the similarities in the forms that the researcher's irritations have taken. Lorenzer here writes of "experiences of evidence" that are tied to interaction patterns: "It is the interaction patterns that allow us to recognize the most diverse experiences as variations of one and the same scenic arrangement" (Lorenzer 1970, 144, our translation). The collection of material is continued in this way until the identification of typical interactional patterns has become saturated.

Thus embarking on the methodological task of "scenic understanding"-a task which we have severely simplified for our summary here-we hope that the reader can see that this task is usually much less specific, as well as more open(-ended), than that of the present article, which is to detect, identify and describe alienation on SNSs. Simply put: while in a fully fledged depth-hermeneutic approach to Facebook identifying interaction patterns as alienated/alienating would be one possible outcome amongst others, we will direct our attention straightforwardly to those aspects in our material that can be linked to the conception of alienation that we put together from the pertaining literature above.

The material that we have chosen for our demonstration is taken from the comment section to the post "Thanks for your feedback" by Facebook's Chief Privacy Officer, Erin Egan, posted on Facebook's Site Governance website on 15 November, 2013. Egan's post seeks to clarify the "changes we proposed recently to our Data Use Policy and Statement of Rights and Responsibilities" (Egan 2013, no page numbers) and obviously tries to deflect accusations put forth by commentators throughout the posts to the page: "We want you to know that nothing about this update has changed our advertising policies and practices. We heard this question a lot so we want to be clear. The goal of the update was to clarify language, not to change policies or practices" (Egan 2013, no page numbers, emphasis in original). From a thread containing 529 comments we chose a mere six for further analysis. From such a rela-

terfragen, um an diesen Stellen eine ideologische Verschleierung des subjektiven Faktors, den erst Freud aufzureißen begann, zu durchschauen." (Lorenzer 1972, 15)

${ }^{4} \mathrm{https}$ ://www.facebook.com/fbsitegovernance/app_4949752878 (accessed on 9 June, 2014). 
tively small number it is not possible to identify consistent patterns of interaction for the site. What we can and want to do nevertheless is to identify certain tendencies that might point us towards more consistent attitudes held on the site.

A first such tendency can be accessed via a set of comments that compare Facebook to Google, the other Internet monopolist. Thus writes a user in the thread under Egan's remarks: "At least Facebook tries to explain what is going on, UNLIKE GOOGLE" (15/11/2013), to which another user seems to add loosely: "At least it doesn't deceive like Google does" (15/11/2013). At the surface level, what are traded here are modes and quantities of an acute dissatisfaction on part of the users with the ways they are treated by social media services. More important, however, are the concrete forms that the articulations of this dissatisfaction take. In order to inquire into this, we would like to shift gears now and present some of our free associations in view of the material. This is a further methodological step of depth hermeneutics; Lorenzer suggests for a group of researchers to come together and freely associate to the collected material in order to refer these associations, as well as the group dynamics arising from the discussion-conflicts between researchers, shared emotional states, images, etc.-back to the material, making them fertile for the material's interpretation; this being a methodological idea that Lorenzer borrowed from psychoanalytic group supervision (Lorenzer 1986, 43; Balint, 1964).

\subsection{A Pleasurable Sense of Importance}

For us the irritations emanating from the two comments above are related to the theatrical posture carried by word choices and choices of phrasing, such as "at least" or the high-case "UNLIKE GOOGLE". What the two posts triggered in us were notions of juvenile intrigue and high school drama of the kind that one frequently encounters in US-American television series catering for teenagers. Facebook and Google appear to be cast in the roles of conniving, two-timing and coolly plotting lovers whose actions command their partners' attention even in their absence.

To be sure, this notion of juvenile drama is by no means intended as a characterisation of the commentators themselves; we do not have any more information about them than what can be gathered from the comments to Facebook's Site Governance page themselves. By contrast, our point is exactly to treat these comments as characteristic of the piece of symbolic interaction they are part of. Putting forth the notion of juvenile drama, then, is meant as a suggestion of a relevant character trait of the relation between Facebook and its users. Granting our two comments some paradigmatic force, what this notion says about this relation is first of all that there is a high expectation of being fooled on part of these users and agreement amongst them about the platform's reputation: "one just cannot trust it". Clearly, the two comments show themselves to be hurt and disappointed. At the same time, however, there is an attempt to find solace in this by emphasising the residual good that can be found in the partner: "at least Facebook tries to explain" (although it does not succeed, one could add); "at least it doesn't deceive" (although sometimes it comes close). The comparison of degrees of immorality renders the less immoral part tolerable and legitimate.

Now, we are aware of how eccentric such formulations as the above ones will appear within a conventional academic paper; however, we find it important to demonstrate some of the free-associative material in order to work out the core point that we want to make about the two comments' meaning for the relation between Facebook and its users. Specifically, we find that the lack and the deficiencies which the comments point to are not simply and exclusively suffered as deficiencies but also enjoyed and celebrated to a degree for their value as scandals in which one is personally involved. It seems to us that the indignation that is being enacted by those comments is experienced as partly pleasurable because it endows both, the ones articulating this indignation and those taken into confidence (the authors of this present article, for example), not only with a sense of righteousness but, not least, with a sense of importance. Venting and calling forth feelings of indignation and having them acknowledged and objectified is thus suffused with narcissistic pleasure. This seems to us to show parallels to a process of coming off age and joining the world of grown-ups, where personal 
relations have weight and importance and one's actions real-life consequences. "Is this really happening to me? Is it really I who is part of this?" At the same time, this sense of maturity and importance emanating from the comments appears to be performed in a playful and somewhat inconsequential manner. Therefore, we feel that the kind of "deception" that the two comments deal with can and will be put aside if the deceiver only shows a quantity of remorse towards his/her partner(s).

In compressed form, what we can thus identify in the pose of the two commentsLorenzer would call them "scenes" (1986, 60-62)-is a pleasurable, narcissistic sense of personal importance derived through the enactment of indignation over an experience of being deceived-an experience of impotence. Turned the other way around, what this constellation could mean for the users' relation with the Facebook platform is that there is a common experience of powerlessness, of being disappointed and hurt, that users render tolerable, even pleasurable, through a mutually affirming performance of indignation that serves to take the consequence-that of impotence, immorality, deception etc.-out of the perceived reality of the relation.

Referring these observations back to Jaeggi's definition of alienation, they allow us a glimpse of what shape the "relation of unrelatedness" (Jaeggi 2005, 19) might take on SNSs. In our two scenes a notion of meaninglessness announces itself in the exaggerated and clichéd theatricality in which the comments fashion their indignation: "at least", "UNLIKE GOOGLE". However, the re-enactment of the relationship seems well rehearsed and devoid of spontaneity and it might just as well be such routine which protects the relation from the insight into its meaninglessness. This form of theatricality also takes the edge off one's powerlessness in the face of the platform's actions. It distracts and glosses over the consequences of one's relation with the SNS, which would otherwise become fully perceivable and experiencable.

\subsection{Staging a Breakdown in Relations}

Giving our above findings of a notion of self-importance and its affirmative effects more relief, we can refer to another, very common type of post which is characterised by the staging of a breakdown in the relation with the SNS that is attacked:

If my posts are glitched anymore..I mean they are actually taken away from me..I will file.a lawsuit against Facebook..my son is a former Atty General..and he is fantastic prosecutor..I WILL FILE CHARGES WITH THE FED. TRADE COMM...I WILL HAVE THEM MONITOR MY POSTS AS I TYPE THEM..AND SEE FOR THEMSELVES..SICK OF YOUR LIBERAL CRAP..ARE YOU EVEN AMERICAN?????????????/ (24/05/2014)

Arguably, what one can identify here is an insight that realises in relational form what Fuchs and Sevignani $(2013,257)$ list as one of the aspects of alienation: "alienation from the objects/material of production" (ibid). After all, the user literally feels that he has been robbed. The persecuted and threatening feel of the post reaches far beyond its reference to consumer rights ("the Fed. Trade Comm.") that are seen as being attacked by Facebook as a corporation. In its attempt at enlisting us (the authors of this paper) as supporters of its accusations and denunciations, by seeking to convince us of its strength and authority ("my son is a former Atty General"), and by trusting us to share in its moral high ground (anti-liberal American) as well as its rage ("crap"), it achieved the exact opposite. Not only did the cultural differences between us (central European, left-leaning) and this post make empathy hard to come by; moreover, its accusations we felt to be poorly informed-a lack of insight into how Facebook works-its assurance of agency seemed to us a sure sign of powerlessness, its rage an unacceptable lack of control and composure, a demonstration of a deep, tormenting melancholia that we did not want to have a part in.

Whereas in the first set of posts, discussed above, what we found is a show of indignation that is half-serious and inconsequential and, in this way, amounted for us to an enjoyable avoidance of the insight into one's own powerlessness vis-à-vis the SNS, this post here 
brings its helplessness to the fore exactly in its attempt at acting up (and out) against the threat of meaninglessness and powerlessness that has befallen it.

Another example containing a very similar interactional pattern we found in a complaint by a user about being harassed by one or more other users-a complaint, which once again slides quickly into "ALL CAPS" mode. The post climaxes with "I MUST ASSUME THAT FACEBOOK HAS ENABLED THEM TO DO THIS, OTHERWISE_ HOW IS IT POSSIBLE!" $(22 / 05 / 2014$, extract). What we find here again is screaming, outrage as a counterpart to the comparatively mild, (self)-ironic form of indignation above. Once more this outrage brings the mode of playing along to a halt which is maintained in the other set of posts. And once more powerlessness is articulated in the empty threat of contacting "the federal authorities if this sort of HARASSMENT of me continues". Finally, in a parallel to the displacement of aggression towards liberalism ("liberal crap") in the former post, we again find rage being channelled towards a conspiracy-theoretical object par excellence: "the Fasclst-in-Chief__t that piece of shit DESPOT OBAMA." (22/05/2014, extract).

Are these two last posts examples of a specific kind of alienated situation? The strong projective tendencies and conspiracy-theoretical fantasies at least point to a condition in which acute feelings of powerlessness and meaninglessness in one's own innermost relations are fought off with considerably desperate means. Despite the posts' strong affinities to some of the variables that Fuchs and Sevignani (2013) deduce from Marx's four dimensions in Grundrisse (i.e. alienation from the instruments; alienation from the objects), it is not clear at all whether this enraging feeling of disorientation which is enacted here, is related to the structuration of labour on Facebook itself or rather to an inability to use the network in the way intended by its owners. This latter case would be one of lacking knowledge, rather than one of a deficiency in the relation to oneself and others due to one's realisation of the specific relations of production on the SNS.

\subsection{A Reified Sense of Entitlement}

There is one more set of posts which we would like to discuss here and with which we would like to return to the sense of importance that struck us in the first set of posts that we presented. In the following posts we find that this sense of importance is frequently attached to another such sense, namely one of entitlement and worth. User "Steve Smith", for example, shares a stylishly laid out message by a user or user group named "Social Fixer". This message provides a list of demands and instructions addressed to Facebook: "Dear facebook," it reads, "The Newsfeed is getting worse! Here is what I want", followed by instructions that are reasonable in content-e.g. "Posts in the order they were posted"-yet, at the same time, curiously impatient and familiar in form, e.g. "[I want] All my friends' posts. Not just what you think I will like" $(08 / 12 / 2013$, our emphasis). In contrast to the second set of posts (staging a breakdown) there is no trace of acting out here; rather, one can draw comparisons to the initially discussed ones whose playful form of indignation also produced notions of proximity and familiarity. Probably the clearest example of the relational aspect that we think is behind this intimate form of demanding things and the sense of entitlement that drives it appears in a comment that was posted a number of times in the threat that we looked at, with the text obviously having been copied, pasted and thus identified with by various users on the page, thus approaching the status of a meme.

Do not collect my data. Do not use my data without my specific hand written permission for each item. Do not release my personal information whether written of me by someone else or by me, past, present and future without my hand written permission or a court order. If you do use my personal and private information I shall be notified immediately and paid for it whether it was used previously or will be used in the future. I DO NOT AUTHORIZE the use or collection of my personal information I have given to or shared with Facebook, past or present, which includes but is not limited to my messages, notifications, friends, texts, photos, images, comments or any other content contained in or on my profile or timeline including information I have shared on anybody's Facebook account whether current or deleted for any reason or purpose other than my own personal use 
and purpose of my Facebook account. I hereby declare that my copyright is attached to everything I have done and will ever do with my Facebook account and my handwritten consent is required at all times with no exception for any purpose other than my own regarding the information contained in it or on it. (e.g. 05/04/2014, extract)

Needless to say, commentators did in fact authorize the use of their personal information when they ticked off the terms and conditions agreement as part of the Facebook membership procedure. Therefore, the post's juridical tone-"I hereby declare"-is not endowed with the authority that it requires. The incongruence between the mode of speech and the actual position of the one speaking produces a somewhat tragicomic effect. Yet, what is important to us in this post is that the distribution of being deserving on part of the comment/commentator and an acute sense of obligation located on part of the other, i.e. Facebook, speaks of the same injury that we found present but glossed over in the posts above. The obsessive desire of being met in one's demands down to the most minuscule detail seems to be a reaction to having been disappointed, utterly and beyond repair, and can thus be seen as a continuation of the indignation performed in the first set of comments. In its repetitive phrasing of "do not" and its strong investment in the "handwritten", the post takes on notions of what early psychoanalytic theory called hysteria, with the idea of the forbidden, catastrophic touch-"I have been soiled"-forming the implicit counterpart of the fetishistic idea of the "handwritten"-the touch of genius. In this way, then, this post seems to conjure up the figure of a disappointed, deceived lover who cannot bring him/herself to leave the deceiver; instead s/he continues the process of hollowing out the relationship by nagging, ranting, and dishing out increasingly harsher rules of conduct, none of which can be expected to be truly followed or to truly change the deceiver's morals, let alone mend the relationship.

Also this last association is meant as an experimental insight into the relation between Facebook and its users and sheds light on the ways in which a lacking ability to identify with one's actions on the SNS and an experience of powerlessness are articulated in it. In this last case, the relation is articulated in an immensely bloated self-image that seems to mistakenly use Facebook's interest in the personally trivial and mundane (Fisher 2012, 177) for the bolstering of personal importance and exceptionality.

\subsection{Inverse Fetishism Revisited}

It is with this relational image in mind that we can return to Fuchs and Sevignani's concept of "inverse fetishism" $(2013,261)$. As stated previously, this concept argues that users deny the reifying aspects in their relationship with Facebook by emphasising the social gratifications they receive from using the service. What our exemplary findings so far brought to the fore in this respect is first of all the aspect of denial, i.e. that the threatening insight of being exploited, coerced and alienated has to be fended off on a mental plane. However, in comparison to Fuchs and Sevignani's example of "Facebook does not exploit me, because I benefit from it by connecting to other users", people in our cases addressed the forlornness of their relation with Facebook full on; at the same time, however, they seemed to use this very performance of indignation to (unconsciously) defend themselves against this knowledge. This means that, in our examples, what was defended against was not so much the knowledge of the deceiving, exploiting nature of Facebook itself, but of the consequences for the users arising from their partnership with Facebook. "Facebook exploits me, but that's not so bad as long as I can benefit from it by connecting to other users."

John Steiner's concept of "turning a blind eye" (1986) seems to offer an adequate umbrella for the various ways of defending against knowledge which Fuchs and Sevignani as well as our own experimental reading of posts has brought forth. Steiner refers to this mechanism as turning a blind eye because, as he writes, "I think this conveys the right degree of ambiguity as to how conscious or unconscious the knowledge is. [...] [W] are vaguely aware that we chose not to look at the facts without being conscious of what it is we are evading" (Steiner 1986, 161). Likewise, when Sebastian Sevignani writes that, "users are kind of aware that people who own and control the SNS are appropriating societally-produced surplus" 
(2013, 331, our emphasis), the phrase "kind of" seems to us to point to the very ambiguity that Steiner observes.

Taking the theme of "inverse fetishism" one step further, we would like to suggest that the commodity form does not simply remain "hidden behind the social use-value of Facebook", as Fuchs and Sevignani argue $(2013,261)$. Rather, it becomes identified with and enacted in the users' interactions with the SNS. Vaguely knowing how dubious their relation with Facebook is, the users' exacting demands and instructions, which we detected in our third set of posts, can be read as attempts at selling themselves as dearly as possible to the platform owners: "Do this, not that"; "Don't give me just what you think I like"; "Don't touch anything without my handwritten permission" etc. It is against this interaction pattern that the notion of the diva is evoked: just as a diva's mannerisms can be interpreted as acting up against a culture-industrial complex, which, paradoxically, is fed and reaffirmed by these actions, so can the exacting demands put forth in the comments we analysed be seen as means for the commentators to adapt to the corporate conditions of socialising on the platform and make themselves at home in an alienating, objectifying relation. "Well, if you want to instrumentalise me," these comments seem to tell Facebook, "then at least treat me as a precious instrument and maintain me well!"

\subsection{Trade-Off Strategies and Narcissism in User-User Relations}

Against the above extension of the concept of inverse fetishism we can also gain a new perspective on the trade-off strategies that Sevignani (2013) identifies as sites of alienation. From his interviews with users, Sevignani learns that the only viable strategy for users to strike a balance between privacy needs and privacy threats is "to limit their information disclosure" (Sevignani 2013, 332). Building on this, from what we found in our cases of users directly addressing Facebook as an imagined/imaginary other, we are rather pessimistic as to the consequences of such trade-off strategies for user-user relations, since the probability seems high that a user's sense of her/his commodification is also being worked into her/his interactions with other users. In this respect, a certain degree of narcissism, which users of SNSs are so often accused of falling prey to in popular journalism, seems system-inherent. Also such narcissism must be understood first and foremost as "a defence produced in response to misrecognition", as Aaron Balick explains from a relational psychoanalytic perspective $(2014,81)$. According to this perspective, then, the narcissist invests in a reified image of her/himself that is not entirely of her/his own making, but a coproduction between the platform and her/him, which works according to our description of instrumentalisation and self-reification above. Part of the trade-off thus seems to be a continuous, automated inspection of oneself along the lines of one's own "preciousness", not only with respect to one's Facebook friends, but also against the vague intuition that one is feeding one's own objectification as well as the return of one's data in disguise (Andrejevic 2011, 2014).

\section{Conclusion}

It was the task of this article to shed further light upon the concept of alienation and how it is experienced, as well as articulated in relational form online. For this purpose we made use of Marx's early formulations on alienation in the Economic and Philosophical Manuscripts of 1844 and Rahel Jaeggi's (2005) reading of the former. With Alfred Lorenzer's method of scenic understanding $(1970 ; 1986)$ we approached an exemplary selection of user posts to Facebook's Site Governance Page. Let us pull together the various threads of our article's argument by briefly reviewing our findings against the literature we used.

While Fuchs and Sevignani (2013) observe apropos their concept of "inverse fetishism" $(2013,261)$ that the object status of users is hidden behind the use value, our experimental, exemplary findings suggest, firstly, what form this latent objectification takes, secondly, what effects it might have on user-platform, as well as user-user relations. Thirdly, and consequently, our findings suggest that this objectification does make itself felt in these relations after all. It is these point that will occupy us in the following passages. 
As might be an expected bias of posts to the Site Governance Page, we found them to be astonishingly aware of them being instrumentalised. Contrary to the ideal statement of "Facebook does not exploit me, because I profit from it by connecting to other users" (Fuchs and Sevignani 2013,261 ), the postures taken in the posts we observed rather amounted to "Facebook does in fact exploit me, but that's not so bad because I profit from it by connecting to other users." Moreover, in a surprising realisation of Marx's early dictum in the Economic and Philosophical Manuscripts that the "estrangement of man [...] is realized and expressed only in the relationship in which a man stands to other men" (Marx, 1844 [1956/2009], 32) these posts seem to realise their estrangement by establishing a quasi-personal relation to Facebook. In the user experience, this notion of Facebook as a palpable Other seems to arise through what Andrejevic (2011) describes as the interchanging relation of users feeding data to the SNS and the SNS feeding these data back to the users in disguised form (through advertising, friend suggestions, selection of posts in the newsfeed, etc). It is in such moments, in which users obtain a glimpse of the SNS's "Iatent desires" as well as their part in them- "...] what you think I will like" (Social Fixer 2013) - that the impression of a certain character type arises, a type that the posts we looked at judged to be a chronically unfaithful, conniving lover- "one just cannot trust her/him".

However, despite the deep running mistrust towards Facebook which the posts demonstrate, we thought to detect in this very demonstration a missing willingness and/or ability to put an end to the relation. The indignation expressed in the posts appeared to us to be strangely clichéd and theatrical-a theatricality that in turn suggested to us the opposite of acting upon a vivid insight. Specifically, the subjects speaking through these posts seemed to derive a pleasurable sense of importance from being instrumentalised which seemed to help users "turn a blind eye" (Steiner 1986) and gloss over the consequences that their instrumentalisation has for them. This aspect of our findings also sheds new light on the dynamics of the concept of "ideological coercion" (e.g. Fuchs 2012, 732), specifically, that the repeated, mutual confirmation amongst users of being victims in the same intrigue takes the edge off the personal sense of powerlessness that the betrayal might otherwise have. In the cases we looked at it is thus the very articulation of indignation that serves as a safety valve and makes the continuation of the relation possible, if not pleasurable. "Oh, you know, it's Facebook-What can you do?" In this relation-affirming staging of indignation we therefore find a moment of alienation from oneself and from others in that the removal of consequence from one's relation with Facebook goes hand in hand with the removal of consequence from one's own symbolic interaction.

In this way, the relation with the deceiving partner is continued on the basis of a fundamental injury that has been derealised. Symptomatically, this injury shows in the increasingly impatient, at times literally hysterical demands with which the deceived unconsciously seeks to take revenge on, as well as control over, the partner and force upon him/her a compromised display of appreciation. This symptomatic finding seems to go most clearly against Fuchs and Sevignani's otherwise immensely fruitful concept of "inverse fetishism" (2013, 261): having reached a certain level of frustration with Facebook as a partner, posts begin to display signs of a regressive, destructive and most importantly: self-destructive reification and instrumentalisation of the self. "If I am an instrument for you, then at least treat me like a prescious instrument!" It is in this protesting act of self-reification that Rahel Jaeggi's reading of Marx's definition of alienation can best be unfolded. When several users copy, paste and post the same comment that displays a striking investment in the "handwritten" we can detect in their misconception of the value which they thus attribute to their symbolic produce that which Jaeggi calls "the inability to identify meaningfully with what one does and with whom one does it with" (Jaeggi 2005, 30). By contrast, the hysterical notions of the forbidden touch-"do not [...]"-in the same post appear clearly related to what Jaeggi calls "the inability to exert control over what one does" (ibid). Both dynamics are related and tied to one another and have their roots in the conditions of production on the SNS.

Extrapolating our findings, we scrutinised the thesis of "inverse fetishism" further, questioning in how far objectification would in fact remain hidden in user-user relations in the face of the symptoms that some of the posts to the Site Governance Page displayed. Drawing on 
the relational psychoanalytic conception of narcissism as a "defence produced in response to misrecognition" (Balick 2014, 81), we asked whether platform-driven objectification and instrumentalisation shows in user-user relations in the form of such narcissism. According to the definition presented here, narcissism can no longer be seen as a creation of which either the user or the SNS alone is responsible. Much rather, narcissism has to be understood as a co-creation between the users, the SNS, as well as the socio-cultural context within which a given user responds to the SNS's capitalist agenda with acts of self-reification-acts which again will have been culturally, as well as biographically rehearsed (ibid, 79). In order to say more about what Stephen M. Johnson calls the "narcissistic style" (1987) and its potential for online alienation, further studies will be necessary. These studies will have to take into consideration not only the relations of production on online SNS, but, as Aaron Balick cautions, both the wider "culture and family constellations" $(2014,79)$.

Rounding off the review of our findings, we see that it was only in the most extreme articulations of frustrations with the SNS-articulations that nevertheless can be seen as typical topoi in online discourse-that the state of alienation described above (a state which in all other posts observed was maintained in the continual hollowing out of the relationship) could be seen to break down. Even though we are unsure in these cases as to what degree we can relate the breakdown to the posts' relation to the SNS directly, the disorientation as well as the strong projective responses tied to this disorientation appear characteristic of the release of psychic energy that was formerly bound by a (self-)destructive relationship. As different as these incidents of acting out might seem from the milder forms of indignation experienced in the other posts, they can still be placed on the same continuum. While the posts that remained attached to the SNS turned this SNS into a quasi-person in order to enter into what psychoanalysis calls a "family romance", the posts staging a breakdown in relations had to rely on a concrete counterpart. In the open confrontation with their powerlessness vis-a-vis the network, these latter posts set up characteristic conspiracy-theoretical objects for their projections, for example "the Fasclst-in-Chief_that piece of shit DESPOT OBAMA" (22/05/2014, extract).

In an article from the late 1990s, the German social psychologist Gerhard Vinnai analysed the affectively charged "family romances" that people in Germany created in relation to Princess Diana after her tragic death in a car accident in Paris in 1997. People fantasised themselves to be on intimate terms with the "people's princess", reporting her to be like a sister, a mother, daughter, best friend, etc. Vinnai interprets these romantic fantasies as compromise constructions: in a socio-cultural, socio-economic set up in which people can no longer see through the complexity of the resulting relations, these romances help them make sense of their own place within them-albeit in a precariously regressive and reductive way.

\begin{abstract}
The tendency of falling back behind once secured stages of social emancipation has its counterpart in a dubious urge for the familiar. That people should turn into social beings, that they should become culturally and politically enabled, demands the loosening of childish ties to one's family origins. It demands that one leave behind the limited orientation towards the family in favour of becoming interested in more complex social interrelations. The urge to escape from burdening social realities facilitates the regression to familiar experiences of relations that are then transferred to social structures for which they are not adequate (Vinnai 1998, no page numbers, our translation).
\end{abstract}

We feel that this theme of family romantic relations standing in for and covering up a much harsher political-economic reality is highly applicable to the users' relations to Facebook and has been an undercurrent in nearly all our observations on online alienation. It is in light of these family romances that we deem Fisher's (2012) neat equation of more-exploitation-lessalienation to be in need of revision. Arguably, social networking online might help a large number of people strengthen their social ties. However, while the generation of exchange value from these networking activities remains mostly hidden to the users, it remains all but ineffective. 


\section{References}

Andrejevic, Mark. 2014. Alienation's Returns. In Fuchs, Christian and Sandoval, Marisol, eds. Critique, Social Media and the Information Society, 179-190. New York: Routledge.

Andrejevic, Mark. 2011. Surveillance and Alienation in the Online Economy. Surveillance \& Society 8 (3): 278-287.

Balick, Aaron. 2013. The Psychodynamics of Social Networking: Connected-up Instantaneous Culture and the Self. London: Karnac.

Balint, Michael. 1964. The Doctor, his Patient and the IIIness. London: Pitman Medical Publishing.

Bereswill, Mechthild, Christine Morgenroth and Peter Redman. 2010. Alfred Lorenzer and the Depth Hermeneutic Method. Psychoanalysis, Culture and Society, 15 (3): 221-250.

Burston, Jonathan, Nick Dyer-Witheford and Alison Hearn, eds. 2010. Digital Labour: Workers, Authors, Citizens. Ephemera: Theory \& Politics in Organization 10 (3): 214-221.

Comor, Edward. 2010. Digital Prosumption and Alienation. Ephemera: Theory \& Politics in Organiza tion 10 (3): 439-454.

Comor, Edward. 2011. Contextualizing and Critiquing the Fantastic Prosumer: Power, Alienation and Hegemony. Critical Sociology 37(3): 309-327.

Dyer-Whiteford, Nick. 2001. Empire, Immaterial Labor, the New Combinations, and the Global Worker. Rethinking Marxism: A Journal of Economics, Culture \& Society 13 (3): 70-80.

Dyer-Witheford, Nick. 2010. Digital Labour, Species-becoming and the Global Worker. Ephemera: Theory \& Politics in Organization 10 (3):484-503.

Elster, Jon. 1986. An Introduction to Karl Marx. Cambridge: Cambridge University Press.

Fisher, Eran. 2012. How Less Alienation Creates More Exploitation? Audience Labour on Social Net work Sites. tripleC_Journal for a Global Sustainable Information Society 10 (2): 171-183.

Fromm, Erich. 1961. Marx's Concept of Man. New York: Frederick Ungar.

Fromm, Erich.1965. The Sane Society. New York: Rinehart and Winston.

Fuchs, Christian and Sebastian Sevignani. 2013. What is Digital Labour? What is Digital Work? What's their Difference? And why do these Questions Matter for Understanding Social Media? tripleC_-Journal for a Global Sustainable Information Society 11 (2): 237-293.

Fuchs, Christian. 2012a. Dallas Smythe Today-The Audience Commodity, the Digital Labour Debate, Marxist Political Economy and Critical Theory. Prolegomena to a Digital Labour Theory of Value. tripleC_-Journal for a Global Sustainable Information Society 10 (2): 692-740.

Fuchs, Christian. 2011. An Alternative View of Privacy on Facebook. Information (2): 140-165.

Fuchs, Christian. 2010. Labour in Informational Capitalism. The Information Society 26 (3): 176-196.

Garnham, Nicholas. 1986. Contribution to a Political Economy of Mass-communication. In

Media, Culture \& Society: A Critical Reader, edited by Richard Collins, et al., 9-32. London: Sage.

Giritli Nygren, Katarina and Gidlund, Katarina L. 2012. The Pastoral Power of Technology. Rethinking Alienation in Digital Culture. tripleC_Journal for a Global Sustainable Information Society 10 (2): 509-517.

Greenhill, Anita and Melanie Wilson. 2006. Haven or Hell? Telework, Flexibility and Family in the ESociety: a Marxist Analysis. European Journal of Information Systems 15: 379-388.

Hermann, Christoph. 2009. Value and Knowledge: Insights from Marxist Value Theory for the Trans formation of Work in the Digital Economy. Rethinking Marxism: A Journal of Economics, Culture \& Society 21 (2): $275-289$.

Horkheimer, Max and Theodor W. Adorno. 1972. Dialectic of Enlightenment, New York: Seabury Press.

Huws, Ursula. 2009. The Making of a Cybertariat? Virtual Work in a Real World. Socialist Register 37: $1-23$.

Jaeggi, Rahel. 2005. Entfremdung. Zur Rekonstruktion eines sozialphilosophischen Begriffs. Frank furt/M.: Campus Verlag.

Kang, Hyunjin and Matthew P. McAllister. 2011. Selling You and Your Clicks. Examining the Audience Commodification of Google. tripleC - Journal for a Global Sustainable Information Society 9 (2): 141-153.

Kücklich, Julian. 2005. Precarious Playbour: Modders and the Digital Games Industry. Fibreculture Journal 5. http://five.fibreculturejournal.org/fcj-025-precarious-playbour-modders-and-the-digitalgames-industryl (accessed on December 02, 2013).

Lorenzer, Alfred. 1972. Zur Begründung einer materialistischen Sozialisationstheorie. Frankfurt/M.: Suhrkamp.

Lorenzer, Alfred. 1970. Sprachzerstörung und Rekonstruktion. Vorarbeiten zu einer Metatheorie der 
Psychoanalyse. Frankfurt/M: Suhrkamp, 1995.Lorenzer, Alfred. 1986. Tiefenhermeneutische Kulturanalyse. In Kultur Analysen:Psychoanalytische Studien zur Kultur, edited by Alfred Lorenzer, 11-98. Frankfurt/M.: Fischer.

Lorenzer, Alfred and Peter Orban. 1978 (1988 edition). Transitional Objects and Phenomena: Socialisation and Symbolisation. In Between Reality and Fantasy. Winnicott's Concepts of Transitional Objects and Phenomena, edited by Simon A. Grolnick, Leonard Barkin and Werner Muensterberger, 1-34. Northvale: Jason Aronson.

Manzerolle, Vincent. 2010. Mobilizing the Audience Commodity. Digital Labour in a Wireless World. Ephemera: Theory \& Politics in Organization 10 (3): 455-469.

Marcuse, Herbert. 1966. Eros and Civilization. Boston: Beacon Press.

Marx, Karl. 1959. Economic and Philosophic Manuscripts of 1844. New York: Prometheus Books. Proofed and corrected version by Matthew Carmody published online in 2009. https://www.marxists.org/archive/marx/works/download/pdf/Economic-Philosophic-Manuscripts1844.pdf.

Marx, Karl. 1873 [1857-61]. Outlines of the Critique of Political Economy [Grundrisse]. London: Penguin. Published online 1997. http://www.marxists.org/archive/marx/works/download/Marx_Grundrisse.pdf.

Mosco, Vincent and Catherine McKercher, eds. 2007. Knowledge Workers in the Information Society. Lanham, MD: Lexington Books.

Musto, Marcello. 2010. Revisiting Marx's Concept of Alienation. Socialism and Democracy 24 (3): 79 101.

Scholz, Trebor, ed. 2013. Digital Labor. The Internet as Playground and Factory. London: Routledge.

Sevignani, Sebastian. 2013. Facebook vs. Diaspora: A Critical Study. In Unlike Us Reader. Social Media Monopolies and Their Alternatives, edited by Geert Lovink and Miriam Rasch, 323-337. Amsterdam: Institute of Network Cultures.

Smythe, Dallas W. 1981. Dependency Road. Norwood, NJ: Ablex.

Terranova, Tiziana. 2000. Free Labor. Producing Culture for the Digital Economy. Social Text 18 (2): 33-58.

Toffler, Alvin. 1980. The Third Wave. New York: Bentam.

Vinnai, Gerhard. 1998. Das Diana Syndrom. Über eine Unfähigkeit zu Trauern. Widersprüche: Zeitschrift für sozialistische Politik im Bildungs-, Gesundheits- und Sozialbereich 68. Published online June 1998. http://www.vinnai.de/diana_syndrom.html.

\section{About the Authors}

Steffen Krüger

is postdoctoral researcher at the Department of Media and Communication, University of Oslo, Norway. He is contributing editor of the journal American Imago. His PhD thesis about the psychoanalyst and-for the time World War II-propaganda researcher Ernst Kris was published as Das Unbehagen in der Karikatur (Fink, 2011). In his current research project, Online Interaction Forms, financed by the Norwegian Research Council (NFR), he analyses forms of digital media discourse from a psychosocial, and specifically, depth-hermeneutic perspective.

\section{Jacob Johanssen}

is a PhD student in Psychosocial Studies at the University of East London (UK). His research interests include digital culture, critical theory, psychoanalysis and media as well as audience research. Publications include "Cyborg Subjects: Discourses on Digital Culture" (edited with Bonni Rambatan, 2013). His PhD thesis explores a psychoanalytic conception of the subject that is both theoretical and epistemological. The research involves interviews with viewers of "Embarrassing Bodies" and explores their investments, fantasies, and viewing practices. 


\title{
Production Cultures and Differentiations of Digital Labour
}

\author{
Yujie Chen
}

\author{
University of Maryland, College Park, the United States, chenyj@umd.edu
}

\begin{abstract}
The article makes two theoretical interventions to engage with current scholarship on digital labour. First, the author complicates the relationship between culture and production by bringing the former from the "superstructure" in the classical Marx's framework to the "base." As various cultural production, consumption, and economic activities converging onto digital, networked media ecosystem, digital labour is indeed the indispensable source for capitals' accumulation of surplus and, more importantly, for cultural differentiations of production process. How labourers perceive their relations and interactions to the digital production process is as crucial as which capacity they rely on to perform their labour. Culturalization of production process (re)draws the boundaries for the meanings of labouring and thus is complicit in constructing ideal digital labourers with exemplary, normative social behaviours. Second, precisely because the production process has become normative construction site, meanings and values of labouring are subject to broader social and cultural context beyond the digital realm. Therefore, to further understand the conditions of digital labour, historical global inequality needs to be articulated. Equally important is to investigate emerging cultural discourses which render certain social behaviours in the digital realm as normal and desirable while others as invisible.
\end{abstract}

Keywords: digital labour, discourse, value, data,

Acknowledgement: The author is grateful to the editors of this special issue, the reviewers, and Dr. Jason Farman, who have all made invaluable comments on earlier drafts of this article.

\section{Introduction: big data and digital labour}

Three headline stories in 2012 draw people's attention to the notion of data and its economic and cultural ramifications for work in contemporary age. Facebook Inc.'s \$16 billion IPO in May 2012 exemplifies the complex relations between the value of labour and the information production on the social media in particular and the new media ecology in general. In spite of the dramatic plummet of its share price in the weeks following the IPO, the market value of Facebook as the day of writing (June 2014) -about $\$ 170$ billion-still baffles those who know this number is larger than the combination of the top two PC manufactures, HP and Lenovo, which are worth $\$ 28$ billion and $\$ 9$ billion respectively. Where is Facebook's enormously economic evaluation coming from? In February 2012, Facebook opened its pitch for public trading by presenting four numbers in a diagram in its S-1 registration statement filed with U.S. Securities and Exchange Commission $(2012,1)$ : 845 million monthly active users, 2.7 billion likes and comments per day, 250 million photos uploaded per day, and 100 billion friendships. While the company did not demonstrate how these figures translate into its potential market value, it managed to generate economic significance of these numbers under one category-user data. As the company explained to advertisers, its biggest revenue source, the ownership and manipulation of such a large size of user data, gives the company a "unique" edge to enhance the effectiveness and relevance of their advertisement. ${ }^{1}$ Facebook believes that user data, be it uploaded photos or friendship connections or "likes", express individuals' interests and disclose details about their social relations. By selling the information about users' interests and social relations to advertisers who can then make the advertisements more relevant to the targeted people, Facebook made one billion dollars in 2011. And the economic anticipation shown in its IPO and its concomitant capitalization on

\footnotetext{
${ }^{1}$ Revenue from advertisers consists of $98 \%, 95 \%$, and $85 \%$ of the total Facebook's revenue in 2009, 2010, 2011, respectively. See (United States Securities and Exchange Commission 2012, 11).
} 
the financial market seem to reinforce the logic Facebook embodies regarding the relationship between data, profit, and market value.

Speculations on anticipative value of information and data are not restricted to market capitalization, but expand to political and cultural realms. Early in November 2012, Barack Obama was reelected as U.S. President with a significant margin of the electoral votes. With Obama's victory, his campaign's less well-known team of data analysts stepped into the public eye (Scherer 2012). Obama's campaign team of data scientists has a massive synthetic database which contains pollsters' information, voters' demographic data, voting records, and voters' consumer records, as well as social media activities. Through data mining and predictive analytics, the data science team was able to identify the key concerns in the undecided voters' mind and help President Obama tailor the political ads to get voters' attention and eventually their ballots. ${ }^{2}$ With President Obama's win, a New York Times blogger and a statistician named Nate Silver who was independent of the Obama campaign attracted tremendous media attention. Like what he did with the election four years ago and even better, Silver correctly predicted not only the victory of President Obama ( $90 \%$ chance of win) but also the outcome of the election in 49 states, when the national polls showed, and most politics observers seconded, it should have been a tied race. ${ }^{3}$ Silver's book entitled The Signal and Noise: Why So Many Predictions Fail-but Some Don't (published before the Election Day) soon became a national bestseller. Silver's precise prediction shares President Obama's secret to successful reelection, namely, the reliance on large datasets about American voters and sophisticated statistical models to analyze the data and predict voters' behaviours. Indeed, President Obama is not the first candidate incorporating data analysis into political campaign. Nor is Nate Silver the first ever statistician who applied statistic models in forecasting. For those who still remember, the "electronic brain", a computer named UNIVAC, was first introduced for election coverage in 1952 presidential election. But the purpose was more to seize the public imagination of computers than actual adoption of computerized prediction (Chinoy 2010). When it comes to political analysis, UNIVAC fell out of favour in the following mid-term election in 1954 and was replaced by human brains.

It took six decades for the mainstream media to finally embrace the power of big data in political forecasting, which seems to be a long overdue justification for a technological turn in the narratives around the presidential election. Nonetheless, President Obama earning the title of "big data" president and big data being singled out as the most identifiable characteristic for 2012 election reveals something deeper about the shifts in cultural attitude toward data and the scope of the fields to which data analysis can be applied. ${ }^{4}$

Along with creating a popular tech-savvy icon out of Nate Silver as "the data wizard" (Hurwitz 2012; Casaretto 2012), some reporters declared that we are entering a new political era, "where data scientists have pushed out the [...] experts" and where hard data proves the invalidity and obsolescence of "political instinct" (Vos 2012; Scherer 2012; Horsey 2012). Several months after the Election Day, Wired magazine speculated on the success formula for the next U.S. president in 2016 as "Big Data + Social Data = Your Next President" (Chahal 2013).

Facebook's IPO, Obama's reelection, and Nate Silver's mythical prediction based on manipulation of massive data and statistical simulations make "big data" one of the most significant terms in economic, political, and cultural expressions.

The problem is, however, there is no consensus on almost any specifics regarding the term big data, from the definition and connotation of the term, to the scope and measurement

\footnotetext{
${ }^{2}$ Data mining refers to a set of data analysis techniques used to discover unknown patterns or correlations among various variables in the given datasets. Predictive analytics are typically defined as the analytics, built upon correlations found via data mining and computerized simulation and modeling, which are used to predict the possible future outcomes so that companies can plan future strategies accordingly.

${ }^{3}$ For more on Nate Silver's blog at New York Times, see http://fivethirtyeight.blogs.nytimes.com/

${ }^{4}$ Data and information are defined differently in the field of information management and computer science, as the former is considered as the base unit for the latter and the latter occupies a higher position in the knowledge production hierarchy. I will return to the difference in the later chapters. For an excellent critique of this hierarchy (Weinberger 2012).
} 
methods, and implications of big data. Nor is there any agreement on how large a dataset or the accumulative amount of data can be considered as "big". The term big data means different things, refers to different contexts, and implies different consequences depending on who deploys the concept. It indicates a cultural trend that involves interplays of technology as well as unfolding myth-construction which promotes faith in quantification and associate quantification with absolute neutrality, objectivity, and truth (boyd and Crawford 2012, 663).

When Mark Poster (1990) examined the implications of electronic communication technologies, he suggested to expand the scope of analysis to "the mode of information"-the cultural experience of the subject, which is configured by new technologies but subject to more possibilities. As information has been elevated to a new level of cultural privilege in the 1980s and 1990s, remarkable discourses around (big) data and pervasive discussions on the implications of big data thus require serious scholarly examination into the mode of data, and more importantly, the role of digital labour in the process of data production.

While it is urgent to have scholarly conversations on digital labour in the fields of new media culture and critical studies of contemporary society, there are several practical and analytical obstacles to theorising digital labour. These obstacles derive from common misunderstandings around digital labour. Nonetheless, leading multi-national companies are committed themselves to instilling these misunderstandings into the society to conceal the way in which digital labour is appropriated for value generation and profit making.

Misunderstanding \#1: "It is fun and it does not feel like work at all." Users of social media like Facebook are producing their personal data for the company, but are they workers for Facebook? Since Facebook does not pay their users for disclosing and sharing their personal information, is it legitimate to describe users' work for Facebook as being exploited or as "free labour"? When Arianna Huffington sold Huffington Post, a blogosphere where freelancer columnists contribute on a voluntary basis, for $\$ 315$ million to $\mathrm{AOL}$, the transaction ignited furious protest from volunteer columnists and online fair labour activist groups. They demanded financial compensation for the bloggers' voluntary work for Huffington Post. In sharp contrast, Facebook's IPO encounters nearly zero protest from its users and labour union organizers, although the former build the Facebook social media empire in almost the same way as freelancer columnists did for Huffington Post. Lack of protest for financial compensation in the case of Facebook can partially be attributed to the experience with social media that, as Trebor Scholz $(2012,2)$ describes, "[it] does not feel, look, or smell like labour at all". In addition, joining Facebook and utilizing social media is optional. The fact that it is optional and not obligatory implies certain degree of autonomy on the part of Facebook users. At the end of the day, they can opt out and stop using the social network site any time they want. This feelings of autonomy is part of the reason for interactions on the social media site to not be felt like labour at all. Statements like "it is voluntary and it is fun so it should be considered as work" misunderstand the relationship between labour and work. What constitutes labour in the digital realm becomes elusive. Nowhere is the elusiveness of the characteristics more visible than the flood of newly emerged scholarly vocabularies to describe the feature of labour in the digital world. Digital labour, cyber coolies, free labour, immaterial labour, cognitive labour, creative labour, knowledge workers, collaborative labour, crowd-sourcing, micro work, and playbor, and the list continues. Various adjectives before the word "labour" attest to scholarly desires and efforts to make sense of the changes information and communication technologies (ICT) have brought to not only the working environment and labour organization, but also the content of work and the nature of working in the mediated environment.

Misunderstanding \#2: Marx's labour value theory is obsolete. Whether social media users' labour is the sole creator of the value of their data as well as Facebook's profits, as Karl Marx might argue, turns out to be a perplexing question. For instance, Adam Advidsson and Elanor Colleoni (2012) contend that Marxist labour theory of value is obsolete in explaining the value creation of social media companies and their relationship to social media users. For the market value of the social media company is determined more by financial market speculation than the productivity and inputs of their users. Furthermore, users' affective investment in maintaining their social interactions via social media platforms cannot be measured by (labour) time which Marx reckons as the single determinant in how much value is created. As 
the company's S-1 form and stock performance evidenced, however, the value of Facebook is largely dependent on the speculations of the potential profits the company can make out of the massive user data.

Misunderstanding \#3: digital labour is immaterial and thus bodiless which has little to do with geographical and temporal barriers. If we take a closer look at Nate Silver's day job and President Obama's team of data scientists, polling information and other social data about the voters certainly constitutes the "raw materials" for their work content. Given the reliance on the data-processing technologies and expertise in statistics and computing modeling, information is both the raw materials and the means of production. Indeed, the source of the data has little to do with the location of these peoples' workplace. As long as they have the access to the Internet and the databases, they can perform their work anywhere they want. However, a division of labour is set in motion between specialized data analyst (and scientist), which Silver represents, and less visible data producers such as Facebook users and millions of consumers who feed into database their social interactions, consumption patterns, political tendencies, and so on. This division of labour is also intimately tied to a bifurcated reward mechanism, symbolically and financially. While the former represents members of "creative class" enjoying public attention and transcending occupational boundaries, the latter is absent from the grandiose narrative about the future of big data and rendered as invisible in data production process. ${ }^{5}$ This misunderstanding greatly distorts the reality of digital labour in the form of data production also because social activities such as consumption habits and online interactions which are coded as data in the social media system largely reflect and/or expand what they are like in the offline world (Rainie and Wellman 2012). The offline world is subject to geographical inequalities which causes the gaps in the Internet access.

Along with Trebor Scholz's aforementioned comment, what should labour "feel, look, and smell like" in the digital age of big data? If user data are the product Facebook is selling and capitalizing upon, how can we delineate the process and to what extent is users' perception of their relationship with Facebook representative of the forms of digital labour? Given Nate Silver's hypervisibility and era of celebrity, what kind of power mechanism is at work to determine the degree of the visibility, earnings, social status, and cultural significances of different types of digital labour?

Digital labour throughout this article is defined as the part of interactions with digital communication devices, which have been appropriated for profit making. This definition differs from conventional concept of work in that it describes more fragmented and ephemeral encounters with digital, networked online environment, which may or may not be recognized as conventional work.

The goal of this article is to further dismantle the aforementioned misunderstandings of digital labour. In the following sections, I will propose the theoretical groundwork to analyze digital labour by establishing connections among the categories of "data", "value", and the notion of "labour". My argument is that we need a discursive-material approach toward analyzing the cultural differentiation, valorization, and the organization of digital labour. It needs to focus on discursive formation as certain activities and online behaviours, which are deemed as not related to work, have been appropriated for private company's profit making. As we migrate more and more social interactions onto the online space and most of the online activities are coded into data forms unknown to us, the line between the social and the economic starts to become blurring. Latest discourse around the value of (big) data which renders labour embodied in the data production invisible shift the definitive boundaries of what needs to be considered as labour in the first place (section 2). One of the most profound implications of challenging dominant discourses around data and value is to make otherwise invisible digital labour visible. In section three, along with analyzing the aforementioned three misunderstandings of digital labour as the key examples of how discourses work, I will focus on discussing the materiality of digital labour. Labour is almost always em-

\footnotetext{
${ }^{5}$ While as data producers, most of the Internet users are invisible, as targets for surveillance and victims for privacy violation, they are hyper-visible (even more so after Edward Snowden's revelation of NSA's surveillance program). Mark Andrejevic (2012) argues cogently that theoretical framework and social advocacy based on privacy as an inalienable right fall short to capture the whole picture of the political economy of data production.
} 
bodied. Labour division is associated with social stratification and cultural differentiation. Without addressing both the discursive formations surrounding data and value and the implications of discourses for the valorization, circulation, and organization of labour, scholars would find it difficult to critically understand digital labour in the informational capitalism as well as in the age of big data.

\section{What can we learn from discourses around value and (Big) Data about Digital Labour}

Due to information and communication technologies (ICT) developments like the computer, Internet, tablets, and mobile phones, the collection and dissemination of information has become more abundant and data gathering comes from almost everywhere in today's world. Information exchanged on the Internet is considered as a significant resource for economic production and indispensable component in people's daily life. International Data Corporation (IDC) predicts a 6.9 per cent increase in worldwide IT spending in 2012 while the projection of the global gross domestic product (GDP) growth is set at 2.5 per cent by the World Bank (IDC 2011; The World Bank 2012). An average daily consumption of 34 gigabytes of information in 2008 shows the crucial position of information in Americans' daily life (Bohn and Short 2009). To put this number in a more meaningful context, a three-hundred-page dissertation stored in portable document format (PDF) and a popular song in MP3 format is about 4 megabytes. The size of a DVD movie varies from 2 to 7 gigabytes each. Since one gigabyte equals 1,024 megabytes, it means in 2008 the average American's information consumption could be anywhere from 256 dissertations or songs to 5 DVD movies per day. In addition to consumption, the pace to produce information is also unprecedented, and information production does not restrict to information technology industries. For instance, Walmart, world retailer industry leader, has to handle more than 2.5 petabytes of data generated from customers' transaction per hour. That is equivalent of 167 times all books in the U.S. Library of Congress (The Economist 2010). Juan Luis Carselle (2013), the former chief information security officer at Walmart South America, points out that Walmart is now an IT company that also does retail.

McKinsey Global Institute $(2011,15)$, a research firm affiliated with McKinsey \& Company, estimated that the information produced and stored by global companies and individuals in the year of 2010 exceeded 13 exabytes, which is 60,000 times larger than the amount of information U.S. Library of Congress stores.

Indeed, information has been assigned with increasing social and cultural significance. Nonetheless, the job of number-crunching clerks or book-keepers was once pictured as monotonous, dispiriting, and a "sorry example of the ongoing massification of modern life" in many literature and popular imaginations (Roszak 1987, 5). It is intriguing to juxtapose this past pathetic image to the tech-savvy, brilliant, and creative figure of statistician embodied by Nate Silver. What is omitted from the attitudinal shift is the fact that the content of their job is pretty much unchanged, namely, making sense of abstracted numbers. The most visible characteristics which distinguish Nate Silver from his occupational predecessor are the amount of data he is analyzing and the fields where he applies the meanings of the numbers. In a very similar gesture, Facebook boasts about the amount of data they are holding on their users, and see those data as the unique assets of the company.

The pure, mathematic reasoning originated from modern information and communication theories has successfully expanded to the social and cultural realms. Scholars and advertisers have developed "trust in numbers" (Porter 1995) and relied upon quantitative analysis for analysis and predictions for decades. What distinguishes the present moment from the past usage of quantitative data is the extent to which new categories of data are invented to describe and measure socio-psychological behaviours, physiological motions, and even sentimental ups and downs. Facebook's knowledge about its users is far beyond basic profiling information and aggregated social interactions data. Through built-in algorithms, Facebook also knows its users' "pace of life", "concentration cycle throughout the day", "preferences between text, photo, and video", "logical thinking", and "the degree of happiness" to name a 
few (Olsthoom 2013). The source for these "knowledge" is a huge engineering project which codes people's social connections and desires as peculiar types of data manageable and manipulable by computers.

Nonetheless how engineers develop viable interpretations of users' Facebook data as indicators for "the degree of happiness" remains unknown to the public. One thing is crystal clear-the power relations between those who set rules for the definition and interpretation of the data and those who produce data are opaque and asymmetric. Andrejevic $(2012,84)$ pinpoints the asymmetric power relations embedded in the commercialized model of the Internet infrastructure, that is, operative in the hands of private companies. As people rely on the Internet to perform more and more activities from getting news and information to interacting with friends and engaging in communities, the Internet infrastructure becomes increasingly constitutive of tens of millions of people's social reality. However, for platform-provision companies, the Internet infrastructure indeed becomes "material resources" for economic profits while for Internet users the Internet is the place for a variety of social and cultural activities.

This functional conflation alerts us to confront the social and cultural ramifications of economic activities based on constructed value of information and data, and how exactly value and labour are defined in those economic practices. To better understand how value of data and labor are socially constructed in the informational capitalism, it is important to first set the concept of the economy back to the social which is subject to discursive formations.

Economic activities are historically specific and should always be understood as "embedded" in the society at large and in the complex social relations and cultural practices. Timothy Mitchell (1998) suggests that the concept of the economy is a recent idea which dated back to the mid-twentieth century. Over the last half a century, scholars and national policy makers jointly constructed and "modelled the economic process as a mechanical apparatus" (Mitchell $1998,86)$ which gradually appeared to detach from the social forces. Karl Polanyi (2001) is perhaps among the first few thinkers who cast skepticism on the naturalness of capitalist market economy and deconstruct the liberal creed of autonomous market as a discursive and social construction. Back in the mid-20th century when political economy theorists were engaged in heated debate on the necessity of governmental regulations in the realm of free market, Polanyi $(2001,3)$ argues that autonomous market economy is historically proved non-existent and liberal economist's belief in laissez-faire is "stark Utopia" and groundless. Prior to the emergence of free market, economic exchanges and market activities, for instance, in the customary practice of barter, were largely rested upon social connections and local communities. The inception of capitalist market economy was not a natural outcome of local markets converging into a national and international one. On contrary, the imagined autonomous market is constructed along with vocal advocacy for free competition, rather violently economic endeavor to expand trade from Europe to across the planet, political lobby for tariff protection and governmental regulation. While lobby for tariff protection and trade expansion is coming from elite business interest groups, often overlooked grassroots resistance which is behind the forces for government regulations is from the mass individuals. Public resistance does not come from intuitive hatred toward market or orchestrated conspiracy against the spread of transnational trade. On the contrary, the construction of autonomous market disregards the deeply rooted, long-term daily practice and social relationships in which individuals make sense of their economic activities, and attempts to subjugate the former to the latter. Public resistance originates from the disruption caused by the sweeping market economy of individuals' habitual daily practice and social relations.

Polanyi's recount of the interplays between the liberal discourse of free market and the economic practices rooted in the networks of social relations, together with his thesis on economic embeddedness in the broad social context, indicates that the boundaries around the economic realm are porous and socially constructed. The line between the economic and the non-economic has been drawn and redrawn throughout the history. Take Facebook as an example again. Several scholars consider the social media giant's worldwide popularity as the ultimate triumph of capital's "real subsumption" of every bit of social interactions and relationships for Facebook users' activities constitute the very informational infrastructure 
and online environment they are inhabiting (Dyer-Witheford 2004; Fuchs 2011; Fuchs 2010). While this kind of criticism pinpoints the economic engines behind the Internet companies such as Facebook, it glosses over the way in which those companies redefine what constitutes economic practices and relations and what are redefined as the non-economic ones. Indeed, discussions on social ramifications of the line redrawn beyond economic exploitations of the Facebook users are nowhere close to the easy resort to the Marxist mode of economic criticism.

The point, however, is not to discard a closer scrutiny of Facebook's profit-generation strategy. Rather, it might be more productive to put its profit-generation strategy within a broader social context and consider what has been redefined as economic activities and what has not. Keep in mind this scenario: when asked on his stance on the privacy issue in early 2010, Mark Zuckerberg (2010), the CEO and founder of Facebook, responded that "[people] have really gotten comfortable not only sharing more information and different kinds, but more openly and with more people...We (the Facebook) view it as our role in the system to constantly be innovating [...] to reflect what the current social norms are...We decided that these would be the social norms now and we just went for it". The social norms Zuckerberg claimed as he and his company detect are exactly the social behaviours and activities they encourage Facebook users to do and perpetuate as the norm. Investigating how social norms around information and data have evolved and been constructed by a cluster of players including Facebook is just as important as disclosing Facebook's reliance on users' shared information for economic profits.

Value is never a pure economic term it almost always has social and cultural register. Oxford English Dictionary documents, besides being quantitative measurement of the material worth of something, often used as the medium for market exchange, value also refers to "worth based on esteem; quality viewed in terms of importance, usefulness, desirability" (Oxford English Dictionary 2013). While social worthiness and desirability has a lot to do with the broad cultural discourses and historical circumstance, they are not immaterial. On the contrary, social desirability and worthiness are established largely upon material practices such as possessions of certain symbolic and substantial goods or the construction of social hierarchies which directly determines the distribution of social, political, and economic resources.

All these entail a material-discursive perspective to look at the information-based economy and labour issues. As cultural studies theorist Lawrence Grossberg urges, we should recognize "that economies are partially discursive [...] [and] completely integrated into the social totality" (Grossberg 2010, 102).

The power of technological discourses in particular takes effect in material forms. Since the invention of scientific management and Fordism way of manufacturing, there are few doubts about technologies' effect on division of labour. However, few notice that technological division of labour can hardly separate from social construction of differentiation among labour types. For instance, the constructed division between women's domestic labour and men's productive labour was tied to the fact that women were paid nothing for the fulfillment of the reproductive labour. And their domestic labour was theorized as unproductive and immaterial making little contributions to economic development. Even if technological advancement and social improvement allow women to step out of home participating in traditionally productive sectors, a "gender-segregated" labour market is awaiting (McRobbie 2011). As a result, a majority of women found themselves concentrated in certain subordinate or feminized occupations such as office clerk. The social hierarchy regarding women and men's labour also involves a spatial division between them which designates the home space to women and public sphere men. I will return to the point on the social and spatial division of labour later.

Along this line, as Zuckerberg tried to dissolve the line between the economic and the social, his claims remind us of how discourses work to construct the line between productive and unproductive labour. In his framework, the social norms are widely accepted social activities (not to mention Facebook's own role in creating the social norms in the first place) which should not be considered as productive since productive activities translate into monetary rewards. By expanding the scope of acceptable online social activities and creating new so- 
cial norms, Facebook and Zuckerberg attempt to redraw the boundaries of being productive or unproductive. Therefore, to thoroughly understand this type of digital labour, ideologies deployed to construct what are socially desirable and acceptable need to take into consideration.

Admittedly, social media users as the sole producers of their social data, their labour is creating the value of those data. However, their data world be worthless if it were thirty years ago when the constructed value of data have not reached the current level. Consequently, it would be meaningless to analyze the value and organization of labour unless situating it in the concrete social, material conditions which are already mediated by cultural discourses. Social differentiations of working bodies (as in the case of men and women) are inseparable from spatial rearrangement of labour division. As this article aims to engage with scholarly conversations on the value and organization of labour in the network society, it is necessary to tease out the threads from the "contextually determined set of material-discursive apparatuses" (Grossberg 2010, 102), which shape our perceptions of the social worthiness of information, the value of labour related to information (from producing to processing to dissemination), and socio-geographical division of labour. Thus, interrogating dominant ideology and rhetoric around work in the information age is foundational to understanding the differentiations of digital labour.

\section{Is digital labour immaterial? The materiality and differentiation of digital la- bour}

Technological discourses surrounding information and labour in the informational network society acts on material basis. In this section, I will mainly dismantle misunderstanding \#3 around digital labour. Namely, nothing is further from the truth than to regard digital labour to be immaterial. The bodily foundations for labouring activities and the materiality of labour are reflected in the interplays among human bodies and information and communication technologies (ICTs) within the social and cultural context. Two dominant forces are noteworthy. The first is capital's constant tendency to make labour invisible in the form of commodities and service. The second is hegemonic ideologies rendering bodies as obsolete in informational and network society, which further distance labouring bodies from our range of vision. Moreover, to recognize bodily materiality of digital labour is crucial also because labouring bodies are the sites where social differences are constructed and spatial divisions are formed.

\subsection{Make invisible labour visible}

Karl Marx's criticism of commodity fetishism is one of first eloquent accounts to identify capital's tendency to invisiblize labour. Marx (1995a) opens Capital with a discussion on commodities, but he directs the readers away from the marketplace where commodities are exchanged to the production site. His real intention is to demystify commodities as inherently valuable and naturally exchangeable goods. Focusing on the production site, Marx argues that commodities are able to circulate on the market because there is something commensurable and common in all commodities despite their varied utilities. And this commonplace is the embodiment of human labour. To unveil how labour is extracted to create value and wealth, Marx identifies the dual-character of labour as being both abstract and concrete. While abstract labour reflects the universalized, interchangeable nature of the expenditures of human bodily practice, concrete labour has to do with the specific subject, raw materials, and instruments in the labouring process. Abstract labour creates the exchange value of the product; concrete labour shapes the utility of certain products, namely, the use value. Both value and use value of commodities are the embodiments of labour. Commodities production site is where labour is turned into the value and where labour is materialized. Commodities are the objectified labour, but at the same time they make labour invisible.

Writing at the dawn of industrial age, Marx already saw, and anticipated more, technological applications into production. He considered massively applied science and technologies as the objectified labour in the forms of tools, instruments, and machineries in the industrial age, and perhaps including today's automated systems. He described machinery as "the 
body of factory" and the stored-up labour in tools and instruments as "dead labour" (Marx 1995b). Thanks to the technological development, capitalistic productivity grows at an exponential rate, relying less and less on the operative labour at the machineries and more and more on the productive tools and instruments (read dead labour). Since capitalists hold the private ownership over tools, instruments, and machineries, it appeared to Marx that the objectified labour taking various shapes (including commodities and tools) eventually would dominate the living labourers who oversight the automated systems. In this way, capitalists' social dominations over wage workers are enhanced and naturalized in the "technological fact" (Marx 1861). Again, the dead labour in forms of technologies, knowledge, and tools and instrument are rendered invisible and independent of, if not alienated from, the contemporary workers. Marx's line of reasoning and endeavour is endorsed and persistently pursued by generations of succeeding scholars. For example, Kathi Weeks $(2011,7)$ protests the oblivion to the central role of work in our current daily life. She sees publicizing work's foundational role is the first step to politicize it and "to counter the forces that would naturalize, privatize, individualize $[\ldots]$ and $[\ldots]$ depoliticize it."

While Marx's focus of study remains at the production site, namely, manufacturing factories, capital's accumulation thrives upon appropriations of activities in the non-production fields where work exists in forms other than wage labour (De Angelis 2001; Huws 2003; Prodnik 2012; Terranova 2000; Virno 2004). The existence of those unrecognized forms of labour challenges Marx's labour value theory since performed outside production site those unrecognized labour is most likely embodied in the forms like service rather than commodities. In addition, the value of various forms of unrecognized labour is subject to social and cultural forces other than marketplace exchange mechanism. Nor can they be measured by the amount of "socially necessary labour" used to produce it, as Marx proposes.

Several scholars have proposed expansive definition of commodification and tactical understanding of work to more accurately understand the relationship between those parasite activities and standard occupational labour. Ursula Huws (2003, 67), for example, defines labour performed outside the standard commodification process as "unsocialized labour". The ongoing commodification constantly affects the forms and scopes of labour that is not yet incorporated into the capitalist cycle of accumulation. The boundaries between unrecognized labour and relatively more standardized forms of labour are porous and keep shifting under the circumstance of capital accumulations. Once the border shifts, the scopes and forms of unsocialized labour change accordingly, which in turn affect the established pool of standard work. Technologies are always deployed to facilitate changes in the scopes and forms of labour. Some skills are rendered obsolete by technologies, new skills are demanded, and still others revive after a period of disappearance. In the rise and fall of certain labour, we notice shifted boundaries are accompanying spatial divisions for the labour and discursive constructions of differences for the bodies that perform the unsocialized labour.

Women's unpaid domestic labour, for instance, is among the first unsocialized labour that was subsumed by capitalist economy. And home appliance is one of the earliest examples of capital's tendency to replace unsocialized labour with massive manufactured commodities and/or paid service provision (Huws 2003; Fortunati 2007). Thanks to technological development, all kinds of home appliances are manufactured. Home appliances are promoted as liberating women from tedious housework with the latter often relegated as outside productive domain and without value. The grandiose mission to replace women's reproductive labour seems to have failed; the one to turn the home space into another market for machinzed goods and standardized service have succeeded. Unfortunately, commercial promotion of home appliance's efficiency conceals new forms of women's reproductive labour, such as operating home appliances and communicating with technicians to maintain the home appliance (Fortunati 2007).

The concept of immaterial labour is also introduced to capture new content of work and the shifting line of labour. As James Beniger meticulously documents, how to improve information processing and communication technologies (ICT) first appeared as an urgent challenge in late 19th century and early 20th century (Beniger 1986). During that period of time, innovations in ICT were badly needed because industrial revolution led to massive reproduc- 
tion of goods and the information concerning inventory and business transactions exceeded the contemporary processing capacities. Information processing capacities prove to be the bottleneck for full realization of manufacturing productivity. Since then, especially in the United States and other developed countries, workers increasingly rely upon their subjectivity and knowledge to fulfil their jobs; and their work content has more and more to do with information, communication and coordination rather than direct contact with manufactured goods. To readdress the composition of wage workers in post-industrial capitalism and the role of workers' subjectivity in processing information to create value, Maurizio Lazzarato (1999) first introduced the concept of immaterial labour. He defined immaterial labour as what produces "informational and cultural content of the product." Informational content consists of the service and the knowledge labourers provide to the customers with respect to the processing, control, and exchange of information in both manufacturing and service industries. Cultural content includes cultural knowledge on norms and customs, aesthetic tastes, fashion and artistic judgment, and affections.

Similarly, Hardt and Negri $(2001,290)$ in Empire used the concept of immaterial labour to describe service (production) industries because "the production of service results in no material and durable good [...] that is, labour that produce immaterial good, such as a service, a cultural product, knowledge, or communication". They further fleshed out three sub-types of immaterial labour activities. The first involves information processing, such as entry-level word processing and electronic inventory keepers; it typically appears when computers are widely applied in manufacturing industries, realizing the shift from commodity production to service provided as goods. The second is engaged with the creative and/or mechanical manipulation of signs and symbols, such as graphic designers and routine web maintenance workers. The last type of immaterial labour requires personal affective contact and interactive communications, which is also known as affective and communicative labour. Customer service providers and maids are among the third sub-type of immaterial labourers.

Indeed, the idea of immaterial labour greatly advanced our understandings that social relations and cultural, ideological environment are embedded in the social production and continuously reproduced through capitalistic economic reproduction. The notion also substantially reframes the approach to labour-value relationship by extending the parameter of value to the social realms. In this way, we are able to disclose what "material production had 'hidden', namely, that labour produces not only commodities, but first and foremost it produces the capital relation" (Lazzarato 2011). When communication networks increasingly converge with social networks and transnational corporation's networks of suppliers from all over the world, traditional non-working space and time become the new territory for value creation. It is hard to tell where the cycle of immaterial production begins and ends, when social relations, workers' subjectivity, cultural and ideological environment are all reckoned as "raw materials". The elusive nature of immaterial labour deeply troubles Marx's equation between value and labouring time. As a matter of fact, the content of the work and the extraction of labour can be nearly anything as long as capital accumulates.

Another prominent example of how technologies are manipulated to trigger changes in labour forms is the construction of consumer's role in the production cycle in the U.S. pervasive customer self-service in U.S. nowadays has demonstrated the triumph of the steady trend to put consumers to work in 20th century. In this steady process, the boundaries of labour have been shifted from paid service workers to consumers; meanwhile we also hear new words to define the role of customers and thus the forms and meanings of their labour. Marketing and management professionals have been encouraging consumers' (voluntary) participation into production by promoting the latter's creativity and contributions with the label such as value "co-creation" and "consumer-led design" (Prahalad and Ramaswamy 2004; Lovelock and Young 2011). When it comes to Internet users on open, interactive, collaborative platforms such as P2P platforms, Wikipedia, Myspace, and Facebook, terms like "produsers", "prosumer", mass collaboration and cloud collaboration become buzzwords to 
describe Internet user's activities. ${ }^{6}$ The rationale is that web content are produced and consumed simultaneously by massive anonymous Internet users without any charge or payment. Some scholars argue that conventional understanding of production as distinct from consumption from the industrial age seems inapplicable to prosumer-created web content. For the latter represents the non-monetary spirit of sharing which makes it qualify for a fundamental alternative to market- and proprietary based economy, or at the extreme end, a new imagination of capitalism (Bruns 2008; Benkler 2007; Bauwens 2011; Ritzer and Jurgenson 2010; Graham 2011). Others believe that the prosumer metaphor blows the work of a slim percentage of Internet users out of proportion (Van Dijck and Nieborg 2009). The overgeneralization of all Internet users as equally active and motivated and making equal contributions ignores those who literally work online such as Amazon's Mechanic Turks. The metaphor of prosumer society also seems to conceal the value extraction by promoting a particular way of online engagement.

Through the examples of YouTube, Wikipedia, Google, and most recently Facebook, we clearly see the power online interactivities have unleashed from Internet users (among them prosumers). It shows great potentials for future social development, too. Meanwhile we also see the continuity in garnering unrecognized labour for value and surplus production in the course of (re)defining the role of consumers and Internet users. Take Google as an example. Its algorithm, PageRank, is created to order the value of webpages by measuring the number and the quality of incoming links. The more incoming links are and the better quality, the more significance a webpage is. PageRank allows Google to tap on every single cognitive decision made by Internet users in clicking the hyperlinks. Users' cognitive labour contributes to the value garnered by Google (Pasquinelli 2009). Their contributions are naturalized not in the form of commodities but built into the online navigation system structured by algorithms like PageRank and Facebook's default setting for disclosing user's information. Different from Marx's point on living labour in the factory enacts on the dead labour concealed in the machineries, Internet online activities are much alive and constitute the very "technological fact". As part of technological infrastructure, users' labour becomes not only invisible, but also ephemeral yet perpetual in value-creation.

Prosumers' labour is not new, and Internet economy has a long tradition of appropriating non-standard forms of labour, ranging from violent exploitation of volunteer's labour to "anarcho-communism"-spirit of sharing (Terranova 2000; Postigo 2003; Barbrook 2007). It is too hasty to conclude if prosumers are the protagonist of the network society and their social significance can rival that of producers in the industrial age and consumers in the postindustrial age. Conscious construction and recognition of prosumer's place in the Internet structure, nonetheless, allow us to be more aware of the shifted boundaries and forms of labour on other realm than the Internet. In sharp contrast, manual labour in the electronics manufacturing industries are almost complete absent from the dominant discourses around online digital culture. Take assembly line workers for digital devices as an example. Manufacturing workers are facing numerous challenges including low wages, excessively long work hours, severe work conditions, constant harms on health, and hostile political environment which prevents them from forming labour union. As Sandoval's (2013) analysis of Apple Inc.'s responses to criticism of its supply chain management has shown, Foxconn workers are excluded from the hi-tech, clean, sleek image Apple, Inc. aims to create. Instead, the statement from the most profitable IT company deflects its responsibilities for poor work conditions and multiple suicides committed by Foxconn workers. Only when these cheap labourers are faded into the shadows, becoming invisible, then the aura of sleekness and cleanness that Apple devices represent can successfully take root in the digital culture.

Along this line, an intriguing new territory for informal labour activities is in online virtual games. For some U.S. based cultural critiques and intellectuals, the global popularity of virtual environment, including massively multiplayer online role-playing game (MMORPG), signals a new form of sociality where virtual leisure is intertwined with real life social interactions

\footnotetext{
${ }^{6}$ The term prosumer is first used by Alvin Toffler in The Third Wave, but it starts to gain momentum in recent years because of the rise of users-generated content on the Internet.
} 
(Boellstorff 2010; Castronova 2006; Corneliussen and Rettberg 2011; Nardi 2010; Turkle 1997). While game playing always implies fun and leisure, in MMORPGs player labour is harnessed to produce economic value. The feelings of play and fun often obscure the fact that play is labouring (Yee 2006). A transnational hierarchy of labour division seems to form when 100,000 Chinese players are recruited to work on the game producing in-game currency so that their American counterparts can purchase the in-game currency acquiring new weapons and enjoying the pleasant synthetic experience (Lehdonvirta and Ernkvist 2011). However, as Chen (2013) argues, Chinese MMORPG play workers are "double marginalized" in the construction of digital gaming culture across the Atlantic. In the American cultural context, they are rule-breakers, "illegal immigrants" who destroy the fun, non-financial atmosphere of online gaming community. On the other hand, in the Chinese cultural context, playing online games is historically associated with problematic youth who lack self-discipline and suffer from Internet addictions. Under this circumstance, the value of digital labour in the case of online game playing is inseparable from the ongoing construction of gaming cultures.

\subsection{Make differentiated labouring bodies visible, immaterial labour material- interrogating normalization of digital labour}

The notion of both immaterial labour and prosumer each defines the feature of labour with the reference to the final product, although the former tends focuses on the added content of the product or service and the latter on a blended production and consumption behaviours. So far both presumes the working bodies as a given without questioning which forces bring them to the kind of labouring activities in the first place, let alone recognizing the differentiations within the same labour category.

Part of the reason for the absence of bodies is that information, and to the same extent informational labour, is often seen as without materiality. The concept of immaterial labour in particular tends to reduce the material to the tangible; and the information, culture, symbols, affection, and feelings are intangible and thus immaterial. Tiziana Terranova (2004, 3-6) singles out "two prejudices" preventing us from fully capturing the materiality and physicality of information, namely "the idea that information is the content" of messages or communication and the notion that information as code exists immaterially in cybernetic space. Information is not formless, bodiless code floating in the cyberspace. On the contrary, not only are information flows shaped by their material media infrastructures and systems, but also the meaning it conveys is to a large extent constituted by that materiality.

A third hindrance is that modern information theories and bioinformatics have caused an epistemic shift which treats human bodies a medium for signal/information transmission, storage, retrieval, and processing, no differently from other media (Hayles 1999a). Thus the missing link I suggest to put back into the picture is the bodily foundations for informationrelated workers. It requires us to centre upon working bodies and acknowledge bodies as the contested sites for cultural inscriptions and potentially subversive articulations. In doing so, we can make sense how the shifted boundaries of labour in the network society associate with social construction of different labour types. The starting point is to unpack the interactions between labouring bodies and communication tools and media.

Elizabeth Grosz (1994) explained how human is able to use tools in general:

Part of the difficulty of learning how to use these implements and instruments is not simply the technical problem of how they are used but also the libidinal problem of how they become psychically invested [...] It is only in so far as the object ceases to remain an object and becomes a medium, a vehicle for impressions and expression, that it can be used as an instrument or tool (Grosz 1994, 80; Lupton 2000).

These embodied extensions of physical strength, motions, emotions, expressions, and cognitive ideas constitute the materiality of interactions between bodies and the medium. Bodily interactions and mental investment help humans convert the tools in hand (and later on machines and computers) to be part of human bodies or the extension of bodily senses and intelligences (Hayles 1999a; Lupton 2000; Thrift 2006). Boundaries between human bodies 
and tools as objects collapse at the moment when human bodies incorporate the tools into bodily coordinate motions. In labouring, the constant interaction between human bodies and the immediate environment of tools, language, interfaces, and media forms an embodied working environment. The intimate relations and interactions between human and technologies not only constitute the material in the global network society, but also add complexity to the already existed mechanisms of divisions, differentiations, and exclusions.

Labouring bodies are the ultimate actors who interface with the devices making sense of the information and initiating communications. Walter Benjamin (1936) presents a convincing account on how massive reproduction of art occur concurrently with the rising desire from the mass to kill the social distance between the privileged and their mundane life by dislodging subsume elements symbolized in the forms of classical arts from their authenticity and originality. In a similar manner, instantaneous communication via interfaces like computer screens and mobile phone pad has profoundly transformed the interactive dynamics between workers and their clients. Mark Poster argues that the fixed cultural identity categories, such as nationality, in the network society are losing their currency. He also shows that contemporary cultural conditions which have been fundamentally transformed by digital/information technologies are materialized by an intimate hybridity of human and machines, or in his words "humanchine" (Poster 2001; Poster 2004, 318). In Poster's eyes, "humanchine" is subject to dominant politico-economic powers and other social trends but simultaneously emerges as the new contested sites of resistance and empowerment. Scott Lash $(2002,15)$ also proposed a "man-machine interface" as an entry point to understand life experience in the informational and technological society. Although Lash insisted that it is an interface rather than cyborg or any forms of fusion, both Poster and Lash implied that bodies are indispensable for the materiality in the information technologies. Hayles (1999b, 193-207) argued that a new kind of "subjectivity" emerges from constant interplays between normative and disciplinary forces from dominant culture inscribing upon bodies and embodied articulations from culture-specific, environment-sensitive experiences. Indirectly echoing Benjamin's elaborations on the relationship between historical circumstance, medium, and the living experience, Hayles (1999a, 291) stated, "human functionality expands because the parameters of the cognitive system it inhabits expand [...] [Therefore] it is not a question of leaving the body behind but rather of extending embodied awareness in highly specific, local, and material ways that would be impossible without electronic prosthesis." Instead of undoing bodies, we should put bodies at a central position in the studies on labouring activities particularly when bodily interactions with electronic devices are constitutive of working environment.

Beyond the interface level, no matter how geographically disperse or complicated the networks are, bodies are always at the network terminals. In the networks of information, labour, and images, human bodies are conflictive yet integrated sites for both inscriptions of cultural differences and potential interventions. The Internet is structured by hierarchical protocols and multi-layered coding system (Hayles 2004; Lessig 2006; Galloway 2001; Gallant and Boone 2011). If we see them together as a newly emerged signification mechanism, two characteristics stand out as compared with the language we use. 1) Computing systems are composed of multi-layered coding-decoding systems which centralize discursive formations and are open to individual-based interventions. 2) At stakes are not merely layers of text and coding systems, but also the physicality of the medium and the material infrastructure of the network. Internet's vast network of nodes across the world and its distributive design promise omnidirectional exchange of information. But critical Internet nodes still concentrate in a few places because the construction and maintenance need stable energy supplies and continuous updates of whatever becomes obsolescent. The access to the network comes before one can participate in the workforce online. In this sense, there are more forces at work other than just pure technological innovations or computing languages. Thus, networks are no longer neutral communication infrastructures. Instead they are deployed in regulating and controlling the labour flows and accumulations throughout the networks. More importantly, cultural differences inscribed upon (working) bodies further differentiate the social value of their labour. 
As Grosz (1994) stresses, when human is used to technological tools, tools extends human physical, psychological, and social reaches. Humans tend to forget where the devices are coming from and thus the labour embodied in the tangible technological devices is rendered invisible. Labour is objectified in the devices. Similar logic goes to social media and informational content production. Increasingly people rely upon social media to interact with each other. Social media and other smart devices become tools in their eyes, and they tend to forget about what is behind the interface. Thus, all labour, including data production labour, equipment manufacturing labour, website maintenance labour, are rendered invisible behind the interface.

It makes much more sense if we see the construction of prosumer identity as a differential technology for social division of Internet labour and compare it with the cultural representation of Chinese game play workers in the American context. Seemingly to be left out from this dominant discourse around online participatory prosumption, online gaming remains firmly in the consumption realm invoking the feelings of leisure and play in the U.S. in particular. Any productive activities and financial compensations are foreign to the gaming world except for contributions to the virtual community (that is the guild individual player belongs to in the games like the World of Warcraft). With non-monetary play set as normal behaviour, Chinese gold farmers break the rules and destroy the fun, innocent aura of the gaming space by making money out of playing without contributing to the community. American leisure players resort to racial profiling of Chinese gold farmers based on in-game behaviours and language abilities in order to protect their gaming space (Nakamura 2009; Nakamura 2012) Under these circumstances, the gaming world is not an isolated, virtual playground independent from the social reality, but part of contentious cultural realms across national borders. And the bodily inscriptions on each group, which are deeply connected to racial formations in American reality, play the role to differentiate the value of labouring bodies.

\subsection{Is spatial division of labour irrelevant to digital labour?}

Meanwhile, we must not forget that transportation advancement and international trade treaties had made it cheaper to relocate manufacturing jobs to underdeveloped countries before prominent American scholars like Daniel Bell and Fritz Machlup started to describe American society as a post-industrial society. As for the rise of network society, sociologists like Manuel Castells and Saskia Sassen explain that the formation of multi-national corporations networks, globally interdependent financial systems, and the emergence of global cities as "strategic sites" for service industries and transnational business transactions are facilitated by a variety of ICT innovations, deepened globalization, and the rise of neoliberalism which prioritizes privatization and "friction-free" market capitalism (Castells 2000; Sassen 1999; Harvey 2007). Based on his analysis of call centre workers, back-office operation, human resources and payroll management, insurance claims processors, and website services workers in India, Aneesh (2006) identifies a key shift in labour mobility in the digital age. Namely, "body-shopping" which transfers programmers to work on short-term projects overseas periodically has been replaced by online delivery of work across national borders without physical traveling. He terms them as "virtual migrant" (Aneesh 2006). Virtual migration underscores the transmission of digital labour and service via online network (virtually) and the confinement of the body within national boundaries. It enables the workers to evade such messy process as visa-applying which is unavoidable in the physical migration (read bodyshopping). However, it remains an open question whether virtual migration signals a new trend of digital labour mobility which makes geographical barriers irrelevant.

Globalization and the rise of network society are not happening evenly on the planet. And technology-triggered international division of labour does not follow the national line neatly. Some scholars recognize that the increasingly significant role played by ICT worldwide implicates a radical shift in economic organization and people's perception of space. Manuel Castells $(2000,442-453)$, for example, labelled the dominant spatial expression of the economic and cultural logic of the network society as "the space of flows", as opposed to the shared, continuous experience in a fixed place. Similarly, Michael Hardt and Antonio Negri pointed 
out that labour control in the informational age is governed by the new logic of a decentralized network, which prevails over the dependence on geographical concentration and proximity, a typical feature of labour management in the industrial manufacturing age. For white collar professionals in the developed countries, constant connectivity presents a mobile and distributed workplace which enables them to work anywhere and anytime (Gregg 2011; Rainie and Wellman 2012; Ross 2012). Geographical boundaries are not the obstacles to finish the jobs in the distributed workplace.

The reality of invisible digital labour seems to prove the opposite. Take Chinese MMORPG play workers as the example again. That China becomes the major destination of MMORPG game workers is not an accident. On contrary, the online virtual gaming world and digital labouring practice have deeply rooted in geographies. The availability of large pool of cheap labour, fast construction of the technological infrastructure, and massive influx of migrant workers from rural regions to urban areas all have paved way for cities in China and Internet cafes in the cities in particular to become the work place for game play workers (Qiu 2009). Another example is rare earth mineral miners. For example, coltan-one of the essential rare earth elements for manufacturing capacitors in laptops, mobile phones, and other electronic devices like game consoles. Although Democratic Republic of Congo possesses 80 percent of the world's coltan, its neighbouring countries like Rwanda, Uganda, and Burundi become major exploiters of coltan in Congo. Because the country has been caught in wars and violent conflicts since 1990s, the conditions of minerals mining in Congo is the "modern forms of slavery" (Fuchs 2014). Congo becomes the most tragic geographical place on the landscape of electronics manufacturing and consumption. Geography brings the country the richest natural resources but fails to bring the country an upgraded digital labour work condition. Or rather, when dominant cultural discourses help consumers in the developed countries construct digital consumption culture, mineral miners in Congo are not part of the picture. They are on the back of it. Their toils and bloody, dirty bodies are not to be seen by common prosumers in the digital age.

As shown by these examples, geography and labourers' bodies, if anything, become more important in the informational society, because geographical locations are deeply connected to information infrastructure which creates geographical segregation in the transnational labour hierarchy (Sassen 1999; Aneesh 2006; Huws 2003). Although for some workers, geographical barriers have been lifted by the technologies, institutions and policies continue erect obstacles to control labour mobility. Moreover, heterogeneous spatial transversal and border-settings accompany labour exchange, of which some become embedded in the workplace. National borders are at best selectively flexible for talented knowledge workers who frequently travel from one global city to another half-way across the planet, but become more strictly monitored for unwelcomed bodies (Sassen 2007; Sassen 1999; Ong 1999; Mezzadra and Neilson 2011).

\section{Conclusion}

When examining the relations between linguistic power and the new economy, Marazzi $(2008,42)$ argues that "the capacity for centralization and subjugation (for private appropriation) of the disseminated forces of production [...] operates now in a less directly visible and material form. It reinforces itself and subjugates by way of communicative and linguistic means, by activating symbolic and normative circuits." This article theorises digital labour by stressing discursive formations around the value of data and the value of labour in the Internet age. The cultural discourses around the potentials of (big) data and labouring activities associated with data production and consumption have been deployed by leading private companies to construct the normative behaviours in the digital realms. By reconstructing what are socially and culturally desirable activities, dominant discourses around digital labour and data have rendered certain types of labouring activities invisible, for instance, low-skilled game play workers, assembly line manufacturing workers, and so on. The feelings of fun and play and the sense of participation and sharing are among the salient features in the normative construction mechanism. Other than desirable digital labour activities, others are 
deemed as marginal and valueless, and they do not belong to the construction of digital culture. Therefore, these digital labourers who include manufacturing workers, mineral miners, and low-skilled game play workers have to be invisible. The power of these discourses not only determines varied value of data but also set new hierarchy for digital labour.

Technology in general and ICT in particular often trigger shifts in the scopes, forms, and content of work, which are almost always deployed favourably to the accumulation of capital and to disciplining workers. Technology-facilitated division of labour should be understood in the framework of social constructed differences regarding labour types and labouring bodies. Therefore, to dismantle ideological myths surrounding digital labour, scholars in particular need scrutinize the role played by cultural discourses and to be attentive to what kind of labour are invisible from the discourses and what kind of activities are constructed as highly desirable. As Fuchs (2014) forcefully argues, informational capitalism takes complex and multiple dimensions which exploits a variety of types of digital labour such as enslaved mineral miners' labour, industrial assemblage workers, as well as free social media users' labour. Indeed, it is time to deconstruct how digital capitalism works and to broaden the meaning and scope of what constitutes digital labour. More importantly, as this article argues, it is time to take into consideration the role of culture in selectively making parts of production process invisible. As we pay more attention to how culture interplays with production processes, conversations on digital labour can be broadened to talk about cultural differences constructed around different labouring bodies.

\section{Reference}

Andrejevic, Mark. 2012. Exploitation in the Data Mine. In Internet and Surveillance: The Challenges of Web 2.0 and Social Media, edited by Christian Fuchs, Kees Boersma, Anders Albrechtslund, and Marisol Sandoval, 71-88. New York, NY: Routledge.

Aneesh, A. 2006. Virtual Migration: The Programming of Globalization. Durham, N.C: Duke University Press Books.

Arvidsson, Adam, and Elanor Colleoni. 2012. Value in Informational Capitalism and on the Internet. The Information Society 28 (3): 135-50. doi:10.1080/01972243.2012.669449.

Barbrook, Richard. 2007. Imaginary Futures: From Thinking Machines to the Global Village. London: Pluto Press.

Bauwens, Michel. 2011. The Social Web and Its Social Contracts: Some Notes on Social Antagonism in Netarchical Capitalism. Re-Public: Re-Imagining Democracy. January 24. http://www.republic.gr/en/?p=261.

Beniger, James. 1986. The Control Revolution: Technological and Economic Origins of the Information Society. Boston, MA: Harvard University Press.

Benjamin, Walter. 1936. The Work of Art in the Age of Mechanical Reproduction. Marxists Internet Archive. http://www.marxists.org/reference/subject/philosophy/works/ge/benjamin.htm.

Benkler, Yochai. 2007. The Wealth of Networks: How Social Production Transforms Markets and Freedom. New Haven, CT: Yale University Press.

Boellstorff, Tom. 2010. Coming of Age in Second Life: An Anthropologist Explores the Virtually Human. Princeton, N.J: Princeton University Press.

Bohn, Roger E., and James E. Short. 2009. "How Much Information? 2009 Report on American Consumers". San Diego, CA.: Global Information Industry, University of California, San Diego Center. http://hmi.ucsd.edu/pdf/HMI_2009_ConsumerReport_Dec9_2009.pdf.

boyd, danah, and Kate Crawford. 2012. Critical Questions for Big Data. Information, Communication \& Society 15 (5): 662-79. doi:10.1080/1369118X.2012.678878.

Bruns, Axel. 2008. Blogs, Wikipedia, Second Life, and Beyond. New York: Peter Lang.

Carselle, Juan Luis. 2013. Security and Online Retailing. Interview by Paul Taylor. http://video.ft.com/2660685618001/Security-and-online-retailing/Companies.

Casaretto, John. 2012. Romney's Project Orca-A Big Data Fail. SiliconAngle. November 12. http://siliconangle.com/blog/2012/11/12/romneys-project-orca-a-big-data-fail/.

Castells, Manuel. 2000. The Rise of the Network Society (The Information Age: Economy, Society and Culture, Volume 1). 2nd ed. Cambridge, MA: Wiley-Blackwell.

Castronova, Edward. 2006. Synthetic Worlds: The Business and Culture of Online Games. Chicago: University of Chicago Press. 
Chahal, Gurbaksh. 2013. Election 2016: Marriage of Big Data, Social Data Will Determine the Next President. Innovation Insights. Accessed November 5. http://www.wired.com/insights/2013/05/election-2016-marriage-of-big-data-social-data-willdetermine-the-next-president/.

Chen, Yujie. 2013. Speculations on Bodies and Embodied Spatial Politics in the Transnational Virtual Labor Mobility: The Case of Chinese Gold Farmers. PowerLines 1 (1). http://amst.umd.edu/powerlines/yujie-chen-speculations-on-bodies/.

Chinoy, Ira. 2010. Battle of the Brains: Election-Night Forecasting at the Dawn of the Computer Age. Ph.D., United States -- Maryland: University of Maryland, College Park. http://search.proquest.com/pqdtft/docview/734386073/abstract/13AA80D09326C7346EF/1?accoun tid=14696.

Corneliussen, Hilde G., and Jill Walker Rettberg, eds. 2011. Digital Culture, Play, and Identity: A World of Warcraft $\circledast$ Reader. Boston, MA: The MIT Press.

De Angelis, M. 2001. "Marx and Primitive Accumulation: The Continuous Character of Capital's' Enclosures'." The Commoner 2: 1-22.

Dyer-Witheford, Nick. 2004. Autonomist Marxism and the Information Society. Multitudes. http://multitudes.samizdat.net/Autonomist-Marxism-and-the.html.

Fortunati, Leopoldina. 2007. Immaterial Labor and Its Machinization. Ephemera: Theory \& Politics in Organization 7 (1): 139-57.

Fuchs, Christian. 2010. Labor in Informational Capitalism on the Internet. The Information Society 26: 179-96.

— 2011. Foundations of Critical Media and Information Studies. 1st ed. New York, NY: Routledge.

2014. Theorising and Analysing Digital Labour: From Global Value Chains to Modes of Production. The Political Economy of Communication 1 (2). http://www.polecom.org/index.php/polecom/article/view/19.

Gallant, Linda M., and Gloria M. Boone. 2011. Communicative Informatics: An Active and Creative Audience Framework of Social Media. tripleC_Cognition, Communication, Co-Operation 9 (2). http://www.triple-c.at/index.php/tripleC/article/view/253.

Galloway, Alex. 2001. Protocol, Or, How Control Exists after Decentralization. Rethinking Marxism 13 (3): 81-88. doi:10.1080/089356901101241758.

Graham, Mark. 2011. Cloud Collaboration: Peer-Production and the Engineering of the Internet. In Engineering Earth, edited by Stanley D. Brunn, 67-83. Springer Netherlands. http://link.springer.com/chapter/10.1007/978-90-481-9920-4_5.

Gregg, Melissa. 2011. Work's Intimacy. 1st ed. London: Polity.

Grossberg, Lawrence. 2010. Cultural Studies in the Future Tense. Durham, NC: Duke University Press.

Grosz, Elizabeth. 1994. Volatile Bodies: Toward a Corporeal Feminism. Indianapolis: Indiana University Press.

Hardt, Michael, and Antonio Negri. 2001. Empire. Reprint. Boston, MA: Harvard University Press.

Harvey, David. 2007. A Brief History of Neoliberalism. 1st, First Edition. Oxford University Press, USA.

Hayles, N. Katherine. 1999a. How We Became Posthuman: Virtual Bodies in Cybernetics, Literature, and Informatics. 1st ed. Chicago: University Of Chicago Press.

- 1999b. How We Became Posthuman: Virtual Bodies in Cybernetics, Literature, and Informatics. 1st ed. Chicago: University Of Chicago Press.

. 2004. Print Is Flat, Code Is Deep: The Importance of Media-Specific Analysis. Poetics Today 25 (1): 67-90. doi:10.1215/03335372-25-1-67.

Horsey, David. 2012. Obama's Data Geeks Have Made Karl Rove and Dick Morris Obsolete. Los Angeles Times, November 14. http://articles.latimes.com/2012/nov/14/nation/la-na-tt-data-geeks20121113.

Hurwitz, Judith. 2012. The Making of a (Big Data) President. BusinessWeek: Companies and Industries, November 14. http://www.businessweek.com/articles/2012-11-14/the-making-of-a-big-datapresident.

Huws, Ursula. 2003. The Making of a Cybertariat: Virtual Work in a Real World. New York, NY: |Monthly Review Press.

IDC. 2011. IDC Press Release. IDC. December 1. http://www.idc.com/getdoc.jsp?containerld=prUS23177411.

Lash, Scott M. 2002. Critique of Information. 1st ed. London: Sage Publications Ltd. 
Lazzarato, Maurizio. 1999. Immaterial Labor. http://www.generationonline.org/c/fcimmateriallabour3.htm.

. 2011. Immaterial Labor. January 24. http://www.generationonline.org/c/fcimmateriallabour3.htm.

Lehdonvirta, Vili., and Mirko Ernkvist. 2011. "Converting the Virtual Economy into Development Potential: Knowledge Map of the Virtual Economy." infoDev/ World Bank.

http://www.infodev.org/en/Publication.1076.html.

Lessig, Lawrence. 2006. Code: And Other Laws of Cyberspace, Version 2.0. Basic Books.

Lovelock, Christopher H., and Robert Young. 2011. Look to Consumers to Increase Productivity. Harvard Business Review, December. http://hbr.org/1979/05/look-to-consumers-to-increaseproductivity/ar/1.

Lupton, Deborah. 2000. The Embodied Computer/User. In The Cybercultures Reader, edited by David Bell and Barbara M. Kennedy, 477-88. New York: Routledge.

Marazzi, Christian. 2008. Capital and Language: From the New Economy to the War Economy (Semiotex. New York: Semiotext(e).

Mark Zuckerberg. 2010. Facebook CEO Mark Zuckerberg: TechCrunch Interview At The Crunchies. Interview by Michael Arrington.

http://www.youtube.com/watch?v=LoWKGBloMsU\&feature=youtube_gdata_player.

Marx, Karl. 1861. Economic Works of Karl Marx 1861-1864: The Process of Production of Capital, Draft Chapter 6 of Capital, Results of the Direct Production Process. Karl Marx Internet Archive. 1863. http://www.marxists.org/archive/marx/works/1864/economic/ch02b.htm.

— _ 1995a. Capital Vol. I Chapter One: Commodities. Marx \& Engels Internet Archive. http://www.marxists.org/archive/marx/works/1867-c1/ch01.htm.

_ 1995b. Capital Vol. I Chapter Fifteen: Machinery and Modern Industry. Internet Archive. Marx \& Engels Internet Archive. http://www.marxists.org/archive/marx/works/1867-c1/ch15.htm.

McKinsey Global Institute. 2011. Big Data: The next Frontier for Innovation, Competition, and Productivity. New York, NY: McKinsey Global Institute.

http://www.mckinsey.com/insights/mgi/research/technology_and_innovation/big_data_the_next_fro ntier_for_innovation.

McRobbie, Angela. 2011. Reflections on Feminism, Immaterial Labour and the Post-Fordist Regime. New Formations 70: 60-76.

Mezzadra, Sandro, and Brett Neilson. 2011. Border as Method, Or, the Multiplication of Labor. January 24. http://eipcp.net/transversal/0608/mezzadraneilson/en.

Mitchell, Timothy. 1998. FIXING THE ECONOMY. Cultural Studies Cultural Studies 12 (1): 82-101.

Nakamura, Lisa. 2009. Don't Hate the Player, Hate the Game: The Racialization of Labor in World of Warcraft. Critical Studies in Media Communication 26 (2): 128-44.

2012. Don't Hate the Player, Hate the Game: The Racialization of Labor in World of Warcraft. In Digital Labor: The Internet as Playground and Factory, edited by Trebor Scholz, 187-204. New York, NY: Routledge.

Nardi, Bonnie. 2010. My Life as a Night Elf Priest: An Anthropological Account of World of Warcraft. Ann Arbor, MI: University of Michigan Press.

Olsthoom, Peter. 2013. It's Complicated: The Power of Facebook. Amsterdam, The Netherlands: Ehio Media.

Ong, Aihwa. 1999. Flexible Citizenship: The Cultural Logics of Transnationality. Duke University Press Books.

Oxford English Dictionary. 2013. Value, N. Oxford English Dictionary. http://www.oed.com/view/Entry/221253?rskey=TT9w5e\&result=1\&isAdvanced=false.

Pasquinelli, Matteo. 2009. Google's PageRank Algorithm: A Diagram of the Cognitive Capitalism and the Rentier of the Common Intellect. In Deep Search: The Politics of Search beyond Google, edited by Konrad Becker and Felix Stalder. Innsbruck: Studien Verlag.

Polanyi, Karl. 2001. The Great Transformation: The Political and Economic Origins of Our Time. 2nd ed. Boston, MA: Beacon Press.

Porter, Theodore M. 1995. Trust in Numbers: The Pursuit of Objectivity in Science and Public Life. Princeton, N.J.: Princeton University Press.

Poster, Mark. 1990. The Mode of Information: Poststructuralism and Social Context. 1st ed. Chicago, IL: University Of Chicago Press.

2001. What's the Matter with the Internet? 1st ed. Minneapolis, MN: Univ Of Minnesota Press. . 2004. The Information Empire. Comparative Literature Studies 41 (3): 317-34. 
Postigo, Hector. 2003. Emerging Sources of Labor on the Internet: The Case of America Online Volunteers. International Review of Social History 48 (Supplement S11): 205-23. doi:10.1017/S0020859003001329.

Prahalad, C. K., and Venkat Ramaswamy. 2004. Co-Creation Experiences: The Next Practice In.value Creation. Journal of Interactive Marketing (John Wiley \& Sons) 18 (3): 5-14.

Prodnik, Jernej. 2012. A Note on the Ongoing Processes of Commodification: From the Audience Commodity to the Social Factory. tripleC-Communication, Capitalism \& Critique 10 (2): 274-301.

Rainie, Lee, and Barry Wellman. 2012. Networked: The New Social Operating System. Cambridge, MA: The MIT Press.

Ritzer, George, and Nathan Jurgenson. 2010. Production, Consumption, Prosumption The Nature of Capitalism in the Age of the Digital 'prosumer'. Journal of Consumer Culture 10 (1): 13-36. doi:10.1177/1469540509354673.

Ross, Andrew. 2012. "In the Search of the Lost Pay Check." In Digital Labor: The Internet as Playground and Factory, edited by Trebor Scholz. New York, NY: Routledge.

Roszak, Theodore. 1987. The Cult of Information. New York, NY: Pantheon.

Sandoval, Marisol. 2013. "Foxconned Labour as the Dark Side of the Information Age: Working Conditions at Apple's Contract Manufacturers in China." tripleC: Communication, Capitalism \& Critique. Open Access Journal for a Global Sustainable Information Society 11 (2): 318-47.

Sassen, Saskia. 1999. Globalization and Its Discontents: Essays on the New Mobility of People and Money. New York: New Press, The.

2007. A Sociology of Globalization. New York: W. W. Norton \& Company.

Scherer, Michael. 2012. Inside the Secret World of the Data Crunchers Who Helped Obama Win. Time. Accessed November 9. http://swampland.time.com/2012/11/07/inside-the-secret-world-ofquants-and-data-crunchers-who-helped-obama-win/.

Scholz, Trebor, ed. 2012. Digital Labor: The Internet as Playground and Factory. New York, NY: Routledge.

Terranova, Tiziana. 2000. Free Labor: Producing Culture for the Digital Economy. Social Text 63 (18): 33-58.

- 2004. Network Culture: Politics for the Information Age. London: Pluto Press.

The Economist. 2010. Data, Data Everywhere (Special Report: Managing Information). The Economist, February 25. http://www.economist.com/node/15557443.

The World Bank. 2012. Global Economy Prospects. Washington, DC: The World Bank. http://web.worldbank.org/WBSITE/EXTERNAL/EXTDEC/EXTDECPROSPECTS/EXTGBLPROSPE CTSAPRIL/0,,menuPK:659178 pagePK:64218926 piPK:64218953 theSitePK:659149,00.html.

Thrift, Nigel. 2006. Re-Inventing Invention: New Tendencies in Capitalist Commodification. Economy and Society 35 (2): 279-306. doi:10.1080/03085140600635755.

Turkle, Sherry. 1997. Life on the Screen: Identity in the Age of the Internet. First Edition. New York, NY: Simon \& Schuster.

United States Securities and Exchange Commission. 2012. Registration Statement on Form S-1 by Facebook Inc. United States Securities and Exchange Commission. http://sec.gov/Archives/edgar/data/1326801/000119312512034517/d287954ds1.htm.

Van Dijck, J., and D. Nieborg. 2009. Wikinomics and Its Discontents: A Critical Analysis of Web 2.0 Business Manifestos. New Media \& Society 11 (5): 855-74. doi:10.1177/1461444809105356.

Virno, Paolo. 2004. A Grammar of the Multitude: For an Analysis of Contemporary Forms of Life (Semiotex. Translated by Isabella Bertoletti, James Cascaito, and Andrea Casson. First US edition. New York, NY: Semiotext(e).

Vos, Dan. 2012. Big Data Spells Death-Knell for Punditry. The Guardian. November 7. http://www.guardian.co.uk/media-network/media-network-blog/2012/nov/07/big-data-us-electionsilver.

Weeks, Kathi. 2011. The Problem with Work: Feminism, Marxism, Antiwork Politics, and Postwork Imaginaries. Durham, NC: Duke University Press Books.

Weinberger, David. 2012. Too Big to Know: Rethinking Knowledge Now That the Facts Aren't the Facts, Experts Are Everywhere, and the Smartest Person in the Room Is the Room. New York, NY: Basic Books.

Yee, Nick. 2006. The Labor of Fun: How Video Games Blur the Boundaries of Work and Play. Games and Culture 1 (1): 68-71. doi:10.1177/1555412005281819. 


\section{About the Author}

\section{Yujie Chen}

Yujie Chen (chenyj@umd.edu) is Ph.D. candidate in American Studies at the University of Maryland, College Park. Her research interest includes digital labour, big data culture, and critical digital media studies. Her dissertation explores how labour is rendered invisible in the ideologies around Big Data and argues to redefine the concept of digital labour for the age of data glut. She teaches courses on diversity and changing cultures of work and play in the digital age. 


\title{
Digital Labour in Chinese Internet Industries
}

\author{
Bingqing Xia
}

\author{
Institute of Communications Studies, University of Leeds, UK, csbx@leeds.ac.uk
}

\begin{abstract}
Digital labour has been the subject of considerable research in recent years (Van Dijck 2009, Manzerolle 2010, Dyer-Witheford 2010). But relatively little research has considered professional workers in digital media. This research addresses this gap by focusing on professional workers in the Chinese Internet industries. This paper asks: How are these digital labourers involved in the digital media production? To what extent should we criticise this involvement?
\end{abstract}

Based on detailed empirical research in China, I argue that the rapid growth of the Internet industries depends on exploiting these Internet workers, such as the workers in Chinese Internet industries-the new 'sweatshop' of the digital era. Chinese Internet workers have been subsumed in the global capitalist system as the new 'sweatshop workers'.

This paper shows that Chinese Internet workers suffer very poor working conditions, and argues that these working conditions are the result of exploitation, a concept explored via using Eric Olin Wright's schema. This paper also argues that most of the Chinese Internet workers are in the lower middleclass class position, in which they are exploited by the upper classes. Their working conditions have seriously deteriorated and they are victims of inequality and injustice-although they also are able to exercise agency and resistance. This paper therefore calls urgent attention to the working conditions of these digital labourers.

Keywords: Quality of Working Life, Exploitation, Chinese Internet Workers, Working Conditions, Digital Labour

Acknowledgement: I express sincere thanks to my PhD supervisors, Prof. David Hesmondhalgh and Dr. Helen Kennedy, for their continuous encouragement, advice and critique, which have greatly contributed to the progress of this paper.

\section{Introduction: The Existing Discussion of Digital Labour}

Digital labour has been discussed from various perspectives in recent years. For example, some theorists regard Internet users as a form of digital labour and highlight productivity and creativity of these users (Barbrook 2005, Hills 2002, Jenkins 2008, Ornebring 2008 and Wang 2008). Terranova (2004) defines active users in the "digital economy" as "free labour", who build a community without great financial rewards and in return, obtain "the pleasures of communication and exchange" (91). The people who carry out "free work" are involved in work such as "building websites, modifying software packages, reading and participating in mailing lists and building virtual spaces" (74). Terranova regards these free labourers as a new productive force of capitalist production, as well as believing that capitalism is increasingly relying on free labour with its emergence.

Barbrook (2005) highlights the contribution of Internet users to the digital economy by analysing the paradoxical relationships between the new form of digital labour and capitalist production. He divides digital economy into three parts: The public element, the commercial sector, and the gift economy. To Barbrook, the gift economy is a free economy based on Internet users' free exchange of information- "anarcho-communist" (2) participation in his term. This gift economy is an alternative to existing capitalism, as it tends to build 'anarchocommunism' via mutual collaboration with the commercial sector: Free exchanges of information between users rely on the "capitalist production of computers, software and telecommunications" (5), and capitalist production depends on the "increasing numbers of people participating within the hi-tech gift economy" (5). In other words, Barbrook believes that the 
gift economy, based on Internet users' activities, would develop via its collaboration with the commercial sector, and ultimately becomes an alternative to existing capitalism.

Terranova (2004) criticises Barbrook for his optimistic emphasis on the autonomy of the gift economy from capitalism. Nevertheless, Barbrook usefully criticises the commodification of hi-tech gifts and anticipates what people today called the "capitalism of communism", where the "communism of capital" (the elements that stem from information production that go beyond capitalism) are reversed and the economy of free gifts becomes a new capital accumulation strategy. As an alternative, Terranova (2004) emphasises the absorption of free labour into capitalist production. Both Terranova and Barbrook highlight the productivity of Internet users, via focusing on their online activities and participation, although they evaluate such activities in very different ways. Their discussions highlight dynamics between Internet users and capitalist production in the new media era. But these theorists do not conceive Internet work in the wider context of capitalist-labour production. They both fail to capture the ambivalent, complex, and dynamic relations amongst labour and capitalism, though the question of 'unpaid labour' is an important issue.

By contrast, some theorists pay attention to the question of professional labour in the digital era, by focusing on professional workers in new media industries, such as web designers and Internet workers (Kennedy 2012, Gill 2002). For example, Kennedy (2012) discusses ethics and values in web designers' working experiences via examining work practices and working conditions of web designers in the UK. She highlights that web designers, who are ethically motivated, make efforts to include web users with disabilities, in order to develop the accessible and perceived web for all people, especially people with disabilities. For instance, she argues that self-regulation in web designers' working experiences provides a different model from self-regulation in other cultural workers' experiences: Self-regulation in some cultural workers' experiences is problematic, because it results in individualisation; by contrast, self-regulation in web designers' experiences is ethically motivated, because it is driven by "a commitment to the founding ideals of the web as open, interoperable and accessible" (20).

Gill (2002) investigates poor working conditions in new media industries by highlighting certain issues, such as pervasive insecurity, low pay, and long working hours. She particularly links these poor working conditions to gender inequalities in new media work, by arguing that female workers in new media industries experience inequalities in terms of education, access to the work, autonomy, flexibility, and pay.

Both theorists' work suggests a need to focus on professional labourers in new media industries, such as examining how these digital labourers are working. Based on such "turn to labour" tendency in the research of digital labour, this paper pays particular attention to professional digital labourers in the Chinese context: Professional workers in the Chinese Internet industries.

According to Noon and Blyton $(2002,5)$, there are several criteria to classify people's work, such as the way jobs are undertaken, the main purpose of the work, job status, temporal pattern, and work location. I classify the Internet workers by the criterion of temporal pattern, such as full-time or part-time and permanent or temporary. Therefore, my PhD project classifies Chinese Internet workers into full-time workers, interns, and agency workers.

However, in this paper, Internet workers particularly refer to the full-time workers, who are paid regularly and hold formal positions in Internet companies. These workers are the main contributors to the creation of cultural products. Some of these workers possess high skill, while some have low skill, though none of them only sell manual labour as manual workers did during industrialist capitalism. Thus, some full-time workers here are understood as technical workers, conducting technical-related work, such as programming, web design and APP (Application Software) developing, etc. Other full-time workers are non-technical workers, who are mainly involved in administrative and routine work, such as HR (Human Resources) recruiting and training employees; marketing workers doing promotional work; and PR (Public Relations) personnel maintaining relationships with government officials, etc. 
By clarifying who are these Internet workers, this paper asks: How are the Chinese Internet labourers involved in the digital media production? To what extent should we criticise this involvement?

The first step to answer these two questions is to examine how Chinese Internet industries have developed in recent years, and to what extent it links to the global economic system. Therefore, in section two, I discuss how Chinese ICT industry and Internet industries have developed in recent years, and how they contribute to Chinese economy. In section three, I explain the methodology of this research. Section four presents the empirical data I collected from fieldwork, in which I discuss certain issues that answer how Internet workers are involved in the digital media production, such as working hours, pay, lay-off, and pension problems. Sections five and six are the theoretical parts that answer to what extent we need to criticise such involvement. Section seven, the conclusion, I indicate a sort of worker agency as a hope in Chinese Internet industries. Below, I discuss the development of Chinese ICT industry, Internet industries, and Chinese economy.

\section{A New Economic Growth Field: Chinese Internet Industries}

\subsection{The Chinese Economy}

David Harvey (2005) defines neoliberalism as "a theory of political economic practices that proposes that human well-being can best be advanced by liberating individual entrepreneurial freedoms and skills within an institutional framework characterized by strong private property rights, free markets, and free trade" (2). This concept had a strong impact to Chinese society in the 1980s with the introduction of market principles, which had close relationships with neoliberalism. Neoliberals appreciate the reform and opening up policy because they believe it emancipates the Chinese market and develops the Chinese economy. For example, Huang (2008) highly appreciates the move towards a more market-oriented economy in 1980s Chinese society, as it improved social welfare. Mok and Lo (2007) point out that, "the policy of decentralization and marketization being adopted to reform the social policy domain has significantly reduced the state provision and financing in social service and social provision" (2).

Admittedly, the Chinese economy has seen a rapid growth since the issuing of the reform and opening-up policy in 1978. Foreign investors have rushed into the Chinese market since China's accession to the WTO (World Trade Organisation) in 2001. However, social problems and tensions are generated from the ongoing economic reform: Inequality between the rich and poor, and injustice between the bureaucratic capitalists and workers have expanded. For example, Zhao (2003) discovers inequalities within the Chinese ICT industry by discussing different access to media between Chinese urban middle-class and rural peasants: "While the rising business and urban middle classes are increasingly using the media to articulate their interests and shape state policies toward their preferred ends, the rally cries of tens of thousands of Chinese workers and farmers in their struggles for economic and social justice, for example, have simply fallen on deaf ears in the Chinese media system" (63). Zhao states that the rising business and urban middle classes increasingly enjoy better quality of life, such as gaining more prestige, better education, and better health care; but workers and farmers, by contrast, are still struggling at the bottom of the social hierarchy. Moreover, Zhao (2007) explains the uneven regional development by quoting Hu, Zhou and Li's work (2001), which depicts China as "one country, four worlds" (102) because of the fragmentation and polarization of "class, region, gender, ethnicity and other cleavages" (101). Here, Zhao highlights inequalities between Chinese people due to the ongoing economic reform.

Some theorists explore the Chinese economy by highlighting the role played by the state. For example, Wang Hui (2003) states that neoliberalism is problematic in the context of China because it denies "the close relationship between the market and the political process" (100). He claims that the state plays a significant role in the Chinese economic system, since the political system in China is highly centralised. As Wang Jing (2008) suggests, the Chinese market is still controlled by the "party-state". Wang Hui (2003) then enriches the concept of neoliberalism with Chinese characteristics: It is "a combination of notions of market 
extremism, neo-conservatism, and neo-authoritarianism" (81). Here, neoliberalism is understood to accelerate the process of delegating economic and political power from the central government to regional governments in a stable manner, to build an authority to guarantee the process of marketization, as well as to help the retreat of the state in the process of globalisation.

As a supplement, some scholars use the concept "crony capitalism" (Andres 2010) or "crony communism" (Dickson 2011) to understand the close relations between the Chinese state and the market. Andreas (2010) claims that contemporary Chinese society is the one labelled as a "state-led urban decade" (65). Dickson (2003) unpacks the reliance of a capitalist economy on close relationships between business and the state by the concept of crony communism. He argues that crony communism in China is different from other contexts, because the political hierarchy is dominated by all levels of officials, rather than a ruling family or central leader as in other East Asian countries, such as Burma. Consequently, the ruling officials are titled 'red capitalists', as many of them are involved in the economic system: "Many of the most wealthy entrepreneurs formerly held high-level party and government posts, and some are even the offspring of China's leaders; a far larger number of private entrepreneurs are former mid-level officials, or simply rank-and-file party members who did not hold formal posts but left their previous jobs to go into business [...]" (Dickson 2003, 45 ). Meanwhile, Chinese crony communism is also distinctive because capitalists are subsumed into the group of officials: "[...] another group [...] [that refers to] those who were coopted into the party after demonstrating their entrepreneurial skills and business success' (Dickson 2003, 4-5).

Such discussion of the Chinese economy is helpful to grasp a sense of how the Chinese economy has developed in recent years, and is a useful way to understand the context in which Chinese Internet industries have developed. Below I introduce the recent development of Chinese Internet industries, and how they reflect certain characteristics and problems of Chinese economy.

\subsection{The Chinese Internet Industries}

In this study, the Internet market is divided into four parts: The hardware market (including companies producing computer hardware, such as Dell); the software market-including companies producing computer software, such as Oracle; the service market-including companies providing Internet services, such as Google; and the content market-including companies producing contents or converging contents provided by Internet users, such as Facebook. The Internet industries I focus on in this paper are part of the emerging content market, like Facebook and YouTube, as well as Chinese equivalents like Sina Weibo and Youku, which accumulate massive economic capital by providing online content.

The Chinese Internet content market has developed exponentially since the end of 2002, when the market was revived from the dot-com crash in 2001. At that time, some portals in the industries, such as Sina, Netease and Sohu began to make profits and to grow significantly. In 2003, the market was developed with the blooming of varied content services, such as search engines (Baidu), online gaming (SNDA), instant messaging (Tencent), and online commerce (Alibaba). According to a research report by the Boston Consulting Group, the Internet industry economy made up 5.5\% of China's GDP in 2010 (The Boston Consulting Group 2012). In 2012, the annual market value had reached 385.04 billion RMB ( $£ 38.5$ billion), an increase of 54.1\% from 2011 (iResearch 2013). As a report from Xinhua News indicates, Internet-related consumption of information and services would be one of the biggest drivers of China's economic growth in the next ten years (iResearch 2013). The development of the Chinese Internet industries can be acknowledged from Figure 1, which indicates the fast economic accumulation of the Internet industries in one minute. 


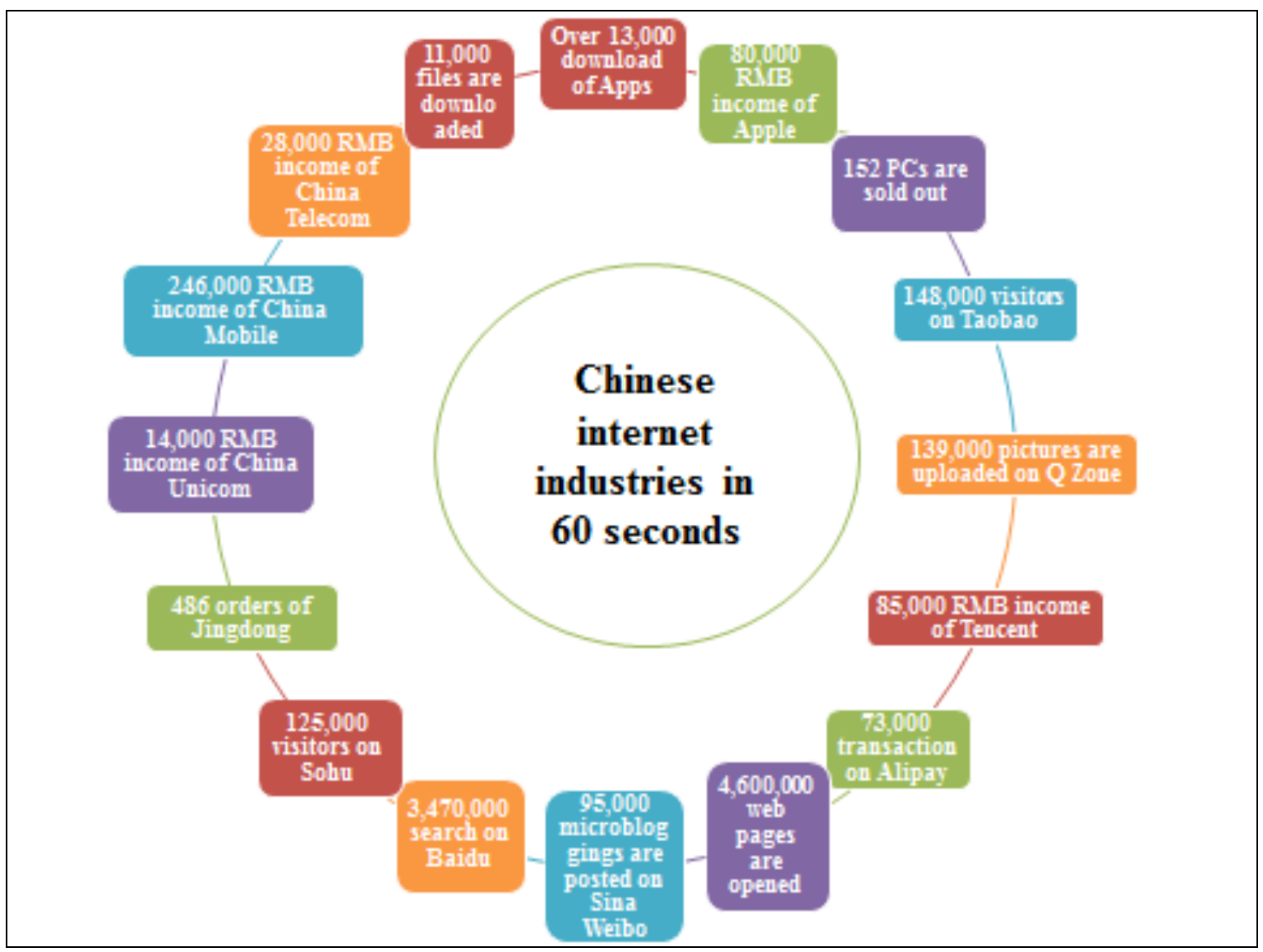

Figure 1: What happens in Chinese Internet industries in 60 seconds? (Translated from Sohu IT 2013)

This fast capital accumulation largely depends on the labour efforts of Internet workers, as huge numbers of workers are involved in the production process. The number of Chinese Internet workers had increased to 12.3 million by the end of 2009 (Liaoning Research Institute of Industry and Information Sciences 2013). Little academic attention has been paid to the class formation of these workers, such as their educational background, socioeconomic status, and social locations, nor to other dynamics and practices, such as their struggles in the industries and society. So how do Internet workers contribute to the fast growth of the Chinese Internet industries? What is their working life like in the industries? How class analysis can help understand Chinese Internet workers?

The Chinese Internet industries have been subsumed into the global value chain via not only its large number of Internet users (for example, Internet users in China had reached 590 million in the first half of 2013, which is more than the number of users in the whole of the Western Europe) (Xinhua News 2013), but also the close relationships between the Internet industries and the Chinese economy: The Chinese Internet industries have become an important economic growth field in contemporary Chinese economic system. As China has become one of the most important players in the global economic system, Chinese Internet industries also have been subsumed into the global economic system.

As I stated earlier, some theorists argue that Chinese economy develops in a different way than other social contexts, because of the close relationships between the market and the state. Likewise, Chinese Internet industries are also distinguished from ones in other geographical contexts because the state holds high authority and intervenes in the production process. For example, Ross (2005) recognises that Chinese government officials are still playing a strong role in the Chinese high-tech industries, even though the central government has not been planning the economy in detail since the 1980 s. 
Figure 2 shows a national conference, Constructing Healthy Network Cultures (gongjian gongxiang wangluo wenhua), organised by the Party, which all CEOs of the dominant Internet companies were required to attend. At this conference, the state intended to ask these Internet leaders to follow its plan for constructing the Internet industries. For example, as the first picture in Figure 2 points out, the state required Internet companies to work in conjunction with it to build a "healthy Internet space", where information security, such as the filtering of all information against the state, would be guaranteed. In this case, the Internet leaders were 're-educated' about the state's plan for constructing online freedom.

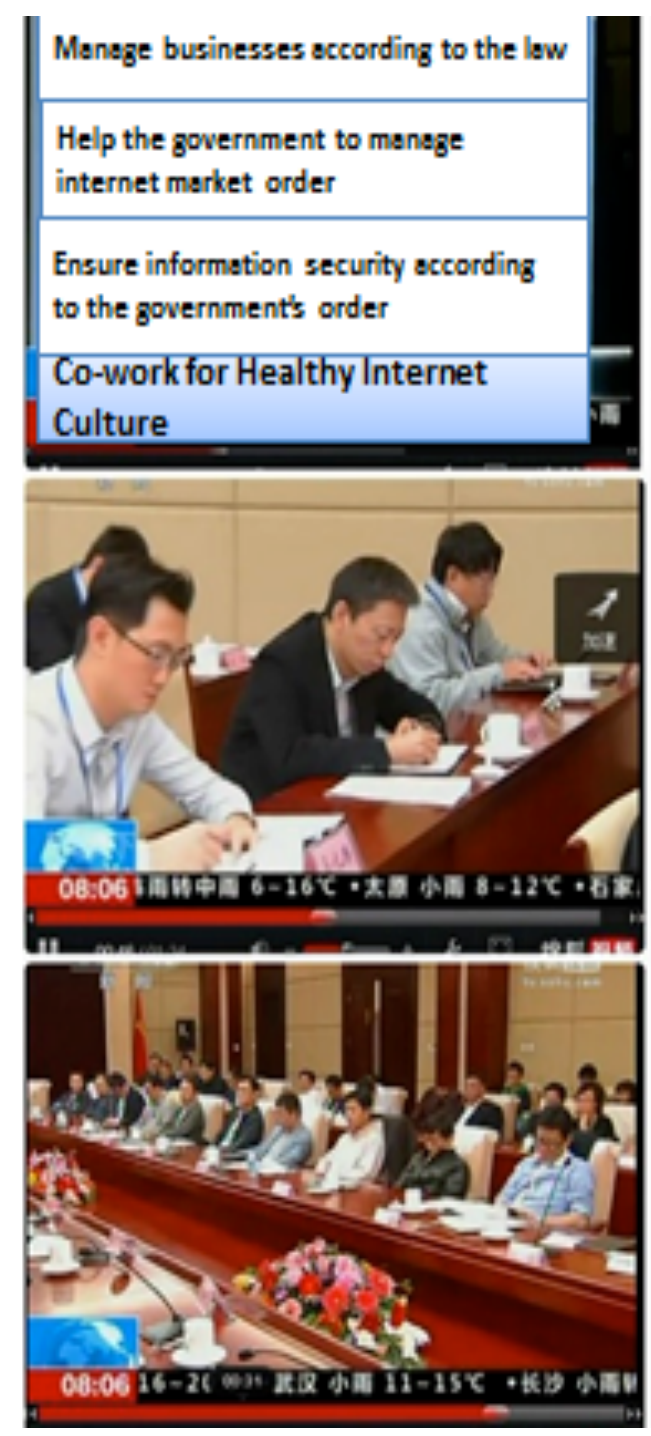

Figure 2: A conference in relation to Internet industries organised by CCP (From an interviewee's personal website on Campus)

According to iResearch, a leading company focusing on in-depth research on Chinese Internet industries, all the top 21 Internet companies in Chinese market are owned by individuals, who are widely known for pulling themselves up by their own bootstraps (iResearch 2013). These leaders were mostly not part of the bureaucratic capitalist class (I explain classes in section five) before their companies became the "large entities" in the industries. The state quickly made attempts to subsume leaders of the large entities by organising such "educational conferences", in which leaders were required to follow the state's rules and plans concerning the Internet industries, in order to realise its slogan "control the big, let go the small" (O'Conner and Gu 2012, 4). 
It would seem that the Internet leaders are not keen to study the state's plan and rules about the Internet industries, as the final picture in Figure 2 indicates-some Internet leaders, such as the leader of Alibaba, the largest online commerce company that has Taobao as its constituent company, and the leader of Netease, one of the largest portals, fell asleep in the conference. But this does not mean that they reject being subsumed into the bureaucratic capitalist class. As an alternative, most times, these leaders choose to stand with the state, because their projected benefits are the same-both seek to maximise their economic benefits from the Internet industries. These benefits are mostly based on the labour efforts of Internet workers.

However, it is not only the relevant industrial policies, through which the central government intervenes in the industries, but also certain direct intervention in Internet workers' daily practices that influence the workers' experiences in the industries. For example, according to Leo, one of my interviewees who worked in the open platform department at Campus in 2010, officials from the State Administration of Radio, Film, and Television visit Campus every month in order to have regular meetings with workers in the Security Department. In these meetings, officials inform the workers about recent sensitive issues, which are usually related to politics and pornography, and ask the workers to delete references to these issues from all Campus' online products, such as forums.

Take the 1989 Tian'anmen Square Protests as an example: According to Alex, another interviewee who also worked in the Open Platform Department at Campus in 2010, at the end of April every year, the company starts to organise a number of meetings to prepare for the coming June 4, where a lot of Internet users usually organise various online activities for the anniversary of the protests. The company makes great efforts to stop these online activities, obeying the rules set by the state. Normally, there are two ways the company filters information relating to this sensitive issue: Filtering key words using censorship technologies and filtering pictures by manual examination.

The user-generated content department and the security department take charge of filtering key words, such as "explosion" and "bomb". The state provides a list of sensitive words as a guideline, which includes millions of words relating to the Tian'anmen Square Protests, and asks the company to delete them from its website. Alex's roommate, who worked in the security department, told him that such lists included 7,300 words relating to AV (Adult Video) actresses, not to mention numerous words relating to the Tian'anmen Square Protests, which remains one of the most sensitive political issues in contemporary China. Moreover, such prohibited words on the list include not only those written in Chinese, but also words from many other languages.

The manual examination of sensitive pictures requires efforts from a lot of workers. According to Alex, almost all workers, including full-time workers and interns, and even the boss of Campus, work day and night around $4^{\text {th }}$ June, in order to filter pictures posted by Internet users and delete the sensitive ones. Normally, online pictures are examined after users have posted them, but, during this special period, these pictures need to be examined before being shown on the website. Thus, more workers are required to have excessive working hours in order to filter all the pictures. The workers, who usually work in relays, are required to work together at the same time around $4^{\text {th }}$ June, in order to deal with the mountain of work. In other words, the workers are forced to have excessive working hours because of the state's requirements concerning sensitive issues.

Sometimes, the state directly stops workers' programs or products, because they may still include certain sensitive issues after the workers have filtered the information. For example, Leo said that the popular online game, Godfather, created by the department in 2010 and ranked as one of the top 10 most popular online games by users, was stopped by the Ministry of Culture, because it was perceived to contain sinister gang and gambling-related content. In this case, the workers' creativity was directly intervened with by the state, when they stopped the product because of sensitive issues.

Put simply, the state assigns the responsibility of filtering sensitive issues to Internet workers, by requiring them to delete sensitive words and pictures in relation to certain issues without giving standards. This increases workers' work intensity. When the state is unsatis- 
fied with workers' practices or programs, it easily stops them. Such rough intervention from the state not only influences workers' practices and creativity, but also increases their work intensity, as the Tian'anmen Square Protests case indicated. Therefore, as an important part of Chinese economy, Chinese Internet industries reflect certain characteristics of the economic model, such as the close relationships between capitalists and the state; the industries also show some problems of the ongoing Chinese economic reform, such as the bad working conditions (excessive working hours without equal pay, which I discuss later) caused by the close relationships between the state and the market. Before I illustrate the working conditions, I explain the methodology I adopted in this research.

\section{Methodology}

I carried out empirical, at times ethnographic, research in two Chinese Internet companies, in order to study the workers in this paper. The first will be called Grand; this company focuses on online entertainment, such as online gaming and online fiction. The second one will be called Campus; this provides social networking services. I used observation and in-depth interviews as my primary methodology.

I conducted in-depth interviews in three periods in Campus: Seven interviews in February 2010; nine interviews in August 2011; and five interviews in December 2011. I also spent three months in Grand conducting participant observation, where I worked as an intern to observe and keep a journal about workers' daily practices. I also invited one worker at Campus, who will be called Galeno, who participated in the interviews in two periods in 2011, to conduct self-observation, by keeping a journal about his working life during the period of August to December 2011. And finally, due to his own habit of keeping work journals, he gave me his work journals from September 2009 to December 2011. I had hoped that more workers would agree to engage with this process, but in the event, they did not. Through these mixed, qualitative methods I explored how Internet workers get involved in the digital production of the Chinese Internet industries, which I discuss in the next section, and in the final section, I outline how I recognised a sort of worker agency as a hope in the industries.

My participant observation in Grand was covert for a number of reasons. Firstly, because Chinese companies tend to reject requests for access to do academic research, unless the research could bring them commercial benefits. Such rejection would certainly have been the case for my research into workers' practices. Secondly, this paper develops from my PhD project, which not only focuses on the quality of working life, but also emphasises acts of worker agency. By using a covert method, I felt that I would be able to witness more "genuine" acts of worker agency, which was an important part of my PhD project.

However, covert research necessarily brings with it ethical concerns. I felt that I was deceiving "participants" as I simultaneously built personal friendships and gathered their stories. Participants told me their personal stories because they saw me as a friend; friendship therefore helped me to gather data. This then presents me with a dilemma regarding sharing the stories that participants confided in me. This dilemma and feelings of deception remain, yet I choose to write about the research in this public domain, because I feel that it contributes to understanding Chinese society and the roles played by Internet workers in that society. Indeed, this is why I chose to pursue the research through what might be seen as an ethically problematic means. I hope that if my participants read this paper and recognize themselves in it, they understand my motivations for carrying out covert research and the benefits it may bring, and that they do not feel deceived by me.

\section{Joy and Tears in the Internet Sweatshop}

\subsection{Working Hours and Wages}

Most of my interviewees, technical workers in both companies, state that overtime work is a common phenomenon in the Internet industries. For example, Tim, a new technical worker who joined Grand at the same time as me, stated that he had worked overnight for several 
days since he joined the department half a month ago. The following are some quotations from other workers who also experienced this issue.

In Campus, the standard working time is $10 \mathrm{am}$ to $9 \mathrm{pm}$ [...] This [long working hours] is quite common in the industries nowadays, where 10 hours are the average working hours [...] I have a friend working in another Internet company, where he usually finishes his work at $11 \mathrm{pm}$ or $12 \mathrm{am} .$. . As far as I know, workers in the $3 \mathrm{G}$ department now are still staying in a hotel to work day and night for a new program [...] (Louis, former technical worker in the Open Platform Department at Campus, $28^{\text {th }}$ August 2011, interview).

Non-technical workers, who deal with administrative-related work, in both companies, also share this overtime experience. For example, Katy, a new HR worker, who joined Grand at the same time as me, worked overtime for nine days, which usually meant she finished work at $11 \mathrm{pm}$ or $12 \mathrm{pm}$, during the first ten days she joined the company. She said that sometimes she even considered sleeping over in the office, because her home was far away from the company. If she was "lucky" to finish work at 11:30pm, she could claim reimbursement for the taxi fee, would arrive home after midnight, and go to sleep by $1 \mathrm{am}$. But she would need to get up at $5 \mathrm{am}$, as she needed to change three times on the underground to arrive at the office. Thus, the overtime seriously reduced her sleeping time and made her very tired.

There are several reasons for Internet workers to choose overtime work, such as rewards they receive from the work-to satisfy themselves when having certain achievements in the work. But one of the important issues that forces Internet workers to work overtime is the high competition given by managers.

Ross (2005) points out that workers in the Chinese outsourcing high-tech industries work overtime because of high competition in the industries: They need to work harder to help avoid the risk of other workers taking their places. Some of my participants show the anxiety caused by high levels of competition.

My leader always reminds me to keep myself in a high competitive status. For example, to think about who will be fired if the company needs to lay off staff, if I were the person, it means I need to work more. (Galeno, technical worker in the Product Administration Department at Campus, $24^{\text {th }}$ August 2011, interview).

Managers and companies play a key role in pushing these workers to work overtime, because they seek high profits with low labour costs. For example, Galeno shows how his manager keeps him in a sense of crisis: He may be laid off if he does not work hard. It is also common to promote "geek culture" in all Internet companies, which encourages workers, especially technical workers, to show their love of the Internet work via working day and night.

Such long working hours causes a striking issue in terms of Internet workers' well-being in recent years: Karoshi, a Japanese term meaning death from overtime work. There were some cases in the Internet industries during the five months that I spent there, where some workers died from exhaustion, which was usually in relation to overtime and high pressure. For example, one of these examples was in Tencent, one of the main portals in China with the famous instant messenger system $Q Q$, where an online editor died because of overtime work. The news was circulated on microblog sites because the editor talked about his overtime situation on his microblog (Sina News 2010). Some of his posts showed that he even worked until 8am in the morning.

Another karoshi case was in Baidu, the main search engine company in mainland China, where a member of staff in the online game department died from overwork on $14^{\text {th }}$ November 2011 (Tencent Technology 2011). According to this news, the average age of death from overtime in the Internet industries is just under 38 (Tencent Technology 2011). Sam, the senior manager in HR department in Grand, says that it is not unusual to find cases of karoshi in the Internet industries. According to him, there were some cases of karoshi in Grand before I joined, but the PR department covered them up. 
All these stories show the serious overtime situation in the industries. The most significant issue here is whether the overtime is reasonably rewarded. According to my participants, indeed, little of this overtime work is rewarded. In Campus and Grand, full-time workers' overtime work during weekdays is not paid. Instead, workers working late during weekdays can benefit from a free dinner or the money to buy a dinner, which is usually offered with certain restrictions. For example, in Campus, workers can only benefit from a free dinner after working for 12 hours. In Grand, workers only receive $£ 1.80$ for a dinner when they work after $8: 30 \mathrm{pm}$, and can be reimbursed for taxi fees after 11:30pm. Working during weekends is paid at double time, but in both companies, weekend overtime work needs to be approved by department leaders, who usually encourage workers to finish their work during weekdays. In other words, full-time workers are forced to work overtime without reasonable rewards. Put the unrewarded overtime work aside, it is important to ask how much Internet workers earn for their hard work. In both companies, technical workers are paid £13,000 per year, and non-technical workers receive $£ 4,500$ per year, compared to SOE (State-Owned Enterprise) workers' yearly salary of over $£ 10,000$. SOE workers also receive various bonuses from the state and some of their living costs are covered by the state. For example, according to my interviewees, workers in a state-owned flight company only need to pay $£ 0.1$ per day for their meals, which are good quality (most food is organic). In comparison, Internet workers, as workers in private enterprises, live only with this fixed salary, without extra bonuses and cheap meals.

My salary is $£ 13,000$ per year before tax [ $£ 1,000$ per month $\times 13$ months] with the endless overtime, while my friend who works in a SOE, earns around $£ 20,000$ per year after tax [ $£ 800$ per month $\times 16$ months, and a bonus of $£ 200$ per quarter $\times 4$ quarters] without any overtime (William, technical worker in the $3 G$ Department at Campus, $19^{\text {th }}$ December 2011, interview).

According to William, SOE workers are paid much higher than Internet workers, even technical Internet workers with high skills. The difference might be assumed to relate to their different work intensity, but the reality is that Internet workers are paid less, and have much higher work intensity. In contrast, SOE workers receive higher pay without working any overtime; neither do they have to devote themselves to their work during work time, as Internet workers do. In other words, Internet workers are paid unequally, compared to SOE workers.

This is what Wright (2010) points out in his research in Chinese society: SOE workers receive benefits from the party-state that "have been unavailable to other poor individuals" (3). Such inequality between SOE workers and Internet workers can also be found in their different benefits: SOE workers and executives in Internet companies enjoy specially delivered organic food as a benefit, whereas most Internet workers do not.

There is another issue relating to Internet workers' benefits: Sharing companies' stocks. Internet work has been fetishised in recent years because the workers are likely to receive companies' stocks as their bonus. For example, Xinhua News reported that the listing of Baidu-the dominant search engine company in China-in the USA NASDAQ stock market in 2005 enabled 400 workers in the company to become millionaires because they were given some stocks before the listing (Xinhua News 2005). It sounds as if every Internet worker is able to become a millionaire once he or she receives stocks. However, large numbers of Internet workers are struggling in difficult working conditions with the dream of being the next hero, but only a small number of individuals achieve the dream.

I had some stocks before I left [Campus]. They were just 3,000 shares. It was $£ 0.4$ per share when I received them, which was evaluated as £1 per share when I left [Campus]. It seemed that I could receive $£ 3,000$ when I left. But, indeed, the company had a rule, which meant we could only sell a quarter of our shares every year. So this year I can only sell a quarter of my shares, which is $£ 750$. But because income from stock sharing is windfall in the income tax law, I need to pay $47 \%$ of this amount of money as tax, which means I only receive $£ 397.50$ after tax. This is even less than my monthly salary [...] 
(Louis, former technical worker in the Open Platform Department at Campus, $28^{\text {th }}$ August 2011, interview)

According to Louis, only some experienced workers who had joined Campus when it was founded could receive certain stocks. These stocks are not worth as much as workers in other industries imagine them to be and few of the more recently employed workers can receive stocks.

\begin{abstract}
Most of us were so disappointed when the company went public. All the managers celebrated it, but it's none of our business. We do not get any benefit from it [...] Even to the workers who received stocks, such sharing does not guarantee them anything. It only becomes a way for the company to stop [workers] job-hopping: once you want to leave the company, HR would suggest you stay for one more year, in order to sell another a quarter of your shares. This does not only happen in our company, but also happens in other Internet companies. It [stock sharing] becomes a way for companies to bargain with us [workers] [...] (Galeno, technical worker in the Product Administration Department at Campus, $20^{\text {th }}$ December 2011, interview)
\end{abstract}

Here, it is evident that stock sharing does not authorise these workers any managerial power; instead, it becomes a way for the company to control workers. With such unequal pay, some Internet workers experience difficulty of housing in cities where they work. For example, most Internet workers are struggling to buy a house in the big cities with their low salary.

I never think about settling down here, in Beijing, because it's too expensive to be here, especially buying a house [...] Now I rent a house with my colleague, each of us pays $£ 200$ per month for a small and old room. It is still not cheap for us [...] (Wynn, technical worker in the Open Platform Department at Campus, $24^{\text {th }}$ August 2011, interview)

In these big cities, it seems that only SOE workers, civil servants, government officials and the rich can afford to buy a house. As Shelly, an intern in Grand, told me, civil servants in Shanghai are usually offered discounted houses by their work units (danwei), for which they only need to pay less than half of the market price, because these houses are built by their work units with free land provided by the local government. Here, the work unit again plays an important role in workers' benefits, as it did in Mao's era. But the difference is that the work unit in contemporary Chinese society only protects and guarantees certain classes benefits, such as the higher middle class-civil servants and SOE workers.

Admittedly, there are some jobs that pay more than others, which cannot be criticised as a result or form of exploitation. But, in this study, Internet workers receive low pay, in spite of their long working hours and high work intensity, because they are controlled by the higher classes, such as the capitalists in this study-Internet companies, via work contracts (Internet workers cannot escape these experiences of unrewarded overtime work, because it is so common in the Internet industries). This shows that Internet workers' labour efforts and skills are appropriated in the capitalist production process via long working hours without equal pay.

As I pointed out earlier, some technical workers receive certain stocks as part of their bonus, but such stock sharing does not guarantee them good pay, nor does it authorise the workers any managerial power. With such limited benefits, Internet workers still need to sell their skills to survive. In other words, Internet workers are forced to sell their labour efforts and skills in the Chinese capitalist market, which has been subsumed in the global value chain as the second largest economic system, with an unreasonable price. I argue that such unjust experience is caused by a sort of exploitation between classes: The bureaucratic capitalist class-the state and Internet companies in this study-dominates the lower-middle class and appropriates the labour of this class, because the former own means of production and the political power to allocate these resources. I explain the way I discuss classes in section five. This exploitation between classes results in an injustice in relation to Internet workers' pay and work time: Internet workers are not rewarded for their high work intensity, 
and in contrast, government officials and capitalists appropriate this part of labour efforts. Therefore, the structural concept, exploitation, is the force behind Internet workers' unequal and unjust experiences in terms of work intensity and pay. I clarify the way I use this concept in the theoretical part-sections five and six.

\subsection{Job-Hopping or Lay-Off?}

Some theorists argue that job-hopping becomes a common phenomenon in Chinese hightech industries (the hardware market). For example, Ross (2005) points out that the average turnover rate in the Chinese high-tech industries is about $20 \%$, which is quite high in his understanding. This high turnover rate was reflected in my fieldwork: Six out of the seven interviewees, whom I contacted in 2010, had left Campus by the time I contacted them again in 2011.

As some of my interviewees say, two years is quite a long period to stay in the same company in the Internet industries.

Most workers in my department, almost 20 people, left in just one and half years [...] Every time we gathered for team building, I saw new faces [...] (Galeno, technical worker in the Product Administration Department at Campus, $24^{\text {th }}$ August 2011, interview)

This obviously indicates a high turnover rate in the Internet industries. Some theorists point out certain problems caused by this high turnover rate. For example, Ross (2007) points out that such frequent turnover, results in high costs for training, because workers usually leave after benefiting from training, and then new workers have to be trained to replace them. Ross (2007) also claims that job-hopping becomes a bargaining tool for experienced workers to negotiate with companies for good pay.

But, indeed, such bargaining experience only applies to certain experienced technical workers, who have the high skills to bargain with companies. In contrast, most workers change their jobs because they struggle to earn good money and want an easier working life.

Now everything's getting expensive, I need to find another position with better pay, so that I can survive in this city [...] But autonomy in work is the more important reason for me to job-hop [...] (Walter, technical worker in the Advertisement Department at Campus, $25^{\text {th }}$ August 2011, interview)

Here, Walter points out that good pay is an important reason for him to change jobs, though autonomy is another important reason for him to consider changing jobs. As in most countries, working and living in big cities is not easy. Some workers, most of whom are in middle class locations, choose the new lifestyle of a "weekly couple" (zhoumo fuqi) or "monthly couple" (yuemo fuqi), which refers to the family gathering that takes place once a week or once a month, because of the high living expenses in big cities. For example, Sam is a senior manager in Grand's Shanghai office, and his family are based in Najing, another city two hours away from Shanghai by train. The high cost of living in Shanghai stops his family from moving there with him, and the terrible traffic jams, means it takes Sam more than four hours to go back home, and stops him from gathering with his family every day. Then, the only choice for Sam is to be a "weekly couple" with his wife by going back home every weekend. This new lifestyle obviously influences workers' life quality, as it separates workers from their families. This goes against the traditional Chinese family life, which is based on living in groups. It is the high cost of living in big cities and their unequal pay that push workers to choose this modern lifestyle and decrease their quality of life.

Put simply, some experienced technical workers do benefit from the frequent job-hopping in the industries, because they have high skills to bargain with companies for good pay. In contrast, many workers choose to change their jobs because they suffer from bad pay and high living costs in big cities. These workers pursue a good job, with good pay and a convenient lifestyle, by frequently job-hopping. Moreover, there is another side to the coin: in some cases, companies lay off workers. 
I had a colleague, who had been at Campus for four years, but he still received the same salary as me, and I had just joined the company... It was one way the company forced us to leave [...] Finally, he left as most new employees were paid higher than him... If I were my colleague, I would also choose to resign, as salary is a way to evaluate a person's talent and to show respect to that person [...] It doesn't matter how much you are paid, but it matters how much more you are paid than others, especially people whom you think are less talented than you [...] (Galeno, technical worker in the Product Administration Department at Campus, $24^{\text {th }}$ August 2011, interview)

In this case, workers are forced to leave by the company in an indirect way, because the company needs "fresh blood" to bring creative ideas. Workers, indeed, are laid off in an indirect way.

Three months after I finished my internship in Grand, I contacted my friends there, and was surprised to find that more than half of the full-time workers in the HR department had been laid off, because the company wanted to cut its labour costs. According to the work contracts, these workers were informed of their redundancy one month before they left and were paid one more month's salary as compensation. These workers did not have the time to find new jobs in one month, but they had to accept the situation. In the Chinese context, work unions do not work well. The Chinese unions usually stand with companies, by persuading workers to accept unfair working conditions, rather than protecting workers.

In both examples, workers were laid off in an indirect or direct way, without any protection from the unions. The lay-off issue addresses an urgent problem in the Internet industries: Internet workers lack protection from the work unions, such as job security. Admittedly, some workers benefit from frequent job-hopping. But, more workers change their jobs because of the bad working conditions they experience. And some workers are even forced to leave without protection, because companies want to save labour costs or employ "fresh blood".

\subsection{Life After Retirement}

As I have discussed in the last two sections, Internet workers experience serious overtime work, unreasonable rewards for their hard work and unstable working status. But this is not the complete picture of their working life. They also experience insecurity in their working life, such as the insecurity of life after retirement.

Similar to some countries, in contemporary Chinese society, certain types of retired workers are protected by the pension system. For example, Internet workers are required to pay $8 \%$ of their salaries into a pension fund, to which the companies give an amount equal to $20 \%$ of workers' salaries. The workers will receive a certain amount of this money every year after retirement. Compared to the pension system in which Internet workers-employees in private enterprises-participate, SOE workers and civil servants benefit from another pension system, in which the work units pay into the pension fund, but they will be given more money than private enterprises' employees after retirement.

After the pension reform in China, workers now need to work longer and pay more into their pension funds than they have done before. Workers deposit part of their salaries in a pension fund, in order to guarantee their quality of life after retirement. However, in China, this amount of money is shrinking, due to the failure of investment in pension funds in recent years. It is reported that the growth rate of pension funds is much slower than the growth rate of the CPI (Consumer Price Index) (Chinese Economics 2013). In other words, the pension that workers will receive after retirement will no longer cover their living costs.

Such inequality surrounding pension systems points to injustice between Internet workers and SOE workers: Internet workers experience difficult working conditions, such as long working hours without reasonable rewards, while needing to pay into their pension fund. In contrast, SOE workers have good working conditions and benefit from a state-secured pension. In this sense, the higher middle class, such as SOE workers, seems to have better working conditions than the lower-middle class, such as the Internet workers, because of the 
support they receive from the state and companies-the bureaucratic capitalist class, which I clarify later.

In the contemporary Chinese context, there is another issue links such inequality to exploitation: pension corruption. Workers are worrying about their lives after retirement, even though they pay into a pension fund every month, because government officials might embezzle their pension before their retirement. For example, there were several scandals regarding pension corruption from 1993 to 2010: around £8.9 million of pension money was embezzled in Guangzhou in 1993 (Youth Times 2012); £0.86 million pension money was embezzled in Taiyuan in 2003 (Youth Times 2012); and $£ 32$ million pension money was embezzled in Shanghai in 2006 (Youth Times 2012).

These news reports make workers feel unsafe and insecure about their work, especially about life after retirement. As Monica says:

I'm not sure whether I could get back my pension after retirement, because it is possible that some officials have already embezzled it before I retire (Monica, HR worker at Grand, observation journal).

This pension corruption indicates that government officials appropriate workers' labour efforts, shown as the money they pay into their pension fund. To take a more structural perspective, this is resulted by an appropriation of labour of a low class by a high class: The low class's labour is "robbed" by the high class via acts of corruption, due to the strong political power to allocate social resources and control the lower classes owned by the bureaucratic capitalist class. This makes "the poor becomes poorer, while the rich becomes richer". In other words, the bureaucratic capitalist class exploitation of labour of the lower-middle class results in insecure experiences in Internet workers' working life.

Some of my interviewees even state that they could not imagine their lives after the age of 40 in the Internet industries:

We [Internet workers] sometimes made jokes that we might die before [our] 40s [...] To be honest, I cannot imagine my life after [my] 40s. Maybe I will leave the industries [...] (Galeno, technical worker in the Product Administration Department at Campus, $24^{\text {th }} \mathrm{Au}-$ gust 2011, interview)

Galeno's recognition of high risks in the Chinese Internet industries echoes Gill's (2002) findings in her study of new media industries: Work in new media industries is characterised by issues of insecurity, low pay, and long working hours. These unsafe and insecure working conditions, such as lay-offs, karoshi, and unguaranteed pension fund, make Internet work unstable and precarious, though there are other things that make it precarious, such as long working hours and low pay. These precarious and risky working-life experiences of workers in the Internet industries, answers my question addressed in the beginning of this paper, specifically how these workers are involved in the global value chain. I now turn to explain why workers still stay in the industries, despite the bad working conditions.

\subsection{Why Don't Internet Workers Leave the Sector? Autonomy?}

As I pointed out earlier, Internet work has been fetishised in recent years because some workers have become millionaires by receiving companies' stocks. Meanwhile, the Internet work is fetishised also because of offering a high level of autonomy to the workers. It is worth to ask, whether autonomy is the force of keeping Internet workers in the industries, despite the difficult working conditions.

Hesmondhalgh and Baker (2010, 39-44) clarify autonomy in the cultural work with two concepts: Workplace autonomy and creative autonomy. Workplace autonomy refers to the degree of workers' self-determination within certain work situations, such as how they balance their work and life in their daily practices. Creative autonomy refers to the freedom in terms of practicing creativity. Here, I focus on workers' self-determination in their work practices and creativity, under the pressures that are exerted by the state and firms. 
It is easy to understand that workers' freedom of self-determining their project is affected by companies. In some cases, Internet workers are forced by companies to conduct some projects that they are not good at. For example, during my observation, all workers in some technical departments in Grand, no matter what areas they were specialised in, were encouraged to learn developing Android-an open source operating system, which was the next key project for the company. It seemed that workers were "encouraged" to pick this option, but the company issued a new rule to process the program: Department leaders would have deductions from their salaries, if $30 \%$ of the employees in their departments could not pass the Android test. Put simply, the workers were forced to learn new technologies and conduct new programs without any consideration of their capabilities and interests. Two workers in the operations department told me that they needed to conduct the Android project at the same time as learning the skill, which was quite stressful to them. Therefore, the workers involved in the Android project did not have any freedom to decide which projects to carry out.

However, in both Internet companies, there are also workers who consider themselves to have a considerable degree of freedom to self-determine practices and creativity, such as workers in the online novel department in Grand.

The main job of workers in the online novel department is online editing. Their work includes managing online writers' writing and emotions. For example, when online writers face difficulties writing novels, these online editors are responsible for cheering them up, providing ideas, searching writing materials, and even suggesting structures for their stories. These editors are allowed a high level of freedom in determining these practices, such as deciding when and how to manage the writers and novels.

Such authorisation of their high level of professional autonomy covers some disadvantages within the work: The workers need to work day and night without receiving equal rewards. In order to maintain good relationships with the online writers, who usually start their writing at night because they are only part-time writers, these editors need to work until late at night. Surprisingly, although they carry out such day and night overtime, these editors receive just $£ 300$ a month without any overtime pay compared to other full-time workers' $£ 1,000$ monthly pay.

Thus, it might be claimed that some Internet workers, such as workers in the online novel department, stay in the industries, because of the high level of autonomy given by companies in their daily practices and creativity, though there are other difficult working conditions along with such autonomy. It then becomes necessary to ask, why Internet workers suffer such ambivalent working conditions-excessive working hours with unequal pay, lay-off without protection from unions, insecure work status, and certain autonomy. Below, I adopt Eric Olin Wright's concept of exploitation to answer this question.

\section{The Class Analysis Approach}

Erik Olin Wright (2009) illustrates three approaches relevant to class analysis in order to clarify his approach towards exploitation: Stratification research, which defines classes with "the attributes and material life conditions of individuals" (102); the Weberian approach, which centres on "the ways in which social positions afford some people control over economic resources while excluding others" (102); and the Marxist approach, which regards classes as "being structured by mechanisms of domination and exploitation, in which economic positions accord some people power over the lives and activities of others" (102).

The stratification approach focuses on class background, which consists of attributes such as sex, race, religion, age, education, and material life conditions, which refers to adequate income, dire poverty, and pleasant suburban houses. This approach identifies the middle class as people who "have enough education and money to participate fully in the vaguely defined 'mainstream' way of life (which might include particular consumption patterns, for example)" (Wright 2009, 103). But, as Wright acknowledges, this approach lacks serious consideration of the injustice and inequalities between different social positions, such as why some jobs are better than others. 
The Weberian approach focuses on the unequal "opportunity hoarding" (104), which highlights the restricted access to certain positions. For example, high levels of education are restricted to the upper classes, because of the high tuition fees. Good education then further benefits the upper classes, as it usually relates to good jobs. In other words, unequal locations within market relations are causally connected to unjust opportunity hoarding among different social positions. According to Wright (2009), this approach is usually adopted by sociologists to analyse American society, where the middle class is defined by "mechanisms of exclusion over the acquisition of education and skills" (106). In Wright's discussion, the Weberian approach has a critical difference to the stratification approach, as it indicates that, "the economic advantages gained from being in a privileged class position are causally connected to the disadvantages of those excluded from such positions" (106). Put simply, the upper class's economic advantages are causally related to the lower class's disadvantages. Breen (2005) has discussed Weberian understandings of the mechanisms sustaining the privileges of advantaged classes in terms of the concept of "life chances". Life chances are chances that "individuals have of gaining access to scarce and valued outcomes" (43). He then claims three aspects of the distribution of power in society, which is widely adopted by Weberian approach, as factors that influence the distribution of life chances: Classes, status groups, and parties. All these dimensions overlap, while none of them can be reduced to others. The status groups imply "some level of identity in the sense of some recognised 'positive or negative social estimation of honour'" (Weber 1978, 932; Wright 2002, 834). In other words, members of a status group are conscious of being members of the group. Wright (2002) points out that Weber distinguishes status from class by highlighting the different mechanisms through which they shape inequalities of the material conditions of people's lives: Status affects people's well-being with 'the monopolisation of ideal and material goods or opportunities' (835); by contrast, class influences people's well-being via economic assets that people bring to market exchanges. Weber distinguishes status groups from classes by highlighting members' consciousness, and regards classes as objective places.

The Weberian approach usually relates inequalities between different classes to different life chances: Workers are given different chances to access to scarce and valued outcomes. Both Weberian and Marxist approaches agree that occupants of different classes enjoy different life chances, though they provide different schemata for understanding class. The Weberian approach might understand these inequalities and injustices in terms of salary and benefits shown above, as a form of exploitation, because they show inequalities in people's daily experiences. But the Marxist approach, especially the neo-Marxist approach adopted by this research, understands exploitation and domination as the mechanism linking different class locations to variations in life chances. According to Wright, the Marxist approach focuses on mechanisms of exploitation and domination. Unequal opportunity hoarding does not only relate to restricted access to certain positions and resources, but also depends on the ability of the exploiting/dominating group controlling the labour of the exploited/dominated group. Put simply, this approach highlights "an ongoing relationship between not only the conditions but also the activities of the advantaged and disadvantaged" (108). The traditional Marxist approach distinguishes three class locations based on ownership of means of production: The capitalist class, the petty bourgeoisie, and the working class.

Some theorists in the 1980s argue against this Marxist approach to class locations, stating that the working class has diminished or even disappeared in capitalism, especially the manual working class, which only occupies a small part of the workforce, and that white-collar workers are already in the position of the middle class. However, Callinicos (1983, 193-195) claims that many people still occupy the position of the working class, even though they are not engaged in manual labour in Marx's industrialist capitalism. For example, because of the industrialisation of office work, clerical workers are doing similar work to manual workers and suffering from a similar working condition to manual workers, with the massive introduction of new technologies. Therefore, they are in the same position as the working class, as manual labourers, because they are "compelled to sell their labour-power in order to live" (193), even if they do non-manual work. As a result, Callinicos argues that the change in class location and class relations since Marx's period is "a shift in the structure of the working class, not its 
abolition" (195). In later work, Callinicos (2004) introduces Wright's work on class to explain the fragmentation of class structure in contemporary capitalism. As Wright (1985) argues, class locations in modern capitalism are contradictory, as some positions share properties of both labour and capital. For example, managers perform some functions of capital by directing others' work, but still sell their labour-power in order to live.

In the book Classes, Wright (1985) explicitly introduces his framework of contradictory class locations and fragmented class structure. He argues that Marxist criteria for class are an approximate framework for class structure in capitalism, rather than an elaborated classification. He then develops a much more complex typology of class in capitalism, where he divides typology into two parts: Owners of means of production and non-owners. Among these non-owners, their locations are divided by organisation and skill/credential assets. The class locations of wage labourers in a capitalist society are classified into expert managers, non-managerial experts, and non-skilled managers, etc. Wright (1996) further modifies this typology of class locations in his later work by specifying three dimensions that clarify class relations: Property, authority, and expertise/skill, which is where questions of symbol making and manipulation come in. The property dimension consists of employers, the petty bourgeoisie, and employees; The authority dimension is divided into managers, supervisors, and non-managerial employees; and the expertise/skill dimension contains professionals, skilled employees, and non-skilled employees (704).

Wright (2009) aims to move beyond the traditional Marxist approach to class analysis by developing a detailed typology of class locations. He identifies certain key aspects that constitute the new class structure of his model: The mechanism of exploitation and domination in the traditional Marxist approach, the mechanisms that sustain the privileges of advantaged classes in the Weberian approach, and the individuals' class locations in the stratification approach. He argues that a completely elaborated class analysis needs to combine the 'macro-model of conflict and transformation with the macro-micro, multi-level model of class processes and individual lives' (111) (see Figure 3). Put in another way, Wright argues that individuals' lives depend not only on the micro-model of attributes and material life conditions, but also on the macro-model of social conflicts and transformations where their lives take place. 


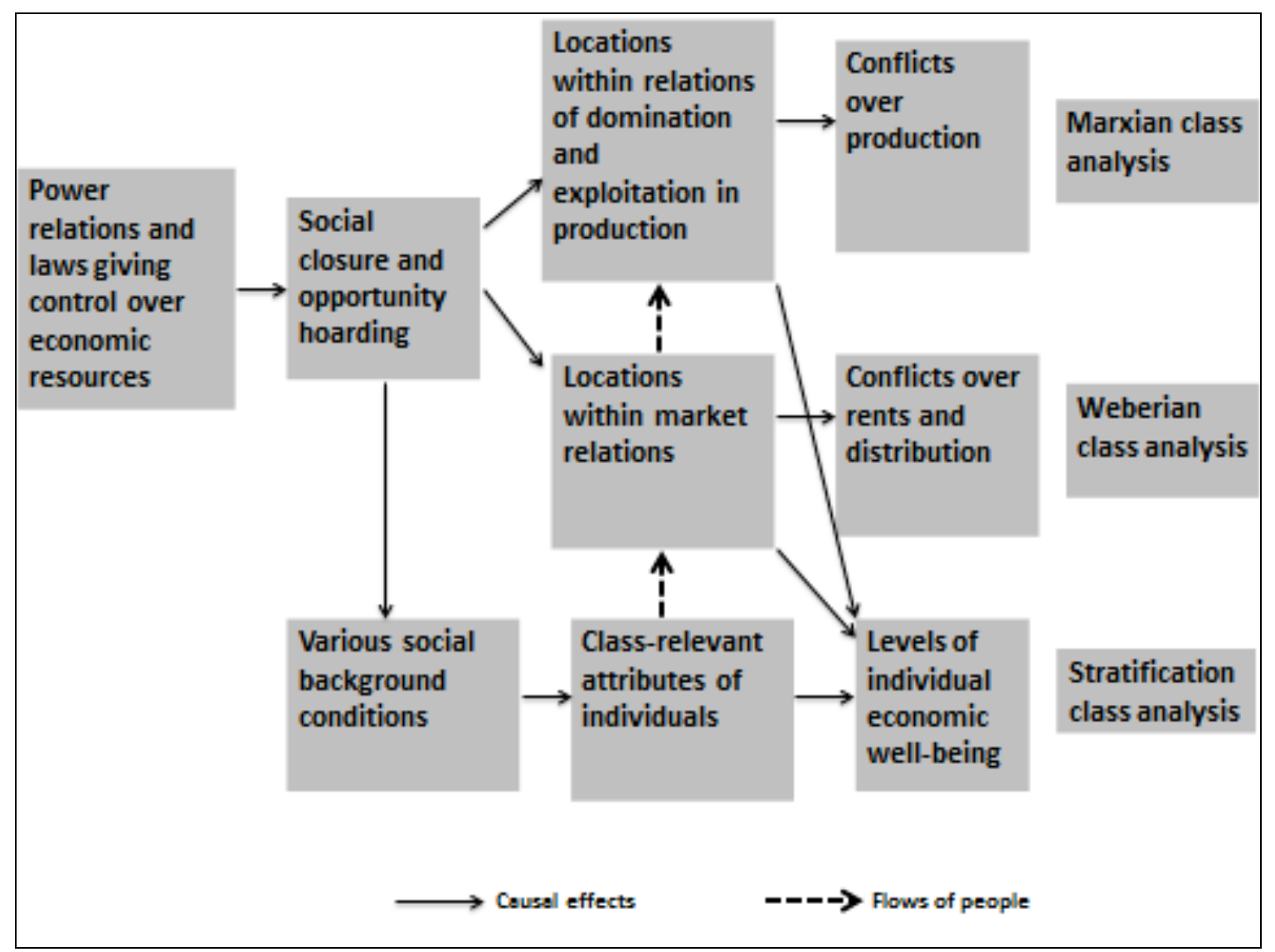

Figure 3: Combined class analysis: macro and micro processes (Wright 2009, p.111)

Wright's work (2009) then suggests a necessity to analyse class locations by locating individuals' lived experiences, such as "class background", in the context of social conflicts and transformations. It is no longer the problem of individuals who fill these positions, but rather, it is important to recognise the mechanisms shaping the privilege of certain class positions. As Wright points out, the middle-class problem is not who is excluded from the position, but is the fact that "there are mechanisms of exclusion that sustain the privileges of those in middle-class positions" (109). Likewise, I adopt a neo-Marxist class analysis approach, which combines both the macro model of transformation and the macro-micro model of individual lives. It is not my interest to just identify the scope of the Chinese middle class, by clarifying who is excluded from the position; rather, my aim here is to recognise the important and unique positions of Internet workers in the general Chinese social structure, and to clarify the mechanisms that sustain and change their unique positions (probably privileged positions) in the Chinese context, which result in inequalities in their working lives, as shown in section four.

\section{Why Bad Working Conditions? Exploitation?}

\subsection{The Lower Middle Class: Chinese Internet Workers}

Based on Wright's model of class structure, here, I explain Internet workers' position in the Chinese class structure, and clarify how certain mechanisms shaping their working lives. So (2003) points out that a new dominant class emerged in China during the process of privatisation of SOEs in 1992: Cadres set up their own businesses, which at times cooperated with foreign capitalists, by usurping resources from SOEs where they had executive positions. Capitalists also joined the existing structure using bribery to access to the market and gain resources. The new partnerships between cadres and capitalists enabled the new private sectors to "save on the additional costs of pension schemes, health and welfare insurance, 
environmental protection facilities" (368), which ultimately led to the deterioration of working conditions in private enterprises.

Such discussion highlights that the bureaucratic capitalist class accumulate capital via appropriating lower classes' efforts, which ultimately leads to the deterioration of working conditions. The class typology in the contemporary Chinese context is complex but my focus in this paper is the low-class location of Internet workers, due to which the labour of this class is appropriated by another higher class. ${ }^{1}$

In the Western context of the UK cultural industries, Hesmondhalgh and Baker $(2010,68-$ 69) place creative workers in these industries principally in a middle-class class location, although they acknowledge that there are various classes involved in cultural production (such as working-class cleaners). Based on Wright's schema, which is also a fundamental framework for this study, they claim that most creative workers in the cultural industries occupy lower-authority, higher-skilled positions, such as skilled workers with little managerial power.

However, in the Chinese context, I argue that creative workers' location in the middle class, such as Internet workers' location, is not only decided by their skills and managerial powers, but also decided by their family backgrounds, work units, education level, and political authority (see Figure 4).

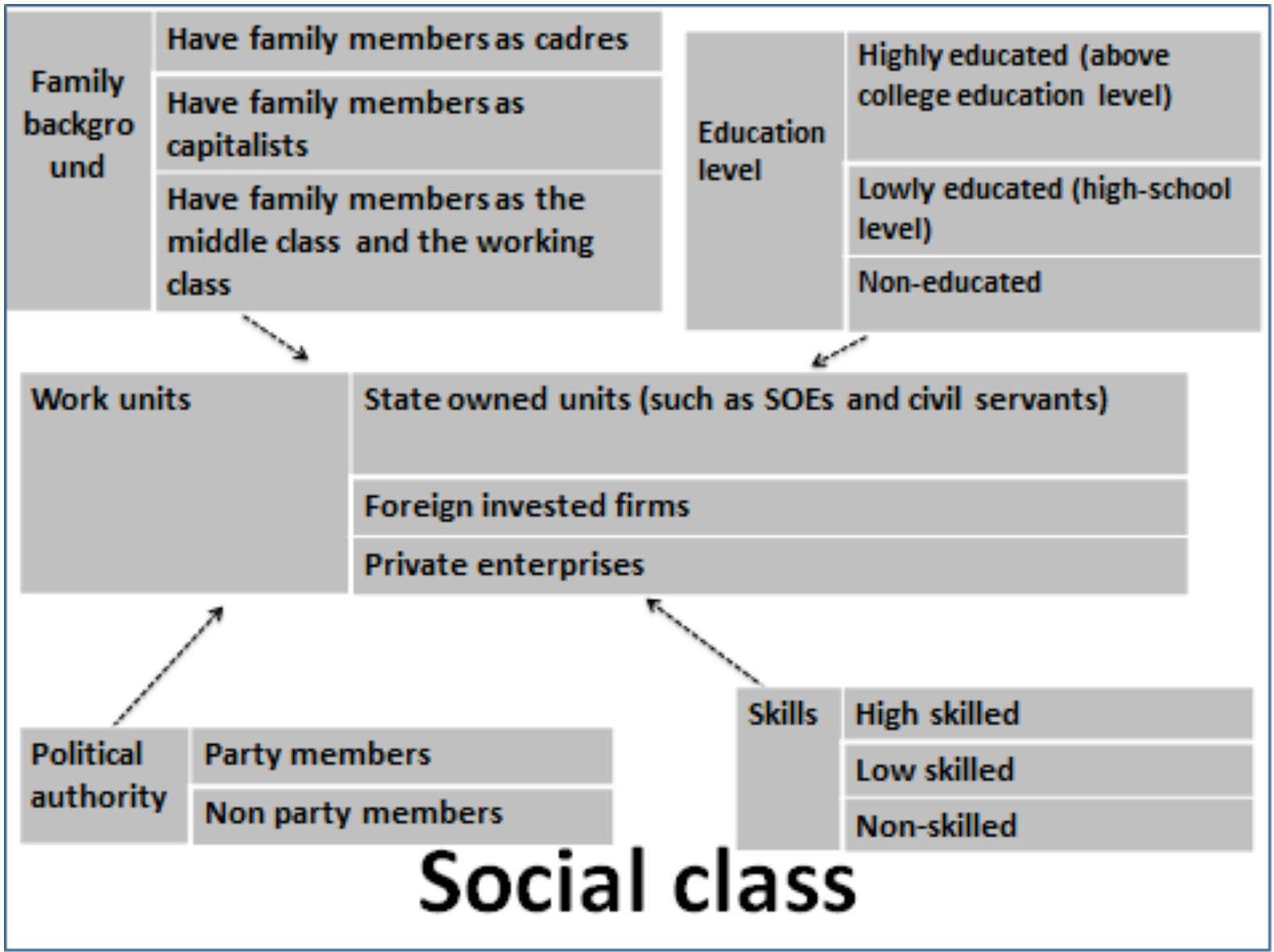

Figure 4: Typology of Chinese middle class

According to Figure 4, education level, family background, political authority, skills, and work units generally decide individuals' positions in the middle class: Workers in SOEs and civil servants have higher positions than private enterprise workers; workers who are highly edu-

\footnotetext{
${ }^{1}$ Also present in the Chinese social hierarchy in the post-1992 period were various other classes, such as the petty bourgeoisie and small employers.
} 
cated have higher positions than workers who are not; workers who have family members as cadres have privileged positions; party members are more likely to be guaranteed stable work and lives than others; and high-skilled workers have more possibility to have well-paid jobs than others. It is hard to quantitatively evaluate the influence of these five factors on individuals' locations in the middle class, such as whether individuals who are not party members but have high skills have higher positions than individuals who are party members but work in private enterprise, and it is not my aim to do so here. Instead, I highlight these issues to give a variation within classes to analyse Chinese Internet workers' social class.

As I stated in section two, most Internet workers in China are working in private enterprises, which are excluded from certain advantageous resources that are only available for SOEs. Due to the inequality between SOE workers and workers in private enterprises, which I illustrated earlier, it is possible to say that large numbers of Internet workers do not have family members in the bureaucratic capitalist class, who have priority to send their offspring to beneficial jobs, such as SOE work and civil servant positions.

Most of these workers still conduct intellectual work, which is defined as middle-career (Zhou 2008, 114-117), though based in private enterprises, which are inferior to SOEs, due to their limited access to certain advantageous resources. As I pointed out earlier, in the existing media reports and academic research, there is no survey conducted amongst Internet workers to report their education background and income. Instead, according to a sample survey conducted amongst IT workers (workers in the hardware market) in some big cities (such as Shanghai, Beijing, Wuhan, and Dalian) in 2010, $97.13 \%$ workers were educated at college level $(\mathrm{Li} 2010,128)$. This figure enables us to deduce that a large number of Internet workers are also educated at colleges. Indeed, according to my qualitative research, all the participants and interviewees are educated at college level, which might help us recognise that generally most Chinese Internet workers are highly educated.

Likewise, as little research investigates Internet workers' income, it is hard to give an authoritative figure about Internet workers' income. Rather, according to an annual report about salary information in various industries, which was conducted by a professional HR service company, PXC, in 2013, the increase in salary rate in the Internet industries was $16.2 \%$, which was the highest among all industries (excluding SOEs and civil servants' positions) (GRlib 2013). Meanwhile, according to Guangzhou Daily, a local newspaper, annual salaries of fresh graduates who find jobs in the top 5 Internet companies are between $£ 10,000$ and $£ 15,000$, which is a middle-level salary for most jobs (Gangzhou Daily 2013). This indicates that Internet workers have a high-level salary among jobs in private enterprises.

Though CCP intends to control big private enterprises via subsuming employees there into its party system, as its managerial slogan in the cultural industries indicates: "Control the big, let go the small" (O'Conner and Gu 2012, 4), it is still hard to find large numbers of party members in the Internet industries. For example, according to one of CCP's official magazines Oriental Outlook, only 9 Internet companies in Beijing had organised Party Committees until 2011, and there were only 2,680 party members in all Internet companies in Beijing, who were mostly in Baidu and Sina. Most of these members joined the Party after 2010 (Oriental Outlook 2013). In other words, large numbers of Internet workers are non-party members.

Unquestionably, as Hesmondhalgh and Baker (2010) claim, just as most cultural workers are highly skilled, either with professional skills or technological skills, Internet workers also have high professional or technological skills. Therefore, the picture here becomes clear: Most Internet workers are based in private enterprises, which indicates that they do not have family members in the bureaucratic capitalist class; most workers are college-educated with low political authorities, as they are non-Party members; and they earn high salaries among people in the middle-class location, as most of them are highly skilled. As I stated earlier, it is hard to evaluate Internet workers' location in the middle class with any sophistication with these figures. However, this generally shows that the Internet workers here occupy an inferior position to SOE workers and civil servants, but this does not indicate these workers have an inferior location in the Chinese social structure-the Internet workers still occupy more 
privileged locations than those in working-class locations. In other words, most Internet workers occupy the lower position in the middle class.

\subsection{The force behind the unequal working-life experiences: exploitation}

After clarifying Internet workers' lower-middle class position, it is necessary to explain how certain mechanisms shaping these workers' working-life experiences, due to their class position. Following Marx's work, many theorists regard injustice as central to understanding Marxist ideas of exploitation (Callinicos 2000; Roemer 1982; Cohen 1985; Wright 1996).

Roemer (1982) pays particular attention to exploitation in existing socialism (Roemer 1982, Chapter Eight). He claims that exploitation still exists in socialism, and that socialist exploitation, based on the inequality in ownership of skills, is socially necessary at a certain stage. The historical task of socialism is to eliminate capitalist exploitation, rather than socialist exploitation. However, Roemer, as a follower of Marx who devotes considerable efforts to explain Marx's work, still shows his ethical concern about socialist exploitation, by asking "if a form of exploitation is socially necessary, what should one's attitude toward it be? Should its existence be endorsed?" (240). Roemer answers these questions with reference to the level of "social consciousness-how the people involved think" (248). He puts it thus: If the exploited fight against injustice, even though the revolution or rebellion is doomed to fail, the social necessity of the exploitation then should be questioned. Because of this, it would be morally wrong to accept a form of exploitation that seems to be socially necessary, without criticising it.

Cohen (1995) argues that Roemer correctly states that exploitation is not based on natural injustice, but that he is incorrect to indicate that an unequal product flow is unjust "only if it reflects an unjust initial asset distribution" (204). The work of exploitation needs to focus on the unjust exploitative allocation. This is because Roemer's work directs our interests to unjust asset distribution, which in Cohen's understanding is caused by the unjust product flow. As an alternative, Cohen states that it is necessary to focus on the "injustice of an exploitative allocation" (207) rather than the "injustice of the initial distribution" (207), as the former generates the primary injustice that drives the latter to be unjust.

Wright (1985) criticises Roemer's work on exploitation because of the elimination of class relations in his analysis of injustice. For example, Wright states that Roemer fails to point out that "real transfers from one actor to another" (74) create unjust inequalities. According to Wright, Roemer fails to introduce the notion of dominance in his game-theory approach to exploitation. As an alternative, Wright defines exploitation as a process that contains both "economic oppression" $(1985,74)$ and the "appropriation of the fruit of the labour of one class by another" (74). As a criterion of exploitation, the benefits of the exploiter must depend on the work of the exploited.

Wright $(1976,28-29)$ claims that exploitation needs to be discussed within varied modes of production, as different forms of exploitation correspond to different modes of production. For example, workers in industrial capitalism are exploited in a way that is distinguished from workers in the earliest capitalism: on the one hand, they cannot control the labour process as producers in cottage industries did, because they are gathered in factories; on the other hand, the labour force is deskilled and the production process is fragmented, because of the introduction of new technologies in factories. Meanwhile, capital is not a commodity in existing socialism as it is traded in capitalism. Burawoy and Wright (2002: 478-480) distinguish existing socialism from Marxist socialism by using the example of Soviet communism, which is entitled "state socialism". They claim that state socialism refers to a central planned system: a class of "planners" take charge of the "redistribution of surplus", which is extracted from a class of "direct producers". This extraction is legitimised in the name of "the superior knowledge of the planner about the needs of the people" (479).

Roemer (1982) divides modes of production into four categories, based on the different forms of exploitation: Feudal exploitation, which is based on injustice generated by unequal distribution of labour power assets, in which lords and serfs are the main classes; capitalist exploitation, which is based on injustice generated by unequal distribution of alienable as- 
sets, in which relations between bourgeoisie and proletariat are the main class relations; status exploitation, which exists in the existing socialism, a historical stage between capitalism and socialism; and socialist exploitation, which is based on injustice generated by unequal distribution of inalienable assets, in which experts and workers are the main classes. In status exploitation, exploiters control labour power and property because of their high status in the social structure. This is different from the injustice generated by either means of production or skills.

Wright (1985) agrees with Roemer that skill-based exploitation would exist in a Marxist socialist society, and it could only be eliminated in Marxist communism. But Wright indicates that Roemer's concept of status exploitation is problematic in two ways: First, it is not necessarily related to production at all and second, it is hard to distinguish it from feudal exploitation. As an alternative, Wright (1985) points out a post-capitalist mode of production that exists between the stages of capitalism and socialism, statism, which is based on organization asset. In this mode of production, bureaucrats and managers occupy the class location of the exploiter.

This is agreed by Callinicos (1983), who interprets that existing socialism is "bureaucratic state capitalism" (183), as "a state bureaucracy, which competes with its Western counterparts" (183) exploits the working class. In the context of the Soviet Union, socialism, or "bureaucratic state capitalism", did not self-emancipate the working class, as it claimed. The followers of the Soviet Union, such as China, reproduce this mode of bureaucratic state capitalism in their societies. Callinicos (2004) further explains his arguments in his later work. He states that the existing socialist societies are "state bureaucratic socialist, combin[ing] the statist and socialist modes of production" (223). This includes multiple occurrences of exploitation based on the unequal ownership of varied resources: "Skills, organisational assets, means of production, labour-power" (225).

Some theorists who work on modern Chinese society have acknowledged this exploiter class, which allies bureaucrats and capitalists. I have demonstrated such research in the last section, in order to clarify the social class of Internet workers. Here, I continue my argument from the last section, based on Wright's and Callinicos' work, recognising the social mode of production in modern China as bureaucratic state capitalism or bureaucratic state socialism. The bureaucratic capitalist class, which I defined in section five, occupies the location of exploiter class, with ownership of the means of production, organisational assets, and political authority. This class accumulates huge wealth by controlling labour power and the skills of the middle class and the working class. This activity of appropriation then generates inequality and injustice between the bureaucratic capitalist class, the middle class, and the working class.

In the Chinese context, the working class sells labour power in order to survive, as their livelihoods are not guaranteed by society. The bureaucratic capitalist class owns the means of production, such as factories/firms, raw materials, and telecommunication, and has the political authority to allocate these means of production. For example, executives in the party-controlled enterprises (the SOEs in contemporary Chinese society) and government departments own the main raw materials and economic resources, such as oil and telecommunication. Officials in the bureaucratic capitalist class with certain political power (similar to Wright's state power) and capitalists with certain economic power allocate these raw materials and economic resources. But what does the middle class own, and what are the relationships between the middle class and these other two classes?

Internet workers, as members of Chinese lower-middle class, own certain means of production, such as professional and technical skills, but this ownership is only helpful when they place it in the capitalists' service. According to Wright $(1997,19)$, this is the general problem of the middle class, who sell their labour power as they lack the means of production, while they do not regard themselves as the working class. Under the movement towards globalisation, precarious and uncertain work and life status are shared by workers in different social contexts, both in socialism and capitalism. Workers in Western societies share the severe economic pressure faced by Chinese Internet workers, which I showed earlier, as it becomes a general problem of the middle class, to use Wright's terms. 
In the Chinese context, the bureaucratic capitalist class dominates the working class and the middle class, because of the ownership of the means of production, and the power to allocate these resources. It is the bureaucratic capitalist class, where officials and capitalists gain benefits from corruption and bribery, which appropriate the labour of other classes. Due to this, the wealth of the bureaucratic capitalist class is based on the labour efforts of the working class, who contribute labour power, and the middle class, who contribute skills.

Due to the inferior position in the middle class, Internet workers lack the power to allocate the resource they own, such as skills; instead, their labour efforts are appropriated by the bureaucratic capitalist class via long working hours, unequal pay, insecure work status, and unguaranteed pension fund. Such structural activity of appropriation, exploitation in Wright's explanation, thus, becomes the force behind Internet workers' poor working-life experiences. Here, exploitation becomes a mechanism to explain why Internet workers suffer bad working conditions, it is worth asking: Why do Internet workers still work in the industries, despite inequalities resulted by the exploitation?

\section{There is still hope: agency of Internet workers}

As I stated in last sections, Internet workers experience inequalities and injustice, such as unequal pays with long working hours, insecure work status, and unguaranteed life after retiring, due to the structural exploitation. But, this does not indicate that Internet workers accept such difficult working conditions, due to certain level of autonomy given by companies, without any acts of agency. Rather, Internet workers use various acts of negotiation and resistance to improve the quality of working life.

For example, some workers regard intervention from the state as influencing their practices and creativity in an unacceptable way:

We [workers] definitely don't like the rule. We prefer to stand with users, who could bring us money. But, as the state could easily stop our service, we still need to follow the rules in certain ways [...] (Galeno, technical worker in the Product Administration Department at Campus, $20^{\text {th }}$ December 2011, interview)

Here, Galeno shows the necessity of balancing the state's requirement and Internet users' needs for free space in his daily practices; moreover, he states that some workers feel unsatisfied with the state's intervention. Thus, these workers, who are unsatisfied with such intervention, apply their professional knowledge and skills to acts of negotiation and resistance, in order to gain more autonomy.

For example, In an Election Meeting of Candidates of the National People's Congress in Shanghai, which took place during my fieldwork, representatives of workers in Grand expressed discontent about current working conditions, and made a case for more work-related benefits. They asked the local government to build a new kindergarten near the company in order to benefit the workers with children. They also raised the issue of overtime work in the industries. Moreover, these representatives questioned whether their voices could be heard by the departments responsible for bringing in changes via the Congress system. Since I left Grand two months after this event, I do not know the result of such bargaining. But the voices of the workers in this event indicate Internet workers' special forms of response towards state control and intervention. It is rare to hear the voices of other workers in the Chinese context, such as SOE workers.

This form of negotiation may not be so unusual in other geographical contexts, such as the UK, but such direct questioning of authority can hardly be found amongst workers in other industries in China. Internet workers' direct expression of discontent and their questioning of authority mark a fundamental shift in attitudes towards worker agency in the authoritarian Chinese context.

Here, I acknowledge workers' subjectivity and agency in such events. As Burawoy and Wright (2002, 474-475) claim, the exploited classes tend to resist the appropriation of their labour efforts. The exploited class-the lower-middle class-has the potential to resist the exploiter class-the bureaucratic capitalist class-via acts which attempt to eliminate inequalities 
and injustice in their working life. This then creates potential for changing working conditions in the Internet industries in the future. I regard this as a sign of hope in contemporary Chinese Internet industries.

\section{References}

Andreas, Joel. 2010. A Shanghai Model? On Capitalism with Chinese Characteristics. New Left Review. 63-85.

Barbrook, Richard. 2005. The High-Tech Gift Economy. First Monday. Special issue 3: Internet banking, e-money, and Internet gift economies.

Breen, Richard. 2005. Foundations of a Neo-Weberian Class Analysis. In Approaches to Class Analysis, edited by Wright Eric Olin, 31-50. Cambridge: Cambridge University Press.

Burawoy, Michael and Eric Olin Wright. 2002. Sociological Marxism. In Handbook of Sociological Theory, edited by Turner Jonathan, 459-486. New York: Plenum Books.

Callinicos, Alex. 1983. The Revolutionary Ideas of Karl Marx. London: Bookmarks.

Callinicos, Alex. 2000. Equality. Cambridge, Oxford, and Malden: Polity Press and Blackwell Publisher.

Callinicos, Alex. 2004. Making History: Agency, Structure, and Change in Social Theory. Leiden: Koninklijke Brill NV.

Cohen, Gerald Allan. 1995. Self-Ownership, Freedom, and Equality. Cambridge: Cambridge University Press.

Dickson, Bruce. 2003. Red Capitalists in China: The Party, Private Entrepreneurs, and Prospects for Political Change. Cambridge: Cambridge University Press.

Dickson, Bruce 2011. Who Consents to the 'Beijing Consensus': Crony Communism in China. In In search of China's Development Model: Beyond the Beijing Consensus, edited by Hsu Philip, Wu Yu-Shan and Zhao Suisheng, 24-36. London: Routledge.

Dyer-Witheford, Nick. 2010. Digital Labour, Species-Becoming and the Global Worker. Ephemera 10 (3/4): 484-503.

Gill, Rosalind. 2002. Cool, Creative and Egalitarian? Exploring Gender in Project-Based New Media Work in Europe. Information, Communication and Society 5 (1): 70-89.

Harvey, David. 2008. The right to the city. New Left Review Sep-Oct: 23-40.

Hesmondhalgh, David and Sarah Baker. 2010. Creative Labour: Media Work in Three Cultural Industries. Abingdon and New York: Routledge.

Hills, Matt. 2002. Fan Cultures: Sussex Studies in Culture and Communication. London: Routledge.

Huang, Yasheng. 2008. Capitalism with Chinese Characteristics: Entrepreneur and the State. Cambridge, New York: Cambridge University Press

Kennedy, Helen. 2012. Net Work: Ethics and Values in Web Design. Basingstoke: Palgrave Macmillan

Manzerolle, Vincent. 2010. Mobilizing The Audience Commodity: Digital Labour In A Wireless World. Ephemera 10 (3/4): 455-469.

Mok, Ka Ho and Yat Wai Lo. 2007. The Impacts of Neo-Liberalism on China's Higher Education. Journal for Critical Education Policy Studies 5 (1): 316-348.

Noon, Mike and Paul Blyton. 2007. The Realities of Work: Experiencing Work and Employment in Contemporary Society. Houndmills, Basingstoke: Palgrave Macmillan.

Ornebring, Henrik. 2008. The Consumer as Producer-of What? User-Generated Tabloid Content in the Sun (UK) and Aftonbladet (Sweden). Journalism Studies 9 (5): 771-785.

Roemer, John E. 1982. A General Theory of Exploitation and Class. Cambridge, Massachusetts, and London: Harvard University Press.

Ross, Andrew. 2005. Fast Boat to China: High-Tech Outsourcing And The Consequences Of Free Trade: Lessons From Shanghai. New York: Vintage Books.

Ross, Andrew. 2007. Nice Work if You Can Get It: The Mercurial Career of Creative Industries Policy. In My Creativity Reader: A Critique of Creative Industries, edited by Lovink Geert and Rossiter Ned. Amsterdam: Institute of Network Cultures.

So, Alvin. 2003. The Changing Pattern Of Classes And Class Conflict In China. Journal of Contemporary Asia 33 (3): 363-376.

Terranova, Tiziana. 2004. Network Culture: Politics For The Information Age. London: Pluto Press.

Wang, Hui. 2003. China's New Order: Society, Politics, and Economy in Transition. Cambridge, Massachusetts, and London: Harvard University Press.

Wang, Jing. 2008. Brand New China. London: Harvard University Press. 
Wright, Eric Olin. 1976. Class Boundaries In Advanced Capitalist Societies. New Left Review 98: 3-41 Wright, Eric Olin. 1985. Classes. London: Verso.

Wright, Eric Olin. 1996. The Continuing Relevance Of Class Analysis-Comments. Theory and Society 25: 693-716.

Wright, Eric Olin. 1997. Class Counts: Comparative Studies in Class Analysis. Cambridge: Cambridge University Press.

Wright, Eric Olin. 2002. The Shadow Of Exploitation In Weber's Class Analysis. American Sociological Review 67: 832-853.

Wright, Eric Olin. 2006. Compass Points: Towards A Socialist Alternative. New Left Review 41: 93124.

Wright, Eric Olin. 2009. Understanding Class. New Left Review 60: 101-116.

Wright, Teresa. 2010. Accepting Authoritarianism: State-Society Relations In China's Reform Era. Stanford, California: Stanford University Press.

Zhao, Yuezhi. 2003. Transnational Capital, The Chinese State, and China's Communication Industries in a Fractured Society. The Public 4: 53-74.

Zhao, Yuezhi. 2007. After Mobile Phones, What? Re-Embedding The Social In China's 'Digital Revolution'. International Journal of Communication 1: 92-120.

Zhou, Xiaohong. 2008, Chinese middle class: reality or illusion. In: Christophe, J. \& Van der veer, P. ed. Patterns of Middle Class Consumption in India and China. Los Angeles: Sage

\section{Websites}

Chinese Economics. 2013. Shouyilv Nan Di Tongzhang, Yanglaojin Ruhe Baozhi Zengzhi. Accessed July 29, 2013. http://finance.chinanews.com/cj/2013/07-09/5017318.shtml

http://finance.chinanews.com/cj/2013/07-09/5017318.shtml

Guangzhou Daily. 2013. Jinnian Yingjiesheng Qixin Gao, Neidi Wuda IT Qiye Nianxin 10wanyuan

Qibu. Accessed April 14, 2014. http://finance.ifeng.com/a/20131209/11234834_0.shtml

iResearch Inc. 2013. iResearch China Internet Economy Research Report 2011-2012. Accessed June 11, 2013. http://www.iresearch.com.cn

Liaoning Research Institute of Industry and Information Sciences. 2013. Zhongguo IT Keji Renyuan Keyan Chengguo Zhuanhua Fenxi. Accessed June 10, 2013. http://www.Iniis.gov.cn/dwdt/ShowArticle.asp?ArticleID=6921

Oriental Outlook. 2013. Hulianwang Qiye Dangjian Diaocha. Accessed April 14, 2014. http://finance.sina.com.cn/roll/20130225/170114639663.shtml

Sina News. 2010. Tengxun 37sui Zhuanti Zhubian Yu Shihong Cusi. Accessed January11, 2014. http://eladies.sina.com.cn/qg/2010/0521/1631993336.shtml

Sohu IT. 2013. Zhongguo Hulianwang Yifenzhongnei Hui Fasheng Shenme? Accessed January 14, 2014. http://it.sohu.com/20130322/n369907395.shtml

Tencent Technology. 2011. IT Chanye Cheng Wu Da Jibing Zhong Zaiqu: Guolaosi Pingjun 37.9 Sui. Accessed July 19, 2012. http://tech.qq.com/a/20111124/000177.htm

The Boston Consulting Group. 2012. Clicks Grow Like BRICS: G-20 Internet Economy to Expand at 10 Percent a Year Through 2016. Accessed June 16, 2014. http://www.bcg.com/media/PressReleaseDetails.aspx?id=tcm:12-100468

Xinhua News. 2005. Li Yanhong Zai Meiguo Chuangzao Shenhua: Baidu 400 Lao Yuangong Yi Ye Bao Fu. Accessed September 23, 2013. http://news.xinhuanet.com/fortune/200508/08/content_3323159.htm

Xinhua News. 2013. Xinhua Insight: Internet Industry Optimism To Buoy China's Economy? Accessed October 10, 2013. http://news.xinhuanet.com/english/indepth/2013-08/17/c_132638709.htm

Youth Times. 2012. Wei He Shuo 'Yanchi Tuixiu' Bu Ke Xing. Accessed October 29, 2012. http://www.qnsb.com/fzepaper/site1/qnsb/html/2012-06/11/content 373983.htm

\section{About the Author}

\section{Bingqing Xia}

Bingqing Xia currently is a PhD candidate in the Institute of Communications Studies at University of Leeds, UK. Her research focuses on labour and the quality of working life in the Chinese Internet industries. Bingqing's background is in history and new media studies. She has presented her work at 
a range of international, annual and biannual conferences such as the Association of Internet Researchers, the International Association for Media and Communication Research, and the Crossroads Cultural Studies conference. 


\title{
Will Work For Free: The Biopolitics of Unwaged Digital Labour
}

\author{
Brian A. Brown \\ University of Windsor, Windsor, Ontario, Canada, bbrown@uwindsor.ca
}

\begin{abstract}
This paper begins with a survey of the literature regarding a particular, yet ever more consequential and profitable, typology of digital labour: 'free labour' (Terranova, 2000, 2004), 'unwaged immaterial labour' (Brown and Quan Haase, 2012; Brown, 2013), and/or immaterial labour 2.0 (Coté \& Pybus, 2007), to name a few of the more common terms. It then moves on to proffer a critical synthesis of this body of work so as to conclude with a much more theoretically nuanced definition of unwaged digital labour than that which has thus far been provided. In sum, the author argues that there are five central facets to unwaged digital labour that defines and differentiates it from its waged brethren. The first is that unwaged digital labour is fundamentally and inherently autonomous. Free of management oversight, the cooperative and creative capacities of content-generators produce massive amounts of digital artefacts that in the majority of cases also yield massive amounts of profit for the owners of Web 2.0 sites and services. The surplus value produced by this first facet refracts into the second. Following the work of Fuchs $(2010,2011,2012,2013)$, unwaged digital labour is (in the majority of circumstances) hyper-exploited. As has been argued elsewhere (Brown, 2013), this hyperexploitation is the primary cause for recurrent 'user' uproar on Web 2.0 sites and services. This kind of exploitation, then, is met with the third facet of digital labour considered herein: resistance or struggle. Facile recourse to nebulous conceptions regarding the invasion of one's privacy on eminently social networks no longer suffices in explaining these instances of 'user' uproar. Thus, a more nuanced consideration of the forms of resistance that occur on social media sites and services is offered. Similar, yet different, to its waged genus, the fourth facet of unwaged digital labour is that it is intrinsically collaborative, cooperative, and generative of social relationships. The differences that obtain between the orientation of the social relationships constituted by waged and unwaged digital labour respectively are indicative of political potentials that have up until this point been under-theorized. Thus, building on the four aforementioned facets, as well as the arguments put forth by Hardt and Negri regarding the biopolitical dimensions of 'immaterial labour' $(2000,2004,2009)$, the fifth and most theoretically provocative facet is that this kind of labour is inspired, guided, and regulated by a radically different amalgam of biopolitical power relationships that point to the potentials of a commons-based political economy existing beyond the hyper-exploitative dimensions of capital.
\end{abstract}

Keywords: Unwaged Digital Labour, Free Labour, Immaterial Labour 2.0, New Media, Social Media, Autonomist Marxism, Biopolitics, Online Privacy.

\section{Introduction: The 6 Million Dollar A Day Man}

On November 7, 2013, Twitter, a wildly popular micro-blogging service that encourages its members to post one hundred and forty character status updates to a list of followers that sometimes numbers in the millions, filed its Initial Public Offering (IPO). In one of the most highly anticipated technology offerings to hit the open market in decades, shares in the micro-blogging service closed on the first day of trading at US $\$ 44.90$ yielding a valuation of roughly US $\$ 31$ billion. At the time of last revision (July 30, 2014), Twitter's share price is US\$46.62 with a market capitalization of US\$22.77 Billion. These massive valuations are particularly noteworthy in that Twitter "is unlikely to make a profit until 2015" (Frier and Picker 2013) and has yet to prove its mettle in terms of its ability to monetize the billions of tweets coursing through its servers every second of the day. These astronomical sums, however, are not without precedent.

In May 2012, Facebook, a social media behemoth founded by Mark Zuckerberg, brought itself to market and, in what is widely regarded as a botched IPO, was initially valued at 
roughly US $\$ 100$ billion. The stock declined precipitously in the coming weeks and months, but eventually rallied behind a new mobile advertising strategy and at the time of last revision is valued at $\$ 74.88$ per share with a market capitalization of US $\$ 194.69$ billion. As far as Facebook is concerned, this colossal sum positions the social media company as more valuable than General Motors and the Ford Motor Company combined. In a telling indication of the shifts in the production of wealth and profit in the contemporary era, Zuckerberg, Facebook's Chief Executive Officer (CEO), is reported to have made more than US\$6 million each and every day in fiscal 2012 (Rushe 2013).

Fewer than 6000 individuals around the world draw a pay packet from Facebook Inc. Once again, in comparison, Ford Motor Company employs roughly 180,000 individuals and General Motors 202,000 people (MacroAxis 2013). However, unlike these industrial giants, Facebook has managed to offload the vast majority of the heavy lifting that generates the profits accrued by them to their unwaged 1.23 billion active monthly 'users.' All of this labour, the work of generating content and posting it to their website, creating and maintaining networks, sharing images, stories, news, videos, sound clips, and personal thoughts is undertaken and accomplished free of the wage relation and, pivotally, free of any kind of management oversight or supervisory directive. Left to their own devices and free to do as much or as little labour as they desire, Facebook's 'users' have proven an inexhaustible source of content and thus of the profits that accrue as a result of leveraging the details of this content into advertising dollars-the primary source of Facebook's revenue.

Different scholars have conceptualized this kind of labour in a variety of manners. By far the most popular way of understanding all of this unwaged digital labour is via recourse to the critical political economy of communications and the work of Dallas Smythe (1977). This paper begins, then, with a critical examination of the ways in which scholars have described and characterized all of the unwaged labour taking place on what have variously been termed New Media sites and services, Web 2.0 sites and services (O'Reilly 2005), or Social Media websites.

One of the first to conceptualize all of the work taking place on Web 2.0 sites and services was Tiziana Terranova. Terranova describes all of the unwaged labour taking place on the Internet as a form of "Free Labor" (2000). Building on the work of Terranova and referencing the highly influential thoughts of Maurizzio Lazzarato and his conception of "Immaterial Labor" (1996), Mark Coté and Jennifer Pybus proffer "Immaterial Labour 2.0" as a typological descendent of Lazzarato's theorization of waged Immaterial Labour and Terranova's conception of Free Labor. Responding to the work of Smythe and the above authors, Nicole Cohen, in an article that has proven highly influential, focuses her critical sights on the "free labour" (2008) responsible for producing the profits generated by Facebook. Finally, Christian Fuchs $(2009,2010,2012,2013)$, one of the most vocal and astute critics of the 'free labour' thesis, argues via Marx's Labour Theory of Value (1976) that all of the work that goes into maintaining one's presence and profile on Web 2.0 sites and services can be considered exploitative in that in its absence, the companies would have to pay employees to generate an equal amount of content. Fuchs' contribution offers a much more theoretically nuanced definition of this concept that encapsulates many of the attributes identified in the aforementioned authors' work. As will be detailed below, what all of the above authors have in common is a consistent focus on the critical political economic dimensions of Web 2.0 and social media. This focus, however, while accurate and theoretically correct, overlooks an equally important definition of the typological distinctions that separate unwaged digital labour from its waged forebear. In what follows, then, I define "unwaged digital labour" via a five-faceted typological theorization that takes into account existing scholarship, but moves beyond it so as to define the concept's core characteristics in much more concrete terms.

The five facets of unwaged digital labour described below are as follows: i) its inherent autonomy, ii) its exploitative nature, iii) instances of resistance and struggle, iv) its intrinsically collaborative and cooperative nature, and v) its biopolitical impact on the constitution of subjectivity. By conceptualizing unwaged digital labour in this way, we gain an appreciation of the relatively unique and progressive political potentials of this form of directed action. This paper concludes by pointing to the influence of unwaged digital labour on the subjectivities of 
those that undertake and accomplish it and argues that without the biopolitical influence on the subjective orientation of unwaged digital labourers, social movements such as Occupy Wall Street or the (dis)organizational forms that inspired the halting 'Arab Spring' in Egypt, for instance, would never have come to pass. It is the inherent autonomy of this form of labour, combined with its biopolitical influence, which provided these social movements with nascent evidence of the viability of a form of leaderless organization that is, on the one hand, highly interconnected, collaborative and cooperative, at the same time as it is, on the other hand, heterarchical, fluid, and dynamic. By appreciating the progressive political potentials evinced by unwaged digital labour, we gain a more robust understanding of the modes of organization that are becoming ever more common in the struggle against capital. It is to a critical analysis of this relatively unique form of labour that the following pages are directed.

\section{2. 'Free' (Unwaged) [Immaterial/Digital] Labour \{2.0\}: Reviewing the Literature}

Nomenclature is important. The ways in which we name, describe, and characterize particular phenomena influence our understanding and critical appreciation of them. As the subheading of this section of the paper indicates, the nomenclatural contortions that have come to characterize our understanding of the subject at hand have been diverse. This paper settles upon and operationalizes the term "unwaged digital labour" to describe all of the "work" being done on Web 2.0 sites and services. It does so with the goal of conceptual clarity foremost in mind.

The first term in the phrase indicates that all of this labour goes "unwaged". In fact, as previous research has shown (Brown 2013), members of Web 2.0 sites and services undertake and accomplish this labour without any expectation of financial remuneration. The second term in the phrase is a more accurate description of the nature of this labour than the "immaterial" qualifier that so often gets attached to this form of directed action. Lastly, "labour" rather than "work" was chosen as a more suitable term in that it acknowledges Marx's "Labour Theory of Value" (1976) and the exploitative dimensions of this form of directed action. (See: Fuchs and Sevignani 2013). The choice of this phrase, however, did not arise in a vacuum. There is a long history of thinking through the various dimensions and instances of unwaged labour (Dalla Costa and James 1973, Wages For Housework 1988). In what follows, however, I will focus strictly on those authors that consider these ideas alongside networked modes of digitized communication.

Writing in an epoch that predated the rapid rise of "social media" and "user-generated content", Tiziana Terranova identified "Free Labor" (2000) as a central component of networked forms of communication and production. She was one of the first to conceptualize and try to better understand the implications of this "free labor" to the Internet. Terranova defines "free labor" as "Simultaneously voluntarily given and unwaged, enjoyed and exploited, free labor on the Net includes the activity of building Web sites, modifying software packages, reading and participating in mailing lists and building virtual spaces on MUDs [Multi-User Dungeon] and MOOs [Object Oriented MUD]" $(2000,33)$. She argues further that, "the Internet is animated by cultural and technical labor through and through, a continuous production of value that is completely immanent to the flows of the network society at large" $(2000,34)$. After assessing the productivity and efficiencies of this work, she argues that our understanding of what it means to labour cannot be myopically confined to that which is exchanged for a wage. She states categorically that "Labor is not equivalent to waged labor [...] [and to] emphasize how labor is not equivalent to employment also means to acknowledge how important free affective and cultural labor is to the media industry, old and new" (46). The point being that "free labor" must be considered as such in that it is not only temporally, affectively, and physically taxing, but it is also productive of enormous sums of profit for those that own the means through which it is monetized.

Projecting Terranova's argument into the Web 2.0 era of social media and user-generated content, and leaning on the work of Maurizzio Lazzarato and his conceptualization of waged "immaterial labour" (1996), Mark Coté and Jennifer Pybus (2007) choose the once dominant, 
but now in decline, social network of MySpace (and subsequently Facebook (Coté and Pybus 2011) as their object of study and posit "Immaterial Labour 2.0" as a significant and meaningful amendment to Terranova's and Lazzarato's "free (immaterial) labor" thesis. They argue that:

What the '2.0' addresses is the 'free' labour that subjects engage in on a cultural and biopolitical level when they participate on a site such as MySpace. In addition to the corporate mining and selling of user-generated content, this would include the tastes, preferences, and general cultural content constructed therein. $(2007,90)$

Immaterial labour 2.0 further blurs the already hazy distinction between labour time and leisure time identified as a primary outcome of the waged immaterial labour thesis initially offered by Lazzarato (1996) and further underscored by scholars like Berardi (2009), Turkle (2011), Hardt and Negri (2004), and Huws (2003). Broadly conceptualized as an instance of the 'real subsumption' (Marx 1975, Negri 1991) of subjectivity to the demands and dictates of a capitalist mode of production that reaches into evermore intimate and private spheres, Immaterial Labour 2.0 was a pivotal publication that helped clarify the terms of debate and elucidate the penetration of the capitalist relation into spaces and places previously undertheorized.

Coté and Pybus argue that on social networks such as MySpace, Facebook, or, by extension, Twitter, the work one does connecting with friends, sending messages or updates, linking to web pages, posting images, "liking" posts, products, and people, uploading songs, sharing, chatting, and socializing, is unquestionably productive of economic value for the owners of these social networks. Social Networks like MySpace and Web 2.0 archetypes such as Twitter are, then,

shaped by the creative imprints of [their] users. However, its political-economic foundation demonstrates how such user-generated content-immaterial labour 2.0-is the very dynamic driving new revenue streams. Thus, it is the tastes, preferences, and social narratives found in user entries which comprises the quotidian mother lode of these new revenue streams. (Coté and Pybus 2007, 100)

In this regard (and similar to the arguments made by Terranova above) Immaterial Labour 2.0 focuses on a critical political economic interpretation of all the work being done on Web 2.0 sites and services. All of this labour, while unwaged, is productive of profit and, thus, exploitative of those responsible for accomplishing it.

Nicole Cohen's work too falls into the critical political economic realm of theorizing unwaged digital labour. Taking Facebook as her central example, Cohen argues that Web 2.0 sites and services would simply not exist without an enthusiastic and highly motivated work force. According to Cohen:

By uploading photos, posting links, and inputting detailed information about social and cultural tastes, producer-consumers provide content that is used to generate traffic, which is then leveraged into advertising sales. [...] In this model, rather than employing workers to create content, Web 2.0 companies [...] profit from the unpaid labour time that producer-consumers spend working on their online identities and keeping track of their friends. $(2008,7)$

She continues later on in her article by referencing the autonomist Marxist conception of the social factory (Negri 1989) as a place where the aforementioned "real subsumption" of subjectivity takes place. "Facebook, a space where both leisure time is spent and labour performed, is an example of how, in the social factory, general social relations become moments of production" (Cohen 2008, 18).

While the above authors point to the exploitative relationship that exists between the owners of Web 2.0 sites and services, they have yet to adequately unpack this relationship in such a way that makes manifest this exploitation in terms of its applicability to Marx's Labour 
Theory of Value (1976). The work of Christian Fuchs supplements the above authors' contentions, then, via a close reading of Marx's original thoughts on exploitation and the ways in which the political economy of Web 2.0 meshes with them.

Fuchs argues his position via reference to the work not only of Marx but also Critical Communications Scholar Dallas Smythe and his concept of the 'audience commodity' (Smythe 1977). Smythe's work will be addressed in more detail below. For the time being, however, it is important to emphasize the exploitative dimensions of unwaged digital labour as Fuchs understands them. He argues:

The users who google data, upload or watch videos on YouTube, upload or browse personal images on Flickr, or accumulate friends with whom they exchange content or communicate online via social networking platforms like MySpace or Facebook, constitute an audience commodity that is sold to advertisers. The difference between the audience commodity on traditional mass media and on the Internet is that in the latter the users are also content producers [...] Advertisements on the Internet are frequently personalized; this is made possible by surveilling, storing, and assessing user activities with the help of computers and databases. This is another difference from TV and radio, which provide less individualized content and advertisements due to their more centralized structure. $(2009,31)$

The labour required to produce all of the content that populates Facebook or Twitter, for instance, is accomplished by a group of individuals who receive no compensation in exchange for this work. Indeed, because this labour goes unwaged and because it is also productive of massive amounts of profit, Fuchs argues, correctly, that this scenario can be described as hyper-exploitative or "one of infinite over-exploitation [...] an extreme form of exploitation" (2011, 298).

As the all-too-brief tour through the nomenclatural history of the unwaged digital labour debates indicates, there has been, from the beginning, a penchant to focus on its critical political economic aspects in tandem with its exploitative dimensions. While this emphasis is accurate and correct, the authors that expound its exploitative dimensions have yet to appreciate or take into consideration a number of other markers that distinguish unwaged digital labour from its historico-conceptual predecessors. In what follows, then, the exploitative conditions under which the vast majority of content generators operate are acknowledged, but a more rounded and nuanced version of this labour is also offered. This is done in an attempt to better conceptualize the place of unwaged digital labour within the current mode of capitalist globalization as well as to point to the manner in which it is also cracking the ahistorical veneer of the capitalist social relation. By doing so, this kind of unwaged labour is allowing some light to be shone on fundamentally autonomous modes of production that posit the faint possibility of one day eclipsing the exploitative dimensions of the wage relation. Even if ever so faint, this possibility requires that we further examine it and reflect upon its radical potential.

\subsection{Facet One: The Autonomy of Unwaged Digital Labour}

It is no coincidence that the theoretical lineage upon which much of the debate surrounding the unwaged digital labour thesis can be broadly described as sympathetic to the perspective provided by autonomist Marxist theory. Originating in Italy in the 1950s, autonomist theory is predicated on the argument that labour and capital are engaged in recurrent and everexpanding "cycles of struggle" (Dyer-Witheford 1999, 62-90) that mutate the composition and form of each party according to the offenses and defenses launched by their other. Catalyzing these cyclical struggles is the ever-present potential autonomy of labour in its relation to capital. That is, whereas capital is ultimately reliant on labour as its primary source of profit, autonomist theory argues that the inverse relation does not apply. While capital cannot survive without labour, labour is capable of organizing and managing its creative and productive capacities free of the capitalist social relation. In other words, "Capital, a relation of general commodification predicated on the wage relation, needs labor. But labor does not need capi- 
tal. Labor can dispense with the wage, and with capitalism, and find different ways to organize its own creative energies: it is potentially autonomous" (Dyer-Witheford 1999, 68). It is the persistent desire to organize these capacities autonomously and beyond the exploitative purview of the capitalist social relation that catalyzes the cyclical struggles between labour and capital. When considered from the perspective provided by unwaged digital labour, nowhere is the potential autonomy of labour more evident than on the self-managed and selforganized networks of Web 2.0 in general and social media in particular. It is for this reason that the perspective provided by autonomist theory is highlighted as the first facet of this theoretical framework.

Free of any kind of management oversight, unencumbered by supervisory directive, absent a structured work environment or duration, removed from the organizational forms so characteristic of hierarchical capitalist command and control, and, undeniably free of the wage relation, unwaged digital labourers devote prodigious amounts of time, energy, intellect, and affect to projects that have the capacity to harness the collective intelligence of a global network of content generators, working at all times of the day, and in every corner of the world. While the owners of Web 2.0 sites and services urgently rely on this globally distributed, yet interlinked, network of content generators, the inverse relation does not hold. This pivotal point is made clear by reference to one of the now ossified fossils of the Web 2.0 era-MySpace.

Rupert Murdoch's News Corporation purchased MySpace at its popular apogee in 2005 for an estimated US $\$ 580$ million (Rushe 2011). Six years later, and in the wake of a mass exodus from the site towards Facebook, News Corporation sold an emaciated MySpace for a paltry US $\$ 35$ million. This example points to the resolute dependence of high-technology capital on the surplus value produced by their unwaged digital labour force. However, it also points to the mobility and elemental autonomy of this same labour force, underscoring their autonomous and peripatetic nature.

The autonomous nature of labour is not unique to its unwaged digital variant. Free lancers and the self-employed, for instance, have some level of autonomy that resembles the autonomous nature of unwaged digital labour. Digital labourers, in general, have much more autonomy in their day-to-day activities than did industrial workers of the past for instance. However, there are two fundamental differences that distinguish the autonomy of unwaged digital labourers from waged digital labourers. The first is the complete and utter lack of a boss, manager, client, or supervisor in the social relations that constitute the work environment. The second is, as the terminology indicates, the absence of a wage.

In regards to the first, unlike waged digital labourers, unwaged digital labourers do not undertake a project, task, assignment, or job in response to the directive provided by a superior-whether that superior be a client, boss, or manager. Unwaged digital labourers work on projects they choose for themselves and in collaboration with a group of networked peers. Regarding the second, the absence of any kind of financial remuneration distinguishes unwaged digital labour from its waged variant. The lack of financial incentive in relation to the amount of labour taking place and the profits accrued is one of the most significant elements of unwaged digital labour that marks it as distinct from its waged other.

Importantly, the fundamental autonomy of unwaged digital labours does not extend into all areas of their existence. For instance, in order to do the labour they do on social networks, these individuals must have a source of income that provides them with the financial resources to purchase the hardware and software required to participate on these same networks. That is, they must exchange their labour for an incommensurate wage on the labour market with an institution that relies on exploiting this labour to turn a profit. And while the relative absence of autonomy in the waged labouring environment is, in a manner of speaking, prerequisite to labouring in the unwaged digital environment, the non-autonomous nature of one environment should not preclude appreciation of the autonomy evident in another.

More than capable of managing and organizing their own labour free from the structures of hierarchical control so characteristic of capitalist enterprise, this labour force assembles, disassembles, aggregates, and disaggregates according to popular whim and fancy. It merits mention, however, that as individual content generators devote more and more time and ef- 
fort to their particular plot of virtual land within a particular social network, the likelihood of them migrating to another platform diminishes as they become further and further tied, or "locked-in," to that domain. The diminishing potential for virtual migration is explained by the fact that if these individuals were to uproot themselves from Facebook's proprietary servers, for instance, the labour required to "start anew" on another plot would be substantial. However, acknowledging the potential of "lock-in" does nothing to negate the fundamental autonomy of this labour force in any way. Whether labouring on a proprietary, for-profit social network or, instead and perhaps, on a commons-based social network organized around an entirely different logic with an entirely different amalgam of social relations, the autonomy of the unwaged digital labour force is resolute.

As alluded to above, one of the most important aspects of the autonomy of unwaged digital labourers that differentiate them from their waged counterparts is the fundamental lack of hierarchical command and control characteristic of industrial or corporate environments. Instead of the chain of command dictating what gets produced, when, how, and where, unwaged digital labour is guided by a much more unstable form of organization. Axel Bruns (2008) argues that instead of hierarchy, projects that rely on a mode of "produsage" (Production + Usage) are organized along heterarchical lines. Rather than having a waged employee assigned to a determinate job and a fixed position within the corporate hierarchy that excludes all of his/her other potential contributions, within a heterarchical form of organization,

there is no prior formal filtering for participation, but rather [...] it is the immediate practice of cooperation which determines the level of expertise and level of participation. [This] does not deny 'authority,' but only fixed forced hierarchy, and therefore accepts authority based on expertise, initiation of the project, etc. (Bauwens, 2005 quoted in Bruns, 2007).

This authority is not based on a rigid model that fixes decision-making and directive in the body and mind of a single individual - the CEO or Supervisor, for instance. It is, rather, a temporary and amorphous form of authority, transferred from one person to the next, which responds to the idiosyncratic nature of the problem or task at hand by calling upon the aptitudes of those self-nominating individuals capable of helping to resolve it. The parallel between Bruns' concept of heterarchy and the organizational form of the Occupy Movement merits brief mention at this point so as to further emphasize their importance below. The contingent and provisional meritocratic qualities of this heterarchical arrangement of 'produsers' is fundamentally different than the hierarchical and rigid division of labour evident in the industrial or corporate sector.

Unwaged digital labourers, then, "lead by example, not by coercion, by merit, not by power inherited from a position in the hierarchy, by consensus, not by decree" (Bruns, 2008, 30). As the above description of the fundamentally autonomous nature of unwaged digital labour indicates, the potentials for this labour force to organize itself and its productive/creative capacities free of the wage relation is enormous. By overlooking this fundamental autonomy, we misjudge the present, though nascent, viability of form of organization that exists beyond the "negative externalities" characteristic of the capitalist relation. However, as the concept of autonomy and the cycles of struggle indicate, capital, a social relation dependent on the exploitation of others, will not slacken its grip without a fight.

The impetus behind these forms of networked struggle is often characterized as having to do with one's 'privacy' on networks that are eminently social. When considered from the perspective provided by the exploitative relationship between owners and labourers, in addition to considering the highly personal registers that absorb the brunt of this exploitative scenario, the second facet of unwaged digital labour is in need of further clarification.

\subsection{Facet Two: Hyper-Exploitation}

Without doubt, the "exploitation paradigm" is the facet that has been addressed most comprehensively by the academic literature regarding unwaged digital labour. The popularity of the "exploitation paradigm" is attributable to the long history of the communications and cultural industries and the manner in which they were, and remain, profitable enterprises. Based 
on the illuminating work of Dallas Smythe and his concept of the "Communications Blindspot" (1977), the modus operandi of corporate entities devoted to the business of media and communications has, despite tremendous alterations in the technologies of production, distribution, and reception, changed very little.

Well before the rise of the Internet, Smythe argued presciently that while the mass media produce copy, stories, images, films, photographs, and videos, they are primarily in the business of producing a commodity far more valuable than these trifling end products. First and foremost, communications industries old and new are in the business of producing and selling audiences. They bring these audience commodities to market so as to sell them to advertisers and marketers eager to ply their wares and services to eyeballs and intellects allegedly interested in the products/services on offer. While the copy, programming, dialogue, photographs, video, etc. are indeed end-products, they are, according to Smythe, but a 'free lunch' meant to entice a captive audience into consuming much more lucrative productsadvertisements and marketing appeals. Similar to the act of offering patrons at a pub free pretzels or peanuts in an attempt to whet their palates so that they might then consume a much more valuable commodity-alcohol primarily-the process of whetting the cognitive and emotive palates of media consumers with an array of 'free' stories or services that in turn then entice the consumption of much more valuable product, advertisements, media industries have long traded in the attention economy. The "audience commodity", however, is becoming ever more valuable as we move from an era defined by broadcasting to one dominated by narrowcasting, niche markets, the long tail, and "big data".

Numerous scholars have charted these developments. One of the most consequential, in terms of the transition from basic demographic research to much more finely tuned psychographic, behavioural, and bio-demographic research, is Mark Andrejevic. In a number of different texts, Andrejevic argues $(2002,2007,2009)$ that the development of more and more personalized media hardware and software has, at base, the intention of producing more and more accurate information regarding the specific predilections of an ever more refined audience commodity. Limiting his analysis to Digital Video Recorders (DVR) and couching his analysis in the Taylorist Paradigm of Scientific Management (Taylor 1915), Andrejevic argues that these digital contrivances are designed with the intention of tracking each and every television program the audience member views and/or records. He argues:

Even as it retrieves programming for viewers, the [DVR] doubles as a monitoring device in the service of the system's operators, creating a detailed 'time and motion' study of viewing habits that can be sold to advertisers and producers. In the panoptic register, the [DVR] becomes an automated confessional: an incitement to divulge the most intimate details of one's viewing habits. $(2002,240)$

By divulging the "most intimate details of one's viewing habits", the audience member is communicating a vast amount of information to a service provider regarding individualized preferences, patterns of consumption, and behavioural predispositions that can be crossreferenced with other demographic information such as location and average household income, so as to compile an accurate composite image of what this individual likes to watch, when s/he likes to watch it, where s/he is located, and how much disposable income s/he might have. This information is invaluable to media industries in that they can then offer their most valuable clients, advertisers, explicit information regarding the audience that is exposed to their commercial pleas.

If we extend this argument into the present day and age, as many of the most well regarded critical political economists of new media have done, what obtains is a composite profile of not only what a particular audience member likes to watch and when, but much, much more. Taking into consideration mobile communications, ubiquitous computing, "always-on" social networking, and the agglomeration of thousands of "likes", "+'s", and "retweets", advertisers not only get a sense of the consumptive patterns of the audience commodity, but are provided with a micro-detailed profile of every movement, purchase, 
habit, and location, in addition to the age, gender, and familial situation of this ever more unalloyed audiece commodity. An automated (digital) confessional indeed!

As mentioned, this situation is accurately described as exploitative. This is especially the case when the responsibility for generating all of the finely tuned information found on social media profiles falls to those same individuals who's attention is being bought and sold by the platforms upon which they labour. The work of Fuchs' is without question the most accurate and rightly acerbic in this regard. He believes that when 'users' begin generating content on Web 2.0 sites and services or data for search engines and, by doing so, generating value for the site,

\begin{abstract}
in terms of Marxian class theory, this means that they also produce surplus value and are exploited by capital as for Marx productive labour is labour generating surplus. Therefore the exploitation of surplus value in cases like Google, YouTube, MySpace, or Facebook is not merely accomplished by those who are employed by these corporations for programming, updating, and maintaining the soft- and hardware, performing marketing activities, and so on, but by wage labour and the producers who engage in the production of user-generated content. $(2009,30)$
\end{abstract}

In fact, according to Fuchs, this situation is better thought of as "one of infinite overexploitation [...] [or] an extreme form of exploitation" $(2011,298)$.

\title{
2.3. Facet Three: Resistance \& Struggle
}

Resistance to forms of exploitation is not unique to unwaged digital labour. However, the form of struggle that manifests within the unwaged digital domain is. For the most part, these struggles have been mischaracterized and, hence, misunderstood as pertaining to the violation of one's privacy. Whenever there is a controversy on a platform that exploits the unwaged digital labour of their "user" base, this controversy is misconstrued as one pertaining to the invasion of one's privacy. This mischaracterization has obfuscated a more accurate understanding of the registers at which this exploitation is experienced and, therefore, the forms of resistance that meet it. Once again, nomenclature is important in this regard.

For the most part, these instances of revolt are recurrently, simplistically, and myopically characterized as controversies pertaining to the invasion of one's 'privacy' on networks that are eminently social. Often, when a social network alters its Terms of Service (TOS) or its End User License Agreement (EULA) so as to extract more information and data from the profiles of a "user", there is resistance to these alterations. For instance, in December 2012, Instagram, a popular photo-sharing platform acquired by Facebook for US\$1 Billion (Raice and Ante, 2012), altered its Terms of Service in such a way that would allow them to sell the personal photographs of its members to advertisers. The member's, whose photograph or likeness would be portrayed in these advertisements, would receive no compensation for the use of said images. The images in question had already been uploaded to the service and were already being shared by these individuals. They were, then, anything but private. Therefore to characterize the ensuing outrage as pertaining to the violation of one's privacy is incorrect. Not surprisingly, Instagram's members resisted this form of exploitation and set about struggling against the changes to the social network's TOS. In an abrupt about-face, Instagram's owners withdrew their amended TOS (see Streitfeld and Perlroth 2012). This reversal further underscores the autonomy of peripatetic unwaged digital labourers and the fact that even in the era of social media, "Capital [...] needs labor. But labor does not need capital" (Dyer-Witheford 1999, 68).

Further evidence for resistance to (or struggles against) this form of exploitation would serve to bolster the claims made by theorists that subscribe to and defend the "exploitation paradigm". Brown (2013) provides such evidence by recasting the multiple instances of "user" uproar and revolt that have occurred, and will surely continue to occur, on Web 2.0 sites and services along more appropriate and accurate theortical lines than those associated with the violation of "user" privacy. However, "Privacy and social media are con- 
ceptually oxymoronic in that adherence to the principles of the former would render pointless the purposes and functionality of the latter" (Brown 2013, 386). Therefore, a more adequate and accurate explanation for the multiple instances of "user" uproar on Web 2.0 sites and services is one that takes into account the exploitative nature of these sites and services and the intimate registers at which this exploitation is experienced. Via recourse to Marx's ideas regarding "so-called primitive accumulation" (1976, 873-940) and Hardt and Negri's characterization of the exploitation of the personal and private moments of one's life as "biopolitical exploitation" $(2009,137)$, the author argues that:

\begin{abstract}
Privacy is inimical to the primary purpose of the networks themselves. These are spaces where individuals share personal moments openly and freely with friends and acquaintances that sometimes number in the thousands. In such realms, concern over privacy may be one way of understanding the kinds of protest and struggle that have taken place but [...] is not the best way. Much better at acknowledging the complexity of this context and the nuance of these struggles is the theoretical framework offered by reinterpretation of Marx's concept of primitive accumulation by way of Hardt and Negri's theory of biopolitical exploitation. (Brown 2013, 398)
\end{abstract}

In this way, when the personal relationships, intimate communicative practices, innermost affective responses, and interrelated social habits of individuals are exploited ever more completely, and "user's" gain an appreciation of these exploitative practices, they rightly (and via the channels they have become so adept at leveraging) vocalize their discontent and begin to reassert their autonomy. While the invasion of one's privacy is one way of understanding the multiple instances of uproar to have occurred on Web 2.0 sites and services, it is not the most accurate. Instead, understanding these instances of uproar as stemming from the biopolitical exploitation of members' affective relations is a more nuanced and appropriate explanation. Therefore, it is not only the presence of the exploitative social relationship that exists between owners and "users", but also resistance to this biopolitical form of exploitation that targets the highly collaborative and cooperative social relationships obtaining between "users" themselves.

\title{
2.4. Facet Four: Collaborative, Cooperative, Communicative \& Communal
}

Unwaged digital labour is inherently cooperative, collaborative, communal and based predominantly on the non-instrumental sharing of digitized content and data between members of Web 2.0 sites and services. Thus, the fourth facet of Unwaged Digital Labour that marks it as a distinct typological category is, on the surface, similar to that which distinguishes waged digital (or immaterial) labour, yet fundamentally different than that which commands and controls one's intimate communicative capacities from above. Lazzarato argues that waged digital labour, what he terms immaterial labour, "produces first and foremost a 'social relationship'" and that it "constitutes itself in forms that are immediately collective" (Lazzarato 1996, 136). Terranova extends the argument put forth by Lazzarato by applying the collective, social, and collaborative nature of waged digital labour to the unwaged realm. She argues:

Unrestricted by physical distance, [unwaged digital labourers] collaborate with each other without the direct mediation of money and politics. Unconcerned about copyright, they give and receive information without thought of payment. In the absence of states or markets to mediate social bonds, network communities are instead formed through mutual obligations created by gifts of time and ideas. (Terranova 2000, 36)

The fundamental difference being, then, that in a situation such as that described by Lazzarato, where the labourer exchanges his or her communicative, affective, and emotive capacities for a wage, "we have here a discourse that is authoritarian: one has to express oneself, one has to speak, communicate, cooperate, and so forth. The 'tone' is that of the people who were in executive command under Taylorization: all that has changed is the content" (Lazzarato 1996, 134). With unwaged digital labour, however, one does not have to do anything. The willingness and eagerness to communicate, collaborate, cooperate, and share 
with others is one that marks "unwaged digital labour" as distinct from its typological and waged predecessor. Members of Web 2.0 sites and services devote vast amounts of time, energy, and emotion to their social network of choice. They collaborate, cooperate, and communicate free of the wage relation, free of any kind of management oversight or supervisory directive, and are responsible for fostering and nurturing a community of peers in such a way that escapes this kind of top-down command and control. This, then, is what differentiates unwaged digital labour and its collaborative, cooperative, communicative and communal qualities from its waged variation.

Don Tapscott and Anthony Williams marvel at the self-organizing and collaborative capacities of unwaged digital labourers, albeit from an ideological position that runs counter to the argument presented herein. Referencing Flickr, a massive photo-sharing social network and early poster-child of the Web 2.0 environment, the authors argue that the owners of Flickr may provide,

\begin{abstract}
the basic technology platform and free hosting for photos [...] Users do everything else. For example, users add all of the content (the photos and captions). They create their own self-organizing classification system for the site (by tagging photos with descriptive labels). They even build most of the applications that members use to access, upload, manipulate, and share their content [...] Flickr is basically a massive self-organizing community of photo lovers that congregates on an open platform to provide its own entertainment, tools, and services. (Tapscott and Williams 2006, 38; emphasis added)
\end{abstract}

The cooperation, collaboration, and non-instrumental sharing immanent to Web 2.0 sites and services is formative of social relations and, thus, as will be discussed in the following section, forms of subjectivity, that allow us to glimpse, even if ever so slightly, the possibility of reorienting our infinitely creative and fundamentally autonomous labouring capacities beyond the exploitation and affective violence immanent to waged digital labouring practices. It is the fundamental autonomy of a self-organizing amalgam of highly cooperative, collaborative, communicative, and communally oriented peers spread throughout the global network, that often work on projects of amazing complexity free of the wage relation and free of any kind of management oversight that distinguishes unwaged digital labour from waged digital labour.

\title{
2.5. Facet Five: The Biopolitical Dimensions of Unwaged Digital Labour
}

The fifth facet of unwaged digital labour is arguably its most significant, certainly its most theoretically complex, and will therefore be examined in more depth of detail than those discussed above. Succinctly, unwaged digital labour is biopolitical. More comprehensively, unwaged digital labour requisitions and activates some of the most personal and intimate faculties of the individual that undertakes and accomplishes it. Over time and through exposure to the autonomous modes of unremunerated cooperation and collaboration that are the prerequisites to participation on these networks of affiliation, the subjectivity of the individual (in communion with the collective) is altered in biopolitically significant ways.

Michel Foucault coined the terms "biopower" and "biopolitics" (1978) in an attempt to provide a more subtle understanding of the myriad relationships of power that discipline and control the production and regulation of individual and collective subjectivity. Less obvious than monarchical power, more subdued than the power of the State and its various 'apparatuses' (Althusser 1971), biopower and biopolitics describe the constitutive influence of a variety of dispositifs (Delueze 1992), apparatuses (Agamben 2009), or biopolitical "machines" (Foucault 1995, 207) on our subjective dimensions. This kind of power over the individual and collective bios (and the political dimensions thereof) discipline and control the actions, behaviours, belief systems, and desires of the individual and collective in such a way that is not immediately apparent, nor recognizable. The various dispositifs, apparatuses, or machines of the biopolitical production of subjectivity arrange "things in such a way that the exercise of power is not added on from the outside, like a rigid, heavy constraint [...] but is so subtly present in them as to increase their efficiency" (Foucault 1995, 206) 
Foucault's most celebrated examples of biopolitical dispositifs, apparatuses, or machines are, of course, the architectural arrangement of light, brick, and glass characteristic of Bentham's panoptic prison, the discourse surrounding sexuality, and the classificatory function of psychiatric institutions. According to Foucault, biopower relies not on physical "force to constrain the convict to good behaviour, the madman to calm, the worker to work, the schoolboy to application, [nor] the patient to observation of the regulations" (Foucault 1995, 202), but on much more diffuse and nascent forms of suasion that discipline and control its subjects as if from the interior. In an infamous description, under regimes of biopower, the relationships of power that produce and regulate our subjective dimensions become "capillary" (Foucault 1980,96 ). Extending this idea into the contemporary era (and in light of the modifications to have taken place at the level of our digital labouring capacities) they have also become neuronal.

There exist a plenitude of biopolitical dispositifs, apparatuses, or machines in addition to those of the prison, the discourse characteristic of eighteenth century sexuality, and/or the psychiatric institution. Each has a consequential influence on the production and regulation of subjectivity. The most important of which, for the present purposes at least, is the biopolitical dimension of digital labour in general and unwaged digital labour in particular. According to Lazzarato, in the opening salvo of what has become, as this special edition of this journal indicates, one of the most hotly contested concepts in academia, waged "immaterial labour" (or what is more accurately referred to throughout this paper as "waged digital labour") is productive of subjectivity and thus can be considered, by extension, biopolitical. In a passage that speaks to the biopolitical impact of waged digital labour without naming it, Lazzarato argues: "[t]he concept of immaterial[/digital] labor presupposes and results in an enlargement of productive cooperation that even includes the production and reproduction of communication and hence of its most important contents: subjectivity." He continues,

[w] hat modern management techniques are looking for is 'the worker's soul to become part of the factory.' The worker's personality and subjectivity have to be made susceptible to organization and command [...] [and] [t]he capitalist needs to find an unmediated way of establishing command over subjectivity itself; the prescription and definition of tasks transforms into a prescription of subjectivities. (Lazzarato 1996, 134)

Making more complex Lazzarato's work, Michael Hardt and Antonio Negri make explicit the link between Foucault's concept of the 'biopolitical' and Lazzarato's argument regarding the subjective dimensions of immaterial/digital labour by claiming that 'waged immaterial/digital labour' is biopolitical. Waged digital labour is,

biopolitical in that it is oriented toward the creation of forms of social life; such labor, then, tends no longer to be limited to the economic but also becomes immediately a social, cultural, and political force. Ultimately, [...] the production involved here is the production of subjectivity, the creation and reproduction of new subjectivities in society. Who we are, how we view the world, how we interact with each other are all created through this social, biopolitical production" (2004, 66; emphasis in original).

In Commonwealth (2009), the authors go so far as to rename "immaterial labour", "biopolitical labour" in an attempt to emphasize its influence of the subjectivities of those who exchange their communicative and cooperative capacities for a wage. In other words, waged digital labour is biopolitical in that it requisitions, purchases, and, over time and through exposure, eventually commandeers one's ability to communicate and cooperate with others. As a result of the personal, affective, and communicative faculties, aptitudes, and talents this kind of work requisitions and progressively comes to control, the subjectivity of those individuals tasked with undertaking and accomplishing it is progressively colonized by the demands and dictates of those persons who have purchased the right to control it via the wage relation. By being forced to repeatedly communicate in the service of someone else, build personal and social relationships premised on the needs and desires of one's superior, conjure ideas, images, and affects that attend to the will of one's boss, and, thus, progressively ex- 
change control over these subjective capacities for a wage, the subjectivity of the worker is ever-more, though not entirely, overtaken by the needs, wants, and desires of their bosses. This kind of labour, then, is eminently biopolitical in that it purchases, commandeers, and regulates some of the most intimately subjective capacities of the individual as if from the interior. The pivotal point not addressed by the body of literature regarding the biopolitics of digital labour in general, however, is that its unwaged variant too is also biopolitical and constitutive of different forms of subjectivity than that of its waged genus. These different forms of subjectivity result from fundamentally different political economic relationships that exist between members of Web 2.0 sites and services themselves, rather than those that exist between waged digital labourers and their superiors.

Based on a fundamentally autonomous mode of digital production that is heterarchical, highly cooperative, collaborative, and founded on the non-instrumental sharing of the fruits of one's unwaged digital labour, the biopolitics of this same labour have opened up a space in our individual and collective consciousnesses that recalibrates "[w] ho we are, how we view the world, [and] how we interact with each other" (Hardt and Negri 2004, 66). The elemental autonomy of the unwaged digital labour taking place on and within these self-organized, selfmanaged, and self-regulated networks of affinity and recognition, passes the subjectivities of those individuals who populate, navigate, and participate in them through a different die. By doing so, they extrude a different form of subjectivity than that present prior to their appearance. This fundamentally autonomous amalgamation of individuals that come together on distributed networks that are, for all intents and purposes, leaderless, heterarchical, selfmanaged, and self-organized, open up a field of cognitive possibility that heretofore was deemed all but impossible. In doing so, they have posited the nascent feasibility of an organization of labouring bodies and minds, working in all corners of the globe, and free of the wage relation. The biopolitical force of unwaged digital labour is such, then, that it cracks the seemingly impenetrable veneer of the ostensible permanence and ahistorical nature of the hierarchical capitalist organization of life, love, and limb.

Possibilities that were inconceivable a few short years ago no longer seem absurd. Potentialities that at one time could not be dreamt appear attainable. Latencies that, in the past, may have remained as such, are thrust to fore and allowed the opportunity to prove their viability. If the biopolitical mechanisms of previous institutional and organizational forms moulded, disciplined (Foucault 1995) and controlled (Delueze 1992) the subjective dimensions of the individual and collective, then new biopolitical mechanisms have the potential to mould entirely different subjects. It is, then, the cognitive dimensions unlatched by the particular biopolitical mechanisms of unwaged digital labour that structure a new field of possibility and make viable a mode of social organization considered unimaginable a few short years ago.

One of the most consequential biopolitical dimensions of unwaged digital labour, therefore, is that it is based on a fundamentally different mode of production that comes to influence the subjectivities of those exposed to it. Referencing Marx, Jason Read argues:

The immanent relation between production and the production of subjectivity announces itself most forcefully at the points in Marx's writing where he deals with the problem of a transition from one mode of production to another. At these points of historical transformation, where one mode of production is destroyed and another is constituted, Marx underscores that such a transformation is impossible without a corresponding transformation of subjectivity. (Read 2002, 131)

He continues, arguing "Reproduction of a social relation is also reproduction of a particular form of subjectivity" (Read 2002, 134). The social relations that exist between members of Web 2.0 sites and services themselves are of a different type and kind than those that exist between owners and members. While others have dealt with the exploitative dimensions of the latter relationships, it is the forms of subjectivity produced by the former that are of particular interest. 
The biopolitics of unwaged digital labour have opened up "a whole field of responses, reactions, results, and possible inventions" (Foucault 1982, 789) that heretofore were never deemed viable. They have subtly rediscovered cognitive, communicative, and affective spaces from which we have begun to imagine new forms of heterogeneously collective, commons-based, and autonomous forms of self-organization and direction. It merits acknowledging that this unapologetically optimistic account of the biopolitical dimensions of unwaged digital labour is not without its sceptics or detractors. There are those who regard social media as emblematic of the further entrenchment of a neo-liberal subjectivity that forces users to self-brand (Hearn 2008) and market themselves as a commodity to others. Alice Marwick, for instance, argues that members of Web 2.0 sites and services "tend to adopt a neoliberal subjectivity that applies market principles to how they think about themselves, interact with others, and display their identities" $(2013,7)$. While these perspectives are important to acknowledge, they approach their object from a vantage point that does not take into account the biopolitical influence of the fundamental autonomy of unwaged digital labour and the evidence provided by another, equally important, set of evidentiary instances.

In an attempt to engage in this important debate, what follows will provide evidence for the impact of the biopolitical dimensions of unwaged immaterial labour on individual and collective subjectivity beyond that which regards it as nothing more than another incursion of neoliberalism into the subjective dimensions of individuals and collectives. This evidence can be found in nascent form within the organizational and communicative spaces leveraged by the multitudinous social movements that burst onto the global stage in 2011 and 2012.

\section{Audacious Imaginings}

In response to the Occupy Movement and the events of the "Arab Spring", Hardt and Negri published Declaration (2012). In the final paragraph of this text, the authors address one of the most consistent and (for some) confounding aspects of these diverse and heterogeneous movements: their apparent disorganization and the putative weaknesses associated with a leaderless movement that lacks a coherent and consistent set of sound-bite-friendly demands or purposes. Taking into consideration the different organizational forms adopted by these movements, Hardt and Negri write:

Don't think that the lack of leaders and of a party ideological line means anarchy, if by anarchy you mean chaos, bedlam, and pandemonium. What a tragic lack of political imagination to think that leaders and centralized structures are the only way to organize effective political projects! (Hardt and Negri 2012, 91)

However, the 'tragic lack of political imagination' associated with regarding a fundamentally heterogeneous and leaderless movement as somehow anarchic, confused, or disorganized, has begun to give way in light of the biopolitical force of unwaged digital labour and the evidentiary feasibility of such associative forms made manifest via the channels of social media and Web 2.0 sites and services. The experience of labouring on autonomous networks of loose affiliation based on heterarchical modes of organization and production "falls back on the process" (Read 2002, 115) and in so doing unhinges the bung on an anything-but-tragic imagination that can perhaps (for the first time) glimpse the feasibility of such an audacious prospect. Put simply, the biopolitics of unwaged digital labour make possible thoughts and imaginings regarding 'a whole field of responses, reactions, results, and possible inventions' that in the past might never have been considered.

The primary argument that this paper has been leading up to is the following. The biopolitical dimensions of unwaged digital labour posit the nascent viability and latent feasibility of a fundamentally autonomous and heterogeneous organization of individuals that remain as such within a collective. They have, in a manner of speaking, freed the imagination of these same individuals regarding the capacity to organize themselves and their creative capacities in a similar fashion in the material world. Dyer-Witheford argues correctly that "It is nothing if not audacious to discern" $(1999,87)$ such a composition of heterogeneous individuals, movements, and alliances, but it is becoming ever less so as a result of the experience and 
biopolitical force of just such an affinity of alliances made and fostered via unwaged digital labouring practices.

According to Jason Read in a passage referring specifically to the changed mode of production characteristic of waged digital labour:

Mutation of the [mode of production] does not simply alter what can be produced, or how, but it falls back on the process, transforming the producer himself or herself. The production of things is also always an autopoieis, a production of the one producing-a production of subjectivity. As Marx writes with respect to the laborer, 'Through this movement he acts upon external nature and changes it, and in this way he simultaneously changes his own nature.' (2003, 115; emphasis added)

The fundamental point being, once again, that we must not limit the application of this theoretical observation too narrowly to the point that it applies exclusively to the waged digital labouring environment. Rather,

we should keep an eye out from the beginning, following Foucault's intuition, for how biopolitical production, particularly in the ways it exceeds the bounds of capitalist relations constantly refers to the common, grants labor increasing autonomy and provides the tools or weapons that could be wielded in a project of liberation. (Hardt and Negri 2009, 137)

In this manner, we need to regard all of the unwaged digital labour taking place on Web 2.0 sites and services as an elementary stage in fashioning the organizational, cognitive, and affective "tools or weapons that could be wielded in a project of liberation".

The radical experimentations with direct democracy and leaderless modes of organization undertaken by a variety of social movements the world over, which on their surface are, indeed, "nothing if not audacious", are made less so because of the experience of activists (and the public at large!) on social media sites and services accustomed to exactly this kind of (dis)organizational environment. The ostensible impossibility of a leaderless organization of heterogeneous and autonomous individuals is, therefore, made much less so as a result of the experiences of activists, protestors, and the public at large on Web 2.0 sites and services. The biopolitics that guide and regulate the production and regulation of subjectivity, that act upon the present or future actions of these same participants, have escaped their virtual confines and have come to influence a jubilant imagination that dares to dream beyond the cybernetic sphere.

The ray of hope shone by the significance of the encounters that individuals have with the various biopolitical dispositifs, apparatuses, or machines that fashion and refashion their subjectivities is that they are in a perpetual process of becoming something and someone other than that which they presently are. According to Hardt and Negri, "The most important fact about human nature (if we still want to call it that) is that it can be and is constantly being transformed" $(2009,191)$. At base, subjectivity and 'human nature' are, then, historical. This fact postulates a malleable subject, one that is in a constant process of change, influenced by the biopolitical "machines" through which s/he passes. According to Read, "transformations in technology, politics, media, and the economy affect each other insofar as they produce new subjectivities and new relations" $(2001,28)$. The importance of this realization is that the transformations evinced by the technologies and social relations emblematic of Web 2.0 "produce new subjectivities and new relations" that inform the means by which we may become something other, indeed something much better, than that which we presently are. Our world, then, "is continually made and remade by the bodies and desires of the many, thus exposing the way in which the world can be made otherwise" $(2001,30)$.

This shift in thinking towards a possible or future self is consequential in that "Once the temporal horizon of a possible future replaces the spatial confines of an existing sphere [...], the standard by which the present is judged could expand to visions of what we might want rather than the defense of what we already have, know, or are" (Weeks 2007, 248). In other words, "Through the production of subjectivity, the multitude is itself author of its perpetual becoming other, an uninterrupted process of collective self-transformation" (Hardt and Negri 
$2009,173)$ that is influenced in a consequential fashion by the experiences and modes of autonomous production characteristic of unwaged digital labour. It is not only that the biopolitics of unwaged digital labour 'fall back upon the process,' then, it is that they provide subtle evidence of a differential mode of existence made manifest in the audacious and jubilant imagination that briefly exerted itself in the global wave of revolt and struggle witnessed in 2011 and 2012.

\section{Conclusion: Audacity Unhinged}

In the fall of 2011, a wave of protests and Occupy Movements that began in Zucotti Park in New York City quickly spread around the world. The demands of these movements were diverse and their goals and tactics equally so, but the variegated forms of inequity and widespread lack of opportunity they were railing against, all too common. Drawing inspiration from the struggles in Egypt, Libya, and Syria, the student protests in the United Kingdom, the antiausterity movements in Greece, the struggles of the Spanish Indignados, and the complete lack of culpability placed at the feet of those persons responsible for the worst Global Financial Crisis (GFC) in world history, the Occupy Movement was concrete evidence that the recomposition of a 'class' hostile to imperial capital was beginning to take embryonic form.

At the technological epicentre of this movement were a number of social networks that enabled the transmission and receipt of digitized messages, photographs, and video. With the so-called "Twitter Revolution" in Tunisia (Zuckerman, 2011), the role of BlackBerry's Messenger application in the student riots in London (Wasik, 2011), and the place of Facebook in Egypt's Tahrir Square, not to mention the "99\%'s" tumblr blog, the relative importance of social networks and communication technologies to forms of struggle and resistance is a hotly debated topic. This paper has no interest in engaging with the argument regarding whether or not these were "Twitter Revolutions" or "Facebook Revolutions". What it is interested in is better understanding the manner in which the biopolitical dimensions of all the unwaged digital labour taking place on these networks has "fallen back on the process" and produced forms of subjectivity that no longer dismiss the feasibility of a leaderless, heterarchical, and unapologetically heterogeneous movement as a practical impossibility. Social networks like Twitter or Facebook, therefore, should not be thought of as determining factors in these struggles, but as dispositifs, apparatuses, or "biopolitical machines" endowed with particular affordances that work upon the subjectivities of those persons who daily use them. In other words, these technologies have not and could never change the world on their own, but, paraphrasing Marcuse $(1978,32-33)$, the biopolitical dimensions of all the unwaged digital labour required to use them are in the process of changing the subjectivities of those individuals who could.

According to a number of different academics, it is at the biopolitical level of the constitution of subjectivity that one of the most important forms of contemporary struggle is focused. For instance, Hardt and Negri, "regard the production of subjectivity [...] as the primary terrain on which political struggle takes place. [They argue,] We need to intervene in the circuits of the production of subjectivity, free from the apparatuses of control, and construct the bases for an autonomous production." (Hardt and Negri 2009, 172). Later on in the same text, they reiterate, "liberation [...] requires engaging and taking control of the production of subjectivity" (Hardt and Negri 2009, 331-332). David Harvey, too, agrees. In an otherwise less than glowing review of Commonwealth, Harvey writes:

Its authors are unquestionably right, for instance, to insist that critical engagement with how subjects and subjectivities are produced is essential if we are to understand revolutionary possibilities and that is something classical Marxism was not adept enough at doing. [...] And they constructively take up Foucault's notion of dispositifs as 'the material, social, affective, and cognitive mechanisms in the production of subjectivity.' Doing so, Hardt and Negri say, 'allows us to conceive the collective production of the common as an intervention in the current relations of force aimed at subverting the dominant powers and reorienting forces in a determinate direction. The strategic production of knowledge 
in this sense implies an alternative production of subjectivity.' And this is where their theory of revolution comes from. (Harvey, Hardt and Negri 2009, 4-5)

Jason Read offers a more succinct variation of the above argument, but he does so by considering the influence of waged digital labour on the production of subjectivity. He argues that,

the stakes of opposing capital are not simply economic or political, but involve the production of subjectivity. In order to oppose capital it will be necessary to engage in a counter production of subjectivity. The tools for this counter-production are already in our hands, in the affective and communicative networks that are created and maintained in our day to day labors. $(2002,141)$

Via an examination of the four-facets of unwaged digital labour in general offered above and a more focused investigation into the biopolitical dimensions of unwaged digital labour in particular, this paper provides a more detailed and nuanced examination of one of the more consequential apparatuses responsible for the production and regulation of a form of subjectivity not only hostile to the 'negative externalities' of neo-liberal capital, but amenable to and capable of imagining a fundamentally different way of organizing individuals and collectives free of said negative externalities.

The audacious and jubilant imagination of a group of autonomous individuals accustomed to heterarchical modes of leaderless self-organization took its first infant steps in 2011 and 2012, then very quickly came crashing to the ground. The knowledge, courage, and strength to take these initial steps, however, did not arise from, nor vanish into, the abyss. Nor did the oppression, violence, and inequality these struggles were railing against. While Web 2.0 sites and services like Facebook and Twitter are often mistakenly characterized as the pivotal implements that brought down the oppressive Gaddafi and Mubarak regimes, for instance, this perspective fails to acknowledge the resolute efforts of the individuals who together fought, suffered, and, in some instances, perished in these struggles. Twitter has never caused a revolution. Neither has Facebook. However, the biopolitics of the unwaged digital labour performed on these networks produce forms of individual and collective subjectivity daring enough to make the demands they did. The viability of just such an amalgam of heterogeneous actors that gathered in virtual and material spaces in an attempt to autonomously organize their infinitely creative capacities was, in the not too distant past, all but inconceivable. Today, however, it has become less so and the struggles that will without question continue are more potent and powerful because of it.

\section{References}

Agamben, Giorgio. 2009. What Is An Apparatus?-And Other Essays. Stanford, California: Stanford University Press.

Althusser, Louis. 1971. Lenin and Philosophy, and Other Essays. Translated by Ben Brewster. London: New Left Books.

Andrejevic, Mark. 2002. The Work of Being Watched: Interactive Media and the Exploitation of SelfDisclosure. Critical Studies in Media Communication 19 (2): 230-248.

Andrejevic, Mark. 2007. Surveillance in the Digital Enclosure. The Communication Review 10 (4): 295-317.

Andrejevic, Mark. 2009. Exploiting Youtube: Contradictions of User-Generated Labour. In The YouTube Reader, edited by Pelle Snickers and Patrick Vondreau, 406-423. Stockholm: National Library of Sweden.

Berardi, Franco. 2009. The Soul At Work: From Alienation to Autonomy. Los Angeles: Semiotext(e).

Brown, Brian A. 2013. Primitive Digital Accumulation: Privacy, Social Networks \& Biopolitical Exploitation. Rethinking Marxism 25 (3): 385-403.

Brown, Brian A. and Anabel Quan-Haase. 2012. A Workers' Inquiry 2.0. tripleC-Communication, Cognition, Cooperation 10 (2): 488-508.

Bruns, Axel. 2008. Blogs, Wikipedia, Second Life, and Beyond: From Production to Produsage. New York: Peter Lang. 
Bruns, Axel. 2007. Produsage: Necessary Preconditions. Produsage. Accessed May 13, 2014. http://produsage.org/node/12

Cohen, Nicole. 2008. The Valorization of Surveillance: Towards a Political Economy of Facebook. Democratic Communique: Journal for the Union of Democratic Communications 22 (1): 5-22.

Coté, Marc, and Jennifer Pybus. 2007. Learning to Immaterial Labor 2.0: MySpace and Social Networks. Ephemera: Theory and Politics in Organization 7 (1): 88-106.

Coté, Marc, and Jennifer Pybus. 2011. Learning to Immaterial Labour 2.0: Facebook and Social Networks. In Cognitive Capitalism, Education and Digital Labor, edited by Michael A. and Ergin, Bulut Peters. New York: Peter Lang.

Dalla Costa, Mariarosa, and Selma James. 1973. The Power of Women and the Subversion of Community. Bristol: Falling Wall Press.

Delueze, Gilles. 1992. Poscript on the Societies of Control. OCTOBER 59 (Winter 1992): 3-7.

Deleuze, Gilles. 2007. What is a Dispositifs? In Two Regimes of Madness: Texts and Interviews, 1975-1995, by Gilles Deleuze and D. Lapoujade. New York: Semiotext(e).

Dyer-Witheford, Nick. 1999. Cyber-Marx: Cycles and Circuits of Struggle in High-Technology Capitalism. Urbana, III.: University of Illinois Press.

Foucault, Michel. 1978. The History of Sexuality. Vol. 1. New York: Vintage Books.

Foucault, Michel. 1980. Power/Knowledge: selected interviews and other writings 1972-1977. Edited by Colin Gordon. Translated by Colin Gordon, Leo Marshall, Leo Mepham and Kate Soper. New York: Pantheon Books.

Foucault, Michel. 1982. The Subject and Power. Critical Inquiry 8 (4): 777-795.

Foucault, Michel. 1995. Discipline and Punish: The Birth of the Prison. New York: Vintage Books.

Frier, Sarah, and Leslie Picker. 2013. Twitter Stays Mum on Profit on Roadshow Ahead of IPO.

Bloomberg. Accessed May 13, 2014. www.bloomberg.com/news/2013-10-31/twitter-stays-mum-onprofit-on-roadshow-ahead-of-ipo.html

Fuchs, Christian. 2009. Social Networking Sites and the Surveillance Society. Vienna: Forschungsgruppe. Online edition: http://fuchs.icts.sbg.ac.at/SNS_Surveillance_Fuchs.pdf.

Fuchs, Christian. 2010. Labor in informational capitalism and on the internet. The Information Society 26 (3): 179-196.

Fuchs, Christian. 2011. Web 2.0, Prosumption, and Surveillance. Surveillance and Society 8 (3): 288309.

Fuchs, Christian. 2012. Dallas Smythe Today-The Audience Commodity, the Digital Labour Debate, Marxist Political Economy and Critical Theory. Prolegomena to a Digital Labour Theory of Value. tripleC_Communication, Capitalism \& Critique 10 (2): 692-740.

Fuchs, Christian. 2012. Google Capitalism. tripleC_Communication, Capitalism \& Critique 10 (1): 4248.

Fuchs, Christian, and Sebastian Sevignani. 2013. What is Digital Labour? What is Digital Work? What's Their Difference? And Why Do These Questions Matter for Understanding Social Media? tripleC_Communication, Capitalism \& Critique 11 (2): 237-293.

Hardt, Michael and Antonio Negri. 2000. Empire. Cambridge, MA: Harvard University Press.

Hardt, Michael and Antonio Negri. 2004. Multitude: War and Democracy in the Age of Empire. New York: Penguin Press.

Hardt, Michael and Antonio Negri. 2009. Commonwealth. Cambridge, MA: The Belknap Press of Harvard University Press.

Hardt, Michael and Antonio Negri. 2012. Declaration. e-book: Argo-Navis.

Harvey, David, Michael Hardt, and Antonio Negri. 2009. Commonwealth: An Exchange. Artforum International (November): $210+$.

Huws, Ursula. 2003. The Making of a Cybertariat: Virtual Work in a Real World. London: Merlin.

Kleiner, Dmytri and Brian Wyrick. 2007. InfoEnclosure 2.0. Metamute. Accessed May 13, 2014. http://www.metamute.org/en/InfoEnclosure-2.0

Lazzarato, Maurizio. 1996. Immaterial Labor. In Radical Thought in Italy: A Potential Politics, edited by Paolo Virno and Michael Hardt, 133-147. Minneapolis, Minnesota: University of Minnesota Press.

MacroAxis. Ford Number of Employees. Macroaxis. November 22, 2013. http://www.macroaxis.com/invest/ratio/F--Number of Employees .

Marcuse, Herbert. 1978. The Aesthetic Dimension. Boston: Beacon Press.

Marx, Karl. 1976. Capital: Volume 1. New York: Penguin Classics.

Marx, Karl. 1975. Marx and Engles Collected Works. Translated by Ben Fowkes. Vol. 34. New York: Intl Publishers. 
Negri, Antonio. 1989. The Politics of Subversion: A Manifesto for the Twenty-First Century. Oxford: Polity Press.

Negri, Antonio. 1991. Marx Beyond Marx: Lessons on the Grundrisse. Brookly, N.Y. : Autonomedia.

O'Reilly, Tim. 2005. What is Web 2.0? Design Patterns and Business Models for the Next Generation of Software. O'Reilly. Accessed May 13, 2014. http://oreilly.com/web2/archive/what-is-web-20.html

Raice, Shayndi and Spencer Ante. 2012. Insta-Rich: \$1 Billion for Instagram. Wall Street Journal. April 10, 2012: B1.

Read, Jason. 2001. The Hidden Abode of Biopolitical Production: Empire and the Ontology of Production. Rethinking Marxism 13 (3/4): 24-30.

Read, Jason. 2002. A Fugitive Thread: The Production of Subjectivity in Marx. Pli 13: 125-146.

Read, Jason. 2003. The Micro-Politics of Capital: Marx and the Pre-History of the Present. Albany, New York: State University of New York Press.

Rushe, Dominic. 2011. Myspace Sold for \$35m in Spectacular Fall from \$12bn Heyday. The Guardian. Accessed May 13, 2014. http://www.theguardian.com/technology/2011/jun/30/myspacesold-35-million-news

Rushe, Dominic. 2013. US CEOs Break Pay Record as Top 10 Earners Take Home At Least $\$ 100 m$ Each. The Guardian. Accessed May 13, 2014. http://www.theguardian.com/business/2013/oct/22/top-earning-ceos-100m-paychecks-record

Smythe, Dallas. 1977. Communications: Blindspot of Western Marxism. Canadian Journal of Political and Social Theory 1 (3): 1-28.

Streitfeld, David and Nicole Perlroth. 2012. Instagram Reversal Doesn't Appease Everyone. New York Times. December 22, 2012: B1.

Taylor, Fredrick Winslow. 1915. The Principles of Scientific Management. New York: Harper \& Brothers.

Terranova, Tiziana. 2000. Free Labor: Producing Culture For the Digital Economy. Social Text 18 (2).

Turkle, Sherry. 2011. Alone Together: Why We Expect More from Technology and Less from Each Other. New York: Basic Books.

Wages For Housework. 1988. Wages For Housework. New Internationalist Magazine. March 1988.

Weeks, Kathi. 2007. Life Within and Against Work: Affective Labor, Feminist Critique, and Post-Fordist Politics. Ephemera 7 (1): 233-249.

\section{About the Author}

Brian A. Brown

Brian A. Brown is an Assistant Professor in the Department of Communication, Media \& Film at The University of Windsor in Windsor, Ontario, Canada. His research and teaching are broadly focused on the progressive political potentials evinced by the rapid adoption and profusion of what can broadly be characterized as 'social' or 'new' media. 


\title{
Toward a Political Economy of 'Audience Labour' in the Dig- ital Era
}

\section{Brice Nixon}

\author{
Independent Scholar, Hermosa Beach, USA, bln222@nyu.edu
}

\begin{abstract}
This article contributes to a political economic theory centred on the concept of "audience labour". First, the previous use of the concept of audience labour is briefly traced and the process of rethinking the concept as the basis of a political economic theory is begun. Second, a theory of the audience labour process is developed, drawing on previous theories of audience activities of cultural consumption as productive activities of signification and adapting Marx's theory of the human labour process to the audience labour process. Third, a political economy of audience labour is outlined. As a theory of the basic processes through which communicative capital can control and extract value from audience labour, it describes the exploitation of audience labour and accumulation of communicative capital through distribution relationships of rent and interest. Finally, the continuing centrality of audience labour exploitation in the digital era is discussed.
\end{abstract}

Keywords: Audience Labour, Communicative Capital, Communicative Production, Signification, Cultural Consumption, Dallas Smythe, Karl Marx, Raymond Williams, David Harvey, Exploitation, Rent, Interest

After nearly fifteen years of theorizing and analysing aspects of digital media use as digital labour, ${ }^{1}$ a consideration of the specificity, and importance, of audience labour remains missing. While interest in the political economy of digital labour has continued to grow, there seems to have been no inquiry into audience labour as a specific kind of digital labour. ${ }^{2}$ The ability of scholars to make sense of the political economy of communication in the digital era remains hindered by the lack of attention to the specificity of audience labour. Dallas Smythe introduced the concept of audience labour to the political economy of communication nearly four decades ago, but the concept remained underdeveloped during debates in the 1970s and 80 s about the supposed "audience commodity". In the twenty-first century, the issue of labour has been a focus of a much larger group of scholars through the concept of digital labour and related concepts. However, the kind of labour specifically described by Smythe and others as audience labour is missing from the discussion. This paper argues that audience labour should be made a more central concept in the political economy of communication and attempts to demonstrate the productive potential of that development through an outline of a political economy of audience labour that describes how the audience labour of cultural consumption is exploited, including in the digital era in which "users" and "prosumers" are presumed to have replaced audiences.

However, it is no simple task to make audience labour the focus of at least some research in the political economy of communication going forward. It can neither simply be inserted into the existing body of digital labour scholarship nor recovered from a previous body of scholarship. Instead, it seems necessary to return to the initial conceptualizations of audience labour, beginning with Smythe, in order to be able to begin anew and then go well beyond existing concepts and theories of audience labour. To do so requires dealing with a number of conceptual, theoretical, and methodological issues in terms of both communica-

\footnotetext{
${ }^{1}$ Terranova (2000) appears to be the first to have tried to theorize digital media use as labour-specifically, a kind of "free labour".

${ }^{2}$ One exception is Shimpach (2005), who argues that audiences have always worked and continue to do so in their use of digital media, although their activity has not been recognized as a kind of labour. However, Shimpach does not further develop the conceptualization of the specificities of audience labour, a development I argue is necessary in order to understand how that labour is an object of control and source of value for various industries.
} 
tion and political economy. In many ways, the concept goes right to the core of the old "political economy vs. cultural studies" debates, since audience activities clearly involve cultural consumption and signification but conceptualizing those activities as audience labour is meant to put them within the terms of political economy. Any attempt to put audience activities of cultural consumption into political economy should be done with the intent of avoiding the dead ends of those past debates (Peck 2006; Schiller 1996). In my view, beginning from the concept of audience labour and developing a theory of the audience labour process and its direct relationship to capital circulation and accumulation is precisely the way to do so. In the sections below, I attempt to follow that path through to a basic political economy of audience labour that provides a starting point for understanding the continuing reality of audience labour exploitation in the digital era.

\section{Conceptualizing "Audience Labour" within "Communicative Production"}

In this section, I first outline the brief history of the concept of audience labour. I argue that the early conceptualizations of audience labour provide a useful starting point for a political economy of audience labour but also leave the concept relatively undeveloped. I also argue that more recent concepts of digital labour ignore or do not specify digital audience labour. Second, I discuss the method of a political economy of communication that enables the theorizing of audience activities of cultural consumption as labour in such a way that capital's control over those activities can be clearly understood as a matter of exploitation. Third, I attempt to revive Raymond Williams' effort to theorize communication as production and incorporate audience labour into that effort.

\section{1. “Audience Labour” in the Political Economy of Communication}

In order to develop a political economy of audience labour in the digital era, audience labour must be reconceptualised and separated from the erroneous concept of the audience commodity. Those activities that are specifically audience activities of reading, listening, and viewing - activities of consumption, in the sense of the consumption of meaning - must be recognized as constituting a specific kind of labour and I will follow Smythe and others in calling it audience labour. Furthermore, those consumption activities should simultaneously be seen as activities of production, which makes it easier to see how they constitute a kind of labour. I will consider the product of audience activities in later sections. In this section, I briefly trace the conceptualization of audience labour within the field of the political economy of communication from Smythe in 1977 to theorists of digital labour in the twenty-first century. I argue that audience labour remained a relatively undeveloped concept in the work of the few scholars who considered it in the late-twentieth century. I also argue that audience labour is completely absent from the recent theories of digital labour because the activities of consumption that are specifically audience activities are ignored (or conflated with other activities) while attention is focused on the cultural production of digital media users and the surveillance-based production of data about digital media users.

Audience labour was first put forward as a concept for the political economy of communication in 1977, when Smythe claimed that "western Marxist analyses" had not asked "what economic function for capital" mass communication systems serve; they had only asked what "ideological" function those systems serve (Smythe 1977, 1). Smythe examined the "economic function" and concluded that, "the threshold question" becomes "What is the commodity form of mass-produced, advertiser-supported communications?" (2). His answer to that question was the audience as a commodity. He then asked a follow-up question: What is the audience commodity? His answer to that question was audience labour-power, or audience members' capacity to "pay attention" (4). Advertisers buy audience commodities from media companies, and audience members then work for advertisers by learning "to buy particular 'brands' of consumer goods, and to spend their income accordingly," ie, "to create demand" (6).

Smythe thereby attempted to simultaneously reorient the political economy of communication toward consideration of the audience commodity rather than other media or cultural 
commodities and communication theory toward consideration of audience labour or audience workers rather than the audience as a mass of consumers. But, in the scholarship on the political economy of the audience commodity from Smythe to the present, the specificity of audience labour has been lost. The result, I claim, is that one of the primary aspects of communication as capital, ie, processes of human communication transformed into processes of capital circulation and accumulation, has gone unexamined: the exploitation of audience labour.

To understand audience activities of cultural consumption in terms of a capital-labour relation, those activities must also be understood as being part of a process of production, but Smythe stopped well short of making that move even though he conceptualized audience activity as labour. Smythe opened the way to consider how capital circulates and accumulates through the exploitation of audience labour, but he did not pursue that path. Smythe's starting point was the commodity, which in this case he determined to be the audience-or, more specifically, the capacity of people to perform audience activities. For Smythe, the activity of audiences was a kind of labour, a type of communicative labour. What was typically seen as audience members' consumption of cultural products-eg, watching television shows or reading newspaper articles-was for Smythe the work of ideology or consciousness production (Smythe 1978, 121, 125). Smythe considered the specific character of audience labour, or "the service performed for the advertiser by the members of the purchased audiences" (Smythe 1977, 6), to be the work of "paying attention" for advertisers as part of a marketing process: "[A]udience members [...] learn to buy particular 'brands' of consumer goods, and to spend their income accordingly" (6). Audience labour is the act of "paying attention," by which audience members "learn to buy," and thus the product of audience labour is demand. He concluded that audience labour amounts to "performing the ultimate marketing service for [advertisers]" $(1977,6)$ as "a do-it-yourself marketing agent" $(1978,121)$. But Smythe did not go any further in elaborating on audience labour. He claimed that audience members' cultural consumption should be seen instead as the work of ideology or consciousness production and then proceeded to theorize how that capacity for audience labour had been commodified, ie, he proceeded to construct a political economy of the audience commodity. Smythe's undeveloped concept of audience labour resulted in fundamental errors of political economy in his theory of the commodification and exploitation of that labour.

For Smythe, however, the significant connection between audience activity and capital accumulation was in the realm of consumption in general, hence his concern for advertising and demand management. He claimed that mass media companies facilitate demand management because they produce and sell audiences as commodities to advertisers. In Smythe's political economy of mass communication, "demand management" rather than communication is the real process that is occurring when audience members consume advertisements. He ignored the audience activity of consuming non-advertising content. Starting from the question of communication as capital, rather than Smythe's concern for how mass media facilitate monopoly capital "economically" rather than simply "ideologically," makes "the threshold question" not, "What is the commodity form of mass-produced, advertiser-supported communications?" (Smythe 1977, 2), but rather, how do capitalists appropriate value from communication processes? How is communication treated as capital? How are communication processes treated as processes of capital circulation and accumulation? Audiences are only one part of that story, although they are an important part-the most essential part, in my view.

Sut Jhally and Bill Livant offered one of the two major alternatives to Smythe's political economy of the audience commodity published in the decade following Smythe's initial article. They explored the concept of audience labour and the value of that labour to capital. ${ }^{3}$ However, Jhally and Livant did not advance much beyond Smythe in considering the specificity of audience labour, which also left them with an inaccurate picture of how audience labour is commodified or exploited. Jhally and Livant co-authored an article in 1986 that drew

\footnotetext{
${ }^{3}$ The second alternative political economy to Smythe's was that of Eileen Meehan (1984). Audience labour is completely absent from her political economy of the audience commodity as a ratings commodity.
} 
attention to what they called "the valorization of audience consciousness." They claimed audience labour is work done for media companies and the commodity in question is watchingtime. They also claimed that audience members are only working during the time they are watching advertising and that only a portion of that working time is surplus watching-time, which equals surplus-value or profit (Jhally and Livant 1986, 136). They also claimed that watching programs is not work because the programs are wages in exchange for the watching-time that is work (136). While Smythe's political economy put the audience commodity in the sphere of consumption, Jhally and Livant's $(1986,125)$ political economy put their "watching-time" commodity in the sphere of circulation: "Through advertising, the rapid consumption of commodities cuts down on circulation time and storage costs for industrial capitalists." They did not consider communication as capital itself, only how communication facilitates the general accumulation of capital.

From that limited approach to the political economy of communication, Jhally and Livant were still able to gain a crucial insight that I build upon in the third section: What they called "industrial capital" or "capital-as-a-whole," as advertiser of all the commodities it has produced, pays "media capital" rent for access to audiences (Jhally and Livant 1986, 125); hence, "[m]edia capital [...] receives a portion of surplus value (profits) of industrial capital" (125). However, they did not pursue the category of rent into the realm of distribution, in which rent is one category of the division of surplus-value, presumably because they believed that theorizing watching as working or labouring required them to work within the bounds of production (ie, Capital Volume I), in which capital appropriates surplus-value after it has been produced by labour. They did not consider the possibility that the appropriation of surplus-value by "media capital" does not occur through production, and that media capitalists in their relation to audience labourers ${ }^{4}$ are not like industrial capitalists but rather are similar to landlords, even though they implied as much by using the concept of rent.

Jhally and Livant also produced another insight that I build upon in the next two sections. It is an insight that was only implicit in Smythe: audience labour involves the production of "audience consciousness." Jhally and Livant did not formulate it quite so directly, but they went further than Smythe in specifying what kind of labour audience labour is and what it produces. Jhally and Livant stated in the title of their article that they were concerned with "the valorization of audience consciousness." They described audience labour as the work of watching, which involves "capacities of perception" and is "guided by our attention" (Jhally and Livant 1986, 126). Watching is the creation of meaning (142), or "the process of consciousness" (143).

Since the beginning of the 21 st century, a number of scholars have contributed to the development of a political economy of digital labour. That political economy has generated significant attention to the issue of communicative labour using digital media, or digital labour. Included in that has been a revival of the political economy of the audience commodity. However, the development of that digital labour scholarship has involved the disappearance of audience labour from the political economy of communication.

Terranova (2000) was perhaps the first scholar to offer a detailed consideration of the political economy of digital labour, which she described as "free labour," but she did not consider the audience work of cultural consumption. In the scholarship on the political economy of digital labour, surveillance has received a significant amount of attention as one way companies can profit from digital communication by collecting data about communicative activities, which the scholarship views as digital labour (eg, Andrejevic 2002; 2007; 2011; Cohen 2008; Fuchs 2011a; 2011b; Kang and McAllister 2011; Manzerolle 2010; McStay 2011). The basic political economic theories put forward involve the sale to advertisers of the data gathered through surveillance. Within the scholarship specifically advancing a new political economy of the audience commodity as an update to Smythe's original idea, the supposed selling of users, prosumers, or digital labourers (or their attention) to advertisers has also been the

\footnotetext{
${ }^{4}$ But not in their relation to the cultural labourers they employ to produce cultural commodities, a relation of production that does resemble the relationship between industrial capitalists and their workers, with the latter creating surplus-value in the production process and the former appropriating that surplus-value.
} 
subject of a significant amount of research (eg, Fuchs 2010; 2012; Kang and McAllister 2011; Manzerolle 2010; Napoli 2010). I argue that this scholarship suffers from many of the same errors of political economy that are present in Smythe's original theory and the work of others in the old political economy of the audience commodity. The appropriation of the usergenerated content created by digital labour has also been a focus (e.g. Cohen 2008; Fisher 2012; Fuchs 2010; Terranova 2000). I define that as the exploitation of digital cultural labour. The basic political economic theories put forward claim that digital cultural labour is exploited, although there is no clear link presented between such exploitation and capital accumulation.

The work that Smythe first drew attention to as audience labour and that Jhally and Livant further considered as including "the work of watching" and the production of "audience consciousness" seems to continue to be the most difficult kind of labour to grasp within the political economy of communication. Neither Smythe nor Jhally and Livant were sufficiently specific in their conceptualizations of audience labour, while theorists of the political economy of digital labour have made audience labour completely disappear from the view of the political economy of communication. None of the recent scholarship noted above addresses the fundamental relationship between communicative capital and digital audience labour-the relationship that defines digital media users as consumers of meaning (although they are often also producers) and thereby enables the direct or indirect exploitation of digital audience labour. ${ }^{5}$ That relationship is the control of the means of communicative production used in the process of cultural consumption.

\subsection{The Problem of Method}

In this section, I consider the methodological adjustments necessary for the development of a political economy of audience labour as a theory of communication as capital, cultural consumption as audience labour within communicative production, and the relationship between capital accumulation and audience labour as one in which control over audience activities can be understood as a matter of exploitation. To focus on audience labour within political economic theory requires a consideration of what audience activity is and how that activity is exploited by communicative capitalists-a consideration of the audience labour process and the way in which that process is related to capital accumulation. Smythe $(1978,126)$ insisted the necessary method of integrating audience activity into political economy is a "historical, materialist, dialectical method." I have previously argued that a dialectical method that is also historical and materialist is the necessary method for political economic theorizing of communication (Nixon 2012). In this section, I will expand on that argument in order to develop a method specifically for producing a political economy of audience labour.

Political economy describes "the general characteristics of production at a given stage of social development" (Marx 1978, 224). Political economy is the science of "the sum total" of "the material conditions of life" or the "relations of production." Political economy, as a theory of production in the sense just described, is concerned with what Marx says classical political economy treats as the "generality" in relation to other aspects of the whole mode of production. Production creates the products that are divided up in distribution and formally exchanged between individuals who finally consume them.

[P]roduction, distribution, exchange and consumption form a regular syllogism; production is the generality, distribution and exchange the particularity, and consumption the singularity in which the whole is joined together. [...] Production is determined by general natural laws, distribution by social accident [...]; exchange stands between the two as formal social movement; and the concluding act, consumption, which is conceived not only as a terminal point but also as an end-in-itself, actually belongs outside economics except in so far as it reacts in turn upon the point of departure and initiates the whole process anew. (Marx 1978, 227)

\footnotetext{
${ }^{5}$ See Nixon (2013) for a more detailed discussion of the undeveloped concept of audience labour in Smythe, Jhally, and Livant, and the disappearance of audience labour in the political economy of digital labour.
} 
Marx critiques the "shallow" coherence of classical political economy's "regular syllogism" from the generality of production to the particularities of distribution and exchange to the singularity of consumption, and he puts forward a dialectical relation between production, distribution, exchange, and consumption as "members of a totality, distinctions within a unity," "moments" of an "organic" rather than syllogistic whole, moments between which "mutual interaction takes place" (Marx 1978, 236). However, David Harvey argues that, to some extent, Marx also attempts to adhere to that syllogism and its shallow coherence (Harvey 2012, 10 ) in his attempt to determine the laws of motion of capitalism. Since Marx's aim is "to reveal the economic law of motion" of capitalist society, the "natural laws" of a capitalist mode of production (Marx 1990, 92), it is easy to see why Marx would have stuck to the level of generality. As Marx himself said in reply to those who criticized classical political economists for overemphasizing production: The task is "the grasping of real relations," not "the dialectic balancing of concepts" (Marx 1978, 228). It is production that "is determined by the general natural laws" (227), that can only be understood theoretically through the power of abstraction. Even when the syllogism is instead thought of dialectically, with each category being a moment in an organic whole or totality, "[p]roduction predominates" over the other moments (236).

In this article, I seek to construct a political economy of communication that explains the laws of motion of capitalist communicative production, a theory of how capital is accumulated through communication. For that reason, I attempt to focus on the generality of production in the form of communicative production, but I also attempt to more fully integrate aspects of distribution and consumption into the political economic theory using what is useful from the syllogism described above as well as Marx's own dialectical conception of the productionconsumption and production-distribution relationships and Harvey's development of those relationships within political economic theory in Limits to Capital (Harvey 2006).

Harvey (2012) describes how Marx's method works in practice as a method of political economy, which requires reconciling Marx's historical, materialist, and dialectical principles of knowledge production with the "weak syllogism" and focus on the "generality" of production found in classical political economy. As Harvey points out, Marx wants to continue the "science" of political economy at the same time that he wants to construct a political economy that fundamentally contradicts the utopian vision of classical political economy by demonstrating the dystopian logic of the laws of motion of the capitalist mode of production (Harvey $2012,6-7 ; 2010,52-53)$. In that practice, Marx rigorously, and perhaps rigidly, sticks to the "regular syllogism" of classical political economy in which production is the "generality" and is, therefore, the object of theorizing if the goal is to produce knowledge of the laws of motion of the capitalist mode of production (Harvey 2012,6). In his process of abstraction, Marx seeks to look at the generality of production as exclusively as possible while still using "organic thinking and dialectical relational analysis" to construct his political economy (10). Harvey explains, "[t]he exclusions are almost always justified on the grounds that they do not lie within the field of generality with which Marx is exclusively concerned" (11).

In constructing a political economy of audience labour in section three, I attempt to approach communicative production with the method described above in order to reconcile the "weak syllogism" and the related focus on the generality of production with a historical materialist dialectical method of theorizing. But I also attempt to further enrich the political economy of communication as a theory of the generality of communicative production by integrating the particularity of distribution and even the singularity of consumption in a dialectical way and to whatever extent is necessary to grasp "the real relations" of capitalist communicative production (Marx 1978, 228). In order to do so, I draw on Marx's own discussion of the dialectical relationships between production and the other "moments"-distribution, exchange, and consumption. Before I can do so, however, I first begin to put the concept of audience labour in a position in which it can serve as the basis of a political economic theory by incorporating Raymond Williams' effort to develop a historical materialist dialectical method of theorizing communication as production. 


\subsection{Renewing Raymond Williams' Project: Theorizing Communicative Production}

Williams is essential to the development of a political economy of audience labour because he conceptualized the "means of communication" as "means of production" and theorized communication and culture as human processes of production (Williams 1980a; 1980b; 1981). Most importantly, human productive activity is at the core of Williams' method of theorizing: "A society is not fully available for analysis until each of its practices is included" (1980a, 44). Cultural practices, Williams insisted, are an aspect of "the general social process" (44). Within what Williams $(1980 \mathrm{~b}, 53)$ called "the whole historical social and material process," by which he meant human existence, both culture and consciousness are produced. Echoing Marx (1978), Williams (1980a, 46-49) insisted on theorizing human activities primarily as processes of production rather than consumption, and he said there had been a particular failure to do so with respect to communicative and cultural practices.

Dan Schiller (1996) has found in Williams' work the basis for developing a 'unified conceptual framework" for theorizing communication. Schiller concluded his history of communication theory by declaring Williams had provided a necessary way forward: a way to unify communication under the concept of labour, i.e. a way to theorize communication as labour. I attempt to build on Schiller's claim by demonstrating how a renewal of Williams' project is productive in the way Schiller claimed through an argument about how it specifically helps advance the project of developing a political economy of audience labour, both by helping to demonstrate the need for such a theoretical development and by providing essential means by which that development can be accomplished.

The first of what I consider to be Williams' most significant contributions relevant to the political economy of audience labour concerns the issue of "base and superstructure in Marxist cultural theory." Rather than approaching culture and cultural activities as an aspect of the superstructure that is determined by the base, as Marxist theory often does, Williams insisted it is necessary to reconsider the base in order to understand "the realities of the cultural process" (Williams 1980a, 33, emphasis added). Williams pointed out that Marx emphasized "productive activities," thus a process of production rather than a static "base" (34-35). Cultural production is, then, clearly not outside the general process of production, and the cultural practices that make up the cultural process of production cannot be seen as "superstructural" simply because they are cultural. It is possible to theorize cultural practices as activities of production rather than simply consumption by discovering "the nature of a practice and then its conditions" (47).

Theorizing culture by looking at "the conditions of a practice" (Williams 1980a, 48) is useful in relation to the method of political economy I discussed in the previous part of section two. That method of theorizing culture can be expanded in a way that is directly productive for a political economy of audience labour by enriching it through Williams' discussion of "productive forces" and, in particular, the means of communication as means of production. In contrast to the received conception of productive forces as "industrial" and by definition excluding supposedly superstructural aspects such as means of communication or other means of cultural production, Williams asserted that a productive force is "all and any of the means of the production and reproduction of real life" (Williams 1977, 91). It is then possible to understand the previously evaded or missed "material character of the production of a cultural order" (93) as "real practices, elements of a whole material social process [...] many and variable productive practices, with specific conditions and intentions" (94, emphasis added). It is possible, finally, to see that it is necessary "to look at our actual productive activities without assuming in advance that only some of them are material" (94). It is in the precise spirit of that statement that a political economy of audience labour can be developed by means of a historical materialist dialectical method. A specific elaboration of the "means of communication as means of production" contributes further to that process.

Williams declared directly and simply that "means of communication are themselves means of production" (Williams 1980b, 50) and, by doing so, produced a powerful insight for the method of theorizing a political economy of communication (exactly the kind of "theoretical revision [...] of the definition of productive forces" he said was necessary (Williams 1977, 
136), and an insight whose significance is missed by one of the few scholars to take it up recently (see Hebblewhite 2012). The concept of "means of production" is an already existing-and central-concept of political economy, but Williams did not attempt to determine how those means of communicative production should be integrated into a political economy of communication through a historical materialist dialectical method. Instead, he explored them historically and in the process generated a second insight for the political economy of communication: the category of "communicative production." In the final section of this article, I attempt to integrate the means of communicative production into a political economy of audience labour. For the moment, I hold off further discussion of that simple but powerful insight because Williams did not discuss it further in terms of political economic theory.

While Williams (1980b) suggests the need for a "history of "communicative production,"' (53-54), I take it as necessary to also theorize communicative production, ie, to develop a political economy of communication by means of the materialist method suggested by Williams. As I want to specifically develop a political economy of audience labour, I want to be able to theorize audience labour within capitalist communicative production. By doing so, I aim to answer Smythe's call for a historical materialist dialectical method of theorizing communication by appropriating Marx's method and using it to theorize communicative production and audience labour with the help of Williams' insights, thereby theorizing communication as capital and determining the nature of the exploitation of audience labour. Williams is a useful resource for one final, but essential, development in my conceptualization of audience labour: theorizing communication as labour.

\section{Specifying Audience Labour: Theorizing the Audience Labour Process as Sig- nification through Cultural Consumption}

In his theory of the audience commodity, Smythe described a theoretical "blindspot" to "the production of ideology" (Smythe 1978, 121) and traced that blindspot to a methodological error. He insisted that a historical materialist dialectical method was necessary to bring into view a theory of "the production of ideology," and he insisted that method would lead to a theory of audience members doing the work of producing their own consciousness as a consumer consciousness, thereby producing "demand" for commodities, while their capacity to do that work is exchanged as an audience commodity that is produced and sold by communication industries and purchased by advertisers (Smythe 1977; 1978). Putting aside, for the moment, my critique of that political economic theory, I argue that in making the connection between audience labour and the production of ideology, Smythe provides an important starting point that can be further developed as the basis for a theory of the audience labour process.

Audience labour is a kind of labour involved in the production of ideology, or audience consciousness more generally. Smythe provided only a vague description of it as paying attention, "learning to buy," and "learning the theory and practice of consumership" (Smythe $1977,4,6,20)$. Jhally and Livant described it a little more specifically as the creation of meaning and "the process of consciousness" (Jhally and Livant 1986, 142-143). Building on Williams' (1980) notion of "communicative production" and what Schiller (1996) derives from Williams as the basis for theorizing communication as labour, it is possible to think of the audience labour process as a process within communicative production, thus a process that produces something. The question that must be answered, then, is what is the nature of that audience labour process?

The process I describe as audience labour is also often described simply as consumption, which suggests that it is necessary to take seriously the "singularity" of consumption, as it is described in the Introduction to the Grundrisse (Marx 1978), in order to theorize the audience labour process. In order to then develop that theory of the audience labour process into a political economy of audience labour, it is then necessary to connect the "singularity" of consumption to the "generality" of communicative production, as well as the "particularity" of distribution. I attempt the latter two theoretical developments in the third section. Here, I attempt to develop a basic theory of the audience labour process by trying to determine, first, how 
theories of audience activity as the creation of meaning contribute to a reconceptualization of the audience labour process beyond the theory of audience labour in the political economy of the audience commodity; second, how Horkheimer and Adorno's theory of the relationship between the commodification of culture and the production of ideology contributes to a theory of the audience labour process; and, finally, how Marx's description of the labour process in Volume I of Capital can be used as a template for a theory of the audience labour process.

\subsection{Audience Activity as Meaning-Making}

Stuart Hall's "Encoding/Decoding" essay provides a useful starting point for enriching the theory of audience labour through an integration of theories of the "meaning-making" audience, despite the fact that Hall's essay is directed precisely against the theoretical development for which I am using it, insisting as it does on the distinction between what Hall described as "discursive 'production' " and "other types of production" (Hall [1980] 2006, 163; Schiller 1996, 149). Hall ([1980] 2006) offered a revised theory of the process of "mass communication" and the relationship between communication industries and their audiences, in the language of semiotics and through the method of structuralism (theorizing, e.g., "articulations" between "relatively autonomous" "determinate moments" [164]). Audience labour can be seen in the process Hall described as "decoding."

Within the whole process of "encoding" and "decoding" in "discursive production," there is the possibility for a "struggle over meanings" (Hall [1980] 2006, 168) precisely because of the necessity for "signs" to be "decoded." In other words, audiences must work to produce meaning from the meanings they consume. Signs are by nature "polysemic" (169), and within the "connotative" aspect of the "decoding" process, signs are not fixed but are instead "more open, subject to more active transformations" (169). Since "decoding" "has its own conditions of existence" (170), there is "no necessary correspondence" between "encoding" and "decoding," and so the meaning for audience members is one they produce, although within certain structural limits determined by the "code" itself (169), and although it is further always possible to "decode" a message with a "negotiated" or even "oppositional" code (172-173).

While, for Hall, the process of "encoding" in the production of messages is a "labour process" (Hall [1980] 2006, 164) and consists of "interpretive work" (169), the audience's activity of "decoding" is not characterized as work at all. Furthermore, for Hall, "discursive production" itself is distinct from other types of production because its products ("sign-vehicles" containing meaning) take on different "discursive forms" as they circulate through the communication process and it must operate within the "formal rules" of language (163-164). Still, the attention Hall drew to the process by which audiences produce meaning using the "encoded" meanings they encounter in messages produced by communication industries opens up the possibility of focusing attention on the process by which audience members produce meaning through their activity of consuming culture. Hall took meaning out of the message itself so that an audience member is not merely the receiver of meaning contained in a message, a perspective that obviously leads to the pursuit of knowledge of the "effects" of that message on the audience, but is rather a decoder of meaning, which leads instead to the pursuit of knowledge of the meanings made by the audience. One such approach is the "active audience."

It is possible to make further progress toward a reconfiguration of the audience labour process by enriching the concept of audience labour with aspects of the concept of "active audiences." The connection to Hall's theory of the "active transformations" of meaning possible in the process of "decoding" is evident in Fiske's description of the theory of the audience as "active" in the sense of being a "social" rather than "textual" subject: "The actual television viewer is a primarily social subject. This social subjectivity is more influential in the construction of meanings than the textually produced subjectivity which exists only at the moment of reading" (Fiske 1987, 62). Further along those lines, Fiske stated that analysis has to pay more attention to "the gaps and spaces that open television up to meanings not preferred by the textural structure, but that result from the social experience of the reader" (64). The theory of the "active audience" "making meanings" makes it possible to enrich the theory of audi- 
ence labour by specifying the audience labour process and the product of that process. The basic theory of audiences making meanings is one that must be adapted to political economic theory in the form of a theory of audience labour. Fiske's emphasis on "how meanings are made by the active reading of an audience" (67) is useful in that process of further developing the theory of audience labour. The product of audience activity is "meanings," thus audience labour is the process of signification through cultural consumption.

\subsection{Audience Activity as the Production of Ideology}

Horkheimer and Adorno's (2002) essay on "The Culture Industry" contributes to a political economy of audience labour through its discussion of the relationship between commodified culture and the production of ideology. Their theory of the production of consciousness is not a political economic theory, but it can be a source of developing the latter because it can be used to reveal something about the audience labour process from the perspective of political economic theory. Horkheimer and Adorno make no mention of audience activity as labour, and their focus is on how the culture industry produces ideology. As such, there is a danger of the audience being lost in the process they describe. But there is a crucial complexity to their argument that makes it a resource for a political economy of audience labour: It is not simply that the commodification of culture in itself produces "mass deception"; there is specifically a relationship between the content of culture and the consciousness that is produced by the consumers of that culture.

The culture industry - the industry that mass-produces culture, ie, the collective system of cultural production comprised by "mass media" industries-produces a certain kind of "sameness" (Horkheimer and Adorno 2002, 94). The sameness Horkheimer and Adorno are concerned with is suggested in the essay's subtitle: "Enlightenment as Mass Deception." They are concerned with what happens to the content of thought when culture is commodified, specifically when cultural production is the mass production of cultural commodities. Without a source for critical thought, as Horkheimer and Adorno assume culture can be, there is mass deception rather than enlightenment. The particular concern of Horkheimer and Adorno is not, in itself, the culture that is produced by the culture industry as a mass of commodities but rather how that culture relates to social consciousness, i.e. mass enlightenment or mass deception. The culture produced by the culture industry, they conclude, is the basis for the production of mass deception, of the "ideology" that reproduces the status quo under the name of freedom of choice, ie, a dialectic of enlightenment in which wider access to culture produces mass deception rather than mass enlightenment specifically because of the content of that culture.

The "insatiable uniformity" of cultural production by the culture industry impoverishes the "aesthetic material" of culture because the production process is that of industrial mass production, which unifies the content of the cultural products that could instead be individual cultural expressions (Horkheimer and Adorno 2002, 97-98). Cultural production prior to the culture industry was not unified in the same way. It did not present a "false identity of universal and particular" (95), so it allowed for details to be emancipated from the cultural products of which they were a part, which allowed for the particularity of every individual work as "the detail [...] rebelled as unbridled expression, as agent of opposition, against organization" (99). Such details are subdued and subordinated to the totality of the formula of the culture industry's cultural production: "the formula [...] supplants the work" (99). The products of the culture industry thereby produce a "withering away of imagination and spontaneity" as the consumers of those products are prohibited from thinking if they are not to miss all the effects that are, however, parts of the formulaic totality (9-100). "Each single manifestation of the culture industry inescapably reproduces human beings as what the whole has made them. And all its agents [...] are on the alert to ensure that the simple reproduction of mind does not lead on to the expansion of mind" (100). To be entertained by the products of the culture industry is only to escape "from the last thought of resisting" a "bad reality," to be liberated "from thinking as negation" (116). 
While the promise of mass enlightenment seems inherent in the increased availability of culture created by the culture industry's mass production of culture-"The public should rejoice that there is so much to see and hear" (130)-the content of that culture, as described in the passages above, ensures that no such enlightenment is imminent. The unifying sameness of the culture produced by the culture industry effects a sameness in social consciousness. "All are free to dance and amuse themselves. [...] But freedom to choose an ideology ... everywhere proves to be freedom to be the same" (135-136). It is clear that, for Horkheimer and Adorno, there is a relationship between culture and the consciousness produced through its consumption. Since audience activity is most obviously the consumption of culture, culture is clearly a resource used in the communicative production process of signification through cultural consumption. Control over culture is the means by which capital can control audience activities. In order to see the contribution of Horkheimer and Adorno's theory, as well as the theories of active, meaning-making audience, make to a theory of the audience labour process and a political economy of audience labour, it is necessary to put those theories in the terms of political economy.

\subsection{Audience Activity as Labour: Marx's Theory of the Labour Process}

Marx's theory of the labour process in Volume I of Capital provides a template for translating the reconceptualization of the audience labour process developed above into a theory of the audience labour process than can serve as the basis for a political economy of audience labour. The human labour process, in its simplest sense and independent "of any specific social formation," has three elements: "(1) purposeful activity, that is work itself, (2) the object on which that work is performed, and (3) the instruments of that work" (Marx 1990, 284). There are then three concepts: labour, object of labour, and instrument of labour. The audience labour process, similarly abstracted from the specific form it takes under capitalism, can also be first theorized as a process involving audience labour, the object(s) of audience labour, and the instrument(s) of audience labour.

Audience labour in that sense is simply the activity of audience members, who engage most obviously in various activities of cultural consumption. The object of audience labour is the object that is typically perceived as being consumed by audience members: culture, or signified objects created to have their meaning consumed (which includes advertisements). However, to speak of "audience labour" is actually to already presuppose labour in a specific social relation and, furthermore, to presuppose specifically capitalist communicative production. To treat audience labour as a fundamental human kind of labour apart from any "specific social formation" is to reify the culture industry that creates audience members out of individuals in the first place. The object of audience labour as "culture" assumes the existence of the production of something that can be identified as a distinctly cultural product; it assumes the existence of the culture industry. It is necessary to abstract further from audience labour to see that it is an aspect of the more general labour of signification, or producing meaning through the consumption of meaning. The object of that labour is then anything perceived by the senses and thus "consumed" in thought. ${ }^{6}$

However, in order to avoid moving way beyond the bounds of a political economy of communication, I return to the already socially specific audience labour process and treat it as abstractly as possible, but with the necessary proviso I have just made. The object of audience labour, then, is what I refer to as culture, by which I mean any signified object that anyone might identify as a cultural product. I use "culture" in the same broad sense that Marx

\footnotetext{
${ }^{6}$ Furthermore, that labour cannot itself be understood as a truly distinct kind of human labour because it is, in fact, an aspect of all human labour. Humans produce their own consciousness in the process of living, not only when they are doing specific activities, such as consuming culture. It is for that reason that a political economy of consciousness is ultimately necessary, but that is a potentially enormous field that would encompasses the consciousness producing aspect of every human activity and the conditions in which consciousness is produced in all of those cases. While that does seem like a necessary development of political economic theory, I limit my proposed contribution to it to the political economy of audience labour within the already defined bounds of the political economy of communication, which requires taking for granted the existence of communication as an activity that is treated as separate from other activities, which is to assume the existence of communicative capital.
} 
used "nature" in describing the human labour process in is most fundamental, and therefore abstract, sense: "Labour is, first of all, a process between man and nature" in which man "appropriate[s] the materials of nature" (Marx 1990, 284); "The labour process [...] is an appropriation of what exists in nature" (290). There is no "nature" in that abstract sense, but at the level of human knowledge of the "universality" of the "metabolic interaction between man and nature, the everlasting nature-imposed condition of human existence" (290), there is. In the process of abstraction necessary to theorize the audience labour process in the most general sense, I similarly use culture as a term that singularly encapsulates what is in reality an endless multiplicity.

A concrete labour process has as its object specific "materials of nature"; in the same way, a concrete audience labour process has as its object specific materials of culture (or, cultural materials). As an object of labour, culture is never available "in nature"; it is always a product of other human labour, which I term cultural labour. As such, culture is what Marx referred to as "raw material": It is an object of labour that has been "filtered through previous labour" (Marx 1990, 284). The contemporary discussion of "remix" culture demonstrates the on-going process of producing culture from the raw material of existing culture (see, e.g. Lessig 2004, 29-30, 61; Gillespie 2007, 280). It is important to specify that the object of audience labour, as cultural raw material, is not, for instance, a book but rather what is signified through the visual representation of language printed on the pages bound together as a physical book. The raw material that is the object of audience labour is, in its most specific sense then, immaterial, but it is always material as part of the material audience labour process: It must always be objectified and materialized, in that it is an aspect of the consciousness of a cultural labourer objectified in a physical form, whether in spoken language, printed language, or even digitized language, which still requires a physical manifestation in order to be, e.g., perceived on a computer screen.

The instrument of audience labour is what is used by audience labour to work on the object of that labour. "An instrument of labour is a thing, or complex of things, which the worker interposes between himself and the object of his labour and which serves as a conductor, directing his activity onto that object" (Marx 1990, 285). The instrument of audience labour is a communication medium, which includes electronic and digital "technologies" but is more generally any and all means of communication used to consume culture. Paper is an instrument of audience labour (e.g. a book, a newspaper), but so are a television, a computer, and a smartphone. Eyes and ears (and, potentially other organs of the body related to the human senses) are the simplest instruments of audience labour. ${ }^{7}$ The "objective conditions necessary for carrying on the labour process" are also instruments of labour (286).

"[B]oth the instruments and the object of labour are means of production" (Marx 1990, 287). Within the audience labour process, it is necessary to add to Williams' description of the "means of communication as means production" that culture is a means of production as the object of audience labour. That formulation allows audience labour and its means of production to be put into the basic model of surplus-value production in Volume I of Capital: A capitalist buys labour-power and means of production, employs them in a production process that produces a commodity that has more value than existed at the start of the production process, and can realize that surplus-value as profit through the sale of that commodity. But the audience labour process and the communicative production that occurs through that labour process do not fit into that model. And because of that, audience labour-power cannot be commodified in the way labour-power is in the model of surplus-value production. To return to the Grundrisse, audience labour, as an act of consumption, is a "singularity." That is all the more obvious when it is understood that the product of audience labour is, in the most fundamental and abstract sense, individual consciousness.

The labour process described by Marx is also a consumption process, but it differs from individual consumption:

\footnotetext{
7 Just as, in gathering fruits, for example, "a man's bodily organs alone serve as the instruments of his labour"
} (Marx 1990, 285). 
Labour uses up its material elements, its objects and its instruments. It consumes them, and is therefore a process of consumption. Such productive consumption is distinguished from individual consumption by this, that the latter uses up products as means of subsistence for the living individual; the former, as means of subsistence for labour, i.e. for the activity through which the living individual's consumption is the consumer himself; the result of productive consumption is a product distinct from the consumer. (Marx 1990, 290, emphasis added)

Productive consumption is the consumption of the objects and instruments of labour to produce a product that is alienable from the producer, who is also necessarily a kind of consumer within the labour process. Individual consumption is the consumption of products in order to produce the consumer (eg, the individual consumption of food as a means of subsistence), who is thereby the producer of him- or herself (Marx 1978, 228-229) also referred to this as "consumptive production"). The audience labour process is a process of individual consumption, as it is the consumption of culture as a means of subsistence for the social subjectivity of individuals.

And yet, the culture industry in the U.S. alone generates billions of dollars in profits from its control of access to culture and from seemingly "selling audiences" to advertisers, which suggests audience labour is exploited in some way in connection with the accumulation of capital by the culture industry. The existing political economy of the audience commodity does not explain that process, in large part because of its insufficient theoretical attention to the audience labour process. Audience labour-power is not commodified, thus it is clearly necessary to look beyond the model of surplus-value production through the commodification of labour-power and the productive consumption of the labour process in order to understand how value is appropriated from audience labour. A political economy of audience labour must be developed in order to understand the relationship between audience labour and capital accumulation, and the exploitation of audience labour. I outline that political economy in the next section.

\section{Contribution to a Political Economy of Audience Labour}

It is possible to develop a general outline of the political economy of audience labour, as a theory within the political economy of communication, on the basis of the reconceptualization of the audience labour process undertaken in the previous section. To do so is also to use Marx's method of political economy as the method of the political economy of communication, and to draw on Williams in order to think of communication as communicative production and communicative activity as labour. It is, furthermore, to narrow that theoretical focus to audience labour within communicative production in order to look at the process of signification through cultural consumption as a process of capital accumulation. To produce a political economy of audience labour in that way is to see communication as capital.

\subsection{Communication as Capital}

Capital that circulates and accumulates specifically through communicative processes I refer to as communicative capital. Communicative capital cannot commodify audience labourpower and appropriate surplus-value through the appropriation of the products of audience labour, because the product of that labour is, in the immediate sense, immaterial and subjective. It is the meaning produced through cultural consumption, and when memory is taken into account, it can also be seen as being meaning that is produced at a later time than when the consumption activities occur. Communicative capital can only control the audience labour process of signification through cultural consumption, and extract value from that process, by controlling the objects of cultural consumption. The political economy of audience labour shows the accumulation of communicative capital to be a process of appropriating value in its distribution. At the level of "generality," surplus-value is produced; at the level of "particularity," that surplus-value is distributed (Harvey 2006, 61, 69).

I will argue below that communicative capitalists essentially seek to redistribute value from the wages of workers as a form of rent payment as well as receive a share of the distribution 
of surplus-value from other capitalists through advertising as a form of interest payment. For that reason, it is necessary to integrate the "particularity" of distribution into the "generality" of production in the construction of a political economy of audience labour. As the labour involved is the audience labour of individual cultural consumption, it is also necessary to integrate the "singularity" of consumption into the "generality" of production in order to construct a political economy of audience labour. I say integrate into production because political economy is a theory of the level of "generality" that is production, thus a more historical approach would be necessary to generate more complete knowledge of the "particularity" of distribution and, especially, the "singularity" of consumption. Political economy can only deal with those aspects as they relate to the "generality" of production. As this paper is an attempt to contribute to the development of the political economy of audience labour within the capitalist mode of communicative production, I will deal with distribution and consumption as they relate to capital accumulation through the communicative production of audience labour.

While advertising offers one potential starting point for examining the relationship between audience labour and capital accumulation, as it seems to be a situation in which communicative capital "sells audiences" to advertisers, the relationship between communicative capital, audience labour, and advertisers actually points to the necessity of uncovering a more fundamental relationship: the social relation that creates audience labourers in the first place, which is a social relation between communicative capital and audience labour. The appearance of communicative capital selling audiences led Smythe to theorize audience labourpower as a commodity and Jhally and Livant to theorize concrete audience labour (time) as a commodity. But, if communicative capital has the power to sell audience labour-power, labour-time, or something else related to audience activities that is as yet undefined, and thereby has the power to direct the audience labour process to work on advertisements so that it can accumulate capital through advertising revenue, then there is some social relation through which communicative capital has that power over audience labour. It is that relationship that defines audience labour as a specific kind of labour related to capital in a specific way. When that relationship has been defined, it is then possible to understand the relationship between audience labour, communicative capital, and advertising, in which communicative capital uses its power over audience labour to appropriate value not directly from audience labour but rather from advertisers.

\subsection{Integrating Critiques of Smythe: Separating the Exploitation of Audience Labour from the Audience Commodity}

As Chen (2003) and Caraway (2011) point out, Smythe's political economy of the audience commodity was fundamentally erroneous in its conception of the commodification of audience labour-power. Building on Smythe, then, as much of the political economy of digital labour does, only compounds that error. In their critiques, however, both Chen and Caraway push audience labour even further from the view of political economy. My aim is to put the focus squarely on audience labour. Chen and Caraway do put forward the concepts of rent and fictitious capital as necessary to explain capital accumulation in relation to (digital) communication, and I agree those concepts are essential to a political economy of audience labour that explains the processes of capital accumulation through the exploitation of audience labour. Pasquinelli $(2009 ; 2010)$ also argues for the importance of the concept of rent. Fuchs $(2012,732)$, however, explicitly rejects the idea that the concept of rent has any applicability to an explanation of the process of capital accumulation through digital labour exploitation. He claims that "using the category of rent for describing commercial media and Internet practices and their outcomes means to assume that activities on the corporate media and Internet [...] are not exploited and are no form of labour."

Jhally and Livant (1986) first suggested rent as a useful category for the political economy of the audience commodity, but they did not then pursue an integration of the relation of distribution defined by rent into their political economy. Chen (2003) follows Jhally and Livant in situating the audience commodity in the sphere of circulation, which implies that audience labour's relation to capital is that it is productive toward an acceleration of the rate of turnover 
and a faster realization of surplus-value for the commodities advertised. Chen does not deal with the fundamental relationship between audience labour and communicative capital. In Chen's theory, industrial capitalists, as advertisers in relation to communicative capitalists, pay rent to communicative capitalists for access to audiences (Chen 2003, 9-10). Communicative capital appropriates a portion of industrial capital's surplus-value as rent, and industrial capital will pay that rent "to prevent a realization crisis" (11). Chen concludes that the audience commodity constitutes a fictitious commodity, accepting Meehan's claim that the audience commodity is actually only the ratings commodity, a "constructed audience image" (13). Thus, communicative capital produces and circulates fictitious capital because it does not (and, I argue, cannot) actually purchase audience labour-power. The value of the fictitious audience commodity is constituted by credit generated through the ratings commodity (13). That credit is what Chen had described as rent: Communicative capital's fictitious audience commodity is purchased by advertisers/industrial capital, with the money paid constituting fictitious capital as credit since the exchange is a process of speculation on the part of industrial capital that purchasing the (fictitious) audience commodity will generate more surplusvalue through faster realization (12-13). For that reason, Chen claims communicative capital actually accumulates debt through the advertising money it receives as credit from industrial capitalists, a claim that seems to negate itself. But, he also claims that, "accumulation of debts can appear as an accumulation of capital as long as audiences/consumers/workers do not seriously challenge the circulation of the fictitious audience commodity" (15).

While Chen generates a useful insight by employing the concept of fictitious capital to understand the relationship between communicative capitalists and advertisers-an insight I will further explore below through Harvey's development of the concept in relation to the capitalization of rent (Harvey 2006) - his depiction of a "credit-sustained television economy" does not hold up to close scrutiny. Chen claims there is no real audience labour performed (14) and so pays no attention to the audience labour process. I contend that audience labour is at the heart of the accumulation of capital through the consumption of culture. The commodity exchanged between communicative capital and advertisers is not a fictitious commodity at all; it is culture as the object of audience labour. Actually, it is access to culture, which is controlled by communicative capital through copyright and other means. I will return to that point after considering how another critique of the political economy of the audience commodity contributes to an elaboration of communicative capital's power over audience labour.

Caraway (2011) proceeds from Chen's claim that the concepts of rent and fictitious capital provide a means to correct errors in the existing political economy of the audience commodity (701). Caraway points to the centrality of rent in the exchange of what Smythe and others had called the audience commodity, but Caraway takes that fact as evidence that there is no "audience labour" and there is certainly no exploitation of audience labour. Caraway claims an a priori need for "a balanced approach to the class analysis of free labour" (694), meaning digital labour, because he assumes, along the general lines of the Autonomist Marxist view, an inherent process of liberating human labour in connection with the increasing significance of "knowledge and social cooperation for the organization of work" (693), i.e. the rise of "immaterial labour," the "general intellect," and the "social factory." He argues that the political economy of the audience commodity "tends to overstate the realization of" capital's efforts to exploit digital labour, which is always "contingent, contradictory, and contested" (694). While I agree with the latter point, a "balanced approach" as Caraway employs it leads to a conclusion that "class antagonism" is driving "the trajectory of capitalist development" in communicative production toward the liberation of communicative labour (706). For Caraway, Smythe's theory of audience labour exploitation is inherently "counter-revolutionary" (702) by its very claim that such exploitation exists.

Contrary to what Caraway claims, Smythe's theory does not dismiss "audience subjectivity" (Caraway 2011 705). Smythe insisted audience subjectivity is put to work in the production of ideology. Caraway also describes audience subjectivity as "working-class subjectivity," which makes clear that, as is true of the political economy of the audience commodity he critiqued, he considers the audience commodity and audience labour from the perspective of 
the circulation and accumulation of capital in general rather than within communication. For that reason, Caraway presents as an argument that undermines Smythe's theory the claim that workers struggled to win the very leisure time that Smythe claimed was transformed into audience work time (702-704). But that debate about leisure time is beside the point of whether audience labour is exploited, which would necessarily be a process within communicative production. What Smythe did not do was to look more closely at the audience labour process, which would have enabled him to see that audience members do not only work when consuming advertisements, when they produce the ideology of consumption that generates demand for commodities, but, more accurately, they work whenever they consume culture, i.e. whenever they are in the position of audience member and cultural consumer in relation to communicative capital. Unfortunately, Caraway also does not look closely at the audience labour process in terms of political economic theory. From the specific perspective of audience labour, and an understanding of the audience labour process as signification through cultural consumption, it is clear that resistance from audience labour has not disrupted capitalist accumulation.

However, Caraway (2011) does pinpoint the fundamental flaw in Smythe's political economy: the theory of the commodification of audience labour-power (697). As Caraway has pointed out, and as I note above, "the audience does not appear as a seller of a commodity in Smythe's formulation" (697), as the labourer does in Marx's formulation of labour-power as a commodity in Volume I of Capital. But Caraway then resorts back to the Capital Volume I model to show that Smythe's theory is wrong by insisting the exploitation of labour can only happen in a process that fits the Volume I model (697), while Caraway claims Smythe's theory is actually a theory of rent and, therefore, cannot be a theory of labour exploitation. Caraway then claims rent is the correct way to theorize the relationship between what I call communicative capital and advertisers (701) and I think his basic formulation is correct, if "rents" is replaced by "lends": "The media owner rents the use of the medium to the industrial capitalist who is interested in gaining access to an audience. The rental may be either for time ... or space" (701). In the process, however, Caraway intentionally eliminated audience labour from the theory. I argue that the concept of rent actually helps clarify the process of audience labour exploitation.

Caraway is also correct in claiming that Smythe provided "no demonstration" that the audience labour process "is under the control of the capitalist; nor is there any attempt to show that the use value is alienated from the audience" (697). It is not possible to demonstrate either without a deeper interrogation of the audience labour process itself, as I argue above. Although Caraway makes no effort to pursue that line of inquiry despite his repeated emphasis on "audience subjectivity" in the face of what he perceived as Smythe's dismissal of that subjectivity, he concludes that audience members do not "work for capital when they interpret media texts" (701) — the audience labour that I describe as signification through cultural consumption-because the "meanings" that he views as the "use values" derived through that interpretation are "not under sufficient control by the capitalist" (701). In fact, copyright is precisely the property relation that puts the interpretation of media texts under capitalist control as audience labour that can be exploited, and is also a relation of distribution that is a "production-determining distribution" (Harvey 2006, 332) by which capital has enough power over communicative production to appropriate value and accumulate capital in relation to that production process. I will expand on that point below.

\subsection{Capital Accumulation through Audience Labour Exploitation}

In order to proceed from the opening provided by Chen and Caraway, it is first necessary to develop a theory of the social relationship that defines audience labour within the capitalist mode of communicative production: the relationship between audience labour and communicative capital. Audience labour, as individual consumption and a process of individual signification, is a "singularity." For that reason, audience labour-power cannot be commodified. Its product, meaning, can never be alienated in the way other products of human labour can. The situation is not one of mind control. That product is, in its most essential form, immateri- 
al, but it can and must always be materialized not just to be "communicated" but also to be the "practical consciousness" of a living person, either as actions guided by thoughts, spoken language expressing thoughts, or cultural objects expressing thoughts in language, images, etc. It is not possible to own another person's capacity to signify, or audience labour-power, in the way that labour-power as the general human capacity to create through conscious activities of material production can be commodified. But that does not leave audience labour free of social determination or even exploitation. It is possible to own the means of communicative production that are means of cultural consumption for audience labour: Both culture, as the object of audience labour, and, in some cases, media, as the instruments of audience labour, can be owned by capital. And through that ownership of the means of communicative production, the "singular" signifying labour of audience members can be brought into the process of capital circulation and accumulation. The same holds true for digital audience labour, and for that reason it is crucial that digital audience labour and digital cultural labour not be conflated in the political economy of communication.

The "particularity" of distribution is also a crucial aspect of capitalist communicative production. The social relation that most immediately defines audience labour is a relation of distribution: rent. Capital's ownership of the object of audience labour, culture, creates audience labour by creating a class relationship between those who own culture and those who do not. That ownership occurs most obviously through copyright. But culture is not a typical commodity. The ownership of culture is determined by the specific qualities of culture as an object of labour and a means of production: Culture is fundamentally immaterial in nature, when culture is considered the "meanings," "ideas," or "information" signified in a cultural product, as I define it here, rather than the material medium through which it is accessed. To be an object of labour, however, it must, of course, be objectified, and thus is must be materialized. But culture is, therefore, "non-rival": the consumption of it by one person does not preclude the consumption of it by another person (Benkler 2006, 36). The consumption of culture is not like the consumption of commodities that are rivalrous. The ideas, meanings, expressions, etc, the consciousness objectified in it, is never fully consumed but is only used-it is used as the object on which audience labour works to produce meaning subjectively-and it remains available for use by another audience labourer or by the same audience labourer in a repeated use (e.g. re-reading a book), as long as it exists in an objectified form and can therefore be an object of labour.

The purchase of a cultural commodity is only ever payment for access. For capital, it is the appropriation of surplus-value in its distribution as rent. There is no exchange of ownership of culture. For example, a book purchaser does not become the owner of the consciousness objectified and materialized in a book. Ownership of the physical object that is a book is purchased, but ownership of the "ideas" expressed in material form as language printed on paper remains with the copyright holder. The copyright holder is a cultural "landlord" who does not accumulate capital through the sale of commodities by rather through the granting of access to a privately owned cultural resource in return for payment, i.e. through rent.

For capitalist communicative production, the consumption process that appears as similar to the typical consumption of a commodity, and thus the completion of the process of capital accumulation in the realization of surplus-value through the purchase of the commodity that is then consumed, is actually the central production process for the accumulation of capital. Even in the book trade that seems to be the historical origins of the capitalist mode of communicative production, audience labour, rather than the cultural labour of objectifying ideas in the physical object of a book, was the fundamental labour process by which the process of communication was a process of capital accumulation. Ownership of culture as the object and raw material of audience labour is the basis for audience labour itself-it is the social (and property) relationship through which individuals are made into consumers of culture whose activities area a source of value to communicative capital because of its control of means of production. In that way, culture is like land, and the use of culture as a means of production in communicative production creates a process of exploitation that, like the process in relation to land, occurs in distribution, through the appropriation of (surplus-)value as rent. 
"Monopoly power" through "private ownership of land is the basis of rent as a form of surplus value" (Harvey 2006, 73). The same can be said of culture. Crucially, Harvey adds that the power of private ownership of land "would come to nought [...] were it not for the fact that land is an indispensable condition of production in general [...] even a means of production" (73). Again, the same goes for culture: It is precisely because culture is a condition and a means of communicative production through social signification that private ownership of culture through copyright creates monopoly power for the copyright holder that can be used to appropriate rent. The appropriation of rent is a relation of distribution that also affects "the conditions of production" (69).

The communicative production of audience signification is affected by rent as a condition under which that production occurs: Access to culture is required for communicative production through the audience labour process, but the copyright holder controls that access. Distribution relations are also class relations: There is not simply "capital," there are capitalists who appropriate surplus-value in different ways (as profit on productive capital, profit on merchants' capital, interest on money capital, and rent) and, therefore, exist as "fractions" or classes: industrial capitalists, merchant capitalists, rentiers, and landlords (Harvey 2006, 7374). The copyright holder, then, is like the landlord, but is also a kind of capitalist. The landowner in a capitalist mode of production does not use the land but instead treats the privately owned land as a pure financial asset (347): "in return for a straight monetary payment," the landlord "confers all rights to the land as both instrument and condition of production" (343). The owner of culture operates similarly, granting the right to use culture in exchange for payment, thereby either appropriating value from the wages of a wage-labourer or surplusvalue from the profit, interest, or rent of another capitalist. Any individual who wants access to culture owned by a communicative capitalist becomes an audience labourer, and that individual's audience activities of signification through cultural consumption become a means by which communication is treated as capital. Cultural consumption thereby becomes an exploited activity.

Harvey $(2002,98)$ argues the specific kind of rent that can be appropriated through copyright is monopoly rent. "All rent is based upon the monopoly power of private owners [...] by virtue of their exclusive control over some directly or indirectly tradable item which is in some crucial respects unique and non-replicable" (94). That certainly seems to describe culture as an exclusively owned resource. When there is no competition for ownership of the privately owned resource, monopoly rents can be realized (Harvey 2006, 350), and that is certainly the case with copyright, which is by definition a monopoly right rather than an ownership right that is subject to competition.

The instruments of audience labour, as means of communicative production, have also existed in the form of ownership by communicative capital, as in the case of a movie screen in a movie theatre (and also the theatre itself). But audience labourers generally own the basic instruments of their labour in the form of a commodity they have purchased, as in the case of a book, a newspaper, a radio, a television, and a computer. Therefore, ownership of the instrument of audience labour is not a significant issue for the general relationship between communicative capital and audience labour, although it is a defining aspect of specific audience labour processes, such as watching films in a theatre. The foundational relationship, however, and the relationship by which the capitalist mode of communicative production is defined, is the "production-determining distribution" relationship created by the private ownership of culture. That social relation of distribution conditions the communicative production process of audience signification.

Audience labour is exploited by communicative capital through a relation of distribution that conditions the production process so that audience labour is dependent on communicative capital for access to the object and "raw material" it needs as a means of signification through cultural consumption. Communicative capital can use its power over audience labour to appropriate value directly from audience labour by, eg, charging a fee for access to its monopoly-owned culture. That extraction of rent is a process of direct exploitation of audience labour by communicative capital, since value is directly appropriated from audience labour. 
Communicative capital can also use its power over audience labour to appropriate surplus-value from advertisers while providing audience labour free access to culture. Advertisers can only achieve their immediate aim, which is to influence the actual meaning produced through audience signification, by turning objects of cultural consumption into signified objects designed to have a specific "effect" when they are consumed and worked on in audience labour processes of signification. But advertisers do not own the objects of audience labour. Communicative capitalists own those objects. Since ownership of culture provides the owner a power to appropriate a constant stream of rent (until the copyright expires), that rent can be treated as capital by being capitalized as "the interest on some imaginary, fictitious capital" (Harvey 2006, 347). This is the case of communicative capital lending culture to advertisers, which is not granting access for use, as it is when rent is appropriated from audience labour, but is rather the lending of a portion of the objectified form of culture itself, as in the lending of space in a newspaper, by which part of the space becomes advertising space, or the lending of time in a television program, by which part of the time becomes advertising time. In return for that loan of cultural space or time as fictitious capital, advertisers pay interest to the lender, a communicative capitalist who thereby generates advertising revenue.

That extraction of interest from advertisers is a process of indirect exploitation of audience labour by communicative capital, since the surplus-value is taken from the advertiser rather than the audience labourer. I argue that the process can still be seen as one in which audience labour is indirectly exploited because communicative capital uses its control over audience activities of cultural consumption to appropriate value and, in the process, directly modifies the audience labour process by transforming part of the object of that labour process into an advertisement. The advertiser will pay the interest because it gains a portion of communicative capital's power over audience labour by doing so. As the owner of culture and, consequently, the possessor of some power over audience activities (but never enough to force audience labour sell its labour-power), the communicative capitalist is in a dominant position in relation to the advertiser, who seeks to gain power to affect the audience labour process. But, to the extent that communicative capital becomes reliant on the interest it appropriates from advertisers as it sole source of surplus-value, communicative capital is in a position of dependence in relation to advertisers.

When culture is exchanged as a commodity, lent by communicative capital and borrowed by advertisers, the process becomes one not simply of appropriation of interest but of the circulation of fictitious capital. It seems that here culture is quite different than land. Rent on land can be capitalized as fictitious capital by selling "title to the [...] rent yielded. The money laid out is equivalent to an interest-bearing investment. The buyer acquires a claim upon anticipated future revenues, a claim upon the future fruits of labour" (367). It seems that advertisers, as borrowers of culture, do not seek ownership of the right to the rent that can be appropriated in the future through control of a specific cultural object. They do not seek to appropriate surplus-value from audience labour in the communicative production process of signification through cultural consumption. Advertisers do seek a claim upon the future fruits of labour, but it is the specific fruits, or products, of audience labour: meaning.

That brings back the issue of "singularity" and the fact that the product of audience labour is, in the most general sense, consciousness. Advertisers are interested in that product. While the ultimate aim of advertising is to facilitate the sale of a commodity that is not a process that occurs within communication as capital. It is not a process that directly involves audience labour. The only way to facilitate the sale of a commodity within communication is to affect the actual consciousness produced by specific individuals-what Smythe described as producing the ideology of consumer consciousness that creates demand for advertised commodities. While communicative capital seeks to appropriate value though ownership of the object of audience labour (whether directly through payment by audience labour or indirectly through advertising revenue), and is only concerned with the specific consciousness produced through signification by it audience labourers to the extent that, e.g. popularity yields relatively higher rent or interest, advertisers are precisely concerned with the specific consciousness they can "effect" in specific individuals by making their advertisements the object of those individuals' audience labour. It seems, then, that Smythe was correct on at 
least one count: for advertisers, audience labour is productive when it produces the specific consumer consciousness that creates demand for commodities.

\section{Conclusion}

The political economy of audience labour outlined above describes the basic processes through with audience labour is exploited in the accumulation of communicative capital. It demonstrates the productivity of a specific focus on audience labour for scholarship in the political economy of communication, making it possible to begin to grasp theoretically one of the fundamental aspects of communication as capital in the digital era: the exploitation of digital audience labour. Although the concept of audience labour was initially put forward in relation to the political economy of communication in the era of print and electronic mass media, it remains a necessary concept for the political economy of digital communication. Every company that operates as a communicative capitalist by generating profit from payments for access to culture or advertising revenue is, in fact, exploiting audience labour, and that describes most of the companies involved in digital communication. It seems that controlling and extracting value from audience activities-which is to say, activities of cultural consumption-is the primary way (though by no means the only way) in which communication is treated as capital in the digital era.

The recent development of a political economy of digital labour has generated many insights into the capitalist mode of digital communicative production, but it has not grasped the central role of digital audience labour. This paper has attempted to begin the process of filling in that theoretical missing piece and pushing scholarship in the political economy of communication toward a political economy of audience labour. It has done so by further developing the concept of audience labour, providing a theory of the audience labour process, and then outlining the most fundamental aspects of the relationship between audience labour and capital, including the basic processes through which communicative capital exploits audience labour. There remains much work to be done to more fully develop the political economy of audience labour and to make use of that theory in the analysis of the capitalist mode of digital communicative production. There also seems to be the potential for tracing a long history of audience labour exploitation within the history of communication as capital, or the capitalist mode of communicative production. ${ }^{8}$

\footnotetext{
${ }^{8}$ I attempt both a preliminary examination of the history of audience labour exploitation and an analysis of two cases of digital audience labour exploitation in Nixon (2013).
} 


\section{References}

Andrejevic, Mark. 2002. The Work of Being Watched: Interactive Media and the Exploitation of SelfDisclosure. Critical Studies in Media Communication 19 (2): 230-248.

—_ 2007. iSpy: Surveillance and Power in the Interactive Era. Lawrence, KS: University Press of Kansas. 287.

2011. Surveillance and Alienation in the Online Economy. Surveillance \& Society 8 (3): 278-

[Is this OK Christian?]

Benkler, Yochai. 2006. The Wealth of Networks: How Social Production Transforms Markets and Freedom. New Haven, CT: Yale University Press.

Caraway, Brett. 2011. Audience Labor in the New Media Environment: A Marxian Revisiting of the Audience Commodity. Media, Culture \& Society 33 (5): 693-708.

Chen, Chih-hsien. 2003. Is the Audience Really Commodity? An Overdetermined Marxist Perspective of the Television Economy. Paper presented at the Annual Meeting of the International Communication Association, San Diego, CA.

Cohen, Nicole S. 2008. The Valorization of Surveillance: Towards and Political Economy of Facebook. Deomocratic Communiqué 22 (1): 5-22.

Fiske, John. 1987. Active Audiences. In Television Culture, 62-83. London: Metheun.

Fuchs, Christian. 2010. Labor in Informational Capitalism and on the Internet. The Information Society 26 (3): 179-196.

- 2011a. An Alternative View of Privacy on Facebook. Information 2 (1): 140-165.

309. 2011b. Web 2.0, Prosumption, and Surveillance. Surveillance \& Society 8 (3): 288

2012. Dallas Smythe Today-The Audience Commodity, the Digital Labour Debate, Marxist Political Economy and Critical Theory. Prolegomena to a Digital Labour Theory of Value. tripleC: Cognition, Communication, Co-operation 10 (2): 692-740.

Gillespie, Tarleton. 2007. Wired Shut: Copyright and the Shape of Digital Culture. Cambridge, MA: The MIT Press.

Hall, Stuart. (1980) 2006. Encoding/Decoding. In Media and Cultural Studies: KeyWorks, edited by Meenakshi Gigi Durham and Douglas M. Kellner, 163-173. Malden, MA: Blackwell Publishing Ltd.

Harvey, David. 2002. The Art of Rent: Globalization, Monopoly and the Commodification of Culture. Socialist Register 38: 93-110. Accessed February 26, 2012. http://socialistregister.com/index.php/srv/article/view/5778/2674

. 2006. The Limits to Capital. London: Verso.

2010. A Companion to Marx's Capital. London: Verso.

2012. History Versus Theory: A Commentary on Marx's Method in Capital. Historical Materialism 20 (2): 3-38.

Hebblewhite, William Henning James. 2012. 'Means of Communication as Means of Production' Revisited. tripleC: Cognition, Communication, Co-operation 10 (2): 203-213.

Horkheimer, Max and Theodor W. Adorno. 2002. The Culture Industry: Enlightenment as Mass Deception. In Dialectic of Enlightenment: Philosophical Fragments. Edited by Gunzelin Schmid Noerr. Translated by Edmund Jephcott. Stanford: Stanford University Press.

Jhally, Sut. 1982. Probing the Blindspot: The Audience Commodity. Canadian Journal of Political and Social Theory 6 (1-2): 204-210.

Jhally, Sut and Bill Livant. 1986. Watching as Working: The Valorization of Audience Consciousness. Journal of Communication 36 (3): 122-142.

Lessig, Lawrence. 2004. Free Culture: The Nature and Future of Creativity. New York: Penguin Books.

Livant, Bill. 1982. Working at Watching: A Reply to Sut Jhally. Canadian Journal of Political and Social Theory 6 (1-2): 211-215.

Marx, Karl. 1978. The Grundrisse. In The Marx-Engels Reader (2nd Ed), edited by Robert C. Tucker, 221-293. New York, NY: W. W. Norton \& Company, Inc.

— 1990. Capital: A Critique of Political Economy: Volume I. London: Penguin Books. 
Meehan, Eileen R. 1984. Ratings and the Institutional Approach: A Third Answer to the Commodity Question. Critical Studies in Mass Communication 1 (2): 216-225.

Nixon, Brice. 2012. Dialectical Method and the Critical Political Economy of Culture. triple-C: Cognition, Communication, Co-operation 10 (2): 439-456.

_ 2013. Communication as Capital and Audience Labor Exploitation in the Digital Era. PhD diss., University of Colorado Boulder. ProQuest (UMI 3592351).

Peck, Janice. 2002. The Oprah Effect: Texts, Readers, and the Dialectic of Signification. The Communication Review 5 (2): 143-178.

2006. Why We Shouldn't Be Bored with the Political Economy Versus Cultural Studies Debate. Cultural Critique 64: 92-126.

Schiller, Dan. 1996. Theorizing Communication: A History. Oxford: Oxford University Press.

Shimpach, Shawn. 2005. Working Watching: The Creative and Cultural Labor of the Media Audience. Social Semiotics 15 (3): 343-360.

Smythe, Dallas W. 1977. Communications: Blindspot of Western Marxism. Canadian Journal of Political and Social Theory 1 (3): 1-27. 1978. Rejoinder to Graham Murdock. Canadian Journal of Political and Social Theory 2 (2): $120-127$.

Terranova, Tiziana. 2000. Free Labor: Producing Culture for the Digital Economy. Social Text 63: 3358.

Williams, Raymond. 1977. Marxism and Literature. Oxford: Oxford University Press.

_. 1980a. Base and Superstructure in Marxist Cultural Theory. In Problems in Materialism and Culture, 31-49. London: Verso.

1980b. Means of Communication as Means of Production. In Problems in Materialism and

Culture, 50-63. London: Verso.

. 1981. The Sociology of Culture. Chicago: The University of Chicago Press.

\section{About the Author}

\section{Brice Nixon}

Brice Nixon is an independent scholar and was previously an adjunct assistant professor in the Department of Communication Studies at Baruch College and the Department of Media, Culture, and Communication at New York University. He received his PhD from the University of Colorado Boulder in 2013. His dissertation is titled Communication as Capital and Audience Labor Exploitation in the Digital Era. 


\title{
Playing, Gaming, Working and Labouring: Framing the Con- cepts and Relations
}

\author{
Arwid Lund
}

Department of ALM, Uppsala University, Uppsala, Sweden, arwid.lund@abm.uu.se

\begin{abstract}
The aim of this article is to define the concepts of playing, working, gaming, and labouring, through a literature study, and to construct a typology. This typology will be used to create a field model that is structured by the horizontal parameters of qualitative-quantitative (characteristics) and the vertical parameters of activity-result (in focus). It is shown how this model can be used to visualise different theoretical positions in empirical material, which connects to the concepts and their relations. Working and labouring are distinguished into a trans-historical and a historical, capitalist, category, and likewise playing and gaming, where the former is the trans-historical category and the latter the historical one. The main focus of the article, since working and labouring is well covered within the critical Marxist tradition, is on playing and its relation to working, with the aim of understanding and criticising the concept of playbour.
\end{abstract}

Keywords: Playing, Working, Gaming, Labouring, Play, Work, Game, Games, Labour, Labor, Cognitive Capitalism, General intellect, Peer Production, Crowdsourcing, Playbour, Playbor, Immaterial labour, Marxism, Marx, Autonomist Marxism, Ideology

Acknowledgement: The labour on this article has partly been made possible by a scholarship from the EU COST Action, "Dynamics of Virtual Work" (see http://dynamicsofvirtualwork.com/), and special thanks go to Christian Fuchs, University of Westminster, and Ursula Huws, University of Hertfordshire Business School

Many thinkers during the capitalist era have touched upon the relation between play and labour. The concepts have been thought of as each other's opposites by some and as sharing certain constructive or creative attributes by others. For some conservatives the political aim has been to keep the two realms apart, and some radicals have wanted to infuse their contemporary society, characterized by labour, with playing modes of life, while yet others have wanted to see a shrinking of the necessary hard labour and an increasing realm of freedom built on a synthesis of play and work into a higher unity characterized by attractive or pleasurable productivity.

The main aim of this article is to contribute to the dismantling of the ideological concept of "playbour". The notion of playbour pretends to point at a higher unity of play and labour within the capitalist mode of production and as such it is a highly ideological concept, which I aim to show with a study of related categories and concepts. In order to succeed with this I will develop my study in two steps: First I will develop and propose a typology of the central concepts, thereby introducing a verb form of the concept 'game', gaming ("spela" in Swedish), that traditionally does not exist in English. The typology will consist of four concepts: playing, working, gaming and labouring. All of them in some way relate to the human condition that Hannah Arendt described in her work Vita Activa (Arendt 1998). The typology will be grounded in a literature study with a special focus on identifying shared dimensions by which to compare the different categories. The result will be visualised in a field model that in a later step can be used to map the position of different statements and opinions. This typology and its model will help me to analyse and criticise the contemporary use of the term playbour, as well as give future empirical research a means by which to visualise and place opinions in empirical materials in the created field structure. In a second part I will focus, also through a literature study, on the character of the relations between the concepts of the typology with the two-fold aim of providing further examples of positions (that can be placed in the field 
model) and identifying statements of synergies and conflicts in the relations to counter the rather static picture of the field model. This last study can hopefully, when more fully developed, lay the foundation for yet another, future model to come, on the direction and forces as well as synergies and conflicts within the processes which build up the relation in the interface between the categories of activities. Such a model, and future studies using it, also holds the promise of helping us to deepen our understanding of the concept of playbour.

The first hypothesis of the investigation is that the concepts of playing and working have a qualitative character. Playing is engaged in for the pleasure of the activity in itself and work for the satisfaction of a qualitative need by the production of a certain use value. Gaming and labouring, on the other hand, are understood as quantitative in that they contain measurement of a person's activities in relation to him- or herself or others in gaming, or in relation to the exchange of values on the market within labouring.

Secondly, both playing and gaming are engaged in by actors mainly for their own sake. The activity in itself is the important thing, even if the result does have some role within gaming. In contrast to this, working and labouring are engaged in for the results of the activity. Working is focused on use values and is performed within society for social needs. Labour is focused on the exchange that it makes possible for the labourer, but on the exploitation needed for the accumulation of profit when it comes to the representatives of the capitalist class.

Behavioural categories like these are often defined in terms of structural or physical descriptions, functions, causations and motivations. It is important to keep these dimensions distinct (Pellegrini 2009, 7). The literary study has convinced me to use structural, functional and motivational dimensions in developing the typology. The structural dimension will be divided in two sub-dimensions that was identified in the study: Degree of voluntariness (distribution of power) surrounding the activity (in both subjective and objective terms), and Form of practice. Function will be understood in the light of the meta-question: is the activity mainly specific to a certain society and time or is it mainly trans-historical?

Anthony D. Pellegrini addresses the problematic of conflating structural aspects with functional aspects when it comes to play, because of the elusiveness of the functions in this field (Pellegrini 2009, 7). The problem complex is even wider. It always involves the risk of circular logic when you trace a function from a structure, or entails that the analyses remains on the surface of phenomena, but sometimes the existing structure is the best thing we have to build our understanding on.

The dimension of causation will not be treated as a dimension in itself. To determine the causation of a certain structure or behavioural pattern is even more difficult. A structure could have functions that can be theorised with the help of empirical observations or more abstract theorisation. The causations behind a certain structure (with several functions), on the other hand, could be any number of unknown factors, which as a rule are not related to the structure as existing structure with certain functions.

Even so I will include causation in the dimensions of function and motivation in this investigation, simply to point to the fact that sometimes the function is the only factor (as in the case with structure above) that can help us theorize about the causation of a structure within a biological or psychological perspective (here I depend heavily on biological and psychological research outside of my field of expertise). But the main focus in the article will be cultural and social in character. If we analyse a structure from a cultural or social perspective, we also have to take into account the motivations of the individuals and groups involved in the processes of the structure. Motives can be empirically accessed through different techniques, and can also be used to theorise about the causation, undoubtedly with other scientific risks involved. The dimension of motivations will be divided into the sub-dimension Organising purpose, and Associated feeling (even if it is hard to see the feelings associated with forced wage labour as a motivating force; here the economic incitement or greed together with the necessity of making a living can be used as alternative descriptions). 
The physical dimension, finally, permeates all of the categories in the sense that humans are physical beings in a physical world, and will not be investigated further here. In total this gives us five dimensions: Organising purpose, Form of practice, Mainly trans-historical or mainly historical, Degree of voluntariness and Associated feeling. In the work on the typology I will use the dimensions identified to highlight the differences and similarities between the categories. The five dimensions will together be necessary and sufficient for defining an activity as belonging to a specific category. The categories of playing, working, gaming and labouring will be visualised in a model in accordance with their relation to quality/quantity and activity/result, resulting in a quadrant (see section 2)

The relations between the categories can for now, to get an overview, be visualised like this:

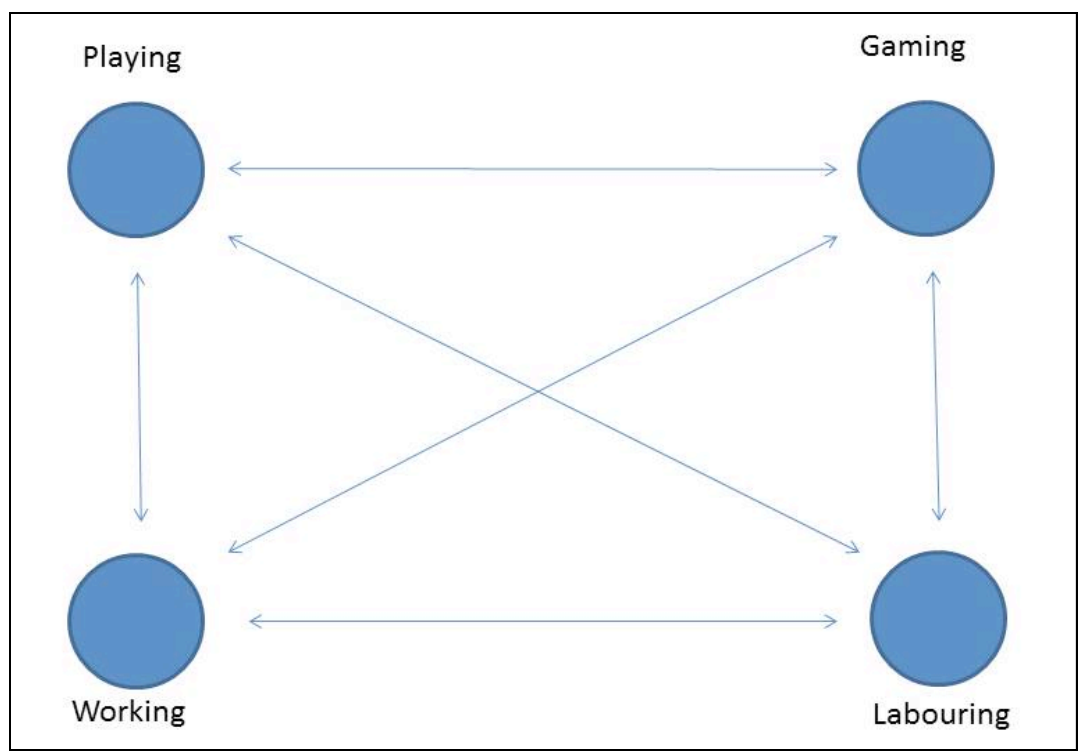

Figure 1: Relations between the four concepts (Arwid Lund 2014)

From the typology, the axes of quality/quantity and activity/result, and the scheme of the relations between the concepts, it is possible to develop a field model for mapping empirical results and the position of various opinions within the field. But this model does not say anything about the character of the relations between the categories. This theme will be touched upon in the last section of the article, together with the ambition of providing examples of empirical positions within the field. ${ }^{1}$ A more in-depth study will have to wait.

Before ending the introductory part of the article it is necessary to comment on the heavy focus on play in what is to come. In the contemporary critical discussion on digital labour, and especially of so-called playbour, there are a lot of influences of Marxism, and work and labour therefore has a theoretical grounding, which play and gaming do not have. When it comes to the relation between play/game and work/labour, central to the understanding of the ideological concept of playbour, the theoretical grounding is lacking to an even higher degree.

\section{The Typology: Playing, Gaming, Working and Labouring}

The conservative Dutch cultural historian Johan Huizinga defined play in an influential way in Homo Ludens: Proeve Ener Bepaling Van Het Spelelement Der Cultuur (1938). He did not distinguish between free-form play and rule-based games, probably because play and game in Dutch, as in German "Spiel", was covered by the one concept "spel". Huizinga was not un-

\footnotetext{
${ }^{1}$ This twofold aim creates some tension in the text. The force and direction characterising a relation, according to some thinker or text, cannot be presented adequately in the field model, which is developed to display static ideological positions. The third and last section should hence be read as a preliminary and tentative attempt to approach rather difficult theoretical questions.
} 
aware of the differences between different languages regarding the word for play, and he concluded that there were as many cultures that distinguished between play and contest as ones which did not (Hägglund 1989, 48). The same word for the two connotations is for example used in Dutch, German, French and Spanish. This fact is commented upon in the Swedish translation due to the fact that in Swedish the two forms have their own terms: leka (playing) and spela ("gaming"), and both in contrast to the English language, are verbs. You play a game in English, you do not game it.

Game is a concept that focuses more on rules, which make it possible to compare and measure performances, and therefore results in more competitive features of the play (and changes the character of playing). I will use the concept gaming in this article and distinguish it from playing.

Jesper Juul comments on the broad definition of play that Huizinga uses and criticises him for his sketchy descriptions of the actual games. Johan Asplund, a social psychologist, instead criticises Huizinga for focusing on rule-based games and not the more informal and improvised forms of play that he counts as an elementary social form (Asplund 1987, 64-65). Given their different scientific missions, both of them are correct.

Juul explicitly focus on games in his study where he provides a definition that he calls the classical game model. He builds on, but also criticises, the former surrealist and scholar, Roger Caillois's categorization of different games into four categories: Agôn (competition), Alea (chance), Mimicry (simulation) and Illinx (vertigo). The categorisation implied according to Juul that you had to make a choice between different sorts of games, but in reality Caillois clearly stressed that the categorisation of a game depended on the category that is dominant in it, hence, that every actual game could contain several categories within it (Juul 2005,10; Caillois 2001).

Caillois in turn criticised Huizingas definition for being both too narrow and too broad (writing in French he did not distinguishing play and game from each other), and contended it was wrong in its connection of play to the secret and mysterious. Play and fiction would be a detriment to the mysterious, not taking it seriously. Play was seen, in contrast to the mysterious, as unserious in the old surrealist's interpretation. He also contended that Huizinga was wrong in denuding play of all material interest. Games of chance contained playing with money even if it was not productive (Caillois 2001).

Johan Asplund makes a sharp distinction between play and game (Asplund 1987). Gaming is organised play, meaning that it is not play anymore. Games are clear-cut in their social responsiveness; one action leads to another action. Play is capricious in its behaviour and does not follow rules in an explicit and uniform way; play involves a lot of negotiations and improvisation; it can even break with some rules. Play is social responsiveness pure and simple according to him (Asplund 1987, 64-67).

Regarding the concepts of work and labour Hannah Arendt claimed that it was necessary to make a distinction between the two. Such a distinction could be traced back to John Locke, who made a distinction between: "The Labour of his Body, and the Work of his Hands" (Locke 1988, 287-288), or even back to Antiquity and the Greek language where cheirotechnes stood for working handicraft men in contrast to the slaves and house animals that were labouring with their bodies (later in Latin called animal laborans in contrast to homo faber) (Arendt 1998,120-126).

Her main argument, though, is that the distinction has been made and maintained in all European languages, with the exception of two nouns in German and French that include both connotations: Arbeit and Travail (Arendt 1998). This has led to some confusion in the interpretation of the works of Karl Marx. Fuchs and Sevignani have shown that insufficient sensibility to this fact has led to grave errors in the English translations of work and labour in Marx oeuvre. This is of some importance because it takes place at the epicentre of Marxian thought. Marx himself claimed that his distinction between concrete and abstract labour in Capital was crucial for the understanding of the rest of that work (Marx and Engels 1972, 73; Marx 1867, 49). Fuchs and Sevignani claim that the dual character of labour reflects the fact 
that Marx was writing both a critique and an economic theory at the same time and that therefore two series of categories were developed on two different levels:

[O]n the one hand that which is specific for capitalism and on the other hand that which forms the essence of all economies and therefore also exists in capitalism and interacts dialectically with capitalism's historic reality. (Fuchs and Sevignani 2013, 247-248)

The trans-historical categories, according to them, are work, use value, concrete labour, labour process (living labour) and necessary labour. The historical categories under capitalism are labour, exchange value, abstract labour, valorisation process and surplus labour (Fuchs and Sevignani 2013,248 ). In the following I will use work for the activity that is mainly focused on the kind of concrete labour which always has to exist, albeit in different historical forms, and labour for the historical form of abstract labour in capitalism which is mainly focused on production of exchange values, surplus value and accumulation according to its own abstract logic. This manoeuver does not in itself answer the question whether some historical mode of production transforms the trans-historical form of work into something qualitatively different. Bands of hunters and gatherers, feudal societies and emergent forms of peer production are historical variations, in the same formal way as capitalism, in relation to transhistorical work.

In line with a critical Marxist perspective I contend that abstract labour introduces a social mechanism that dominates work, the societal production of use values, in a negative way that is uncontrolled by the producers themselves and not in their interest. This not mainly as a result of existing social inequalities in class society, but as a result of a domination of the mode of producing ${ }^{2}$ under abstract standards and an abstract logic of permanent growth and accumulation for the few. This dynamic and expanding character makes capitalism qualitatively different than other historic forms of deviations from the trans-historical work, which also have been built on forced and alienated forms of work. ${ }^{3}$ In order to point to the progressive potential that exist today (as well as yesterday) I reserve the trans-historically inclined concept of work for historical modes of production and distributions of use values that are not characterised by, to borrow a concept from Moishe Postone, run-away growth for un-social private or partial economic interests (Postone 1993,17, 312, 314) but for societal needs that has been decided upon in at least freer forms of associations between (wo)men.

This is a political choice of mine that is also historically evidenced to some degree. On the one hand I tend to agree with Max Horkheimer when he states in 1937 that particular judgements about what is human or not can only acquire their correct meaning in a historical context. The problem is that in an unjust society criminals are not inferior according to Horkheimer, whereas they will be viewed as un-human in a fully just society. In today's society this justifies a political critique against a naturalised run-away growth. In this it helps to remember that the association of free men is not an abstract utopia: "the possibility in question can be shown to be real even at the present stage of productive forces" (Horkheimer 1972, 242). On the other hand, I do believe Postone has a point when he concludes that capitalism is a mode of production that is qualitatively different from all other modes of production in human history. Circulation of commodities and money existed before capitalism, and it was only in capitalism that the commodity became universal, when labour power became a commodity and introduced wage labour. Prices thus preceded value. "Value as a totalizing category is constituted only in capitalist society" (Postone 1993, 270-271).

To complicate the discussion further it has to be stressed that capitalism, in being qualitatively different in all of its quantitative logic, also creates the possibilities of its own abolishment when the quest for relative surplus value results in "a growing disparity" between the

\footnotetext{
${ }^{2}$ The connotation of the term is the form of practice of abstract labour and should not be confused with the concept of mode of production. The term Mode of producing was coined by Moishe Postone (1993, 67-68).

${ }^{3}$ Feudalism and slavery are arguably also modes of productions which deviate from the trans-historical version of work in a qualitatively and radical way. However the notion of labour will be reserved for abstract labour within capitalism in this article.
} 
conditions for the "production of wealth" from "those for the generation of value" (Postone 1993, 298):

In the course of capitalist development a form of production based upon the knowledge, skills, and labor of the immediate producers gives rise to another form, based upon the accumulated knowledge and experience of humanity [...] the social necessity for the expenditure of direct human labor in production gradually is diminished. Production based upon the present, upon the expenditure of abstract labour time, thus generates its own negation-the objectification of historical time. (Postone 1993, 298)

In the contemporary field of digital labour peer production forms an emergent and historic mode of production that is growing in the emancipatory potential that has its material ground in the developments described here. Peer production has a pronounced focus on the concrete labour and the production of use values in free associations of (wo)men, and can thus be associated with the trans-historic aspect of human work. Hence, the instrumental reason of Horkheimer, that is the "constitution in alienated form of social general modes of knowledge and experience" which have a negative effect on much immediate labour, gives rise to the possible emancipation of people "from the sway of their own labour" and their "reappropriation of the socially general knowledge and power first constituted historically in alienated form" (Postone 1993, 328).

This leaves us with four concepts to understand and define in the typology: Playing, Working, Gaming and Labouring.

\subsection{Playing}

In Grundrisse Marx concludes that not-labour under capitalism appears as freedom and happiness, but when labour has created the subjective and objective conditions for itself it would instead transform itself into attractive work in the realm of freedom. He does not say anything about play in this context, apart from criticising Charles Fourier for believing that labour could become play. Self-realisation through attractive work was not to be understood as "mere fun, mere amusement" (Marx 1973, 611-612). Play was thus mere fun and amusement and unproductive to him.

Against this stands the idealistic tradition, which goes back to the ancient Greece. Johan Huizinga especially liked Plato when it came to the long tradition of viewing (wo)man and society sub specie ludi. Plato stated that:

A man ought to be in serious earnest about serious things, and not about trifles; and that the object really worth of all serious and blessed effort is God, while man is created [...] to be the plaything of God, and the best part of him is surely just that; and thus I say that every man and woman ought to pass through life in accordance with this character, playing at the noblest of pastimes, being otherwise minded than they now are (Plato, Laws, Cf. Anchor 1978, 63)

Friedrich Schiller added that man was truly a man when playing. Robert Anchor contends that not just Hegel, Nietzsche and Heidegger, and several other modern philosophers, had similar thoughts, but also, though wrongly it seems, Marx (Anchor 1978, 63). Marx's view of play as something non-important and unproductive will partly be challenged in this article. His perspective does not contribute to a full understanding of the importance of the category. This will demand that we scrutinize playing as a proper category with its social importance in relation to working, but also how it affects and becomes affected by gaming and labouring under capitalism.

Johan Huizinga explicitly pointed to the relation between play and culture/civilisation. He thought that civilization "arises and unfolds in and as play", it was not about placing play within culture but ascertaining "how far culture itself bears the character of play". He was fiercely anti-Marxist and claimed that the $19^{\text {th }}$ century had left little room for play with its utilitarianism and industrialism thus resulting in a luxation of the intellects that helped the misconceptions 
of Marxism, that "economic forces and material interest determine the course of the world", to be put about and believed (Huizinga 1955, 191-92). He also stated that "where there is no visible action there can be no play" (Huizinga [1938] 1955,166).

In contrast with this idealistic perspective I assume that play has to be understood historically and socially, even if this article mainly focuses on this when it comes to the historical period of capitalism, as well as pointing to play's both constructive and destructive powers.

Sigmund Freud first viewed play from the biological and psychological perspective and first only indirectly connected play to culture as an intermediary sphere of compensation either for a deficiency of energy or compensation (as a release of surplus energy) for the instinctual renunciation that the reality principle inflicted upon the pleasure principle. This sublimation of the libido became in later phases of his theories more explicitly connected-as the instinct Eros-to the construction of culture (Reich 2012; Fromm 1988) This biological and psychological perspective has inspired me to theorise play's trans-historical character as being part of our constitution. The perspective of Freud almost equates play with work. This demands some discussion. Play is not mere fun as Marx thought, but neither is it the same thing as work. Asplund's distinction between playing and gaming is important. Play is not well planned but capricious, spontaneous, social responsive and not uniformly produced. I would therefore say that play can be productive, very similar to work in its effects, but it is also something else and more immediate. Humans indeed have a need to play, but the reason for playing is not primarily and consciously to produce a use value that satisfies a consciously felt need; play is not thought of in an instrumental way. You can play just for the fun of it, as well as a child un-consciously can work his/her way through a problematic experience by play. And is it not true that play also, for various reasons, can be destructive in relation to use values? Breaking the rules can be fun.

At any rate play has been of major interest to a whole spectrum of disciplines-from biologists and psychologist to philosophers, anthropologist and cultural historians-during the last hundred years.

\subsubsection{Play as the Meeting Point of Biological Energies and Psychological Needs}

Let us start the discussion of play as part of the human constitution with a comparison between Friedrich Schiller and the Greek philosophes of Antiquity. Friedrich Schiller saw human play as an intermediary expression between the purely sensuous and the rational. As a synthesis of the sensuous and formal impulses, play cancelled the authority of both and liberated man physically and morally. In the play experience (wo)man's dual nature was harmonized and humanized (Hein 1968, 67). Play was then freedom, and freedom humanized the human.

Johan Asplund stresses that it is hard to speak of a theory of play in the case of Schiller. The key concept Spieltrieb (play drive) had the power to reconcile the two essential drives (matter and form) that in turn were the foundation of many dichotomies like affects and reason, life and representation, transformation and identity, passivity and activity and so on. The matter drive was life and total presence in the senses; the form drive was connected to reason. In play humans were truly humans and as soon as mankind could spare some energy in the quest for survival they started to mould, perform and play. Asplund stresses that play had an abundant, exuberant and redundant character for Schiller. In play humans and animals went outside of themselves and created an object for him/herself (Asplund 1987, 5658).

For Schiller the nearby aesthetic activity was "the contemplative one rather than the creative experience", the highest form of which was the "free exercise of the imaginative and intellectual faculties rather than the physical" (Hein 1968, 67) According to Hein this largely imaginative play contributed to the "moral enhancement of the individual and society" (Hein $1968,67)$. This effect was unintended on the part of the individual because the player was perceived as voluntarily engaged in the activity "for its own sake" (Hein 1968, 67). "In this respect play is properly contrasted with work, which is stimulated by deprivation and is en- 
gaged in as a means to a practical end" (Hein 1968, 67). Play was a non-utilitarian activity, (Hein 1968, 67) even if it was essential for the creation of works of art. Artistic activity resulted in an end but did not produce use values, and for the artist the activity was important in itself. Art was understood as derived from play, but not as synonymous to it.

Schiller did not articulate the relation between play and art in a clear way. Hein assumes that both play and art were manifestations of the play impulse for Schiller. She interprets Schiller's view of art as "gratuitous exercise of higher level, i.e. intellectual, faculties" that was a "more mature or complex form of play" (Hein 1968, 67). This is also the primary deficiency in Schiller's theory on play according to her:

[T] he alleged value of play lies in a feature which is not merely nonessential but is in fact in direct conflict with the essential nature of play. If play by definition is spontaneous activity engaged in exclusively for its own sake, then to value it for its possible consequences is a denial of its essence. (Hein 1968, 67-68)

The productivity or non-productivity of play was a recurrent theme even in ancient Greece. Plato and Aristotle regarded play as "sensuous in character and as a natural expression of animal restlessness" (Hein 1968, 68) Play was not necessary to rationality, but neither necessarily antagonistic to it. It "could be used to both good and evil ends, but was in itself nonserious and of little consequence."(Hein 1968, 68) Play was thus not an intermediary synthesis, but was sensuous, even though not antagonistic to reason in the referred view. More parallel to Schiller's view was the opinion that play itself was of little consequence, but could be manipulated for productive or non-productive ends. But playfulness had an ethical dimension for the Greeks. Aristotle's Eudaimonia, happiness, was an end in itself but only philosophers - a leisurely attitude was needed to engage in theories - were "properly predisposed to play" with the right "seriousness and disdain for decadence" (Nagel 1996, 2-3). The same contradiction as Hein point at in the thought of Schiller thus existed in Antiquity, even if Schiller's and Kant's concepts of play as creativity, freedom and autonomy were alien to the world view of Aristotle according to Nagel (Nagel 1996, 2).

But the ancient Greeks also made a distinction between Apollonian, logocentric, and Dionysian, tragic and bacchanalian, forces when they discussed play. Play was both matter and form in the words of Schiller. Aristotle clearly favoured Apollonian play, and it was with him that the abjection of play as the Other to reason originated - the Other being mere play and nothing serious. The tragic Dionysian play became eclipsed by Aristotle rather than Plato (Nagel 1996, 4-5). For Plato play and seriousness could instead, in the right moment (kairos), be contiguous. This was not so for Aristotle, even if he stressed "the instrumental value of paidia for children's and young men's education" (Nagel 1996, 1, 4, 6-7).

These two examples, Schiller and the ancient philosophers, show a certain ambiguity with the concept of play. The major stress is put on it being engaged in for its own sake and not for any other serious reason. Play is thus the opposite of reason or an intermediary synthesis between the senses and the reason. At the same time it could have unintended good effects, even being the foundation of the arts, according to the former, but it could also have bad effects according to the ancient philosophers. Play could be manipulated as an instrument in the hands of external parties, even if the result was unintended by the player.

Neither Schiller nor the major Greek thinkers gave any importance to the ludic Dionysos. It was Hegel who first gave equal importance to the Apollonian and Dionysian play impulses, when he compared truth with a Bacchanalian whirl "which is the interplay of drunken frenzy and calm repose". This trope was a central category in Hegel's play theory (Nagel 1996, vii, 2,7 ). Hegel thus pre-empted Friedrich Nietzsche's ludic perspective (Nagel 1996, 3), even if it becomes clear that Nietzsche view on Apollonian and Dionysian play has a lot to do with gaming rather than playing.

Friedrich Nietzsche stressed play as part of the instincts of life and the drive to enhance life, and he affirmed the frenzied and irrational aspect of Dionysian play which he thought of as intertwined dialectically with Apollonian play in a unity of opposites (Nagel 1996, 3). If play 
was part of a truth-seeking in the thoughts of Hegel, Nietzsche stressed that play in its Dionysian forms was something in it itself, being life. Life was inherently affirmative according to Nietzsche, very much in the same way as play has been understood, but he also advocated values and actions which enhanced life (Mitcheson 2013) in a rather specific way as competition with other wills to conquer power. This latter focus is better conceived of as gaming than playing. Nietzsche dismissed scientific reason in the name of life and affirmed art in a playful manner, much like Schiller. An artist brought something forth that did not exist until then (Heidegger 1991, 69). The creativeness of art and play was practised outside of the rules, in a world that was not ruled by the existent.

Evolutionary biologists in the $19^{\text {th }}$ century like Karl Groos and Konrad Lange, and social Darwinist Herbert Spencer, broke with the picture of play as a joyous expenditure of surplus energy and instead explained play as having evolutionary functions, rather than involving pleasure. Play was for them a conscious self-deception as a compensation for disappointments in life and as preparation for serious activities. Play thus got a teleological orientation as a preparation for life or as a conscious self-deception that compensated for disappointments in life. Play could function as a safety valve for these biologists, "enabling the organism to discharge the emotions which experience generates but for which it provides no adequate outlet". Play according to Herbert Spencer had "survival value" and yielded "rewards beyond itself" (Hein 1968, 68).

It was at this time that the classical play theories were developed according to the drama pedagogue Kent Hägglund: 1) The Energy Surplus theory of Herbert Spencer (who was inspired by Schiller) that claimed that the play of children could be categorized as sense-motor (body movements), artistic-aesthetic play, rules-based play, and imitation play. 2) The Recreational Theory developed by Moritz Lazarus and Heymann Steinthal that stressed that play was relaxing. 3) The Exercise Theory developed by Karl Groos that stated that play was an instinct that directed the child to play out things and abilities that they were going to need as adults. An animal high up in the developmental chain needed a longer period of adolescence. 4) The Recapitulate Theory of Granville Stanley Hall (see above) who was influenced by Ernst Haeckel's theory of Recapitulation and the thesis that ontogeny recapitulates phylogeny (Hägglund 1989, 12-13, 5-61; Wikipedia contributors n.d.; Schwartzman 1978, $21-$ 22, 4-42).

Helen B. Schwartzman, the social anthropologist, has criticised many of the abovementioned notions of the defining characteristics of play. Her point of departure is that the systematic study of play was born at the same time as the discipline of social anthropology in the late $19^{\text {th }}$ century. Many scholars were influenced by Charles Darwin and Social Darwinism under this period of time and identified parallels between the development of the species and societal development. All highly developed animals had developed from single-celled organisms, and contemporary civilisation had developed from hunter-and-gatherer societies. Social anthropologists took this view with them when they went out to study primitive societies. They thought they were studying the origins of their own society. Taking on this perspective it was easy to see other societies and cultures as less mature, less valuable and childish, which fitted the interests of imperialist powers of the day. This perspective also made it easy to see children as more primitive, and their development proceeded in the same manner as civilisations were thought to make progress in stages (Hägglund 1989, 12 13, 5-61; Schwartzman 1978, 2-22, 4-42).

Schwartzman especially criticises early anthropologists like Granville Stanley Hall that thought that they could find remnants of old cultures in the play of children, but also Sigmund Freud and Jean Piaget, whose theories about children's mental development in stages (see below) had remnants of this thought pattern (Hägglund 1989, 13, 5-61; Schwartzman 1978, 21-22). Sigmund Freud continued within this biological paradigm but also transcended it by stressing the psychological dimensions of play (Hein 1968, 68). In his first phase Freud argued that play had the same function for the child as dreams and phantasies. (S)he could create his/her own world with objects from the real world in play and repeat pleasurable 
events at will. In play it was also possible to change events so that they met the expectations of the child. These first theories did not answer the question why children in role plays repeat unpleasant experiences (Hägglund 1989, 14-15).

Play's relation to these unpleasant experiences was at the centre of Freud's concerns in his second theoretical phase. Play was now one of the first normal methods applied by the child's mental apparatus and in Beyond the Pleasure Principle he told the story of a year-and a-half-old boy that threw away things that he a moment later, to his great joy, found again. Freud interpreted the story as a great cultural achievement of the child and saw it as a compensation for an instinctual renunciation that the boy had made earlier when he had allowed his mother to go away without protesting. The boy thus staged the disappearance and return of his mother (Freud 1974, 8-9). Hein stresses that play according to Freud could only superficially be described as a "joyous and spontaneous expression of vital freedom" or simple analysis of play "in terms of imitation or aimless pleasure-seeking or instinctive satisfaction of biological needs"; instead, play is regarded as an assimilative activity where the agent "gains active mastery of a situation" and Freud understand play as a form of conquest which also is the reason for the experienced pleasure: "As such it is as serious as any of man's activities and as purposive in its nature as his work" (Hein 1968, 69). Play is thus a psychological technique or mechanism to handle the pleasure principle in its contact with reality.

But at the same time children's play is still seen in Beyond the Pleasure Principle as part of the pleasure principle. Small children cannot get enough of repeating pleasurable experiences, a behaviour that later in life was changed when only new jokes were funny (Freud 1974, 1-11, 29). Freud thus stressed that play at least had two functions for the child: First, helping it to master situations in which (s)he had been passively subordinated, and second, to amuse the child in a joyous manner. Play was then both a drive- "At the dawn of the development of the human soul, free and unhampered play is enjoyed by notions, feelings, desires that at later stages of development would horrify the conscious" (Vološinov 2012, 52) and a function to deal with clashes between it and the reality.

The pleasure principle connected (wo)man with sex, enjoyment and play. In play between adults, jokes and wit could function as a relief for the repressed impulses of Eros: "Jokes and witticism have the tendency to bypass reality, to provide relief from the seriousness of life, and to secure an outlet for repressed infantile impulses, whether sexual or aggressive" (Vološinov 2012, 91-92).

In his final theoretical phase Freud focused on the relation of the instincts and the psyche to the cultural and societal sphere, and stressed the socially constructive roles of humans according to Fromm who emphasises that Freud made a clear connection between Eros and Civilisation, at the same time as the destructive forces were internalised and transferred to Thanatos rather than existing between people in society-which was the point of criticism of the social psychologist Wilhelm Reich (see below) (Fromm 1988; Reich 2012). In contrast to Fromm, Hägglund claims that the relation between Eros and Thanatos was a dialectical one where the former was the drive for sexuality, lust and life in general, and Thanatos the drive for death, destruction and aggression. Hägglund maintains that play here did not belong to Eros but to Thanatos, and its will for repose and balance. Especially repetitive play was part of death drive (Hägglund 1989, 16).

Herbert Marcuse, on the other hand, claimed that the mental forces "opposed" to the reality principle were relegated to the unconscious and therefore could "provide no standards for the construction of the non-repressive mentality, nor for the truth value of such a construction". Instead Freud according to him singled out fantasy as a mental activity that "retains a high-degree of freedom from the reality principle even in the sphere of the developed consciousness". Fantasy links "the deepest layers of the unconscious with the highest products of consciousness (art), the dream with reality; it preserves the archetypes of the genus, the perpetual but repressed ideas of the collective and individual memory, the tabooed images of freedom" (Marcuse 1955, 140-141). 
This theory of the human instincts thus went through several changes. From the beginning when he made a distinction between the pleasure principle (containing play) and the drive for self-preservation or the reality principle, to the middle period when he included the latter within the former, making the pleasure principle all-encompassing (including play as both drive and sublimation), before finally ending up with the final version (Marcuse 1955; Fuchs 2013; Vološinov 2012, 50-54; Reich 2012).

Freud's view on play was further developed in the field of psychology by Lili E. Peller, Erik Homburger Erikson, Daniel Woods Winnicott, and Jean Piaget. Lev Vygotsky and George Herbert Mead were more interested in the relation between the individual and the social world and will therefore be presented in the next sub-section.

Peller was active within the movement of Montessori pedagogy, and systematized Freud's views on play. She maintained that play was about dreams coming true, enjoyment, relief and well-being, but it did not have to do with pleasure all the time; it could also be about compensation. She introduces stages in the use of play of the child. In the last period between seven and twelve years of age the rules becomes more strict and rule-based plays substitute freer forms of play, but even in this period it is more important to follow the rules than to win the game (Hägglund 1989, 18-19). Erik Homburger Erikson assumed that the psychological development of humans did not end in the juvenile period but continued through life. Play was a universe of experiments. (Hägglund 1989, 21-22). Donald Woods Winnicott made the point that psychoanalysts had failed to focus on the function of play in itself and favoured play as an instrument in therapy and research. He speaks of play as the third room, a special mental space between the subject and the external world. This third room still exists for adults, and it is there that play and fantasy that we use for creating art, music and theatre, continue to develop (Hägglund 1989, 26-28).

Jean Piaget started off as a zoologist and later became interested in philosophy and psychology. His main focus was to analyse and systematise how human intelligence developed. $\mathrm{He}$ based his investigation on close and intensive studies of relatively few children, including his own. He was primarily interested in play's central role in the development of the mental processes, rather than play in itself. He worked out the concepts of assimilation, accommodation and adaption. Assimilation meant that the child regulated the world in accordance with his/her needs, accommodation meant the opposite like in plays of imitation. Finally adaption formed a synthesis constructed on the interaction between the former two in a kind of Hegelian dialectic. For the child, whose developmental stages were divided into sense-motor stage, the stage of concrete operations of thought, and the stage of formal operations of thought, the assimilation process was the most important. The stages followed each other in a strict sequence. Piaget identified the exercise form of play in the first stage and symbolic play and role playing in the second one, and finally, the more mature the child, the more organised the play became, leading up to rule-based play and sports (Hägglund 1989, 30-34).

Instead of stressing the difference between autotelism (play meaningful in itself) and heterotelism (the goal of the activity takes the form of a result) Piaget saw play in the contradiction between assimilation and accommodation: "When assimilation and accommodation is not differentiated, as at the beginning of the first year, there seems to be autotelism without there being play in the strict sense, but as assimilation gains on accommodation play is divorced from the corresponding non-ludic activities.". He also stressed that play is not simply spontaneous and it was possible to identify two poles between which play existed: "the one truly spontaneous, since it is uncontrolled, the other controlled by society or by reality" (Piaget 1962, 148). This fits the distinction that I am stressing even though Piaget himself did not distinguish between play and games. In contrast to Piaget the Swedish child psychologist Birgitta Knutsdotter Olofsson contends that play forms do not develop in stages, but coincide or are played out side by side. All forms of play are learnt under the first year of the child according to her (Hägglund 1989, 69-71). 
During the second half of the $20^{\text {th }}$ century biological and psychological research has continued to develop as academic disciplines like sociobiology, evolutionary psychology, behavioural ecology and epigenetic, all of which offer different views on play.

Anthony Pellegrini stresses in The Role of Play in Human Development (2009) that most theorists posit that play is observed only in situations "that are safe and where the organisms are well fed". According to him, Gordon Burghardt has advanced the Surplus Resource Theory of Play, not to be confused with Schillers notion: "While the concept of 'surplus energy' no longer is viewed as scientifically valid, the idea that play is supported only after organisms' basic needs are met is still current" (Pellegrini 2009, 9-16). Pellergrini, himself invested in the field, stresses that evolutionary devlopmental psychology has reached the conclusion that play differs between individuals and contexts in its alternative, and not pre-programmed, responses to the environment. (Pellegrini 2009, 20)

Stephen Miller argued in 1973 that even if it seems difficult to define play there seem to be, funnily enough, a great deal of consistency between untrained observers at the zoo, about, for example, when the animals are playing and not. He concludes that there exists a lot of intuitive knowledge about play that is hard to articulate. He then proceeded in his argumentation with the claim that very much the same patterns or motifs appear in almost all form of play, justifying the use of the one word play for such a "motley bunch of activities". Play, animal and human alike, involves according to him activities that in one situation can be play and in others not. A child's use of a pen can be strictly utilitarian, but also play. Miller cites Piaget, who stressed that a "schema" was never ludic or non-ludic and that it all depended on the context and the actual functioning. Miller suggested that if organized behaviour is viewed as involving some co-ordination of means and ends, then play could be distinguished by the way these are handled by it (Miller 1973, 92):

Play involves a relative autonomy of means. Ends are not obliterated, but they don't, as in some other modes of organization, determine the means. Furthermore this state of affairs implies a degree of autonomy for the actor who manipulates the processes at his disposal, which makes for freedom to assume roles otherwise unreal. Finally, means are elaborated by a psychological process that we thus far referred to as "galumphing"-in general, the voluntary placing of obstacles in one's path. (Miller 1973, 93)

Galumphing is defined as a shorthand term for patterned, voluntary elaborations or complications of processes where the pattern is not under the dominant control of goals (Miller 1973, 92).

Practice play, the word of Piaget for the exercise of activities just for the pleasure of the exercise (confined to individuals in his view and characteristic of the sensory-motor period in a child's development), applies equally well to the patterns in primate play (Miller 1973, 91). Studying the play of baboons, he concludes that it is easy to compile a list of characteristics that delimit play from non-play. The activities involve both animate and inanimate portions of the environment, and the motor-patterns involved resemble patterns from other contexts involving aggression, sex, feeding and so on. The motor-patterns in play can be performed in new and different sequences that are not possible in the other contexts, repetition is normal, and the motor activity is often exaggerated and uneconomical in comparison with the nonplay contexts (Miller 1973, 89). Practice play does involve rules but they are freely chosen and flexibly adapted. "Play is not means without the end; it is a crooked line to the end" (Miller 1973, 93).

Play between baboons also involved the reversal of roles where the mock fights often were initiated by the smaller of two animals. Miller concluded that "[s]uch ahierarchical activity seems to be a general feature of play" (Miller 1973, 89-90).

\subsubsection{Play as the Foundation for Socio-Cultural, Communicative and Political Practice}

Lev Vygotsky, professor in psychology in the Soviet Union was critical towards bourgeois psychology, and in his view on play he focused on it as social process, but like Piaget he 
stressed that each stage in the development of a child has its own drives and motives regarding play - even if the child does not start to play until its third year. The things which are interesting to a one-year-old child are uninteresting to a three-year-old that does not simply exists in a continuous now. Play for Vygotsky was the child's method of coping with the conflict between what you want and what is feasible (Hägglund 1989, 36-38).

In preschool years play encompassed almost all of the activities of the child, but in school "play and work or play and school tasks" become separate and form "two basic streams along which the activity of the schoolchild flows and finally, in the transitional age [...] work moves to first place, putting play in a subordinate and secondary position" (Vygotskij 1987, 27). This is the strongest connection between play and work I have found in my study of the literature on play, and it affirms my claims that play and work are trans-historical categories, implying that we should not treat the two categories dualistically but dialectically focusing both on similarities and differences in the interaction between them.

Vygotsky discusses both the research of Karl Groos and the theory of serious play that a scholar named Stern had proposed. Vygotsky admitted that the latter was not totally wrong. According to him Stern stressed that adolescence occupied a "middle ground between the play of the child and serious activity of adults".(Vygotskij 1987, 27) The theory pointed out two basic areas: the erotic and social relations. For the adolescent play was completely serious and satisfaction came from the functioning of the process and not the result of it. But for Vygotsky the theory had several severe flaws. The first and most important it shared with the theories of Groos. They were "purely naturalistic" teachings that could not establish the "principal difference between the play of an animal and the play of a human child." Play formed a complex developmental process in the child that mixed the natural, biological and organic evolution with the "social-cultural formation" in a complex "synthesis that makes up the real process". Therefore it was too simplistic to claim that the play of the child and the serious activities of the adults could be used to derive "an arithmetical average of both components in the form of serious play." It is in this context that he proposed the definition of play as selfeducation. (Vygotskij 1987, 27-28)

Vygotsky did not base play in the pleasure instinct, with the argument that all play was not pleasurable, for example the one that involved contests (Hägglund 1989, 37-39). The consequence of this observation, and the fact-I would like to add-that he did not distinguish between play and games, is that it is impossible to base play in pleasure.

All the basic activities of the child were not play, even if it looked that way for the adults when the child did things like opening and closing doors, playing with hobby-horses, without thinking of getting anything out of it. He favoured the idea of Groos that experimental play differed so much from other forms of play that it was not play at all. He wanted to break up the concept of play: "Play must be considered as a completely original activity and not as a mixed concept that unites all types of children's activities, particularly those that Groos called experimental play". Early childhood saw games as drinking from an empty cup, but Vygotsky did not want to compare that play with the creation of "imaginary situations". The former was more like the serious play of adolescents, where the play is not totally separated from real situations. It was in this context that he proclaimed that play had a unique relation to reality that for example involved the transference of properties of some objects to others within these imaginary situations. (Vygotskij 1987, 266-267) The claim that play is a kind of selfeducation could be seen as contradictory to the dismissal of experimental play as not-being play.

Hägglund claim that all forms of play contained rules according to Vygotsky. Imaginary situations in play demanded rules and rule-based play always created imaginary situations: role play was rule play and the other way around. And on a very abstract and general level play always performed or represented situations in life within the imaginary situations that lacked the conditional constraints of real situations. In play the cognitive process was separated, within limits, from the objects of the real world, and it was the will of the child that governed 
the rules of the play and not the situation. Rules even made the play more fun (Hägglund 1989, 37-39).

Working in the same tradition as Vygotsky, Aleksei Nikolaevich Leontiev developed a theory that saw human activity as socially situated and complex. He maintained that the active lives of human beings were characterised by play, memorisation and work. These activities dominated different periods in life. Play was most important for children and it functioned as a reflection of the objective real world. The content of play was directed by the children's will to copy the actions of the adults. Play's most important function was then socialization and accommodation (using Piaget's concept) to reality (Hägglund 1989; Wikipedia contributors n.d.). Also, George Herbert Mead, who had his major impact on sociology and social psychology with his theory on symbolic interaction, stressed the role playing for the development of our identities. When the child played being someone else it could see him/herself from the outside. This also led to internalisations of social roles. In contrast to Vygotsky he distinguished games from play. For him play was the spontaneous activity of the little child and games were the co-ordinated activity between several children (Hägglund 1989, 42-43).

Johan Huizinga ridiculed the naïve optimism of enlightenment and its worship of reason, homo sapiens, but also the preference of homo faber in his own time. He concluded that there existed a third function, applicable to both humans and animals, namely playing. "It seems to me that next to Homo Faber, and perhaps on the same level as Homo Sapiens, Homo Ludens, Man the Player, deserves a place in our nomenclature"(Huizinga 1955, Foreword). He conceived of play more as a cultural phenomenon than a biological one; play transcended the physiological and pure biological, and he approached it historically, with a focus on adults, and not in the spirit of the natural sciences (Huizinga 1955). Play was not understood as a utility and was passionate, but play was certainly also rooted in a biological instinct-play had a "primordial quality" in its intensity, tension, absorption, mirth, power of maddening and its fun, that was given by nature (Huizinga 1955, 2-3).

Huizinga focused on play as a function of culture and in this he drew attention to the relation between language and play.

\begin{abstract}
The great archetypal activities of human society are all permeated with play from the start. Take language, for instance- - that first and supreme instrument which man shapes in order to communicate, to teach, to command. Language allows him to distinguish, to establish, to state things; in short to name them and by naming them to raise them into the domain of the spirit. In the making of speech and language the spirit is continually "sparking" between matter and mind, as it were, playing with this wondrous nominative faculty. Behind every abstract expression there lie the boldest of metaphors, and every metaphor is a play upon words (Huizinga 1955, 4).
\end{abstract}

Johan Huizinga defined play as a free and voluntary activity; play that was ordered was not play (at best it was a "forcible imitation"), which had its aim in itself and was consciously performed outside of ordinary life as non-serious, but at the same time it absorbed the player intensely and was accompanied by feelings of tension and joy; play was an activity without material interest and proceeded within its own "boundaries of time and space according to fixed rules and in an orderly manner" that he called play's magical circle, which made play resemble a ritual. The rules were "freely accepted but absolutely binding". Play also demanded "visible action". In all of this it promoted the "formation of social groupings which often surrounded themselves with secrecy and disguises (Huizinga 1955, 7, 10, 13, 28, 166).

Johan Asplund criticises Huizingas definition in a work that aims at grounding his discipline in (wo)man's characteristic sociality and responsiveness (Asplund 1987, 29). Asplund exemplifies with (wo)man's predisposition to "answer" when a question is posed to him/her. In his eyes Huizinga stressed the rules and organisation of play too much. Even if Huizinga did not distinguish between play and game, his focus was on rule-based activities within a certain time and place that assumes a fixed character, according to Asplund. These activities can be repeated and guarded in the same form and become a tradition, and as such they exclude all forms of play that have the character of a one-time event. Improvisation, Asplund 
contends, appears as play only when it is formalised and thus is ended as improvisation. He opposes this and the view that some forms of creativity are not seen by Huizinga as necessary components of play (and to a lesser degree for games). He points to everyday language and contends that we are prone to conceive of capricious behaviours with no perceivable rules as play. Play could even be to break the rules. Asplund even assert that if we could conclude from a stimulus what the response would be, then it is not play, but maybe a game (see gaming). Play, to Asplund, in line with Gadamer (see below), is sheer responsiveness, but he agrees with Huizinga that play is voluntarily engaged in and that you do not do it for useful purposes or any other external goal or purpose. This means that play is exuberant or abundant. You play for the fun of playing, and play is its own reward. The opposite of play is not seriousness but utility, according to Asplund. Play and games are non-productive activities according to him, but they are social activities that can take place in many different places and situations (Asplund 1987, 29, 55, 63-66).

$\mathrm{He}$ admits that Huizinga is correct when he says that play in this improvised sense cannot found or construct a culture, but for Asplund it is play that contributes the matter for the cultural work of sorting and organising. The play precedes the game, the irregular the regular. Culture is created and completed as soon as play has disappeared and everything has become a game, one big treaty. A living culture according to Asplund needs redundancy, useless and exuberant action. The only thing that is left to do when all of such useless and exuberant actions have been persecuted and done away with by legislation is to "flip out" according to him. If Huizingas definition is too narrow in not taking into account non-regulated play (and in that process reifying a complex notion with solid and static concepts), it is too broad in its non- distinction of play from gaming. Asplund contends that a game is not a game unless it, in principle, allows cheating. Play does not allow cheating; it is a kind of cheating (Asplund 1987, 63-66). Asplund's understanding of play correlates well with my use of the concepts playing and gaming in this article.

Michail Bakhtin investigates the relation between play and culture in another way than Huizinga. He maintains in Rabelais and his World (1965) that the serious and the comic aspects of the world and of the deity, in early societies of a "preclass and prepolitical social order", were equally official, but that such equality was impossible in societies where the state and class structure had consolidated itself (Bachtin 2007, 6). "All the comic forms were transferred, some earlier and others later, to a nonofficial level. There they acquired a new meaning, were deepened and rendered more complex, until they became the expression of folk consciousness, of folk culture" (Bachtin 2007, 6-7). The relation of laughter, minstrels, festivities and carnivals to play is central according to Bakhtin. The basis of the laughter in the medieval carnival is free from religion and dogmatism, mysticism and piety, and they "do not command nor do they ask for anything". Some carnival forms parody the cults of the church in such a way that it is clear that the laughter belongs to an "entirely different sphere":

Because of their obvious sensuous character and their strong element of play, carnival images closely resemble certain artistic forms, namely the spectacle. [...] But the basic carnival nucleus of this culture is by no means a purely artistic form nor a spectacle and does not, generally speaking, belong to the sphere of art. It belongs to the borderline between art and life. In reality, it is life itself, but shaped according to a certain pattern of play.

In fact, carnival does not know footlights, in the sense that it does not acknowledge any distinction between actors and spectators. Footlights would destroy a carnival, as the absence of footlights would destroy a theatrical performance (Bachtin 2007, 7).

Bakhtin describes the carnival as play, rather than a game, in a class society. In the turning upside down of the highly hierarchical medieval society, this was not done in a spirit of competition. The festive laughter of people was also directed at those who laughed. "The people do not exclude themselves from the wholeness of the world." They, too, were incomplete (Bachtin 2007, 12). 
Bakhtin stressed that every feast was an important primary form of human culture that could not be explained "merely by the practical conditions of the community's work" and neither, more superficially, by pointing at the psychological demand for "periodic rest". Rather it was connected with the ideological dimension or the "world of ideals" (Bakthin, Rabelais, 8) The Swedish translation mentions here that the feast is no "workplay"' (Bachtin 2007, 9, 20).

If we compare the views of Huizinga, Asplund and Bakhtin with the view of the psychologists and Vygotsky on child play, as well as the perspective of game scholars like Jesper Juul, it seems that the concept of play, like the concept of culture, has two basic definitions: one broad and one narrow, the first focusing on life and social interaction in itself and the other focusing on specific activities (even if they are grounded in psychological development and needs). It is important to remember this difference, and especially the focus on the narrow definition, when we now look at Hans-Georg Gadamer's view on play and later on compare it with the views of language and communication of Hannah Arendt, Jürgen Habermas and Ludwig Wittgenstein.

Play could not exist without action according to Huizinga. Hannah Arendt mainly associated action with communication in a way that resembled Huizinga's view of play (it was meaningful in itself). Remembering the latter's argument about our nominative faculty and its play with words behind every metaphor, we can ask ourselves if play can be necessary for communicative action. Communication and speech acts in their turn have also been described by Jürgen Habermas in a way that has similarities to Huizinga's definition of play and Ludwig Wittgenstein has analysed speech-acts and primitive language in terms of language games. On a cultural level Michail Bachtin has written on the playful character of language and communication in terms of the dialogical word.

But before we examine in depth how communicative action relates to play, we will study Hans-Georg Gadamer's critique of the subjective notion of the concept of play, which saw play as something that happened in the mind or in the impulses of the subject (Vilhauer 2010, 32). In Warheit und Methode (Truth and Method) from 1960 Gadamer makes a distinction between the attitude of the player and play. For the player play is not serious in an ordinary way, like the serious purposes which dominate the world, and this is in fact why he plays. More important than the recreational side of play is play's own sacred form of seriousness. "Play fulfils its purpose only if the player loses himself in his play." Gadamer contends: "only seriousness in playing makes the play wholly play" (Gadamer 1975, 91-92). This mode of being of play does not "allow the player to behave towards play as if it were an object" (Gadamer 1975, 92). But even if (s)he knows what play is, and that (s)he 'only plays a game', the player does not know what exactly (s)he 'knew' in knowing that (Gadamer 1975, 92).

This convinces Gadamer that it is impossible to find an answer regarding play in the "subjective reflection of the player". Instead we should look at the play's very mode of being. He maintains that play has its own essence that is independent of the players. He points to the use of metaphors like play of light, play of the waves, play on words, play of forces to stress that play exists even "when the thematic horizon is not limited by any being-for-itself of subjectivity, and where there are no subjects who are behaving 'playfully'." Play reaches presentation through the players in a movement that has "no goal which brings it to an end" but rather "renews itself in constant repetition". He talks of this as a "to-and-fro movement" that "accords with the original meaning of the world spiel as 'dance"' (Gadamer 1975, 9-93).

The movement backwards and forwards is obviously so central for the definition of a game that it is not important who of what performs this movement. The movement of play as such has, as it were, no substrate. It is the game that is played-it is irrelevant whether or not there is a subject who plays. The play is the performance of the movement as such (Gadamer 1975, 93).

The most original sense of the word play is a "medial one", as in "something is "playing"'. If language is concerned, the subject of play is the play itself. The savage does not according 
to Gadamer know of any "conceptual distinction between being and play" (Gadamer 1975, 9-94). Gadamer holds that even the experiences of play that anthropologists and psychologists have get illuminated by acknowledging the primacy of play over the player's consciousness and by treating play in the medial sense of the word 'spielen':

\begin{abstract}
Play obviously represents an order in which the to-and-fro motion of play follows of itself. It is part of play that the movement is not only without goal or purpose but also without effort. It happens, as it were, by itself. The ease of play, which naturally does not mean that there is any real absence of effort, but phenomenologically refers only to the absence of strain, is experienced subjectively as relaxation. The structure of play absorbs the player into itself, and thus takes from him the burden of the initiative, which constitutes the actual strain of existence (Gadamer 1975, 94).
\end{abstract}

Different games have different spirits as a result of different organisations of the to-and-fro movement. Gadamer stresses the importance of rules and claims it is characteristic of human play that we chooses to play something, a structure of movements, with a definite quality that the individual submits her/himself (Gadamer 1975, 96). Vilhauer interpret this as the pattern of movements in play surpassing the players. All the players belong to the play and "the game tends to master the players" (Vilhauer 2010, 32; Gadamer 1975, 95). The movement of the game is not a free area in which the player plays him/herself out (Gadamer 1975, 96) But at the same time play's character is variable in Vilhauer's interpretation of Gadamer. It cannot be "fully determined or mechanical" and involves "the possibility of spontaneity and variety" that demands that the players' moves "are not identical to each other or totally predictable in advance" (Vilhauer 2010, 33).

The player's submission to play involves at the same time a unique pattern, a subject matter in which the player presents him/herself by playing something that (s)he wants to play. Within this "readiness to play" the player also chooses what (s)he is to play. Human play involves intention or the "choice to constrain one's own freedom to the rules of a game", and is not simply instinctive or caused by external forces according to Vilhauer's interpretation (Gadamer 1975, 96; Vilhauer 2010, 34). Gadamer speaks of the self-discipline and the order we impose on ourselves with effort, ambition and commitment in play and calls this selfpresentation (Hans-Georg Gadamer, "The Relevance of the Beautiful", 1977 cf. Vilhauer 2010, 34).

The form of play is near the mobile form of nature according to Gadamer. Rather than saying that the animals plays too and metaphorically that the lights play, we should instead say that man too plays-a natural process. Play for man, as a part of nature, is "pure selfpresentation" (Gadamer 1975, 94). In an essay from 1973 he contends that play as a phenomenon is blurring the distinction between man and animal. Play is presented as an elementary phenomenon that pervades the animal world and is determinant of man as a natural being. Monica Vilhauer claims that play is essential to all living things in the thinking of Gadamer (Vilhauer 2010, 33).

Gadamer here succeeds in managing both the narrow and broad definition of play at the same time. His focus on the importance of the rules stresses the close connection between play and game and he does not distinguish between them, but I would argue that he implicitly also shows the differences between the two-as in this argumentation:

Every game presents the man who plays it with a task. [...] Thus the child gives itself a task in playing with the ball, and such tasks are playful ones, because the purpose of the game is not really the solution of the task, but the ordering and shaping of the movement of the game itself (Gadamer 1975, 97).

This is clearly about playing. Playing is a natural and creative self-presentation that is not aimed for anyone other than the players, but play could also be play or representation for others. I contend that this latter category of playing is better understood as gaming. 
Gadamer's theoretical understanding of play has some similarities with Lewis Hyde's understanding of the gift. Hyde holds that the creation of a work of art is a gift, not a commodity; work of arts can survive without the market, but if there is no gift involved then there is no art. What does this mean? Hyde claims that gifts are bestowed upon us and point to the fact that we speak of 'talent', intuition and inspiration as gifts; a gift "is a thing we do not get by our own efforts" (Hyde 2012, xxii):

\begin{abstract}
Usually, in fact, the artist does not find himself engaged or exhilarated by the work, nor does it seem authentic, until this gratuitous element has appeared, so that along with any true creation comes the uncanny sense that ' $l$ ', the artist, did not make the work (Hyde 2012, xxii).
\end{abstract}

Play and gifts seems central to the arts, but the art is often performed in front of an audience. If Hyde's remarks above point to the "inner life of art" there is also an "external life" after the work of art has left its maker. Hyde maintains that the gift is central also in this external life. "The art that matters to us-which moves the heart, or revives the soul, or delights our senses, or offers courage for living, however we choose to describe the experience - that work is received by us as a gift is received" (Hyde 2012, xxii). Hyde proceeds by claiming that this feeling is not changed by an entrance fee at the museum, but the way we treat a thing or work of art can change its nature. It is possible to destroy a work of art by converting it into a pure commodity. "The gift portion of the work places a constraint upon our merchandising" according to him (Hyde 2012, xxiii). This roughly correlates with my analysis that play can contain indeed contests and performances, but that external actors have the power to change or destroy its character as play. This possibility is also admitted by Gadamer (Gadamer 1975, 98).

From these inner and external processes we also can conclude that there exists a movement within the world of art, a kind of communication, or inter-play of gifts. Building on anthropological research and aesthetic theory, Hyde asserts that the cardinal property of the gift is that it should always be in motion. What we have been given is supposed to be given away again, not kept: it is even better if the gift is not returned, but instead given to some new "third party". If someone instead commercialises the gift relationships, the whole social fabric of a group is "invariably destroyed" (Hyde 2012, 4-5).

As with Gadamer's play, the gift of Hyde's moves on in a dynamic process. Like a free conversation (exchange of meanings back and forth between humans) rather than a static or reified thing. Asplund's contention of play as an elementary social form, Hyde's proclamation of the gift as a source of social life, and Jürgen Habermas' focus on the importance of a public sphere created by free communication all share a focus on a similar dialectical movement. The sociality and the ease of the performance of the activities stem from them being part of the human constitution.

To this family of theories we can also include Michail Bakhtin who stressed the continuous dialogue going on in the world literature around the most essential questions concerning human existence. The poet, novelist, prophet, leader or scientist all have to justify their words, they have to represent something for someone (Bachtin 1988).

Bakhtin stressed that the reader or listener contributes something proper and new to the work of art. The reader of the work does not exist in the same time and work as the author; there is a distinction. The other is alien to the author, but they are connected through a dialectic relation. Bakhtin did not conceive of any first word or last word, or any boundaries for the context of dialogue in the past or in the future. Not even the dialogues of the past can be seen in fixed forms according to him, they will always keep on changing in the coming dialogues of the future. In every moment of the development of the dialogue there exist huge unlimited masses of forgotten significations that someday will be activated and come to life again in a new context (Bachtin 1988, 269-270).

After this presentation of Gadamer's concept of play as a movement back and forth, as a kind of dialogue, it hopefully seems less strange to connect the thoughts of Huizinga, 
Asplund, Bachtin and Gadamer to the thoughts of Hannah Arendt and Jürgen Habermas on (communicative) action. The main purpose of this section of the article is to examine the connection between communication and play. I will first show how, in the thinking of Hannah Arendt, speech and action are connected in an idealised way to Huizinga's and Gadamer's definition of play, and then I will make some critical remarks of the importance of bringing in history into the equation. After this I will compare and identify the links between Arendt's concepts of speech and action (praxis) with Jürgen Habermas' concept of communicative action.

The idealistic focus of Huizinga on thoughts and mentalities as the driving force of history corresponds to Hannah Arendt's highlighting and appreciation of action and speech in an idealised and truly political sphere, at the cost of work (as in creating lasting works and use values) and labour (as in ceaseless and repetitive toil for survival) that she contends have been endorsed by modern political economy. Arendt was inspired by ancient Greek thought and the focus on political dialogue within the polis.

Arendt stresses, very much as how Gadamer describes the conditions for play, that the plurality of humans is the condition for action, as well as for speech. Plurality is displayed as similarity and difference; without the former, people would not be able to make themselves understood in social interaction or between generations (or to make plans for the future); without the latter, language and action would not be needed. (Arendt 1998, 237-238) Hence, for Arendt, speech is an especially important form of action and through it action acquires meaning. Action is the human activity that requires words to the highest degree; in work and labour they have a subordinated role (Arendt 1998, 242).

Play needed action according to Huizinga; communication is action in itself, but also forms part of other action to a high degree according to Arendt. Communication then goes together well with play, especially if playing with words is an integral part of it, and communication gives play its meaning. Hypothetically I propose that communication can be performed within play in an instrumental way, but then it forms part of a larger play form that is funny in itself, just as it can be playful in itself on other occasions. The proper playfulness of communication can be used, or attempted to be used, within work and labour in instrumental ways: to make the work more attractive, concealing tedious labour or exploitive mechanisms' and alienation. Playing with words in work and in labour will be treated in the section regarding these relations further on in the text. For now I just would like to point to the similarity between play and communication. Playing with words can be playful in connection to the reality and in relation to language's own rules, very much in the same way as Asplund speaks of play as including the joyful breaking of the rules in a pure social responsiveness.

An action puts something in motion according to Arendt; it acts, and it commences and regulates. The new beginning does always contradict the static possibilities and is therefore always improbable, (Arendt 1998, 241) much in the same way as play is open to spontaneity and the freedom of variations according to Gadamer. Arendt argues that the individual takes communicative initiatives without there being any particular decision involved. In opposition to other activities within Vita Activa no man or woman can let go of speech and action.(Arendt 1998, 237-238, 242) Thus speech and action are also essential to humans if we are to believe Arendt. This theory fits well with Asplunds notion of social responsiveness. Initiatives and responses come naturally to (wo)man.

Speech and action, when it is used between people who speak and interact with each other, rather than against and for something, also tell us something about the speaker and (wo)man of action (Arendt 1998, 243), much in the same manner as Gadamer writes that the player to a certain degree plays out him/herself in the play. But here we find a rather big difference. The risk associated with appearing as Someone in the communicative presence of others is something that only a person who is ready stay in that presence will do, according to Arendt (Arendt 1998, 24-244), but in playing you can always say that it was only play. In play you are free to experiment with roles, identities and communicative actions out of the ordinary. Communication is a broader concept, with a broader range of functions, than play 
in the social life. Speech and action need a surrounding world to address and project onto, but this can be for different purposes, and they take on another, not so risky and freer character within play than otherwise. Hence, there exists a relation of mutual influence between play and communication, two concepts that share many similarities but also differ from each other.

The lived action and the pronounced word are expressed in the Aristotelian term 'energia' according to Arendt, which, in contrast to my thinking, stresses an idealised and more narrow version of communication that simply includes the activities that do not have a purpose and do not leave any end result except themselves, and whose meaning is exhausted in their practice. Arendt points to Plato and Demokritos who repeatedly claimed that techne politiche should not be compared to work but with practical arts like medicine, dance and theatre, where the end product is the process in itself. Speech and action was "virtuosic" to her (Arendt 1998, 279-281). This is the origin of Paolo Virno's proposition of the virtuosic character of labour in post-Fordist capitalism.

Habermas' focus on the communicative action in the life world shares this idealism in charging an idealized free communication in the life world with progressive power. One crucial distinction, between Habermas and Arendt is that he situates the life world within the structural transformation of society as a whole, which includes a conflict between the life world and the system world. There is thus a moment of struggle in the vision of Habermas, which is lacking in Arendt's work. This struggle, with its focus on ideas and communication, carries similarities with the Situationist's project of revolutionising everyday life with play, even if the latter was involved in more tangible strategies in a more direct attack on abstract labour.

If action was a necessary (but not sufficient) condition for play according to Huizinga, and communication was the highest form of action, to Arendt, play indeed has fertile soil to expand in when it comes to the trans-historical social life that Jürgen Habermas theorises about with his concept of communicative action (Habermas 1987, 119). In Habermas formalpragmatic analysis, the concept of life world aims at grasping the structures "that in contrast to the historical shapes of particular lifeworlds and life-forms are put forward as invariant". He is engaged in a separation of form and content where he is focusing on the structures of the life world in general. I will here follow his analysis of how the life world is related to the "worlds on which subjects acting with an orientation to mutual understanding base their common definitions of situations" (Habermas 1987, 119-20).

Habermas contends that there exist three different "actor-world relations" in which the subject relates to the world as an object, something social and something subjective (Habermas $1987,120)$. The speech acts' references thus appear to the speaker as something objective, normative, or subjective. Habermas also calls them for formal world concepts and makes a distinction between them and the life world. "[T]he lifeworld is constitutive for mutual understanding as such whereas the formal world-concepts constitute a reference system for that about which mutual understanding is possible" (Habermas 1987, 126). "Communicative actors are always moving within the horizon of their lifeworld; they cannot step outside of it. [...] The structures of the lifeworld lay down the forms of the intersubjectivity of possible understanding (Habermas 1987, 126). A situation, finally, consists of a segment of "lifeworld contexts of relevance" (Habermas 1987, 122).

Hannah Arendt stressed the creative flux of communication and Habermas put more emphasis on the cultural context surrounding each life world and used the formal worldconcepts to show how communication ideally functions in the search for mutual understanding. Communicative actors do not understand their communication as a means to intersubjective agreement about the life world. This instrumental and cultural effect is hidden from them, but they do, Habermas argues, understand that they need a common ground to interpret facts, norms and subjective realities in concrete situations. They need to create common rules and the rule-negotiation appears as rewarding in itself in the context of the life world - a thought that is similar to Gadamer's view of play. 
Ludwig Wittgenstein's theories on language games can perhaps give us a more concrete picture of how communication is used in the situations of real life. In his Philosophical Investigations, paragraph 2, Ludwig Wittgenstein takes the example of a primitive language between a builder $A$ and an assistant $B$. A is building with building stones as blocks, pillars, slabs and beams. They use a language with the words 'blocks', 'pillars', 'slabs' and 'beams'. A calls them and B brings the stones he has learnt to bring at such an such call to A (Wittgenstein 1967, 3).

\begin{abstract}
In the practice of the use of language (2) one party calls out the words, the other acts on them. In instruction in the language the following process will occur: the learner names the objects; that is, he utters the word when the teacher points to the stone-And there will be this still simpler exercise: the pupil repeats the words after the teacher-both of these being processes resembling language.

We can also think of the whole process of using words in (2) as one of those games by means of which children learn their native language. I will call these names "languagegames" and will sometimes speak of a primitive language as a language-game.

And the processes of naming the stones and of repeating words after someone might also be called language-games. Think of much of the use of words in games like ring-aring-a-roses.

I shall also call the whole, consisting of language and the action into which it is woven, the "language-game" (Wittgenstein 1967, 5).
\end{abstract}

Speech and tangible action are here connected to each other in language-games. These language games can have pedagogical and productive results, but the way in which this is done, with repetition and imitation, seems to be playful in a deeper sense to Wittgenstein who in this comes near Gadamer and sees communication as a social "to-and-fro" interaction that is determined by the concrete and situational language games in a sense that resembles Gadamer's view that the players were absorbed by the play. Hence Wittgenstein seems to support the idea of play being central to language that in turn is both central to tangible forms of goal-oriented action as well as determined by the concrete situations (for our purposes speech can then be characterised by play, work or labour).

Wittgenstein makes the point that people do not always have a definite purpose with what they say, and he asks rhetorically if talk without purpose has to be meaningless, implying that it is not (Wittgenstein 1967, 188).

Another play element is touched upon by Wittgenstein when he discusses how we can know what the phrase 'I am frightened' really means. He refutes the possibility of introspection "[W]hat am I referring to when I say it?" Instead the right question is "In what sort of context does it occur?" Asking the first question, the subject repeats the expression of fear at the same time as she attends to herself, as she was observing her soul "out of the corner" of her eye. Wittgenstein asserts that this will not give you an answer to the question on the ground of "observing what accompanied the speaking." The answer given by introspection can only supplement and paraphrase the earlier utterance. The best thing to do in this case, if you want to understand what 'fear' is in a "single shewing", is to "play-act fear".(Wittgenstein $1967,188)$ From this we can learn that Wittgenstein really stressed the relation between play and action and saw play as a way to be in the moment and to re-live the (communicative) action in itself - instead of observing and representing it from the outside. He thus shared the definition of play as something that absorbs you and that you have to lose yourself in to practise.

Gregory Bateson, a social anthropologist that both used cybernetic theories and investigated animal behaviour was explicitly interested in play from a communicational perspective and was interested in the question of how we learn to learn things. The concept of text and context was important for him. Text signified the action at hand in the context. The third concept was meta-communication, meaning unconscious messages that we send to each other at all time to clarify what kind of context we are in-if we are playing or being serious for example. Communication thus labelled the situations. This meta-communication, assigning dif- 
ferent meanings of words and actions in different contexts, was something that the child could train in play according to Bateson. Once again the connection between play, communication and situation seems to be of central importance to humans. Bateson held free play with its intricate meta-communication, higher than (fixed) rule-based play (Hägglund 1989, 54-56).

We cannot leave the category of play without saying something about the surrealists' and situationists' position on play. The latter's position will largely be treated when the relation between play and labour is examined below.

Georges Bataille was part of the surrealist international, but broke with André Breton before the second world war, after it he and some friends and surrealist followers developed an Encyclopaedia Da Costa (1947) that in a joyous but also critical way (against the dialectic of enlightenment) started in the middle of a sentence and just contained words beginning with the letter E. Under the entry "Encyclopedie" you can read that encyclopaedias are going to great trouble to explain words that are no longer in use but that they do not care about the unknown words that are burning to be pronounced. For a child the discovery of language is not the realization of the present but foreknowledge of the future: "Words tell him, not what he is, but what he will be." For each and every new word invented, a link is broken with the effective presence according to the unknown author (Anon 1995, 122, 124). The present now and the creative and experimental power of language to understand the world in new ways was at the core of Bataille's interest in reviving rather than reifying social life with the help of play.

During this time Bataille was writing a major work called The Cursed Share in which you can detect connections between his group's critique of the reified view on knowledge and the reifications of capitalism. In the book Bataille makes a new interpretation of Marcel Mauss essay The Gift, which is centred on how different cultures deal with the question of abundance through rites founded on destruction and euphoric social dissolution. Bataille's thesis was that capitalism gradually had established an economy built on the principle of scarcity and that this had created an imbalance in the social structures manifesting themselves in the accumulation of social wealth by the bourgeoisie. Bataille claimed that accumulation was profanity, homogeneity and stasis, and in contrast to science which depended on homogeneity between its elements, the method of Bataille was a negation that suspended common denominators (Brotchie 1995, 21-23; Bataille 1991, 168-69). Bataille, in contrast to Jorge Luis Borges who delivered his critique against encyclopaedias and classifications by converting the library to a labyrinth, seems more positive about the insecurity of the labyrinth, claiming that trying to understand it would devaluate its presence (Hollier 1989, 60-61; Borges 1998, 35). This celebration of collective rites and the importance of being present in the moment, together with the critique of the stasis and reifications of positivist facts, imply the ideal nonexistence of instrumentality and the abundance of resources, interpretations and communicative inventions, which stresses the same themes as touched on in our discussion of the relation between play and communication.

Roger Caillois, a former Surrealist who together with Bataille broke with the Surrealist International before the war, published a classic account on play and games in 1958 called Man, Play and Games (Les jeux et les homes). He started the book with a critique of Huizinga's definition, but only after first having stressed that Huizingas book Homo Ludens opened up very fruitful avenues for research and reflection, even if most of its premises were debatable. The definition of play that Huizinga presented was both too broad and too narrow. It was meritorious and fruitful to grasp the "affinity which exists between play and the secret and mysterious", but this relation had nothing to do in a definition since doubt, mystery and travesty, when transformed into play tended to lose their very nature of mystery. The old surrealist, taking secret societies and mysteries seriously, in his mission to counteract the dialectic of Enlightenment, thus agreed that play was the opposite of the serious in its exposing and publishing of the mysterious in a way that somehow expended it (Caillois 2001, 4-5). Play had a deconstructive and destructive power according to him, and in this his thought 
came close to Freud's early claim that play was a way to be the master of a situation, implying here that play had the power to take the seriousness out of a threat or mystery, and perhaps also dethrone authorities.

This view of the power of play was taken up by the Situationists in a new way as a strategic means to change everyday life. Play's socialising function has been stressed by many in this overview, especially the psychologically inclined thinkers, but play's subversive function has also been stressed by, besides by the Situationists, Bakhtin and the social anthropologist Helen B. Schwartzman.

Two of Caillois' famous categories of play: Mimicry (simulation) and Ilinx (vertigo), presented in the introductory section, can be easily included in the description of play. The other two are focused on competition and chance. The Situationists of the Situationist International (SI) wanted play to be ridded of all gaming and competition. For them play was perceived as the central category of play and work, perhaps even implying that play had the power to replace socially necessary work as well as labour of capitalism in their struggle to revolutionize everyday reality through creating situations in which all attendants participated in non-reified and playful ways. (Post)modern critics have criticised this both broad and deep perspective, but it has also been defended. In this stress on play the Situationists shared Herbert Marcuse's reading of Freud and Marx. The Situationists will be thoroughly discussed in the sections on the relations between the categories.

Finally, to connect this discussion of play to contemporary cognitive capitalism with its emblematic digital form of work and labour, some words on what Pekka Himanen has called the hacker ethic. Linus Torwalds claims in the foreword to Himanens book that the computer is entertainment for the hacker (Torvalds 2001, xvii) and Pekka Himanen places this statement at the centre of what he calls the hacker ethic. The hacker is enthusiastic about interesting things; they give him energy, according to him. Hacking is joyous and often has its origin in "playful explorations". Himanen thinks that the description of hacker activity as passionate is even more apt, because it conveys the three levels of interesting, inspiring and joyous, which is an improvement on the word entertaining. This kind of "passionate relationship to work" is also seen in academia, among artists, artisans and information professionals according to him (Himanen 2001, 3-4, 6-7).

We leave the category of play with this teaser on constructive play, or is it playful work? Playbour?

\subsubsection{Conclusions}

In this study of the relevant literature on play, I have found that play, as the concept of culture, has both a narrow and broad meaning. Further, I have identified five different dimensions. 1) The purpose of play is the activity itself for the player, but goals can be used within play to form the activity; behind the backs of the players it can have productive outcomes, and Vygotsky stressed that the play of the child later became the work of the adult. 2) Play is a mobile dialectical process which is not reifiable on the subjective level; it is similar to life itself in some ways but free (between the players and in relation to reality) in its use of rules, the processes are open to re-negotiations and improvisations under the activity and therefore play is not totally predictable; play can take place within the solitary player or in his/her relation to the world, or within a playing community, and can often have the character of exaggerated or uneconomical "galumphing". 3) Play occurs when all other needs are satisfied and because the player voluntarily takes the initiative. 4) Play is fun, enjoyable and pleasurable, and can be characterised by a certain ease and relaxation or by a luxury feeling of superfluous abundance, as well as by passion and tension. 5) Play is part of nature and the human constitution, and it is crucial for social life as well as our communication, but in contrast to Gadamer I also stress the importance of the involved subjects' understanding and feeling of play.

There is a fine line between playing and working in some of the digital, or so-called immaterial labour, going on in the general intellect, but it is a crucial one. When it comes to the 
question of activity or result, working always involves production of a use value for yourself and other people (even in the sense of pleasurable or fun for others), and playing involves the pleasurable and fun activities you perform just for the fun of it. It is not play when you perform a pleasurable activity to obtain the relaxation or concentration that the play results in. In the last example the activity is focused on the result that is a use value to satisfy a need. You have to lose yourself in the play, for it to be play.

\subsection{Working}

Lev Vygotsky maintained that the child's play developed into the work of the adult. Play and work were trans-historic. Hannah Arendt as well as Fuchs and Sevignani make a distinction between work and labour, but in different ways. Arendt contrasts labour, as something that is never finished, to work, which always ended up in the world of things. She named the workers homo faber and the labourer animal laborans (Arendt 1998, 126, 140). Fuchs and Sevignani use a Marxist perspective, which is also the perspective used for work and labour in this article.

Work is determined by its concrete aim of producing a value "in use of its product". (Marx $1867,49)$ Hence, work is an instrumental activity, which results in a product, which does not need to be tangible, but could consist of signs, speech, affects and services, that is, useful. It is this aim of producing a use value that makes the work "useful labour"(Marx 1867, 49). Useful labour is a "specific kind of goal-oriented labour", private in character, which "movements and efforts" are conditioned by the use value being produced. Useful labour is defined by Marx as a "productive activity of a definite kind" and is contained in the use value (Marx 1867, 49). Qualitative and particular in its character, work cannot be compared to another particular work (Karlsson 2013, 46), and therefore there can be no competition between different forms of concrete work and their use values; there is not a common ground for them to be measured by as they have different qualitative characters.

To make the point clear: let us imagine a plain barter between two individuals, in which two qualitatively different wants can be met by the exchange of two qualitatively different use values. No competition takes place in the barter, because the use values are as qualitatively different as the wants and needs involved. In the realm of use values and concrete labour, competition can only occur between qualitatively similar use values and activities.

With all this in mind I contend that the activity in itself cannot be a use value for the worker him/herself. This would change the character of the activity. Only the resulting product (or state of mind) can be a use value for the actor, but the activity can be useful in itself for another person who, external to the activity, can use it as a product. This distinction is important for my further study of the relation between play and work/labour.

Moishe Postone interprets Karl Marx's position on work as one where there are two kinds of necessity involved: one that is trans-historical and one that is social and historical (Postone 1993, 381). Some necessity will always exist in a society. Even when the realm of freedom is expanding under communism, some necessity will continue to exist:

One remaining constraint is nature. Although the labor of individuals need not be a necessary means for acquiring means of consumption, some form of social production is a necessary precondition of human social existence. The form and extent of this transhistorical, "natural", social necessity can be historically modified; this necessity itself, however cannot be abolished. (Postone 1993, 382)

Marx himself wrote quite explicitly in volume one of Capital on the topic of man's metabolism with nature. As far as it was work as a creator of use values, useful work, it was a necessary condition that was "independent of all forms of society, for the existence of the human race". It was an "eternal nature-imposed necessity" without which there could be no "material exchanges between man and Nature, and therefore no life" (Marx 1867, 50). Later on in volume three he wrote: 
Just as the savage must wrestle with nature, in order to satisfy his wants, in order to maintain his life and reproduce it, so civilized man has to do it, and he must do it in all form of society and under all possible modes of production. With his development the realm of natural necessity expands, because his wants increase; but at the same time the forces of production increase, by which these wants are satisfied. (Marx 1909)

Work was in the first place a process in which both man and nature participated. Actually man was nature. In his critique of the Gotha program that was adopted by the German Social Democratic Party in 1875, work had a central role. The first paragraph stated that labour was the source of all wealth and culture, and Marx furiously wrote: "Labour is not the source of all wealth. Nature is just as much the source of use values (and it is surely of such that material wealth consists!) as labour, which itself is only the manifestation of a force of nature" (Marx\&and Engels 1991, 301). Work was thus determined and effectuated by nature according to Marx. (Wo)man started the process on his/her own accords and regulated and controlled the material interaction between himself and Nature:

He opposes himself to Nature as one of her own forces, setting in motion arms and legs, head and hands, the natural forces of his body, in order to appropriate Nature's productions in a form adapted to his own wants. By thus acting on the external world and changing it, he at the same time changes his own nature. (Marx 1867, 173)

In this purposeful interaction with nature man transforms himself and "develops his slumbering powers and compels them to act in obedience to his sway" (Marx 1867, 173)—which is yet another parallel to how play functions within the child's development. It is on this selfreflexive dynamic of the work process that the development of the historical phases of labour depends, which in turn allows for the development of new needs, activities and new theoretical knowledge about the world. It is a dynamic that contrast sharply with the instinctive behaviour of animals (Carver 1987, 93-94; Karlsson 2013, 48). Marx was thus not writing about the primitive, instinctive forms that "remind us of the mere animal" in this passage of Capital. He stressed that there was an "immeasurable interval of time" that separated the situation in which a man "brings his labour-power to market for sale as a commodity", from the time when human labour was still in its first instinctive stages (Marx 1867, 173-74), and he therefore presupposes work in a form that stamped it as "exclusively human" (Marx 1867, 173-74).

A spider conducts operations that resemble those of a weaver, and a bee puts to shame many an architect in the construction of her cells. But what distinguishes the worst architect from the best of bees is this, that the architect raises his structure in imagination before he erects it in reality. At the end of every labour-process, we get a result that already existed in the imagination of the labourer at its commencement. He not only effects a change of form in the material on which he works, but he also realises a purpose of his own that gives the law to his modus operandi, and to which he must subordinate his will. (Marx 1867, 174)

The conclusion that work is a trans-historic feature of the human condition carries with it the potentiality that its historical forms of appearances may not necessarily have to be related to hierarchical class societies. The social necessity of work is mainly developed by Marx in relation to work in capitalism, that is labour, but you just have to think of gift economies, like those described by the classics of anthropology, to see that a gift of a use value demands its counter or return gift under different forms of reciprocity (Mauss 1972; Malinowski 1922; Hyde 2012). Social bonds that demand certain behaviour thus exist in all societies.

Marx was clear about the fact that the social character of work was not confined to capitalism. Work as the "special sort of productive activity" that was "determined by its aim, mode of operation, subject, means, and result" was qualitative in kind and not dependent on the production of commodities: 
To all the different varieties of values in use there correspond as many different kinds of useful labour, classified according to the order, genus, species, and variety to which they belong in the social division of labour. This division of labour is a necessary condition for the production of commodities, but it does not follow, conversely, that the production of commodities is a necessary condition for the division of labours. (Marx 1867, 49)

Marx wrote on work in the anthropological way in the Introduction to a Contribution to the Critique of Political Economy, which was later published as the introduction to Grundrisse (Marx 1973). It starts with a famous critique of the Robinsonades of bourgeois political economy:

Individuals producing in a society, and hence the socially determined production of individuals, is of course the point of departure. The solitary and isolated hunter or fisherman, who serves Adam Smith and Ricardo as a starting point, is one of the unimaginative fantasies of eighteenth-century romances a la Robinson Crusoe /---/[?]This is an illusion and nothing but the aesthetic illusion of the small and big Robinsonades. It is, on the contrary, the anticipation of "bourgeois society," [...] The individual in this society of free competition seems to be rid of natural ties. (Marx 1857; Marx 1973, 83)

Work, hence, was social in its character. "Production by a solitary individual outside society" was rare, and if an individual by accident ended up in the wilderness he had already before absorbed the dynamic social forces. It was thus equally absurd to think of these Robinsonades as to perceive of the development of speech and language without assuming "individuals who live together and talk to one another" (Marx 1857; Marx 1973, 84).

But being social, work had the potential of taking on freer and more voluntary forms. "Really free working, e.g. composing, is at the same time precisely the most damned seriousness, the most intense exertion" (Marx 1993, 611). The enjoyment associated with using the bodily and/or mental powers, especially in what Marx called the realm of freedom point to the existing potential of feeling self-fulfilment and self-expression through work. Marx wrote like this of this mixture of necessary work with self-fulfilling and self-expressing work in a way that relates to the close relation between freer forms of work and effortless play:

The less he is attracted by the nature of the work, and the mode in which it is carried on, and the less, therefore, he enjoys it as something which gives play to his bodily and mental powers, the more close his attention is forced to be. (Marx 1867, 174)

When work was enjoyable its character transformed into something similar to art. This potentiality of increasingly more attractive modes of producing which merged work with play into a synthesis of attractive work which seems light and easy also existed as a real potential within the logic of capitalism (Marx and Engels 1991, 306) Marx, finally, wrote in the Introduction to a Contribution to a Critique of Capital about the complex relation between trans-historical and historical forms of work/labour:

Some determinations belong to all epochs, others only to a few. [Some] determinations will be shared by the most modern epoch and the most ancient. No production will be thinkable without them [...] just those things which determine their development, i.e. the elements which are not general and common must be separated out from the determinations valid for production as such, so that in their unity-which arises already from the identity of the subject, humanity, and of the object, nature-their essential difference is not forgotten (Marx 1973, 85).

Marx contends that production is always a particular sort of production, there is thus no production in general, but this must not stop us from assuming there is a common and essential form of work derived from the identity of the human individual and nature (Marx 1973, 85). Herbert Marcuse wrote that work has a Last-Charakter (load-character) that was independent of both the historical mode of producing and the worker's experiences of it. This LastCharakter was founded in the fact that the individual was dependent on the conformity to the laws of the object or Nature (Karlsson 2013, 49). Karel Kosik's understanding of work as de- 
fined by its objectified result (Karlsson 2013,48 ) is more problematic today when we see that work can also express itself in non-tangible and virtuosic forms (Lazzarato 1996; Virno 2004), but it could also be said to confirm my earlier statement that an actor external to the activity could objectify it under the performance, though not the actor himself.

\title{
1.2.1. Conclusions
}

The same five dimensions as in play were found in the view of the Marxist tradition on one of its central concepts: work. 1) Work is goal-oriented and focuses on its result; the goal is to produce use values. 2) Work is a specific and concrete activity, characterized by a specific quality, which changes in its specificity according to what use value it is producing. There is no competition between different concrete work processes due to the fact that they are not commensurable and focus on different social needs. 3) Work is a necessary activity in the human condition; someone has to work if we are to survive as humans. 4) Work is connected to feelings associated with it being necessary and having to be useful, but also to feelings associated with it satisfying social needs through the use of capabilities and knowledge to transform nature in accordance with them. The first category correlates with seriousness, the second with self-expression and self-fulfilment, but also with feelings of social belonging and "togetherness". 5) Work is at the core of human metabolism with nature, connected to survival, and is thus trans-historical.

\subsection{Gaming}

Studies to understand games have frequently been conducted in sociological, anthropological, and philosophical research, to a lesser degree in the aesthetic field, but game theory has also been used within economics and computer sciences (both John von Neumann and Claude Shannon have used so-called game theory to understand strategic choices in economics and in developing the work of the computer). Structuralism has been influenced by game theory. Claude Lévi-Strauss and Vladimir Propp discussed the relation between rules and representation claiming that narratives were based on formal structures, and Ferdinand de Saussure compared chess to language. In psychology Adriaan D. De Groot (1965) studied the psychology of playing chess and Allen Newell and Herbert A. Simon (1972) used game-like problems for studying human problem solving. Marcel Danesi has also shown that games and puzzling solving have yielded many insights in mathematics, for example in the field of graph theory. Hence, the purpose has often been to understand other issues through the study of games and gaming (often called play) (Juul 2005, 8-10).

\begin{abstract}
When rational choice theories attempt a concept of game that excludes play, the Situationists attempt a concept of play outside the game: from each according to their abilities; to each according to their desires" (Wark 2007, paragraph of Cuts (Endnotes) 021). The game, unlike play, is relatively easy to define. Play is what is excluded from any definition of game to give it the appearance of self-consistency (Wark 2007, Cuts [Endnote ] 090).
\end{abstract}

Jesper Juul provides us with a definition of the concept game in which he tries to identify similarities between the things that we call games. He calls his model the classic game model and is building on seven game definitions by previous writers (Juul 2005, 6-7, 23).

The model consists of six features working on three different levels, the levels being the "level of the game itself, as a set of rules; the level of the player's relation to the game; and the level of the relation between the activity of playing the game and the rest of the world" (Juul 2005, 6). The features are then: 1) the rule-based formal system, 2) the variable and quantifiable outcomes, 3 ) the assignment of different values to different outcomes, 4) the exertion of effort from the part of player to influence the outcome, 5) emotional attachment of the player to the outcome, and 6) optional and negotiable consequences of the activity. Juul claims that the six features together are necessary and sufficient conditions for something to be a game (Juul 2005, 6-7). 
Juul focuses on what he calls video games. Video games are defined by the two things they are made up of: real rules and fictional worlds. The rules are real in the sense that players interact with them and that winning or losing is "a real event". The slaying of the dragon, however, is the slaying of a fictional dragon. "To play a video game is therefore to interact with real rules while imagining a fictional world". This fictional world is more concrete than the fictional worlds connected to traditional, non-electronic games that used to be more abstract. Media and video games are a quite new cultural form connected to the appearances of the computer, but as games they have a very long history. (Juul 2005, 1, 3)

When Roger Caillois published his classic account of play, Man, play and games (Les jeux et les hommes), in 1958, he criticised, as we have seen, the definition of play that Huizinga presented. It was both too broad and too narrow according to Caillois (even if he himself did not distinguish between the play and game dimensions). One central problem with Huizinga's definition according to Caillois was that it viewed play as "action denuded of all material interest". This excluded bets and games of chance "which for better or worse, occupy an important part in the economy and daily life of various cultures", according to Caillois, who stresses that "the constant relationship between chance and profit" is striking:

It is certainly much more difficult to establish the cultural functions of games of chance than of competitive games. However, the influence of game of chance is no less considerable, even if deemed unfortunate, and not to consider them leads to a definition of play which affirms or implies the absence of economic interest. Therefore a distinction must be made.

In certain of its manifestations, play is designed to be extremely lucrative or ruinous. This does not preclude the fact that playing for money remains completely unproductive. /---/[?] [The entrepreneur] alone does not play, or if he plays he is protected against loss by the law of averages. In effect, he is the only one who cannot take pleasure in gambling.

Property is exchanged, but no goods are produced. What is more, this exchange affects only the players, and only to the degree that they accept, through a free decision remade at each game, the probability of such transfer. A characteristic of play, in fact, is that it creates no wealth or goods, thus differing from work or art. At the end of the game, all can and must start over again at the same point. Nothing has been harvested or manufactured, no masterpiece has been created, no capital has accrued. Play is an occasion of pure waste: waste of time, energy, ingenuity, skill, and often of money for the purchase of gambling equipment or eventually to pay for the establishment (Caillois 2001, 5-6).

We can here see that play for Caillois is an activity that gives pleasure, but also that he has a strong focus on rule-based games, an attribute that also Huizinga heralded as play, but that I, in the following and partly in line with the critique of Jesper Juul, think it is necessary to understand as gaming. The two forms of play that Caillois here refers to are Agôn (competition) and Alea (chance), and these forms are more inclined toward gaming than Mimicry (simulation) and llinx (Vertigo).

In this section I will focus on the former two and will speak of gaming when I refer to Caillois theories. I also want to claim that Juul goes too far in his criticism of Caillois for his categories to be overlapping. Caillois simple stated that the categorisation of a certain game depended on which category of competition, chance, simulation and vertigo was dominant in it (Caillois 2001, 12).

Caillois's insistence on gaming (agôn and alea) being able to include material interests at the same time as it is not a productive activity, neither in producing use values or use values carrying exchange values that can lead to accumulation of capital, is important for my understanding of gaming and its relation to instrumentality. Gaming is different from both work and labour. Gaming can be in the speculative circuit (but the result is not always M-M'), but takes no part in the capital circuit, M-C...P....'-M', at all. This emphasis on the non-productivity of gaming (as well as playing) is supported by Asplund, who contends that they are redundant and superfluous activities (Asplund 1987, 55) 
Caillois, like the protagonists of psychoanalysis and much in the same manner as Bataille, recognises the relation between gaming and biological energies. He sees gaming as a waste of energies, time and skill for the fun of it, without this excluding money as such. Money could even be a game thing or a toy. This view has some similarities to that of Nietzsche (see below).

The clear separation of gaming from art in the quotation of Caillois above is also an interesting novelty. Taken literally this would lead to concepts like gamework or gamelabour but it is quite clear that the purpose of Caillois investigation is to understand a purer form of gaming, which is pleasurable and fun. The claim in the quotation that it is harder to point to the cultural function of games of chance than of the games of competition elucidates in my eyes the intimate relation between gaming and capitalist logic. When you compete you compete in doing something in accordance to a fixed logic. But at the same time there is gaming in peer production projects as well. Gaming is at work in Wikipedia where the elected administrators are gameworking or workgaming (see below for explication). But maybe we can understand the activities of so-called vandals as being elements of a game of competition with these admins?

Finally, when it comes to the central notion of Caillois, about the goals' peculiar character within games, this view is supported by McKenzie Wark in his work Gamer theory. In what he calls gamespace, which pretends to adhere to Darwins 'the survival of the fittest', the reverse is happening, "the demise of the unfit": "Survival has no positive value. Gamesspace is pure nihilism. The best one can hope for is merely being undefeated" (Wark 2007, 214). Some games, like SimEarth fail because they are too realistic and do not survive. This is then not because they bump up against the reality principle of bare life, but quite the reverse. They fail the fantasy principle (Wark 2007, 214)

Nietzsche's version of the Dionysian play was as a will to power, to overcome yourself, conquering and being master of yourself, your surroundings and all that you meet, and as such this will have more of a distinct goal and a specific form of competitiveness to it even if it does not adhere to any rules but life's enhancement with its irrational frenzy (Nagel 1996, 3 ). This version of play has many resemblances to gaming, even though the breaking of all rules seems rather playful. MacKenzie Wark writes about digital games from the example of the failure of the too realistic SimEarth: "The inclusion of almost everything within the game leaves little by way of topos in which to conquer, expand, colonize, transform. [...] There is no frontier along which a storyline might traffic the unknown into the realm of the known" (Wark 2007, 216). Not that this ever restrained Nietzsche in his fantasies.

The preferred form of gaming of Nietzsche was connected dialectically to the logocentric Apollonian play that Aristotle's saw as a serious and intellectual business when used for good ends by philosophers. The latter profession, thanks to their casual lifestyle (as free citizens and slave owners), engaged as we know from Plato's dialogues, in verbal battles that I also think are more reminiscent of gaming rather than playing.

In reality the Apollonian side of play was less important to Nietzsche. The only thing given as real to him was the "world of desires and passions". Man could only rise or sink in relation to the reality of his drives (Nietzsche 1990, 66). The falseness of a judgement was not the same as an objection to it. Nietzsche affirmed the passions before reason, and the falsest judgement was life-advancing, life-preserving, species-preserving or species-breeding because, in short it is indispensable to us in that it does not need the false logic and falsification of the world by numbers (Nietzsche 1990, 35).

In contrast to this dismissal of scientific reason in the name of life, Nietzsche's affirmation of art was more playful, as mentioned above.

One central theme that Katrina Mitcheson claims is recurrent in the works of Nietzsche is his concept of life as a will to power. This means that the human wants to be the master of him/herself, his/her surroundings. She concludes that the will "is to power in that it strives to feel power in overcoming itself and what it encounters" (Mitcheson 2013). As I see it the dominant feature of this understanding of life is power and competitiveness, and the explora- 
tive dimension is subordinated. Therefore I contend that Nietzsche writes about gaming rather than playing.

The same dual view on play can be seen in three of the maxims in Beyond Good and Evil where Nietzsche mentions play. The first: "Mature manhood: that means to have rediscovered the seriousness one had as a child at play" (Nietzsche 1990, 94). Taking play seriously fulfils you as human. The second: "Around the hero everything becomes a tragedy, around the demi-god a satyr-play; and around God everything becomes - what? Perhaps a 'world'?" (Nietzsche 1990, 102). The world here seems a boring place without the free play of the drives. And finally a quotation from the third maxim: "The industrious races find leisure very hard to endure: it was a masterpiece of English instinct to make Sunday so extremely holy and boring that the English unconsciously long again for their week- and working days", (Nietzsche 1990,112). This witty defence for leisure against labour tells us that labour which is forced upon you, labour before which you are powerless, is contrary to life and gaming in the thought of Nietzsche, who sees gaming as a trans-historical category. Nietzsche's view fits quite well into capitalist society with its stress on competition, expansion and conquering, even if he had nothing but contempt for industrial society.

On a less philosophical and more concrete level of the actual gaming experience, Juul's argument parallels play and that of flow theory within psychology (Csíkszentmihályi 1996). Juul claims that, "the rules of a game provide the player with challenges that the player cannot trivially overcome". The rules are easy to use but present challenges that are not easy. It is this challenge that makes the game enjoyable, and playing a game means improving your skills in order to overcome these challenges. This learning experience takes different forms in different games, but Juul outlines two basic game structures: the structure of emergence (a number of simple rules combining to form interesting variations) and the structure of progression (separate challenges presented serially) (Juul 2005, 5).

Jesper Juul here shows a clear relation between learning and the playing of games. Learning is the unintended consequence of playing games on the subjective level, and this has repercussions on the sociological level in terms of what people learn in society. It also means that games, just like play, can be used as a pedagogical tool and indirectly be made productive. The consequence of an overly blunt manipulation for the sake of an external goal outside of the game affects it in the same way as play. The game becomes more serious from an ordinary life perspective (and thus less fun as the instrumental goal becomes more important), but at the same time games and gaming are already competitive in character like capitalism. Gaming, with its focus on contest, shares the same mentality as capitalism and has the potential of producing a competitive labour force for the capitalist production process. At the same time gaming could also influence capitalism by accentuating the voluntary and enjoyable character, but, on the other hand even Weber saw similarities between the Protestant ethic and gaming - that is between the pursuit of accumulation and a game.

The differences between gaming and playing are still of such importance that I agree with Anthony D. Pellegrini that we must distinguish between play and games. The reason for the common confusion between the two according to him is that they share certain "design features" like organising rules. Pellegrini shares the theories of Piaget that the rules governing games are typically a priori and codified, while rules governing play are flexible and negotiated in communication between the players during the activity. This theoretical understanding is supported by Asplund. The negotiation in pretend play often takes more time than the playing per se according to him. In games the violation of the rules results in some form of sanction rather than any form of renegotiation. He asserts that a large part of the research on games in different playgrounds has tended to support this dichotomization (Pellegrini 2009, 184-185; Asplund 1987, 64).

From this literary overview we can extract three more reasons why we should make the distinction between playing and gaming. First, in playing the activity is all that is important; you cannot objectify it; play is an activity that you lose yourself in. Gaming is different in that 
the goal does have some importance, even if this goal, as Caillois points out, is nonproductive, and erased and annulled before the next game takes place.

Second, playing and gaming are performed between the individual and the context surrounding her/him or between different individuals and their context, while gaming more often (but not always) involves spectators as a central feature of its context. The increased use of media representations of games and the commercialisation of sports, theatre acts and movies in the last hundred years have accentuated the presence and importance of spectators watching the game from the outside. Gaming today, due to this development, stresses the goal or end to a higher degree than before. The involvement of capital together with the spectators and other external actors have driven the character of gaming from, in Gadamer's words, being self-presentation within a community of gamers to being a representation for others (Gadamer 1975, 97). In this movement the similarity with play weakens. If play originates in the biological and psychological constitution of human beings as well as being constructed in social life, gaming is derived from play but socially constructed in a way that marks a rupture with play. According to Gadamer there exists a potential conflict between spectators and play where the threat is that play will lose its "real play character as a contest precisely by becoming a show" (Gadamer 1975, 98). He continues:

Their [the players] mode of participation in the game is no longer determined by the fact that they are completely absorbed in it, but by their playing their role in relation and regard to the whole of the play, in which not they, but the audience is to become absorbed. When a play activity becomes a play in the theatre a total switch takes place. It puts the spectator in the place of the player. He-and not the player-is the person for and in whom the play takes place. (Gadamer 1975, 99)

This difference between 'playing within a community of players' and 'playing for someone else' is stressed here, and religious rites, theatre plays, and sports or other forms of contest, which are played in front of others, are seen as either stressing the goal and result, or the quality of the performance and representation, in an accentuated and qualitatively different way than in play. Play has left the magical circle, and the activity is measured in accordance with social standards that are fixed within a larger societal body.

Play could contain contests (and performances) that have the same "to-and-fro" movement through which "the victor emerges" (Gadamer 1975, 95), but not if they have a focus on the result and on being measured and successfully evaluated by an audience rather than on the to-and-fro movement in itself. Gaming therefore has more in common with sports and arts, but in the distinction of the categories we have to use more criteria than one.

Third, play is explorative and often stresses the co-operation and negotiation between all involved players, while games instead are competitive and stress differences (which are often quantified) between the involved gamers in performances according to fixed rules.

Pellegrini claims that the two categories have different feelings associated with them and shows pictures of an individual who is out cross-country skiing. In one picture the face of the former expresses strain and utmost seriousness; the person is gaming. In another picture the skiers face shows a big smile, the person is out playing and are having fun rather than being concerned about his place in a race. The same individual, the same activity, which are the same in some aspects with totally different attitudes attached to them (Pellegrini 2009, 184185).

This last characteristic of gaming connects it to what Robert Stebbins has called serious leisure: "In an age in which the quest for spectator and sensual diversions dominates the world or leisure." Implying that gaming dominates playing, he contends rather naively that the phrase 'serious leisure' "has a rather curious ring" (what is curious about gaming becoming a commodity and converting leisure activities into unpaid work on your labour or gaming power?) since historically seriousness has been associated with work "whereas leisure has been seen as the happy, carefree refuge from our earnest pursuit of money and the social standing supposedly provided by a paying job". Today this view is losing ground according to 
Stebbins: "Current values and behaviour patterns in work and leisure hint at the presence of a serious orientation toward leisure among a significant proportion $/ . . . /$ of the population in today's postindustrial society." (Stebbins 1992, 1). According to Stebbins, play should, on the other hand, be categorised as casual leisure (Stebbins 2009, 622-623; Stebbins 2013, 340).

This view is confirmed by Pekka Himanen who connects it to the development of capitalism and asserts that the relation to time in the Protestant ethic, time is money, still permeates today's society in what he calls the fridayization of Sunday. There was no play in the work of the Protestant ethic, and today this logic and its time optimization have extended even to "life outside the workplace (if such life still exists)". It has begun to eliminate "the playfulness of free time of Sunday". One of his examples is that today only a beginner relaxes "without having taken a class in relaxation techniques", and he concludes that it is considered an embarrassment to be just a hobbyist "in one's hobbies". Leisure time thus assumes the "patterns of work time" (Himanen 2001, 20, 2-27 32). This comment stresses the competitiveness of both gaming and labouring. Leisure involves more of gaming on the social scene.

The different spirit between playing and gaming is important in two ways. First, players or gamers contribute to the play and game forms being applied in society. Second, the players and gamers construct themselves in their playing and gaming (with different psychological, cultural, social, economic and political effects).

\subsubsection{Conclusions}

Gaming as playing seems to have many implications for other scientific fields. These kinds of consequences, as with play, mainly occur behind the backs of the gamers. The dimensions in gaming are represented by the following treats: 1) Gaming is more goal-oriented than playing, meaning: in gaming goals are set up to structure the activity and make possible the comparison of the activities of the gamers, whereas in playing it is the activity in itself which is the goal. The goals are often quantitative and variable in character, depending on the actions of the gamer. Gaming is not necessarily confined to the gamers and can be performed in front of a public of spectators, which strengthens the importance of the result. But games are also gamed for the fun of the activity. 2) Games have an a priori structure of formal rules governing them. The breaking of rules results in sanctions of some kind. Goals are annulled before a new game is begun. 3) Gaming is voluntarily engaged in, but social pressure (gaming involves competition between gamers in society) is more pronounced than within playing. 4) Gaming involves, like playing, feelings of fun, tension and passion, but also feelings of effort, strain and risk. 5) Gaming is socially constructed in societies that relates to competition for social distinction, as the example of !Kung suggests. Gaming is not trans-historical in character. The increased focus of and on the spectators fundamentally transforms the creative or gaming's affinity with play, much in the same way as Moishe Postone claims that abstract labour transforms the mode of producing of concrete labour (Postone 1993, 6-68).

\subsection{Labouring}

According to Hannah Arendt labour was characterised by never being finished, and the labourer was an animal laborans (Arendt 1998, 126, 140). Karl Marx stated that labour under capitalism had a dual character as concrete and abstract labour. This dual labour produced use values with exchange value (Marx 1969, 3-42) This does not mean that the resulting commodity contains two separate kinds of labour; rather it contains one labour, but that labour can be "regarded from two radically different viewpoints". Johan Fornäs uses the words "contradictory" and "oppositional" to describe the two different perspectives on labour. Every commodity was built with labour characterised by a dialectical unity of these two contradictory aspects; every exchange value had to be a use-value at the same time (Fornäs 2013, 335). Hence, abstract labour is dependent on concrete labour in a way that concrete labour is not in relation to abstract labour. The concrete and qualitatively specific form of labour, with its specific result, is the reason for the exchange of equivalent use values by the social mediation of abstract labour in capitalism (see the section on labour) (Fornäs 2013, 3-35). Marx 
describes this as a coat and linen that have two different use values which can be exchanged and relate to each other as equivalent commodities only because they are the products of qualitatively different concrete work. A coat would not be exchanged for another coat (Marx 1867, 49). On the other hand, concrete labour is dominated, but not erased, by abstract labour under capitalism, that is, work is dominated by labour under capitalism.

The labour we speak of here is thus not the same as the specific and concrete work that is the common denominator of all human labours in all times. Labour is here understood as a historical and particular mode of producing characteristic of the mode of production called capitalism. In capitalism the social production and distribution is organised by the exchange of commodities on markets. This social mediation by markets and exchange is made possible through the common trait of all the commodities-human abstract labour:

\begin{abstract}
Whatever kind of labour is involved, it is spending of labour-power. At the end of the workday the worker is tired, whatever he has spent his force on in the workday. This expenditure of labour-power constitutes the common denominator for all commodities. (Fornäs 2013, 34)
\end{abstract}

This expenditure of a socially standardised and general labour power in all kinds of concrete works is the reason why it is called abstract labour. It is the exchange that expresses the similarity between two different kinds of labour, their shared quality of being values, through the measurement and comparison grounded in the amount of societally necessary abstract labour.

To be a value is no natural property that can be perceived with our common senses. It is a societal characteristic that only becomes 'visible in the exchange process. [...] Value is social, not a natural activity. [...] Yet, they are no pure mental ideas, since the commodity exchange itself shows that values exist. [...] This only happens in the historical conditions of private property, division of labour and exchange. (Fornäs 2013, 35)

Moishe Postone contends that if value is a historically specific form of wealth, then the labour producing it also must be historically determinate. The value form structures the sphere of production as well as that of distribution. And if it is so, it is not enough to simply to abolish class society; you would have to change the mode of producing as well (Postone 1993, 45, 67-68).

In capitalism this particular and historical form of labour is characterised by having an abstract form that can be measured by the amount of societally necessary labour time or the necessary labour force being expended on them. This societal character has, as we shall see, two different meanings. First it points to the historical level of the forces of production in society, and second, it means that it is a production aimed for others-the society in its totality. This abstract and quantifiable value is only expressed in the exchange of commodities and in the prices on the market.

The notion of societally necessary labour time is crucial, and it is often misunderstood due to fact that many scholars confine their analysis of Marx's theory of value to the first volume of capital. If we take in volume two and three, moving up from the deep abstractions of the first volume (where the supply and demand did not affect the value), then supply and demand affects also the values and not only the prices:

For, what is 'socially necessary labour-time, and how is the total labour-time in the whole society allocated onto the production of various kinds of goods? That the labour-time is social means that it is geared towards satisfying other people's needs, which on the surface are expressed through demand. Imagine that far too many TV sets are made, compared to what people have need for. In such a case, a too big part of the total social labour-time has been invested, so that the value of each TV set is much lower than the actual time spent, since it contains superfluous labour-time [...] that the degree to which the labour-time spent on making TV sets is in reality socially necessary is co-determined by 
the social need for TV sets. This need is not visible in the production process, but first on the market. (Fornäs 2013, 37)

The socially mediating market (in capitalism) then affects the production of values according to Marx. It is not simply the amount of socially necessary labour-time according to a certain historical society's average level of the forces of production, the average skill and intensity of production and so on, that determines the value. What is "socially necessary" does not simply change in relation to the development of the forces of production, but also in relation to the development of the needs within a society (as expressed in the demand).

According to Moishe Postone's reading of Marx, as we have seen above, Marx used the notion of 'societally necessary' in two different ways: the trans-historical and the historical form of necessity. The relation between the two is dynamic in the sense that Marx identifies a tendency within capitalism to create the potential for a realm of freedom to grow and prosper at the expense of the realm of necessity, even if it is not possible to get rid of all necessary work. In this section of the article I aim to focus on what Moishe Postone speaks of as the 'mode of producing' (to distinguish it from mode of production) (Postone 1993, 6-68) in an effort to address the effect of capitalism on the immediate labour process in contemporary society.

But before that, some words have to be said about what Ivan Illich has called shadow work. This is a form of unpaid work, not badly paid and not unemployment, that is typical for industrial capitalism. Illich mentions that in most societies "men and women together have maintained and regenerated the subsistence of their households by unpaid activities", but he stresses that this is not what he means by shadow work. The unpaid work of industrial society is an "entirely different form of unpaid work", which is demanded as a "necessary complement to the production of goods and services", which "comprises most housework women do in their homes and apartments, the activities connected with shopping, most of the homework of students cramming for exams, the toil expended commuting to and from job" and so on (Illich 1980, 8). Illich contends that without this "apartheid based on sex or pigmentation, on certification or race, or party membership, a society built on the assumption of scarcity cannot exist."(Illich 1980, 7)

Back to the main track: Postone maintains that the traditional Marxist critique, from the standpoint of labour, that class domination is the fundamental form of social domination, is not enough for a real critique that has to include a social critique of labour per se in capitalism. This latter critique characterises the most fundamental form of domination as "an abstract, impersonal, structural form of domination underlying the historical dynamic of capitalism". In this mode of producing, people are dominated by their labour (Postone 1993, 53, 68). The precondition for the dignity of work is then the abolition of capital and abstract labour, instead of the traditional Marxist view that work accorded dignity to labour, which was fragmented and alienated under capitalism, as soon as class society was abolished. From the traditional position follows: "the perpetuation of such labour and the form of growth intrinsically related to it", whereas Marx saw the "historical overcoming of the 'mere worker' as a precondition for the realisation of the full human being" (Postone 1993, 71).

Production under capitalism of exchange values meant for valorisation and accumulation focuses on processes rather than on the product outcome. The capitalist mode of production results in a two-folded form of practise, depending on which class you belong to, which is mediated through markets. Roughly: for the majority it means forced, alienated and exploited labouring (surplus labour) for which they paradoxically often compete with other labourers to get on the labour market: this being their only way to make a living for themselves; and for the few, that is capital, the practice of non-labouring and appropriation, including managing, of the result from the valorisation and accumulation processes. The exploitation of labourers results from the systematic sale of labour power on the market by the workers and the systematic appropriation of the result of the actually performed labour in the immediate labour process; this exploitation results in the internal contradictions, struggles and recurrent crises of the capitalist system (Postone 1993; Marazzi 2011, 8-81; Marazzi 2008, 11-119). 
In a classic critique of traditional or positivist theory Max Horkheimer claimed that the conceptual and classificatory systems into which living and dead things, psychological, social and physical phenomena, were sorted in this theory, together with the judgements on them, formed an "apparatus of thought as it has proved and refined itself in connection with the real work process" (Horkheimer 1972, 219). This instrumental reason of traditional theory not only contributed to the prevailing habits of thought and carrying on the business of an out-dated model; it also changed capitalism. The liberalist period of capitalism was connected with the dominance of legal ownership of the means of production, but in the period of monopoly capital (with the start in second half of $19^{\text {th }}$ century), which was caused by technological development that resulted in a concentration and centralisation of capital, the legal owners were excluded from management (Horkheimer 1972, 219, 235). Technology rather than accumulation seemed to be the stronger socio-economic force to Horkheimer, and the powerful managers became the ones dominating whole sectors of industry. "The influence of management, which may initially be exercised only over lower judicial and administrative authorities, finally extends to the higher ones and ultimately the State and its power apparatus" (Horkheimer 1972, 235). The instrumental logic of capitalism thus, according to Horkheimer, extended itself to society and social life, where the individual did not have any ideas of his/her own and the content of mass belief was the immediate product of the "ruling economic and political bureaucracies". These in turn were guided by "atomistic and therefore untrue interests" (Horkheimer 1972, 235. 237). Here we can see how abstract labour and people's thinking changes within a dynamic capitalism, even without agreeing about the primacy of science and technology in relation to capital's own logic of accumulation. Horkheimer contends that this process of an expanding instrumental reason increasingly alienates people from their human condition and social relations.

This ideological pressure and pro-active management of our thought increases, but in a different way within what the autonomist Marxist calls the social factory of the general intellect, where the practice of social life is integrated to the labour processes even when it is not paid for, in the new shadow work of prosumption, prosuming or playbour. Christian Fuchs and Tiziana Terranova have argued that all of the time and effort devoted to generating "digital content" on the Internet should be considered a form of immaterial labour, and they emphasize the exploitive character of this unwaged labour. Brian Brown here makes a contribution when he analyses the biopolitical dimension of this shadow work, thus aiming at linking Fuchs' and Terranova's criticism of unpaid labour to the biopolitical critique of waged immaterial labour of Lazzarato, Hardt and Negri (Brown 2012, iii, 13-134, 18-186). His study of "Factory Flickr" is organised around six themes. Of interest to me are the first and second of the site's "ludic roots"- "the impact of these roots on whether or not all of the work done by members is considered as such"-and the public-by-default nature of all photographs and profiles (Brown 2012). Brown offers some arguments, such as the risk of social isolation, in favour of seeing this seemingly voluntary activity as a new kind of, in practice, forced labour.

But can we still speak of instrumental reason if all of our social life has become productive? Games have an instrumental logic, and our leisure time is being perceived more seriously, but what about play? This subject will be elaborated when the relation between playing and labouring is treated below.

\subsubsection{Conclusions}

The dimensions that have been found in the other categories can also be found within the category of labour. 1) The main purpose of labour is the accumulation of capital by an alien power in relation to the producer-that is the capitalist. The process of valorisation, not the use value, is the purpose that controls all involved parties; for the wage labourers it is a question of survival. 2) Labour is the production of exchange values for the market by the bought labour power (from the same market) of wage labourers. Exploitation makes him/her labour more than is needed for his/her own reproduction (surplus labour is systemic). 3) Labour is historically forced upon the labourer and involves the exploitation of him/her, which 
makes possible the non-work of the few. 4) Labour's associated feelings are competitiveness and alienation, together with feelings of being cheated and bossed around (dominated and used as an instrument for someone else's interest). 5) Labour is a historical form that involves trans-historical work, but that is qualitatively different than all other historical versions which have existed, also compared to other class societies dominated by un-economic factors but equally grounded and determined by economic concerns as Althusser would have it (Larrain 1991, 46), due to its abstract character and growth logic. Labour is dependent on concrete and specific work but also dominates it.

\subsection{The Typology}

The conclusions from these discussions of the four categories can be summarised in the form of a typology of the following character:

\section{Playing}

(1) Purpose: activity in itself is the goal

(2) Form of practice: Mobile, not-reifiable, "life-like", presence in the moment, free relation to rules, non-competitive, isolated within the self of the player or within a playing community

(3) Degree of voluntariness: Voluntarily engaged in; performed when you are experiencing well being

(4) Feelings: Fun, enjoyable, pleasurable, ease, tension, passion

(5) Transhistorical/Historical: Transhistorical: biological and psychologicaldrives; but also originating from communication, social and cultural interaction

\section{Gaming}

(1) Purpose: Goal-directed but both activity and result are important, the result/achievement is annulled before a new game starts, not productive in itself or in its outcome

(2) Form of practice: Rule-based, character of contest, competitive, can involve audiences

(3) Degree of voluntariness: Voluntarily, but social pressure or threat of social isolation can potentially be a reason for activity

(4) Feelings: Fun, "serious leisure", self-fulfilment, strain, risk

(5) Transhistorical/Historical: Historical. Socially constructed in societies focused on competition and social distinction

\section{Working}

(1) Purpose: Goal-oriented, production of useful values (use values) others; productive in its outcome

(2) Form of practice: Concrete labour, living labour, noncompetitive

(3) Degree of voluntariness: Necessary labour

(4) Feelings: Seriousness, self-fulfilment in the productive activity and in an objectified sense in the produced use values, "togetherness" and social belonging

(5) Transhistorical/Historical: Transhistorical; human process of metabolism with nature, for the satisfaction of human needs

\section{Labouring}

(1) Purpose: Accumulation of capital by production of exchange values by wage labourers; the process of valorisation (controlled by an alien power in relation to the production) and not the use value is the goal

(2) Form of practice: Twofold, depending on class division and mediated through markets; exploitation competition, accumulation and run-away growth under abstract standards, crises-ridden

(3) Degree of voluntariness: Forced labour due to class society

(4) Feelings: Alienated seriousness, instrumental reason, feelings of being exploited on one part, greed on the other, feelings of superiority or subordination

(5) Transhistorical/Historical: Historical. Socially constructed due to historic reasons

Figure 2: The typology (Arwid Lund 2014)

\section{Field Models for Mapping Positions and Opinions Relating to the Typology}

The activities within peer production as well as other activities on the Internet, which take place within an overall system dominated by a capitalism that is turning cognitive (its most dynamic sectors), can be visualised and mapped in the following model. The model merges the earlier model of possible relations between the categories with the developed typology above, and adds two structuring principles. The first structuring principle is the relation between the qualitative character of playing and working and the quantitative character of gaming and labouring. The second structuring principle is the relation between a focus on the activity, characteristic of playing and gaming, and a focus on the result of the activity, characteristic of working and labouring: 


\section{Activity in focus}

\section{Playing}

(1) Purpose: activity in itself is the goal

(2) Form of practice: Mobile, not-reifiable, "life-like", presence in the moment, free relation to rules, non-competitive, isolated within the self of the player or within a playing community

(3) Degree of voluntariness: Voluntarily engaged in; performed when you are experiencing well being

(4) Feelings: Fun, enjoyable, pleasurable, ease, tension, passion

(5) Transhistorical/Historical: Transhistorical: biological and psychologicaldrives; but also originating from communication, social and cultural interaction

Qualitative character

\section{Working}

(1) Purpose: Goal-oriented, production of useful values (use values) others; productive in its outcome

(2) Form of practice: Concrete labour, living labour, noncompetitive

(3) Degree of voluntariness: Necessary labour

(4) Feelings: Seriousness, self-fulfilment in the productiveactivity and in an objectified sense in the produced use values, "togetherness" and social belonging

(5) Transhistorical/Historical: Transhistorical; human process of metabolism with nature, for the satisfaction of human needs

\section{Gaming}

(1) Purpose: Goal-directed but both activity and result are important, the result/achievement is annulled before a new game starts, notproductive in itself or in its outcome

(2) Form of practice: Rule-based, character of contest, competitive, can involve audiences

(3) Degree of voluntariness: Voluntarily, but social pressure or threat of social isolation can potentially be a reason for activity

(4) Feelings: Fun, "serious leisure", self-fulfilment, strain, risk

(5) Transhistorical/Historical: Historical. Socially constructed in societies focused on competition and social distinction

\section{Labouring}

Quantitative character

(1) Purpose: Accumulation of capital by production of exchange values by wage labourers; the process of valorisation (controlled by an alien power in relation to the production) and not the use value is the goal

(2) Form of practice: Twofold, depending on class division and mediated through markets; exploitation, competition, accumulation and runaway growth under abstract standards, crises-ridden

(3) Degree of voluntariness: Forced labour due to class society

(4) Feelings: Alienated seriousness, instrumental reason, feelings of being exploited on one part, greed on the other, feelings of superiority or subordination

(5) Transhistorical/Historical: Historical. Socially constructed due to historic reasons

\section{Result in focus}

Figure 3: The field model (Arwid Lund 2014)

If we understand the relations between playing, working, gaming and labouring in this way, grounded in the literature on the subject, the next step is to use this model for visualising and mapping the positions (regarding opinions on performed activities) that results from the analysis of empirical material:

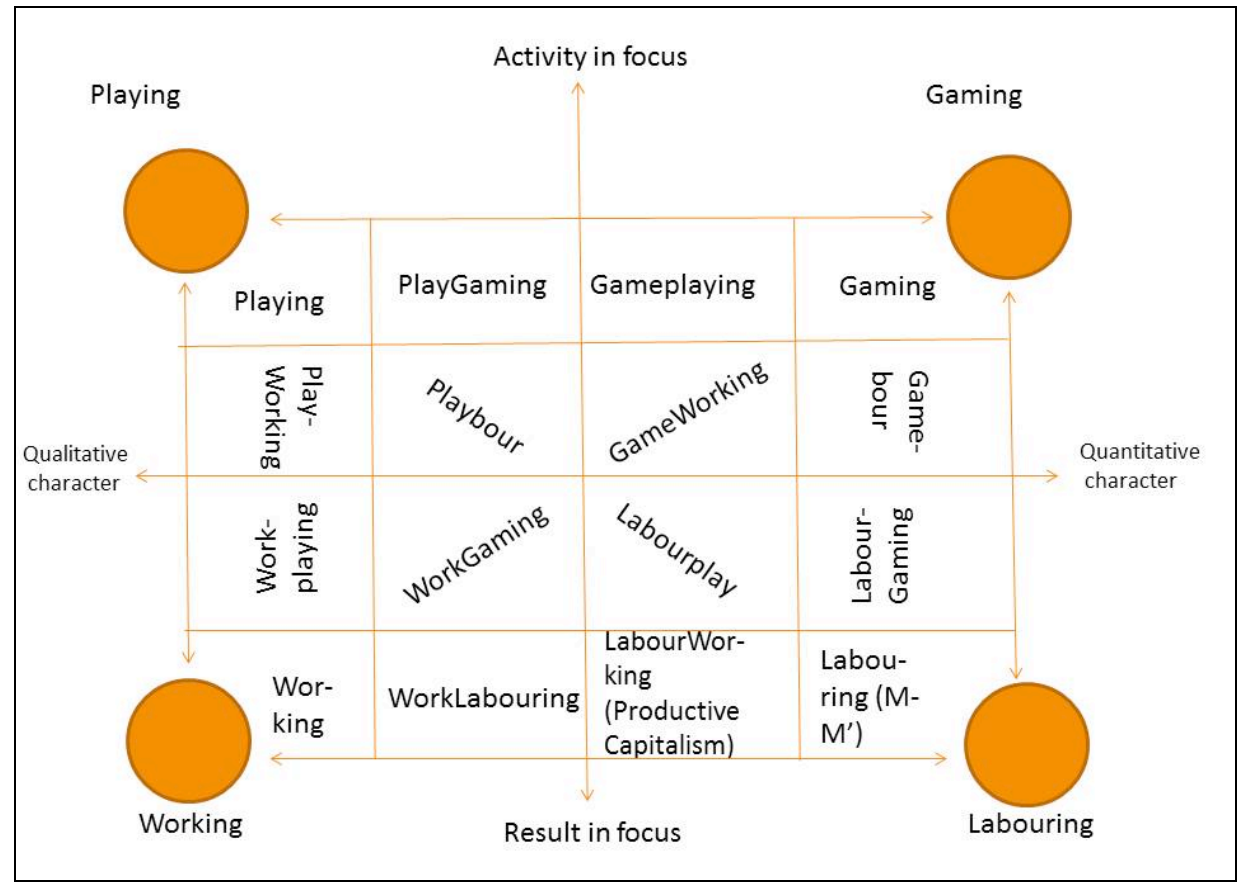

Figure 4: Model for mapping identified positions in empirical material (Arwid Lund 2014) 
In the model above it is only possible to visualise static positions. It is of great interest to understand the relations between these categories in the era of what Marx called the general intellect, but instead of developing a model that can visualise their character I will (for now) limit myself to a study of some of the relevant literature on these relations under capitalism, with the aim of providing some examples of different opinions and statements that can be placed in the field model above, but also of identifying synergies and conflicts that can be departures for future studies and the elaboration of models on the directions, forces and characteristics of the relations.

\section{The Relation between the Categories}

We have six different relations to scrutinize: playing and gaming, playing and working, gaming and working, gaming and labouring, working and labouring, and, finally, playing and labouring. The study has a theoretical focus and does not contextualise the theoretical positions described and analysed as much as would be appropriate. The contextualising that does exist is mainly focused on the contemporary situation.

The ideological concept of playbour focuses on some privileged parts of contemporary labouring life, in so-called creative branches-especially those included in the notion of Web 2.0_-of digital labour (or immaterial labour as some call it). In the new economy of the California ideology (Barbrook and Cameron 1996; Barbrook 2005) flexible exploitation and commodification is combined with a casual life style and creative "gaming" without standardized rules, without there being any friction and conflicts. Everybody is happy in the new economy, capitalists and workers alike.

The notion of playbour is not grounded in a discussion of the concepts of playing and gaming, and it conflates the notions of work and labour. We have here a complex camera obscura image of reality, camera obscura being the word Marx used in the German Ideology to speak of ideology (Marx and Engels [1845] 1998, 42). The term playbour in the discourse of the Californian ideology disguises the reality of its position by using the word play but in reality stresses gaming and competition, and conceals, by way of putting play as the first part of the concept of playbour, that Web 2.0 companies, like all capitalist firms, stress valorisation, labour, rather than even gaming. Hence, it is a gaming that is subordinated to the instrumental reason of capital. Thus a twofold-inverted picture of reality emerges through this criticism of the ideology of the concept of playbour. The activity going on is really about labourgaming, or (maybe sometimes) about, gamebouring rather than playbouring. The two models below are meant to function as examples for how I perceive of the hybrid concepts in this part of the article focusing on the directions and forces that join each other in synergies or clashes. The following two examples of models do not functioning well with the field model that is static in its construction. ${ }^{4}$

\footnotetext{
${ }^{4}$ It is problematic to visualise, for example, an argument about labouring invading gaming almost totally, in the field model. The best way to visualize this argument would be to show how gaming's square turn into a part of labouring's square, but this is not possible at the moment. The analysis is, hence, tentative in character, and explicit in its invitation to critique and further development.
} 


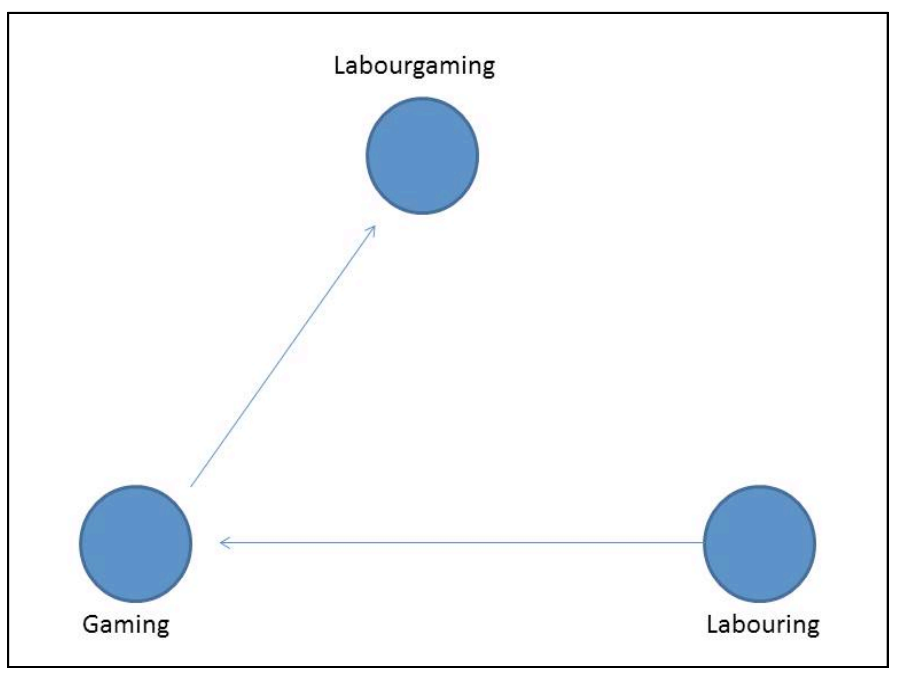

Figure 5: Labourgaming (Arwid Lund 2014)

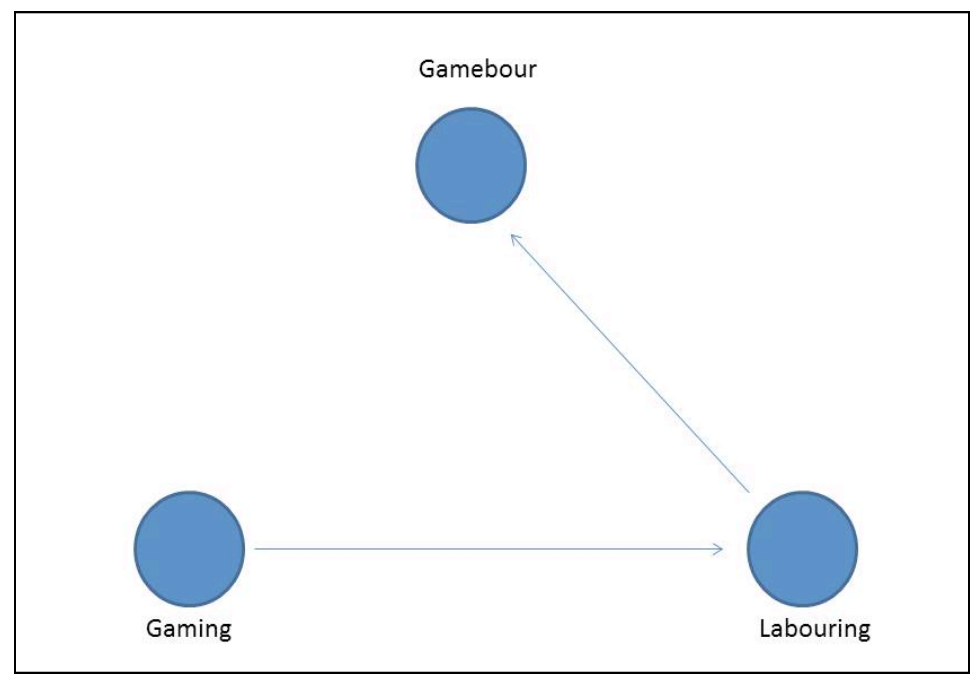

Figure 6: Gamebour (Arwid Lund 2014)

Taking into account commons-based peer production as an embryo of a new mode of production in the centre of capitalism (Rigi 2013, passim), we can use the hybrid concepts of playwork and workplay to describe the core of this mode of production. But now I am getting ahead of myself.

\subsection{Playing and Gaming}

The play of Charles Fourier often had an erotic character and took on the character of gaming. In the Phalanstery, his ideal community, the real contest for prestige and renown was not centred on work or labour but rather on sexual politics, which was a "game of sensual largesse" and the currency of the game was attraction: "but the point of the game is not to hoard and covet, but to dispense and distribute the favours of the favoured" (Wark 2013, 73). Fourier did not see anything un-playful in this competitive gaming and according to himself he would be placed in the middle of playing and gaming on the horizontal axis, but the gaming feature is too accentuated for that. Gameplaying seems more appropriate.

The Situationist International (SI), active about a hundred years later, was instead very much against the game element in play. The play forms in society were according to SI linked to the development of the very forces and modes of production that they tried to chal- 
lenge with play. Play had been dominated by the realm of necessity and labour under capitalism. The political mission of SI was to change the power relations between the two, and the most important question involved a project to make the element of competition disappear from the concept of play and the play actions. According to the organization the question of winning and losing had previously been almost inseparable from ludic activities as a result of "all other manifestations of tensions between individuals for the appropriation of goods" in capitalism. Play was perceived as a "wretched product of a wretched society" and as such was it exploited by all conservative forces, to mask the monotony of the life they themselves imposed (Situationist International 1958a). This statement could be placed in the lower parts of the square for gaming.

According to SI playing was something different from gaming. The former was a propensity of humans and the latter an effect of a class society. The political mission was to infuse everyday life in class society with play instead of play being infused with gaming. The relation between the playing and gaming was an antagonistic one: "[t]he element of competition must disappear in favor of a more authentically collective concept of play: the common creation of selected ludic ambiances" (Situationist International 1958a).

The American anarchist Robert "Bob" Black's anthology of essays from 1985, containing the article "Abolition of Work", is characterised as having a post-Situationist and individual Anarchist perspective by English Wikipedia (Wikipedia contributors 2013b; Wikipedia contributors 2013a). Playing means doing things and being active, to Black: "To be ludic is not to be ludicrous. Play doesn't have to be frivolous, although frivolity isn't triviality; very often we ought to take frivolity seriously. I'd like life to be a game-but a game with high stakes. I want to play for keeps" (Black 1991). He does not distinguish between play and gaming as it seems, and equates both of them with life itself. Play could be competitive, and with high stakes. This position, surprisingly coming from a Situationist, can be placed in the middle between playing and gaming, in its stress on both frivolity and gaming, but at the same time, as we will see more clearly below, the categories of working and labouring have disappeared entirely in Black's ideal society.

The same thing goes for Nietzsche's view of play and wage labour in the third maxim above. He perceived of a conflict between relaxing play and labouring, at the same time as he was in favour of gaming and conquering.

The individualistic approach of Black and Nietzsche contrasts sharply with the playing of the !Kung Bushmen of Southwest Africa. They do not play competitive team games, with the exception of tug-of-war, which re-enacted the founding myth of !Kung, and the idea of winners and losers "is not emphasized as this is a culture that stresses the importance of group and not individual performance". The children's play could otherwise be both free and structured, and conflict, satire and mimicry of animals, other !Kung and other societies formed part of many play activities (Schwartzman 1978, 129-130). This view is firmly placed within the square of play.

Helen B. Schwartzman identifies an important difference between playing and gaming which has the potential to create conflicts between the two forms of activities:

An understanding of game rules provides one with an understanding of the event, and too much spontaneity or individuality (ie, too much play) can spoil the game for all participant. This may mean that, in order fully to understand, we will have to separate it clearly from games. Games rule out the ambiguity, spontaneity, and flexibility characteristic of play, and it may be that it is only our language that is playing tricks on us when we are led to say that we 'play games.' This may actually be a contradiction in terms. (Schwartzman 1978, 327)

\subsection{Playing and Working}

In Grundrisse and the section called the Fragment on Machines Karl Marx stressed that the development of productive power develops both the capabilities of production and the means of consumption. Saved labour time is not the effect of abstinence from consumption, but the 
result of the development of the productive forces. The saving of labour time equals an increase in free time that according to Marx is free time for the full development of the individual which further develops the forces of production (Marx 1973, 71-12). This was a picture of how it could be when, in the era of general intellect, capitalism became so obsolete that the regime of value producing abstract labour potentially could be challenged and defeated. But the increased productivity within capitalism did not produce free time for the labourer. Instead relative surplus value was produced for a capital that struggled to maintain the wage form. Marx thus looked at the relation between playing and working through an historical lens.

In the realm of freedom under communism free time was possible, but it was a free time, which was productive in developing the forces of production. The two phenomena free time and work could no longer be separated as in a bourgeois economy were the labour time is held as the abstract antithesis to free time. Instead free time and work time were dialectically connected then (Marx 1973, 711-12).

These statements are complex. Labour under capitalism, though, just leads to more of the same, placing it in the square of labour, but the tricky question that interests us here is where to place the statement of the shrinking realm of necessity and growing realm of freedom in communism? In terms of what is favoured as positive, the realm of freedom ends up in between play and work as attractive work, while proportions between this and the necessary work, with corresponding positions, depends on the level of historical development.

\subsubsection{Liberating Work Is Serious and Not Mere Fun}

Free time is productive and work has liberating qualities under communism. According to Marx, Adam Smith maintained that labour never changed its value. An hour of labour was always an hour that the worker had to give up the "identical portion of his tranquillity, his freedom, and his happiness" to realize (Marx 1973, 610-11). Marx comments that labour seems like a curse to Smith ${ }^{5}$ in contrast to the tranquillity, freedom, and happiness of free time. For Marx free time could be time for recuperation and reproduction of the labour force under capitalism, but also, as we have seen, productive under communism. In either case it could not be isolated from work. Smith's view of work in turn was flawed in defining it in accordance with its historical form under capitalism:

It seems quite far from Smith's mind that the individual, 'in his normal state of health, strength, activity, skill, facility', also needs a normal portion of work, and of the suspension of tranquillity. Certainly, labour obtains its measure from the outside, through the aim to be attained and the obstacles to be overcome in attaining it. But Smith has no inkling whatever that this overcoming of obstacles is in itself a liberating activity -and that, further, the external aims [...] become posited as aims which the individual himself positshence as self-realization, objectification of the subject, hence real freedom, whose action is, precisely, labour. (Marx 1973, 611)

This view of work as a challenge, obstacles to be overcome, has a structural similarity to the practice of play, even if, in the latter case, it is about inventing imaginary obstacles for the fun of it. The feeling of self-fulfilment when you have overcome an obstacle seems to correlate to play, but has a different form of seriousness added to it if we are to believe Marx. The feeling of freedom is combined with the production of something useful.

Marx agrees that labour in its historical forms as slave, serf or wage labour always appears as repulsive and as externally forced labour, and that not-labour therefore appears as freedom and happiness in these historical realities (Marx 1973, 611). Hence, our way of looking at free time suffers from ideological distortions. Free time is imagined through the camera obscura, upside down (Marx and Engels [1845 ]1998, 42). Maybe it is possible to draw some conclusions of Marx's—not so positive then—view of play from this.

\footnotetext{
${ }^{5}$ The distinction between work and labour, as Fuchs and Sevignani have argued, is of crucial importance to understand this passage. Marx uses the German word Arbeit that can signify both work and labour and does not qualify which he is using at each moment. See the introduction to the typology.
} 
One reason for "not-labour" being idealised under capitalism is that labour has not yet "created the subjective and objective conditions for itself [...] in which labour becomes attractive work, the individual's self-realization (Marx 1973, 611). It is in this context that Marx concludes that Charles Fourier was wrong when he believed that labour could be play (Marx 1973), a statement that also hit the position of SI and implies that play is unproductive. It is in the same spirit that he continues his argumentation after the quotation above: self-realization through attractive work was by no means to be understood as "mere fun, mere amusement" (Marx 1973, 61-12). Play is here assigned a rather minor role by Marx. Play appears as something unimportant in the margins of societal life, with its enjoyable and amusing character. "Really free working", like composing, is instead, at the same time, "precisely the most damned seriousness" and "the most intense exertion" (Marx 1973, 611-12), but the condition for it is that it is social, scientific and at the same time, general in its character. It cannot just be "human exertion" but has to be "exertion as subject" (Marx 1973, 611-12).

Work could, thus, be a really free and liberating activity, giving self-fulfilment in the overcoming of external obstacles. Play, on the other hand was unproductive amusement in itself. Marx did not give importance to the similarities between play and work in any deeper sense, like seeing play as an imaginary variation of work or as a serious and constructive variant of play, but at least favoured play as a subordinated aspect of work when this became attractive.

Marx put the word play in the mouth of the utopian Charles Fourier as a proof of his naivety, although, as we shall see, this was not entirely true. Even so, Marx was not totally negative in his attitude to Fourier. He contended that Fourier's greatest contribution was to express the suspension of the mode of production itself as the ultimate political goal-implying that Marx was not in favour of necessary labour for some moral or political reasons.

We get a closer picture of how play relates to the realm of freedom in a famous passage in volume three of Capital. Work in the sphere of material production is here described as it can never be unconditionally free, it will always exist on the basis of a realm of necessity, but there can be more or less of such necessary work, and it can be executed in a more dignified and joyous way, and thus be more attractive. Freedom within this sphere can only be forwarded by a truly social mode of production of associated producers that in common cooperate to control the interchange with nature in a rational way, which accomplishes its task with the least effort and in the most dignified way for the humans-a statement that places itself in the upper area of the square of working. Beyond this realm of necessity begins the development of human power "which is its own end, the true realm of freedom". The central aim for the freely associated producers should therefore be the shortening of the working day in order to make the realm of freedom, where the human subject could act as a subject and realize and express her/himself, increasingly larger (Marx 1909, 954-55).

Marx, then, even if he in principle was in favour of Fouriers proposition of abolishing the mode of production in itself, did not think that it would be entirely possible. Work in the realm of freedom, on top of the realm of necessity, was attractive, but not pure play. The productive end was still dominating. We do not get closer to Marx's view on play than this. We cannot know if he wanted humans to spend all their time, even if the necessary material production had been suspended by technical development, in his realm of freedom with its "damned seriousness" and "really free work" of social and scientific character, or if there was also room for some play, fun and amusement not focusing on the result of the activity.

The activities in the realm of freedom can be labelled workplay (work is dominating and play is dominated), but not playwork. In his critique of the Gotha programme Marx wrote of the first phase of communism when this society emerges from capitalist society and is "stamped with the birth-marks of the old society" (Marx and Engels 1991, 305-306) In this phase your amount of work according to Marx will render you an equal share in the total amount of social work (that you can exchange for individual means of consumption). The exchange of equivalents in capitalism only exists on the average, here it also exists in the individual case - theory and practice are no longer "at loggerheads" (Marx and Engels 1991, 
306). The problem is that this equal right in theory is an "unequal right for unequal labour" due to the fact that some workers are physically or mentally superior to others (Marx and Engels 1991, 306). This system is abolished in the higher phase of communism:

\begin{abstract}
In a higher phase of communist society, after the enslaving subordination of the individual to the division of labour, and thereby also the antithesis between mental and physical labour, has vanished; after labour has become not only a means of life but life's prime want; after the productive forces have also increased with the all-round development of the individual, and all the springs of common wealth flow more abundantly-only then can the narrow horizon of bourgeois right be crossed in its entirety and society inscribe on its banners: From each according to his abilities, to each according to his needs! (Marx and Engels 1991, 306)
\end{abstract}

Work, thus becomes a "prime want" in the higher phase of communism. Here we have a situation of certain abundance, but what is stressed is that everybody should work in accordance with his/her capability. Of course the realm of necessity is shrinking but nowhere is there anything said about amusement. Instead the prime want was to engage in "really free work".

Let us, in a last effort, visit the Fragment of Machines in Grundrisse again, where Marx follows the logic of capital into the future and the general intellect as the full development of capitalism. The development was based on the total, real subsumption of labour under capital (dead labour) where the worker's activity was reduced to an abstraction of activity and was on all sides determined and regulated by the movements of the machines. The science that was objectified in the machines did not exist in the consciousness of the worker, who was merely supervising the activity as "conscious linkages" of the automated machine system (Marx 1973, 692-93). As gloomy as this appears, the general intellect was also a moment of liberating possibilities. The real subsumption of labour under capital and dead labour, which is manifested in the effectiveness, the intensity and the quantitative extent to which capital is developed as fixed capital, also signifies that science plays a central role in giving production a scientific character that reduces direct labour to "a mere moment" (Marx 1973, 699). This development of fixed capital "indicates to what degree general social knowledge has become a direct force of production, and to what degree, hence the conditions of the process of social life itself have come under the control of the general intellect and been transformed in accordance with it" (Marx 1973, 706).

We now have a situation according to Marx where direct labour is reduced and subordinated (but still an indispensable moment, without it no surplus labour, surplus value and profit-hence no capitalism) to general scientific labour and the general productive force "arising from social combination" (Marx 1973, 706, 709).

This opens up a possibility in history of re-configuring and re-ordering the relations between the realm of necessity and realm of freedom, but also here the argument simply leads to the conclusion that necessary work would be minimized (Marx 1973, 705) and nothing is said of play. Throughout Marx's life his ideal was the well-rounded (wo)man who is not tied to one profession. In communism nobody has an exclusive sphere of activity, and people can therefore take part in any pursuit (s)he wishes. Society regulates general production and makes it possible to "to hunt in the morning, fish in the afternoon, rear cattle in the evening, criticise after dinner [...] without ever becoming hunter, fisherman, shepherd or critic" (Marx and Engels [1845] 1998, 53). The focus is still on "really free work". Could it be that Marx meant that "mere fun, mere amusement" was just an offspring of the mode of producing in class societies, with its dichotomy of free time and labour, and, thus, would necessarily disappear in the classless society where work became attractive work?

This examination of Marx's view of the relation between play and work has revealed that in line with his historical materialist perspective he historicised and contextualised his views on work, free time and attractive work, but that play and the play drive itself never was of any theoretical interest to him. Let us now instead see if we can find some discussions on play and its relation to work in works of Charles Fourier and William Morris. 


\subsubsection{Charles Fourier}

What did Charles Fourier actually say? The question is tricky because Fourier performed a thought experiment with his own unique concepts. In short he argued that labour "forms the delight" of animals such as beavers, bees, wasps and ants, which had the liberty to prefer inertia instead, which led him to conclude that god surely had done the same for the humans. Labour under capitalism was repulsive and odious and what he called "associative labour" had to fulfil "seven conditions" to be attractive. The division of labour would be adapted to the needs and skills of people, not abolished, and the work assignments would rotate before they became "tedious". These short sessions would be "sustained by cabalistic impulses and by friendly union with selected associates", and it could not fail in bringing and finding "cheerfulness everywhere" (Fourier n.d.). Necessary but tedious work was here compensated for by play rather than merged with play.

However, beside the fact that pure play existed for Fourier as an important social phenomenon, play often merged with work in the writings of Fourier rather than substituting for work. Fourier, regardless of the fantastic character of his theories, then seems to have more or less the same opinions on the relation between play and necessary labour as Marx. The differences were located mainly in the attitudes and preferences of the two. Work of the phalanstery, the ideal community of Fourier, was a delight but also tedious and had to rotate and be interrupted by play in the form cabals and orgies. Play and work was distinct categories even for Fourier. Work could be passionate, attractive and social, but not play, on the other hand this work and workplay (when merged with play) sometimes had to be interrupted by pure play. And in some writings the dynamic in the relation between play and work was reversed into some kind of playwork and finally qualitatively changed into other hybrid phenomena.

The nature of the passions was of the utmost importance to Fourier. The passions had been and "will remain invariable among all nations of men". Fourier concluded that these passions and impulses could entice us to evil if "we yield to them individually", but he came up with the following solution:

[A]s soon as the number of associates [...] has reached 1600, the natural impulses, termed attractions, tend to form series of contrasting groups, in which everything incites to industry, become attractive, and to virtue, become lucrative. (Fourier, On the role of the passion.)

The passions were integral to the organization of the Phalanstery, which was characterised by a mixture of playing and gaming; both competition and exchange were central processes in the Phalanstery (see above). McKenzie Wark portrays what he calls the queer theory of Fourier as one of the inspirational sources for leading members of Situationist International. Wark contends that Fourier more than a political economist, wanted to be an "erotic umpire" and characterises his New Amorous World as a "unique kind of philosophy of the orgy, or systems-theory porn". It is the polymorphous play of as much as twelve passions that interests Fourier. "Amour" is not private and not at odds with the world, and he dreamt of a new way to harmonize desire and the social life. In this decentralised word of plenty there was nothing to fight over and no need to build empires. Instead capital, labour and talent cooperated. Fourier was no egalitarian and, as we have and shall see his play was more of a gaming kind, opening up for positions like gameworking and workgaming (Wark 2013, 7-73).

\subsubsection{William Morris on Useful Work and Useless Toil}

William Morris, who initiated the Arts and Crafts Movement in the $19^{\text {th }}$ century in England, made a distinction between useful work and useless toil. Toil signifies an activity connected to certain suffering and pain. In line with Marx he contended that the "race of man must either labour or perish"; humanity had to win its livelihood by work or toil to some degree. Morris claimed that some (not all) work was a curse. Useless toil was an effect of class society and the difference between useful work and useless toil is that one has hope in it and "the other 
has not". The hope is threefold: hope of rest, hope of product and hope of pleasure in the work itself (Morris 1884c). He commented on the hope of rest:

\begin{abstract}
Whatever pleasure there is in some work, there is certainly some pain in all work, the beast-like pain of stirring up our slumbering energies to action, the beast-like dread of change when things are pretty well with us; and the compensation for this animal pain is animal rest. We must feel while we are working that the time will come when we shall not have to work. Also the rest, when it comes, must be long enough to allow us to enjoy it; it must be longer than is merely necessary for us to recover the strength we have expended in working, and it must be animal rest also in this, that it must not be disturbed by anxiety, else we shall not be able to enjoy it. If we have this amount and kind of rest we shall, so far, be no worse off than the beasts (Morris 1884c).
\end{abstract}

Here we can see a relation between free time and work time, which is similar to one of Fourier's positions but different from the way Marx perceived it. This time the word "enjoy" is mentioned in combination with the word "rest". Morris stresses the importance of enjoyment and careless being (no anxiety) in free time as a necessary outside to equally necessary work, which had to be done in all societies and all modes of production. This is quite close to saying that play is the dialectical antithesis to work, but without any synthesis being created in the process. Morris also wanted machinery to be used for cutting down on useless toil (Morris 1884a)-which we can place in the square of labour.

But Morris also spoke of pleasure in work, within the realm of necessity. The argument was ontologically based. All "living things" feel "pleasure in exercising their energies" and even the "beasts rejoice in being lithe and swift and strong". The difference between beast and man was that the latter also exercised the energies of his mind were the memory, imagination and the thoughts of "men of past ages" helped him in his work as part of "the human race" (Morris 1884c). In contrast to Marx he stressed the anthropology or essence of the human race more than historicity and sociality of existing humans, but he still had a clear view of the uneven distribution of labour in capitalist society.

Morris view of the relation of play to the subjectively, scientifically or self-fulfilling work, which Marx spoke about, is expressed in his writings on art and handicrafts and in his involvement in the arts and crafts movement. These accounts also speak of attractive or artistic work as something that should be evenly distributed. Some of the originality of the arguments lay more in its criticism of artistic works degradation under capitalism and it will therefore be treated in the section on play and labour.

In a speech titled Art and Socialism 1884 he contended that capitalism's "haste to gain a very inequitably divided material prosperity" had "entirely supressed popular Art" by excluding the "greater part of the people" that had no share at all in art (Morris 1884b). In another speech titled Art and Labour he developed his view in the relation between the two in a way that tells us something of his view of play's relation to work. Morris defined art as "beauty produced by the labour of man both mental and bodily, the expression of the interest man takes in the life of man upon earth with all its surroundings, in other words the human pleasure of life is what I mean by art" (Morris 1884a). After defining art he focused on the relation of the pleasures of life to labour that produced "all the means of human life" through a historical survey that in the end highlighted the medieval guilds with their roots in the communal life and co-operation of the German tribes. The craft guilds developed in an effort to "free the individuals from the domination and protection of the feudal lord" and mutual protect the associated "guild-brethren". "For a time", Morris concludes, "the constitution of these guilds was thoroughly democratic; every worker apprenticed to a craft was sure [...] to become a master" if he met the standards. Pleasure is here connected to some sort of freedom in the work processes, placing the activity in the middle of play and work, with an extra stress on the non-alienated existence in the world, but, as we saw above, "the animal rest" was also important and could be enjoyed in itself. 


\subsubsection{Psychoanalysis and its Social Critics}

Eros was not only vital and dynamic as a component in psychological life, it was life itself according to Freud. The living cell was dominated by the physiologically constructing force of Eros and not only the individual's life, but also social and cultural life, especially art, had Eros as its "productive source of psychical forces and energies" (Vološinov 2012, 51, 67). Culture grew when Eros was regulated, suspended and sublimated or de-sexualised (by repression of the sexual drives) into creative labour (Fuchs 2013).

Wilhelm Reich and Erich Fromm criticised Freud from a social perspective but came to different conclusions. Erich Fromm contended that the change in Freud's theory of instincts from a mechanistic and physiological approach, where chemically produced tension provoked a need to reduce it to the normal threshold, to the new biologically based theory of Eros as the constructing force of the cell and the unification of all cells and even beyond the cells to the society, was a major one. Freud had discovered non-sexual love and in contrast to earlier, when man was seen in isolation and related to other humans as if in the market, the Eros theory related people primarily to other people with whom the subject needed union. Life, love and growth are one and the same, more deeply rooted and fundamental than sexuality and "pleasure" according to Fromm's interpretation (Fromm 1988, 106-07). The pleasure drive was here something more constructive in the shape of Eros that in this interpretation could be seen as playwork.

Wilhelm Reich, to whom love, work and knowledge were the wellsprings of life, and therefore should govern life, valuated the work of Freud radically differently. He favoured the original theory of the pleasure principle whose limit was set by the reality principle, thus suffering came from society rather than from the biological will to suffer (Thanatos). Reich stressed that Freud's theory of the death wish blocked:

[T] he difficult path into the sociology of human suffering, into which the original psychological formula on the psychic conflict had made considerable headway. /---/ The original formulation of the psychic conflict [...] leads to a critique of the social system (Reich 1990, 23--233).

Reich firmly believed that the pleasure principle was a basic law of the human psyche "according to which pleasure is sought and unpleasure is avoided" (Reich 1990, 233). What this implies when it comes to play and its relation to work is not totally clear, but my guess would be that Reich looked more to the enjoyment of play than its constructive character and relation to work. The former reality principle was not antithetical to the pleasure principle according to him; it only meant that the psyche had to get used to the fact that the external world sometimes demanded that it had to postpone momentary gains of pleasure "and to forgoing some entirely" (Reich 1990, 233), but capitalism certainly created psychological problems. Reich position in contrast to Fromm's was more in the line of Marx's view on attractive work as a kind of workplay.

\subsubsection{The Frankfurt School and the Situationist International}

Theodor Adorno wrote in the essay Free Time (Freizeit), under the counter-culture years, that leisure time was an older concept than 'free time' and 'spare time.' Leisure time stood for the privilege of an unconstrained and comfortable life style, and Adorno thought that its connotations were "qualitatively different and far more auspicious". The concept was not so tied to capitalism, abstract labour and consumption, as the other concepts that were shackled to its opposite, abstract labour (Adorno 1991, 187). The aristocratic pessimism of the statement implied that there once, for the privileged, had existed less friction in the relation between the realm of necessity and realm of freedom, than in the era of free time. The relation between play and work was degrading.

Herbert Marcuse took a more proactive stand. He expressed his political mission in stark contrast to Freud, who maintained that instinctual liberation "would explode civilization itself, 
since the latter is sustained only through renunciation and work (labour) -in other words, through the repressive utilization of instinctual energy" (Marcuse 1955, 175). To meet this position he recalled "certain archetypes of imagination which, in contrast to the cultureheroes of repressive productivity, symbolized creative receptivity". These archetypes "envisioned the fulfilment of man and nature, not through domination and exploitation, but through release of inherent libidinal forces". Marcuse's political mission was then to influence people to set themselves "the task of 'verifying' these symbols [...] demonstrating their truth value as symbols of a reality principle beyond the performance principle" (see section on playing and labouring) (Marcuse 1955, 17-176).

The Situationist International (SI) confronted the state of affairs more proactively and practically, but from very much the same analysis. Instead of being critical to the notion of free time, they wanted free time or play to invade societal life. In contrast to Huizinga, who saw actual play as a temporary and "perfection" limited in space and time, they wrote: "The central distinction that must be transcended is that established between play and ordinary life, play kept as an isolated and provisory exception". The problem of survival, the realm of necessity, was not a problem and could be rationally managed. "[T]his possibility is at the heart of every conflict of our time" (Situationist International 1958a). This means that the realm of freedom was already an existing potential for them and that play and playwork formed a central role in this realm.

In his manifesto, post-Situationist Bob Black claims that we have to stop labouring if we want to stop the sufferings of life, but this does not mean that we have to stop doing things. Play could replace labour in the future society. He describes his position as both "joking and serious" and stresses play's resemblance with work. He equates the will to do things with the drive for play, rather than calling it pleasurable work or attractive work: "Play isn't passive. Doubtless we all need a lot more time for sheer sloth and slack than we ever enjoy now, regardless of income or occupation, but once recovered from employment-induced exhaustion nearly all of us want to act". Play is characterized as vitality and a higher form of existence and not "mere survival". It is also voluntarily engaged in. (Black 1991). Black thus opposes the notion of play as non-instrumental and without consequences:

The point is not that play is without consequences. This is to demean play. The point is that the consequences, if any, are gratuitous. Playing and giving are closely related, they are the behavioural and transactional facets of the same impulse, the play instinct. They share an artistocratic disdain for results. The player gets something out of playing, that's why he plays. But the core reward is the experience of the activity itself. /---/ Conversation, sex, dancing, travel-these practices aren't rule-governed but they are surely play if anything is (Black 1991).

Black interestingly here points to the possibility of the indirect consequences of play (engaged in for the sake of the activity itself) being enough for maintaining society. Necessary work was not needed: all could be managed within the realm of freedom in the form of play. Black here conflates the two categories play and work under the total dominance of the former. The statement that playing and giving is closely related reveals the influence of gift theories and Black concludes that Sahlin's anthropological writings on the original affluent society where the work "is hard to distinguish from what we regard as play" fits well into Schillers definition of play (Black 1991). This understanding also answers Helen Schwartzman critique of the categorical distinction between play and work. Black contends in another version of the text that activities which would be play if performed voluntarily are labour if forced, implying that there is no independent category of work, even though he also stresses in the same context that today's labour is "historically original and horrible" (Black n.d.). Black's position, according to his own statements, should be placed totally within the category of play, especially since he denies the existence of work as an independent category. 


\subsubsection{Contemporary Critics of the SI Concept of Play}

Tom Tenney, describes SI as an intellectual avant-garde collective that rightly used the concept of Homo Ludens to inform a revolutionary praxis, but in doing so also ignored some essential features of the original thesis. Tenney investigates how SI played out the ludic principles in practices as Détournement and Situations and contends that SI ignored the separated realms between play that could represent and anticipate an ideal social order (but still stand apart from it separated in time and space) and real life. The goal of SI was to "create play as real life, as a way of transforming the everyday into a continual play that is seamlessly integrated with quotidian activities, not as something that stands apart" (Tenney 2012, 3). Huizinga's theory perpetuated the division of societal life that SI wanted to eradicate.

The claim of SI that play had been co-opted by consumer culture and the spectacle with the consequence of a "bastardization of play" which had obviated the dichotomy of work and leisure and turned play into an "amusement that carried the same forms that dominate the working life" and simply was used to alleviate tensions created by a mechanized culture, was according to Tenney a misreading of Huizinga, who clearly stressed that competition was "part and parcel of play" (Tenney 2012, 5). In a work called In the shadow of tomorrow, written by Huizinga just before the second world war, he himself spoke of "the perversion of play" as the major malady of his time (Anchor 1978, 78):

The most fundamental characteristic of true play, whether it be a cult, or a festivity, is that at a certain moment it is over. The spectators go home, the players take of their masks, the performance has ended. And here the evil of our time shows itself. For nowadays play in many cases never ends and hence is not true play. A far-reaching contamination of play and serious activity has taken place. The two spheres are getting mixed up (In the shadow of tomorrow [1938] 1964) cf (Anchor 1978, 77).

Huizinga thus saw fascism as a force perverting play much in the same way as SI wanted to use play for changing everyday life, albeit for the opposite political reasons.

Finally Tenney admits that SI implemented the play theory in a successful practice when they used the egalitarianism and freedom experienced in play to challenge established social forms and form a critique that according to him can be "interpreted as active resistance" (Tenney 2012, 5). Some of the mistakes of SI were later corrected according to Tenney by the cultural jammers of the 70 s and 80 s who created a more playful culture rather than demanding a change in the whole of society. Tenney wonders if we could not start to think of the spectacle as a kind of play and SI as the spoiler of its game (Tenney 2012, 14-15). Tenney here precludes the possibility of theorizing about the perversion and bastardization of play, and in this he opens up for the use of ideological concepts like playbour. A management scholar, Douglas Smith, heads in the same direction when he claims that the Situationists gave the game away (Smith 2005, 433). It is hard not to see this critique as a consequence of a change in how the play concept is being used. Lourens Minnema concludes that the $20^{\text {th }}$-century formation of the play concept "reflects the transition from modern to postmodern culture" and a change in interpretation "from play as founding mediation to play as a possible means" that is "closely connected to the failure of mediation efforts which are critical of culture, to overcome radically the disintegration that goes with functional differentiation" (Minnema 1998, 39). This analysis is confirmed by Guy Debord twenty years later when he returns to and comments on The Society of Spectacle (1967). This time play is not positively mentioned as a mediating and progressive force, and the spectacle with its instrumentalism has gained strength (Debord 1990, passim, 4-5). Tenney and Smith are thus directing their critical eyes towards the defeated part rather than against those groups gaining from the expansion of the spectacle.

Helen B. Schwartzman, a social anthropologist, is known for having questioned the dichotomies of play/reality and play/work. In her book Transformations she shows that this understanding of the activities is not a given. In many non-western cultures it is common to mix play with work in a way that does not fit easily within a dichotomous relation between the two. 
In these cultures the more important contrast could instead be between sacred and profane work. Schwartzman connects the difficulties of westerners to perceive of work as playful to the Protestant ethic. This same ethic is also the reason why play sometimes can be perceived of as work, especially when it comes to games (Schwartzman 1978, 4-5, 156). ${ }^{6}$ The activities described by her can be seen in the perspective of this article as either workplay or playwork. Csíkszentmihályi points out here that Max Weber never understood that ascetic withdrawal from all pleasures could in itself be enjoyable, but that he came close to seeing how arbitrary the "work-play distinction" is when he stated in the concluding chapter that the Protestant ethic in its highest form of development, as the pursuit of wealth, took on the character of a game (Csíkszentmihályi [1975] 2000). In line with the argument here this is not so much an example of the relation between work and play as an example of the "gamification" power, which is inherent in capitalism and affects both play and work.

Schwartzman's conclusion is important:

There is nothing to be found in the anthropological literature to suggest that play is not pleasurable or enjoyable except the realization that this is not always the case, and, of course, it is also true that activities that are generally understood to be not-play are also pleasurable and enjoyable. This means that we cannot be satisfied with definitions or theories that argue in a curiously tautological fashion that 'the play element in play is playfulness' (or joy or pleasure or fun) (Schwartzman 1978, 327).

Hence, to define play we have to involve several other criteria than fun, joy and pleasure. ${ }^{7}$

\subsubsection{Hans-Georg Gadamer's view compared to Christian Fuchs' view on Play, Work and Communication}

Gadamer maintained both that play was essential to the animal world, including (wo)man, and had characteristics that differentiated human play from the play of animals. Monica Vilhauer states that human play for Gadamer had the special quality of human freedom, which was not simply a freedom of variability or caprice, but a freedom that involved the intentional "self-restraint that goes along with any effort to accomplish something, do something, play something". Human beings play themselves out by playing something (Vilhauer $2010,34)$. Gadamer's view is very close to substituting play for work. We can call it for playwork.

To this we have to add that Gadamer equates play with understanding. A work of art becomes complete in the inter-play with the spectator, but the same would go for communication based in a free, variable and spontaneous interplay between communicating subjects. Play, the movement of interpretation and communication, is understood as something larger than the individuals and things involved. In short the whole of the hermeneutic circle could be understood as play.

Christian Fuchs, in contrast, contends that communication is work at the same time as, and here Fuchs refers to Marx, sensuousness, speech and communication are taken as signs of a possible non-instrumentality of humans. Hence, Fuchs gives a hint of a possible non-instrumental version of work. Marx also, according to Fuchs, points out "that work is not always and not necessarily a necessity and an instrument to achieve goals, but under communism it becomes a free activity beyond necessity and instrumentality" (Fuchs 2014, 253). I do not totally agree with this. First, in his line of argument on communication being work, Fuchs himself stresses that language is the result of human social activity and that this language is used to co-operate in the production of information (Fuchs 2014, 248). This argument involves a kind of instrumentality, the production of a use value, information. The strength of the argument that sensuousness, speech and communication are signs of noninstrumentality according to Marx is diminished in Fuchs interpretation. Second, I have tried

\footnotetext{
$6 \quad$ Hägglund adds that adults in western cultures also introduce playful moments in their work (Hägglund 1989, 67).

${ }^{7}$ The categorization here depends on the five dimensions mentioned in the beginning of the article.
} 
to show earlier that even when Marx spoke of communism or the realm of freedom, play played a subordinate role under work. Work could indeed be attractive and self-fulfilling, but still focused on use and use value. Hence, work still had an instrumental character in the realm of freedom. Third, this is the time and place to admit the obvious, that communication not only resembles play, but also resembles work. Humans do not always use language and communication in a playful and non-instrumental way. Often they want to accomplish some effect in the outer world with their words. But if communication sometimes is work and sometimes is play, where does that leave us when it comes to the relation between play and work? Should we conflate the concepts under some kind of workplay (or attractive work) or should we just agree that the two share some characteristics and differ in others? That they exist a part from each other as well as being mixed up in different hybrid combinations? I would argue for the second alternative.

An interesting difference between Gadamer and the Situationist International's position on play is that the former sees the spectator as active in the art of work or the event (Gadamer $1975,31-32$ ), and the latter wants to include people in a social situation in a more direct way, implying that being a spectator is a passive mode of being similar to consumption in the "free time". These arguments have ramifications for our understanding of the concept of playbour as well as for the theory of audience labour advanced by Dallas Smythe (audiences were institutionalised by the mass media and their "audience-power" was sold to the advertisers) (Smythe 1984; Smythe 1977) and today used by for example Christian Fuchs (Fuchs 2012), and the theory of the social factory of the autonomist Marxists. This will be dealt with more in detail in the section on playing and labouring.

\subsection{Gaming and Working}

In Rules of Art: Genesis and Structure of the Literary Field (1992) Pierre Bourdieu advances a theoretical understanding of the literary field that can elucidate the relation between working and gaming. In the literary field you can find, argues Bourdieu, an inverted form of economy that is grounded in the dual character of symbolic assets. First there is the commodity aspect, secondly the signification or the symbolic value of the asset. The latter is relatively independent of the exchange value of the former. A developmental process (leading to modernism) resulted in a cultural production for the market and as a reaction against this a production of "pure" works of art intended for symbolic appropriation characterised by the objective and subjective distance of the cultural producers to the market. The strategies of the producers are distributed between two extremes in ways that do not constitute total subordination either under the market or absolute independence. Interestingly there exist two antagonistic forms of production and circulation within these fields (Bourdieu 2000, 215).

The anti-economy of pure art cannot recognise any other demand than the one, which creates itself in the long run. It is focused on the accumulation of symbolic capital as a denied, but generally accepted and legitimate capital, that under certain conditions can be transformed into economic profit (Bourdieu 2000, 216). The culture industry, satisfied with adapting to the existing demand and producing for economic profit as a regular company, has to keep clear of the worst forms of self-interest, if they are to conquer any symbolic capital at all (Bourdieu 2000, 216).

Symbolic capital depends on social and cultural prestige; it can be hoarded but is also easily lost; it is acquired in ways that resembles gameworking or workgaming, with its focus on the quality of the use value and gaming for prestige. This general perspective can be applied to explain peer production in Free and Open Source Software (FOSS) and Wikipedia. If you want to be esteemed and get a position of status within these communities you will have to show that you are committed to the project in deeper sense than in a narrow economic way. This acquisition of social status within a community of practice has a lot to do with gaming. Positions between working and gaming can then be placed according to the same logic as Bourdieu developed in his field theory (Bourdieu 2000, 191, 193). The proportions between working and gaming decide where a phenomenon is positioned. 
Bob Black thinks in the same direction as Bourdieu, but for him working and gaming seems to be the same thing. Playing and gaming mean to be active and do things and can substitute work at the same time as they are voluntary activities for him. He did not see any contradiction between this and his longing for this activity to be competitive in its character. He wanted life, as seen above, to be a game "with high stakes" (Black 1991). But probably the !Kung would not agree with him, being against competition and favouring co-operation as they are.

\subsection{Gaming and Labouring}

Charles Fourier differed from Marx in his view of competition between different groups in the utopian scheme. Where Marx saw co-operation, Fourier saw healthy competition. It would help rotation of work assignments to come about and would arouse such inspiration and passion in the members of the competing groups that the fanaticism would give them the power to do things that seemed humanly impossible (Fourier, Attractive labour.). Fourier, as we have seen above, included capitalists and exchange in his vision of the Phalanstery, hence this analysis of his thoughts on gaming in relation to labouring, although Fourier himself thought of it as non-alienated utopian work. The gaming element is clear in his descriptions; competition between the groups involved a measuring of the achievements, without fostering the usual imbalances of such a system (almost like the vision of the New Economy of the nineties). The point of view can be categorised as gamelabouring and stresses the synergies between the competition of gaming and labour, downplaying the exploitation within capitalism or seeing it as a part of the game. McKenzie Wark speaks of Fourier's philosophy as a heretical reversal of liberalism: "Rather than sacrifice the body to labor in order to sustain a survival in which some modest pleasure might be endured at the margin, the whole social field can engage all of the passions all of the time" (Wark 2013, 75).

This liberal character, albeit heretical, is shown by the fact that the process of exchange was of central importance to the functioning of the Phalanstery. The Exchange would be an institution where the work and pleasure sessions for the following days were planned and all individuals had to attend it with all its "animation and intrigues" (Fourier, The exchange.). Conflicts and intrigues were thus not looked upon as something negative, partly due to the fact that in the Phalanstery everybody desired to manifest his/her intentions and to make them publicly known (Fourier, The Exchange.). These passionate intrigues in the Exchange have a lot of gaming in them but they are portrayed as play, as Fourier did not distinguish between the two (in the earlier analysis his position was categorized as gameplaying, but in the expressed views here it seems appropriate to specify the position as also quite close the labouring square).

The Situationist International ( $\mathrm{SI})$, as we have seen, stressed that the question of winning and losing dominated the ludic activities in contemporary society as a result of all other tensions "between individuals for the appropriation of goods" in capitalism. This way of perceiving gaming as a corrupted, by labour, version of playing (and its ideological function for conservative forces in society) (Situationist International 1958a) that can be placed in the middle of labouring and gaming. The strengthening the competitive part of play seems to be a technique to introduce an alien instrumentality in play.

Helena B. Schwartzman refers to a study which points at some potential weaknesses in the positions of $\mathrm{SI}$ and Caillois on gaming, but in two different ways. In the Kpelle society gaming in the form of ritual speech-acts is ritually used as a regulator in the social distribution of wealth and prestige. Interestingly there exist a number of children's play activities in this community that "serve to develop the verbal and acting skills necessary to engage successfully in 'talking matter"' (Schwartzman 1978, 109). Gaming is not confined simply to capitalism, as could be seen as implied in the position of SI, but rather used in many different historical and cultural contexts, although still this is probably a feature of hierarchical societies as the example of !Kung's collective and their non-competitive perspective on gaming signals. When it comes to Caillois' view on gaming, the gaming of the Kpelle society did not 
simply result in lasting material results, but also affected prestige and future wealth acquisitions of the gamer. In these cases it is better to speak of gameworking than gamebouring.

From now on I will focus on the relation of gaming with capitalist labouring within the general intellect of contemporary society. The autonomist Marxist Paolo Virno stresses, that many of the characteristics of political and communicative action have been absorbed by post-Fordist labour (Virno 2004, 49-51). Hence, poiesis has taken on many characteristics of praxis. At the core of contemporary labour we find the being in the presence of others and "the beginning of new processes, and the constitutive familiarity with contingency, the unforeseen and the possible", and Virno maintains that post-Fordist labour "brings into play the talents and the qualifications" which according to a traditional view had more to do with political action (Virno 2004, 51). Capital activates several new forms of instrumental actions by involving former autonomous actions within alienated and exploited labour.

Virno gives the name of Virtuosity to this hybridisation, meaning "the special capabilities of a performing artist", and proceeds by defining the activity of a performing artist or virtuosic activity which "finds its own fulfilment (that is, its own purpose) in itself without objectifying itself" into an end product or finished product that could survive the performance. Secondly, it is "an activity which requires the presence of others, which exists only in the presence of an audience" (Virno 2004, 52).

This definition opens up for an interpretation in which labour has absorbed characteristics that in many ways also are the characteristics of gaming (more than play-see below) if we follow in the footsteps of Gadamer. If anything connects Virnos' argument to playing, it is his general characterisation of the cognitive character of today's labour that through the mediation of communicative action (see above Arendt, Habermas and Wittgenstein) connects to play. The playful communication on digital platforms is appropriated by the fraction of capital owning the vectors of information; the vectoralist class, as McKenzie Wark named them (Wark 2004). This kind of playbour will be discussed in the section on play and labouring.

One of the most important functions of immaterial labour in post-Fordist capitalism, besides activating productive co-operation, is to develop the social relation to the consumer by communication (Lazzarato 1996, 136-137, 140). This is an additional twist to a performance in the presence of others that corrupts playful communication and converts it into a game for profit, thus gamelabouring or labourgaming. At the same time the consumer is already inscribed in the manufacturing of the product offered. "[H]is or her consumption should be productive in accordance to the necessary conditions and the new products." (Lazzarato 1996, $136-137,140)$ Lazzarato advances the hypothesis that what is productive "is the whole of the social relation" (here represented by the author-work-audience relationship) (Lazzarato 1996,145$)$.

How should we evaluate this new form of work? Lazzarato's claim supports my position on gamelabouring and labourgaming within the general intellect and gives it a material foundation. But at the same time he asserts that immaterial labour starts where the social labour power is independent and able to organize both its own work and its relations with business entities. "Industry does not form or create this new labor power, but simply takes it on board and adapts it." He concludes that this "cooperation can in no case be predetermined by economics, because it deals with the very life of society." Economics can just "appropriate the forms and products of this cooperation, normalizing and standardizing them" (Lazzarato 1996, 137). Here we have progressive potential that will be dealt with, together with my criticism of it, in connection to playing and labouring.

Where we are in this process can only be judged by empirical studies.

On a more concrete level, suggesting that there is also a broad and narrow definition of gaming (as of culture and playing), the autonomist Marxists Nick Dyer-Witheford and Greg de Peuter describe the birth of digital gaming some 50 years ago by Pentagon programmers "who killed away tedious hours tending giant military computers by transforming the electronic screens of nuclear war preparation into whimsical playgrounds". Only years after Atari, the first commercial games company, "converted this bold experiment in computer liberation into 
an entertainment commodity" (Dyer-Witheford and De Peuter 2009, xv). Witheford, de Peuter and Stephen Kline contend that the fetishism of commodities (production processes are hidden and the relations between the products seem to be properties of the commodities in themselves) is "peculiarly intense" when it comes to commercial game products:

Play after all, is the opposite of work. Games are "fun" experiences. Every bit of game marketing and promotion actively discourage us from associating them with such mundane and boring realities as jobs, management, and labour relations (Kline et al. 2003, 197).

In gaming magazines and on the game boxes we find information of how "cool" it is to labour "in the gaming industry":

In such depictions making games is itself shown as play-work as fun. The blurring of boundaries between labour and leisure so that not only consuming games but also producing them is represented as a continuum of endless fun is part of the interactive game industry's hip self-image (Kline et al. 2003, 197).

But there is also another connection between gaming and labouring. Not all of us buy our games but download them with the help of the Warez scene or swap our games with our friends. The authors contend that we are pirates that reckon the chances of getting caught are small.

We may even get a charge out of cracking the various technological systems while Microsoft and Sony try to keep us out: hell, it's just another level of the game. If work-as-fun is the interactive game industry's wet dream, then piracy-as-play is its worst nightmare (Kline et al. 2003, 198).

Here we have both synergies and conflicts represented in the relation between gaming and labouring. It is the first time that some gaming is portrayed as being antagonistic to capital and its labour, placing it between playing and gaming.

\subsubsection{Bruno Latour and Steve Woolgar}

Labourgaming and gamelabouring could perhaps also be said to have been touched upon in a study by Bruno Latour and Steve Woolgar. In Laboratory life from 1986 they developed a theory on the motives of the labouring scientists. In one of the essays, Cycle of Credits, they stress that the scientist has to be understood as part of the laboratory rather than as an individual, even if there are differences between the scientists. When one scientist claimed that a scientific finding was made through them personally, other scientists claimed that the finding was the result of a collective process. Some scientists were more closely tied to the laboratory in their career compared with other more successful ones (Latour and Woolgar 1986, 18189). Four different kinds of credit were discovered in their study, which generally showed that quasi-economic reasoning was common in, for example, the counting of published papers. These credits could be used in several ways: 1) they could be exchanged as commodities, 2) they could be shared, 3) they could be stolen, and 4), they could be accumulated or wasted (Latour and Woolgar 1986, 18-192). Latour and Woolgar stressed that it was difficult to interpret the testimony of the informants, but that it seemed that they were economic in a very broad sense. The informants did not seem to make any distinction between internal and external motives for their actions (Latour and Woolgar 1986, 19-192)

References to credit can frequently be found, but it only assumes prominence in discussions of the past, or of group structure, or of issues of priority. Consequently, credit as reward cannot adequately account for the behaviour of a scientist practising science (Latour and Woolgar 1986, 193). 
Scientific practice was not only about the pursuit of recognition and trustworthiness, it was also about personal interest, curiosity, dedication and playfulness, but the authors also point to the possibility of converting one form of creditworthiness into another within a similar logic as the logic of capital accumulation (the M-C-M' formula): credit-investment-credit' (C-I-C'). This is what they call the cycle of credit. A scientist is more than anything (more than pecuniary rewards) interested in getting more credit to use for their advancement within their field, but this also involves a risk of wasting the credits already accumulated (Latour and Woolgar 1986, 19-198, 201). To me this sounds like the accumulation of symbolic capital of Bourdieu, but without the inverted character of the economy. No conflicts are at hand here, only synergies. The credits could be sold as commodities or stolen as property as well as be used as symbolic capital, at the same time as it was the intrinsic motives that spurred the research activities. Playbour as a phenomenon seems to be confirmed by the study of Latour and Woolgar even if it seems to be labourgaming, when looked at from the structural perspective of post-Fordism entering the attention economy (Davenport and Beck 2001).

\subsection{Working and Labouring}

Here we have at least three themes within Marxist tradition. The first stresses that the concept of abstract labour involves several abstractions from the concrete forms of labour, that is, work: an abstraction from the physical properties of the use values, an abstraction from the single products to establish social relations through the exchange of commodities, an abstraction from simple to more complex activities, and finally, an abstraction "from specific qualities under which specific labour processes took place (such as bad working conditions, low payment, etc.) so that common properties of commodities are foregrounded by the value concept" (Fuchs and Sevignani 2013, 248). This leads up to a one-dimensional perspective focused on realized, fixed and crystalized social labour (Marx 1865, cited through Fuchs and Sevignani 2013, 248).

The second theme, represented by William Morris, asserts that the class society is at the root of creating useless toil that does not produce socially necessary use values. The middle classes do not produce utilities, but they consume utilities "out of all proportion to their due share". The commercial and manufacturing classes "spent their lives and energies in fighting amongst themselves for their respective shares of the wealth which they force the genuine workers to provide for them". The rest of the middle classes are "almost wholly the hangerson" and "parasites of property". The middle class as a whole had one common aim, and that was:

[N]ot the production of utilities, but the gaining of a position either for themselves or their children in which they will not have to work at all. It is their ambition and the end of their whole lives to gain [...] the proud position of being obvious burdens on the community. For their work itself, in spite of the sham dignity with which they surround it, they care nothing (Morris 1884c).

Morris here portrays a large part of labour as antagonistic to work at the same time as he shows the connection between the two. This traditional critique-which position many activities in capitalism within the square of labouring alone-from the standpoint of labour (or rather work) is complemented by Postone's claim that the abstract and self-propelling logic of capital and its labour affects the mode of producing on even deeper levels. Postone's position leads us to the third theme, here represented by Harry Braverman. He wrote a seminal work on the degradation of work under monopoly capitalism in the seventies focusing on, among many things, the extreme division of labour under Fordism (Braverman 1974). With this degradation of the concrete work process, the attractiveness of producing use values disappeared with the impoverishment of skills and knowledge of the labourer-leading to feelings of increased alienation rather than self-fulfilment. Today such work, which can be placed within the category of labour, could be exemplified by the new forms of division of labour through Amazon's Mechanical Turk. 


\subsection{Playing and Labouring}

Labour is dependent on social standards in two ways. The use value produced that carries the exchange value needs to meet social standards, and the exchange of different use values has to be standardised and conducted in the form of equivalents, by the mediation of societal necessary labour time, when it comes to value, and market prices. This abstract logic changes and determines production together with it being conducted within a system that maximises growth and accumulation on a basis of uneven social distribution of the produced value that in turn forces some to labour and leaves other free not to labour. Play is very much the opposite thing. It is spontaneous and improvisational and open for negotiations and renegotiations in a careless, voluntary, but also serious way. It can function as socialisation and a learning process, but also as a critique of social norms.

If we are to believe William Morris it was not simply the work process per se that was degraded under capitalism. The artistic aspect, which was the connecting element between the enjoyment of play and self-fulfilment of work, also disappeared due to the abstract logic of machine-based capitalism.

The degrading processes started as early as with the freeing of the serfs that crowded the medieval craft guilds and led the complete workmen to employ wage labourers. Later on with the introduction of big machinery and "the rise of producing for profit" the workman got "robbed of one pleasure which as long as he is a workman is perhaps his most important one: pleasure in his daily work: he is now only part of a machine, and has indeed little more than his weariness at the end of his day's work." (Morris 1884a) In such a situation it was better, according to Morris, if either all pretence to art was abandoned in the wares produced or that pleasure and interest were added to the necessities of work (Morris 1888).

This picture fits very well with capitalism up until Fordism with its extreme separation of the cognitive content of the production process and the execution of it, but how does it apply to the quite self-sufficient immaterial labour of Maurizio Lazzarato?

\subsubsection{The Frankfurt School and the Situationist International}

Herbert Marcuse connected Marx's theory of capitalism with Freud's pleasure/Eros principle. Alienated labour, domination and accumulation under capitalism constituted a surplusrepression of Eros that exceeded the cultural needs. The demand for surplus labour within capitalism created a surplus repression of the Eros, transforming the reality principle into the performance principle. Marcuse was not against all suppression of Eros and claimed that societally necessary labour for subsistence in society was equal to the necessary suppression of Eros. The reality principle was founded in the basic fact that scarcity ruled in a world too poor for "the satisfaction of human needs without constant restraint, renunciation, delay" (Marcuse 1955, 35). The problem was exploitation under capitalism.

Creatively he proposed turning play and Eros against capitalism, instead of attacking capitalism on part of labour from the site of production-thus attacking capitalism from its periphery or outside rather than from its centre (Marcuse 1955, 175-176)

Marx claimed that material production in the realm of necessity was the foundation for the realm of freedom. A shorter working day would improve the conditions for the development of the realm of freedom and its playful, social, scientific, artistic, dare I say creative, work, characterised by being "damned serious". These two concepts, the realm of necessity and the realm of freedom, were connected to the issue of free time by Marx, who concluded that it was productive in itself under communism when it could not be separated from work. Theodor Adorno made another analysis of the free time of his day. In the essay Free Time (Freizeit) we learn that 'free time' in capitalism was shackled to its opposite: labour.

Indeed the oppositional relation in which it stands imbues free time with certain essential characteristics. What is more, and far more importantly, free time depends on the totality of social conditions, which continues to hold people under its spell. Neither in their work nor in their consciousness do people dispose of genuine freedom over themselves /---/ 
the existence foisted upon people by society is identical neither with people as they are in themselves nor with all that they could be (Adorno 1991, 187).

The character of free time was historically and socially constructed in ways that formed the innermost articulations of human characteristics and this in an age with unparalleled social integration to ascertain anything in humans that is not functionally determined. Free time was neither free nor spare, and it was occupied by labour which one "could designate as heteronomous" (Adorno 1991, 188). This free time was not only productive and connected to the realm of necessity, as Marx had stressed it would be in communism, but invaded by capitalism. The focus of Adorno on heterogeneity makes this a forerunner to the autonomist Marxist notion of the Social Factory. In Marxism concrete work is heterogeneous and carries a onedimensional exchange value. In free time social life itself in its heterogeneity had become concrete work subordinated under abstract labour.

Even when the spell of social conditions was relaxed and the individuals felt themselves acting as free subjects, their will was shaped by the very forces they were trying to escape during their hours of non-labour. He asks himself what the future of free time would be when productivity keeps on rising under persisting conditions of unfreedom.

Free time has already expanded enormously in our day and age. And this expansion should increase still further [...] If one were to try and answer the question without ideological preconceptions, one could not avoid the suspicion that 'free time' is tending toward its own opposite, and is becoming a parody of itself. Thus unfreedom is gradually annexing 'free time', and the majority of unfree people are as unaware of this process as they are of the unfreedom itself (Adorno 1991, 188).

The term 'hobby', in continuation with this, was understood as a paradox by Adorno. The human condition, traditionally seen as the opposite of reification and an "oasis of unmediated life within a completely mediated total system" (Adorno 1991, 187-188), had itself been reified. This was according to Steven Connor's interpretation contrary to real freedom and play:

\begin{abstract}
Adorno's judgement on hobbies and free time will in fact turn out to be another version of the grim verdict supplied in his long, contemptuous condemnation of 'The Culture Industry' of two decades earlier, that 'Laughter is the fraud practised on happiness'. For Adorno, the very distinction between the seriousness of work and the irresponsibility of free time is to be understood as an extension of the remorseless drilling of the bourgeois subject into the required rhythms of modern life (Connor 1998)
\end{abstract}

Happiness and play were manipulated into hobbies and consumption by capitalism. Due to these constraints and manipulations the people were not free in relation to themselves and their potentialities.

The Situationist International (SI) was more practical in its opposition to capitalist society than Adorno, but nonetheless also theoretically interesting. Under the banner of homo ludens they turned Marcuse's theories into practice with the aim of revolutionizing everyday reality with play and gaining their strength from a playful free time. SI attacked the commodifying and reifying processes under the name of the spectacle (this being "a social relationship between people that is mediated by images") (Debord 1967, 12). Slogans such as "Fantasy to the power" and "Demand the impossible" were the anarchic banners of the 1968 rebellion in France. Guy Debord, the author of The Society of Spectacle (1967) both praised collective anarchism for "the merit of representing the refusal of existing conditions from the standpoint of the whole of life" and criticized it for its systematic contempt for method and for ignoring the question of how political ideas should become practice (Debord 1967, 6-64). The rejection of capitalism in the whole of life meant that politics, art and everyday life were seen as a whole. Debord concluded that revolutionary organizations cannot "combat alienation by means of alienated forms of struggle" (Debord 1967, 86, 89). 
Homo Ludens was put up against the consumption society and free time became an important battleground for the new social movements of the time. The contemporary society of spectacle was according to Debord without festivals, even if it presented itself as "essentially made up of many frequently recurring festivities" and as an "enormous positivity where everything that appears is good". Mass-pseudo-festivals made a parody of the gift and dialogue and incited people to excessive spending producing disillusionment with its false promises (Debord 1967, 15, 113) The revolutionary project, in a time when the ruling order by means of the spectacle endlessly discoursed upon itself in an "uninterrupted monologue of self-praise" (Debord 1967, 19) was also: [T] he project of a withering away of the social measurement of time in favor of an individual and collective irreversible time which is playful in character and which encompasses, simultaneously present within it, a variety of autonomous yet effectively federated times - the complete realization, in short, within the medium of time, of that communism which "abolishes everything that exists independently of individuals."(Debord 1967, 116-117).

This is a clear statement on how play was thought to counteract the abstract and selfpropelling logic of value-producing labour. Debord continues the same line of thought in his critique of human geography. Individuals and communities had to create places and events commensurate not just with the appropriation of their labour but with their total history and resulting in a "mobile space of play" that "by virtue of freely chosen variations in the rule of the game" would restore the authentic journey to humanity (Debord 1967, 126). Play for Debord, together with work, had a trans-historical character that was central to social life. The lines between the two were blurry, but rather in the way that play was productive, than work (never labour) playful. The merger of play with work is perceived much as Marx perceived it, but Debord departs from play rather than necessary work. Thus playwork seems an appropriate placing in the field model. Debord stressed play in social life, outside of material production and the realm of necessity, and it is hard to tell in what proportions play pointed to the realm of freedom and sheer playful sociality.

SI was buried in its original form some years after 1968. In 1972 Debord and Raoul Vaneigem broke with each other. Asger Jorn's younger brother, the poet and painter Jörgen Nash later founded the Second Situationist International (SSI), also called the Bauhaus Situationists, and developed a special form of happening, or anti-happening, as an artistic and playful method to radically change society. They wanted to break away from the bourgeois way of using happenings and instead focus on people coming together and creating in cooperation. The new form of activity was called Co-Ritus. Nash held that labour in capitalism stole human lust and imagination to create and stimulated us to use our free time for consumption of mass-produced commodities. This second coming of Situationism contended that emotions came before actions. To Nash the emotions were the ludic element, but the Bauhaus Situationists did not so much develop a new definition of the situation, as a new practice (Thelin 2010, 7, 12; Wikipedia contributors 2013).

The constructed situation was in 1958 understood theoretically by SI as: "A moment of life concretely and deliberately constructed by the collective organization of a unitary ambiance and a game of events" (Situationist International 1958b). Debord wrote that a 'situation' required that one person functioned as a kind of director forcing passive spectators to action (an idea that later was developed by Brazilian dramatist Augusto Boas in his Theatre of the Oppressed) (Debord and SI cf. Tenney 2012,11). In the artistic field SI defined the situation as the "integration of present or past artistic productions into a superior construction of a milieu" and the play-character of the activities in these situations could only be understood properly as movements or processes. There could be "no situationist painting or music", only the use of painting and music as practices (Situationist International 1958b).

Hans-Georg Gadamer holds the same general perspective on play as SI. Vilhauer claims that according to Gadamer you cannot truly participate half-heartedly in play, creating a distance to it by saying it is "just a game". When you do this, you objectify the game by looking at it from the outside, and this takes you out of "true involvement in play" which demands "that the players be completely engaged with other players in the game" (Vilhauer 2010, 36). 
The Fordist capitalism of the sixties and seventies, as well as the mass media of that day, was built on the dichotomy of the artist and the spectator. The question is how relevant the critical mission of the Frankfurt School, SI and Gadamer are today when the mass media have turned into interactive so-called social media?

\subsubsection{Perspectives on the Postmodern Spectacle}

Position one: Adorno was in a sense right in his essay on free time, according to Steven Connor. Connor mentions the nineties as a period when pleasure became "repressively desublimated" into grotesque, compulsive and compulsory pleasures like the "alleged ecstasies of the cyber-body through to the stem delights of fin-de-siècle sadomasochism". He claims that these activities are the subject of "much postmodernist "celebration", but asks himself whether a vital element is not missing in a political and ethical philosophy that has been able to make so little accommodation to the powers of laughter (Connor 1998).

Connor tackles the question of laughter and its co-option by capital and instrumental logics by discussing an essay written by Terry Eagleton in 1983 when the latter, by the analysis of a single line in Yeats's 'Easter 1916', tried to achieve a theoretical knowledge of the mechanisms of pleasure so that it could be used for political objectives. The project became a parody:

The essay is comically poised between convincing its reader of the possibility of subsuming pleasure within cultural politics and acknowledging that such a work of analysis could never be complete or sufficient, would always remain comically, laboriously retarded with respect to its object (Connor 1998).

Hence, laughter and play could not be made instrumental, neither for post-Modernists nor for Marxists. It was fun in itself and stayed in its own square, but at the same time social life lost touch with it.

Position two: Play for Bob Black was the antithesis of labour. Play was always voluntary and what "might otherwise be play is work if it's forced. This is axiomatic". Play was not passive, but at the same time we also needed more time "for sheer sloth and slack than we ever enjoy now". Leisure according to Black was "nonwork for the sake of work", and it was spent "recovering" from labour in a "frenzied but hopeless attempt to forget about work". But something in Situationism's attitude had changed. Labour was even better than leisure, because it was at least paid (Black 1991). The reason that was the foundation for the degradation of leisure time, the social necessity of earning a wage in a market economy, had now some positive effects.

Black's recognition of money and wages as something good is a telling sign of an altered mentality in society, even if we need more time for "sloth and slack", getting money for labour is better than leisure activities in waiting for work. Black confirms the criticism of Connor and Adorno, of leisure time being invaded by capital. The play of today's leisure time is subordinated to labour and under such circumstances it is better for the individual to at least get paid. Labouring dominates this vision totally and playing dissolves as a category.

Position three: Wark concludes that the spectacle of the SI has been replaced by a Spectacle of disintegration today. In Gamer Theory he concludes regarding Guy Debord's treatise on the society of spectacle that the term 'separation' was of key importance to an understanding of the spectacle:

Some argue that the "interactive" quality of contemporary media can, or at least might, rescue it from separation and its audience from passivity. One could with more justice see it the other way around: whatever has replaced the spectacle impoverishes it still further, by requiring of its hapless servants not only that they watch it at their leisure but that they spend their leisure actually producing it. Play becomes work (Wark 2007, Cuts (Endnotes) 111). 
The spectacle of disintegration is explained historically by Wark. If Debord had identified two spectacles in 1967, one the concentrated one of Stalinism and Fascism and the other the diffused one of "endless pictures of models and other pretty things", he identified the integrated spectacle in his Comments on the society of spectacle from 1988. The integrated spectacle had subsumed the earlier two into a new spectacular universe "which molds desire in the form of commodity" and became less and less transparent with its most emblematic concentration in the 'occulted state' that was "occult even to its rulers". Since Debord died in 1994 Wark claims that the spectacle has evolved into an ever more "fecund and feculent form" that "integrates both diffusion and concentration":

\begin{abstract}
The spectacle speaks the language of command. The command of the concentrated spectacle was: OBEY! The command of the diffuse spectacle was: BUY! In the integrated spectacle the commands to OBEY! and BUY! Became interchangeable. Now the command of the disintegrating spectacle is: RECYCLE! Like oceanic amoeba choking on granulated shopping bags, the spectacle can now only go forward by evolving the ability to eat its own shit (Wark 2013, 2-3).
\end{abstract}

Wark's criticism of playbour as unpaid labour, that has an already alienated world as its raw material, comes close to Black's position in portraying social life and play as totally invaded by capital.

Position four: Wark mentions the writings of Pat Kane, who in The Play Ethic describes the consulting business and concludes that once you have got a taste for un-alienated labour "then even the slightest alienation comes to seem like a temporary stay in the prison house" (Pat Kane, The Play Ethic cf. Wark 2007, Cuts (Endnotes) 112). This position gives some hope of play being able to influence labour, rather the other way around, and as such it places itself within labour, but closer to the square of play.

Position five: Pekka Himanen portrays the fourth position in a slightly more independent form, with what he calls the hacker ethic. But he still does not understand it within an anticapitalist framework (with such a framework the position would be positioned in relation to work instead). According to him, we do not have to use new technology to further workcentredness (labour-centredness). This development could be turned around and transformed into the sundayization of Friday. "Hackers optimize time to be able to have more space for playfulness", that is programming that does not have "immediate goals"-work is not always the most important thing (Himanen 2001, 32). This playful motivation is mainly based in human nature and the development of technology and can be placed as a complement to labour (placing it as a dependent position-on labour-in the square of play?).

Position six: Wark focuses on the fifth perspective in a more radical way in his Hacker Manifesto, where the social relations of hacker production, the hacker's interest in the free expansion of the vectors of information as well as freely accessible knowledge and culture (for them to explore and tinker with) are taken into account. He stresses the hackers antagonist interest vis-à-vis the commodity and its production of scarcity (Wark 2004, 381-382) placing it within the category of play.

\title{
3.6.3. Autonomist Marxists on Playing and Labouring in the General Intellect
}

The question for Paolo Virno is if the public character of the intellect, which is a technical requirement of the contemporary production process, can be the basis of a "radically new form of democracy, a public sphere antithetical to the one anchored in the state and its 'monopoly on political decision'"? He highlights two interdependent aspects in his answer: 1) "the general intellect can affirm itself as an autonomous public sphere only if its bond to the production of commodities and wage-labour is rescinded", 2) the subversion of capitalism "can only manifest itself today through the institution of a public sphere outside the state and of a political community that hinges on the general intellect" (Virno 2007, 8). Why is this so?

Virno claims that we have to criticize Marx's position in the Fragment. Marx conceives of the general intellect in a way that "fully coincides with fixed capital", even if it also is an exter- 
nal and collective, public good. Marx also "neglects the way in which the general intellect manifest itself as living labour" (Virno 2007, 5; Virno 2004, 37-38). Post-Fordist production, exemplified by the radically innovated Fiat factory in Melfi, show that the system of machinery does not exhaust the relation between knowledge and production:

\begin{abstract}
In post-fordism, conceptual constellations and logic schemata that cannot be reduced to fixed capital play a decisive role, since they are inseparable from the interaction of a plurality of living subjects. The 'general intellect' comprises formal and informal knowledge, imagination, ethical inclinations, mentalities and 'language games'. In contemporary labour-processes there are thoughts and discourses that function as productive 'machines' in their own right, not needing to take on a mechanical body or even an electronic soul (Virno 2007, 5).
\end{abstract}

This rupture between the general intellect and fixed capital, and the "partial redistribution of the former within living labour" is the cause of small and great disorders. Virno calls this the mass intellectuality to the extent that it is "the depository of cognitive competencies that cannot be objectified in machinery". This is also the prominent form today of the manifest general intellect. He stresses that he speaks of competencies, not the work that is produced by thought, when he talks of the general intellect. "In this regard, mass intellectuality has nothing to do with a new 'labour aristocracy'; it is actually its exact opposite." The general intellect is furthermore conceived as a different real abstraction (with an operational materiality) than the typical ones of modernity: "which embody the principle of equivalence" (Virno 2007, 6).

Whilst money-precisely as the 'universal equivalent'-embodies in its independent existence the commensurability of products, labours and subjects, the general intellect establishes the analytical premises for any kind of praxis. The models of social knowledge do not equate varied labouring activities; rather, they present themselves as 'immediately productive force'. They are not units of measure; they constitute the immeasurable presupposition of heterogeneous operative possibilities (Virno 2007,6).

This is why the homogenising state is not the political body of the general intellect. Social relations are built on communicative actions that are immediately productive and ordered by general cognitive processes and not by the exchange of equivalents. This is not only good, according to Virno. If the public character of the general intellect "does not yield to the realm of a public sphere, of a political space in which the many can tend to common affairs, then it produces terribly effects" (Virno 2004, 40).

Virno here speaks of playful communication within the general intellect and the need for a truly public sphere in this epoch. But post-Fordist capitalism is doing what it can to impose an instrumental logic by commodifying communication. Sharing of linguistic and cognitive habits is at the core of post-Fordist production, but even if this sharing is opposed to the logic of the division of labour, capital still uses segmentation of duties even if they no longer correspond to technical criteria, leaving us with arbitrary hierarchies that are reversible and changeable (flexible). At the same time a personal dependency develops when it is a "person's basic communicative and cognitive habits" that is being subsumed under the logic of capital (Virno 2004, 41).

Maurizio Lazzarato asserted that the old dichotomy of manual and mental labour, or material and immaterial such, had taken on new properties under post-Fordism. Labour could now be defined as the "capacity to activate and manage productive cooperation"; workers were expected to become 'active subjects' in the coordination of the various functions of production" and the "collective learning process" became the heart of productivity, because it was "no longer a matter of finding different ways of composing or organizing already existing job functions, but of looking for new ones" (Lazzarato 1996, 134). Lazzarato's description fits the so-called creative industries well, but his theory also applies to the service and care-industry.

Lazzarato describes how the organization of the cycle of production of immaterial labour is designed. The organisation is not "obviously apparent to the eye" because it is not "defined 
by the four walls of the factory". Immaterial labour is active in the society at large, in what he calls the basin of immaterial labour. Small productive units are organised for specific ad hoc projects and often exists only for the duration of those particular jobs. "Precariousness, hyperexploitation, mobility, and hierarchy are the most obvious characteristics of metropolitan immaterial labor" Lazzarato concludes and points to the self-employed labourer as an "intellectual proletarian" (Lazzarato 1996, 136).

Virno asserts that the contemporary cynicism is connected to the character of the general intellect. The equivalents in Fordist capitalism, despite their constitution of hierarchies and inequalities, ensured a visibility "for the social nexus as well as a simulacrum of universality" that ideologically was the prospect of "unconstrained mutual recognition", "the ideal of egalitarian communication" and "theories of justice". Instead the general intellect "occludes the possibility of a synthesis, fails to provide the unit of measure for equivalence and frustrates all unitary representations". Cynicism is reflecting this and the cynic sets aside aspirations for dialogical communication and relinquishes the search for inter-subjective foundation as a guide for his praxis. This being the "subaltern adaption" to the central role played by the general intellect. In the same manner the growth of the state administration is the example of an "authoritarian concretion of the general intellect" (Virno 2007, 7).

From this we can draw the conclusion that Virno claims that the theory of value has problems, if it is not outright out-dated, in contemporary society. This also means that our society is ready for communism understood as a plurality of individuals interacting in free associations rather than in the state. For the purpose of this investigation we have to dwell a bit on Virno's understanding of living labour's subsumption as activity under capital. He asserts that the classical, threefold division of the human experience in poiesis (labour), praxis (political and communicative action) and intellect (life of the mind) is in crisis. For the multitude who populate the era of the general intellect these spheres are juxtaposed or hybridise. It is this tripartitioning, "the boundaries between pure intellectual activity, political action, and labour", that has dissolved today, but not in the way Arendt theorized it as political action imitating labour in producing the state, party and history. Instead, Virno stresses, as we have seen above, that many of the characteristics of political action has been absorbed by post-Fordist labour (Virno 2004, 4-51). Hence, poiesis has taken on many characteristics of praxis (Virno 2004, 51).

The question is then if the commodification of communication also leads to the commodification of play?

Lazzarato turn the argument around and claims that capital can only "appropriate the forms and products of this cooperation, normalizing and standardizing them", but is not needed in organising and producing them (which is a question of social life) (Lazzarato 1996, 137). This would point to a progressive potential, but I wonder to what degree Lazzarato's argument holds true, and the same goes for the arguments of Hardt and Negri, and Carlo Vercellone below. Is not normalising and standardising becoming increasingly important, as well as an intrusion into the autonomy of the subject's living (and manipulation of it) in cognitive capitalism? On the other hand Yann Moulier Boutang has a point when he stresses that cognitive capitalism is interested in the "valorisation of intelligence and innovation" and not in the "valorisation of information", which according to him was already broadly taking place under mass industrial capitalism. "In order to be productive, cognitive capitalism is condemned to live with the new and unprecedented degrees of freedom" (Moulier Boutang 2011, 41). Such a position seems to imply that capital would have to yield at least a bit to pleasure and play.

Brian Brown points to newer research by Christian Fuchs and Tiziana Terranova who stress that "all of the time and effort devoted to generating digital content on the Internet should be considered a form of immaterial work" even if it is unpaid, and thus "broaden the concept of immaterial labour to include both its waged and unwaged variants" of productive work in post-Fordist capitalism. In so doing they emphasize the "exploitive dimensions" rather than the biopolitical one of Lazzarato. Brown develops an understanding of the biopolitical 
dimensions of unwaged immaterial labour through a case study of Flickr. His research questions focus on how the members of the community look upon their 'labours of love' and what kinds of subjectivities the biopolitics of unwaged immaterial labour produces (Brown 2012, iii, 93).

The majority of his research subjects do not see their activities on Flickr as labour, not even after they had been informed of the commercial character of the project and the business model. Labour was associated with hard toil, and Flickr-activities felt recreational, like a hobby, diversion, escape from there being no "real work" and as an addiction. Several of them experienced enjoyment and felt that they got more than they gave to Flickr; someone stressed the voluntary character. The majority of the few critical remarks stressed that they knew they technically were being exploited, but did not really care about it, not even the ones that had paid for their accounts. Only one of them felt a bit exploited and therefore did not tag the images with a lot of details. He also usually advises people not to geo-tag them (Brown 2012, 135-138). In his analysis Brown stresses that Flickr's early history as Game Neverending that was a "decidedly non-competitive game that prioritized sociality and play over accumulating points or advancing to the next level", and makes the claim that this influenced the ties between the "players" to assume a non-instrumental logic that did not "regard others as tools to advance one's position or competitors to be vanquished, but were based on friendship or companionship". Also contributing to the playful character of the Flickr activities was the public character of the site (Brown 2012, 134).

At the same time the research subjects were well aware of the variety of copyright licenses that they used for marking their content. They were generally happy to share the fruits of their labour, but they wanted to be compensated if someone else could make profit from them, but if no money is to be gained as in relations to non-corporate or not-for-profit status of the collaborators, then they were happy to give away the content for free. There exists an ethos of "non-proprietary sharing", and Brown concludes that Flickr "complicates the traditional notion of the commons in that those working within it regard it as a common resource, at the same time as it is a privately owned domain where profits must be made". He coins the concept of quasi-commons (Brown 2012, 146). The argument could also be turned around: the business model may be weakened by building on voluntary active members with an ethos of free sharing.

Tiziana Terranova conceptualized and tried to understand free labour as early as 2000 , and she defined it as simultaneously voluntarily given and unwaged, enjoyed and exploited (Brown 2012, 93; Terranova 2013). So, once again, how should we explain and problematize that an individual is exploited in his/her labour, which is voluntarily given and enjoyed? How should we understand the subjective side and cultural meaning of it?

Brown answers the criticism about the need for further explanation of the notion of exploitation if the labour going on is not appropriated under threat of force, with the claim that the "threat of social isolation and communicative seclusion may be compulsion enough" to get people 'working'. This is a biopolitical influence of normalized action and behaviour that compels individuals into joining these networks because it is where their peers are (Brown 2012, 97). This statement is supported by Robert Stebbins, who emphasizes that not all "intentionally-productive" and unpaid labour is voluntarily engaged in. He identifies a "domain of nonwork obligation" that contains activities that are "by definition disagreeable". But he also think it is "possible to marry the economic and leisure understandings of volunteering"-which sounds quite like playbour-and stress that volunteering, like serious leisure, combine several instrumental logics regarding career, benefits, ethos and identity issues, which include ten "personal and social rewards" (Stebbins 2013, 34-341). It seems like people in contemporary society have instrumental reasons for everything, according to Stebbins. Even the activity of doing disagreeable and unpaid work because of a perceived obligation is done in a voluntary way because of its rewards. In such a reality it is hard to be unhappy.

Still, the argument that your commenting on Facebook should be seen as forced labour is not entirely convincing. Still we have to further explain the funny and giving activity that the 
users, members, fans, or whatever they are called, feel they are engaged in, besides being exploited. But having said this, it could well be that we are in the beginning of a process of commodification of our social interaction and communication that ultimately will change our view of them from one of play to forced labour. If communication and social interaction increasingly get used for commercial ends, there is a possibility that even the tensions within the quasi-commons will increase, raising questions about privacy but also questions of guaranteed basic income or the abolishment of the wage system all together.

Let us instead approach the relation of playing and labouring from the standpoint of the progressive potentials in contemporary society, remembering Lazzarato's claim that capital is losing its grip over the immediate production process. Can play and work exert their autonomy and free themselves from labour?

Michael Hardt and Antonio Negri develop a political strategy in their Commonwealth. In the preface we read that both socialism and capitalism were "regimes of property" that excluded the common(s). According to them the perspective of the common cuts "diagonally across these false alternatives-neither private nor public, neither capitalist nor socialistand opens a new space for politics" (Hardt and Negri 2009, ix). The contemporary forms of capitalist production is dominated by sectors that involve information, code, knowledge, images, and affects and their producers "required a high degree of freedom as well as open access to the common", especially in the forms of "communication networks, information banks, and cultural circuits " (Hardt and Negri 2009, x). The transition to a "social and economic order grounded in the common" was already in progress, and contemporary capitalism's addressing its own needs created the bases for emancipation (Hardt and Negri 2009, $\mathrm{x})$.

Virno also sees the possibility of a radically new democracy not anchored in the state, a vision that Carlo Vercellone instead calls communism. The latter claims that Marx's idea of the general intellect designates a "radical change of the subsumption of labour to capital and indicates a third stage of the division of labour" surpassing the division of industrial capitalism and making possible the "direct transition to communism" (Vercellone 2007, 15).

The change of the technical composition of capital and of the social labour process in contemporary society is a qualitative change that overturns the subordination of living labour under "dead knowledge incorporated in fixed capital". Vercellone characterizes it as "the tendential fall of the capital's control of the division of labour" (Vercellone 2007, 1 ). When the productive value of intellectual and scientific labour becomes the dominant productive force, knowledge re-socialises everything, and this presents a problem for capital (ultimately ending capitalism). According to him, the cognitive labourer, still dependent on wages (and thus not voluntarily engaged) has his/her autonomy in the labour process, like the craftsmen under formal subsumption, but this also leads to a more brutal capitalism (the use of extra economic methods) or a focus on financial ways of getting hold on surplus value/labour (Vercellone 2007, 2-22, 31-32).

It is possible to criticise Vercellone for downplaying the class aspect in this argument, and also to pose the question: what were the real consequences of the growth of the middle and managerial classes in the $20^{\text {th }}$ century? Could it not be that the privileged strata of today also get "bought" or "corrupted" by capital?

To me the alternative of peer producing (PP) seems more promising. It is an emerging mode of production that is based in the form of commons, built on voluntary, potentially global, yet quite horizontally organised, co-operation online and the free access to the (digital) material under copy-left licenses. ${ }^{8}$ According to Jacob Rigi the "logic of equivalents" is absent in peer production and he does not see any gift economy going on (by focusing on formal rules and using a rather limited view of that phenomenon) within it (Rigi 2013, 397-398, 400,

\footnotetext{
${ }^{8}$ Copy Left is a play with words. In contrast to traditional uses of copyright law, the copy-left licenses use the copy-right to shortcircuit copy-right, and open up the distribution and use of the licensed content. You are free to distribute, use, tinker with and make derivative works of the licensed material as long as the derivative works are distributed under the same "free" or "open" license.
} 
403). More important for our purposes is that PP "negates alienation by transcending the division of labour and replacing labour with joyful and creative productive activity". The new mode of production is not identical to Hardt and Negri's the common which is "ubiquitously present everywhere". PP is instead emerging as "islands within the capitalist social formation" and its generalisation "will require a social revolution" (Rigi 2013, 4). Such a revolution would primarily realize a generalised workplay or playwork.

\section{References}

Adorno, Theodor W. 1991. The Culture Industry: Selected Essays On Mass Culture, edited by J. M. Bernstein, London: Routledge

Anchor, Robert. 1978. History and Play: Johan Huizinga and his Critics. History and Theory 17 (1): 63-93.

Arendt, Hannah. 1998. Människans Villkor: Vita Activa. Göteborg: Daidalos.

Asplund, Johan. 1987. Det Sociala Livets Elementära Former. Göteborg: Korpen.

Bachtin, Mikhail. 1988. Det Dialogiska Ordet. Gråbo: Anthropos.

Bachtin, Mikhail. 2007. Rabelais Och Skrattets Historia : François Rabelais' Verk Och Den Folkliga Kulturen Under Medeltiden Och Renässansen. Gråbo: Anthropos.

Barbrook, Richard. 2005. High-Tech Gift Economy. First Monday (Special Issue Update Nr. 3). http://firstmonday.org/article/viewArticle/1517/1432

Barbrook, Richard and Andy Cameron. 1996. The Californian Ideology. Science As Culture 6 Part 1 (26): 44-72.

Bataille, Georges. 1991. Den Fördömda Delen: Samt Begreppet Utgift, Stockholm; Stehag: B. Östlings Bokförl. Symposion.

Bataille, Georges, Waldberg, Isabelle, and lain White. 1995. Ecyclopaedia Da Costa. In Encyclopaedia Acephalica. London: Atlas Press.

Black, Bob. 1991. The Abolition of Work. Accessed November 18, 2013. http://inspiracy.com/Black/Abolition/Abolitionofwork.html

Black, Bob. The Abolition Of Work. Accessed January 6, 2014. http://Deoxy.Org/Endwork.htm

Borges, J.L. 1998. Biblioteket I Babel: En Antologi Sammanställd Ur Novellsamlingarna Ficciones Och El Aleph. Stockholm: Bonnier.

Bourdieu, Pierre. 2000. Konstens Regler: Det Litterära Fältets Uppkomst Och Struktur. Stockholm/Stehag: Brutus Östlings Bokförlag Symposion.

Braverman, Harry. 1974. Labor And Monopoly Capital: The Degradation Of Work In The Twentieth Century. New York: Monthly Review Press.

Brotchie, Alastair. 1995. Introduction. In: Encyclopaedia Acephalica. London: Atlas Press.

Brown, Brian. 2012. Will Work For Free: Examining The Biopolitics Of Unwaged Immaterial Labour. University Of Western Ontario-Electronic Thesis And Dissertation Repository. http://ir.lib.uwo.ca/Etd/620

Caillois, Roger. 2001. Man, Play and Games. Urbana, Illinois: University Of Illinois Press.

Carver, Terrell. 1987. A Marx Dictionary. Oxford: Polity Press.

Connor, Steven. 1998. Art, Criticism and Laughter: Terry Eagleton On Aesthetics. In Aesthetics, Gender Nation Organised, by The Raymond Williams Trust. Oxford.

Csíkszentmihályi, Mihaly. 2000. Beyond Boredom and Anxiety. San Francisco, Chichester: JosseyBass Publishers; Wiley.

Csíkszentmihályi, Mihaly. 1996. Creativity: Flow And The Psychology Of Discovery And Invention. New York: Harper Collins.

Davenport, Thomas, and John Beck. 2001. The Attention Economy: Understanding The New Currency Of Business, Boston, Mass.: Harvard Business School Press.

Debord, Guy. 1990. Comments On The Society Of The Spectacle. London: Verso.

Debord, Guy. 1967. The Society Of Spectacle. New York: Zone Books.

Dyer-Witheford, Nick, and Greig de Peuter. 2009. Games Of Empire: Global Capitalism And Video Games. Minneapolis: University Of Minnesota Press.

Fornäs, Johan. 2013. Capitalism: A Companion To Marx's Economy Critique. London: Routledge.

Fourier, Charles. Attractive Labour. Charles Fourier, Selections From His Writings. Accessed November 9, 2013. https://www.marxists.org/reference/archive/fourier/works/ch26.htm

Fourier, Charles. On The Role Of The Passions. Charles Fourier: Selections From His Writings. Accessed November 9, 2013a. http://www.marxists.org/reference/archive/fourier/works/ch01.htm 
Fourier, Charles. The Exchange. Selection From Charles Fourier. Accessed November 9, 2013c. http://www.marxists.org/reference/archive/fourier/works/ch24.htm

Freud, Sigmund. 1974. Beyond The Pleasure Principle. London: The Hogarth Press and The Institute Of Psycho-Analysis.

Fromm, Eric. 1988. Greatness and Limitations of Freud's Thought. New York: Meridian.

Fuchs, Christian. 2012. Dallas Smythe Today-The Audience Commodity, the Digital Labour Debate, Marxist Labour Debate, Marxist Political Economy and Critical Theory. Prolegomena to a Digital Labour Theory of Value. tripleC_-Cognition, Communication, Co-operation: Open Access Journal for a Global Sustainable Information Society 10 (2): 692-740.

Fuchs, Christian. 2014. Digital Labour and Karl Marx. New York: Routledge

Fuchs, Christian. 2013. Social Media and Capitalism. In Producing The Internet: Critical Perspectives Of Social Media, edited by T. Ollsen. Göteborg: Nordicom.

Fuchs, Christian, and Sebastian Sevignani. 2013. What Is Digital Labour? What Is Digital Work? What's Their Difference? And why do these Questions Matter for Understanding Social Media? tripleC-Cognition, Communication, Co-operation: Open Access Journal for a Global Sustainable Information Society 11 (2): 237-293.

Gadamer, Hans-Georg. 1975. Truth and Method. London: Sheed and Ward.

Habermas, Jürgen. 1987. The Theory of Communicative Action: The Critique of Functionalist Reason. Cambridge: Polity Press.

Hägglund, Kristopher. 1989. Lekteorier. Solna: Esselte Studium.

Hardt, Michael, and Antonio Negri. 2009. Commonwealth. Cambridge, Mass: Belknap Press Of Harvard University Press.

Heidegger, Martin. 1991. Nietzsche. New York: HarperCollins.

Hein, Hilde. 1968. Play As an Aesthetic Concept. The Journal Of Aesthetics And Art Criticism 27 (1): 67-71.

Himanen, Pekka. 2001. The Hacker Ethic, and the Spirit of the Information Age. New York: Random House.

Hollier, Denis. 1989. Against Architecture: The Writings Of Georges Bataille. Cambridge, Mass: MIT Press.

Horkheimer, Max. 1972. Critical Theory: Selected Essays. New York: Continuum.

Huizinga, Johan. 1955. Homo Ludens: A Study of the Play-Element in Culture. Boston: Beacon Press.

Hyde, Lewis. 2012. The Gift. Canongate.

Illich, Ivan. 1980. Shadow-Work. Philosophica 26 (2): 7-46.

Juul, Jesper. 2005. Half-Real: Video Games Between Real Rules and Fictional Worlds. Cambridge, MA: MIT Press.

Karlsson, Jan Ch. 2013. Begreppet arbete: definitioner, ideologier och sociala former. Lund: Arkiv.

Kline, Stephen, Dyer-Witheford, Nick, and Greig de Peuter. 2003. Digital Play: The Interaction Of Technology, Culture and Marketing. Montreal: Mcgill-Queen's University Press.

Larrain, J. 1991. Base and Superstructure. In A Dictionary Of Marxist Thought, $2^{\text {nd }}$ edn, edited by T.

Bottomore. Malden: Blackwell Publishing

Latour, Bruno, and Steve Woolgar. 1986. Laboratory Life: The Construction of Scientific Facts. Princeton, New Jersey: Princeton University Press.

Lazzarato, Maurizio. 1996. Immaterial Labor. In Radical Thought in Italy: A Potential Politics. Minneapolis: University of Minnesota Press.

Locke, John. 1988. Two Treatises of Government, edited by P. Laslett. Cambridge: Cambridge Univ. Press.

Malinowski, Bronislaw. 1922. Argonauts of the Western Pacific: An Account of Native Enterprise and Adventure in the Archipelagoes of Melanesian New Guinea. London: Routledge and Kegan Paul.

Marazzi, Christian. 2011. Capital and Affects: The Politics of the Language Economy. Los Angeles, CA: Semiotext(E).

Marazzi, Christian. 2008. Capital and Language: From The New Economy to the War Economy. Los Angeles, CA: Semiotext(E).

Marcuse, Herbert. 1955. Eros and Civilization. Boston: Beacon Press.

Marx, Karl. 1867. Capital: A Critique of Political Economy. London: Lawrence and Wishart.

Marx, Karl. 1909. Capital: A Critique of Political Economy. 1909th edn. Chicago: Charles H. Kerr and Company.

Marx, Karl. 1973. Grundrisse : Foundations of the Critique of Political Economy: (Rough Draft). London: Penguin. 
Marx, Karl. 1857. Introduction to a Contribution to the Critique of Political Economy. Economic Manuscripts: Appendix I: Production, Consumption, Distribution, Exchange. Accessed November 28, 2013. https://www.marxists.org/archive/marx/works/1859/critique-pol-economy/appx1.htm

Marx, Karl. 1969. Kapitalet: kritik av den politiska ekonomin. Bok 1. Kapitalets produktionsprocess. Lund: A-Z.

Marx, Karl. 1865. Value, Price and Profit. Economic Manuscripts: Value, Price And Profit, Karl Marx 1865. Accessed November 11, 2013. http://www.marxists.org/archive/marx/works/1865/valueprice-profit/

Marx, Karl, and Friedrich Engels. 1972. Karl Marx/Friedrich Engels: Brev I Urval. Stockholm: Gidlunds Förlag.

Marx, Karl, and Friedrich Engels. 1991. Marx Engels: Selected Works. London: Lawrence and Wishart.

Marx, Karl, and Friedrich Engels. 1998. The German Ideology: Including Theses on Feuerbach and Introduction to the Critique of Political Economy. Amherst, NY: Prometheus Books.

Mauss, Marcel. 1972. Gåvan. Uppsala: Argos.

Miller, Stephen. 1973. Ends, Means, and Galumphing: Some Leitmotifs of Play. American Anthropologist 75 (1): 87-98.

Minnema, Lourens. 1998. Play and (Post)Modern Culture: An Essay on Changes in the Scientific Interest in the Phenomenon of Play. Cultural Dynamics 10 (21).

Mitcheson, K. 2013. Nietzsche's Concept of Life. Journal of the University of York Philosophy Society. http://dialecticonline.wordpress.com/issue-04/nietzsche's-concept-of-life/

Morris, William. 1888. Art and its Producers. William Morris-Art And Its Producers. Accessed January 7, 2014. https://www.marxists.org/archive/morris/works/1888/producer.htm

Morris, William. 1884a. Art and Labour. William Morris-Art And Labour. Accessed January 7, 2014. https://www.marxists.org/archive/morris/works/1884/art-lab.htm

Morris, William. 1884b. Art and Socialism. William Morris-Art And Socialism. Accessed January, 7 2014. https://www.marxists.org/archive/morris/works/1884/as/as.htm

Morris, William. 1884c. Useful Work Versus Useless Labour. William Morris-Useful Work Versus Useless Toil. Accessed November 9, 2013. https://www.marxists.org/archive/morris/works/1884/useful.htm

Moulier-Boutang, Yan. 2011. Cognitive Capitalism. Cambridge: Polity.

Nagel, M.E. 1996. Philosophical Perspectives on Play from Homer to Hegel. Amherst: University Of Massachusetts Amherst, Department Of Philosophy.

Nietzsche, Friedrich. 1990. Beyond Good and Evil. London: Penguin Books.

Pellegrini, Anthony D. 2009. The Role of Play in Human Development. New York: Oxford University Press.

Piaget, Jean. 1962. Play, Dreams and Imitation in Childhood, New York: Routledge and Kegan Paul.

Postone, Moishe. 1993. Time, Labor, and Social Domination: A Reinterpretation of Marx's Critical Theory. Cambridge: Cambridge University Press.

Reich, Wilhelm. 1990. Character Analysis. New York: The Noonday Press.

Reich, Wilhelm. 2012. Sex-Pol : Essays 1929-1934, edited by L. Baxandall. London: Verso.

Rigi, Jakob. 2013. Peer Production and Marxian Communism: Contours of a New Emerging Mode of Production. Capital And Class 37 (3): 397-416.

Schwartzman, Helen B. 1978. Transformations : The Anthropology of Children's Play. New York: Plenum Press.

Situationist International. 1958a. Contribution to a Situationist Definition of Play. Situationist International Online. Accessed November 12, 2013. http://www.cddc.vt.edu/sionline/si/play.html

Situationist International, 1958b. Definitions. Situationist International Online. Accessed November 12, 2013. http://www.cddc.vt.edu/sionline/si/definitions.html

Smith, Douglas. 2005. Giving The Game Away: Play and Exchange in Situationism and Structuralism. Modern and Contemporary France 13 (4): 421-434.

Smythe, D. W. 1977. Communications: Blindspot of Western Marxism. Canadian Journal Of Poliitical And Social Theory 1 (3): 1-27.

Smythe, D. W. 1984. New Directions for Critical Communications Research. Media, Culture and Society 6 (3): 205-217.

Stebbins, Robert. 1992. Amateurs, Professionals and Serious Leisure. Montreal, Quebec, Canada: McGill-Queen's University Press. 
Stebbins, Robert. 2013. Unpaid Work of Love: Defining the Work-Leisure Axis of Volunteering. Leisure Studies 32 (3): 339-345.

Stebbins, Robert. 2009. Leisure and its Relationship to Library and Information Science: Bridging the Gap. Library Trends 57 (4): 618-631.

Tenney, Tom. 2012. (I Will) Never Work: A Critique of the Sitautionists' Appropriation of Johan Huizinga's Theory of Play. Academia.edu. Accessed November 14, 2013. http://www.academia.edu/1564331/It_Will_Never_Work_A critique of the Situationists appropri ation of Johan Huizingas theory of play

Terranova, Tiziana. 2013. Free Labor. In Digital Labor: The Internet as Playground and Factory, edited by Trebor Scholz, 33-57. New York: Routledge.

Thelin, C. 2010. Fantasin till makten! Om 60-talets situationistiska happenings och 00-talets aktions-

konst. Bachelor Thesis. Uppsala University. Department Of Art History.

Torvalds, Linus. 2001. What Makes Hackers Tick? a.k.a. Linus's Law. In the Hacker Ethic and the Spirit of the Information Age. New York: Random House Trade Paperbacks.

Vercellone, Carlo. 2007. From Formal Subsumption to General Intellect: Elements for a Marxist Reading of the Thesis of Cognitive Capitalism. Historical Materialism 15 (1): 13-36.

Vilhauer, M. 2010. Gadamer's Ethics of Play. Lanham: Rowman and Littlefield Publishing Group.

Virno, Paolo. 2004. A Grammar Of The Multitude. Cambridge, MA: MIT Press.

Virno, Paolo. 2007. General Intellect. Historical Materialism 15 (1): 3-8.

Vološlnov, Valentin. 2012. Freudianism: A Marxist Critique. London: Verso.

Vygotskij, Lev Semonovich. 1987. The Collected Works of L.S. Vygotsky: Volume 5, Child Psychology. New York: Springer.

Wark, Mckenzie. 2004. A Hacker Manifesto. Cambridge, MA: Harvard University Press.

Wark, Mckenzie. 2007. Gamer Theory. Cambridge, MA: Harvard University Press.

Wark, Mckenzie. 2013. The Spectacle of Disintegration. New York: Verso Books.

Wikipedia Contributors. Activity Theory. Wikipedia. Accessed December 18, 2013. http://en.wikipedia.org/wiki/Activity theory

Wikipedia Contributors. 2013a. Bob Black. Wikipedia. Accessed November 18, 2013. http://en.wikipedia.org/wiki/Bob_Black

Wikipedia Contributors. Recapitulation Theory. Wikipedia. Accessed December 18, 2013. http://en.wikipedia.org/wiki/Recapitulation theory

Wikipedia Contributors. 2013. Second Situationist International. Wikipedia. Accessed November 14, 2013. http://en.wikipedia.org/wiki/Second Situationist International

Wikipedia Contributors. 2013b. The Abolition of Work. Wikipedia. Accessed November 18, 2013. http://en.wikipedia.org/wiki/The_Abolition_of_Work

Wittgenstein, Ludwig. 1967. Philosophical Investigations. Oxford: Basil Blackwell.

\section{About the Author}

\section{Arwid Lund}

PhD-student in Library- and Information Science at the Department of ALM (Archival, Library \& Information, Museum \& Cultural Heritage Studies), University of Uppsala, Sweden. He is the author of three books in Swedish, and has worked as a librarian with digital publishing and digital repositories. He was an activist in social movements during the 1990's, and the first years of the new millennium. You can contact Arwid Lund at arwid.lund@abm.uu.se. 